A major purpose of the Technical Information Centur is to provide the broadest dissemination possibie of information contained in DOE's Research and Development Reports to business, industry, the academic community, and federal, state and local governments.

Although a small portion of this report is not reproducible, it is being made available to expedite the availability of information on the research discussed herein. 


\section{Diselaimek}

This report was preparat as an account of wort sponsored by an agenry of the Uniled States Government. Neither the United States Government nor any agency thereof, nor any of their employees, makes any warranty, express or implied, or assumes any legal liability or responsibility for the accuracy. completeness, or usefulness of any information, apparatus, product, or process disclosed, or represents that its use would not infringe privately owned rights. Reference herein to any specific commercial product, process, or service by trade aame, irademark. manufacturer, or otherwise does not necessarily constitute or imply its endorsenent, recommendation, or favoring by the United States Governfient or any agency thereof. The views and opiaions of authors expressed herein do sio! secessarily state or reflect those of the United States Gor trnmesi or ar.y agency thereof.

PHYSICS DIVISION

PROGRESS REPORT

for Period Ending September- 30, 1984

\section{J. B. Ball Director}

S. Datz Section Head

E. E. Gross Section Head

C. M. Jones Section Head

J. B. McGrory Sectil.. Head

R. L. Robinson Section Head

Edited by: A. B. Livingston

Date Published: January 1985

OAK R:OGE NATIONAL LABORATORY

Oak Ridge, Tennessee 37831

operated by

MARTIK MARIETTA ENERGY SYSTEMS, INC.

for the

U. S. DEPARTMENT OF ENERGY

under Contract No. DE-ACO5-840R21400

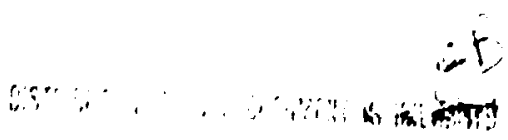


Reports previously issued in this series are as follows:

\begin{tabular}{|c|c|}
\hline ORTL-2718 & Pertod Ending March 10, 1959 \\
\hline OPAR-2910 & Pertod Ending February 10, 1960 \\
\hline ORUL-3085 & Period Ending February 10, 1961 \\
\hline ORAL-3268 & Period Ending January 31, 1962 \\
\hline ORtel-3425 & Period Ending May 21, 1963 \\
\hline ORNL-3582 & Period Ending January 31, 1964 \\
\hline ORNL-3778 & Period Ending Decmber 13, 1964 \\
\hline ORALL-j924 & Period Ending December 31, 1965 \\
\hline ORAL-4082 & Period Enixing Decenber 31, 1966 \\
\hline ORNL -4230 & Period Ending Deceiber 31, 1967 \\
\hline ORNL-4395 & Period Ending Decenber 31, 1968 \\
\hline ORNL-4513 & Period Ending Decenber 31, 1969 \\
\hline ORNL-4659 & Period Ending Deceuber 31, 1970 \\
\hline ORNL-4743 & Period Ending Decenber 31, 1971 \\
\hline ORNL-4844 & Period Ending Deceuber 31, 1972 \\
\hline ORKL -4937 & Period Ending Decenter 31, 1973 \\
\hline ORRL-5C25 & Perfor Ending December 31, 1974 \\
\hline ORNL-5137 & Period Ending Decenber 31, 1975 \\
\hline OAML-5306 & Perfod Ending June 30, 1977 \\
\hline ORNI.-5498 & Perind Ending December 31, 1978 \\
\hline ORKL - 5787 & Period Ending June 30, 1981 \\
\hline ORNL -6004 & Period Ending September 30, 1983 \\
\hline
\end{tabular}




\section{Joseph Lee Fowler}

Joe Fowler, director of the Mysics Division from 1957 to 1973, died on June 3, 1984.

Moving from Los Alamos to Oak Ridge in 1950 , Joe served as director of the high voltage laboratory from 1951 to 1953 and associate director of the Physics Division from 1954 to 1957 before becoming division director. In 1978, he retired from 0ak Ridge Mational Laboratory but continued his appointment as professor of physics at the University of Tennessee. Joe's broad understanding of physics and his skill as an dainistrator served as an inspiration to a'l of those privileged :0 work with him. 


\section{SPECIAL DEDICATION}

This issue of the Physics Division Progress Report is dedicated to the memory of two of our colleagues, Janes L. C. Ford, Jr., and Clyde B. Fulmer. who died during this past year.

Jim Ford joined the Physics Division in 1962. He played a lead role in nui'ear structure studies with Coulonb excitation, inelastic scattering, and transfer reactions. He was especially active in the design and implementation of new experimental devices such as the time-of-flight facility, the gas-jet target, and a series of position sensitive focal-plane detectors. since June 1983, Jic had served as Lfatson Officer to the Holifield Facility Users Group.

Clyde Fulmer joined the Electronuclear Oivision (later to merge with the Physics Division) in 1958. Best known for his extensive studies of the elastic scatterín process and its interpretation via the muclear optfical model, he also ade significant contributions to measurement prograns for accelerator shielding and residual radiation problems. For a number of years, Clyde served as the Division Safety Officer and as the Radiation Control cfficer.

Both Clyde and Jim brought to the Division not mly an ethic of hard work and dedication, but a special love of physics and an intense interest in their work. Between thew, their studies resulted in more than two hundred published papers. Above all, their work was marred by an outstanding spirit of cooperation and good humor. They will be wissed both as valued colleagues and as good friends. 


\section{CONTEIS}

\section{MOLIFID REAT IOW RESEMCH FACILITY}

Overview - R. L. Robinson, J. A. Martin, C. M. Jones

\section{ACCELERATOR OPERATIOUS AND OEVELOPEET}

Operations - 6. D. Alton, J. A. Benjain, J. A. Biggerstaff, M. R. Dinehart,

D. T. Dowl ing, H. D. Hackler, C. L. Haley, D. L. Haynes, D. E. Hoglund,

E. D. Hudson, C. A. Irizarry, C. M. Jones, M. L. Jones, R. C. Juras, S. $N$.

Lane, C. T. LeCroy, R. S. Lord, C. A. Ludemann, J. E. Mann, C. A. Maples,

R. L. Mcpherson, P. Meszaros, G. D. Mills, S. M. Mosko, S. M. Murray,

E. G. Richardson, P. T. Singley, C. L. V:zr, M. F. Ziegler

Tanden Accelerator - G. D. Alton, J. A. Benjanin, J. A. Biggerstaff, D. L. Haynes, D. E. Moglund, C. M. Jones, R. C. Juras, J. E. Mann, E. G.

Richardson, N. F. Ziegler ..................................................

ORIC Accelerator - D. T. Dowling, F. D. Hudson, S. ․ Lane, R. S. Lord, C. A.

Ludemann, J. A. Martin, S. W. Mosko ........................................ S

Negative Ion Source Development - G. D. Alton, T. J. Kvale, D. H. Olive,

D. J. Pegg .............................................................. 9

Beam Bu..cher Development - J. A. Martín, M. T. Milner, M. F. Ziegler .................. 14

Efforts to Improve Tandem Accelerator Tube Performance - P. H. Stelson,

J. R. Raatz, R. D. Rathmeli, N. F. Ziegler

\section{FACILITY OPERATIOUS AMD OEVELOPMENT}

Experimental Apparatus - R. L. Auble, E. E. Gross, M. L. Halbert, J. H. Johnson, P. D. Mfller, N. R. Johnson, F. E. Obenshain, D. Shapira, E. H. Spejewsk1,

H. J. Kin ............................................................. 16

Computer Systems - J. A. Biggerstaff, W. H. Atkins, J. R. Beene, J. W. Mcconnell,

J. B. MeGrory, W. T. Milner, R. 0 . Sayer, C. N. Thomas $\ldots \ldots \ldots \ldots \ldots \ldots \ldots \ldots \ldots \ldots \ldots \ldots$

Factlities - R. L. Robinson, R. L. Auble, F. E. Bertrand, M. L. Halbert, D. Shapira,

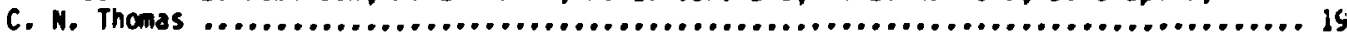

Users Support - R. L. Auble, J. A. Biggerstaff, R. P. Cumby, J. W. Johnson, R. W.

Miles, C. A. Reed, R. L. Robinson, C. N. Thomas, H. T. Milner ................... 20

Joint Institute for Heavy Ion Research - R. L. Robinson, L. L. Riedinger,

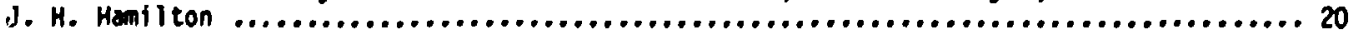

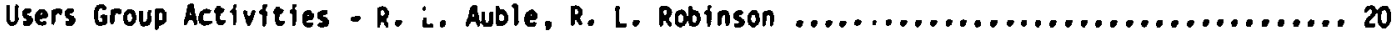

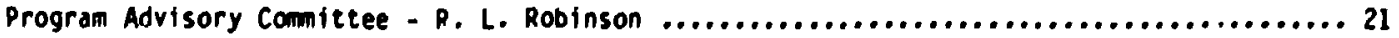




\section{EXPERIICTIM MCLENR MTSICS}

Optical Mode! Malysis of 200-400 MeV Proton Elajtic Scattering on 208pb -

E. E. Gross, F. E. Bertrand, D. "ren, J. Lisintti .................................. 22

Inelastic Excitation of Low-Lying Staces in 20dpb by 334-MeV Protions -

F. E. Bertrand, E. E. Gross, D. J. Horen, T. P. Sjoreen, J. Lisantti. J. T. Tinsley, D. K. Mcoanie?s, L. H. Swenson, T. Carey, K. Jones, J. $B$.

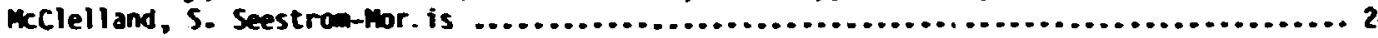

Comparison Between Experimental and Calculated Cross Sections and Asymantries for Spin Excitations in $40048 \mathrm{Ca}$ - D. J. Horen, F. E. Bertrand, E. E. Gross, T. P. Sjoreen, D. X. McDaniels, J. R. Tinsley, J. Lisantti, L. M. Smensen,

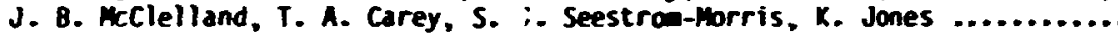

1 High Resolution Study of Giant Resonances in 2040 Using Inelastic Scattering of 334-meV Protons - F. E. Be.trand, E. E. Gross, D. J. Horen, T. P. Sjoreen, J. Lisantti, J. T. Tinsley, D. K. Acoaniels, L. W. Smenson, T. Carey, K. Jones, J. B. KClell and, S. Seestrom-morris, R.0. Sayer

Excitation of Giant Multipole Resonances in sd-Shell Muclei via s00-MeV Proton Inelastic Scattering - B. L. Burks, F. E. Bertrand, R. L. Auble, E. C. Gross, D. J. Horen, R. O. Sayer, L. H. Swenson, D. K. Kcoaniels, J. Lisantti, J. R. Tinsley, r. W. Jones, J. B. HeClel Iand, S. Seestrom-Morr is

Electromagnetic Decay of Giant Resonances - J. R. Beene, F. E. Bertrans, M. L. Halbert, D. C. Fiensley, R. L. Auble, D. J. Horen, R. L. Robinson, T. P. Sjoreen, R. 0. Sayer ..... 32

Meutron Decay of Giant Resonances - J. R. Beene, F. E. Berti and, M. L. Halbert,

O. C. Hensley, R. L. Auble, D. J. Horen, R. L. Robinson, T. P. Sjoreen, R. 0. Sayer ..... 34

Fission Dacay of Giant Resonances in $233 \mathrm{~J}$ - J. R. Beene, C. E. Beais, F. E. Bertrand, R. L. Auble, 8. L. Burks, E. E. Gross, D. J. Hor 2n, I. Y. Lee, R. O. Sayer ............ 35

Elastic and Inelastic Proton Scactering from 160 at $200 \mathrm{MeV}$ - C. W. Glover ...................

Measurements of Gamow-Teller Strength Distributiors in Mass 13 and 15 -

D. J. Horen, C. D. Goodman, R. C. Byrd, I. J. Van Heerden, T. A. Carey,

J. S. Larsen, C. Gaarde, J. Rapaport, T. P. Welch, E. Sugarbaker, T. N.

Taddeucci

The ${ }^{19} F(p, n)$ I Ne and $39 x(p, n)^{3} C_{d}$ Reactions at Intermediate Energies and querching of the Ganow-Teller Strength - D. J. Horen, J. Rapaport, C. Gadrde, J. Larsen, C. Goulding, C. D. Goormen, C. Foster, T. Mastersun, E. Sugarbaker, T. N. Tadueucci

The $5 I V(p, n)^{5 I C r}$ Reaction at $E_{p}=I 60$ meV - D. Horen, J. Rapaport, R. Alarcon, B, A. Brown. C. Gaarde, J. Larson, C. D. Goodman, C. C. Foster, T. Masterson, E. Sugarbaker, T. N. Taddeucci ......................................................44

Measurement of the Transverse Spin Transfer Coefficient $D_{\text {NM }}\left(0^{\circ}\right)$ for $\left(\vec{D}, n^{*}\right)$ Reactions at $160 \mathrm{MeV}$ - D. J. Horen, T. N. Taddeucci, T. A. Carey, C. Gaarde, J. Larson, C. D. Coodinan, T. Mastersor., J. Rapaport, T. P. Welch, E. Sugarbaker ..................... 46

$36 \mathrm{~S}(n, y)^{37} \mathrm{~S}$ Reaction with Thermal Heutrons and Decay of ${ }^{37} \mathrm{~S}$ to Levels in ${ }^{37} \mathrm{Cl}$. S. Raman, H. Ratynski, E. T. Jurney, M. E. Bunker, J. H. Starner

${ }^{36} S(d, p)^{37} S$ and $34,365(d, 3 \text { He })^{33.35 p}$ Reactions - C. E. Thorn, J. W. Olness, E. K. Harburton, S. Raman

Decay Scheme of $1165 n$ from $\left(n, n^{\prime} y\right)$ and $(n, y)$ Results - 2. Gácsi, J. L. Weil, J. Sa,

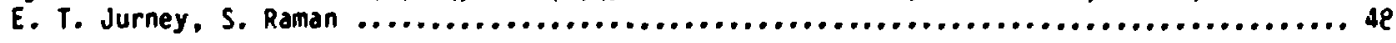

Observation of Extremely Low s-Wave Strength in the Reaction $136 x_{e}+n-B$. Fogelberg,

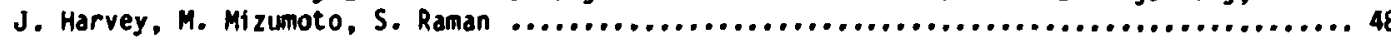

Electric Dipole Transitions from Meutron Capture in 167 Er Resonances - S. Kahane, S. Paman, G. G. Slaughter, C. Coceva, M. Stefanon 
The (3te,t) Reaction at 197 meV on 12C, 24h, 285i and taca - S. L. Tabor, 6. Neuschaefer, J. A. Carr, F. Petrovich, C. C. Chang, R. Guternan, M. T. Collins, D. L. Friesel, C. Glover, S. Y. van der Merf, S. Raman ..........................

Therial Meutron Capture Ga Rays from Sulfur Isotopes: Experiment and Theory S. Rzan, R. F. Carlton, J. C. Mel is, E. T. Jurney, J. E. Lymn .......................... 50

The s- and d-Wave Scattering of Meutrons on tha - J. A. Harvey, R. F. Carlton, C. H. Johnson, Boris Castel ....................................................... 50

Coupled-Channel Optical model for 0-0.5 MeV Meutrons in 69mi - R. R. Mackellar.

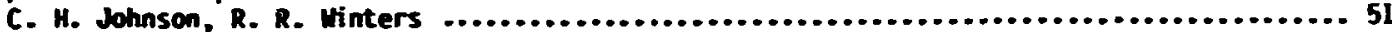

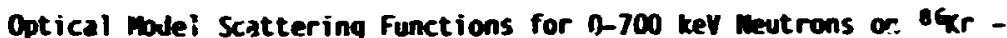
R. F. Carlton, J. A. Harvey, C. H. Johnson ....................................... 52

Measurenents of the heutron Transaission and Capture Cross Sections in 204pb D. J. Horen, R. L. Mackl in, J. A. Harvey, M. H. Hill ................................ 54

Spherical Optical Model Description of $204 p+n$ Scattering Mear Threshold -

D. J. Horen, C. H. Johnson ........................................................ 54

Coupled-Channel Calculation of S-Wave Cross Section for 20epb+n: $E_{n}=40-1005$ keV A. D. Mackellar, D. J. Horen, C. H. Johnson ..................................... 56

Elastic and Inelastic Scattering of 180 from 285 at $19.5 \mathrm{MeV} / \mathrm{au}-$

B. L. Burks, D. J. Huren, R. L. Muble, F. E. Bertrand, J. L. Bl ankenship,

J. L. C. Ford, Jr., E. E. Gross, .. C. Hensley, R. O. Sayer, O. Shapira,

T. P. Sjoreen ......................................................... 56

Spectroscopy of $27 \mathrm{Al}$ via the $205 \mathrm{i}(180,19$ ) $27 \mathrm{Al}$ Reaction - B. L. Burks,

D. J. Horen, R. L. Auble, F. E. Bertrand, J. L. Blankenship,

J. L. C. Ford, Jr., E. E. Gross, D. C. Hensiey, R. O. Sayer,

D. Shapira, T. P. Sjoreen

Inclusive Studies of Heavy Products from $28 \mathrm{Si}+12 \mathrm{C}$ Induced Reactions with Single Unit Mass and Charge Identification - D. Thapira, J. Gomez del Campo.

M. Beckerman, 8. A. Harmon, S. T. Thornton ..................................... 59

Twofold and Threefold Coincidence Messurements of Complex Fragnent Emission in ${ }^{28} \mathrm{Si}+12 \mathrm{C}$ Collisions - D. Shapira, R. Novotny, S. T. Thorntion ....................61

Mass and Charge Flow during Orbiting of Light-Heavy Nuclei. Study of the 28Si + I 4N System - D. Shapira, J. Gomez del Campo, J. L. C. Ford, Jr., B. Shivakumar, P. H. Stelson, B. A. Harmon, R. A. Parks, S. T. Thornton

Crystal Blocking Measurements for the $160+G e$ and $285 i+G e$ Systems J. Gomez del Campo, R. Ribas, D. Shapilia, J. A. Biggerstaff, C. D. Moak, . D. Miller, N. Neskovic ...................................................6 65

Renoving Surface Absorpticn from the Optical Model: CCA Fits to $60 \mathrm{Ni}+60_{\mathrm{Ni}}$ Elastic and Inelastic Data - K. A. Erb, M. J. Rhoades-Brown

Fusion Cross Sections for Beams of $46,50 \mathrm{Ti}$ on Targets $90 \mathrm{Zr}$ and $93 \mathrm{Nb}$ -

P. H. Stelson, H. J. Kim, M. Beckerman, D. Shapira, R. L. Robinson

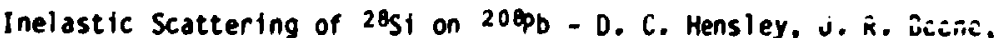

F. E. Bertrand, M. L. Haibert, G. Vourvopoulos

Masses of ${ }^{77} \mathrm{Kr}$ and $75_{\mathrm{Kr}}-$ D. M. Moltz, K. S. Toth, J. P. Sullivan,

R. E. Tribble, C. A. Gagliardf, F. T. Avignone, II

Delayed Proton Decay of I45Dy and of the New Isotope 15 lYb -

K. S. Toth, F. T. Avignone, D. i. Moltz, R. S, Moore ........................... 70

Single-Neutron and Single-Proton States in 14 gr and $11 \%$ Ho - K. S. Toth,

Y. A. Ellis-Akovali, R. S. Moore, D. M. Moltz, R. L. Mlekodaj, D. C. Sousa 
Initial Results with the Use of the UisoR las-Jet Ion Source; Electron-Capture Decay Properties of I5*Ta - K. S. Toth, D. M. Moltz, R. L. Mlekodzj, H. K.

Carter, E. H. Spejewski, C. R. Bingha ......................................... 72

Evidence from a Decay that $Z=82$ is not Magic foi Lig,nt Lead Isotopes -

K. S. Tuth, Y. A. Ellis-Nkovali, C. R. Binghan, D. M. Moltz, A. K. Carter,

R. L. Mlekodaj, E. it. Spejersti, D. C. Sousa

Beta-Delayed Proton Actirities: 1470y and 149r - K. S. Toih, Y. A. Ellis-Akovali. F. T. Avignone, III, D. M. Moitz, E. C. Schloeser, M. D. Cable ........................ 75

Gama-Ray Decay Pathways of a Compound Mucleus with High Angular monentu and Higi Excitation Energy - I. Y. Lee, C. Baktash, J. R. Beene, H. Kin, R. O. Sayer, M. L. Halbert, M. R. Johnson, F. K. McGowan, M. T. Mi !ner, D. G. Sarant ites, M. P. Fewell

An Investigation of the coliectivity in the Yrast Band of 15 ter by Lifetine Measurements - M. Oshima, F. K. McGowan, C. Baktash, Y. Schutz, H. R. Johnson,

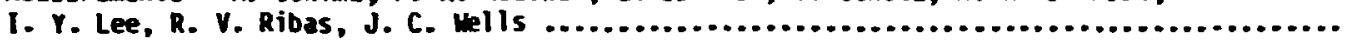

Quasivibrational Bands at High Spins in $15 \% \mathrm{rb}-C$. Baktash, Y. Schutz, I. Y. Lee, F. X. Mc Cowan, M. R. Johnson, M. L. Halbert, D. C. Hensley, M. P. Fewell,

L. Courtney, A. J. Larabee, L. L. Riedinger, A. M. Sunyar, E. der Mateosian,

0. C. Kistner, D. G. Sarantites, I. Ragnarsson

Coulonb Excitation of 1600y with Lead Ions - R. V. Rioas, N. R. Johnson, I. Y. Lee, D. R. Haenni, L. L. Riedinger, R. M. Diamond, F. S. Stephrms, S. Shi, H. i.luge

Discrete and Continuum y Ray Study of $15 \mathrm{kEr}$ at High Spir.s - C. daktask., I. Y. Lee, N. R. Johnson, 0. C. Xístner, D. Horn, C. Y. Chen, J. X. Saladin, C. G. Sarantites, A. J. Larabee, Y. Schutz, M. Oshima, E. der Mateosian, A. M. Sunyar, C. J. Lister, 0 . Dietz: ch, $k$. Horkanen, T. Semkov ........................................... 82

158Yb Gapma Ray Spectroscopy as a Function of Spin and Temperature - Y. Schutz, C. Baktash, I. Y. Lee, F. K. McGowan, M. R. Johnson, M. L. Halbert, D. C. Hensley, L. Courtney, A. J. Larabee, L. L. Riedinger, D. G. Sarantites

E2 and E4 Determinations in 233,234,235,230 - J. D. Zumbro, E. B. Sherd, Y. Tanaka, C. E. Bemis, Jr., R. A. Maumann, M. V. Hoehn, W. Reuter, R. M.

Lifetimes of High-Spin States in $162 \gamma b$ - F. K. McGowan, N. R. Johnson, $Y$. Schutz $I_{0} Y$. Lee, $C$. Baktash, A. J. Larabee, J. $C_{0}$. Hells

Spin and Temperature Dependence of the Multipolarity of the r-Ray Continuum -

D. G. Sarantites, F. A. Dilmanian, M. Rajagopalan, M. Jdiskeláiinen, H. Puchta, R. Hocdward, K. Honkanen, T. Semkow, B. Herskind, G. B. Hagemann, G. Sletten, J. J. Gaardhoje, J. D. Garrett, S. Pontoppidan, P. J. Nolan, Th. Lindblad, J. R. Beene, M. L. Halbert, D. C. Hensley, I. Y. Lee, W. T. Milner, F. Plasil, J. Hattula $\ldots \ldots \ldots \ldots \ldots \ldots \ldots \ldots \ldots \ldots \ldots \ldots \ldots \ldots \ldots \ldots \ldots \ldots \ldots \ldots \ldots \ldots \ldots \ldots \ldots \ldots \ldots \ldots \ldots \ldots$

Reaction Mechanism for One and Two Particle Transfer with Very Heavy lons M. W. Guidry, S. Juutinen, X. T. Lfu, C. R. Bingham, A. J. Larabee, L. L. Riedinger, L. H. Courtney, C. Baktash, I. '. Lee, M. L. Halbert, M. P. Fewell, D. Clíne, B. Kotlínskt, A. Baklin, D. Sarantites, T. M. Senkow, K. Honkanen, M. Rajagopalen

Cross Section for Population of High Spin States in Transfer Reactions M. W. Guidry, S. Juut inen, X. T. Liu, C. R. B!ngham, A. J. Larabee, L. L. Riedinger, L. H. Courtney, C. Baktash, I. Y. Lee, M. L. Halbert, M. P. Fewell, D. Clíne, B. Kotlínski, A. Baklin, D. Sarantites, T. M. Senkow, K. Honkanen, M. Rajagopalen .........................................................

Angular Momentum Transfer in Very Heavy Iorl Direct Reactions M. W. Guidry, R. W. Kincaid, R. Donangelo .....................................

A , todel for Heavy Ion Transfer to the Quasicontinuum - M. W. Guidry, R. W. Kincaid,

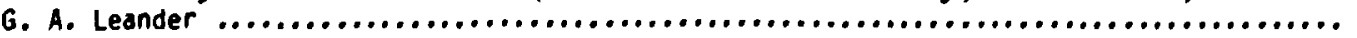


Fission Fragment Angular Distributions from ${ }^{12 C}$ - and 160-Induced Reactions A. Gavron, P. Eskola, A. J. Siert, J. Boissevain, H. C. Britt, K. Eskola, M. H.

Fowler, H. Oh, J. B. Milheligy, S. Wald, R. L. Ferguson .............................

Coincidence Measurewents Between Evaporation Residues and Light Particles Produced

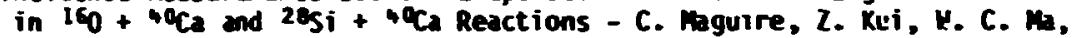
S. Robinson, D. Hatson, 6. Hord, H. Ikezoe, D. G. Kovar, 6. Rosner, G. Stephans,

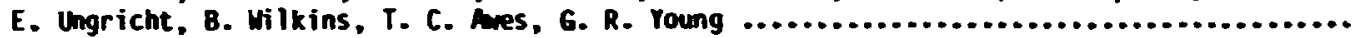

Meutron jaission in Inelastic Reactions $12 C+158$ Cd and 20 we $+150 \mathrm{nd}-$ 6. h. Petitt, A. Gavron, J. R. Beene, B. Cheynis, R. L. Ferguson, F. E. Obenshain, F. Plasil, G. R. Ycung, M. Jääskeläinen, D. 6. Saruntites, C. F. Maguire

Azinuthal Correlations Between Light Particles Enitted in IE-Induced Reactions on $12 \mathrm{C}$ and $197 \mathrm{hu}$ at $400 \mathrm{HeV}$ - H. B. Tsang, W. 6. Lynch, C. B. Chitwood, D. J. Fields, D. R. Klesch, C. K. Gelbke, G. R. Young, T. C. hes, R. L. Ferguson, F. E. Obenshain, F. Plasil, R. L. Robinson

Final-State Interactions Between Moncompound Light Particles for 160 -Induced Reactions on 197 Au at E/anu $=25$ MeV - C. B. Chitwood, J. Aichel in, D. H. Boal, G. Bertsch, D. J. Fields, C. K. Gelbke, W. G. Lynch, H. B. Tsang, J. r.. Shillcork, T. C. Mres, R. L. Ferguson, F. E. Obenshain, F. Plasil, R. L. Robin ..., G. R. Young .........................................................

Structure in the Asymetric Reaction 5ffe + 238y at 15 MeV/anu - A. Weston-Dankes. A. Mignerey, C. Merouane, S. Bradley, K. Kwiatkouski, V. E. Viola, Jr., H. Breuer, D. Benton, F. E. Oberishain, R. L. Ferguson 104

Inclusive Cross Sections for $\pi^{0}$ Emission in 25-MeV/anu Heavy-Ion Reactions P. Braun-Munzinger, R. Freifelder, J. Stachel, F. E. Obenshain, F, Plasil, G. R. Young

Determination of the Rest Frame for Mear-Threshold $\pi^{\circ}$ Emission in Heavy-Ion Reactions - J. Stachel, P. Braun-Munzinger, P. de Young, P. Paul, T. C. Awes, F. E. Obenshain, F. Plasil, G. R. Young

Heavy-Ion-Induced Fission at Energies up to $20 \mathrm{MeV} / a n u$ - T. C. Awes, J. Boissevain, H. C. Britt, K. Eskola, P. Eskola, R. L. Ferguson, M. M. Fowle:- F. E. Obenshain, H. Ohm, F. Plasil, J. B. Wilhelmy, G. R. Young

Nucleus-Hucleus Reactions at Energies up to $200 \mathrm{GeV}$-amu - Plans for an Experiment at CERN - T. C. Awes, C. Baktash, J. R. Beene, R. L. Ferguson, T. A. Gabriel, E. E. Gross, J. H. Johnson, I. Y. Lee, F. E. Obenshaín, F. Plasil, G. R. Young ..........112

Performance of Recoil Mass Separator - H. J. Kim, C. E. Bemis, Jr,., P. H. Stelson ........... 113

Development of a Polarized Eu Target - B. Shivakumar, J. R. Beene, C. E. Bemis, Jr.

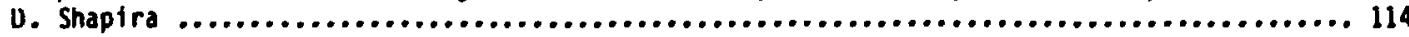

A Logarithmic Total Event Counter System for the Study of Heavy Ion Induced Reactions -

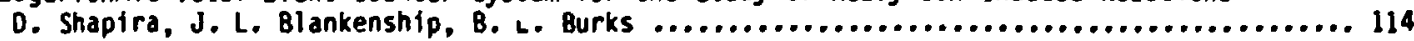

Large Ionization Detecto-s for Heavy Ion Reactions - J. L. Blankenship, F. E. Obenshain, A. it. Snell .....................................................116

Design of an Evaporation Restdues Detector - J. L. Blankenship, F. E. Obenshain ............ 116

Afterpulses from Residual Gases in Photomultiplier Tubes - N. W. Hill, D. J. Horen,

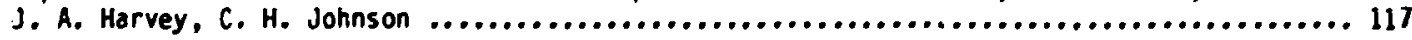

Preparation of Taryets for Nuclear Physics Research - D. M. Galbrafth, F. K. McGowan ........ 118 Special E.רhancements to the Event Handler -0 . C. Hensley ........................... 119 


\section{The unIson moenam}

The InISOi Mass Separator - R. L. Mlekodaj, E. H. Spejenski, H. R. Earter.

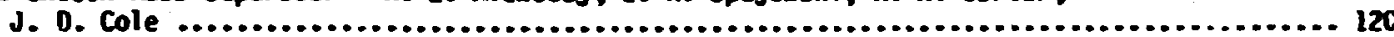

huclear Orientation Facility - E. H. Spejenski, F. T. Arignone, III,

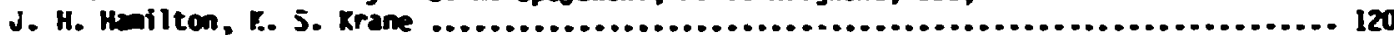

Sea.ch for Deformation in the Very Light Samariua Isotopes - R. L. Mlekodaj, 6. A. Laander, R. A. : Iaga, P. Semes, R. H. Fink, K. S. Tuth, B. D. Kein ............. 12:

The Decay of Mass-Separated $19 \mathrm{king}$ and $19 \mathrm{kghg}$ to $19 \mathrm{ldu}$ and Odd-hass hu Systematics - J. L. Uood, C. D. Papanicolopulos, E. F. Zganjar,

E. Van Mijie, R. L. Metodaj

Dynamical Supersymmetries and the Positive-Parity States in 194/W - J. L. Wood, C. D. Papanicolopulos, E. F. Zganjor, J. D. Cole, X. S. Krane, R. L. Ml ekodaj, L. Vanneste, E. Van wille, M. Huyse, A. V. Ranyya, J. H. Hailton, J. Vervier

Ground State Shape and Crossing of Near Spherical and Ceforned Bands in 102 Hg - W. C. W, A. V. Ramayya, J. H. Hemilton, S. J. Robinson,

K. Thau, J. D. Cole, E. F. Zganjar, E. H. Spejewski, H. E. Barclay 122

Shape Coexistence in $107 \mathrm{hw}$ and 105 w - E. F. Zganjar, J. L. Wood, M. A. 6rian, H. K. Carter ................................................................. 122

A New Class of Low-Energy Structure at Closed Shells: Levels in 187-I9iTl E. F. Zganjar, I. D. Cole, J. L. Wood, 6. M. Gondy, L. L. Riedinger, C. R. Bingham, G. Campbeli, E. H. Spejewski

Measurement of the Isotope Shifts and Hyperfine Structures of $19 \mathrm{2m} \mathrm{TI}$ and $201 \mathrm{TI}$ at the UinsoR Laser Facility - J. A. Bounds, H. K. Carter, C. R. Binghan, R. L. Mlekodaj, E. H. Spejewski, W. M. Fairbank, Jr., P. Juncar

New Method to Measure Relativistic Doppler Shifts: First Results and a

Proposal - P. Juncar, H. K. Carter, R. L. Mlekodaj, C. R. Binghan, D. J. Pegg, J. D. Cole, J. A. Bounds

4. EXPERIRENTAL ATOMIC PHYSICS

\section{ACCELERATOR-BASED ATOMIC PHYSICS}

Correlated Two Electron Effects in Highly Charged Ion-Atom Collisions - S. Datz, C. Bottcher, L. H. Andersen, H. Frost, P. Hvelp? und, H. Knudsen ................... 126

Electron and Positron Channeling Radiation - S. Datz, B. L. Berman, H. S. Park, R. H. Pantell, J. O. Kephart, R. H. Kiein .

Resonant Coherent Excitation of Heavy Ions Moving in Crystalline Channels - $C$. $D$. Moak, J. A. Biggerstaff, 0. H. Crawford, S. Datz, P. F. Dittner, J. Gomez de Campo,

H. F. Krause, P. D. Miller, P. L. Pepmiller, H. D. Brom ........................ 131

Ion-Ion Collision Study Using the Folded Bean Apparatus - H. J. Kim ................... 133

Dielectronic Recombination: A Status Report - P. F. Dittner, S. Datz, C. M. Fou, P. D. Miller, C. D. Moak, P. L. Pepmiller .................................... 134

Charge Transfer to Multicharged Recoll lons ir, a Penning Trap - D. A. Church, R. A. Kenefick, W. \&. Burns, C. S. 0., R. Holmes, S. Huldt, S. Berry, M. Breinig, S. B. Elston, J.-P. Rozet, I. A. Sellin, D. Tayler, B. Thomas ....................... 135

The Effect of Transverse Correlaticns in Ion-Channeling in Very Thin Crystals: Experimental and Theoretical Results - H. F. Krause, S. Datz, P. F. Dittner, J. Gomez del Campo, P. D. Miller, C. O. Moak, N. NeskoviC, P. L. Pepmillier 
Anomalous hean-Free Paths of Convoy Elertrons Produced by 25 a.u. Highly

Stripped $\mathrm{ni}$ Ions in C and Al Targets - C. Bottcher, R. Latz, M. Burkhard,

H. J. Frisschkorn, K. -0. Broeneveld, D. Hofinanr, P. Koschar, S. D. Berry,

M. Breinig. I. A. Sellin

The Regative Ion Source Test Facility as a Negative Ion Atomic Ptysics Research Facility -

G. D. Alton, T. J. Krale ........................................................... I38

Progress Toward Measuremeat of the Properties of Metastably Bound Megative Ions -

G. D. in'ton, T. J. Kuale, R. M. Compton, D. J. Pegg, J. S. Thompson .................... I39

Shape of the Electron Capture of the Continum Cusps for $H, K^{2}$, and He Targets in

the Velocity Range 6.3 to 18.0 a.u. - 5. D. Berry, 6. A. Glass, I. A. Sellin,

K-0. Groeneveld, D. Mefiann, L. H. Andersen, S. B. Elston, P. Engar, K. Stolterfoht,

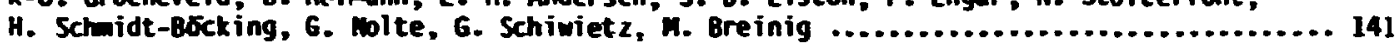

En Tandew Operation - P. L. Pepailler, 6. F. Wells, P. D. Miller ........................ 142

\section{ATOAIC mitsICS FOR FUSIOn mosen}

ECR Multicharged Ion Source - F. W. Meyer, J. H. Male, J. W. Johnson ..................... 143

Elec:ron Capture Cross Section Measurements - F. H. Meyer, A. M. Howald,

C. C. Havener, R. A. Phaneuf ................................................ 146

Ior-Atom Merged Beams Experiment - C. C. Havener, H. F. Krause, R. A. Phaneuf ............ 147

Electron-Impact Ionization of Multicharged Metallic Ions - D. C. Gregory, A. M. Howald ............................................................. 149

Single, Double, and Triple Electron-Impact Isizization of $\mathrm{Xe}^{\mathrm{Gt}}-$ A. M. Howald,

D. C. Gregory, D. H. Crandall, R. A. Phaneuf .,................................. 150

New Electron-Ion Crossed-Beams Apparatus - J. C. Gregory, F. W. Meyer ..................... 152

Radionetric Standard for the Extreme Ultraviolet - P. 4. Griffin, C. C. Havener,

J. W. Johnsoin ............................................................ 153

5. THEORETICAL PHYSICS

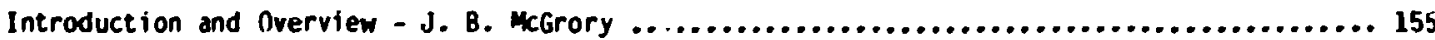

REAYY-ION DYMNICS

Folding-Model Analysis of Elastic and Inelastic oparticle Scattering Ising a Density-Dependent Force - A. M. Kobos, B. A. Brown, R. Lindsay, G. R. Satchler

Potencial Models and Resonances in the 160+285i System - A. M. Kobos,

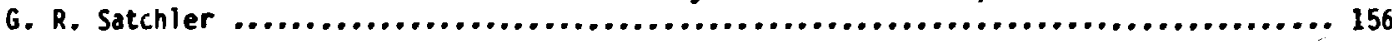

A Global Optical Potential Analysis of $160+285 i$ Elastic Scattering - A. M. Kobos, G. R. Satchler ...............................................................156

One Effect of Using Relativistic Kinematics in the Analysis of Heavy-Ion Elastic Scattering - M. El-Azab Farid, G. R. Satchler .................................. 156

Some Optical-Model Analyses of the Elastic Scattoring of ${ }^{40 \mathrm{Ar}}$ at, $1760 \mathrm{MeV}$ -

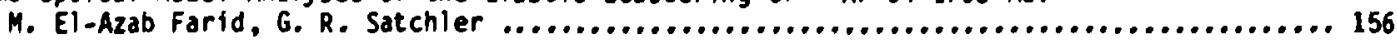

Folding-Model Potentials for Heavy-Ion Scattering Using a Semirealistic DensityDependent Force - M. El-Azab Farid, G. R. Satchler .................................. 156

Effects of Potential Variations on One-Dimensional Barrier Penetration and Fuston Cross Sections Below the Coulomb Barrter - M. M. Shalaby, G. R. Satchler... 
Coupled Elastic and Inelastic Scattering of $160+285 i$ Near the Coulonb Barrier, with a Weakly Absorbing Optical Potential - M. M. Shalaby, G. R. Satch?or.

A Dispersion Relation and the Energy Depender it of the Heavy-Im Optical

Pjtential - C. Mahaux, G. R. Satchler .......................................... 158

Path Integral Approach to Multidinensional Quantum Tunneling and Sub-Barrier Fusion - A. B. Balantekin, N. Takigawa ....................................... 158

Effect of Dissipation on the Eigensolutions Near the Fission Saddle Fsint K.T.R. Davies, J. R. Mix, h. J. Sierk ........................................ 158

St.udies of Conditional Saddle Foint Configurations - K.T.R. Davies, A. J. Siere ........... 158

Macroscopic and Microscopic Fusion studies of the 208b+5qe Reaction - J. R. Mix, A. J. Sierk, K.T.R. Davies, M. R. Strayer, A. K. Dhar, A. C. Merchant .................. 160

Geometry and Dynamics of a Zero-Temperature, Fermi-Gas Model for Pre-Equilibrium Emission of lucleons, with Application to $160+937 b$ at $E_{L a b}=204 \mathrm{MeV}-$

K.T.R. Davies, B. R:Inaud, M. R. Strayer, K. R. Sandya Deví, Y. Raffray

Time-Dependent Hartree-Fock Studies of the Sensitivity of Dynamical Fusion Thresholds to the Effective Two-Body Interaction - J. A. Maruhr, K.T-K. Davies,

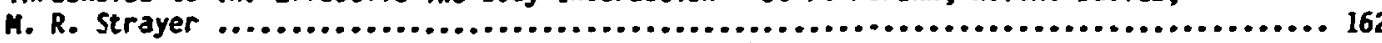

Tỉme-Dependent Hartree-Fock Calculations of Nuclear Molecular Resonances M. R. Strayer A. S. Umar, R. Y. Cusson, P.-G. Reinhard ..........................163

1. Time-Dependent Mean-Field Theory for Prompt Muclson Emission in Heavy-Lon Reactions - A. S. Umar, H. R. Strayer, D. J. Ernst ............................... 164

N-N Correlations in Inclusive Nonequilibrium Particle Emission - A. S. Umar,

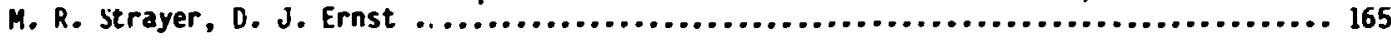

Chaos in Time-Dependent Hartree-Fock Collisions of Heavy lons - M. R. Strayer,

R. S. Umar, R. Y. Cusson, P. G. Reinhard .................................... !56

Mean-Field Calculations of Fluctuations in Nuclear Collisions - J. B. Marston,

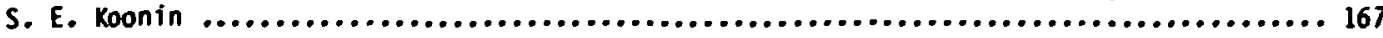

Rotating Toroidal Nuclei in Heavy-Ion Reactions - Cheuk-Yin Wong $\ldots \ldots \ldots \ldots \ldots \ldots \ldots \ldots . \ldots . \ldots 16$

Limits on the High-Density Behavior of Heuristic Nuclear Matter Equations of

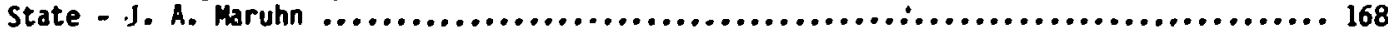

Density as a Constraint and Excitation Energy in TDHF - R. Y. Cusson,

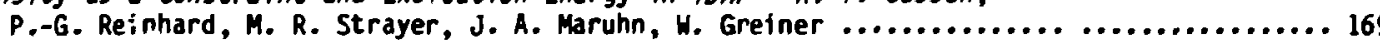

Relativistic Mean-Field Dynamics Applied to $160+160$ Scattering - R. Y. Cusson, P.-G. Reinhard, H. Stócker, M. R. Strayer, H. Greiner ............................. 170

Subthreshold Pion Production in Heavy-Ion Collisions - D. J. Ernst, M. R. Strayer,

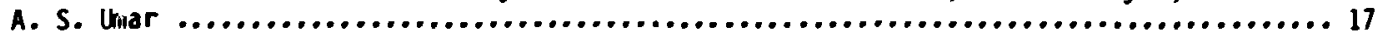

NUCLEAR STRUCTURE

THE UNISOR MUCLEAR STRUCTURE THEORY PROGRAM

Introduction - 6. A. Leander ............................................... 172

Low-Energy Structure - A. F. Barfield, F. Dónau, J. Dudek, B. E. Gnade,

W. M. Howard, G. A. Leander, P. Molier, H. Nazarewicz, J. R. Mix, Ph. Quentín,

P. B. Senmes, J. L. kivod .................................................

Intrinsic Reflection Asymmetry - G. F. Bertsch, J. Dudek, G. A. Leander, P. Moller, W. Mazarewicz, P. Olanders, Ph. Quentin, I. Ragnicrson,

E. Ruchowska, M. R. Stráyer 
High Spins - F. Arve, T. Bengtsson, Y. S. Chen, J. Dudek, S. Frauendorf

M. H. Cuidry, G. A. Leander, H. Kazarewicz, P. 01 anders, I. Ragnarsson ................... 175

quasicont inuum Spectroscopy - T. Bengtsson, G. A. Leander ................................ 175

Acridental Degeneracies and Dynanical Supersymetries - A. B. Bal antekin .................. 176

Character Expansions for Unitary Groups - A. B. Balantekin ............................... 177

\section{RELATIYISII: HEATY-IOM PHTSICS}

Initial Energy Density of Quark-Gluon Plasna in Relativistic Heayy-Ion

Collisions - Cheuk-rin Hong ...................................................... 177

Baryon Distribution in Ultra-Relativistic Heavy-Ion Reactions - Cheuk-Yin Hong ............ 178

Nucleon-Kucleus Reactions at Ultra-Relativistic Energies - Cheuk-Yin Wong ................. 178

The Hydrodynamic Phase in Ultra-Relativistic Heavy-Ion Collisions - M.-C. Chu ............. 178

\section{THEORETICAL ATOMIC MHSICS}

Multiple-Vacancy Production in the Independent-Ferwi-Particle Hodel - R. L. Becker,

A. L. Ford, J. F. Reading ..................................................... 17

Inclusion of Electron Transfer in the Calculation of $K^{n} L^{v}$ Multiple Vacancy

Production by Ion Impact - R. L. Becker, A. L. Ford, J. F. Reading .................... 180

Theory of Multiple L-jhell Vacancy Production in Coincidence with Electron

Transfer - R. L. Becker .......................................................... 181

Collisional Vacancy-Rearrangement in the First Magnus and Coupled-Channeis

Collision Theories - R. L. Becker ............................................ 182

Depencience of Ion-Rydberg Atom Cross Sect ons on the Orientation of the Rydberg

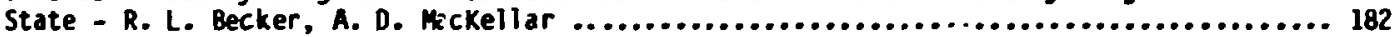

Numerical Solution of the Time-Dependent Dirac Equation - C. Bottcher,

M. R. Strayer .............................................................. 19.

Phenomenclogy of Nuclear Collisions wth Long Time Delays - M. R. Strayer,

V. E. Oberacker, C. Bottcher .......................................................... 185

Time-Dependent Hartree-Fock Theory for Heavy Ion-Atom Collisions - C. Bottcher ............ 185

Threshold Ionization Theory - C. Bottcher .......................................... 187

Formal Relationship Between Maser Theory and Quantum Fluid Mechanics -

C. Bottcher, C. Feutllade ................................................... 187

Low-Erergy Charge Exchange - C. Bottcher, T. G. Heit ................................. 188

Sumary of Code Development for Electror.-Ion Scattering - C. Bottcher,

D. C. Griffin, M. S. Pindzola ..................................................... 189

Excitation-Autoionfzation Processes in the Electron Impact Ionization of Singly-

Charged Ions - D, C. Griffin, M. S. Pindzola, C. Bottcher ................................ 189

Direct and Indirect Ionization of Transition Metal Jons - M. S. Pindzola,

D. C. Griffin, C. Pottcher ........................................................ 190

Dielectronic Recombination in the Lithium Isoelectronic Sequence - D. C. Griffin,

M. S. Pindzola, C. Bottcher ................................................. 191

The Effect of Electric Fields on Dielectronic Recombination in lons of the Lithium and Sodium Isoelectrontc Sequences - D. C. Grifitin. M. S. Pindzola, C. Bottcher 


\section{6. mafia science applicat ms}

Ac:ivities in Support of the US/UK Joint Experiment in the Dounreay Prototype Fast Reactor - S. Raaan, D. A. Costanzo, H. L. Mdair, J. K. Dickens.

J. L. Botts, J. F. Enery, B. L. Broadhead, R. L. Walker ........................... 193

Ultrahigh-Resolution Studies of Heav;-Ion-Induced $X$-ray Satellite Emission -

C. R. Vane, G. Horford, E. Kalin:, 5. Raman, J. Katine, M. S. Suith

Effect of the Chesical Environent on the $L$ and $M$ Heavy-Ion-Induced $X$-ray Satell ite Enission Spectra - T. H. Rosseel, P. L. Pepmiller, J. H. Dale, S. Raman, L. D. Hul ett, C. R. Vane, H. F. Krause, J. P. Young

Simulation of Cosmic-Ray upset of Microelectronic Devices - M. A. Kolasinski,

M. Knoll, J. Adolphsen, R. Koga, G. Bı :ıcker, S. Rzanan, C. R. Vane

\section{PLASM dIAENOSTICS FOR FUSION PROERAM}

Multichannei Polarimetry Using Faraday Rotation - C. H. Ma, D. P. Hutchinson,

P. A. Staats ............................................................... 198

Diagnostics Development Center - C. F. Barnett, E. W. Thomas, K. D. Legg, P. Baxshi ....... 200

Feasibility of Alpha Particle Diagnostics by $\mathrm{CO}_{2}$ Laser Thomson Scattering -

D. P. Hutchinson, K. L. Vander Sluis, 2 . Sheffield, D. J. Sigmar

8. HIGH ENERGY PHYSICS

H. O. Cohn, J. E. Baru, H. M. Bugg, G. T. Condo, T. Handler

9. COMPILATIONS ND E. LUATIONS

Controlled-Fusior. Atomic Data Center - C. F. Barnett, H. B. Gilbody, D. C. Gregory.

P. M. Griffin, C. C. Havener, A. M. Howald, H. T. Hunter, R. K. Janev, M. I.

Kirkpatrick, C. R. Mahon, E. H. McDaniel, R. H. McKnight, F. W. Meyer, T. J.

Morgan, R. A. Phaneuf, M. S. Pindzol a, E. W. Thomas .......................... 206

Nuclear Data Project - S. J. Ball, Y. A. Ellis-Akovali, M. J. Martin, M. R. McGinnis,

M. R. Schmorak .......................................................201

i0. ACCELERATOR-COLLIDER STUDY

G. D. Alton, H. H. Atkins, C. Baktash, J. R. Beene, J. A. Biggerstaff, E. D. Hudson, C. M. Jones, R. C. Juras, I. Y. Lee, J. A. Martín, J. M. McConnell, J. B. MeGrory, W. T. Milner, S. W. Mosko, F. E. Obenshain, D. K. Olsen, F. Plasil, R. L. Robinson, M. R. Strayer, C. N. Thomas, C. Y. Hong, G. R. Young ........................ 208

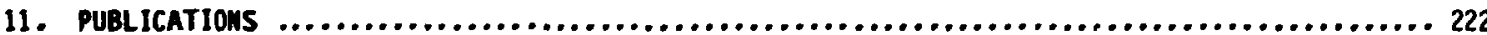

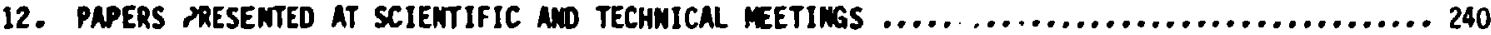

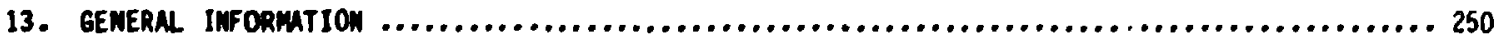


INTRODUCTION

The reporting period covered by this progress report is the 1984 fiscal year beginning October 1, 1983, and ending September 30, 1984. This report arks a return by the Physics Division to issuing these reports on an annual cycle. The research activities of the Division are centered pricarily in three areas: experimental iuclear physics, experimental atoaic physics, and theoretical nuclear and atoaic physics.

The largest of these efforts, experimental nuclear physics, is dominated by the heavy ton research program. A major responsibility under this progran is the operation of the Hol ffield Heavy Ion Research Facility as a national user facility. During the period of this report, the facility has begun routine operation for the experimental program. The Holifield Facility and the internal experimental nuclear physics prograns are described in chapters $1-3$ of this repcrt.

The experimental atomic physics program has two components: the accelerator.. based studies of basic collisional phenomena and the studies in support of the controlled fusion program. These efforts are described in Chapter 4. Also associated with the fusion-related studies are a plasma diagnostics program and the operation of an atonic physics data center. The former is described in Chapter 7 , and the latter is discussed in Chapter 9, along with our effort in support of the nuclear data comptlation effort.

The theoretical physics program, both nuclear and atomic, is covered in Chapter 5. This program has benefited this year from the success of the Vak-AP computer system and from the increase in manpower provided by the ORNL/University of Tennessee Distinguished Scientist Program.

Smaller programs in applications and high-energy physics are summarized in Chapters 6 and 8.

During the perfod of this report, we continued to explore possible future extenstons of the Holiffeld Factlity. He retain a strong interest in a relativistic heavy-ion collider in the $10 \times 10 \mathrm{GeV} /$ nuclear eneray range. The ideas for such a facility, described in last year's. report, have been modified to utilize the HHIRF 25 w tandem accelerator as the first stage. This study is sumarized in Chapter 10.

Finally, the report concludes with some general information on publications, Division activities, and personnel changes. 


\section{HOLIFIELD HEAVY ION RESEARCH FACILITY}

\section{OVERVIEW}

\author{
R. L. Robinson J. A. Martin \\ C. M. Jones
}

Beginning January 19, 1984, the Holifield Facility began its first period of what is considered typical speration. Since that time, the division of time between beam available for research, development, mintenance, and tuning was near that projected for a normal year. Another indicat ion of normality has been the scheduling of the Program Advisory Committee meetings on a sixmonth interval.

The three and one-half month; prior to January 19 were devoted to (1) a major scheduled maintenance period in wich 40 of 54 tandem acceleration tube units were renoved, reconditioned, and replaced, and in which several major maintenance tasks were performed on the cyclotron, (2) repair and modification cf several tandem azcelerator column vacuum relatef components, and (3) initial conditioning of the tandem acce'erator. Following 2 perion of operation for the experimental program and another conditioning period in March, the tandem accelerator was operated with beam at a potential of $22.5 \mathrm{MV}$, a value which exceeds that achieved both at ORNL and at other laboratories by more than 2 MV. Since then, the facility has provided beams for several experiments at $20 \mathrm{MV}$ or slightly above.

Refinements in the calculations used for determining cyclotron operating parameters have made it possible to increase the extraction radius from 77.5 to $79.2 \mathrm{~cm}$. This seemingly modest change has the impressive effect of reducing power for the main cyclotron magnet at the maximum mass-energy product by $20 \%$. resulting in a $15 \%$ reduction in total power use for cyclotron operation. This is especlally significant because power is the major incremental cost for coupled operation and because coupled operation has and apparently will continue to be budget-1imited.

A total of 3,177 research hours were provided during this one-year reporting perfod, of which 1,325 were in coupled-mode operation. Some statistics relating to research hours are given in Tables 1.1 - 1.3. Research hours/ month are shown in Fig. 1.1. Research hours/ month for three of the months in this reporting period exceeded that for any month pritor to this period.
Table 1.i. Division of research hours by research activities for the period October 1, 1983 through September 30, 1984

\begin{tabular}{|c|c|c|c|}
\hline \multirow[b]{2}{*}{ Activity } & \multicolumn{3}{|c|}{ Research Hours } \\
\hline & Tandem & Coupled & Total \\
\hline $\begin{array}{l}\text { High-spin states } \\
\text { Fusion } \\
\text { Damped reactions } \\
\text { Incomplete fusion } \\
\text { Atomic } \\
\text { Charge exchange } \\
\text { Tests of apparatus } \\
\text { Pion production } \\
\text { Quasi-elastic } \\
\text { Giant resonances } \\
\text { Fission } \\
\text { Low-:ying level } \\
\text { properties } \\
\text { Tests of detectors } \\
\text { Applications } \\
\text { a-decay rates } \\
\text { Crystal blocking }\end{array}$ & $\begin{array}{l}214 \\
171 \\
105\end{array}$ & $\begin{array}{r}132 \\
286 \\
274 \\
171 \\
\\
168 \\
49 \\
123 \\
98\end{array}$ & $\begin{array}{r}847 \\
291 \\
286 \\
274 \\
214 \\
171 \\
171 \\
168 \\
154 \\
173 \\
98\end{array}$ \\
\hline Total & 1,852 & $1,325^{*}$ & 3,177 * \\
\hline
\end{tabular}

- These numbers differ slightly from those in Tables 1.5 and 1.6 due to rounding errors.

The Spin Spectrometer has clearly proven to be the most popular research tool of this year (see Table 1.3). However, it was recently discuvered that nearly half of the $70 \mathrm{MaI}$ crystals have extensive cracks which have caused significant deterioration of the detector parameters. Although the Spin Spectrometer is stfll adequate for most experiments, those requiring high resolution (such as study of y-ray emission from giant resonances) must be postponed or compromised.

The highlight for the experimental apparatus took place at UNISOR. The long-awatted atomic hyperfine structure spectrometer demonstrated its capability to investigate mass separated nuclef produced in heavy-ion induced reactions. This was tested with both the ground state and isomeric state in 192T1. (See Section 3.) 
Table 1.2. Number of researchers from different institutions who participated in experiments at the MIRF in the 12-month period October 1, 1983 through September 30, 1984

Institutions $\begin{gathered}\text { Number of } \\ \text { Researchers }\end{gathered}$

\section{UNIVERSITIES}

Eastern Kentucky Univ. Emory Univ.

Georgia State Univ.

Georgia Inst. of Tech.

Indiana Univ.

Louisiana State Univ.

Massachusetts Inst. of Tech.

Michigan State Univ.

ORAU

Research Inst. of Physics

(Sweden)

State University of Hew York

Tennessee Tech. Univ.

Texas AM Univ.

Univ. of Claude Berriard Lyon (France)

Univ. of Florioa

Univ. of Frankfurt (Germany)

Univ. of Houst on

Univ. of Kentucky

Unfv. of Kōln (Germany)

Univ. of Maryland

Univ. of Michigan

Univ. of Pittsburgh

Univ. of kochester

Univ. of South Carolina

Univ. of Tennessee

Univ. of Virginia

Univ. of Washington

Vanderbilt Univ.

Washington Univ.

Western Kentucky Univ.

Yale Univ.

1

2

3

4

2

2

1

3

3

3

1

6

1

6
2

2

3

1

7

2

3

17

2
5

10

5

107

\section{MATIONAL LABORATORIES}

Centro Atomico Baralache

$$
\text { (Argentina) }
$$

LANL

MASA/Goddard

Maval Surface Heapons Lab.

ORNL

SANDIA

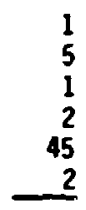

56

IMDUSTRY

Aerospace Corp.

RCA

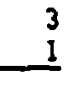

Table 1.3. Use of experiment target stations for the period

October 1, 1983 through September 30, 1984

Research Hours (No. of Runs)

\begin{tabular}{|c|c|c|c|}
\hline & & & \\
\hline Target S & Tandem & Coupled & Total \\
\hline $\begin{array}{l}\text { Y-ray spectrometer } \\
\text { (iandea) }\end{array}$ & $221(3)$ & na & $221(3)$ \\
\hline $\begin{array}{l}\text { y-ray spectrometer } \\
\text { (ORIC) } \\
\text { Velocity filter }\end{array}$ & $\begin{array}{l}\text { na } \\
189(4)\end{array}$ & $\begin{array}{l}46(1) \\
\mathrm{na}\end{array}$ & $\begin{array}{r}46(1) \\
189(4)\end{array}$ \\
\hline $\begin{array}{l}\text { Atomic physics } \\
\text { (tandea) }\end{array}$ & $263(4)$ & na & $263(4)$ \\
\hline $\begin{array}{l}\text { Atomic physics } \\
\text { (coupled) } \\
\text { Split-pole agnetic }\end{array}$ & na & 0 & 0 \\
\hline $\begin{array}{l}\text { spectrometer } \\
\text { Applications (Bean }\end{array}$ & $97(1)$ & na & $9^{\prime}(1)$ \\
\hline $\begin{array}{l}\text { line } 31) \\
\text { Beam line C-9 }\end{array}$ & $\begin{array}{l}82(4) \\
\text { na }\end{array}$ & $\begin{array}{l}\text { na } \\
272(2)\end{array}$ & $\begin{array}{r}82(4) \\
272(2)\end{array}$ \\
\hline & $e^{\text {na }}$ & 0 & 0 \\
\hline $\begin{array}{l}\text { spectrometer } \\
\text { Tipe-of-flifot }\end{array}$ & $34(1)$ & $244(1)$ & $278(5)$ \\
\hline system & $12(1)$ & $189(2)$ & $201(3)$ \\
\hline chanber & $25(2)$ & $318(3)$ & $343(5)$ \\
\hline UKF $\$ O R$ & $293(15)$ & 0 & $293(15)$ \\
\hline Spin spectrometer & $636(7)$ & $256(3)$ & $892(10)$ \\
\hline Total & 42) & (15) & $(5)$ \\
\hline
\end{tabular}

na $\equiv$ not accessible to beam

ACCELERATOR OPERATIOAS AND IEVELOPMENT

OPERATIONS
G. D. Alton
S. N. Lane
J. A. Benjaminl
C. T. LeCroy
J. A. Biggerstaff
R. S. Lord"
M. R. Dinehart
C. A. Ludemann
D. T. Dowling
J. E. Mann"
H. D. Hackler
C. L. Haley
D. L. Haynes
D. E. Hoglund ${ }^{2}$
C. A. Maples
R. L. McPherson
E. D. Hudson
C. A. Irizarry
P. Meszaros 5
G. D. Mills
C. M. Jones
N. I.. Jones
S. H. Mosko
S. N. Murray
R. C. Juras 3
E. G. Richardson
P. T. Singley 6

N. F. Ztegler

The first three and one-half months of this reporting pertod were devoted to a long scheduled matintenance perfod for both the 
ORNL-ONG-83-17475R

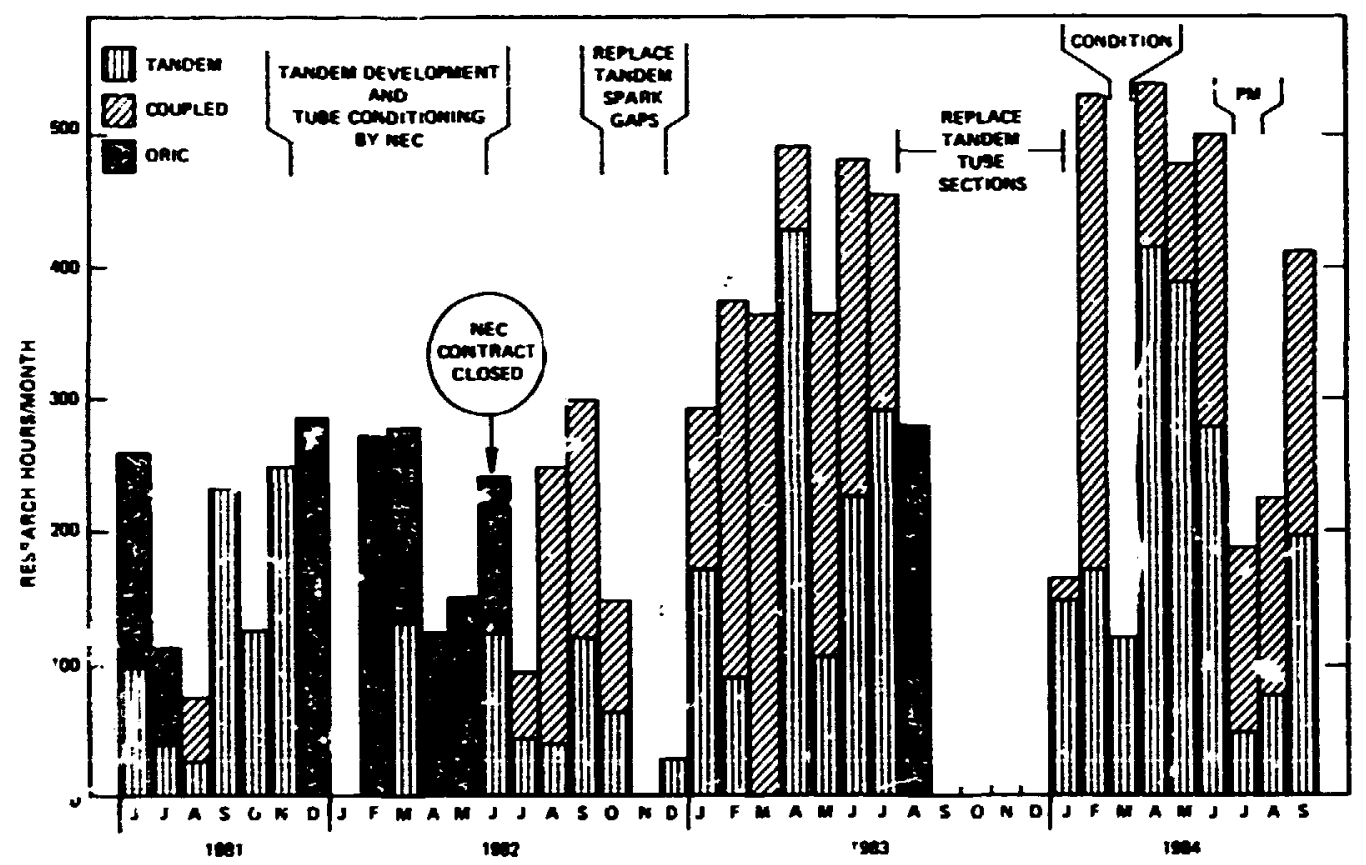

Fig. 1.1. Research r.n.rs per wonth for experiments utilizing the HHIRF acselerators. The interval denoted as PM was a programmed maintenance period.

tandem acceierator and the ORIC, repairs of vacuur related components in the tandem accelerator, and conditioning of the tandem accelerator. No beans were provided for research during this first period. Operation for the research program began in mid-January and continued until the end of this report period with interruptions only for a conditioning period in March, scheduled and unscheduled maintenance, and two major holidays. Thus, "typical" operation was provided for approximately the last three-fourths of the year.

The nost striking feature of operation during this reporting period has been a further improvement in reliability and quality of a latiun. As shown in fig. 1.1, we experipnced three months in wich over 500 hours of beam time were provided for research. We have also achieved a noticeable improvement in the degree to which our research schedule is maintained. Major contributors to these improvements were engineering modifications to both the tandem accelerzior and the ORIC, installation of the improved radial geometry cestum plasma source desr ibed in the last progress report, ${ }^{B}$ a'id inc. eased experience of the operations staff.

A summary of beams provided during this reportinn pericd is presented in Table 1.4. As noted in the table, four ion species $\left(10_{B},{ }^{4} \mathrm{SC}\right.$, $106 \mathrm{Ti}$, and $150 \mathrm{Nd})$ were provided for the firs: time. The most notable of these is probably is No which was provided with coupled operation using a separated tsotonptc sample in the nega$t$ tre ton source. A: an fllustration of the utility of the tandem accelerator, this beam was provided for a total of 45 hours while using only approximately $2 \mathrm{mg}$ of separated tsotoptc matertal.
Table 1.4. Beams provided for research for the period October 1, 1983 through September 30,1984

\begin{tabular}{ccc} 
Ion & $\begin{array}{c}\text { Maximum } \\
\text { Energy } \\
\text { (MeV) }\end{array} \quad$ Mode* & $\begin{array}{c}\text { Provided for } \\
\text { first time } \\
\text { in this period }\end{array}$ \\
\hline
\end{tabular}

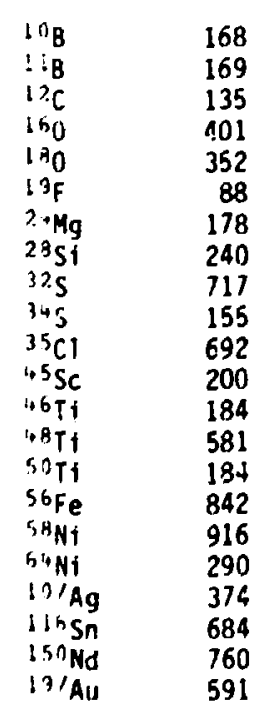

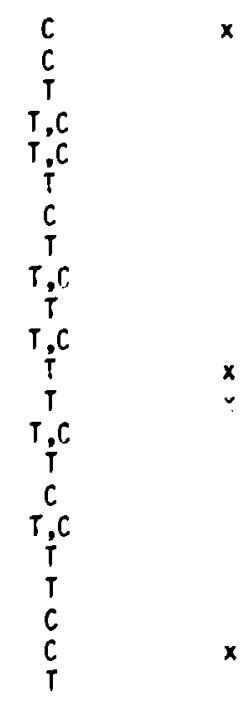

-T = Tandem alone; $C$. Coupled mode. 
Utilization analyses for the tandem acielerator and cyclotron are presented in Tables 1.5 and 1.6, respectively. Electric power budietary limitations continue to restrict cyclotron operation to about 2000 hours per year. Efficient accelerator utilization has been achieved by using the OAIC only in coupled mode ard only for energies beyond the capability of che tandea accelerator.

1. Present address: Br iokhaven Mational Laboratory, Upton, WY 1197 ?

2. Co-op student, Virginia Polytechnic Institute and State University, Blacksburg, VA 24061.

3. Instrumentation and Controls Division.

4. Consultant.

5. Computing and Telecomunications Division.

6. Energy Division.

7. Ret ired.

8. Physics Division Progress Report for Period Ending Septeaber 30, 1983, ORla-6004 (1983).

Table 1.5. Tandea arcelerator utilization for the period $0=$ tober 1,1983 through September 30 , 1984

Yours Percent

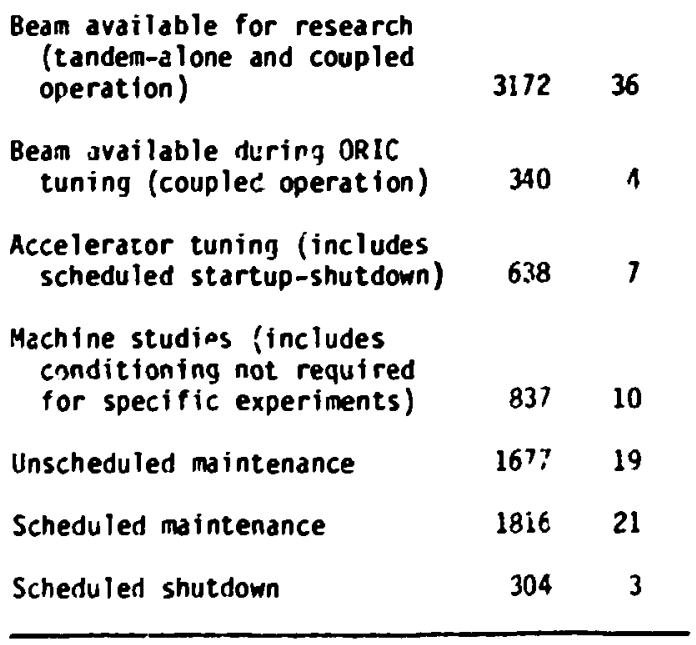

\section{TANOEM ACCELERATOR}

$\begin{array}{ll}\text { G. A. Alton } & \text { C. M. Jones } \\ \text { J. A. Benjamint } & \text { R. C. Jurss } \\ \text { J. A. Biggerstaff } & \text { J. E. Mann } \\ \text { D. L. Haynes } & \text { E. G. Richardson } \\ \text { D. E. Hoglund2 } & \text { N. F. Ziegler }\end{array}$

\section{Voltage Performance}

As discussec in the previous progress report, 5 inftial voltage performance of the tandem accelerstor was somewhat disappotnting. Thus, a major maintenance period was planned for the period August throug? October 1983
Table 1.6. Cyclotron utilization for the period October 1, 1983 tinrough September 30, 1984

Hours Percent

\begin{tabular}{lcc}
$\begin{array}{l}\text { Beam available for research } \\
\text { (coupled operation) }\end{array}$ & 1331 & 15 \\
$\begin{array}{l}\text { Accelerator tuning (includes } \\
\text { scheduled startup-shutdown } \\
\text { and operation during tandem } \\
\text { tuning) }\end{array}$ & 494 & 6 \\
$\begin{array}{l}\text { Machine studies } \\
\text { Unscheduled mintenance }\end{array}$ & 130 & 1 \\
Scheduled mintenance & 205 & 2 \\
Scheduled shutdown & 1376 & 16 \\
\hline
\end{tabular}

with the primary motivation of improving tandem accelerator roltage performance. Ouring this period, 40 of 54 acceleration tube inits were removed, reconditioned at the National Electrostatics Corporation nlant in Madison, hisconsin, and reinstalled in the accterator. Alsn during this period, all magnetic electron traps were removed and ports were installed for possible future hydrogen arc discharge cleaning of the accelerator.

Following repatrs and modifications of vacuum related components in the colum, conditioning of the accelerator began in midDecember 1983, and continued until mid-January 1984. Progress during this period was encouraging and the accelerator was operated at the end of the period with beam at a terminal potential of 20.? WV, a new ORML and world's record. This conditioning period was terminated in order to begin operation for the experimental program and to allow further improvement in vacuum. A second conditioning perfod was scheduled for March 1984. At the end of this period the accelerator was operated with beam at $22.5 \mathrm{MV}$, aga in a new record.

Although operation at unusually high terminal potentials was not a primary goal for our experimental program in Fy 1984, voltage performance of the accelerator during this period was alsu encouraging. As shown in fig. 1.2 , six experimental runs have been performed at or above 19 MV and three experimental runs have been performed at or above $20 \mathrm{MV}$. The highest of these was $20.3 \mathrm{MV}$. During all operation in FY 1984 (both conditioning and operation for the experimental program) only 21 full column sparks occurred of wich only two resulted in observable deconditioning. This low spark rate, the ease with which we have operated for experiments at or above $20 \mathrm{MV}$, and the absence of observed deterioration in voltage performance with time, all sugnest that the voltage capability of the 


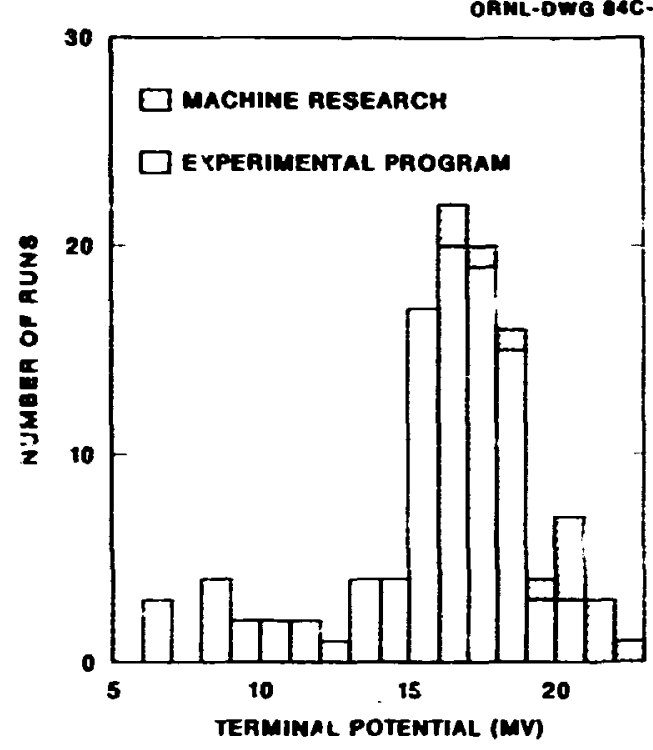

Fig. 1.2. The number of iuns in L-MV-wide intervals is shown as a function of tandem accelerator terminal potential for the period October 1, 1983 through September 30, 1984.

accelerator in its present configuration has not been fully exploited.

Two further initiatives to improve voltage performance are now plannes. These are. hydrogen arc discharge cleaning or conditioning procedure and a modification of the present acceleration tube geometry wich increases the active insulator length by $18 \%$. Both are discussed in greater detaij in other contributiuns to this report. The acceleration tube modification has been proposed as a FY 1986 AIM project.

\section{Improvements and Modifications}

Although our primary focus in this reporting period was on improved voltage performance and operation for the experimental program, a number of important improvements and modifications were also made to the accelerator system.

Within the column, ventilation and cooling were improved for both terminal and dead section electronics. Positive sublimator slug selection readout was provided for the terminal gas rtrippr.r pump and voltage monttoring was arded for column ton pumps. Vartable apertures, Faraday cups, and the terminal gas stripper were all modfied to mintmize motion feedthrough bellows fatlures. Three of six charging chatns were removed to increase reliabtlity and to provide an immediate source of spare parts in the unlikely event of chain bieakage.

Outstide the accelerator vessel, cryopumps were installed on both the low-energy and high-energy beam lines for maintenance use. In addition, a 3-foot diameter by 7-foot long pressure vessel was purchased to enable off-itne pressure testing of accelerator components.

Control system improvements include expanston of the system to include additional injector parameters and all parameters $n n$ expertmental beam itne 21 . In addition to a number of detatled improvements to the control systew operating program, two other important software tasks mere completed. The automatic logging program was extensively revised so as to operate more easily and reliably. New programs were written to provide information on stripper foil parameters such as equilibrium thickness, angular dispers: un, energy loss, and lifetime.

One of the most important improvements of Fy 1984 was final commissioning and installation in the injector of the new radial geometry cesium plasma ion source described in the previous progrezs report. 5 While retaining the versatility and prolificacy of its predecessor, th. "Mark I" ORNL version of the Aarhus radial extraction Penning Source, ${ }^{6}$ the new source has proved to be moice reliable, easier to service, and simpler to cperate. It was used exclusively during FY 1984 and was a significant factor in the improvement in accelerator system utilization described above. The ior: species provided during this period are listed in Table 1.4.

\section{$\mathrm{SF}_{6}$ System Operation}

Operation of the $\mathrm{SF}_{6}$ storage and recirculation system in FY 1984, including fifteen and one-half gas transfers to ind from storage was withou:- incidenc. Within the approximately 27 accuracy of the $S_{6}$ weighing system, no $S_{6}$ loss was observed.

1. Present address: Brookhaven National Laboratory, Upton, NY 11973.

2. Co-op student, Virginia Polytechnic Institute and State University, Blacksburg. VA 24061 .

3. Instrumentation and Controls Division.

4. Consultant.

5. Physics Division Progress Report for Period Ending September 30, 1983, DRNL-6004, (1983).

6. G. D. Alton and G. C. Blazey, Nucl. Instrum. Methods 166, Jj5 (1979).

\section{ORIC ACCELERATOR}

$$
\begin{aligned}
& \text { D. T. Dawling } \quad \text { R. S. Lord! } \\
& \begin{array}{ll}
\text { E. D. Hudson } & \text { C. A. Lisdemann } \\
\text { S. N. Lane } & \text { J. A. Martin } \\
& \text { S. W. Mosko }
\end{array}
\end{aligned}
$$

\section{Coupled Operation}

Ouring the period of this report, 16 experiments have been completed using ccupled operation of the cyclotron and tandem accelerator. Some detilis of coupled operation are given in Table 1.7. The heaviest ion accelerated was $150 \mathrm{Nd}^{+33}$; the highest energy obtained was $916 \mathrm{MeV}$ for $\left\{8_{\mathrm{Ni}}+2.3\right.$. Beam currents obtained were generally consistent with predictions, taking into account the expected fraction of beam in the destred charge state and the buncher efficiency $(\sim 50 \%)$. The highest tandem voltage used was $18.8 \mathrm{MV}$.

Coupled operation was generally more reprodurtble and beam extraction effictenctes were $h t$ ther than experienced last year. These improvements ale a result of refined computathonal methods for beam setup and the use of 
Table 1.7. Coupled operation for research for the period October 1, 198: through September 30, 1984

\begin{tabular}{|c|c|c|c|c|c|c|c|}
\hline Date & Ion & $\begin{array}{l}\text { Desired } \\
\text { Energy } \\
(\text { MeV) }\end{array}$ & $\begin{array}{l}\text { Actual } \\
\text { Energy } \\
\text { (Mev) }\end{array}$ & $\begin{array}{l}\text { Extraction } \\
\text { Efficiency } \\
(x)\end{array}$ & $\begin{array}{l}\text { Injected } \\
\text { Ion }\end{array}$ & $\begin{array}{l}\text { Injection } \\
\text { Energy } \\
\text { (MeV) }\end{array}$ & $\begin{array}{l}\text { Tanden } \\
\text { Voltage } \\
\text { (INV) }\end{array}$ \\
\hline $\begin{array}{l}2 / 5 / 84 \\
2 / 19 / 84 \\
4 / 7 / 84 \\
4 / 9 / 64 \\
5 / 8 / 84 \\
6 / 15 / 84 \\
6 / 19 / 84 \\
6 / 22 / 84 \\
6 / 27 / 74 \\
7 / 6 / 84 \\
8 / 8 / 84 \\
8 / 14 / 84 \\
8 / 17 / 84 \\
9 / 11 / 84 \\
9 / 13 / 84 \\
9 / 25 / 84\end{array}$ & $\begin{array}{l}32 \mathrm{~S}^{+15} \\
18 \mathrm{O}^{+8} \\
16 \mathrm{O}^{+8} \\
16 \mathrm{O}^{+8} \\
56 \mathrm{Fe}^{+22} \\
32 \mathrm{~S}^{+12} \\
11 \mathrm{~B}^{+5} \\
16 \mathrm{~B}^{+5} \\
16 \mathrm{O}^{+8} \\
35 \mathrm{Cl}^{+16} \\
16 \mathrm{O}^{+1} \\
24 \mathrm{Mg}^{+8} \\
150 \mathrm{Md}^{+33} \\
58 \mathrm{Ni}^{+23} \\
48 \mathrm{Ti}^{+1} \mathrm{I} \\
116 \mathrm{Sn}^{+29}\end{array}$ & $\begin{array}{l}700 \\
350 \\
400 \\
400 \\
820 \\
320 \\
170 \\
170 \\
400 \\
700 \\
208 \\
180 \\
750 \\
900 \\
576 \\
760\end{array}$ & $\begin{array}{l}717 \\
351.7 \\
400.7 \\
401.1 \\
841.7 \\
317.8 \\
168.9 \\
168.3 \\
399.7 \\
692.1 \\
206.6 \\
177.6 \\
760.7 \\
916.0 \\
581.4 \\
684.1\end{array}$ & $\begin{array}{r}48 \\
65 \\
68 \\
60 \\
76 \\
55 \\
68 \\
78 \\
\sim 100 \\
75 \\
61 \\
64 \\
67 \\
68 \\
56 \\
78\end{array}$ & $\begin{array}{l}32 \mathrm{~S}^{+6} \\
18 \mathrm{~J}^{+3} \\
16 \mathrm{O}^{+3} \\
16 \mathrm{O}^{+3} \\
56 \mathrm{Fe}^{+8} \\
32 \mathrm{~S}^{+3} \\
11 \mathrm{~B}^{+2} \\
10 \mathrm{~g}^{+2} \\
16 \mathrm{O}^{+3} \\
35 \mathrm{Cl}^{+6} \\
16 \mathrm{O}^{+2} \\
24 \mathrm{Mg}^{+2} \\
150 \mathrm{Nd}^{+13} \\
58 \mathrm{Ni}^{+9} \\
48 \mathrm{Ti}^{+6} \\
116 \mathrm{Sn}^{+1}\end{array}$ & $\begin{array}{r}122.8 \\
67.9 \\
67.5 \\
67.5 \\
170.0 \\
66.9 \\
38.4 \\
39.6 \\
68.0 \\
124.5 \\
32.6 \\
20.1 \\
211.0 \\
170.4 \\
32.9 \\
144.9\end{array}$ & $\begin{array}{l}17.5 \\
16.9 \\
16.8 \\
16.8 \\
18.9 \\
16.6 \\
15.9 \\
16.0 \\
16.9 \\
17.7 \\
10.8 \\
6.6 \\
15.1 \\
17.0 \\
13.3 \\
18.8\end{array}$ \\
\hline
\end{tabular}

more systematic methods in tuning the beam through the extraction systen.

It is well known that when the value of the radial focusing frequency, $v_{r}$, is close to unity, the separation between orbits can be enhanced at a given azimuth by the introduction of a first harmonic component in the magnetic field. Such enhanced turn separation, if achieved at the electrostatic deflector entrance, normally leads to higher extraction efficiency, but, if it occurs at the wrong azimuth, it can drastically reduce the extracted beam. Turn separation enhancement would normally be achfeved by the use of harmonic coils; however, essentially the full capacity of the harmonic colls is used in canceling the harmonics introduced by the extraction system.

By computation with the beam setup programs, it has been discovered land confirmed by beam position measurements) that the compensated-iron extraction channel can produce a first harmonic component that suftably enhances turn separation when the channel currents are unbalanced. Using this technique, we have been able to: (1) extract beam at larger radif, reducing magnet power usage for a given energy; (2) improve average extraction efficfency from about $30 \%$ to about 70x; (3) improve the accuracy of the prediction of extracted beam energy: and (4) by careful adherence to the computer predicted settings, substantially reduce the time renutred to achfev $\geq$ extracted beam.

\section{Cyclotron Development}

Power Supplies. A progran to upgrade dc power supplies throughout the Holfffeld factlity is continuing. Several new SCR regulated power supplfes, including those for the cyclotron harmonic cotls and beam line bending magnets, were put in service during the past year. Thus far, our resilts with this type power supply have been very encouraging. The SCR regulated units rely upon high load reactance and the use of precision external sponsing elements such as high voltage shurits (1 volt or greater) or dc current transformers (transductors) to achieve good regulation and low ripple. The motor generator set (two 1.75 mVA generators), wich provides excitation for the cyclotron main field and compensated-iron magnetic channel outside coil, was overhauled. Its commutators were turned, ground, and undercut. This was the first major commutator servicing since the machine was installed about 24 years ago. Prior to the overhaul, we were experiencing magnet instability and short brush iffe (about 30 to 60 days). Stability has improved, and the brushes installed nearly a year ago are still in service.

RF System. The dee aperture was reduced from $2.5 \mathrm{cr}$. to $2.2 \mathrm{~cm}$ in order to increase dee liner spacing on each side by $0.15 \mathrm{~cm}$. The resultant reduction in dee to liner capacitance produced a slight shift in resonant frequency (an increase of $\sim 0.2 \mathrm{MHz}$ ) and a reduction in excitation power at the upper end of the tuning range. The rf system is now operable over a 6.69 to $19.8 \mathrm{MHz}$ tuning range. Previously it was tuneable but unstable above $18.0 \mathrm{MHz}$. An additional $0.2 \mathrm{~cm}$ aperture reduction is being consfdered with the hope of achieving a full 3:1 tuning range.

Vacuum system. Efforts by the manufacturer to achieve satisfactory performance from the 20-inch cryopump on the if resonator tank were unsuccessful. The pump and compressor made several return trips to the factory for modiflcations and repairs, but were still showing symptoms of excessive heat loading. Consequent ly, added a 16" aperture plate between the pump and gate valve wich serves as a heat, shield with only slightly increased pumping 
imedance. The pubs has now demonstrated several weeks of operation with acceptable pumpirg speed and base pressure.

\section{New onic Control Computer}

A mooconp Classic/55 has been purchased to replace the 12-year-old ORIC control computer. This processor was selected because approximately $90 x$ of the existing control programing will operate oit this sof tware compatible machine. The new hardware will insure the long-range availability of spare components as well as reliability of the system. of equal importance, the increased size of memory and disk storage will enhance the capebility for software development. This will relfeve the bottleneck that has been experienced in improving the operation monitoring software. Furthermore, operation parameter analysis prograns will eventually lead to improved understanding of machine performance. This will be achieved by making detailed comparisons between paraneters predicted by our wathewatical cyclotron model and the actual settings used to produce an ion beas. Additional prograns will be written that provide a data base for evaluating achine performance. This system is expected to be in operation by mid-1985.

\section{Improved Bean Ext.action tagnets}

We have shown that it is posstble to use the compensated-iron anetic extraction channel to provide a desirable first harmonic in the cyclotron magnetic field to provide tum-spacing enhancement for improved beam extraction. However, the computations required to do this are tedious and very timeconsuming and the deriveu settings usually require modification during cyclotron tuning.

The difficulty in accurately predicting settings of the cyclotron extraction system and haruonic colls resules principally from the imperfect design of the compensated-iron magnetic shannel and, to d lesser extent, of the coaxfal magnetic channel. The arrangement of the bean extraction system is shown in Ffg. 1.3. The bean extraction channels must pro$v$ ide magnetic field reductions of $\sim 6 \mathrm{~kg}$ along the extracted beam path wile producing very low external manetic fields (ideally $(10 \mathrm{G})$ in the beam acceleration region. Presently, both channels produce excessive first harmonic external magnetic field com-

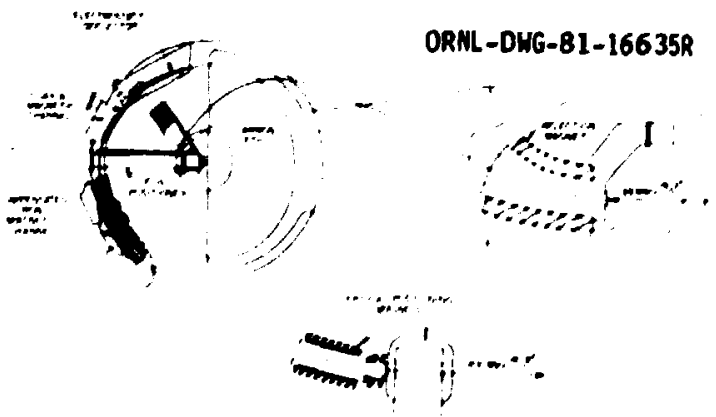

Fig. 1.3. Cyclotron beam extraction system, ponents. Design improvements are now in progress wich will result in a factor of 5 reduction in the harmonic contribution of the coaxial channel and an even greater reduction of the harmonic contribution of the compensatediron itannel. These improvements will effectirely separate the function of field reduction for extraction from the function of turn separation enhancenent, shifting the latter to the harmonic cotls as was originally intended. Separation of functions should simplify corputation and tuning procedures. Reduction of the external field of the coaxial megnetic channel can be accomplished by modifying only the portion of the channel known as the insert." This is a remable section wich contains the conductors closest to the bean. Reshaping the cross section and longitudinal configuration of these conductors, as show in Fig. 1.4 (a) and (b). reduces the first harmonic coefficient of the external field close to the channel by factors of 5 to 9 depending on distance from the channel (Table 1.8). Design studies and computations for this change are complete and engineering drawings are being prepared.

The proposed new design for the compensatediron channel (Fig. 1.5) is based on a cosine distribution of currents in two circuits inside and outside a cylinder of iron - with modifications to accomodate the realities of fabrication. The present design, in use since 1963, exploys a linear distribution of currents about a rectangular fron box ( $\mathrm{Fig}$. 1.6). The external fields of the two channel designs are compared in Fig. 1.7. For this

ORNL-DHG-84-16128

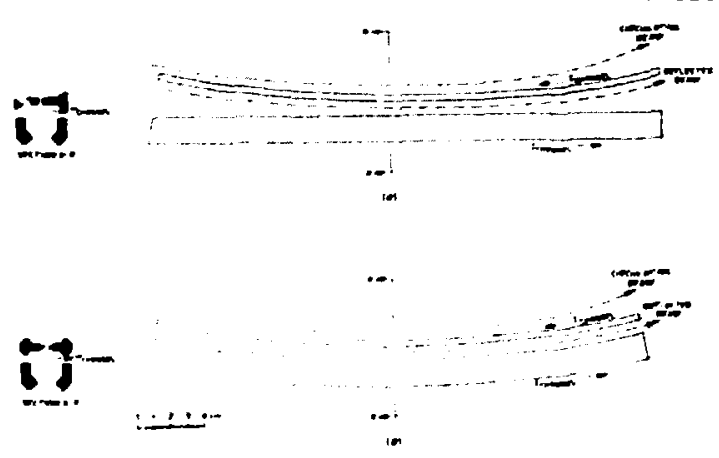

Fig. 1.4. Configuration of the present coax insert conductors (a), and of the proposed insert design (b). Both the detalied cross section and the longitudinal shape have been changed to achieve a lower field ill the circulating beam region.

Table 1.8. External field of coaxial magnetic channel

\begin{tabular}{lcc}
\hline & $\begin{array}{l}\text { 1st harmonic-gauss } \\
\text { Rresent } \\
\text { Radius-centimeters }\end{array}$ & $\begin{array}{c}\text { Proposed } \\
\text { coax }\end{array}$ \\
\hline 76.2 & 1.0 & 0.2 \\
81.3 (coox entrance) & 14.3 & 1.6 \\
\hline
\end{tabular}




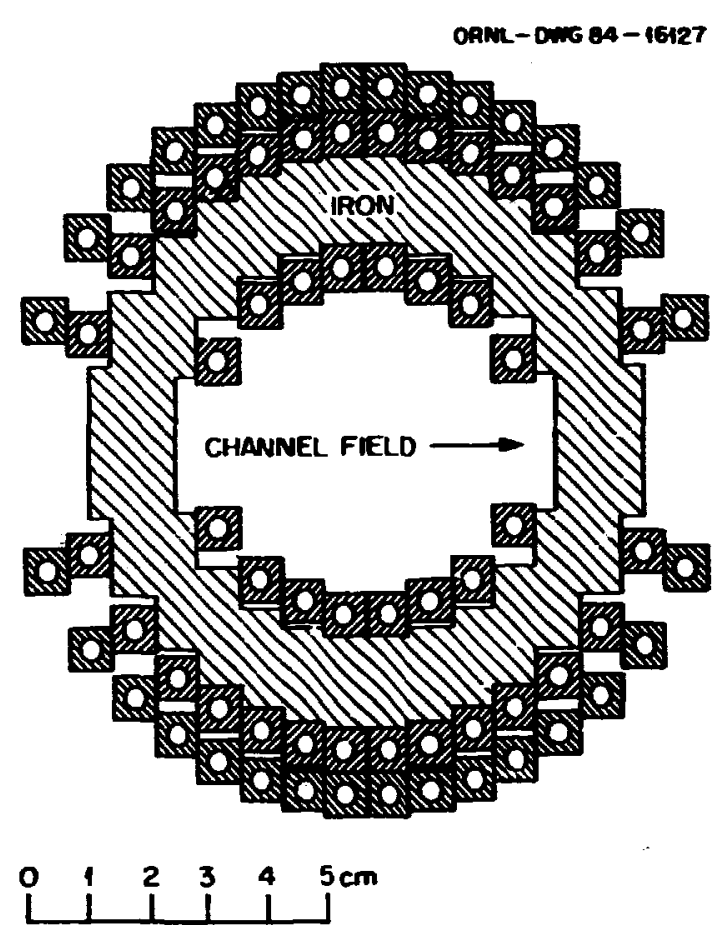

Fig. 1.5. Cross section of proposed new compensated-iron channel. Two layers of coils in one circuit are used inside the fron cyl.nder. The layer of colls zutside the iron forms a second circuit.

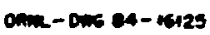

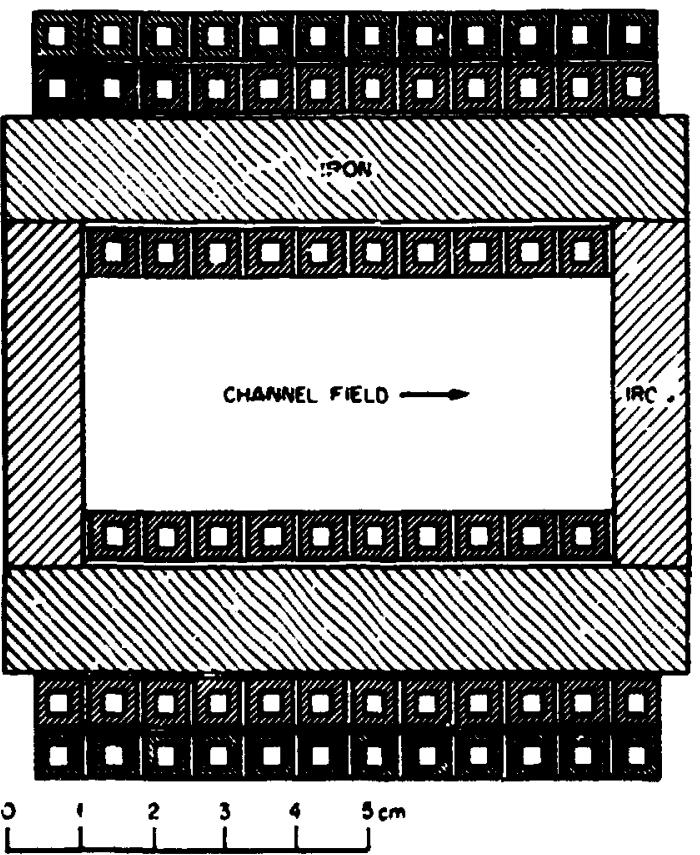

Fig. 1.6. Cross section of the existing compensated-iron channel with inside and outside colls.

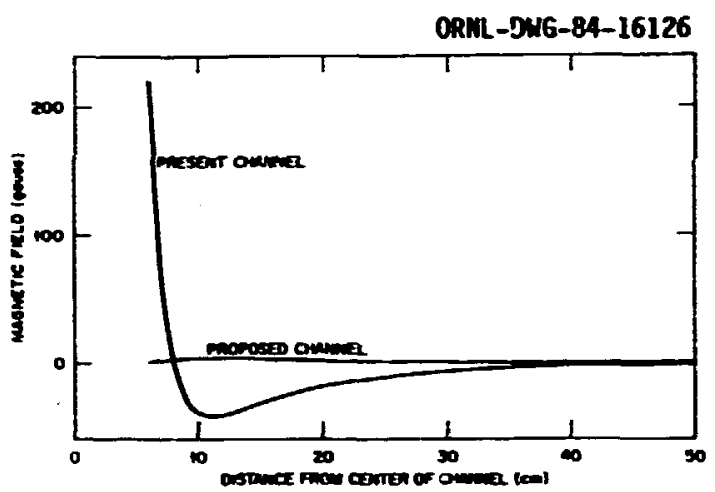

Fig. 1.7. External field of existing and proposed compensated-iron channels.

figure the currents of the proposed channel have been adjusted to produce zero field $6 \mathrm{~cm}$ from the center of the channel - approximately the location of the closest circulating beam orbit. Using the present channel, the best that can be achieved in minimizing the external field gives zero field at $B$ ca and a substantial deviation from zero orer a wide region. The external field of the present channel varies with the level of field reduction required for the extracted beans and must be largely compensated by the harmonic colls, leaving little or no ability to control turn separation with these colls.

Design studies for the new compensated-iron channel are nearly complete and engineering will begin soon.

\section{Dual Are Penning Ion Source Gas Flow Experfments}

Previous experiments have shown that the addition of an easily ionized heavy gas (krypton or xenon) to the arc chamber of a Penning discharge fon source increases the output of aulticharged lighter fons, for example, nitrogen and oxygen. 2,3 In a refinement of this technique, an auxiliary arc chamber, for support gas, was added to the ORIC internal ion source. This also results in an increase in the beam intensity for miticharged fons such as $160^{5 t}$. To clarify the mechanisa of this intensity increase, gas flow rates from the auxiliary chamber to the wan chamber have been measured by using the ORIC cyclotron as a mass spectrometer. The results (Fig. I.8) show that only about three percent of the gas admitted to the auxiliary chamber reaches the main chamber. One can then infer that the improved operation probably results from the stabilizing effert of heating the common cathodes with the ouxiliaiy arc and/or the more favorable distribution of the support gas to the part of the moin arc close to the cathodes.

1. Consultant. 
2. E. D. Hudson and H. L. Mallory, Mucl. Instrum. Methods, 141, 381 (1977).

3. E. D. Hudson and H. L. Mallory, IEEE Trans. Mucl. Sci. MS-24, No. 3, 1590 (1977).

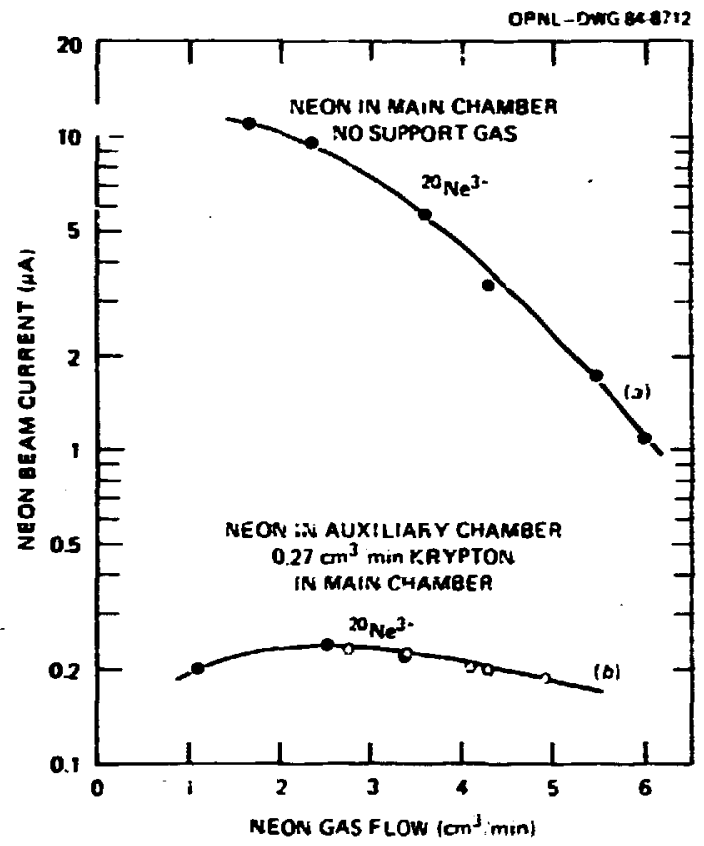

Fig. 1.8. Curve (a) is the intensity of $20 \mathrm{ke}{ }^{3+}$ wen neon is admitted to the min chaber with no support gas. Curve (b) is the ${ }^{20} \mathrm{Ne}^{3+}$ intensity with krypton support gas in the main chamber and neon in the auxiliary chamber.

\section{MEGATIVE ION SOURCE DEVELOPNENT}

\section{Evaluation of the Axial Geometry} Negative Ion Source

\section{G. D. Alton}

The radial geometry negative ion sourcel used in routine tandem accelerator operations can be readily converted to an axial geometry source by an almose trivial modification. The source, wich has been briefly described previously. i is the second iteration of a sintlar source, destgned and developis in 1978, with superior mechanical destign features and rather different performance characteristics. The present source utilizes a solid rungsten annular tonizer with a noninductively-wound tungsten heater instead of the helicallywound tantalum heater wich was incorporated in the orfginal source for producing the postefve cestum inn bedm used to sputter the moterial of intertst. This fontzer was chosen because it offers a simple but well-defined ton generation surface wich is more amenable to numerted andysis than the conventtonally used helical geonetry ionizer. In the present design, ions are generated fron the inner surface of the ionizer and all other heated surfaces are obscured from the electric field region of the source. Consequentiy, the sputter pattern exhibits only a meak halo surrounding the usual concentrated mear pattern located on axis.

The geametrical configuration is readily asenable to simulation by solving Poisson's equation numerically for the electrode systea. Computer assisted design techniques were exployed by performing detailed calculations of the positive and negative ion trajectories through the electrode system prior to expenditure of funds for engineering or construction of component parts of the scurce. An example of the positive ion optics of the ionizer/sputter probe region of the source is show in Fig. 1.9. The computed positive ion current density resulting from the positive ion fapact-is shom in Fig. 1.10.

ORHL-DWG 83-7648BR

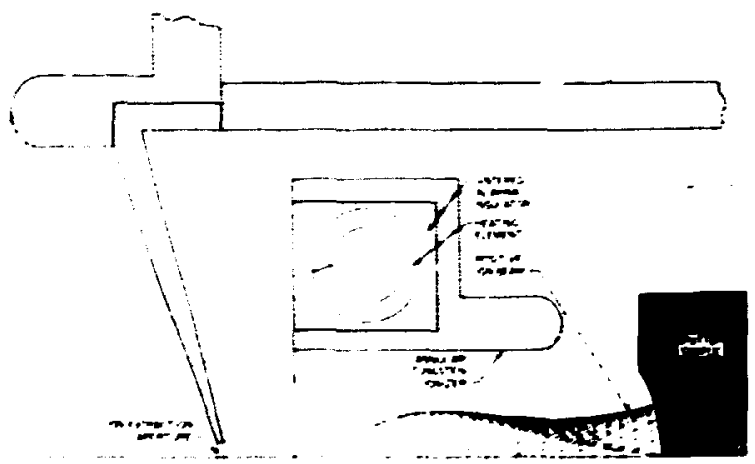

Fig. 1.9. Positive ion cotics of the ionizer/sputter probe region of the axial plases negative ion source.

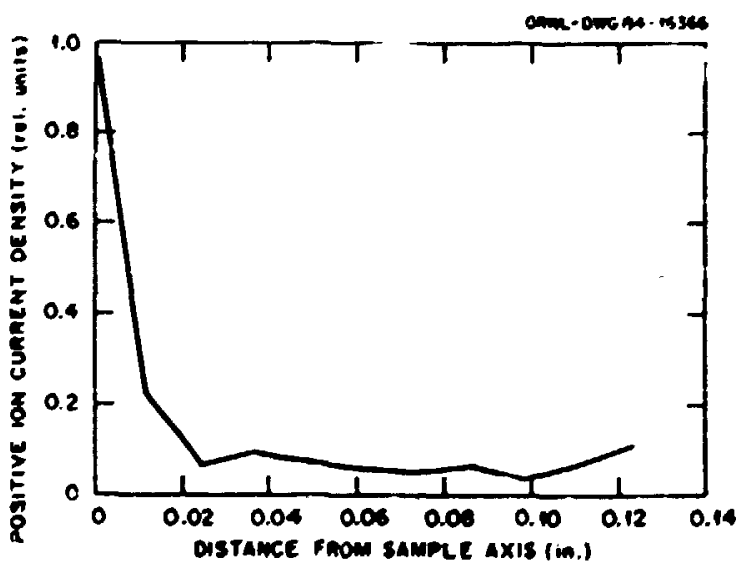

Fig. 1.10. Varlation of the postetve ion current density with distance from sample axts in the artal geometry negative ion source. 
The source performed imediately and as predicteo, without further development. For example, the observed sample mear pattern was found to be identical in almost all details with that predicted fron Fig. 1.10.

Test stand operational experience with the source indicates that the source is reliable, long-lived, stably overating and a prolific producer of a wide spectrum of negative ions. The source has been used to produce beans of $\mathrm{Ag}^{-}, \mathrm{Au}^{-}, \mathrm{Cu}^{-}, \mathrm{Lu}^{-}, \mathrm{No}^{-}, \mathrm{Mi}^{-}, \mathrm{Tm}^{-}$, and $\mathrm{Kb}^{-}$, with respective total negative ion bean intensities of $16,50,57,0.37,1.4,59$, 0.10 , and 5 wh then operated at cptimim cesium oven temperatures and at 1000 volts ic impressed on the sputter probe. Negative ion yields exhibit axim at an optinu cesium oven temerature of $\sim 260^{\circ} \mathrm{C}$. This behavior is identical to that observed for the radial geometry source. The total negative ion yields from this source increase strongly with mitage over a range of probe voltages up to 1100 id according te there $V$ is the probe potential and $h$ is a muber with value 3.5 to 4.0 .

The source has been incorporated as an onIine source along with the radial gecuetry source for routine $25-\mathrm{ml}$ tandea operation.

Pulsed Inegative Ion Source Developments

$$
\text { 6. D. Alton }
$$

Consideration of a synchrotron as an energy booster for HHIRF tandea accelerator fon beans and as a high energy, heavy ton collider for which the tandem accelerator would serve as an injector has stimulated interest in developing pulsed-iode negative ion sources. During the period of this report, a project was inttiated to divelop and evaluate negative ion sources and the assoctated pulsing technology required for injection into such accelerators. Typical pulse widhs and repetition rates for synchrotron infection applications are $\sim 100$ usec and a few $\mathrm{Hz}$, respectively. Oue to interbean scattering effects in the synchrotron, maximum bean intensities will be linited to pesk currents of - 200 uh at injection into the tandem accelerator - an intensity wich. however, far exceeds the of megative ion bean intensities of existing negative heavy ion scurces. This requirement poses a challenge for the production of a wide variety of negative ion beans of this monitude for pulsedmode operation. However, it is known from previous work at the Brookhaven Iattonal Laboratory 2 that such oulsed bean intensities can be produced for a linited number of elements with high electron affinities and that si ch large, low duty-cycle beams can be fi.jecred into type tandem accelerators without detrimental effects to the operational stabflity of the accelerator. The pulsed source development project presently underway was initiated with the objectives of evaluating the performance of existing negseive ion sources in pulsed mode and development of pulsed beams for tests using the HHIRF tandem accelerator.
An off-ground platform was acquired for housing standard power supplies as well as the spectal pulsed high-roltage power supply required for source operation in the pulsed code. A pulsed high-voltage power supply with the desired cutput-characteristics, in terns of wltage aplitude, pulse width and repetition rate, was located within the Physics Division.

Both the radial and axial geosetry cesiun plasm negative ion sources, described in the previous report, were evaluated. A schematic diagran of the power supply arrangenent utilized during testing is shom in Fig. 1.11.
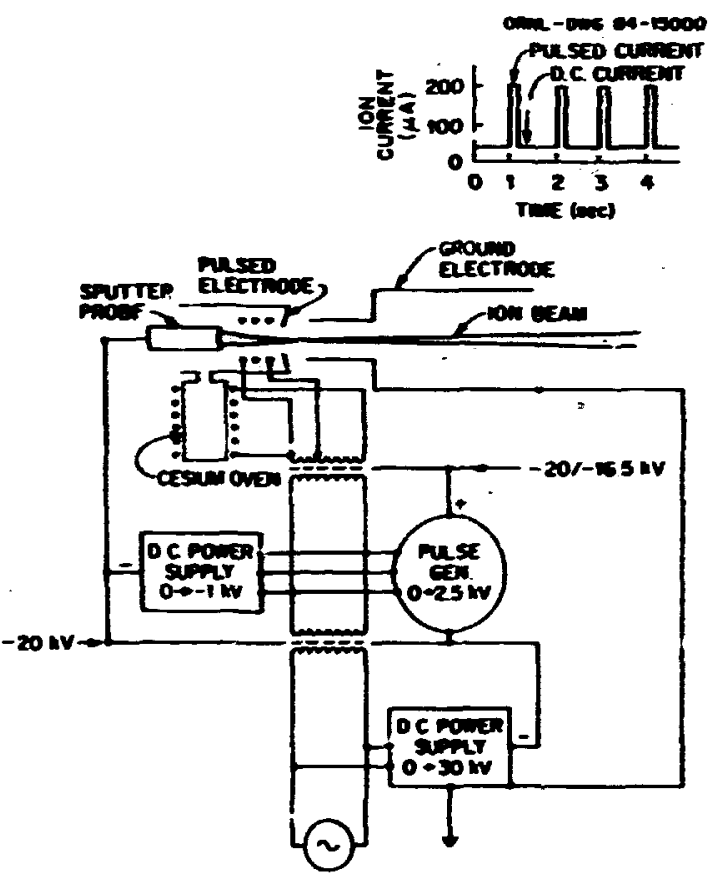

Fig. 1.11. Scheeatic electrical diagram of the pmer supply arrangement used during pulse testing of megative ton sources.

hegative foas are gerated in these sourres by cesfum ton sputtering of a probe containing the aterial of interest which is biased negative by 1 - $3 \mathrm{kV}$ relative to the source housing. The probe potential is wintafined at a de blas level for standard operation and a combination of a de potential and superposed ac potential from the pulser power supply for pulsed operation. With the arrangement show, a de bean of modest intensity (6 1 wo. eypically) can be generated for use as a signal for tandem teriminal voltage conerol and as a bean for cpefinization of beaw line optical elements to assist in eransatssion of the superposed pulsed bean through the accelerator and bean transport system.

'reliminary comparative pulse te- ing of the radial and axtal geometry sources showed the distinct superiority of the axial geometry 
source for puised-mude generation of $\mathrm{Au}^{-}, \mathrm{Ki}^{-}$ and $\mathrm{Cu}^{-}$negative ion beams. Uuring testing, the pulser amplitude voltage was operated at $1.2 \mathrm{kV}$ with a pulse width of $\sim 100 \mathrm{usec}$ and a repetition rate of $10 \mathrm{~Hz}$. The negative ion yields from the radial geometry source for the indicated elements were typically $\sim 60$ uA/pulse while those from the axial geometry source were much higher. Negative ion yield spectra, shown in Fig. 1.12, from the indicateu elements were 100,250 , and $350 \mathrm{uA}$ reak pulse intensity for $\mathrm{Au}^{-}, \mathrm{Ni}^{-}$, and $\mathrm{Cu}^{-}$, respectively.

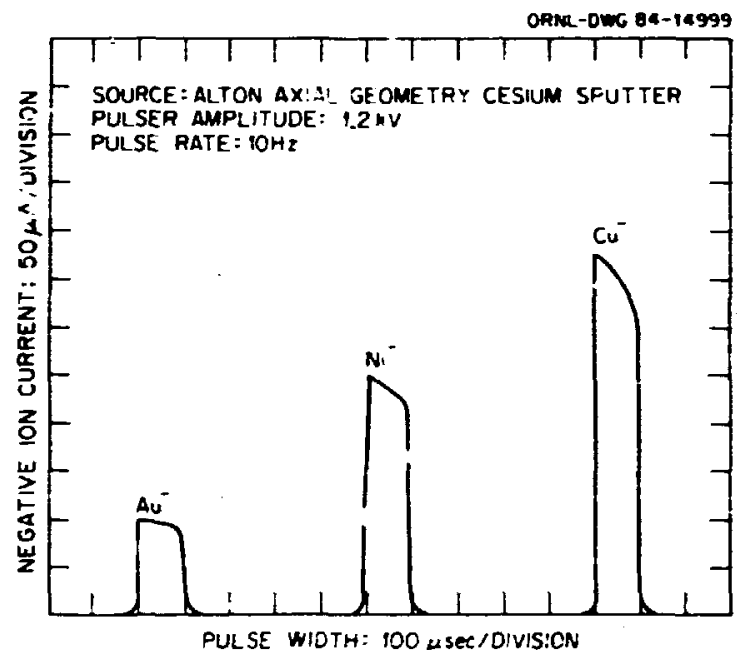

Fij. 1.i2. Negative ion yields of $\mathrm{Au}^{-}, \mathrm{Ni}^{-}$ and $\mathrm{Cu}^{-}$from the axial geometry negative ion source operated in the pulsed mode.

The negative ion yields from the axial geometry source were found to vary linearly with pulse voltage amplitude and, in most cases, the pulsed amplitude and dc amplitude were found to be essentially independent of each other, i.e.. the dc beam could be reduced without affecting the amplitude of the ac component. This independence is very important in practice because a weak dc beam component is desirable for control and mnicoring of the accelerator while transmitting the largest possible ac beam component wich is compatible with synchratron reouirements. The voltage amplitude from the pulsed power supply was limited to $-1.5 \mathrm{kV}$ during testing due to stress limitations of the high-voltage cabling. Future tests will include operztion of the source at pulse voltages up to $4 \mathrm{kV}$ and injection through the 25. MV tandem accelerator. Much higher beam intensitfes are anticipater at the higher voltages and therefore we expect a wider range of negative ion species with intensities adequate for zynchrotron utilization.

Production of $\mathrm{Ca}^{-}$Throisgh Double Charge Exchange With L+ Vapor

$$
\begin{aligned}
& \text { G. D. Alton T. J. Kuale } \\
& \text { D. J. Pegg }
\end{aligned}
$$

Neyative fon formation through sequent tal double charge exchange interact fons between :a initially positive energetic ion and a suitably chosen exchange vapor of fers perhaps the most universal and efficient means of producing negative ions known to date. Production efficiencies depend primarily on ion energy, the electron affinity of the element under consideration, and the electron binding energy and density of the exchange vapor. This mechanism offers, as well, a practical and efficient means of producing useful negative ion beams tiom elements which have negative electron affinities. These ions of ten may be formed with: relatively high efficiency in metastably-bound excited states wich may live long enough to be of use for accelerator applications. The Group IIA elments are examples of jons that are well suited for charge exchange production since they are difficult to produce by other means.

The Group IIA elements (Be, Mg, $\mathrm{Ca}, \mathrm{Sr}$, and $\mathrm{Ba}$ ) have negative ground-state electron affinities and thus do not form stable groundstate atomic negative ions. Therofnre, other techniques must be employed for the production of these elements. For example, some of these elements ( $\mathrm{Be}^{-}$and $\mathrm{Ca}^{-}$) can be formed through charge exchange in metastably-bound, autoionizing states wich have lifetimes long enough to be of practical value. 5,6

A major experimental effort has been initiaced during this fiscal year using the Negative Ion Source Test Facility (NISTF) ${ }^{7}$ and the universal charge exchange source. I These experimental investigations are designed to measure production eficiencies as a function of exchange vapur density and projectile energy as well as the atomic properties of the negative ions generated. Calcium was chosen as the first of the Group IIA elements to be investigated because of the considerable interest in this species for nuclear and atomic physics research. Production efficiencies as a function of exchange vapor density and projectile energy over an extensiv': energy range have never been reported for this ion.

The Negative Ion Source Test Facility has been extensively modified to accommodate research on the charge exchange mechanism and the properties of negative ions generated by this mechanism. In addition to the universal charje exchange source described previously, the facilitty has been equipped with a post momentum analysis charge exchange cell, the ancillary equipment necessary to perform such measurements, and a CAMAC-based data multichannei analysis/microprocessor system for data acquisition and analysis.

A schematic diagram :- the experionental equipment used in measuring the production efficiency of $\mathrm{Ca}^{-}$is shown in the acceleratorbased atomic physics section of this report and therefore will not be included here. A momentum analyzed beam of $\mathrm{Ca}^{+}$is focused by means of a lens through the charge exchange cell and the emerging positive, neutral and negative ion components are separated by means of a specially designed electrostatic deflection system. The negative and positive ion beams are monitored in biased and shielded Faraday cups immediately following the deflection plate system. Two experiments 
are in progress wich deal with the measurement of the production efficiencies and autoand collisional-detachaent properties of $\mathrm{Ca}^{-}$ ions formed in double-charge exchange with neutral lithium vapor. The first of these experiments is described here; the latter e:periment is discussed in the acceleratorbased atomic physics section of this report.

Two principal determinations are of practical importance in the production of negative ions through charge exchange: (1) the measurement of is oroduction efficiency as a function of iarget thickness, and (2) the dependence of the efficiency on prcjectile energy.

Preliminary results frow these measurements for the production of $\mathrm{Ca}^{-}$in lithium vapor are shown in Figs. 1.13 and 1.14. The optiman efficiency for this interaction occurs at a cell temperature of $\sim 625^{\circ} \mathrm{C}$ for these data, as noted in Fig. 1.13. However, the optinum cell temperature is expected to be projectile energy dependent. This dependence is presently being investigated. The efficiency versus projectile energy is shown in Fig. 1.14. Hork on this project will continue during the next fiscal year.

ORNL-DWG 84-46101

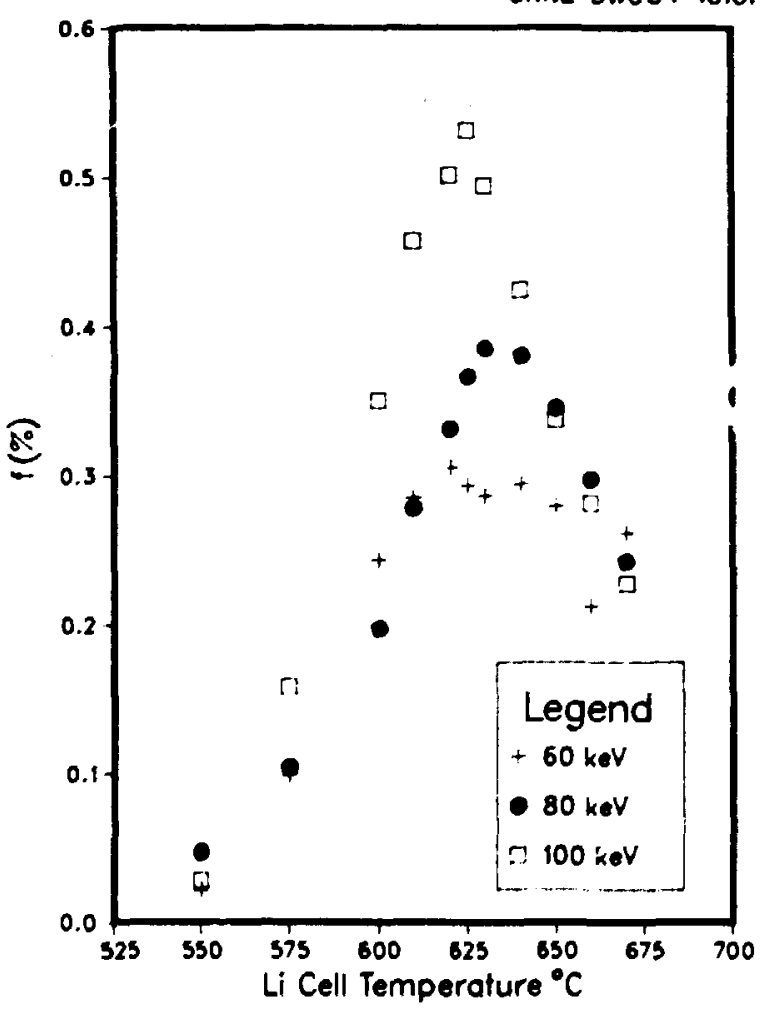

Fig. 1.13. Ca- production efficiency of as a function of $\mathrm{Lf}$ charge exchange cell temperature.

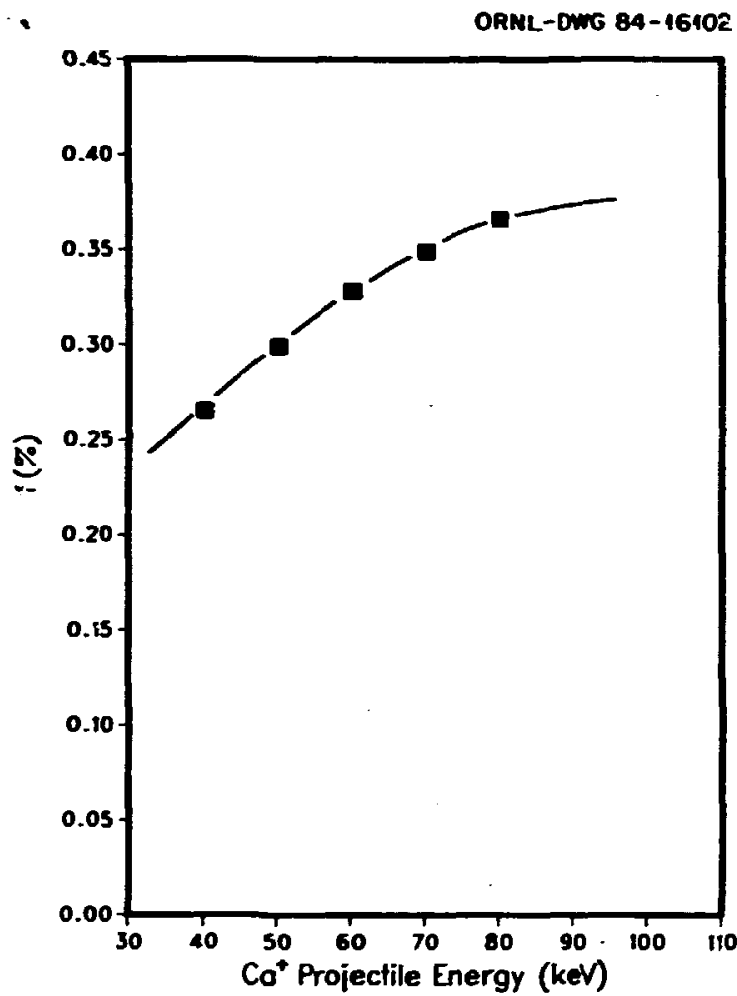

Fig. 1.14. Dependence of the optimum efficiency on $\mathrm{Ca}^{+}$ion energy.

\section{Sputter Ratios for $0.4+50 \mathrm{keV} \mathrm{Cs}{ }^{+}$ Ions Using Sigmund Theory}
G. D. Alton
D. H. 01 ive

In recent years, the sputter process has been utilized in positive ion source applications to provide vapor for subsequent electron impact ionization and in negative ion sources as a means of ejecting neutral particles through a surface covered with minute amounts of Group IA elements in the production of negative ions through surface ionization processes.

The type and quantity of secondary ions emitted as a consequence of sputtering is affected by the work function as well as by damage-induced effects. Posftive surface ionization is enhanced by raising the surface work function, wille negative surface fonization is enhanced by lowering the surface work function. In recent years, the sputter process, in combination with the work function lowering aspects of Group IA elements, has been utilized as a versatfle and relatively effictent means of producing negative ions. In such sources, cestum is usually utilized hecause of ease of vapurization, high sputtering coeffictent and greater work function 1 , iering capabilities. 
Although there is a considerable amount of -xperimental sputtering data available for a variety of energetic projectile-target combinations. there is little data available for the sputtering of solids with cesium ions. Such data is important to the negative ion source specialist wo mst estimate the lifetime of samples subjected to sputtering and minimize the amount of terial require.J for a particular negative ion generation period. Hence, for such applications, there is a distinct need for a relatively accurate means of predicting cesium ion sputter coefficients - the primary objective of this report.

To date, the most widely accepted theory of sputtering is the Sigmund theory ${ }^{9}$ wich assumes that the sputtering of target atoms is a consequence of the formation of cascades of elastic atoafic collisions set in motion by energetic ions as they slow down in a homogeneous medium.

By solving the Boltzmann transport equation and using results from linear cascade theory and elastic scattering theory, Sigmund was able to extract andytical formulas appropriate for low- and high-projectile energies.

The sputter coefficient, $S$, relations appropriate for the indicated ranges of projectile energies are

$S(E, \theta)=\frac{3 \lambda a E}{\pi^{2} 4 U_{0}(\cos \theta)^{F}}($ for $E \leqslant 1000 \mathrm{eV})$

$S(E, \theta)=\frac{0.042 a S_{n}(E)}{U_{0}(\cos \theta)^{F}}$ (for $\left.E>1000 \mathrm{eV}\right)$

where,

a is a tabulated function of the ratio of the target mass $M_{2}$ to projectile mass $M_{1}$,

$U_{0}$ is the heat of sublimation of the target,

6 is the angle between the incident projectile and the surface normal.

+ usually nas values beticen 1 and 1.7 , depending on the ratio of target to projectile masses.

$$
\lambda=4 M_{1} M_{2} /\left(M_{1}+M_{2}\right)^{2},
$$$$
S_{n}(E)=4 \pi Z_{1} Z_{2} e^{2} a_{12}\left[M_{1} /\left(M_{1}+M_{2}\right)\right] S_{n}(\varepsilon),
$$

$Z_{1}, Z_{2}$ are the respective projectile, target atomic numbers,

$\partial_{i 2}=0.8853 \mathrm{a}_{0}\left(z_{1}^{2 / 3}+z_{2}^{2 / 3}\right)^{-1 / 2}$,

ao is the radius of the first Bohr orbit,

$S_{n}(\varepsilon)$ is the projectile stopping power in the target.

$$
c=\frac{M_{2} E /\left(M_{1}+M_{2}\right)}{Z_{1} z_{2} \mathrm{e}^{2} / \mathrm{a}_{1} ?}
$$

While the theory predicts shapes of target sputter coefficients versus projectile energy in good agreement with experimental data. calculated magnitudes are generally in poor agreement with experfmental observation.
Experimentaliy, sputter coefficients are found to vary periodically wile monitonically increasing with projectile ass. 10 These deviations are, in part, attributable to the many approximations and deletions made in the derivation and the assumtion of a homogeneous distributed target wen in reality most targets are polycrystalline.

A computer code, 11 based on Sigmund theory, was used to calculate sputter ratios for cesium projectiles and a variety of targets is a function of energy for an incident angle of $\theta=0^{\circ}$. A scaled version of the high-energy relation (Eq. 2) was selected as the more appropriate expression to cuver the total range of interest. The exi ressions $\alpha$ and $S_{n}(E)$ were extrapolated to include the total array of naturally occurring projectiletarget coabinations.

Since there are few experimental data available for $C^{+}$ions on arbitrarily chosen targets and since there is a pronounced dependence of the scaling fartor on projectile, the task of determining the scaling factor was rendered wo difficult. However, the ratios of scaling parameters determined for $\mathrm{Cs}^{+}$ions and those obtained for $\mathrm{Xe}^{+}$ions at a particular energy were found to be fairly constant from target to target where both sets of experimental data were available. Over the range of experimental data a mean value $(R)$ of the ratio was found to be

$$
\langle R\rangle=0.83
$$

with a standard deviation $\sigma=0.1$.

This value of $(R)$ as used to determine scaling factors for the sigmund theory and, in turn, sputter coefficients for more than 20 targets over an energy range between 0.4 and $50 \mathrm{KeV}$. For targets where experimental data were avaflable, scaling factors were determined by comparing experimental results with the unscaled theoretical results. Figure 1.15 makes comparisons between experimentally determined and calculated sputter ratios for cestum ions on Al and Cu targets.

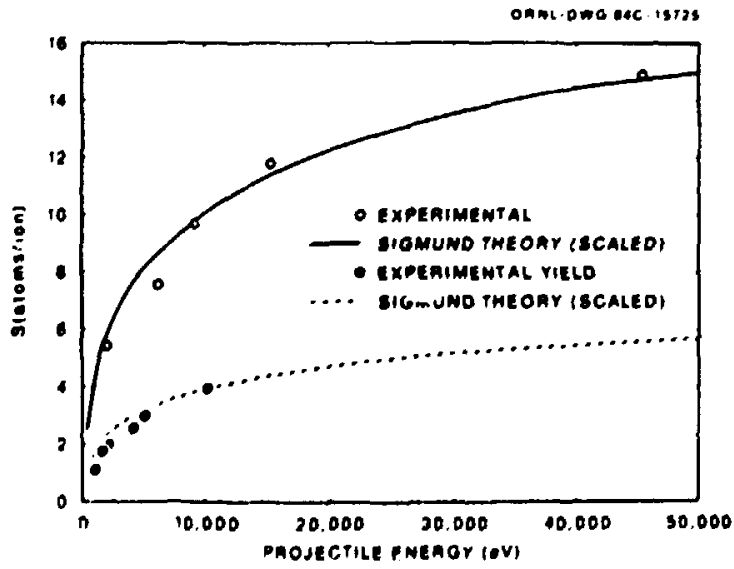

Fig. 1.15. Comparison of experimental and calculated sputter coefficients of ncrmally inctdent fons on $A l$ and $C u$ targets. (Calculated values are based rn scaled Sigmund theory.) 
Nort cont inues on this project wich is directed toward determining scaling parameters for arbitarily chosen projectile - target combinations.

1. Physics Division Progress Report for Period Ending September 30, 1983, ORHL-6004, (1983).

2. Thieberger, M. Mckeown, and H. E. Megner, IEEE Trans. Mucl. Sci. MS-30, Mo. 4, 2746 (1983).

3. CRN graduate laboratory participant frow the University of Missouri-Rolla, Rolla, mo 65401 .

4. Mdjunct staff amber from the University of Tennessee.

5. J. Heinemeier and P. Tykesson, Mucl. Instrum. Methods 141, 182 (1977).

6. W. Kutschera and G. Korschinek, Proc. Int. Conf. Ion Sources, 2nd, Vienna, 1972, D. 908 (1973).

7. G. D. Alton, et al., Physics Division Progress Report for Period Ending Decewber 31, 1974, ORML-5025, (1975), P. 192.

8. Sumier research participant, Oak Ridge Associated Universities.

9. P. Sigmund, Phys. Rev. 184, 383 (1969).

10. D. Almen and G. Bruce, Mucl. Instrum. Methods, 11, 257 (1961).

11. G. D. Alton (unpublished).

\section{BEAM BUICHER DEVELGPMENT}

$$
\begin{gathered}
\text { J. A. Martin W. T. Milner } \\
\text { N. F. Ziegler }
\end{gathered}
$$

A new beam buncher-chopper system is befing designed for the tandem accelerator wich wil provide pulsed beams for time-of-flight work in addition to bunching the beam for injection into ORIC. An fmoroved rf control system will also be added. The chopper will operate at two discrete frequencies of 0.5 and $2.0 \mathrm{MHz}$ providing beam pulses at intervals of 1.0 or 0.25 microseconds. Deflection plates for the chopper will be located i we low-energy beam line between the mass analyzing magnet and the buncher. A double-drift buncher simflar to the present system will be used in the new design; however, three tube lengths will be provited for more effictent operation. An expanded frequency range of 4 to $16 \mathrm{MHz}$ will be avaflable in the buncher. Hith the chopper operating at $2.0 \mathrm{MHz}$, about 25 percent of the dc beam will be compressed into pulses of 1 to 3 ns while operation at $0.5 \mathrm{MHz}$ will reduce the fraction of beam which is compressed to about 6 percent.

Engineering of the new pulsing system should be complete by December 1984, and installation will proceed in 1985.

\section{EFFORTS TO IMPROVE TANEA RCCELERATO: TUBE PERFORHAREE}

In recent wonths the voltage performance of the tandem accelerator tubes has shom encouraging improvenent. However, the accelerator tubes are still the principal Ifattation to achieving higher tandew woltages. In this section we sumarize work done wich could lead to eventual improved accelerator tube perfornance. Much of this wort was inspired by the results obtained at the Munt :h ip Tanden Laberatory.

Hydrogen Arc Discharge Conditioning

$$
\begin{aligned}
& \text { P. H. Stelson J. R. Raatzl } \\
& \text { R. D. Rathueli }
\end{aligned}
$$

Korschinek et al. 2 have shown that REC accelerator tubes can be more effectively conditioned by the use of a ?ou-voltage hydrogen arc discharge than by the standard methods used previously. Results from tests in a sall accelerator showed substantial improvements in voltage gradients from typical values of $20-25 \mathrm{kV} / \mathrm{cm}$ to values of over 40 $\mathrm{kV} / \mathrm{cm}$.

With Korschinek's advice and help, we assembled equipment to carry out hydrogen arc discharge conditioning at ORML. After a period of fawiliarization, the equipment was transferred to the MEC 3-MV test accelerator where voltage tests could be carried out. However, the MEC accelerator could not achieve the colum voltage gradient of $\mathbf{4 0}$ $\mathrm{kV} / \mathrm{cm}$ of the Munich test accelerator but was limited to a maximum value of slightly under $30 \mathrm{kV} / \mathrm{cm}$.

With the reservation that accelerator tube tests suffer from very limited statistics and many vartables, we sumarize our results with the following points.

1. The difference in the inttial behavior of arc discharge condtioned tubes and normal tubes is striking. The familiar microdischarges exhibited by normal tubes are largely el iminated in arc discharge conditioned tubes. There is essentfally no vacuum activity.

2. The maximum voltage gradieit is quickly achieved with tubes conditioned by the are discharge. The column gradient proved to be the linitation.

3. Cont fnuous $X$ rays were frequently observed when high-voltage gradients were applied to arc discharge conditioned tulues. The intensity of these $X$ rays could abruptly change. Once the continuous $X$ rays were initiated, they persisted at lower voltage gradients, where they had not inftially been observed. It is not clear mether these continuous $X$ rays will prove to be troublesome in the use of arc discharge conditioned tubes to accelerate ions. 
However, experience with operation of the HHIRF tande accelerator, wich is equipped with accelerator tubes wich also show cont inuous $x$-ray activity, suggests that continuous $x$-ray activity does not adversely affect operation of the accelerator.

4. The deterioration of accelerator tube insulators by sputtering of electrode material by the arc discharge was very slight. It is important to operate the discharge at a pressure of about 100 mTorr in order to keep the mean-free path of the ion short so that they s' rike the electrodes with loner energi 5 , thus reducing the sputtering yield.

5. Arc discharge conditioned tubes ma be exposed to air with no obvious degradation of the vitage gradient performance.

An explanation for the effectiveness of the arc discharge in conditioning accelerator tubes is that it causes the inner parts of the tube electrodes to be heated to temperatures as high as $500-700^{\circ} \mathrm{C}$, and that this procotes outgassing and cleaning of the electrode surfaces. However, based on our test results, ve prefer a somewhat different explanation. The most ilear-cut result from our tests is the almost complete el imination of microdischarges following arc discharge conditioning. Although hydrogen gas was originally choser. to minimize electrode sputtering, it has other important virtues. From Tokamak wallcleaning research, it is known that the hydrogen discharge is a very effective way to clean carbon and heavy hydrocarbons from surfaces by chemically producing methane, whereas, simple heating of surfaces does not remove these substances. The good removal of carbon and hydrocarbons from the electrode surfaces eliminates the most likely source of negative ions needed to initiate the familiar aicrodischarge.

\section{Compressed Geometry Accelerator Tube Tests}
J. R. Raatz:
R. D. Rathmell
P. H. Stelson
N. F. Ziegler

The success of the hydrogen low-voltage arc discharge conditioning of NEC accelerutor tubes suggested to Assmann et al. " the possibflity of using a "compressed geometry" by elimfnating the heater plates prevfously used for tube conditionting. This compressid geometry provides about 18 percent mo 'e insulation length in NEC accelerators. Tests in the small Munich test accelerator with this new configura: i un were encouragitig.

$\therefore$ :ana.irat stmilar "compressed geometry" accelerator tube was installed in the NEC 3-MV test accelerator. The tube was first subjected to the normal conditioning procedure of gradually increasing the voitage to gradually elfminate microdischarges. This procedure alone was quite successful and demonstrated that the tube would support total voitages witch were about 18 percent higher than those for a tube of standard geometry. Since the accelerator tube had achieved a well-conditioned state without using a hydrogen arc discharge, the result from running an arc discharge in the tube is somewhat inconclusive. Hevertheless, the hydrogen arc discharge conditioning was carried out on this tube and we could conclude only that it did not in any way liait the tube's performance. A detailed accovint of these tests is given in REC Internal Report Mo. 183, June 7, 1984.

The Munich iP Accelerator Are Discharge Conditioning Tests

$$
\text { P. H. Stelson M. F. Ziegler }
$$

In December 1983, the Munich 19 Laboratory performed a hydrogen arc discharge conditioning test on one section of the ip tandea accelerator. For this test they chose the section of the low-energy tube which is next to the terminal. This section had always been the weakest section for holding woltage gradients, typically supporting $3.5 \mathrm{~m}$. After conditioning by the hydrogen arc discharge, this section supported $3.9 \mathrm{~m}$, waking it the strongest section in the accelerator.

Encouraged by these results, plans were formulated to condition and test the whole accelerator during a four-week period beginning in July 1984. ORNL was invited to participate in this program.

The hydrogen arc discharge was run on one half of the MP accelerator tubes at a time. First, the low-energy tube was conditioned by operating a hydroyen arc of about 4 amperes for three hours. The high-energy tube was conditioned in a sinilar way. In both tubes the hydrogen gas was admitted at the opposite end of the tube from were the electron enitting filament and pump were located. During both discharge operations, an unanticipated difficulty was encountered. Gas wich evolved from the tube during the initial operation of the discharge pois ned the $f 3_{3}$ ment. For the low-energy end, the cathode filament was replaced by a new one. For che high-energy end, the cathode was operated at a much higher than normal temperature to achieve a current of 4 amperes. In future conditioning $c^{*}$ tubes, it would be advisable to admit the hydrogen gas at the end of the tube where the ffiament is located to avofd exposing the f $\neq$ lament to the evolved gases.

After two weeks of testing the accelerator, the general concensus of the Munich accelerator staff was that the arc discharge conditioning had only slightly improved the accelerator tube performance. A possible reason for this disappointing result is that such a long accelerator tube system may requite a proportionately longer time for oper an of the are discharge in order to clear the tube. In contrast to our results with much shorter tubes, the Muntch tests showed that microdischarges were still encountered after the arc condtitioning.

1. Nattonal Electrostatics Corporation, Middleton. WI 53562 . 
2. G. Korschinek, J. Keld, A. Isoya, $H$. Assmann, and $H$. Minzer, Mucl. Instrum. Methods Phys. Res. 220, 82 (1984).

3. W. Assann, G. Korschinek, H. Minzer, Mucl. Instrum. Methods Phys. Res. 220, 86 (1984).

\section{FACRITY OPERTION AD IEVEOPFET}

\section{EXPERIMETIAL APPARATUS}
R. L. Auble
M. R. Johnson
E. E. Gross
F. E. Obenshain
H. L. Halbert
D. Shapita
J. H. Johnson
E. H. Spejewskil
P. D. Miller
H. J. Kin

The major experimental devices at HHIRF were described in detail in the previous Progress Report ${ }^{2}$ and only those having significant changes in the past year are discussed in this section. For reference, the layout of the experimental facilities is given in Fig. 1.16.
Split Pole Magnetic Spectroneter

During the past year, a new dedicated power supply for the dipole mgnet ws delivered and installed. At present, the spectrometer scattering chamer and associated bean line serve as the staging area for a polarized target facility which is now nearing completion.

\section{On-Line Isotope Separator}

Since the last report, significant progress has been mide on the He-jet and laser systems. both of which have been under development for several years. Briefly, tests of the He-jet successfully dewonstrated the power of this device for studying short-lived ( 1 sec) activities. Further development is needed, however. to reduce the helium current in the separator and to improve the overall effictency. The laser facility, wich was developed to weasure the atomic hyperfine structure (hfs) of separated isotope beans, ws used successfully in a study of $192 \pi$. In this case, a bean of about $10^{5}$ atoms/sec allowed measurement of the hfs spectrum in a tter of minutes.

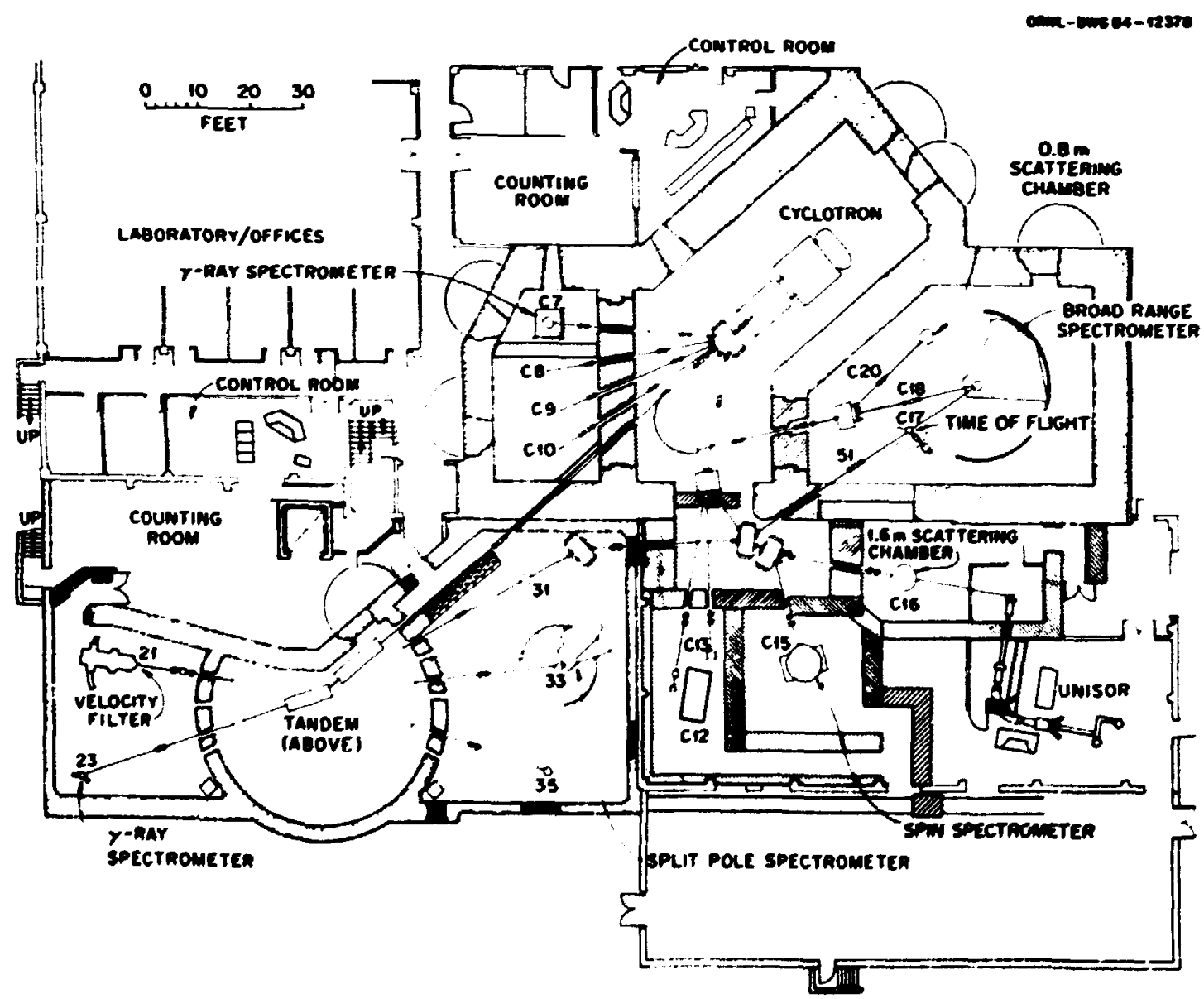

Fig. 1.16. Floor plan of the experimental areas at the HIRF. 
The results of the initial experiments on these devices are described in mre detail el sewhere in this report.

\section{Broad Range Wagnetic Spectrometer}

Improvenents include the installation of a $1500 \mathrm{i} / \mathrm{s}$ cryopuin on the manet vacuun chander and the addition of dedicated gas handling systens for the focal plane detectors. A test run has now been ade to demonstrate the usefulness of the pas for studying heavier particles. Normally, a parallel plate avalanche detector, located between the scattering chamber and the agnet, is used for time of flight measurements. However, for heavier projectiles, in this case $58 \mathrm{Ki}$, the energy loss in this detector becomes unacceptable. Therefore, the PPND ws replaced by a microchannel plate detector wich detects secondary electrons from a 10 $\mu \mathrm{g} / \mathrm{cm}^{2}$ carbon foil located near the exit port of the scattering chamber. This detector, in conjunction with the large PPAD located at the focal plane, provides $\sim 700$ psec time-of-flight resolution and allows us to separate irdividual Mi isotopes.

\section{The Spin Spectrometer}

Inprovenents. The spin spectrometer, the HHIRF"'s "Crystal Ball," has undergone a number of improvements in the past year. The principal ones are as follows.

1. The electronics equipment has been moved to the new south counting room, wich is described in this report. This has made experimental setup much more convenient.

2. Six pentagonal kaI Compton-suppression shields for Ge detectors are now available and have already been used in several experiments. Additional shields of both $\mathrm{NaI}$ and $\mathrm{BGO}$, are under construction. A more complete description of these units is given elsewhere in this report.

3. The exit beam pipe has been modified to allow rotation of the scattering chamber and facilftate installation of auxiliary detectors.

4. The 15 radial rods supporting the $\mathrm{NaI}$ detector array from the oster dodecahedral frame were replaced by largerdiameter rods, and the mechanica: coupling to the frame was improved. The result is a much stiffer and more accurate support system.

Problems. From October 1981 to May 3983 we observed a gradual deterforation of the resolution of the Nal detectors. During this time the average resolution over all units for $898-\mathrm{keV}$ $r$ rays increased from $8.4 \%$ to $9.2 \%$. However, from April to August 19B4, a rapid degradation of performance was observed for most of the detectors. An inspection in August 138: revealed that about 50 untts show moderate to severe cracking of the Nal that appears to have originated at the interface between the $\mathrm{Nal}$ and the glass window.
It is likely that the cracks are the result of two factors, possibly acting synergistically, nameiy rapid thermal cycling and shrinkage with age of the epoxy-cement interface. Evidence for the thermal effect is based on the differing history and degree of cracking of the two halves of the spin spectroneter ball. During the winter, the MaI units from the beam-right segment were stored in an area which suffered a failure of the heating system. Nearly all the MaI units from this talf were later found to be severely cracked. During this episode the beanleft half remained in the rave, well in the interior of the building, where the temperature fluctuations were smaller and slower. Only about $22 \mathrm{z}$ of the detectors on this half of the ball were severely cracked and 142 were moderately cracked.

Evidence for the importance of age is as follows. In the bean-right half of the ball. the two detectors that did not crack were both new or rebuilt within the previous year.

Serondly, it took several wonths after the original assembly of the ball before an air conlitioning unit as installed and during this tixe the temperature fluctuated with the seasons. Yet in the first two years of use, only one detector in the ball showed severe cracks; furthermore, its past performance suggested that it was already cracked before asseably of the ball. Also, at the MaI detector laboratory at Stanford University, this kind of cracking has occurred in all lal detectors with hard-epoxy joints after they aged about five years.

Megotiations are in progress with three firms to replace or repair sost of the detectors in the ball. The interface between the $\mathrm{NaI}$ and the glass will be a silicone-based elastomer instead of a hard-epoxy cement.

\section{Velocity filter}

Calibration of the power supplies has been completed for the maximum deflection angle $\left(10^{\circ}\right.$ electrostatic, $20^{\circ}$ magnetic) using an $\alpha-$ particle source and beams of $\mathrm{Ti}$ and $\mathrm{Zr}$. The electrostatic deflectors have al so been tested at $250 \mathrm{kV}$ ( $70 \%$ of the design limit) without breakdowns or excessive $X$-ray production. Hardware additions include a $10.2-\mathrm{cm} \times 12.7-\mathrm{cm}$ Breskin-type start detector for making time-offlight measurements, an ion chamber plus Sidetector counter telescope for $\Delta E-E$ measurement of the selected particles, and installation of new power supplies wich make the RMS operation independent of shared supplies.

\section{Neutron Chamber}

A recent addition to the experimental apparatus available to HAIRF users is a chamber designed primarily for the study of neutrons emitted in heavy-ion reactions. The chamber, which was designed by G. R. Young with technical support from C. A. Reed, seeks to minimize the amourit of material traversed by the neutrons before reaching the externally mounted neutron detectors. It consists of two spun aluminum hemispheres joined by an 0-ring seal to form a sphere $80 \mathrm{~cm}$ in diameter with $0.3-\mathrm{cm}$ wall 
thickness. The chamber is shom in Tis. 1.17 with the back henisphere rewoved to show the internal structure. Two independently rotatable rings are located inside the chemer to support charged particle detectors, and a target ladder is located on the vertical axis to provide space for mounting four targets. Several feedthrough and vieaning ports are also available, and custon flanges can be readily fabricated. As is erident in the figure, the chaber has been used successfully in several experiments.

1. Oak Ridge Associated Universities.

2. J. L. C. Ford et al.. Physics Division Progress Report, September 30, 1983, ORM-6004, P. 20.

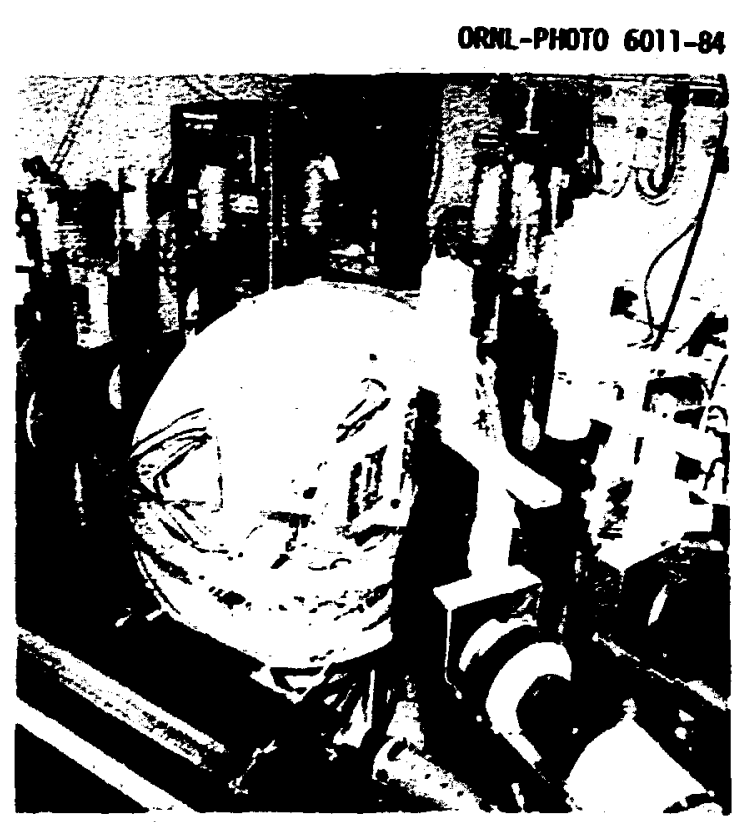

Fig. 1.17. Photograph of the BO-cm neutron chamber with the back hemisphere removed. Liquid scintillator neutron detectors are arranged around the outside of the chamber.

\section{COMPUTER SYSTEMS}

$\begin{array}{ll}\text { J. A. Biggerstaff } & \text { J. B. MeGrory } \\ \text { W. H. Atkins } & \text { W. T. Mflner } \\ \text { J. R. Beene } & \text { R. O. Sayerl } \\ \text { J. W. MCConirell } & \text { C. H. Thomas }\end{array}$

Data Acquisition/Reduction Computer System

The hardware configuration of the data acquisition/reduction system is essentially unchanged from that reported previously. 3 He have added 6250-bpf tape drive (for atal of 7) and color graphics terminal (for total of 6). A monochrom. printer-piotter has been acquired and is dedicated to service of a new counting station in the "deep-south" annex.

We have fully comissioned a new data processing software package called "CHIL" (for Comprehensive Histogra-ing Instruction Language). CHIL provides ajor improvements in both the performance and the ease of use of our processing software without changing the basic structure of the systen described previously.3 Development of CHIL began with a description of a desirable achine instruction set for data processing with ephasis on tools for "gating" and "histograming." These new instructions were implemented in wicrocode for our processors and assembly language routines generated to invoke thes. A CHIL compiler ws witten to translate users" FoRTRA-like gating, histograming and control (EOTO, If, etc.) statements into a "achine-language" progran using the new instruction set. An thportant feature of chll is its robustness. Responsibility for "crash-proofing" the system was divided into two areas. First, the microcode as hardened against unexpected (fllegal) data values. secondly, to elininate the need for timeconsuming run-time tests, the CHIL compiler was desionned with thorough symtax and logic checking to insure that invalid instructions are never generated. User acceptance of the new package was so imediate and so trouble-free that CHIL has completely superseded all cur older histograming software.

\section{The VAx-FPS Dual Processor Computer System}

The VAX-FPS Dual Processor Computer System described in last year's report has become a widely used facility by both nuclear theorists and experimentalists to perform the extensive calculations needed to analyze and understand the heavy-ion physics experimental data produced on HHIRF. Several significant improvements have been made on the systew in the past year. A new unibus adapter has been adted to the $V A X 11 / 780$ to increase the mumer of ports avaflable to users to 32, and the muber of stations with direct access to the system has been increased accordingly. A new fast, sealed, $480-16$ disc has been installed, bringing the total disc storage capacity directly accessible from the vaX $11 / 780$ to almost 1 billition bytes. The fast menory of the FPS-AP 164 attached processor has been increased from $2 \mathrm{Mb}$ to $6 \mathrm{Mb}$. This will enable the system to handle much larger calculations, such as TDHF calculations for medium mass nuclef, and the heavy-ion coupled channels code ECIS will now fit on the NP 164. Hardware is now on order to provide ready access to the system from the Joint Institute for Heavy ion Research.

1. Compesting and Telecomincations Division.

2. Oak Ridge Assocfated Unfversities.

3. J. A. Biggerstaff et al., Physics Division Progress Report, September 30, 1983. ORNL-6004 p. $24 f f$. 


\section{FACILITIES}
R. L. Robinson
M. L. Halbert
R. L. Auble
D. Shapira
F. E. Bertrand
C. M. Thomas

\section{JIHIR Building}

The Hoifield complex was enlarged by coapletion of the second building of the Joint Institute for Heavy Ion Research in April, 1984 (Fig. 1.18). This $6000-\mathrm{ft}^{2}$ building has 12 offices, 2 laboratories, and an 80-person conference roon. Four of fices in this building are occupied by the UNISOR staff. The two picturesque trailers wich had served as their offices have been removed from the Hol ifield site.

\section{Counting Roons}

The tanden counting roon has been reconfigured to provide better access to the electronics racks and more work space. Patch panels linking the counting area with experimental stations $23,31,33$, and 35 are presently in place and a panel for station 21 (velocity filter) will be installed in the near future.
A third counting roow (south counting room) is now available. This counting room is located in the South Annex insediately outside the entrance to the spin spectroneter cave. Conputer terminals, a graphics display unit, and a hard ropy device are installed, and a link to the orIC counting room provides access to the data acquisition computers. Cables connecting this area with the spin spectrometer are in place and the spin spectrometer electronics have been woved from the experiment roon to this counting room. The new counting room has been used in several experiments on the spin spectrometer and has enormously improved the ease of experiment setup and iata acquisition. Expansion of this counting roon to service the $1.6-m$ scattering chamber is being considered.

A cable linking the tandew and orIC counting rooms will soon be installed. This link will make it easy to connect any counting roon with any of the three data acquisition computers, pernitting rapid switching to another system in the event of conputer probleas.

1. Oak Ridge Associated Universities.

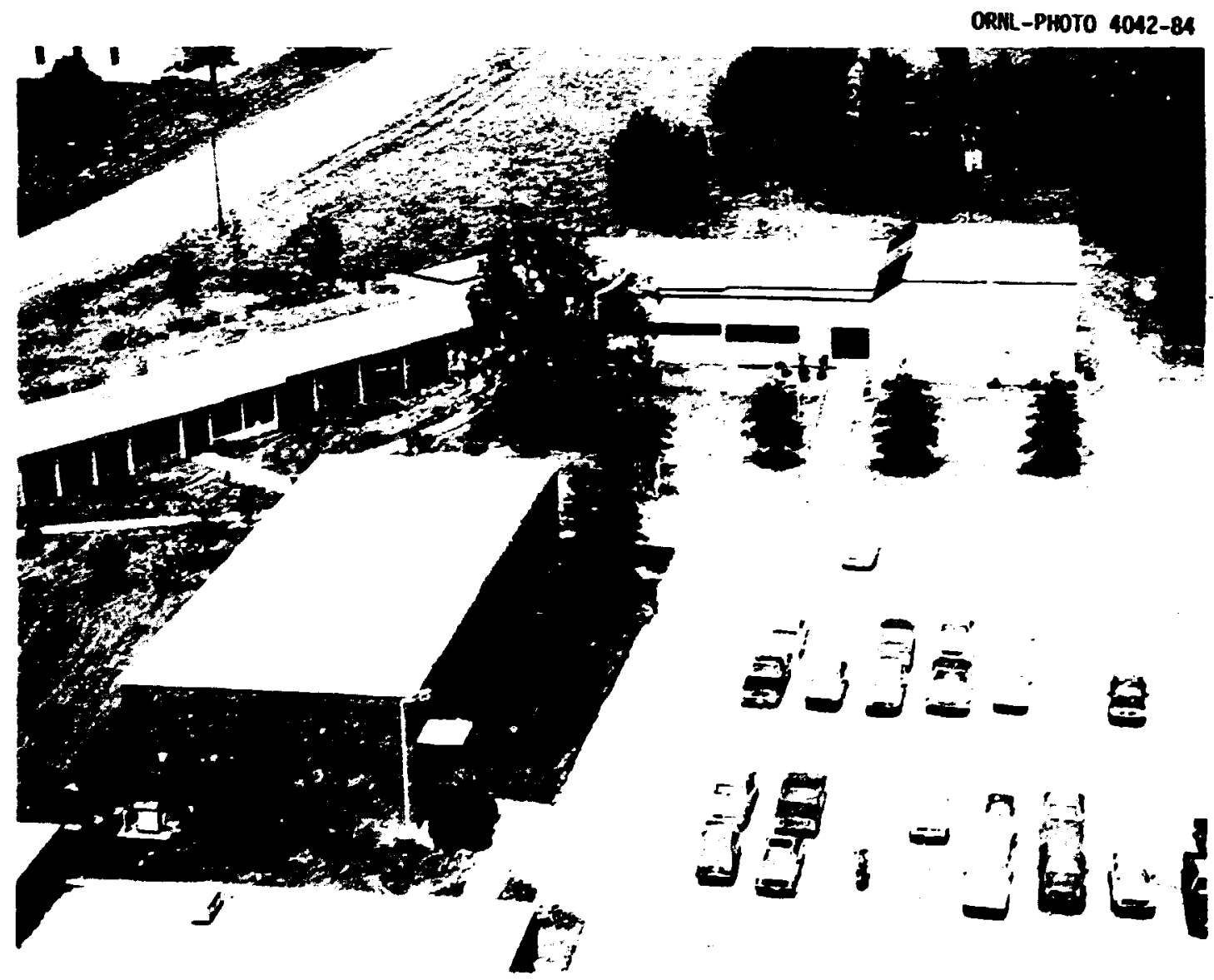

Fig. 1.18. Vtew of the two bufldings of the Joint Institute for Heavy Ion Research taken from atop the HHIRF tower. Butlding two is at the upper right with butiding one at the upper left. 


\section{USERS SUPPORT}
R. L. Mble
J. A. Biggerstaff
R. P. Cumby
R. W. Miles
J. U. Johnson
H. T. Filner
C. A. Reed
R. L. Robinson C. M. nowas

Experimenters routinely call upon the resources of the Users Support Group in setting up their experiments. Mormally, a technician (R. U. Miles or C. A. Reed) is assigned to a specific experiment wen the run schedule is established. They assist the experimenters with mardware related weters such as installing aligiment telescopes, diagnosing and orerseeing repair of racuon problems, and destgning and supervising fabrication of parts wich ay be needed for a particular setup. On the electronics end, R. P. Cuby wintains the HIRF electronics pool (consisting of mearly 1000 instrumen's and a mititude of cables). helps to locate wodules meeded for each experibent. diagnoses problems in the electronics, and tests the semiconductor and scintillation detectors to ensure that they meet specifications. Interfacing of the users electronics with the data acquisition system is manded by Charles Thomas. This includes connecting the caMc isterface to the apropriate CPU, installing the Event-Handler and associated modules, and setting up peripherals (MIn terninals. Chromatics terminals, Trilogs).

In addition to these rout ine tasks, the technicians assist the mentors with mintenance and adification of the various experimental facilities and provide technical support to the research staff in the developent of new equipment. Examples of the projects in wich the technicians have been particularly active are: design of an improved colliator for the velocity filter (R. Miles), design of the $80-\mathrm{cm}$ neutron chaber (C. Reed), development of an operating system for the 68000 Branch Driver (C. Thomas), and cevelopmert. of new high voltage supplies for the velocity fi i: $\circ$ ( $R$. Cumby).

1. Dak Ridge Associated Universities.

\section{JOINT IUSTITUTE FOR KEAVY ION RESEARCH}

$$
\begin{gathered}
\text { R. L. Robinson L. L. Riedinger } \\
\text { J. H. Mawiliton }
\end{gathered}
$$

Although the Jolnt Institute for Heavy Ion Research (JIHIR) legally became an entity with the signing on June, 1982, of the Agreewient of Understanding by representatives of Union carbide corp. (then operating coneractor of ORNL), the University of Tennessee, Vanderbilt University, and the Department of Energy, it did not become a fully operating Institute until it was provided operating funds July 1, 1984. The budget for the year beginning then is about $\$ 250,000$. Two-thirds of this is frum the state of Tennessee via a Centers-of-Excel lence grant through the University of Tennessee - Knoxville.
Contributors to the reaining one-third are ORIL. Vanderbilt University, and the Department of Energy.

Eeginning a nex Institute has required considerable involvenent of the JIHIR Policy Council mich is responsible for establishing policy and overseeing operation. These have included a arriad of apdane details: establishing ctility, janitorial and telephone services by com to a building not a part of Can: - developing a mechanisa for providing support to guests of the Institute; procuring furniture; assuring that minor building problens are rectified, etc. Mubers of the policy Council are J. H. Hailton (also serving as Directur of JIHIR), L. L. Riedinger and

R. L. Robinson.

The mojor portion of the budget is to support guests of the Institute with assigniments ranging from a few days to a year. Persons already supported since July 1 are C. M. Ko, J. A Mruhn, and $J$. Eberth. Thirteen others are expected before the end of this calendar year. The Inst itute has al so hired a half-time secretary.

Initially the second building will provide offices for staff persons of Unison, of the University of Tenmessee, and of Vanderbilt University, and in addition, offices for nine guests of the JIHIR. There are 12 desks for wore Holifield users and JIHIR guests in the first building.

$A$ dedication for the JIHIR is being planned for October 15, 1984. It will be followed by a one and one-haif day symposium on Directions in Muclear Structure Research.

The first building of the Joint Institute. mich was opened for occupancy in May 1981, continues to meet needs by providing conveniently located dormitory accomadations for Holifield Users from outside the laboratory. During FY 1984, its eight dormitory beds accomodated 213 visitors with an average length of stay of 6.1 nights (1308 person-nights). The occupancy rate strongly coupled to acelerator operation. Because of the ireyt ont requests that could not be met when the acielerators were operating (22) of the time the dormitory rooms were fuli), we are adding two wore dormitory rooms during the next month.

\section{USERS EROUP ACTIVITIES}

$$
\text { R. L. Auble and R. L. Robinson }
$$

The MIRF Users Group presently includes nearly 500 meabers representing over 150 inst tutions. Nembers are represented by the Users Group Executive Comittee, whose memership for 1983-84 is given in Table 1.9.

Two new nembers of the Executive cominitee are elected each year by the Users Group from a slate of candidates selected by the Nominating Comittee. For 1984, this comittee consisted of L. Grodzins, M. Guidry. J. Kamiliton, H. Lynch, L. Riedinger (Choirmon), R. Stokstad, and $K$. Toth.

The Executive Committee normally meets about four times year in order to provide input regarding factility operation, equipment needs, and other motters wich impact the users. 
Table 1.9. Users Group Executive Comittee Mubers for 1983, 1984, and 1985.

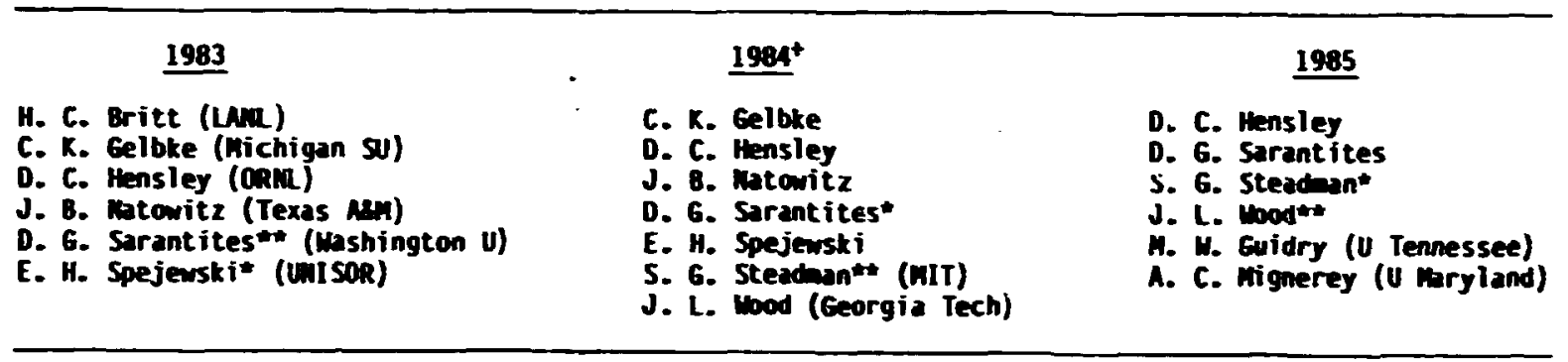

*Chairperson

* Chairperson-elect

+Seven memers this year only due to change from 2 year to 3 year tenure

During the past year, the Committee met on October 14, 1983; My 3, 1984; June 23, 1984; and September 18, 1984; providing mimerous recomendations wich have been taken into consideration in the operation of HIIRF. Minutes of these meetings are available from the Liaison office. The meting of Jure 23, 1984 ws notable in that it ws the first on-site meeting of the comittee. This proved to be very informative for both WIRF and the comittee and it is planned to hold similar meetings on an annual basis.

\section{PROGRAY AOVISORY COMITTEE}

\section{R. L. Robinson}

Table 1.10 gives statistics on the PAC meetings held during FY 1984 . The heavy oversubscription of research hours for PAC-3 is attributed to the long time (13 months) between this PAC and the preceding one. The requests shown for PAC-4 are suspected to be more typical since the time between meetings of PAC-3 and PAC-4 (5 minths) is more nearly like the average 6 month interval that is planned between future PACs.

As outlined in the Users Handbook, the intent, normally, is to complete experiments within a year after their approval. Table 1.11. wich summarizes status of the experiments recommended by all HIRF PACS, indicates that, in practice, this objective is nearly being accomplis shed.
Table 1.10. Statistics on PAC-3 and PAC-4

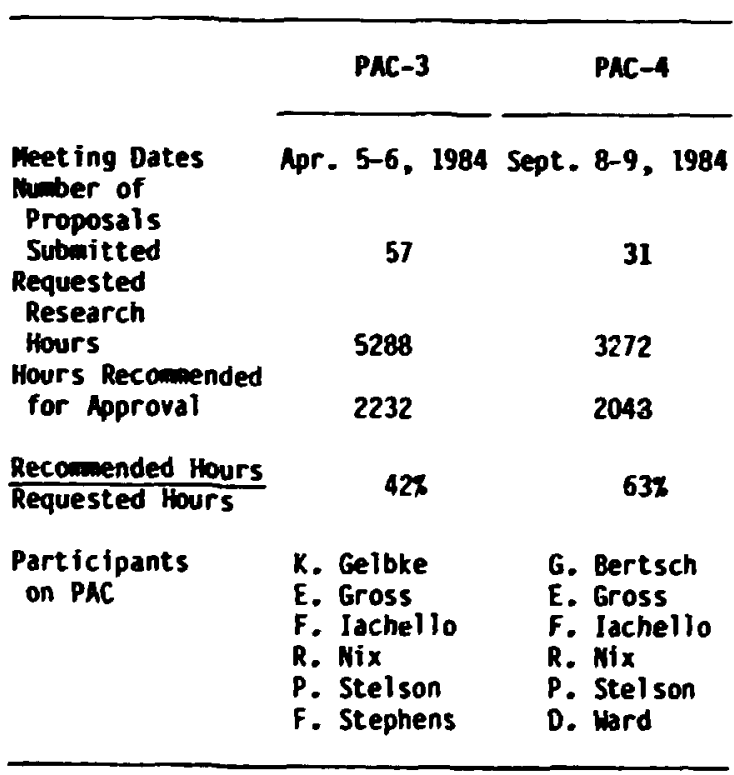

Table 1.11. Status of experiments recommended by HIIRF PACS

\begin{tabular}{|c|c|c|c|}
\hline & \multirow[b]{2}{*}{ Dates Met } & \multicolumn{2}{|c|}{ Hours } \\
\hline & & Recommended & Remaining \\
\hline \multirow{3}{*}{$\begin{array}{l}\text { PAC,-1 } \\
\text { PAC-1 } \\
\text { PAC-2 } \\
\text { PAC-3 } \\
\text { PAC-4 }\end{array}$} & $\begin{array}{l}\text { Nov. 10-11, } 1984 \\
\text { June } 5,1981 \\
\text { Feb. } 28-\end{array}$ & $\begin{array}{l}2120 \\
1032\end{array}$ & $\begin{array}{l}0 \\
0\end{array}$ \\
\hline & $\begin{array}{l}\text { Mar. 1, } 1983 \\
\text { Apr. 5-6, } 1984 \\
\text { Sept. 8-9, } 1984\end{array}$ & $\begin{array}{l}1656 \\
2232 \\
2048\end{array}$ & $\begin{array}{r}184 \\
1184 \\
2040\end{array}$ \\
\hline & Total & 9088 & 3408 \\
\hline
\end{tabular}




\section{EXPERIMENTAL NUCLEAR PHYSICS}

Our win reseirch program in heavy-ion physics is carried out using the beans

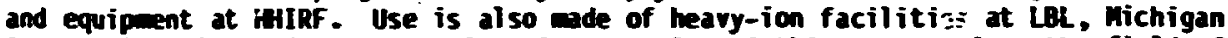
State University and at Texas NAM. An extension of this progra into the field of relativistic heavy-ion reactions is being pursued through a collaborative experiment to utilize 800 6eV to $3200 \mathrm{GeV} 160$ beans at CERM. In addition, we cont inue to carry out a vigorous and productive program in medium energy physics using facilities at UNPF, TRIU.F, and IUCF and a saller progran in neutron physics using the ORELA facility.

noteworthy accomplishents for this period include: new results from the Spin Spectrometer on neutrons and $r$ decay from giant resonances; the appearance of a quasivibrational transition in $15 \%$ b above spin $24 K_{;}$further ieasureants on : production from heavy-ion collisions; and the measureant of time-delayed processes from $100+6 e$ reactions using the crystal blocking technique. This period has also been sadly marked by the losses of two long-tine staff memers, J. L. C. Ford, Jr. and C. B. Fulimer.

OPTICA mODEL Mreysis of 200-400 Mer PROTON ELASTIC SCATTERIIIS ON 20EP
E. E. Gross
D. Horen
F. E. Bertrand
J. Lisantil

Among the results of the Oak Ridge-OregonOregon State proton inelastic scattering program conducted at TRIUMF are: (1) direct evidence for the excitation of the GOP by high energy proton inelastic scattering, 2 (2) corifimation 2 of $L=3 \mathrm{COR}$ resonances in $90 \mathrm{Zr}$ and $120 \mathrm{Sn}$, (3) discovery of $L=4 \mathrm{CHR}$ strength 3 in 206,20 $\mathrm{Pb}$, and (4) an improved understanding of the "background" underl jing the giant resonance region in terms of quasifree scattering. 4

In order to assign strengths (in terms of ener jy-weighted sum rule depletion) to the various giant resonances excited by $200-\mathrm{MeV}$ proton inelastic scattering, we carried out DWBA calculations using an optical potential developed for proton elastic scattering in the 60-160 MeV range.5 This analysis 2,3 led to strengths some $50 \%$ smaller than had been reported from previous lower energy measurements. Using the same analysis, the cross sectton for exciting the low lying 3 - state (2.61 $\mathrm{MeV}$ ) in 208pb was also under-predicted by $50 \%$ and we therefore renormalized the DMBA strength estimates for the observed giant resonances by a factor of about two.2,3 This procedure is not entirely satisfactory and brings into question the applicability of the OWBA method for the higher proton energies.

Subsequently, wave measured 6 differential cross sections for $334-\mathrm{MeV}$ proton elastic and inelastic scattering on $208 \mathrm{pb}$ using the HRS magnet facility at LAMPF. Rather than rely on optical model systematics developed at a lower energy, 5 we could use these new data to generate a more appropriate optical potential. Aithough covering a very linited angular range $\left(2.5^{\circ}\right.$ to $13^{\circ} \mathrm{C}$.m.l, a coupled channels analysis ${ }^{6}$ of the 334 MeV data yielded $B$ (E3) values and giant resonance strengths for $208 \mathrm{pb}$ wich were in excellent agreentent with previous measurements.

Hore recently we have become awara of very extensive differential cross section and polarization mearurenents for $200-, 300-, 400-$ and $500-\mathrm{HeV}$ proton elastic scattering from $20 \mathrm{epb}$ performed by Hutcheon and collaoorators. 7 These data have been subjected to an optical model analysis using the autonatic search routine in the ECIS computer progran. 8 The best fit parameters at each energy are summarized in Table 2.1. The best fit geometry parameters for the 200-, 300-, and 400- $\mathrm{MeV}$ analyses were averaged and the cross section and polarization data were again fitted by varying the optical potential strengths. The average geometry :earch results are also included in Table 2.1. The 500-MeV data could not be satisfactortly represented by an average geometry potentfal. espectally the 500-MeV polarization data. The increasing importance of meson production and relativistic effects may account for the change in conventional optical model systematics in the 400-50n MeV range.

Ftgure 2.1 shows the average gemetry fit to the $200-\mathrm{MeV}$ elastic scatterting data and Ftg. 2.2 shows the same thing for polarization (soltd curves). Also shown are predictions from the Nadesen et al.5 potential (dotted curves). Using the new potential of Table 2.1 tn an analysts of our previous 200 -MeV-protons inelastic scattering data ${ }^{3}$ from $208 \mathrm{pb}$ now provtdes agreement with previcus results for $B(E 3)$ for the $2.613 \mathrm{MeV}, 3^{-}$, level. It is reassuring that the DUBA method can be safely apolied in the 
Table 2.1. Proton +20 sp optical paraneters

\begin{tabular}{|c|c|c|c|c|c|c|c|c|c|}
\hline & \multicolumn{2}{|c|}{$334 \mathrm{keV}$} & \multicolumn{2}{|c|}{$200 \mathrm{nel}$} & \multicolumn{2}{|c|}{$300 n=1$} & \multicolumn{2}{|c|}{400 ney } & \multirow{2}{*}{$\frac{500 \mathrm{HeV}}{F_{\text {it }}}$} \\
\hline & Arg.geo. & Fit & Avg.sco. & Fit & Avg-geo. & fit & Avg-g=0. & Fit & \\
\hline$v_{R}$ & 4.452 & $12.4^{5}$ & 11.17 & 11.11 & 4.37 & 4.934 & 0.292 & 0.20 & -10.11 \\
\hline$r_{R}$ & 1.315 & 1.121 & 1.315 & 1.299 & 1.315 & 1.304 & 1.315 & 1.341 & 1.103 \\
\hline 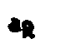 & 0.599 & 0.370 & 0.539 & 0.623 & 0.599 & 0.605 & 0.599 & 0.568 & 0.469 \\
\hline$v_{I}$ & 19.67 & 30.00 & 21.17 & 20.30 & 25.19 & 24.48 & 34.12 & 33.49 & 30.81 \\
\hline$r_{I}$ & 1.149 & 1.090 & 1.149 & 1.167 & 1.149 & 1.165 & 1.149 & 1.116 & $1.15 i$ \\
\hline $\mathbf{I}$ & 0.799 & 0.765 & 0.799 & 0.827 & 0.799 & 0.762 & 0.799 & 0.800 & 0.597 \\
\hline$v_{s 0}$ & 2.11 & 1.89 & 2.20 & 2.65 & 2.32 & 2.105 & 2.211 & $2.23 n$ & 2.972 \\
\hline$r_{50}$ & 1.104 & 1.138 & 1.104 & 1.095 & 1.104 & 1.098 & 1.104 & 1.123 & 1.008 \\
\hline se & 0.689 & 0.777 & 0.689 & 0.685 & 0.689 & 0.651 & 0.689 & 0.730 & 0.874 \\
\hline$v_{I}$ & -0.528 & -0.770 & -2.859 & -2.88 & -3.24 & -3.314 & -4.894 & -3.630 & -0.412 \\
\hline$r_{1 s 0}$ & 1.051 & 1.135 & 1.001 & 1.050 & 1.061 & 1.058 & 1.061 & 1.076 & 1.347 \\
\hline 2130 & 0.801 & 0.687 & $0.80 \mathrm{I}$ & 0.821 & 0.801 & $0 . .85$ & 0.801 & 0.199 & 0.100 \\
\hline$x_{0}^{2}$ & 956 & 554 & 3710 & 1670 & 1530 & 2110 & 1580 & 1040 & 1420 \\
\hline$x_{n}^{2}$ & 644 & 246 & 1820 & 2000 & 663 & 820 & $\$ 150$ & 3030 & 885 \\
\hline
\end{tabular}

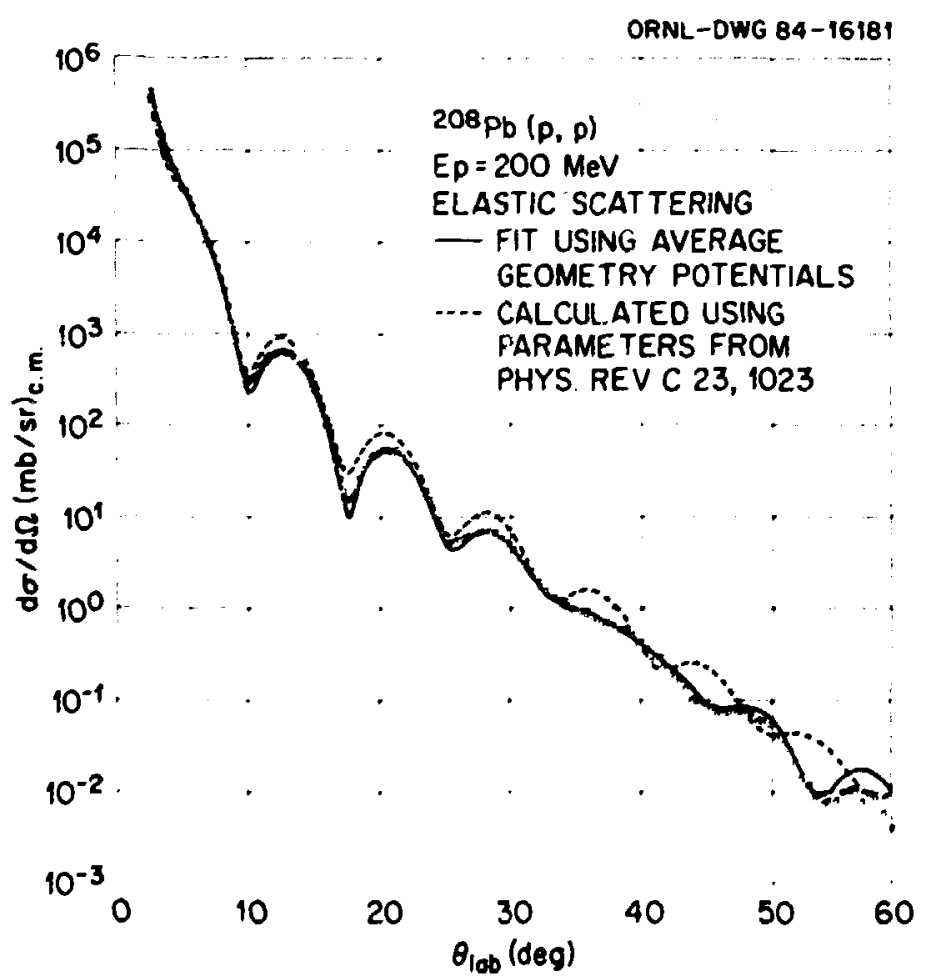

Fig. 2.1. Elastic scattering data 7 for 200-, 300-, and 400-MeV protons incident on a $208 \mathrm{pb}$ target. The solid curves are "average geometry" fits using the potenitals in Table 2.1. The dotted curves are predictions using the Nadasen et al. Systematics. ${ }^{3}$ 


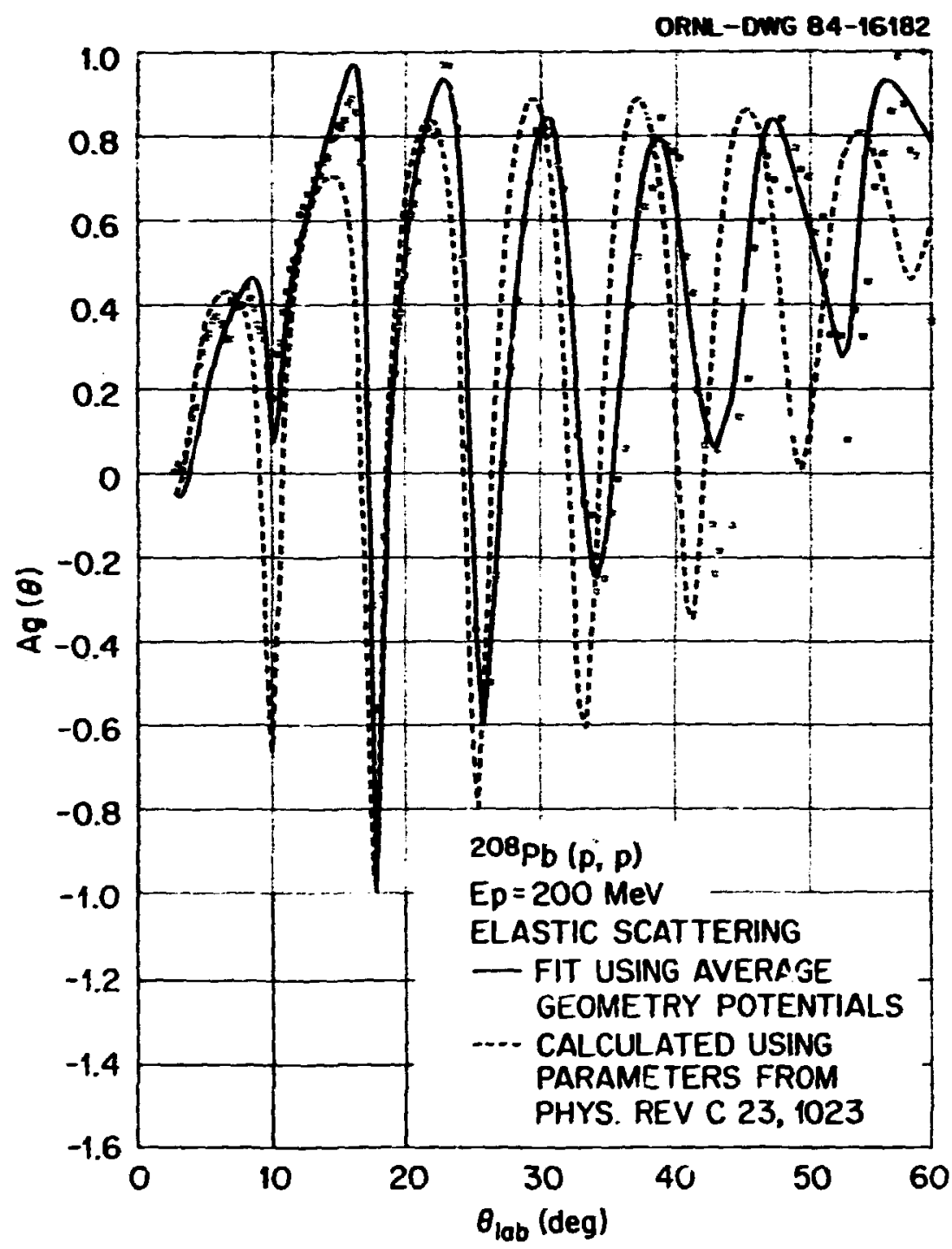

Fig. 2.2. Elastic polarization data 7 for $200-, 300-$, and 400 -MeV protons incident on a 20apb target. The solid and dotted curves correspond to those in Fig. 2.1 for the differential cross sections.

200-40r MeV proton energy range provided care is taken to determine the optical potential.

1. University of Oregon, Eugene, OR 97403. 2. F. E. Bertrand et al., Phys. Lett. 103B, 336 (1981).

3. J. R. Tínsiey et al.. Phys. Rev. C 28 , 1417 (1983).

4. D. K. MCDaniels et al., to be publishied.

5. A. Madasen et al., Phys. Rev. C 23, 1023 (1981).

6. F. E. Bertirarj et al., to be publtshed and following contribution.

7. D. A. Hutcheon (private communication).

B. J. Raynal (private comunication).
INELASTIC EXCITATICN OF LOW-LYING STATES IN 208pb BY 334-HeV PROTONS
F. E. Bertrand
E. E. Gross
D. J. Horen
T. P. Sjoreen 1
J. Lisantti 2
D. K. MCDaniels ${ }^{2}$
L. H. Swenson ${ }^{3}$
T. Carey
K. Jones"
J. B. McClelland4
J. T. Tinsley 2
S. Seestrom-Morris 4

The strength of a giant resonance excitation is generally stated in terms of the percentage depletion of mergy weighted sum rule (EWSR). The EWSR is directiy assoctated with the transition rate $[B(E L)]$ of the resonance as:

$E W S R=B(E L)\left(f_{f} \cdot E_{j}\right)=\frac{L(2 L+l) K^{2}}{4 \pi^{2}(m} A e^{2}<r^{2 L-2}$, 
where $E_{f}$ and $E_{j}$ denota the energies of the final and initial states in the transition and $\langle r L\rangle$ is the rms charge radius of the ground state. For electromagnetic interactions the determiriation of $B(E L)$ is direct. However, in inelastic hadron scatter: na $B(E L)$ is not directly obtained; rather, a deformation parameter, B. is deduced wich is related to $B(E L)$ in a mode dependent way. In order to insure that the coriect EMSR is being deduced for giant resonances excited in inelastic scattering, it is important to check the determination of $B(E L)$ for low-lying nuclear states having know: spin, parity, and transition rate.

In our recent, high resolution measurements of giant resonances in $208 \mathrm{pb}$ using inelastic scattering of 334-MeV protons, we obtained data for the elastic scattering and inelastic excitation of low-lying $3^{-}, 2^{+}$, and $4^{+}$levels. The data have been compared with DWBA calculations which utilize optical model parameters from energydependent potentials obtained from fits to 200 -, 300-, and 400-MeV proton elastic scattering on $208 \mathrm{pb}$ as Jescribed in the preceding contribution. Figure 2.3 shows the elastic scattering data (both differential cross section and ana(yzing power) and the optical model calculations for $334-\mathrm{MeV}$ proton scatteining from $208 \mathrm{pb}$.

Figure 2.4 shows the differential cross sections for inelastic scattering to the $2.613-\mathrm{MeV}$, $3^{-}, 4.086-\mathrm{MeV}, 2^{+}$, and $4.323-\mathrm{MeV}, 4^{+}$, states in $208 \mathrm{~Pb}$. The $\mathrm{B} R \mathrm{R}$ values jeduced by normalizing the calculations to the data are shown in the curves. From these $B_{L} R$ values we derivi the $B(E L)$ values shown in Table 2.2 compared with values adopted in the Nuclear Data Sheets. 5 It is clear from these results that results of inelastic scattering of medium energy protons can be used to deduce a correct $B(E L)$ value. Through this comparison we gain confidence that our EWSR measurements for the giart resonance states in $208 \mathrm{pb}$ are reliable.

1. Solid State Division, ORNL.

2. University of Oregon, Eugene, OR 97403.

3. Oregon State University, Corvallis, OR 97331 .

4. Los Alamos National Laboratory, Los Alamos, NM 87545.

5. M. J. Martin, Nuclear Data Sheets for $208 \mathrm{pb}$, in preparation (private communication).

COMPARISON BETWEEN EXPERIMENTAL AND CALCULATED CROSS SECTIONS AMD ASYMATTRIES FOR SPIN EXCITATIONS IN $40.48 \mathrm{Ca}$
D. J. Horen
J. Lisantti 2
F. E. Bertrand
L. W. Swensen ${ }^{3}$
E. E. Gross
J. B. MCClell and 4
T. P. Sjoreen
T. A. Carey
r. K. McDantels ${ }^{2}$
S. J. Seestrom.Morris 4
J. R. Tinsley ${ }^{2}$
K. Jones ${ }^{4}$

The $40,48 \mathrm{Ca}\left(\vec{p}, p^{\prime}\right)$ reaction has been studied utilizing the HRS facility at LAMPF. The incident protons had an energy of $334 \mathrm{MeV}$, and the

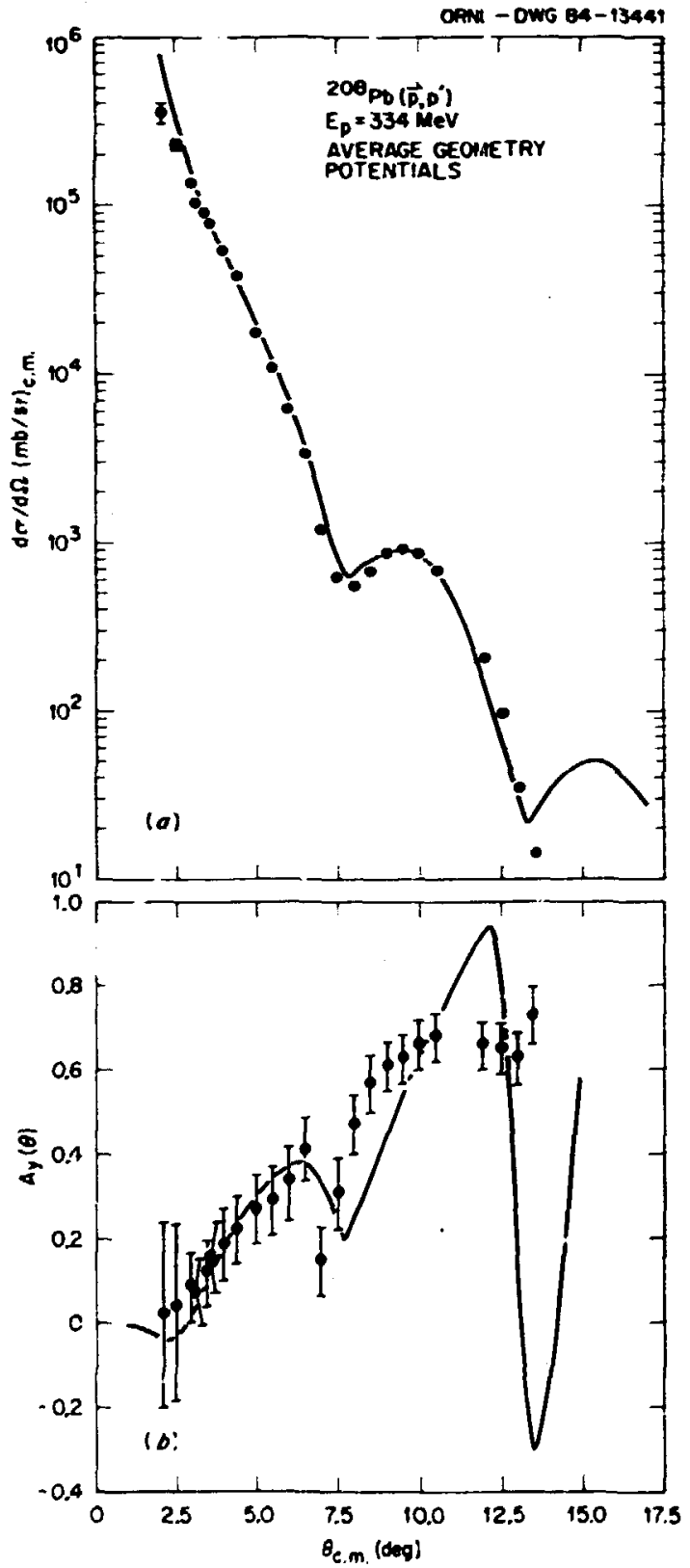

Fig. 2.3. Differential cross section and analyzing power for elastic scattering of $334-\mathrm{MeV}$ protons from $208 \mathrm{pb}$. The solid curve is an optical model calculation.

overall resolution achieved was $B$ nev. Measurements included the angular region $\mathrm{c}_{\mathrm{cm}}$ $\sim 2^{\circ}-10^{\circ}$. The data were analyzed and differential cross sections and asymmetries were determined for spin-isospin excited states at 10.24 $\operatorname{MeV}\left(J^{\pi}=1^{+}, T=4\right)$ in ${ }^{48} \mathrm{Ca}, 8.42 \mathrm{MeV}\left(\mathrm{J} \pi=2^{-}\right.$ $T=1)$ and $10.31 \mathrm{MeV}\left(\mathrm{J} \pi=1^{+}, T=1\right)$ in ${ }^{40} \mathrm{Ca}$. The experimental cross sections and asymmetries are shown in Figs. 2.5, 2.6, and 2.7 . 


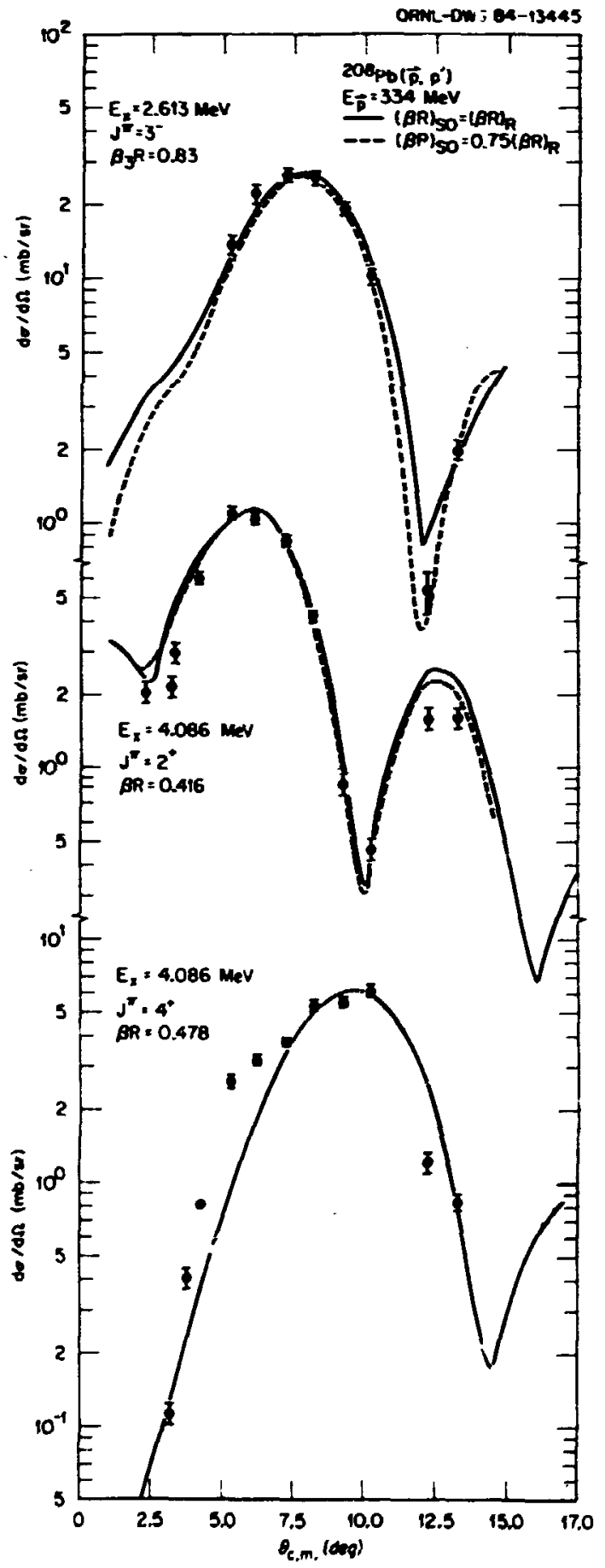

Fig. 2.4. Differential cross sections for tnelastic scattering of $334-\mathrm{MeV}$ protons to three low-lying levels in 208pb. The solid curves and dashed curves are from DWRA calculations.
Table 2.2. Properties of levels studied

\begin{tabular}{|c|c|c|c|c|}
\hline $\begin{array}{l}\text { Level } \\
\text { Energy } \\
\text { (Mev) }\end{array}$ & $\begin{array}{c}\text { Level } \\
\mathbf{J}\end{array}$ & $\mathbf{Q R}$ & $\begin{array}{c}B(E L) \\
\text { Deduced } \\
e^{2 b L}\end{array}$ & $\begin{array}{c}\text { B(FL) } \\
\text { Muclear } \\
\text { Data Sheets }\end{array}$ \\
\hline 2.613 & 3- & 0.83 & $0.67^{6}$ & $0.611^{9}$ \\
\hline 4.086 & $2^{+}$ & 0.42 & $0.33^{1}$ & $0.318^{16}$ \\
\hline 4.323 & $4^{*}$ & 0.48 & $0.12^{2}$ & $0.135^{11}$ \\
\hline
\end{tabular}

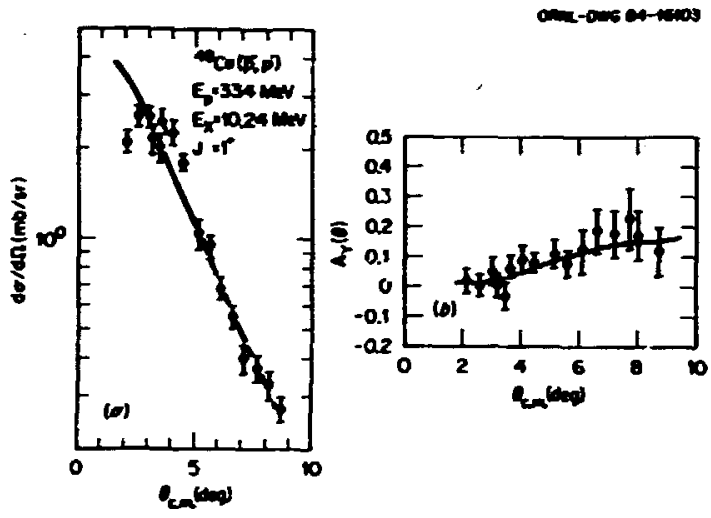

Fig. 2.5. Differential cross section and asymetry for the $10.24-\mathrm{MeV} 1^{+}$state in $48 \mathrm{Ca}$. The co!,d lines are from DilA calculations.

Our measured cross section for the 10.24-MeV state in ${ }^{8} \mathrm{Ca}$ is in good agreement with that obtained by Nanda et al. 5 with protons of energy $319 \mathrm{MeV}$. However, we do not observe the sharp minimum at $\sim 5^{\circ}$ in the asymmetry as reported by those workers. Our data for the $10.31-\mathrm{MeV}$ state in ${ }^{\circ} \mathrm{Ca}$ is consistent with the results obtained wit!) 200 -MeV protons. 6 Our asymetry data are the first measured for this state at intermediate energies. The data at these energies for the $8.42-\mathrm{MeV}$ state in ${ }^{40} \mathrm{Ca}$ is the first to our knowledge.

The quenching of spin transftions (e.g., Ml. GT) is well established, although a complete understanding of its phystcal basis is still befing debated. Two mechanisms wich have been proposed to explain such quenching have been ground-state configuration $m i x i n g$ and $m i x i n g$ with $\Delta \mathrm{N}^{-}$excitations. Both mechanisms would result in pushing some spin strength to the region of higher excitation. For $42-48 \mathrm{Ca}$, MeGrory and Wildenthal ${ }^{7}$ have shown that an ip space can account for a considerable part of the 

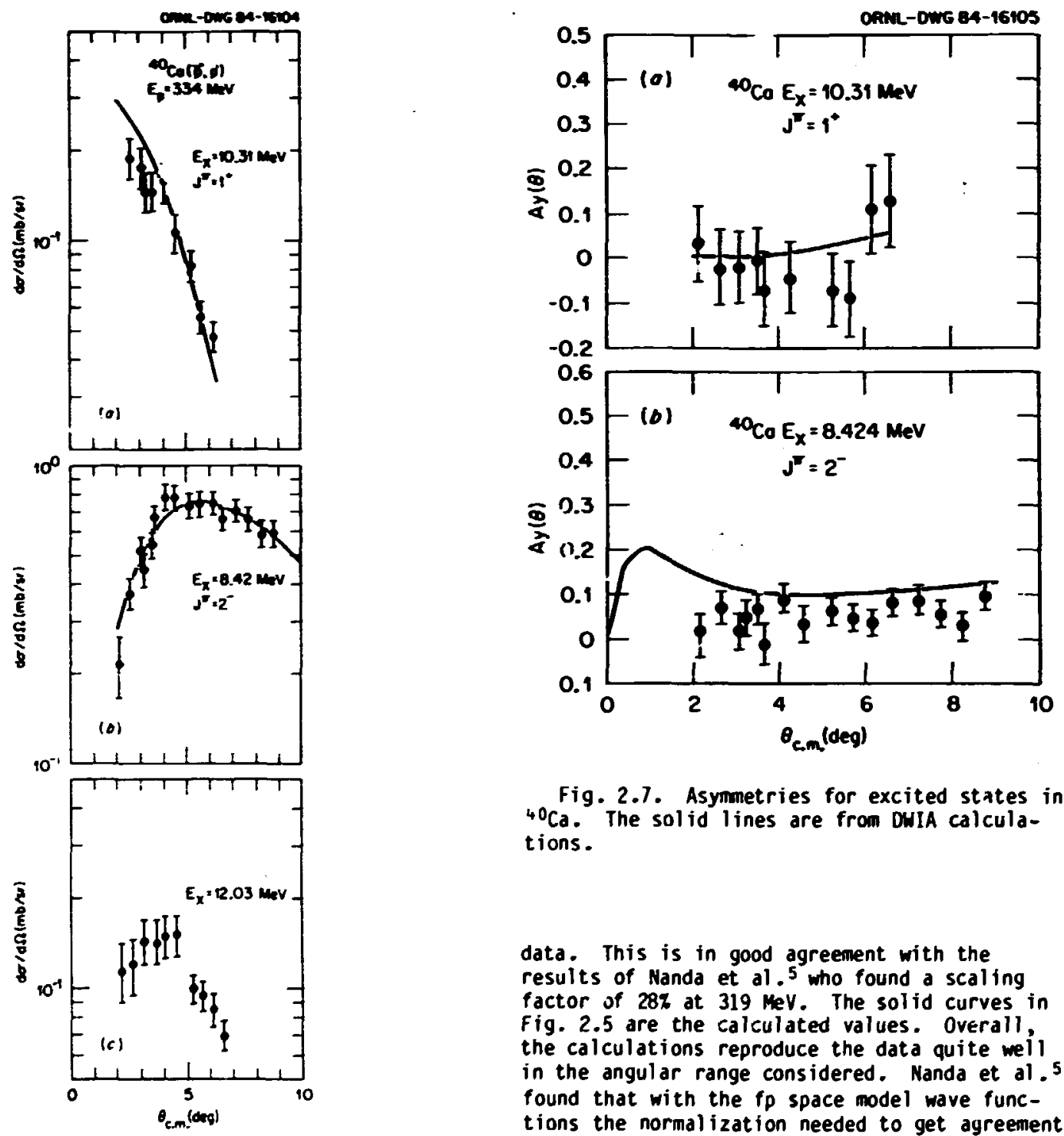

Fig. 2.7. Asymmetries for excited states in ${ }^{\circ} \mathrm{Ca}$. The solid lines are from DNIA calculations.

Fig. 2.6. Differential cross sections for excited states in ${ }^{40} \mathrm{Ca}$. The solid lines are from OWIA calculations.

quenching, but stfll have to quench the o operator by $-80 \%$ as is needed to reproduce the ground-state magnetic moment of ${ }^{4} \mathrm{C} \mathrm{Ca}$. Brown et a1.8 have discussed the qualitative aspects of core breaking.

We have perfurmed microscopic OWIA calculations using the Love-Franey ${ }^{9}$ interaction and the code 10 DWBA-81. For the $1^{+}$excitation in ${ }^{4} \mathrm{C}_{\mathrm{C}}$ we have used a pure $f_{5 / 2} f 7 / 2$ neutron particlehole transition. Our optical potential parameters were deduced by fitting our elastic cross section and polarization data over the angular range $\theta_{\mathrm{cm}} 2^{\circ}-15^{\circ}$. At $334-\mathrm{MeV}$, the reaction is dominated by the or component of the interaction. He find that the calculated cross section must be multiplied by -0.29 to fit the data. This is in good agreement with the results of Nanda et al. 5 who found a scaling factor of 287 at $319 \mathrm{MeV}$. The solid curves in Fig. 2.5 are the calculated values. Overall, the calculations reproduce the data quite well in the angular range considered. Nanda et al.5 found that with the fp space model wave functions the normalization needed to get agreement with the data was $47 \%$. This is about the same ratio obtained from $\left(e, e^{\prime}\right)$ results which is as expected for a pure neutron Ml excitation. From measurements at $E_{p}=201 \mathrm{MeV}, \mathrm{Crawley}$ et al.1l deduced a normalization of only $30 \%$.

For the $10.31-\mathrm{MeV} 1^{+}$state in ${ }^{40} \mathrm{Ca}$, the ground state configuration was taken as

$\left[\left(f_{7 / 2}\right)^{2}\left(d_{3 / 2}\right)^{-2}\right]_{0+} ; f_{\eta / 2}+f / 2$ and $d_{3 / 2+} / 2$ transition densities provided by Brown 12 were used in a DWIA calculation. Normalization of the calculation to the data resulted in $B(M 1)$. $(0.39 \pm 0.08) \mathrm{H}^{2}$. This is in excellent agreement with value of $0.55 \mathrm{u}_{N}^{2}$ deduced from $B(G T)=0.21$

as reported by Taddeucci et al .13 from $(p, n)$ measurements. The calculated $A y(\theta)$ for this transition is in good agreement with the data, as can be seen in Fig. 2.7.

The DWIA calculations for the 8.42-MeV 2state were done with $1 K_{w}$ particle-hole excitation transtition densities provided by Brown.12 
The calculated cross section was multiplied by 0.35 to normalize to the experimental data.

This yields $B(\mathrm{HR})=281 \mathrm{u}_{N}^{2} \mathrm{fm}^{2}$ wich is in good agreement with a value deaced from excitation of the analogue state via the $(p, n)$ reaction, i3 and also a value of $235 \pm 20 \mu^{2} \mathrm{fm}^{2}$ measured ${ }^{i 4}$ by inelastic electron scattering. The calculated $A y(\theta)$ is in reasonable agreenent with the data althoigh it appears to be slightly high (see Fig. 2.7).

1. Solid State Division, ORNL.

2. University of Oregon, Eugene, OR 97403,

3. Oregon State University, Corvallis, OR 97331.

4. Los Alamos Mational Laboratory, Los Alamos, M 87545. (1984).

6. N. Anantaraman et al., Phys. Rev. Lett. 46. 1318 (1981).

7. J. B. McGrory and B. H. Wildenthal, Phys. Lett. 103B, 173 (1981)

8. B. A. Brown et al ., Phys. Lett. 1278, 151 (1983).

9. H. G. Love and M. A. Franey, Phys. Rev. C 24, 1073 (1981); update, January 1984 .

10. Program DuBA70, R. Schaeffer and J. Raynal (unpublished); extended version DWBI by J. R. Confort (unpublished).

11. G. M. Crawley et al., Phys. Lett. 1278, $322(1983)$

12. B. A. Brown, private comunication.

13. T. N. Taddeucci et al., Phys. Rev. C 28, 2511 (1983).

14. A. Richter, private comunication in ref. 13 .

A HIGH RESOLUTION STUDY OF GIANT RESONANCES IN 208pb USING INELASTIC SCATTERING OF 334-MEV PROTONS

$\begin{array}{ll}\text { F. E. Bertrand } & \text { D. K. MCDaniels }{ }^{2} \\ \text { E. E. Gross } & \text { L. W. Swenson } \\ \text { D. J. Horen } & \text { T. Carey4 } \\ \text { T. P. Sjoreen 1 } & \text { K. Jones } \\ \text { J. Lisantti2 } & \text { J. B. McClelland }{ }^{4} \\ \text { J. T. Tinsley } 2 & \text { S. Seestrom-Morris }\end{array}$

Even though giant multipole resonances have been studied for over a decade, there remain numerous uncertainties and discrepancies between measurements on some nuclei. One of these cases is a disagreement between inelastic electron scattering ${ }^{6}$ and inelastic hadron scattering measurements or. ${ }^{208} \mathrm{~Pb}$. The electron scattering results do not show the presence of a broad giant quadrupole resonance (GQR) peak as is observed in all hadron measurements, 7 but rather show a spectrum comprised of a large number of rather narrow peaks. Furthermore, the integrated E2 strength between 8 and 12 MeV of excitation energy is found to be $29 \pm 1+2$ of the energy wighted su rule (EMSR) via the electron work and $80 \pm 15 \%$ in the hadron experiments. The electron experiments were performed with an energy resolution of $50-60 \mathrm{keV}$, considerably better than achieved with any of the hadron measurements.

In order to make a direct comparison with the electron easurenents, we have studied giant resonances in $208 \mathrm{pb}$ using the high resolution spectrometer (HRS) at LAMPF. The resonances were excited using 334-meV polarized protons and were detected in the spectrograph with an energy resolution of $\rightarrow 70 \mathrm{keV}$, comparable to that obtained in Ref. 1 using 50-MeV electrons.

Measurements were made at one degree intervals between 2.25 and 13.25 degrees. An inelastic excitation energy range in $20 \mathrm{pb}$ of 1)-26 MeV was obtained by using three spectrograph anetic field settings.

Figure 2.8 shows 2 epp spectra at several angles. The spectra are dominated by several large and generally broad peaks. The various peaks have been fitted with Gaussian shapes and an estimate has been made for a smooth extrapolation of the nuclear continuum under the resonance reaks. At the smallest angles, 2.25 and 3.25 degrees, the spectra are doainated by a broad peak at $13.6 \mathrm{MeV}$. This peak arises from Coulanb excitation of the giant dipole resonance (GOR). At larger angles the GOR cross section falls off rapidly and a large peak is observed at $10.6 \mathrm{MeV}$. This peak is located at the energy where the GQR peak has been observed in other inelastic scattering mork. The peak at $-14 \mathrm{MeV}$ persists at larger angles but is likely to arise from excitation of both the GOR and giant monopole resonances (GrR). At 9.25 degrees, a peak centered at $\sim 20 \mathrm{KeV}$ is observed. This peak energy is in agreement with the location ${ }^{8}$ of the giant octupole resonance. The spectrum at 10.25 degrees shows the need for inclusion of a peak located at $12.0 \mathrm{MeV}$. This result is in complete agreement with our work' at $200 \mathrm{MeV}$ wich showed the presence of $L=4$ excitation at $12-M e V$ in both $208 \mathrm{~Pb}$ and $206 \mathrm{pb}$. One of the more interesting features of the spectra in Fig. 2.8 is the large number of rather narrow peaks observed below the GQR excitation energy. These peaks are observed consistently at all angles of measurement.

Figure 2.9 shows the extracted angular distribution for the GQR peak ( $10.6 \mathrm{MeV})$. The cross sections are compared to a DUBA calculation for $L=2$ transfer. The calculation lises optical model parameters obtained from a fit to the elastic scattering. Furthermore, as discussed in the preceding contribution to this report, use of the same parameters in DWBA calculations yield, then compared to angular distributions for low lying states in $208 \mathrm{pb}$, excel lent agreement with the known $J \pi$ and $B(E 2)$ for these states. From the comparison between the calculations and data shown in Fig. 2.9, we determine that $70 x \pm 14 \%$ of the EWSR is depleted in the 2.0-MeV-wide GQR peak. The lower part of Fig. 2.9 shows the measured analyzing power for the GQR peak. The comparison between the analyzing power calculation and data is rather good except near 11 degrees. 


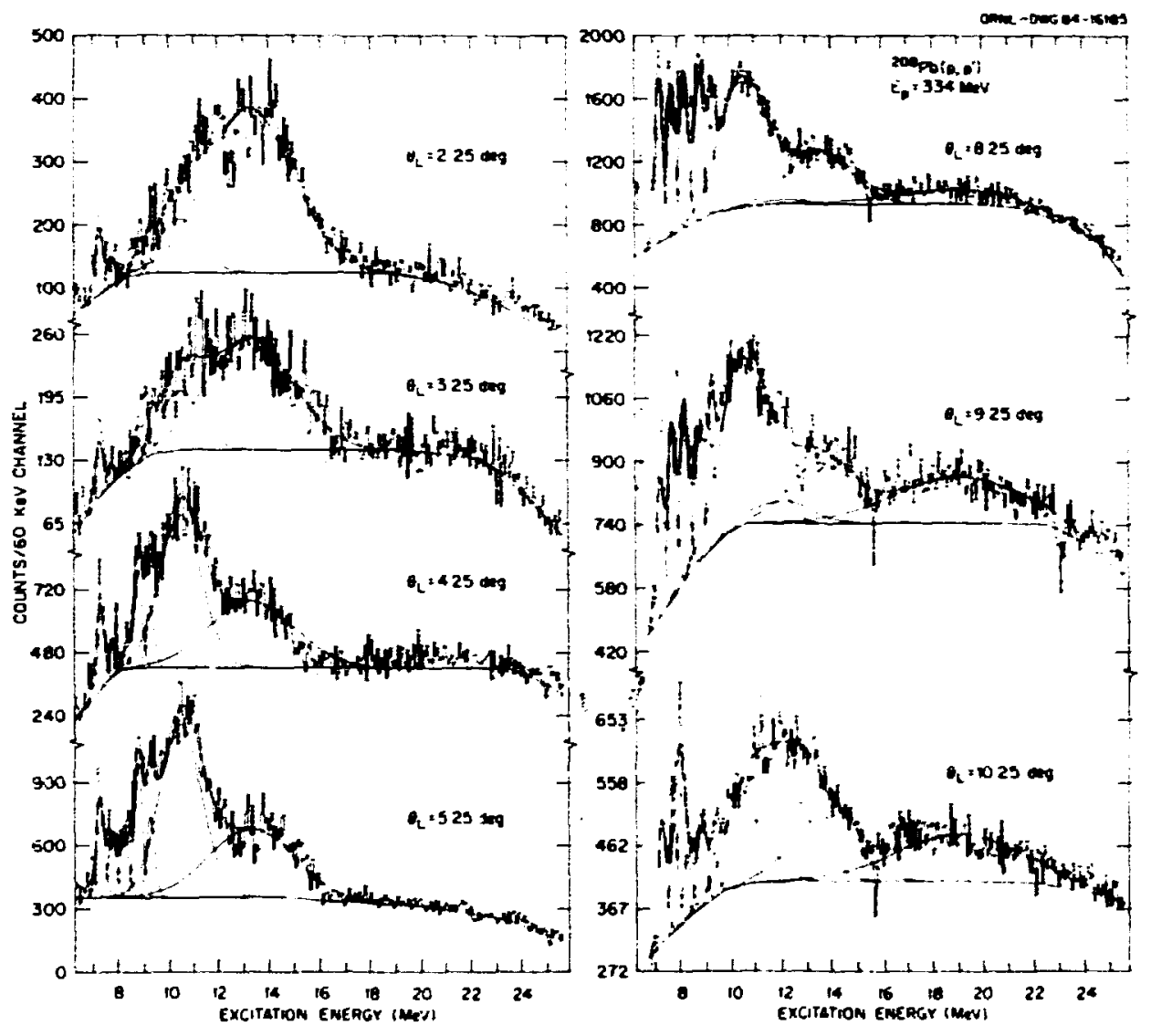

fig. 2.8. Spectra at several angles from the reaction $208 \mathrm{pb}\left(\rho, p^{\prime}\right)$, for $E_{p}=334 \mathrm{MeV}$.

Figure 2.10 shows measured and calculated angular distributions for the narrow peaks found between $\rightarrow 7$ and $\rightarrow 9.5 \mathrm{MeV}$ of excitation energy. We find that most of the peaks are best Jescribed by $L=2$ transfer although one $L=3$ and one $L=4$ peak has been ident if ied. The four $2^{+}$states deplete a total of $\sim 23 \%$ of the $T=0, L=2$, EWSR. This strength, when added to that found in the main GQR peak (Fig. 2.9) accounts for $93 \% \pm 15 \%$ of the $T=0$, quadrupole sum rule strength in $208 \mathrm{pb}$.

It is clear from these measurements that the existence of a localized peak containing a large fraction of the quadrupole EWSR in $20 \mathrm{Bpb}$ persists in hadron scattering, even when studied with an energy resolution of $70 \mathrm{keV}$. Furthermore, these results indicate that $200 \%$ of the $L=2$, EWSR is located in the giant resonance region of $208 \mathrm{pb}$. This result is in excellent agreement with that obtained from our recent measurements of the photon decay of the $208 \mathrm{pb}$ giant resonance region (discussed el sewhere in this report).

1. Solfd State Division, ORNL.

2. University of Oregon, Eugene, OR 97403.

3. Oregon State University, Corvalits, OR 97331 .
4. Los Alamos National Laboratoiy, Los Alamos, 87545 .

5. Computing and Telecommunications Division, ORHL.

6. G. Kuhner et al.., Phys. Lett. 104B, 189 (1981).

7. F. E. Bertrand et al., Phys. Rev. C 22 , $1832(1980)$.

8. F. E. Bertrand, Nuil. Phys. 1354, $129 \mathrm{C}$ (1981).

9. J. R. Tínsley et al., Phys. Rev. C 28, 1417 (1983).

\section{EXCITATION OF GIANT WULTIPOLE RESOMANCES IN SO-SHELL WUCLEI VIA 500-HEV PROTON INELASTIC SCATTERIMG}
B. L. Burks
L. H. Swenson 2
F. E. Bertrand
D. K. MCDanieis ${ }^{3}$
R. L. Auble
J. Lisantti 3
E. E. Gross
J. R. Tinsley
D. J. Horen
K. W. Jones 4
R. 0. Sayer 1 J. B. MCClelland" S. Seestrom-Morris 4

Over the past few years constderable research effort has been expended toward tine establishment of the location of vartous multifole giant 

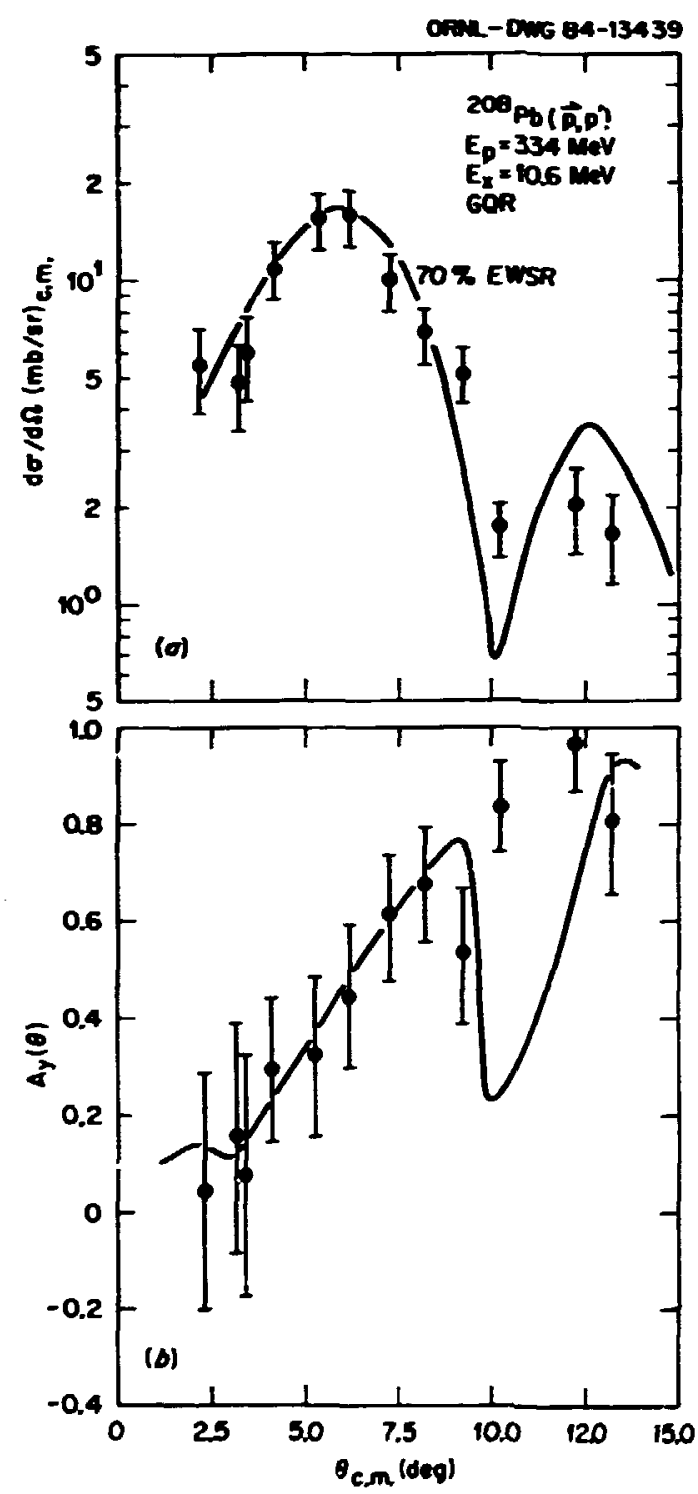

Fig. 2.9. Angular distributions and analyzing powers for the GQR in 208pb from inelastic scattering of $334 \mathrm{MeV}$ protons.

resonances. For nuclef havting $A>40$ systematics of the energy, width, and strength of isoscalar monopole, quadrupole, and octupole resonances have been rather mell established.5 for nuclef in this mass region the isoscalur giant resonances appear as broad (2-6 MeV FWH peaks rising above a generally flat nuclear cont f nuum.

For nuclei lighter than $A-40$, specifically sd-shell nuclei, the character of the gtant quadrupole resonance is dramatically different. No longer is a broad peak observed but with adequate energy resolution many individual peaks are observed in the $2 K_{\omega}-3 K_{\omega}$ region of excitation energy. This fine structure was initially observed via inelastic scattering of $60-\mathrm{MeV}$

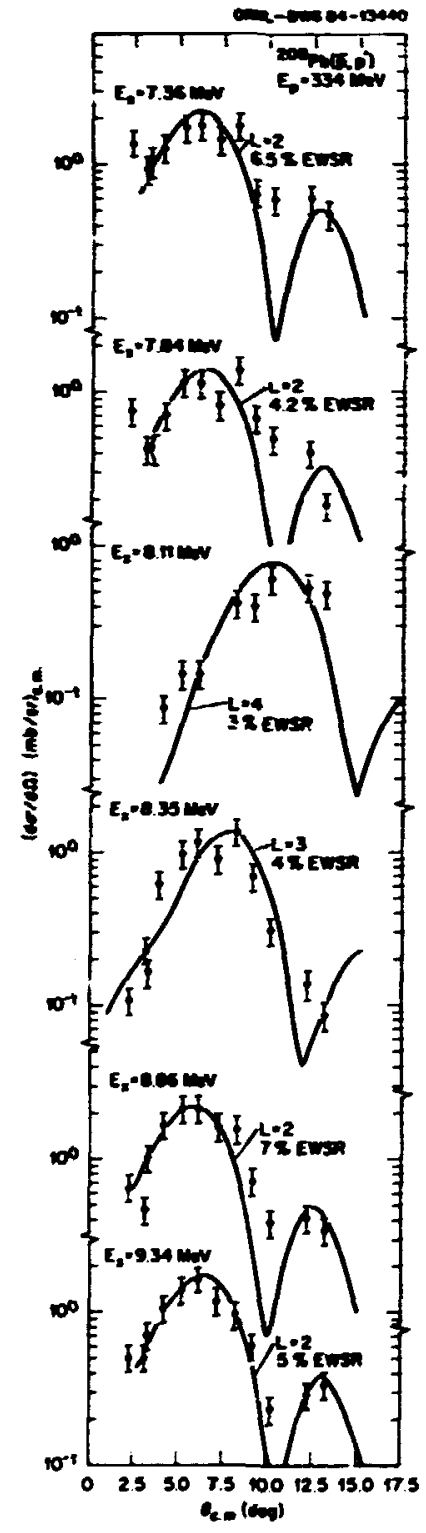

Fig. 2.10. Angular distributions for narrow states observed below the giant quadrupole resonance.

protons 6 and later using the $\left(a, a^{1}\right)$ reaction 7 with 120-MeV alpha particles. The sd-shell offers a unique opportunity for compartson of GOR excitation via proton and alpha inelastic scattering, electron scattering, ${ }^{B}$ and alpha capture reactions.9 A comparison of giant resonance resuits from several different reactions is necessary to unfold the complicated observed resonance structures. Studies in the sd-shell offer the maximum number of reactions for comparison.

We report here a study of giant resonance states in sd-shell nuclei excited by inclastic scattering of 500-MeV polarized protons using 
the HRS facility at LUPF. Data were measured from $4^{\circ}$ to $20^{\circ}$ for scattering from ${ }^{\circ} \mathrm{Ca}$ and from $4^{\circ}$ to $12^{\circ}$ for scattering from $285^{\circ}$. We plan to request additional bea time to extend the $28_{5 i}$ angular distribution and measure additional data for $24 \mathrm{~kg}$ and $26 \mathrm{mg}$. Two settings of the agnetic field were required to obtain composite spectra covering an excitation energy range from 0.5 to - $40 \mathrm{MeV}$. Elastic scattering data mere measured separately at a third agnetic field setting. Figure 2.11 shows a spectrua obtained for the low excitation range in $4 \mathrm{C} C \mathrm{C}$ at a laboratory angle of $7^{\circ}$. Typical spectra have an energy resolution of 80 keV fun ano show essential!y no "bogus" background.

DuBA calculations (see Fig. 2.12) indicate that inelastic scattering of $500-\mathrm{HeV}$ protons should be an effective probe for deterwining the multipolarity of the various observed peaks in the giant resonance region. Significant differences exist between the angular distributions of neighboring L-transfer values. For example, the GOR $(L=1)$ will be excited strongly and nearly exclusively for angles less than -6 degrees and will provide almost no contribution to the spectra for angles greater than -10 degrees. Previous measurements with 60-MeV protons and 120-MeV alphas yielded angular distributions wich were not particularly discriainating between neighboring L-transfer values. With the high resolution of our proton
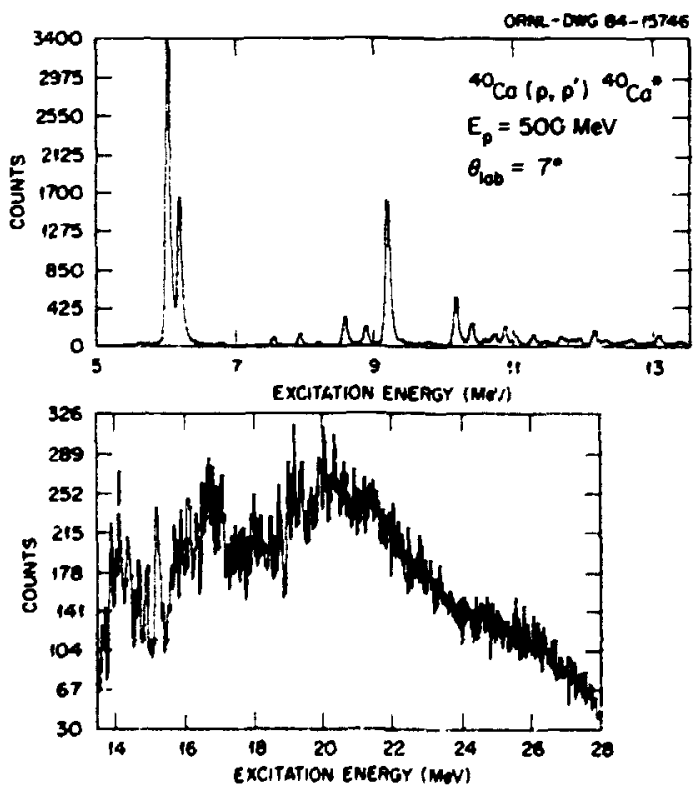

Fig. 2.11. An excitation energy spectrum for ${ }^{4} \mathrm{Ca}(p, p)^{40} \mathrm{Ca}$ at $E_{p}=500 \mathrm{MeV}$ and $0 \mathrm{ab}=7^{\circ}$. (a) shows low-lying discrete states in $4 \mathrm{Ca}$; excitation energies are labeled on the abscissa. (b) shows a compressed view of the giant resonance region in ${ }^{\circ} \mathrm{Ca}$. Note in (b) that the efficiency of the focal plane detector at this magnetic field setting decreases for excitation energies above $25 \mathrm{MeV}$.

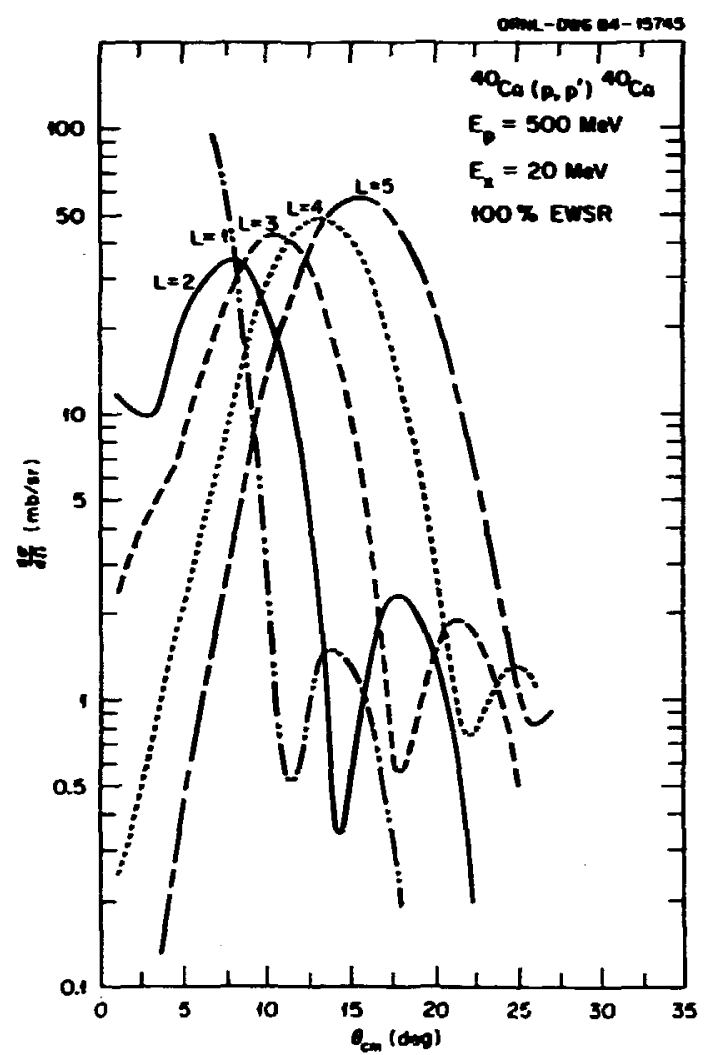

Fig. 2.12. DWBA calculations for excitation of various multipoles by the reaction ${ }^{\circ} \mathrm{Ca}\left(p, p^{\prime}\right){ }^{40} \mathrm{Ca}$ for $E_{p}=500 \mathrm{MeV}$. Each calculation assumes $100 \%$ depletion of the energy weighted sum rule (EWSR).

scattering data and strong L-dependence of the cross section angular distribution, this analysis, when completed. Should improve multipolarity assignments for peaks in the giant resonance region of the sd-shell nuclei.

In order to test the DWBA calculations, data must be taken for low-lying states of known spin and parity. A large number of low-lying states were well resolved in both the ${ }^{40} \mathrm{Ca}$ and $28 \mathrm{Si}$ spectra, see, for example, Fig. 2.11(a). These data have been analyzed using the QSYSTEM replay package on the VAX computers at LAMPF. Peakfitting and DWBA calculations for low-lying states and peaks observed in the giant resonance region are in progress at Oak Ridge.

1. Computing and Telecommunications Division, ORNL.

2. Oreyon State University, Corvallis, OR 97331

3. University of Oregon, Eugene, OR $974^{n}$,

4. Los Alamos National Laboratory, Los Alamos, M 87545.

5. F. E. Bertrand, Nucl. Phys, A354, $129 \mathrm{C}$ (1981). 
6. F. E. Bertrand et al ., ORM -5137, F. 63 (1975), unpublished.

7. F. E. Bertrand et al., Phys. Rev. Lett. 40. 635 (1978) - K. Yan der Borg et al .. Phys. Lett. 678, 405 (1977). K. Van der Borg et al.. mucl. Phys. A365, 243 (1981).

8. A. Richter, Lectures presented at "The International School on muclear Structure," Alushta, USSR, April 14-25, 1980, IKOA 80/4 (1500).

9. E. Kuhleann et al., Phys, Rev. C 11. 1525 (1975).

\section{ELECTROAENTIC DECAY OF GINT RESOMUNES}

\author{
J. R. Beene \\ R. L. Auble \\ F. E. Bertrand \\ D. J. Horen \\ M. L. Halbert \\ R. L. Robinson \\ D. C. Hensley \\ T. P. Sjoreenl \\ R. O. Sayer 2
}

Current theoret ical investigations of giant resonances -icus on erforts to understand the microscopit itructure of the resonances and the damping of se resonance states into the continum. 3 Experimental data required to guide and constrain these investigations include detailed studies of the decay of the resonances. Studies of electromagnetic decay are particularly appealing because of the relative simplicity of interpreting the results, and in the case of the direct decay of the resonances to the ground state, because of the intimate relationship of this process to the definition of the giant resonances.
We have carried out coincidence experiments to investigate photon and neutron exission fro the giant reionance region in $204 \mathrm{~Pb}$ and $90 \mathrm{Zr}$ using the Cad Spin spectraneter, a 72 segrent, ainost 41 mal detector systen. States in 201pb and ${ }^{90 Z r}$ were excited by inelastic scattering of 300-itev 170. 170 ws chosen as a praject ile because its low neutron binding energy (4.1 hey) prevents projectile excitation from being an important source of background in the vicinity of the resonances.

Details of the experiment and the nethods developed to malyze the data have been discussed extensively el senhere. ",5,6 In this report w wil concentrate on results for $20 \% \mathrm{pb}$. The ${ }^{90} \mathrm{Zr}$ experiment produced insufficient data to be useful in investigating the region of the giant quadrupole resconance (ER), but will produce veluable results on decay of lawer lying states, such as the low energy octupole resonance at $7.5 \mathrm{MeV}$.

Table 2.3 somarizes current knowledge of strongly collective states in the 8-18 hey region of $20 \mathrm{pb}$, based largely on high resolution, nediu evergy proton scattering data.? The last to colums refer to the present experiment. The colven labeled Expected of gives the cross section for 170 scattering calculated with the code ECIS, assuing the strengths from proton scattering. The last colum gives the cross sections actually observed in the current experiment, obtained from fits to the $17_{0}$ inelastic spectru with peak parameters fixed in accordance with the information from $\left(p, p^{\prime}\right)$. The 170 data appear to be in excellent agreewent with the proton data.

Table 2.3. Properties of states above $B$ meV in $208 \mathrm{pb}$ observed in $208 \mathrm{pb}\left(p, p^{\prime}\right)$, from Ref. 7. The last two columins refer to the present $20 \mathrm{Bb}\left({ }^{1} 7_{0}, 1^{1} 0^{\prime}\right)$ experiment. Expected $\sigma$ is the cross section expected for 170 scattering based on the proton results. observed $\sigma$ is the cross section which we observe. Uncertainties of about $15 \%$ apply to woth the observed and expected cross sections.

\begin{tabular}{|c|c|c|c|c|c|}
\hline $\begin{array}{l}\text { Excitation } \\
\text { Energy } \\
\text { (MeV) }\end{array}$ & $L$ & $\underset{(\mathrm{MeV})}{5}$ & $\begin{array}{c}\text { EUSR } \\
\text { Fraction } \\
x\end{array}$ & $\begin{array}{c}\text { For }( \\
\text { Expected } \\
\sigma \\
(m b / s r)\end{array}$ & $\begin{array}{c}\frac{\left(7^{\prime}\right)}{\text { Observed }} \\
(\mathrm{mb} / \mathrm{sr})\end{array}$ \\
\hline $\begin{array}{l}8.11 \\
8.35\end{array}$ & $\begin{array}{l}4 \\
3\end{array}$ & $\begin{array}{l}0.4 \\
0.4\end{array}$ & $\begin{array}{l}3 \\
4\end{array}$ & $\begin{array}{l}9 \\
5\end{array}$ & 13 \\
\hline 8.86 & 2 & 0.4 & 7 & 8 & 6 \\
\hline 9.34 & 2 & 0.4 & 5 & 5 & 13 \\
\hline 10.6 & 2 & 2 & 70 & $5 c$ & 60 \\
\hline 12.0 & 4 & 2.4 & 10 & 17 & 18 \\
\hline $\begin{array}{l}13.6 \\
13.9\end{array}$ & $\begin{array}{l}1 \\
0\end{array}$ & $\begin{array}{l}4.0 \\
2.9\end{array}$ & $\begin{array}{l}100 \\
100\end{array}$ & $10 !$ & 20 \\
\hline
\end{tabular}


Figure 2.13 shows the relative ground state branching (i.e... ground state gamas/total gamas) as a function of excitation energy in 20sp. A rapid fall-off in ground state decay above the meutron bindirg energy $(7.4 \mathrm{MeV})$ is evident, as is a strong clustering of ground state strength in the 9-15 MeV region. This provides a graphic illustration of the special relationship that states in this excitation energy region have with the ground state. This broad peak contains contributions from both the GQR and the giant dipole resonance (EOR), wich though very weakly excited (see Table 2.3) has a unch larger ground-state gama decay width. The ground-state gama decay spectru was decomposed using the peak information from table 2.3. assuaing that only dipole and quadrupole states contribute. The decomposition was confirmed by gama-ray angular distribution data wich showed 708 of the ground-state radiation in the 9.6-11.6 MeV region to be quadrupole. The ground state decay and yields from the singles spectra provide an estinate of the ground-state gama branch of the GQR:

$$
\frac{\Gamma_{Y_{0}}}{{ }^{I_{T}}}=(3.27 \pm 0.45) \times 10^{-4} .
$$

This result can be used to obtain an absolute value for $\Gamma_{T_{0}}$ if we assume $\Gamma_{T}=\Gamma^{+}$(mere $\Gamma^{+}$is the spreading width) and identify ${ }^{+}$with the experimentaliy observed resonance width iExp from Table 2.3:

$$
\Gamma_{P_{0}} \times \frac{\Gamma_{Y_{0}}}{\Gamma_{T}} \Gamma_{\text {EXP }}=654 \div 91 \mathrm{eV}
$$

or, taking $E_{\text {xo }}=10.6$

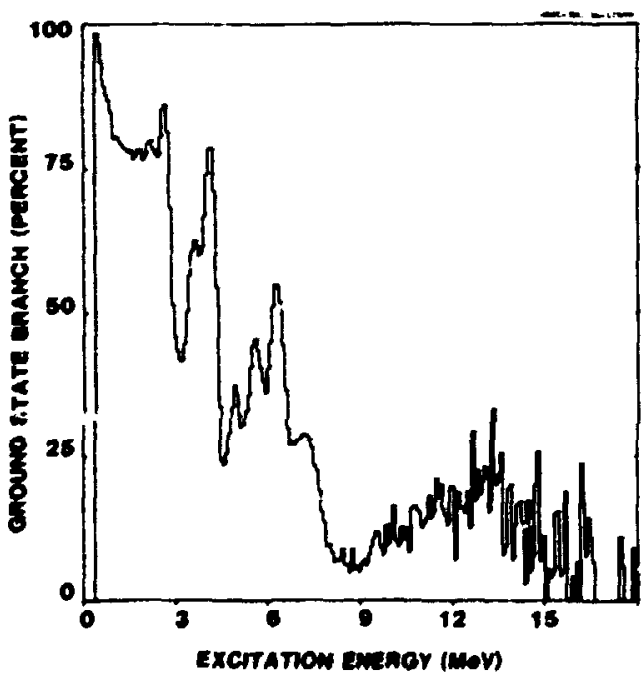

Fig. 2.13. The yield of gamma rays directly to the ground state divided by the total gammaray yield, as a function of $20 \mathrm{Bpb}$ excitation energy.
$B(E 2 t)=(5.81 \pm 0.81) \times 10^{3} \mathrm{e}^{2} \mathrm{fo}^{4}$.

This should be compared with the energy wighted sure rule (EusR) value, s

$B(E 2 t)_{\text {ENSR }}=49.9 A^{5 / 3} /\left(5 \times E_{p}\right)=6.9 \times 10^{3} e^{2 f_{m}^{*}}$.

Therefore.

${\frac{B(E 2 t)}{B(E 2+)_{\text {EUSR }}}}=0.85 \pm 0.12$

The yield of dipole ground state transitions from the vicinity of the $E D R$ is consistent within large uncertainties ( $-50 \mathrm{~s}$ ) with the pure Coulow pred.ction of COA excitation obtained from the direct reaction code SCIS, and a decay strength corresponding to 1007 of the EUSR strength.

Gan decay branches from the resonance region other than the ground state decay are also of great interest, in particular, direct decays to the low-lying collective states at $2.0 \mathrm{MeV}\left(3^{-}\right)$and $4.08 \mathrm{MeV}\left(2^{+}\right)$. Figure 2.14 shows the relative strength of gama ray branches to a nuber of low lying states: (a) is for the ground state, (b) the $2.6 \mathrm{MeV} 3^{-}$, (c) the 4.00 MeV $2^{+}$, and (d) the $4.97 \mathrm{MeV} \mathrm{3 \%}$. The yield distributions in (b)-(d) aust be considered semi-quan:itative, especially where they indicate small strengths, because adequate backgraund subtraction has not been performed. Mevertheless they are valuable to indicate general features. One of tne nore striking aspects of these data is the marked ausence of strength to the 2.61-MeV and 4.08-MeV states across the entire resonance region. Another interesting feature is the strong yield of decays to the 2.61-MeV state at $-5.2 \mathrm{MeV}$ of excitation energy. This might be an indication of the long-sought, two-phonon octupole vibrational state. In contrast to the collective 3state, yield to the 4.97-MeV 3- state (which is thought to be dominated by a single neutron particle hole configuration) is significant across the entire igR resonance, but with its greatest yteld $-1.5 \mathrm{MeV}$ below the peak of the GQR. A very similar strength distribution is seen for population of a $5^{-}$state at $3.97 \mathrm{MeV}$, which we take to indicate the presence of high spin $\left(4^{+}\right.$or $\left.6^{+}\right)$strength underlying the GOR.

$A$ more quantitative analysis of the decay of an excitation energy bin centered on the GQR is given in Table 2.4. It should be noted that the absence of decay to the 2.6-KeV 3- state, wich appears remarkable at first sight, was predicted by Bortignon. Broglia, and Bertsch, is

The data presented in this report are only a sampling of a rich body of information now hecoming avaflable from spin spectrometer-based studies of giant resonance decay.

1. Solid State Division, ORNL.

2. Computing and Telecommunications Division, ORML. 
3. 6. E. Bertsch, P. F. Corticnon, and R. R. Broglia, Rer. Mod. Phys. 5, 201 (1983).

4. J. R. Beene et al .. Euclear Physics with Heovy Ions, ed. P. Braun-munzinger (harnood Teadelic, i.Y., 1983).

5. F. E. Bertrand, J. R. Beene, and T. P. Sjoreen, Journal de Physique 45, C4-99 (1994).

6. J. R. Beene et al .. Proceedings of Fifth International Symposium on Capture Gua Ray Spectroscopy. Sept. 19\%4, to be published.

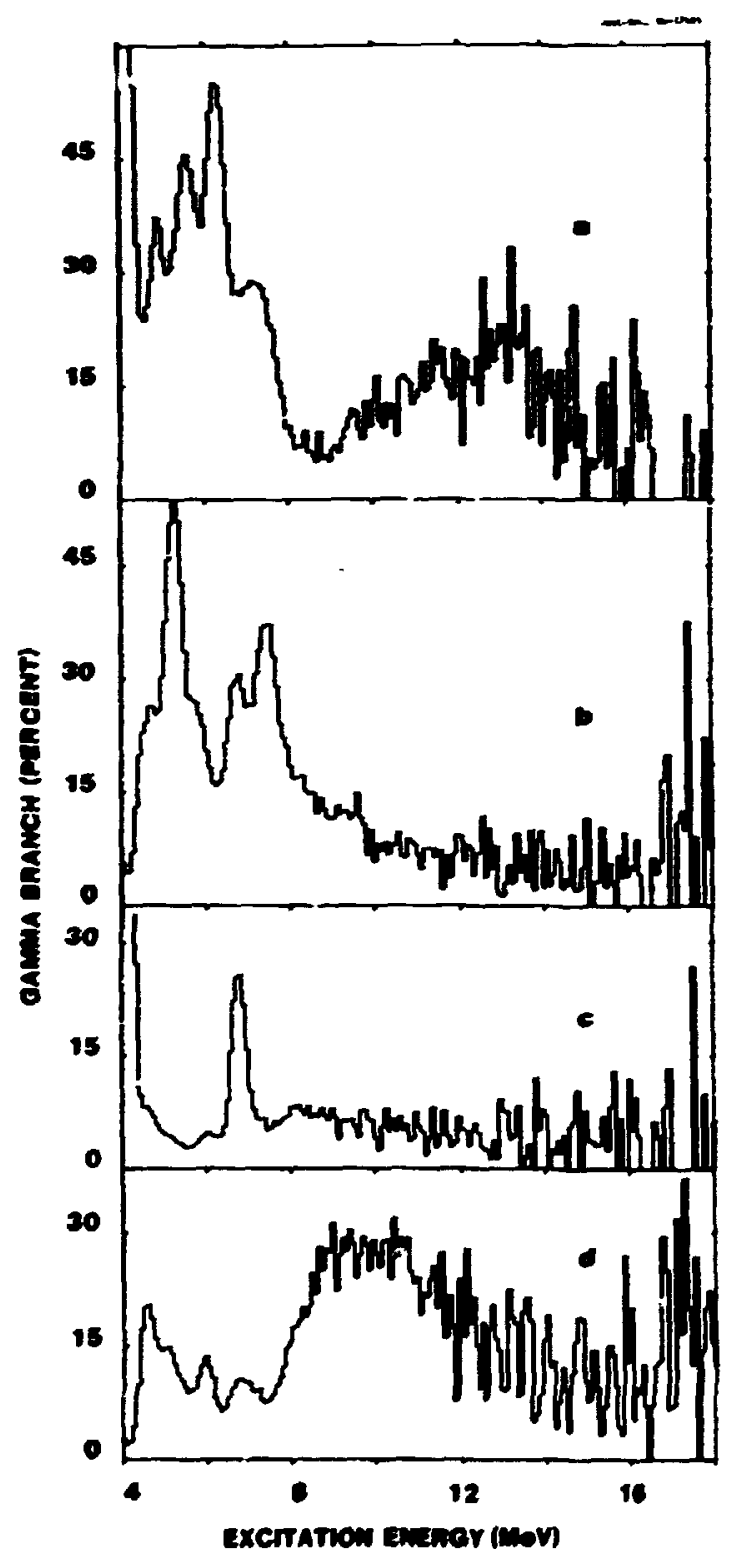

Fig. 2.14. The yield of gamm rays populating low-lying states in $208 \mathrm{pb}$, relative to the total gami-ray yield, as a function of excitation energy in $200 \mathrm{pb}$. Figure $2 \mathrm{a}$ is for the ground state, $2 b$ the $2.61 \mathrm{MeV} \mathrm{3-}, 2 \mathrm{c}$ the $4.08 \mathrm{MaV} 2^{+}$. and $2 d$ the 3.97 MeV 3- state.
7. F. E. Bertrand et al., private commications.

8. F. E. Bertrand, Mn. Rey. Mucl. Sci 26, $457(1976)$.

9. A. Bohr and B. Mottleson, muclear Structure, Vol. II (eenjwin, Rending hass, 1975).

10. P. F. Bortignon, R. Brogilia, and G. F. Bertsch, to be published.

Toble 2.4. Relative gamo branching to lowIying states in 2elpo fro ar excitation eneryy region 9.5-11.5 MeV [E(EQR) \pm (EQR)/2]. The 5-7 MeV 1 - states refers to a group of 1 - states in that region toun from T.T' experiments.

\begin{tabular}{lll}
\hline Energy & $j *$ & $\begin{array}{c}\text { Relative Ean } \\
\text { Branch (S) }\end{array}$ \\
\hline 0 & $0^{+}$ & $20 \pm 2$ \\
2.61 & $3-$ & $0.8 \pm 0.8$ \\
3.97 & $5-$ & $-5-10$ \\
4.08 & $2+$ & $0.2 \pm 0.3$ \\
4.97 & $3-$ & $36 \pm 5$ \\
$5-7$ & $1-$ & $23 \pm 9$ \\
\hline
\end{tabular}

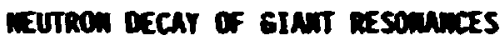
J. R. Beene
R. L. Iuble
F. E. Bertrand
D. J. Horer.
M. L. Halbert
R. L. Robinson
D. C. Hensley
T. P. Sjoreen 1
R. 0. Sayer ${ }^{2}$

Experimental studies of the decay of giant resonances are required to guide and constrain theoretical efforts to gain a more complete understanding of the microscopic structure of giant resonances and their daping into the underlying continuuw of more complex states. We have performed experinents to study in detail all decay modes of the giant resonance region of 208pt and $90 \mathrm{Zr}$. Both 20ept ano 90zr were excited by inelastic scattering of $381-\mathrm{heV} 170$. Decay of the giant resonance region up to $20 \mathrm{WeV}$ was studied using the CRim Spin Spectrometer. 3 The experimental techniques and methods of analysis employed in this study have been discussed in dotafl el sewhere, $4,5,6$

Meutron enission makes up the bulk of all decays in the 9-20 $\mathrm{MeV}$ region in both systems studied. The goal of the present study is to obtain as complete information as possible on the distribution of decay strength to low-lying states in $207 \mathrm{~Pb}$ and $89 \mathrm{zr}$ as a function of $20 \mathrm{mb}$ and $90 \mathrm{zr}$ excitation energy. The residual excitation energy in the daughter nucleus is 
cbtained fron the total gana-ray energy observed in the Spin Spectroneter in coincidence with a neutron (distinguished from gamas rays by its longer tice of flight to the spectrometer). Neutron angular distributions can also be constructed, utilizing the alwost ax solid angle and relatively fine segmentation ( 70 elements in our case) of the Spin Spectraneter. 3 The kinetic energy of the emitted neutrons is obtained froa the initial excitation energy in the parent mucleus (i.e., from the inelastically scattered 170 energy). the residual excitation energy in the daughter nucleus (sum gama-ray energy), and the neutron binding energy.

The analysis of neutron decay has, so far, concentrated on the $20 \mathrm{Bpb}$ data. We find neutron angular distributions up to acout $12-13 \mathrm{meV}$ of excitation energy wich are very nearly $5 y$ metric with respect to the 2040 recoil direction. sugesting the dominance of statistical or cumpoun: actay. The neutron energy spectra obtained from this excitation region, however. are significantly different from the Maxwellian shape normally associated with statistical decay. In apparent 10-152 excess of high energy neutron enission is observed, in agreement with earlier work.7 It is dangerous, however, to conclude that these high energy neutrons represent a genuinely nonstatistical fraction without more careful investigation. The high energy neutrons result primarfly from population of the ground state and $t_{1}$ ? first two excited states in $307 \mathrm{pb}$. The level density in this region is far too low to generate axwellian neutron spectra. Careful statistical nodel calculations, including explicitly the experimental level scheme of $20 \%$ b are required before a significant comparison can be made. 9 Such calculations are in progress.

The data show significant yield to high spin states $\mathrm{Cf} 207 \mathrm{pb}$ (I $>9 / 2$ ) across the region doainated by the giant quadrupole resonance (9-12 MeV). This is in agreenent with earlier work ${ }^{7}$ and also supports evidence for high spin strength in this region found in the electromagnet ic decay data. $4,5,6$

Above $-13 \mathrm{meV}$ of excitation in $20 \mathrm{Bpb}$ we see a rapidly rising (with increasing excitation energy) fraction of strongly forward peaked neutrons. These neutrons appear to be associated with processes which preferentially populate the $5 / 2^{-}$and $3 / 2^{-}$states at 570 and $898 \mathrm{keV}$ in $207 \mathrm{~Pb}$. At $\mathrm{E}^{*}$. $15 \mathrm{MeV}$, approxiastely $30 \%$ of the population of the $3 / 2^{-}, 898 \mathrm{keV}$, state corresponds to these forward peaked neutrons. Preliminary analysis indicates that these neutrons are more tightly correlated with the direction of detection of the 170 ejectile than with the beam direction. It is likely that most of them arise from the reaction $208 \mathrm{pb}(170,180) 207 \mathrm{pb}$, in wich both the $207 \mathrm{pb}$ and the ion pronducts are left excited, the ${ }^{A} 0$ having suffictent energy to decay in tlight by neutron emission ( $\mathrm{Sn}=8.04 \mathrm{MeV}$ for $1 \mathrm{~B}_{0}$ ) . This process has, of course, nothing "o do with decay if the giant resonance region in $20 \mathrm{Apb}$. These and other interesting aspects of the data are under active study.
1. Solid State Division, ORKL.

2. Computing and Telecomunications Division, ORn.

3. 0. 6. Sarantites, R. Lovett, and R.

woodward, Nucl. Instrum. Methods 171,503 (1980), and $M$. Jajuskel ginen et al., Mucl. Instrum. Methods 204, 385 (1983).

4. F. E. Bertrand, J. R. Beene, and T. P. Sjoreen, Journal de Physique 45, C4-99 (1984).

5. J. R. Beene et al., Nuclear Physics with Heavy Ions, ed. P. Braun-hunzinger (Harwood Rcademic, N.Y., 1983).

6. J. R. Beene et al., Proceedings of fifth International Symposium on Capture Gama Ray Spectroscopy, Sept. 1984, to be published.

7. H. Steuer et al., Phys. Rev. Lett. 47, 1702 (1981).

8. H. Dias and E. Wolynec, preprinz IFUSP/P-469.

\section{FISSIOM DECAY OF GIAMT RESOMnnces In 23\%}

$$
\begin{aligned}
& \text { J. R. Beene B. L. Burks } \\
& \text { C. E. Benis E. E. Gross } \\
& \text { F. E. Bertrand D.J. Horen } \\
& \text { R. L. Auble I. Y. Lee } \\
& \text { R. O. Sayer I }
\end{aligned}
$$

In an earlier study ${ }^{2}$ of the fission decay of the giant quadrupole resonance in 238u, using the $23 \mathrm{BU}\left(a, a^{2}\right)$ reaction at $152 \mathrm{MeV}$, the data suggested tha: the fission branch was consistent with that of the underlying continuum and the GDR. A ajor shortcoming of that study was the limited angular range covered by the fission fragment detectors. This introduced a considerable uncertainty in the deduced fission branch due to the lack of inforation regarding the fission fragment angular distribution. A value $T_{f} / r=0.25 \pm 0.10$ was obtained by assuming $K$ was preserved in the decay process. This disagrees with an earlier measurement ${ }^{3}$ which gave an upper liait of about 0.04 . Thus. additional measurements mere indicated to resolve this discrepancy.

The present measurements were performed using the $238 u\left(160,160^{\circ}\right)$ reaction at $400 \mathrm{MeV}$. This reaction was chosen on the basis of measurements on $20 \mathrm{spb}$ where a favorable peak/cont inuum ratio is obtained in GQR region using heavy ion inelastic scattering. " The scattered $160^{\prime}$, and other reaction products, were detected in elght Si-detector telescopes oriented in a ring surrounding the beam line and at a scattering angle of :2 21.5 degrees (a maximum of the GOR angular distribution). The fission fragments were detected by two $12 \mathrm{~cm} \times 12 \mathrm{~cm}$ position sensitive avalanche detectors (PSAO's) located at $90^{\circ}$ on either side of the beam line. The detector arrangement is show in Fig. 2.15. The data will provide a measurement of the complete fission fragment angular distribution, relative to the recotl axis, and will answer the question regarding $K$ conservation as well as provide a more precise measurement of the CQR fisston 
Oall-Photo 2730-84

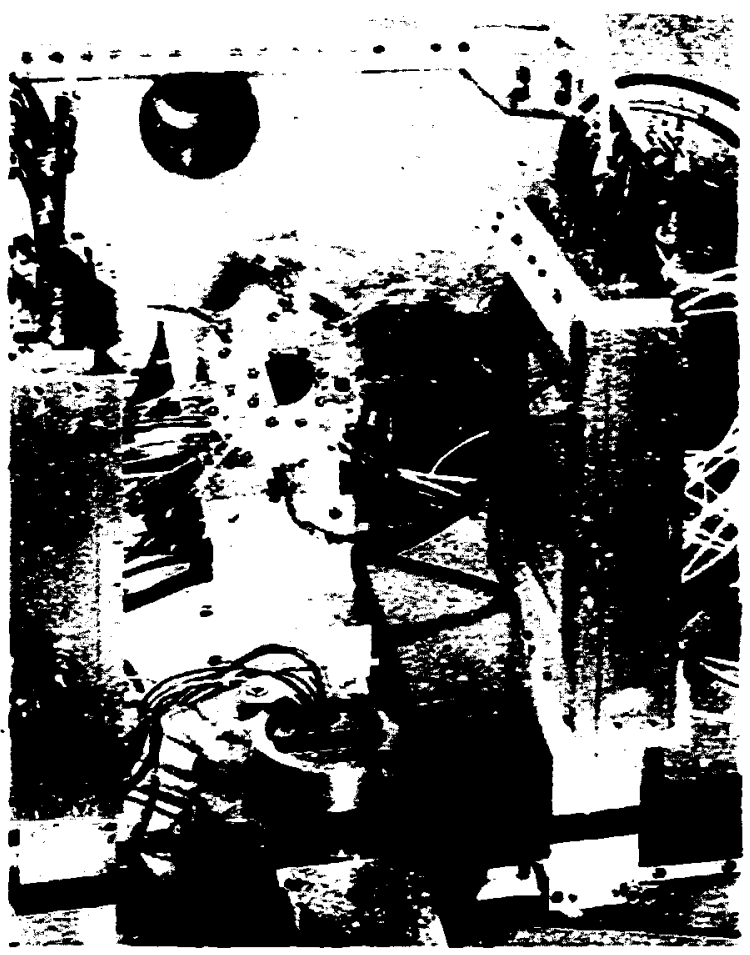

Fig. 2.15. Detector arrangement used to study the fission decay of the giant resonance region in 23\%. The parallel plate aralanche counters are located on either side of the bea line and a ring containing eight si-detector telescopes is located near the center of the photograph. The target, wich normally sits between the two aval anche detectors, has been reaved.

branch. The PSAD position calibrations are now complete and the data analysis is in progress.

1. Computing and Telecomunications Division, ORM.

2. F. E. Bertrand et al., Phys. Lett. 998, $213(1981)$.

3. J. van der Plicht et al., Phys. Rev. Lett 39. 1188 (1977).

4. T. P. Sjoreen et $31 .$, Phys. Rev. 29, 1370 (1984i.

\section{ELASTIC AND INELASTIC PROTON SCATTERIMG FROM ISO AT $200 \mathrm{MeV}$}

$$
\text { C. H. Glover }
$$

A growing number of nonrelativistic distorted wave approximation (OWA) analyses of elastic and inelastic proton scattering indicate that the effective free nucleon-nucleon inceraction ( $t$-matrix) must be modifted to include effects due to the nuclear edive for froton bobarding energies below $400 \mathrm{MeV} 1$ and the need becomes essential for bobarding energies below 200 MeV. 2 The most sophisticated modifications to date have been those derived from 6-matrices based on realistic in potential ${ }^{3}$ and calculated in the presence of axclear atter mere ane of the ton interacting nucleons has an energy greater than the Fermi energy. The resulting 6-matrices are local, energy- and densitydependent. In particuldr, the central isoscalar spin-independent componet of the effective $m$ interaction is the cost sensitive to these nuclear medium indifications. 2,3 These 6atrices are aplied to transitions in finite muclei by invoting the local density approxiantion (LOA).2

The study of proten induced elastic and inelastic isoscalar matural pe-ity transitions has show that these transitions are drived priartily by the central isoscalar spin-independent component of the effective interaction at sall I menten transfer ( $\leq 1$ fir-1). by the spin-orbit part of the effective interaction for internediate $q$ values ( 1 fr- $1 \leq q \leq 2.5(n-1)$ and both comonents have roughly the sane mogittude in the high-q region. 4,5,7 Calculatioas for these inelastic transitions in the 100-200 hev energy regive which use the free t-atrix and phenomenological distorted waves indicate that the low-q cross section is overpredicted by typically 503 and the high-q part is underpredicted. The calculations using the G-matrix are in closer agreenent with the cross section data because the low (high)-q components of the central isoscalar spin-independent part of the in-interaction is reduced (enhanced) as compared to the free $t \rightarrow a t r i x .2,5$ Moreover, when malyzing power data are considered in 2-ition to the cross section data, they appear to prefer distorted wes generated from a folding model optical potential using the G-matrix interaction over tinose generated from the phenomenological Woods-Saxon optical potentials which are fit to the elastic data.2 However, the folded G-matrix optical potentials with the characteristic pocket of attraction in the nuclear surface do not adequately reproduce the elastic scattering data. Even so, all calculations (both elastic and inelastic) using the folded G-matrix are still far supertor to those using the free $t$ matrix interaction.

An explanation, given by xelly, ${ }^{2}$ for this apparent lack of consistency in the G-matrix description of the elastic and inelastic data focuses on the radial localization of the transition." Radial localization can be understood in the distorted we approxination (Duk) by recalling that the inelastic transition anpli. tude is proportional to the average of the produce of the transition density, distortion factor, and the effective $\mathrm{NW}$-interaction. Inelastic natural parity transttions involving large angular mentum transfers $(-2-4 k)$ tend to have transition densities wich peak near the low densily nuclear surface," Monopole and isoscalar electric dipole transitions tend to peak more toward the high density nuclear intertor ${ }^{2}$ Hence, the offferent, inelastic transitions tend to wight different regtons of the 
distortion factor (i.e., optical potential). wile elastic scattering is averaged over the entire muclear volune. Thus, some ine!ast ic transitions $m y$ be more sensitive to the interior properties of the cotical potential than el astic scattering.

This can be realized by considering the fact that in fitting a thencmenological boods-Saxon optical potential to elastic scattering data neasured over small wantu transfers (g) $\leq 3$ fa-l) results in a potential mich is biased tomer the nuclear surface region and does not adequately ref lect the contributions $t r$. elastic scattering fro the nuclear iaterior. Hence, measurements to large q would constrain. those partial wes emating from the nuclea: interior. Also, the lloods-Saxon s.lape presumes the eptical potential reasirs constant in the nuclear interior and this is contrary to what is expected fro the microscopic nuclear atter calculations.

An approach, the one tu be reported here, to renedy this inconsistency is to measure elastic scattering data to large monentum transfer, so the contributions to the optical potential frem the nuciear intex inr are reflected in the date. Thes, fit the data $t y$ using a phensmenological paranetrization for :he real central cotical potential that is flexible enough to reproduce those characteristics expected fro aicroscopic derivations of the single-particle potential in muclear atter. Finally, a comparison of the Dul analyses of the inelastic data for surface and interior states using these potentials for the distorted wes against those using the folded G-atrix optical potential should indicate to that extent our expectations from nuclear matter are borne out phenomenologically.

In Ref. 6, optical potentials were reported for $D \pm 160$ at 200 meV miere the data mere ceasured to $4-6$ fa-l. The standard single woods-Saxon (SUS) and a monstandard double loods-Saxon (Dis) optical potential parametrizations --2 applied to the data, see fig 2.16 . In the Dus parametrization, the central real potential was adified to be the sum of two Woods-Saxon prtentials, one atcractive and one repulsive. The more flexible ous parametrization reproduced all the data better than the SuS parametrization. The Dus parametrization proved to be so flexible that two vastly different optical potentials were found. These pocentials are not related to one another through a phaseshift abiguity. The best description of the data (inclinding total and reaction cross sections) is obtained with the Dus(-) parametrization, where the real central potential is attractive over the entire radial range and exhibits an enhanced attraction inside $2 \mathrm{fm}$. The data are described nearly as well, however. by the Dus(t) representation in wich the real central potential has an attractive pocket near the nuclear surface and a repulsive core inside 2 fin (hence the ciaracterizations Dus $(+)$ and Dus( $(-)$ with the $(+)$ and $(-)$ symbols obviously referring to the repulsive or attractive real central core]. The results of the latter carametrization are more in accord with expectations from various microscopic theories of nucleon propagation in a many-body nuclear
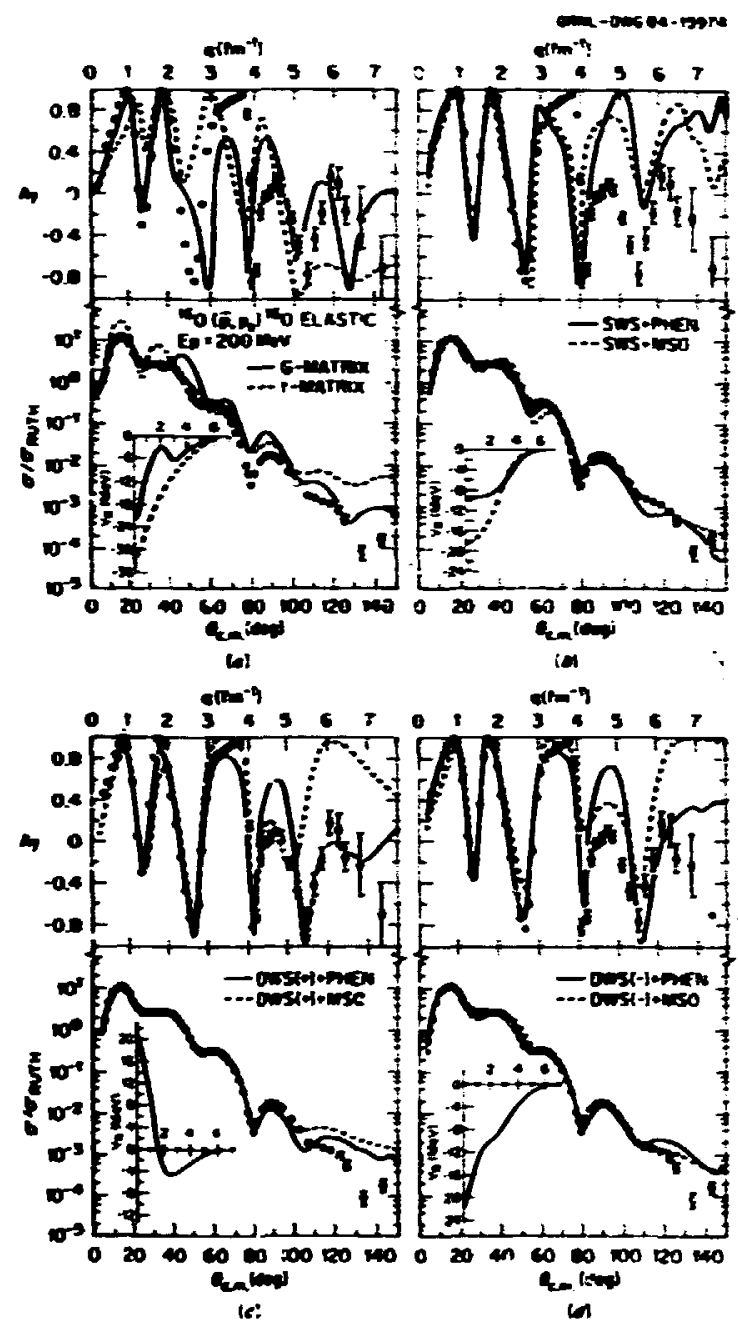

Fig. 2.16. The $\vec{p}+160$ elastic scattering cross section and analyzing power data are compared with those microscopic and phenomenological optical potentials that are discussed in the text. The data are displayed as a function of center-of momentum angle $\theta \mathrm{cm}$ on the bottom axis and as a function of momentum transfer $q$ on the top axis. Inset in each figure are the resulting real central optical potentials. In Fig. 2.16(a), the r-ratrix optical model calculation is based on the Paris in interaction.

enviroment. Each potential implies that a different scattering mechanism is responsible for the increase in the elastic flux at large $q ; a$ particle can be scattered to large angles efther from the near side of the nucleus by reflection from strongly repulsive potential, or by a strongly attractive potent, fal causing the particle to orbit around the far side of the nucleus. The results of the present analysis indicate that it is impossible to distinguish between these scattering mochanisms on the basis of the available elastic scattering data alone. 
These phencme.:ological optical potentials have been used along with those generated by folding the 6 and $t$-atrix in interactions with the Ifo ground state censity distribution in a oul analyses for the inelastic transitions to the first $2^{+}$state (a surface state) and to the first 1- state (an interior state). The ground stite and inelastic transition leasities were measured to -3 fi- -1 by electron scattering. (Hence, any critical assessment of the calculations will be lieited to the data formard of 3 fo-l). The free in t-matrix cones from the parzetrization of love and franey at $210 \mathrm{meV}$. The two 6-atrix interactions cone fro the paranetrizations of the Paris and knada-Johnson potentials by won Gerab. 3 The calcilations were perforned with computer code MULid-Thuma as modif ied by Carr, Kelly, and Petrovich. a The results of these calculations are displayed in Fig. 2.17. The data mere taken at the Indiand university crelotron facility and the experinental procedure is described in Ref. 6.

In Fig- 2.16, we can see that the paraneterfree microscopic aptical ootential derived fro the a-matrix interaction does not reproduce all of the elastic data as well as the phercomenologi=al potentials, but is in closer agreement with the forward angle cross section and analyzing power data chan those from the t- merix interaction. The dempening of the cross section at the first and second wixim and the enhancesent of the third and fourth axima in the Gatrix calculations over that from the $t$-atrix calculations is a result of the low-q (high-q) suppression (enhancenent, of the isoscalar spin-independent $n$ interaction mich ws discussed bove.

The consistent on alyses of the first $2^{+}$ (suriace) traasition using the Gatrix interaction are in closer agraenent with all of the data than the alcolations using the free $t$ atrix interaction. This is quite evident in the andyaing power ata (see Fig. 2.17a). (Here the tern consistent means the optical potentials were calculated using the same in interaction as that used in the inelastic form factor calculations.) Surface transitions, such as the $2^{+}$. are sensitive to the low density part of the 6-matrix interaction. The 6-matrix calculation bused on the Paris interaction is core representative of the $2^{+}$cross section data than the alculations using the Haxde-Johnson (HW) interaction. Mis indicates that the magitude of the low-density low-q component of the $6 \mathrm{~W}$ interaction is too simall and the lowdensity high-q compont is too large. while the consistent calculation using the Paris (HJ) interaction under (over) predict the 1- cross

CONSISTENT L. $\mathbf{W}$.

and-0.06 84-45977

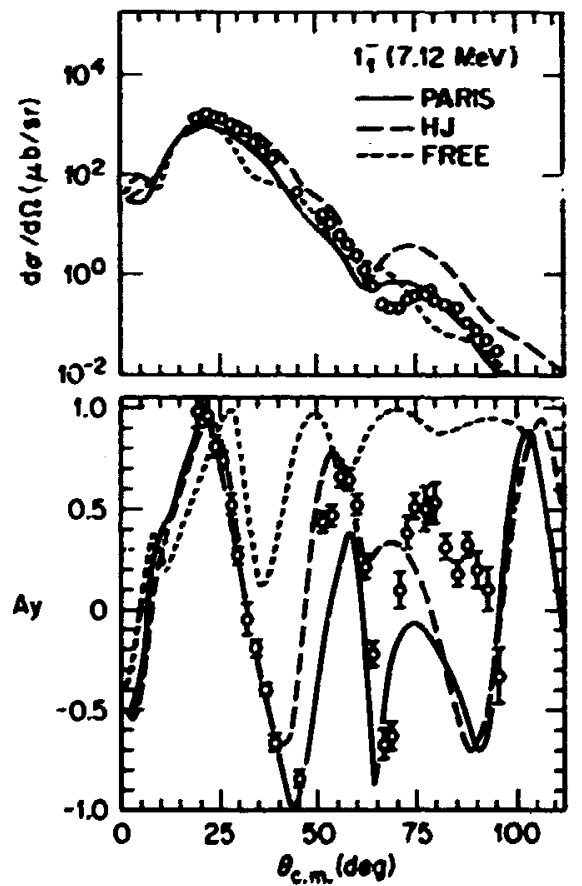

(a)

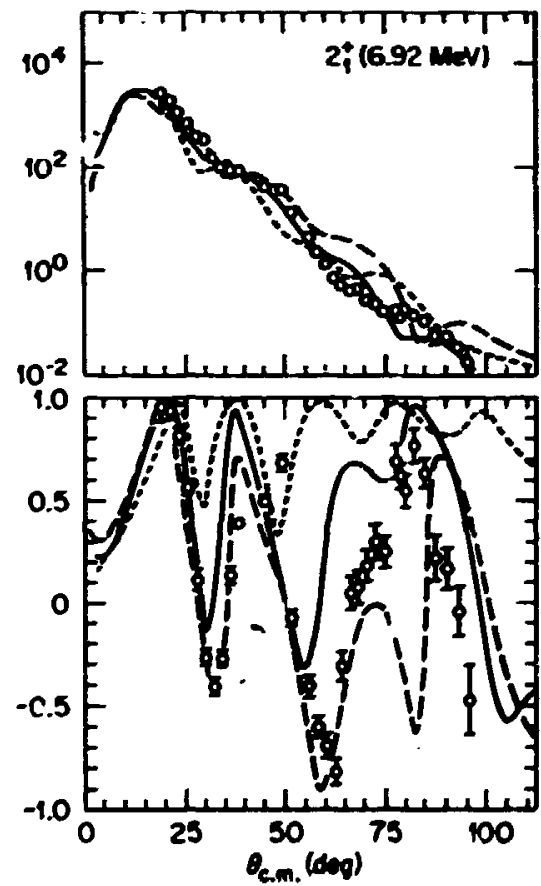

(b)

Fig. 2.17. The completely consistent microscopfc calculations are compared with the $2^{+}(1.12$ MeV) and 1-(6.92 MoV) inelastic transition data in (a) and (b), respectively. Here the distorted waves were generated from a folding model optical potentfal wich uses the same um interaction as that used in calculating the inelastic form factor. 
section (see Fig. 2.17b). This indicates the high-density component of the Paris (HJ) Gmatrix is too sall (large). However, all the analyzing power data appear to prefer the $\boldsymbol{H J}$ interaction. It is apparent from these calculations that the data require the use of a modified $\mathrm{w}$ interaction in the high density nuclear interior were Pauli blocking must be taken into account. Thus, in order to ascertain which phenomenological distorting potential the i.jelastic data prefer, we shall use only those form factors generated irom the G-matrix interactions.

We shall use our arguments on the radial localization of the transition densities to infer the properties of the surface and interior foris of the optical potential. Displayed in Figs. 2.18a and 2.19a are the calculations for the $2^{+}$state enploying the $\mathrm{HJ}$ and Paris Gmatrix interactions for the form factor and the phenomenological distorting potentials. Their counterparts for the $\mathrm{I}^{-}$state are shown in Figs. $2.19 \mathrm{~b}$ and 2.18b, rispectively. One can see the effects of the different optical potentials by comparing the calculations using the consistent distorted waves with those using the phenomenological distorted waves. ihe first thing to be noticed is that the calculations using the SWS and DWS(-) optical potentials are not very dif- ferent except at large $q$, where the calculations are more sensitive to the difference between the two potentials. Second, the calculations using the $\mathrm{HW}$ form factor are in closer agreement with the analyzing power data for both transitions than those using tle Paris based interaction. Third, the phenomerological optical potentials have little effect on the forward angle calculations and damp the oscillations at the large: angles. In the sense that the calculations for the cross section data are more sensitive to the imaginary central optical potential than the real central potential and the reverse is the case for the analyzing powers, 2 one would expect tr. high-q part of the analyzing power's angular distribution to be a very sensitive indicator as to which phenomenological real central optical potential is preferred by the inelastic data. From Figs. 2.16 and 2.19, one can see that the analyzing power data is best described by the DWS( + ) optical potential, thus confirming our expectations from nuclear matter about the nature of the single particle potential.

This analysis, along with our understanding of the nuclear matter single particle potential, may indicate one possible source for the inconsistent G-matrix description of the elastic and inelastic data. A particle moving in the folded G-matrix real central optical potential sees a

ORNL-UWG 84-15976

HJ-FORM FACTOR

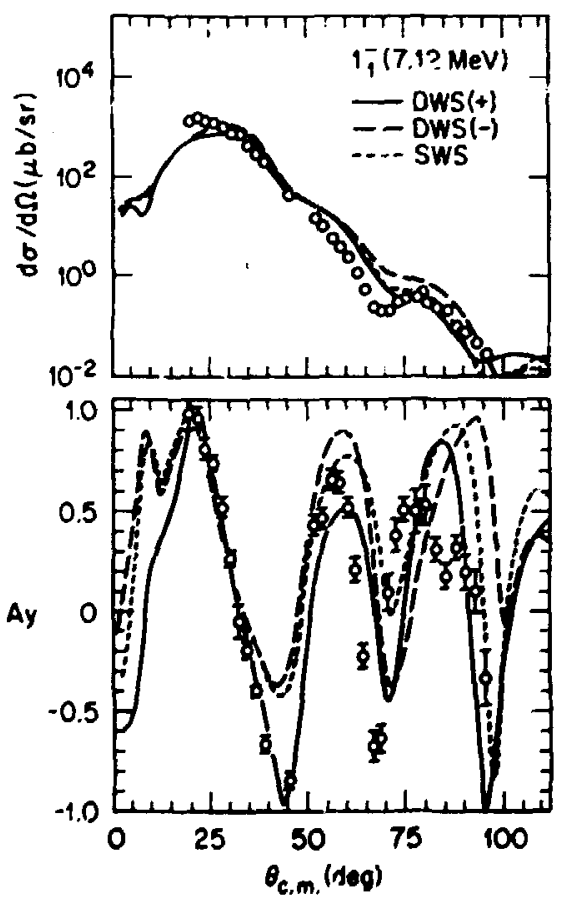

(o)

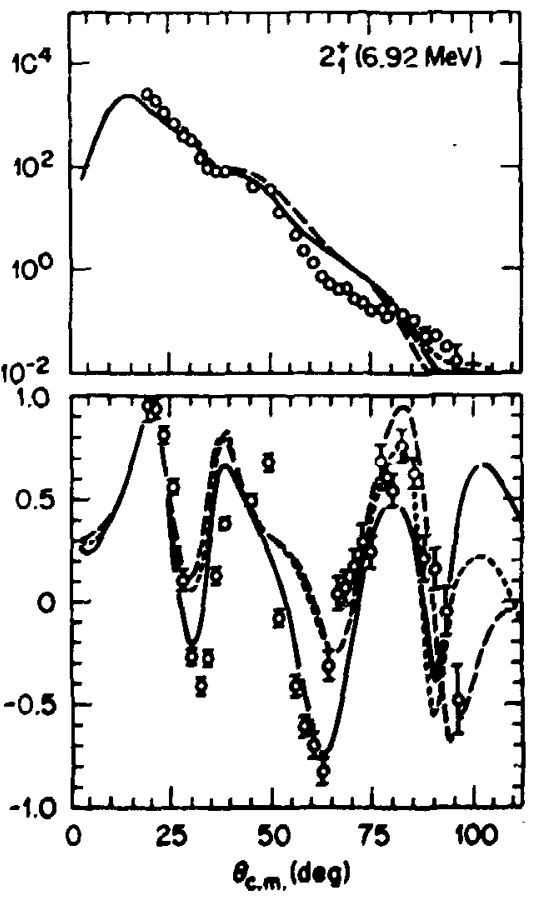

(b)

Fig. 2.18. The inelastic data are compared with calculations employing phenomenological optical potentials to generate the distorted waves and the form factor was calculated using the HJ based G-n.dtrix. 


\section{PARIS FORM FACTOR}

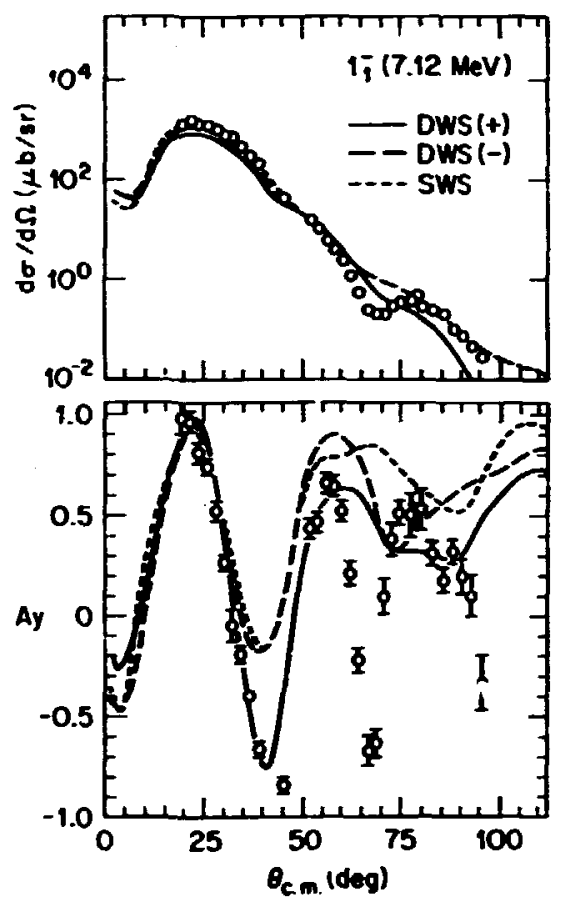

(a)

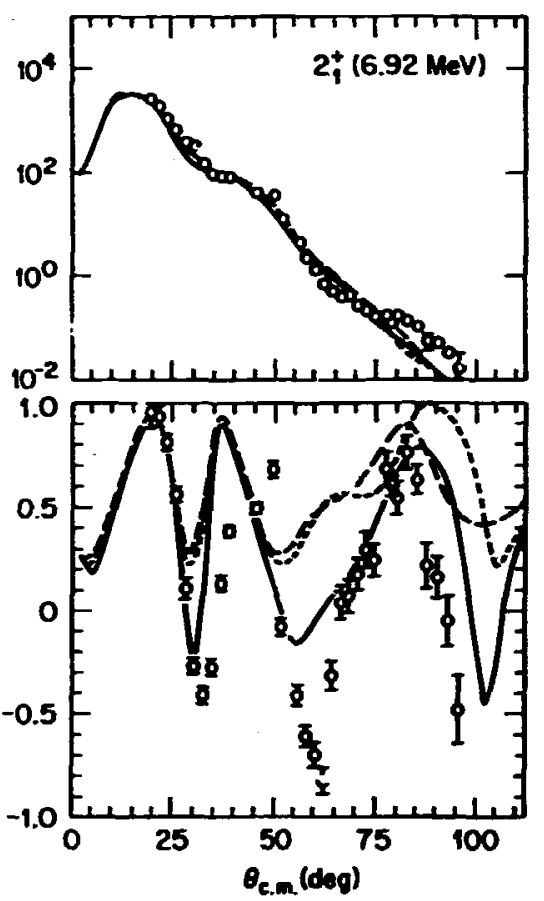

(b)

Fig. 2.19. This figure is the same as Fig. 2.18, except that the form factor was calculated using the Paris based G-mairix.

pocket of attraction near the nuclear surface. The attraction decreases as the particle approaches the nuclear interior. In the extreme nuclear interior the particle experiences a second pocket of attraction. This second pocket of attraction is contrary to what is expected from nuclear matter calculations. In the case of nuclear matter, as particles are added to the nuclear volume they attract each other until saturation is reached. Bcyond this density the particles are squeezed so tight that the hard core repulsion of the AN interaction dominates. It is at this point the single particle potential becomes repulsive and stays repulsive. The phenomenological OWS(+) reaT central potential has this quality. Also, it provides a more consistent description of the elastic and inelastic data, especially in the back angle inelastic analyzing power data wich is very sensitive to the interior of real central optical potential.

An alternate approach, successfully taken by Kelly, ${ }^{9}$ to rectify this inconsistency is to parametrize the isoscalar spin-independent Gmatrix and search on the parameters until a simultaneous description of the elastic and inelastic data can be found.
1. L. Ray, in The Interaction Between Medium Energy Nucleons in Nuclei - 1982, edited by H. 0. Meyer (AIP Conf. Proc. No. 97), P. 121 and references cited within; in Studying Nuclei with Medium Energy Protons, edited by J. M. Green (Univ. of Alberta/TRIUMF Workshop - 1983), p. 101 and references cited within.

2. J. Kelly, in The Interaction Betweel Medfum Energy Nucleons in Huclei - 1982, edited by H. O. Meyer (AIP Conf. Proc. No. 97), D. 153 and references cited within.

3. H. Von Geramb, in The Interaction Between Medium Energy Nucleons in Fuclet - 1982, edited by H. 0. Meyer (AIP Conf. Proc. No. 97), p. 1 and references cited within; in, Studying Muclei with Medium Energy Protons, edited by J. M. Green (Univ. of AtbertaliRIUwF Workshop - 1983), p. 44 and references cited within.

4. F. Petrovich et al., Phys. Lett. 918, 27 (1980).

5. H. G. Love, in Siudying Nuclei with Medium Energy Protons, edited by J. M. Green TUniv. of Alberta/TRIUMF Workshop - 1983), p. 29 and references cited with? $n$. 


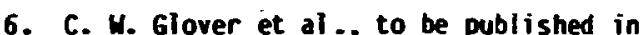
Phys. Rev. C.

7. M. G. Love and M. A. Franey, Phys. Rev. C 24, 1073 (1981)

8. J. C. Carr, J. Kelly, and F. Petrovich, the computer code ALLWRL, to be published.

9. J. Kelly, private comunication.

\section{REASURERENTS OF GANW-TELLER STRENGTH} DISTRIBSTIONS IN MSS 13 NDO 15
D. J. Horen
J. S. Larsen
C. D. Goodman
C. Gaarde 4
R. C. Byrdi
J. Rapaport
I. J. Van Heer
T. P. Nel chs
T. A. Carey ${ }^{3}$
E. Sugarbaker 6

T. K. Taddeucci $i^{7}$

The analysis of beta decay between mirror states has been used extensively for comparing shell model calculations with Gamow-Teller (GT) matrix elements extracted from asured decay rates. The essential simplicity of these transitions is that the parent and daughter states differ only in the isospin projection, $T_{Z}$. The transition rate between mirror states is the incoherent sum of the rates for the Fermi and Gamow-Teller components. The Fermi (F) operator causes changes only in the isospin projection and converts the parent state exactly to the daughter state. Thus, the square of the Fermi matrix element is simply $2 \mathrm{~T}_{z}$. The Ganow-Teller operator changes the projections of both isospin and spin. Due to the spin-orbit interaction, the GT strength is distributed between the spinorbit pair states, and only a fraction of the GT strength is contained in the mirror state transition. In these cases, which can be explored through beta decay, the fraction of the total GT strength that appears in the mirror transition is typically very small, thereby making deductions about missing strength much more model-sensitive than would be the case if a large part of the sum rule strength were seen. To investigate a possible aj dependence of the GT quenching, we have studied the mass 13 and 15 systems. The cross sections and transverse spin-flip probabilities for $13 C(p, n)$ and $15_{N}(p, n)$ were measured at zero degrees using a $160 \mathrm{MeV}$ polarized proton beam from the Indiana University Cyclotron Facility and a neutron polarimeter consisting of bars of plastic scintillator at the end of a 60-meter flight path. The polarimeter is described brifefly el sewhere ${ }^{\theta}$ and $w 1 l$ be described more fully in a future publication. The targets were pressed wafers of carbon (>957 $\left.{ }^{13} \mathrm{C}\right)$ and $\mathrm{Mel}$ amine $\left(\mathrm{C}_{3} \mathrm{H}_{6}{ }^{1{ }^{3} \mathrm{~N}_{6}}\right.$, >997 enrichment in $15 \mathrm{~N}$ ).

The results of our measurements are displayed in Table 2.5 mich gives the calculated and experimentally deduced values of $B(G T)$. The shell model calculations are based on the assumption that these nuclei can be characterized as p-shell nuclef.9 The ground state to ground state $B(G T)$ values are deduced from beta decay ft values.

Excited-state transition strengths can be extracted by first decomposing the ground state
Table 2.5. Cross sections, spin-flip probabilities, and GT transaission strengths for ${ }^{13} \mathrm{C}(p, n){ }^{1}{ }^{K}$ and $15_{N}(p, n) 150$ at $\theta=0^{\circ}$ and $E_{p}=160 \mathrm{HeV}$.

\begin{tabular}{|c|c|c|c|c|}
\hline $\begin{array}{l}\text { Final } \\
\text { state }\end{array}$ & 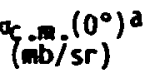 & $S\left(\operatorname{Mr}\left(0^{\circ}\right)\right.$ & $\begin{array}{l}\text { B(GI) } \\
\text { expt. }\end{array}$ & $\begin{array}{l}\text { B(GT) } \\
\text { theory }\end{array}$ \\
\hline $\begin{array}{l}13 K\left(0.00,1 / 2^{-}\right) \\
13 N\left(3.51,3 ! 2^{-}\right)\end{array}$ & $\begin{array}{r}4.4 \\
11.1\end{array}$ & $\begin{array}{l}0.46 \\
0.66\end{array}$ & $\begin{array}{l}0.206^{b} \\
0.83^{c} \\
0.75^{d}\end{array}$ & $0.323^{\mathrm{e}}$ \\
\hline $\begin{array}{l}\text { i50(0.00, } 1 / 2-) \\
150(6.18,3 / 2-) \\
=50\left(8-12,3 / 2^{-}\right)\end{array}$ & $\begin{array}{r}4.7 \\
11.5 \\
3.4\end{array}$ & $\begin{array}{l}0.53 \\
0.70 \\
0.68\end{array}$ & $\begin{array}{l}0.261^{b} \\
1.00^{c} \\
0.80^{d} \\
0.30 c \\
0.26^{d}\end{array}$ & $\begin{array}{l}1 / 3 \\
8 / 3\end{array}$ \\
\hline
\end{tabular}

astatistical uncertainty only. Absolute normalization uncertainty is $\pm 15 \%$.

bTransition strength determined from beta-decas ft values.

$C_{B}(G T)$ determined from $(p, n)$ data and Eqs. (2) and (4). Note that the value of $B(G T)$ for $13 N(3.51$ Mel) differs slightly from that in Ref. 10 because we have not averaged in values obtained from data at other energies.

${ }_{B}(G T$ ) determined from $(p, n)$ data and Eqs. (3) and (4).

eShell-model transition strengths, Cohen-Kurath "POT" wave functions, Ref. 9.

cross section into Fermi and Gamow-Teller parts by using the observed relationship between GT and $F$ transitions ${ }^{10}$ :

$\sigma_{G T} / \Phi=\left(E_{p} / 55 \pm 1 \mathrm{MeV}\right)^{2}[B(G T) / B(G(F)]$.

where $B(F)=N-Z$. Once the fraction $f_{G T}=$ $\sigma_{G T} /\left(\sigma_{G T}+\sigma\right)$ of the cross section attributable to the GT part of the mirror transition has been determined, the cross section per $B(G F)$ for that target is known. Values of $B(G T)$ for excited states are then extracted with this proportionality factor.

The spin-flip probability measurements give a serond (independent) determination of the GT fraction in the ground state cross sections, the spin-flip probability for these transitions is the weighted sum of the pure GT value of $S_{N N}\left(0^{\circ}\right.$, $G T)=0.66 \pm 0.03$ and the Fermi value of zero. The "pure GT" value represents an average obtatned from measuraments of $S_{N N}$ for many GT transitions at $160 \mathrm{MeV} . \theta$ This value is consistent with the value $1 / 2$ expected for a pure $L=0$ transition. The uncertainty represents not only experimental uncertainties, but also real deviations from the nominal value that can ve attributed to $L \neq 0$ amplitudes in the transition. The two procedures for extracting the GT fraction in the ground state and $B(G T)$ for excited states can be summarized in the following formulas:

$$
f_{G T}=\left[1+B(F) / B_{M}(G T) R^{2}\right]^{-1}
$$


or

$$
f_{G T}=S_{W N}\left(0^{\circ}, M\right) / S_{K N}\left(0^{\circ}, G T\right)
$$

and

$$
B_{x}(G T)=\left(g_{x} / q_{H}\right) f_{G T}^{-1} B_{H}(G T) F(q)
$$

where $R=E_{p} /(55 \pm 1$ MeV $), n$ refers to the mirror (ground state) transition, and $x$ refers to the excited state. The factor $F(q)$ is a correction for the momitu transfer dependence of the differential cross sectio.. and is less than 1.10 for the cases studied nere. The values of $B(G T)$ for the excited states show in Table 2.5 indicate that the methods of Eqs. (2) and (3) are more or less consistent. If anything, the spin-flip probability procedure makes the quenching of the $3 / 2^{-}$transitions look even greater.

For the strongest transitions in both wass 13 and mass 15 , the values of $B(G T)$ extracted by the al-zye procedures are reduced from the shellwodel values by factors much larger then typical GT quenching. A striking feature is that the model predicts a ratio of $8: 1$ for the $1 / 2+3 / 2$ to $1 / 2+1 / 2$ transitions in mass 15 , yet the observed ratio is only about 4:1. Similarly, in ass 13 , a shell model calculation' predicts a ratio of 7.5:1 for the ratio of the strongest $1 / 2+3 / 2$ transition to the mirror states $1 / 2+1 / 2$ transition, wile the observed value is about $4: 1$. Stated in other words, it appears that the major $1 / 2+3 / 2$ transition is significantly more quenched than the $1 / 2+1 / 2$ transition.

In submary, wave measured the cross sections and transverse spin-flip probability for $1^{3} C(p, n)$ and $15_{N}(p, n)$ at $E_{p}=160 \mathrm{MeV}$. $A$ surprising feature of the results is that the relative cross sections for the $\mathrm{pl} / 2+\mathrm{pl} / 2$ and $p \rightarrow p 3 / 2$ transitions suggest that the $1 / 2+3 / 2$ GT transitions are more quenched than the $1 / 2+1 / 2$ transitions. It is not clear at this time whether the apparent extra quenching of the $p 1 / 2+p 3 / 2$ transitions tends to favor explanattons of GT quenching in terms of delta $(3,3)$ coupling or in terms of configuration mixing with multiparticle-multihole states. In either case, some of the appealing simplicity of the nuclear shell model in providing a guide to a valid truncation of the space seems is be iost. Even for one of the simplest shell nodvi nuclet, the simple verston of the model seems ic fail.

1. Indiana University, Bloomington, IN 47405 .

2. Permanent address: University of Western

Cape, Private Bag X17, Bellville, South Africa.

3. Los Alamos National Laboratory, Los

Alamos. M 87545.

4. Niels Bohr Institute, Unfversity of

Copenhagen, DK-2100, Copenhagen, Denmark.

5. Onis University, Athens, OH 45701.

6. Ohfo State University, Columbus,

OH 43214.
7. Ohio University, Athens, 45701 and Indiana University Cyclotron Facility, Blocington, IM 47405.

B. T. M. Taddeucci et al ., Phys. Rev. Lett. 52, 1960 (1984).

9. T.-S. H. Lee and D. Kurath, Phys. Rev. C $21,293(1980)$; S. Cohen and D. Kurath, Mucl. Phys. 73, I (1965); 0. Kurath, private conmunication.

10. T. N. Taddercci et al ., Phys. Rev. C 25, 1094 (1981).

\section{THE $1 \%(p, n)^{19}$ we ND $3 \%(p, n)^{34}$ Ca REACTIOAS AT IMTERIEDIATE ENERGIES MD QUEICHIMS OF THE CUMN-TELLER STRENGTH ${ }^{1}$}
D. J. Horen
J. Rapaport 2
C. D. $\cos 0 \operatorname{ton} 3 \pi^{5}$
C. Gaarde ${ }^{3}$
C. Foster 5
J. Larsen ${ }^{3}$
T. Masterson 6
C. Goulding"
E. Sugarbaker?
T. N. Taddeucci ${ }^{8}$

Beta decay transitions between $T=1 / 2$ ground states have a special sfimplicity because the initial and final states are isospin mirror images of each other. The $T=1 / 2$ states, wich have half-integral spins, can transform to their isospin wirrored states through either feini or Gamow-Teller transitions, and the observed beta decay is an incoherent sum of these two camponents. Since the Fermi operator transforms a $T=1 / 2$ to its wirrored state, the Fermi atrix element is unity. This information allows one to decompose the total transition probability into its two components and to extract the GT matrix element from the ft value derived from the measured half-life and decay energy. The deduced enpirical GT strength is 597 of the shell model value for the ITe $\left(\beta^{+}\right) 19$ (gs) transition and $46 \%$ for the ${ }^{3 \%} \mathrm{Ca}\left(\mathrm{B}^{+}\right)^{3{ }^{2} \mathrm{~K}}$ (gs) transition. Several authors have reported on the corrections needed to the calculated single particle GT matrix element. Recently Towner and Khanna" have done a very extensive study on these corrections; Brom and Wildenthal 10 have al so reported on a comparison of shell-model predictions with sd-shell data.

In the case of mass 19 the model calculation shows that wost of the strength should reside in the airror transition. In the case of mass 39 only one-fifth of the sum strength is expected in the mirror transition, but the model is especially simple, that is, to first order the nucleus can be descriked as single $d_{3 / 2}$ hole in an L-S closed shell core.

The Indiana University Cyclotron and bean swinger factlityll provided proton beaws with energies $E_{p}=120 \mathrm{MeV}$ and $E_{p}=160 \mathrm{MeV}$. The time of fllght $(t .0 . f$.$) of heutrons was measured$ over a 100-m flight path with three large-volume time-compensated plastic scintillators. 12 Data were obtained for the angular range $0^{\circ}<\theta<25^{\circ}$ with a typical time resolution of $800-900$ psec.

A commercially avatiable teflon tape 35 $\mathrm{mg} / \mathrm{cm}^{2}$ thick was used as a 1 target. A nat $\mathrm{kF}$ target $34.6 \mathrm{mg} / \mathrm{cm}^{2}$ thick was used for the 120 MeV experfment and a nominal 3 $\mathrm{KHF}_{2}$ target, 28.7 $\mathrm{mg} / \mathrm{cm}^{2}$, $99.96 \%$ enriched in $39 \mathrm{~K}$, was used for the 
$160 \mathrm{meV}$ run. The potassiu targets were covered with a thin (about $1 \mathrm{mg} / \mathrm{cm}^{2}$ ) double layer of polyethylene. Thus the $12 C(p, n)^{12}$ (gs) transition is present in all the observed spectra wich helped in detersining possible target danage during the runs. A ${ }^{12} \mathrm{C}$ target, 34.9 $\mathrm{mg} / \mathrm{cm}^{2}$ and 99.91 enriched, was also run under identical experimental conditions.

The contribution from the $C(p, n)$ s reaction was removed from the t.o.f. spectra taken with the teflon target at each angle, oy substracting normalized observed t.o.f. spectra taken with the graphite target. In the case of the potassium spectra, subtractions had to be made for both if and $c(p, n)$ contributions. For the natural $k$ target the neutron group representing the ${ }^{39} \mathrm{~K}(p, n){ }^{39} \mathrm{Ca}(g s)$ transition it $120 \mathrm{MeV}$ has also contributions fro the $4 i x(F, n)^{4 i} \mathrm{Ca}$ (IAS) transition. The difference in Q-value ( $(B)$ between these two transitions is $(259=20) \mathrm{keV}$. A comparison of the spectrun at $120 \mathrm{MeV}$ with the one at $160 \mathrm{MeV}$ indicates a slight relative enhancement for the gs transition observed at the lower energy due in part to the $41_{x}(p, n)^{4}$ Ca $_{\text {? }}$ (IA) contribution. Also the cross section value for the Fermi transition, which contributes to the gs transition, increases with decreasing energy with-respect to the cross section value for the GT transition, wich remains rather constant in this energy region. ${ }^{3}$ The 120-MeV zero degree spectrum obtained with the thin ( 32 $\mathrm{mg} / \mathrm{cm}^{2}$ ) natk target resulted in a better resolution and the 78 contribution of $4 !_{K}$ in the natural $k$ target was resolved.

The analyzed spectra are shown in Fig. 2.20 for the $1 F(p, n)$ ise reaction and in Fig. 2.21 for the ${ }^{39} \mathrm{~K}(p, n)^{39} \mathrm{Ca}$ reaction and the deduced cross sections are given in Tables 2.6 and 2.7 .

For 1 we we observe that the gs transition carries abost $84 \%$ of the total observet GI strength. The observed tota: GT strength is only $66 \%$ of the sum rule lower limit. In the case of ${ }^{3} \mathrm{Ta}$, the observer sum GT strength up to $10 \mathrm{MeV}$ excitation is only $36 \%$ of the sum rule lower limit. The distribution of GT strength among excited states agrees with the distribution of $d_{5 / 2}$ hole strength reported in neutron pick-up reaction studies. The relative ground state to excited states GT strength ratio agrees with the reported $d_{3 / 2}$ to $d_{5 / 2}$ hold strengths. The case of mass 39 represents the lowest quenching factor (i.e., 365) for the total GT strength as reported from $(p, n)$ reactions. This anomalously lnw value is also reported in the gs transition but calculated corrections to the shell model ${ }^{9}$ are unable to predict the measured value. It is possible that weak transitions carrying additional GT strength are located above $9.0 \mathrm{MeV}$ excitation energy in ${ }^{9}{ }^{9} \mathrm{Ca}$, mich were not detected in the present experiment.

1. Summary of paper submitted to Nuclear Physics.

2. Ohto Intversity, Athens, $\mathrm{OH} 45701$.

3. Niels Bohr Institute, Biegdamsvej 17, OK2100 Copenhagen, Denmark.

4. Los Alamos Natforial Laboratory, Los Alamos, 87545 .

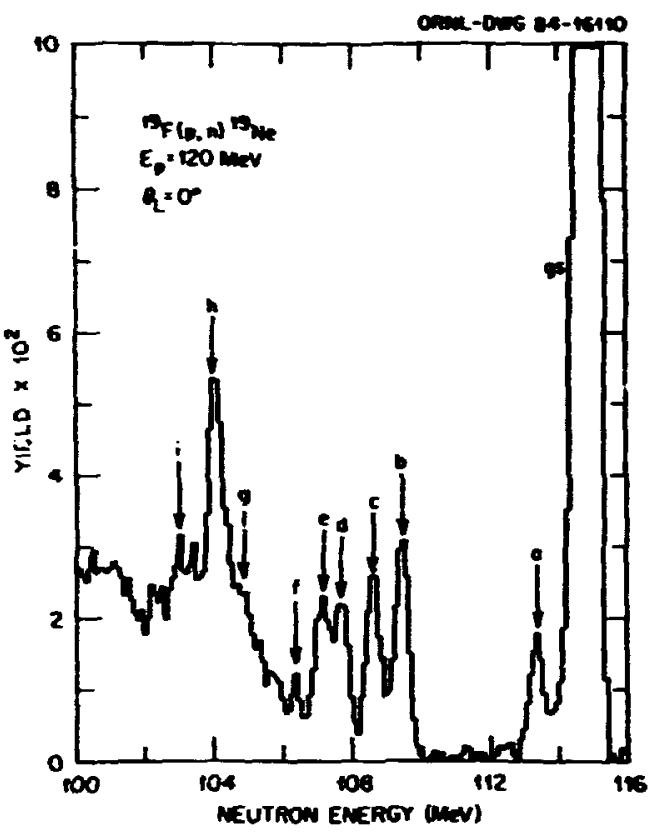

Fig. 2.20. Spectrum for the $1 \%(p, n) 1$ we reaction at $E_{p}=120 \mathrm{MeV}$ and $\theta=0^{\circ}$. Cross sections for the labelled neutron groups are reported in Table $\mathbf{2 . 6}$.

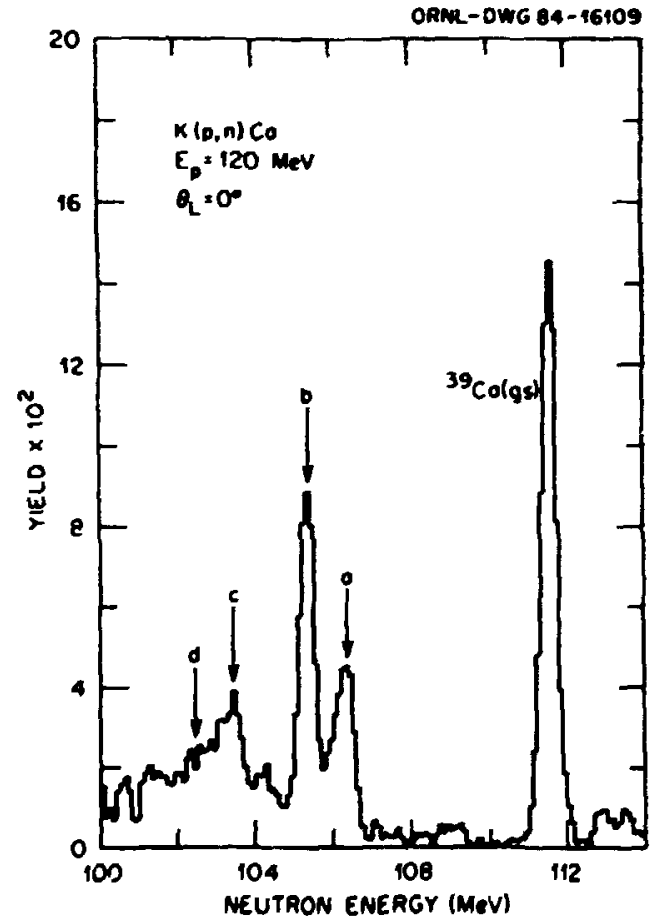

Fig. 2.21. Zero-degree spectrum for the nat $_{K}(p, n) \mathrm{Ca}$ reaction at $E_{p}=120 \mathrm{MeV}$. Cross sections for the labelled neutron groups messured at $E_{p}=160 \mathrm{MeV}$ are reported in Table 2.7 . 
5. Indiana lniversity Cyclotron Facility, Blocmington, In $45 \% 05$.

6. Colorado University, Boulder, CO 70309.

7. Ohio State University, Columbus, OH 43210.

8. Ohio University and Indiana University Cyclotron Facility.

9. I. S. Towner and F. C. Khanna, Mucl. Phys. 1399, 334 (1983).

10. B. A. Brom and B. H. Wildenthal, Phys. Rev. C 28, 2397 (1983).

11. C. D. Goodanan et al .. IEEE Trans. Mucl. Sci. is-26, 2248 (1979).

12. C. D. Coodman et al ., ucl. Instrum. and methods 151, 125 (1978); C. D. Goodman, J. Rapaport and D. E. Bainu, IEEE Trans. Mucl. Sci. 15-25, 577 (1978).

13. T. N. Taddeucci et al., Phys. Rev. C 25 , 1094 (1982).

Table 2.6. Summary of cross sections for the ${ }^{1} F(p, n)$ igre reaction

\begin{tabular}{|c|c|c|c|c|c|}
\hline $\begin{array}{l}\text { Neutron } \\
\text { group } \\
\text { Ex }_{x}(\mathrm{HeV})\end{array}$ & n) & $\begin{array}{l}E_{p}=120 \\
c_{b / s r}\left(0^{\circ}\right) \\
c_{b / s r}\end{array}$ & $B(G T)$ & $\begin{array}{l}E_{p}=160 \\
K_{e V} \\
q_{b / s}\left(0^{\circ}\right)\end{array}$ & $B(G T)$ \\
\hline $\begin{array}{c}95^{b} \\
1.54 \\
3.4 \\
6.2 \\
7.1 \\
7.7 \\
8.60 \\
10.2 \\
11.0 \\
12.1\end{array}$ & $\begin{array}{c}0 \\
(0+2) \\
0 \\
(0+1) \\
(0+1) \\
(0+1) \\
(0) \\
(1) \\
0 \\
(0+2) ?\end{array}$ & $\begin{array}{l}15.5 \pm 0.5 c \\
0.34 \div 0.1 \\
0.48 \div 0.05 \\
0.04 \div 0.05 \\
0.34 \pm 0.05 \\
0.34 \div 0.15 \\
0.10 \div 0.05 \\
0.15 \div 0.05 \\
0.58 \div 0.06 \\
0.18 \pm 0.03 \\
\Sigma 8(G T)=\end{array}$ & $\begin{array}{l}1.64 d \\
0.04 \\
0.06 \\
0.05 \\
0.04 \\
0.04 \\
(0.01) \\
0.08 \\
0.03 \\
1.97 \pm 0 .\end{array}$ & $\begin{array}{c}18.2 \pm 0.5 c \\
0.47 \pm 0.1 \\
0.54 \pm 0.15 \\
0.38 \pm 0.1 \\
0.17 \pm 0.01 \\
0.27 \pm 0.1 \\
\cdots-. \\
0.2 \pm 0.05 \\
0.8 \pm 0.2 \\
0.25 \pm 0.05 \\
06\end{array}$ & $\begin{array}{l}1.64 d \\
1.05 \\
0.05 \\
0.03 \\
0.02 \\
0.03\end{array}$ \\
\hline
\end{tabular}

aRelative uncertainties; to obtain absolute

values add $15 \%$ in quadrature.

b At angles larger than $10^{\circ}$, the contribution of the $0.24 \mathrm{MeV}(\Delta \mathrm{L}=2$ ) has been estimated.

$C$ Includes cross section for fermi transition, t.e., $B(F)=1.0$.

dkormalized to B-decay value.

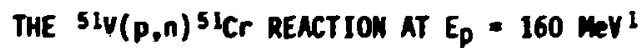

$\begin{array}{ll}\text { D. Horen } & \text { J. Larson } \\ \text { J. Rapaport }{ }^{2} & \text { C. D. Goodman } 5 \\ \text { R. Alarcon } 2 & \text { C. C. Foster } 5 \\ \text { B. A. Brom } & \text { T. Masterson } 6 \\ \text { C. Gaarde } & \text { E. Sugarbaker } \\ \text { T. H. Taddeucci } & \end{array}$

The $\operatorname{siv}(p, n)^{s i} \mathrm{Cr}$ reaction has been studied at $E_{p}=160 \mathrm{MeV}$ using the Ind $i$ and University beam swinger factlity. Data have been obtained at several angles up to $\theta=20^{\circ}$. The zero degree spectrum is used to obtain a 4 - 0 response function from inich Gamow-Teller strength is derived. A shell model calculation of the GT
Table 2.7. Sumary of cross sections for the $3 \times(p, n)$ jeca reaction $E_{p}=160 \mathrm{HeV}$

\begin{tabular}{|c|c|c|c|c|}
\hline $\begin{array}{l}\text { Neutron } \\
\text { group } \\
\text { (MeV) }\end{array}$ & $\Delta$ & 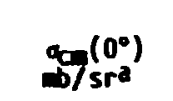 & $\mathrm{B}(\mathrm{GT})$ & $B(F)$ \\
\hline $\begin{array}{l}95 \\
5.3 b \\
6.2 \\
8.1 \\
9.0\end{array}$ & $\begin{array}{c}0 \\
0 \\
(0+1) \\
(0+1) \\
(0+1)\end{array}$ & $\begin{array}{l}3.6 \pm 0.2 \\
1.7 \pm 0.1 \\
2.8 \pm 0.2 \\
1.7 \pm 0.15 \\
0.9 \pm 0.1\end{array}$ & $\begin{array}{c}0.27 c \\
0.19 \pm 0.02 \\
0.31 \pm 0.03 \\
0.20 \pm 0.03 \\
0.10 \pm 0.02\end{array}$ & 1.0 \\
\hline
\end{tabular}

aRelative uncertainties; to obtain absolute values add in quadrature $20 \%$.

blieutron groups corresponding to excitation energies of $5.1 \mathrm{MeV}$ and $5.5 \mathrm{MeV}$ are observed in the $120 \mathrm{MeV}$ experiment; the relative strength of the 5.1 Mel state is bout $30 \mathrm{z}$ larger than the strength of the $5.5 \mathrm{MeV}$. CNormalized to o-decay value.

strength distribution is presented and compared with the experimental results. Ine $M$ strength is also calculated and compared with available results from $\left(e, e^{\prime}\right)$ and $\left(p, p^{\prime}\right)$ experiment. A comparison is ade with other $N=28$ nuclei. Effects of a truncated shell model space are presented.

In a recent paper. Bender et al. 9 report on a search for $M$ l strength in 'SlV via inelastic electron scattering. No strong Ml excitation was detected, in contrast to a $51 v\left(p, p^{\prime}\right)^{51 y}$ experiment by Djalali et al. 10 in wich a concentration of MI strength was observed in the 10-HeV excitation energy region. This clear discrepancy has prompted us to report on the Gamow-Teller (GT) strength obtained via the 5 lv $(p, n)$ si Cr reaction.

Here the experimental GT strength distribution is compared with that obtained in a shell model calculation done in a truncated model space. Using free nucleon g-factors, B(MI) values are calculated in the same model space and compared with avatlable data. 11 The effects of a truncated shell model space are discussed by making an analogy between $N=14$ (sd-shel) nuclei) and $N=28$ isotones nuclei. A full basis calculation can be done for the sd-shell nuclet as well as for teca but not for other $N=28$ isotones. Data are presented for the ${ }^{4} \mathrm{~B} C \mathrm{C}(p, n)^{48} \mathrm{SC}$ reaction and the total GT strength is compared with the sum rule limit, $3(N-2)=24$. The Indtana Untversity Cyclotron was used to obtain procon beams of $159.4 \mathrm{MeV}$. The daca were obtaines with the beam swinger facility. 12 A natural self-supported $V$ target with a thickness of $5 \mathrm{C}+3 \mathrm{mg} / \mathrm{cm}^{2}$ was sed and an energy resolution of approximately $600 \mathrm{keV}$ was achieved. The absolute values and energy dependence of the efficiency of the neutron detectors were obtained by taking data on a set of $\mathrm{hi}_{i}$ and $12 \mathrm{C}$ targets under identical experimental conditions. 
The $A=0$ contributions to the zero-degree $(p, n)$ spectra have been estimated in Fig. 2.22 . The 9.5. of the nucleus $51 y$ has $\mathrm{j}^{\pi}=7 / 2^{-}$. $T=5 / 2$. Thus $G T$ transitions will excite states in ${ }^{51} \mathrm{Cr}$ with $\mathrm{J}^{\mathrm{T}}=5 / 2^{-}, 7 / 2^{-}, 9 / 2^{-}$and $\mathrm{T}=3 / 2$, $5 / 2,7 / 2\left(T_{2}=3 / 2\right)$. The IA of 5 ly (G.5.) in 5lCr occurs at $E_{X}=6.61 \mathrm{HeV}$ and it has to be subtracted fros the $\alpha=0$ spectrum in order to obtain the $6 T$ strength-function. The estimated total $G$ strength corresponds to $S_{G}=12.6 \pm 2.5$ in units such that the free neutron has aT strength, $B(6 T)=3$.

In order to interpret the experimental results, shell model calculations have been perforned. Many of the low-lying levels of 5iv and ${ }^{51} \mathrm{Cr}$ are fairly well described with the simple $\left(1 f_{7 / 2}\right)^{11}$ model space ${ }^{13}$ (model space A). However, this basis is clearly not adequate for the calculation of the GT strength distribution because of the iaportance of the $f_{7 / 2}$ to $f_{5 / 2}$ transition. The only model space truncation wich is at present practical allows for at most one particle to be excited from the $f_{7 / 2}$ shell to the other $f_{p}$ shell orbitals and we refer to this as model space B.

In a full space calculation, It the GamowTeller sum rule $S(G T)=B(G T)-B(G T+)=3(N-2)$ must be obtained. In terns of the isospins of the final states, this sum rule in our case is

$$
\begin{aligned}
S(G T)= & B(G T-)-B(G T+) \\
= & B(G T-, T=3 / 2)+B(G T-, T=5 / 2)- \\
& 20 B(G T-, T=7 / 2)=15 .
\end{aligned}
$$

The sum rule obtained in model space $B$ is, in fact, 13.9 rather than 15 . The reason for this

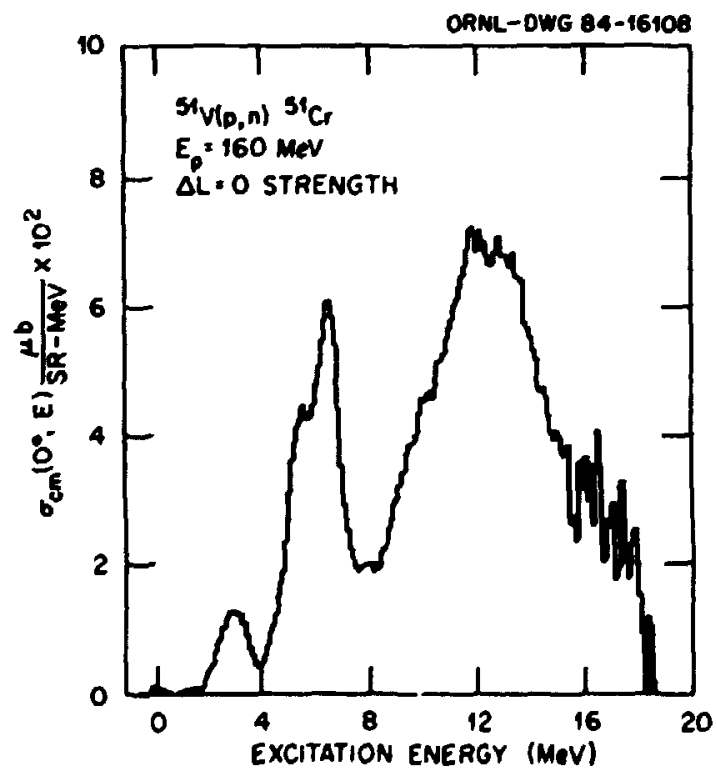

F19.2.22. $\mathbb{N}=0$ strength obtatned at $q=0^{\circ}$ for the $\operatorname{siv}(p, n)^{s i c r}$ reaction at $E_{p}=160 \mathrm{MeV}$ versus excitation energy in ${ }^{5} \mathrm{C} \mathrm{C}$. is that the 6T operator acting on the initial state can also go to a state with two particles excited into one of the $f_{5 / 2}, P_{3 / 2}$ or $p_{1 / 2}$ orbits and this state lies outside of model space B. Since we ust make some truncation, it seems most consistent to restrict the $51 \mathrm{~V}$, $J=7 / 2$ ground state to the $\left(f_{7} / 2\right)^{l l}$ (the model space A) configuration. Then the sum rule for the $A+B$ type transitions is satisfied. The readining discussion will be concerned only with A + B calculations.

The theoretical $B(G T-)$ distribution is presented in Fig. 2.23 where it is compared with the empirical results (shaded). The histograms have been obtained by suming over $1 \mathrm{MeV}$ intervals. The centroids and widths of the experimental and theoretical spectra are in excellent agreement with each other. The integrated experimental strength of $12.6 \pm 2.5$ is $(63 \pm 13) \mathrm{x}$ of the total theoretical strength of 20.14. This quenching factor is consistent with the global quenching factor of about $60 \%$ observed on a number of even-even targets in the region $40<A<208$. Mithin our model space there is essentially no GT strength above $20 \mathrm{MeV}$ in excitation. This is consistent with the present multipole decomposition of the experimental strength above $20 \mathrm{MeV}$ which shows

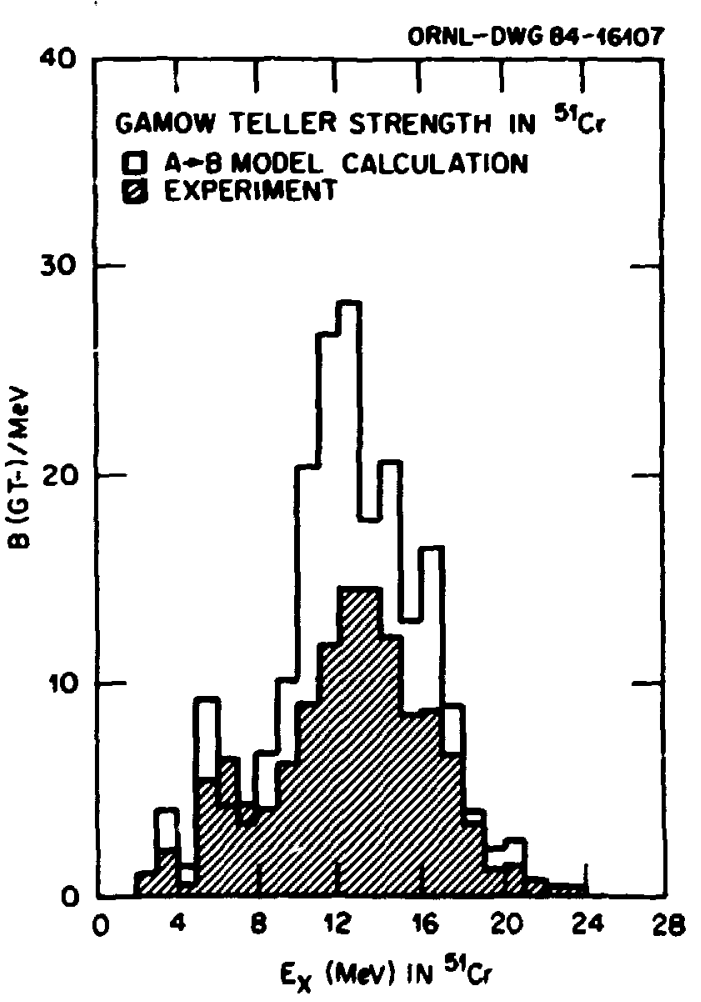

Fig. 2.23. Gamow-Teller strength in ${ }^{51} \mathrm{Cr}$. The strength in energy bins of $1 \mathrm{MeV}$ is presented versus excitation energy in ${ }^{5 i} \mathrm{Cr}$. The experimental B(GT-; (integrated in $1 \mathrm{MeV}$ uins), shaded area, is compared with shell model calculations. 
$\Delta=1$ and $L=2$ transitions to be dowinant in this region.

The major effect of going from a truncated model $A+B$ type calculation to a full fp shell codel calculation is the adaixture of two quasiparticle $f_{5 / 2}$ states in the ground state. The full $f p$ shelf wodel calculation is, in general, inpossible to carry out. However, for the Ca isotopes, the full basis is tractible and the $M l$ strength have been calculated by Hicfrory and Uildenthal. 15 The ratio $R$ (the su in the fullbasis calculation divided by the $A+$ calculation) varies monotonically from 0.62 to 0.75 on going from teca to tea.

Using an analogous argument for the $N=14$ isotones in the sd shell going from 220 to $265 i$, one can deduce a similar ratio for the $\left(f_{7 / 2}\right)^{11}$ proton nuclei. We then find the quenching for $B(6 T-)$ in $51 y$ to be $0.70 \div 0.14$.

The $G T$ strength distribution obtained from the ${ }^{4} \mathrm{Ca}(p, n)^{48} \mathrm{Sc}$ reaction has been reported 16 and has been the subject of several papers.17,16.19 However, the value of the total 6T strength normalized to beta-decay measurements has not been reported. We have measured a spectrum at $\theta=0^{\circ}$ were used to estimate the $\alpha=0$ cross section at $\theta=0^{\circ}$ for the 4 Ca $(p, n)+8$ Sc reaction. From our analysis we find the quenching for $8(6 T)$ in 4 ba to be $0.71_{-0.15}^{+0.08}$ in good agreewent with that deduced for the 5ly.

8 (M1) values were calculated from wave functions using free nucleon g-factors and a truncated model space. The sumed Ml strength for $51 \mathrm{~V}, 2 \mathrm{~B}(\mathrm{ML})=20.19$ is in disagreewent with the experimental $\left(e, e^{\prime}\right)^{9}$ value <l bi. A siailar comparison with results obtained from the ${ }^{5 l}\left(p, p^{\prime}\right)$ experiment 20 indicates that only about $13 \%$ of the calculated strength has been observed. These results may be oue to a high degree of fragmentation of MI strength wich may be difficult to detect above background.

1. Sumbary of paper to be published in Nuclear Physics.

2. Ohio University, Athens, of 45701 .

3. Michigan State University, East Lansing, MI 48824 .

4. Niels Bohr Institute, University of Copenhagen, OK-2100 Copenhagen D. Denmark.

5. Indiana University, Bloomington. If 47405 .

6. University of Colorado, Boulder, CO 80309 .

7. Ohio State University, Columbus, OH 43214.

8. Ohio University, Athens, $O H 45701$ and Indiana University, Bloomington, IN 47405.

9. 0. Bender et al.., Nucl. Phys 1398, 408 (1983).

10. C. Ojalalf et al.. Nucl. Phys, A388, I (1982).

11. G. M. Crawley et al., Proc. of the 1983 RCNP International Symposfum on Light Ion React. Mech., editert by H. Ogata, T. Kammurf, I. Katayama, May 1983, D. 153.
12. C. D. Goodan et al., IEEE Trans. Mucl. S:i. is-26, 2248 (1979)

13. W. Kutschera, 8. A. Brom and K. Ogawa, Revista del Movo Cimento, Serie 3, Vol. I. No. 12 (1978).

14. C. Gaarde et al ., Mucl. Phys. 1334, 248 (1980).

15. J. B. HeGrory and B. H. Mildenthal, Phys. Lett. 1038, 173 (1981); Mucl. Phys. A399, 334 (1983); Phys. Rev. C 28, 2397 (1983).

16. B. D. Anderson et al ., Phys. Rer. Lett. 45. $(1980)$.

17. F. Osterfeld, Phys. Rev. C 26, 762 (1982).

18. F. Osterfeld et al., Phys. Rev. Lett. 49. 11 (1982).

19. M. Weise, Mucl. Phys. A396, 373C (1983). 399 (1983).

. C. Djalali et al .. Mucl. Phys. M10,

\section{MEASTREEMT OF THE TRUSVERSE SPIM TRASTER COEFICIENT Dun $\left(0^{\circ}\right)$ FOR $(\vec{p}, \hat{n})$ RERCTIOUS}
D. J. Horen
C. D. Goodman 5
T. A. Taddeucci 2
T. Masterson 6
T. A. Carey ${ }^{3}$
J. Rapaport?
C. Gaarde
T. P. Welch?
J. Larson"
E. Sugarbaker ${ }^{8}$

The first measurements of the transverse spin transfer coefficient $D_{m a}\left(0^{\circ}\right)$ for intermediate energy $(p, n)$ reactions to well resolved nuclear states are reported. Results are presented for $\left(\vec{p}, \hat{n}^{*}\right)$ reactions on $6=7 \mathrm{~L}, 12,13,14 \mathrm{C}$, and $90 \mathrm{Zr}$ at $E_{p}=160 \mathrm{meV}$. The average value of $\mathrm{ONW}_{1}\left(0^{\circ}\right)$ for pure Ganow-Teller transitions at this energy is show to be close to the expected value of $=1 / 3$. The utility of spin transfer measurements for investigating aspects of the nuclear spindependent response not otherwise observable is pointed out.

Despite the siccessful use of $(0, n)$ differential cross sectiris to identify and measure spinflip strength, in some cases important detafls of the reaction are not adequately revealed by differential cross section measurements alone: In particular, there is the continuting problem of distinguishing giant resonances excited by the $(p, n)$ reaction from the background of continuous excitations or competing giant resonances with wich they are mixed.9-13 This separation mainly depends upr..1 distinguishing between components that have differing spin-filp characteristics.

A direct measure of the spin-flip character of a $(p, n)$ transition is provided by the transverse spin transfer coefficient $D_{N M}(\theta)$ [or, equivalently, the transverse spin-fifp probability $\left.S_{\text {HM }}=\left(1-D_{M M}\right) / 2\right]$. Measurements of $D_{M N}\left(0^{\circ}\right)$ are potentially important tests of the effect fue nucleon-nucleon interaction as well as a means of investigating the nuclear spindependent response.

Data were obtained using the Indiana University cyclotron and beam-swinger factifty. 14 polarized protons with energy $E_{p}=160 \mathrm{MeV}$ bombarded self-supporting targets 
with thicknesses ranging fro $107 \mathrm{mg} / \mathrm{ca}^{2}$ to $232 \mathrm{~m} / \mathrm{cm}^{2}$. The bea polarization was typically $0.65</ \mathrm{pi}_{\mathrm{i}} \mid \mathbf{0 . 7 5}$ and was cycled between up and dom orientations at two-minute intervals. The nectron polarimeter consisted of six $15 \sigma \times 15 \propto \times 100 a$ plastic scintillators arranged in two parallel stacks of three. The long axes of the scintillators were parallel to the horizontal plane and perpendicular to the incident neutron flux. The separation between stacks was aproxiately $1.4 \mathrm{a}$. The polarization of incident neutrons ws determined by scattering from hydrogen [ ${ }^{1} H\left(t, n^{\prime}, 4\right)$ and carbon [12c( $\left.\left.f^{\prime} n^{\prime} x\right)\right]$ muclei in one of the formard scintillators. The scattered neutrons (denoted as $n^{\circ)}$ were subsequently detected by one of the trailing scintillators. A thin plastic scintillator between the stacks was used to reto events associated witn forward-going protons. Time signals derived from each end of the detectors furnished both the TOF of the incident neutron and positron information from wich the event geometry was reconstructed. Meutrons that scattered at polar angles of $14^{\circ} 40 \mathrm{c3} 1^{\circ}$. at azimuthal angles within $45^{\circ}$ of the horizontal plane, and with velocity $v_{n} \% / \cos \theta \times 0.91 v_{n}$ were accepted as valid events. The measured instrumental analyzing power for these conditions (which includes the average value of $\cos \phi$ ) is $A_{p}=0.34 \pm 0.02$ and is primarily due to scattering from hydrogen. The neutron-energy dependence of the analyzing power was calculated using the N-N phase shift solutions of Arndt 15 and acounts to a change of Jess than $5 \%$ in the magnitude of $A_{p}$ for $140 \mathrm{MeV}<E_{n} \triangle 160 \mathrm{MeV}$.

At $\theta=0^{\circ}$ the spin transfer coefficient is given by

$$
D_{M N}\left(0^{\circ}\right)=p_{f} / P_{i}
$$

(where $p_{i}$ is the proton and $p_{f}$ the neutron polarization), and may be defined in terms of measured quantities as

$$
D_{H W}\left(G^{\circ}\right)=\left(\bar{P}_{j} A_{p}\right)^{-1}(R-1) /(R+1)
$$

where $\bar{p}_{i}=\left(p_{j}{ }^{+}-p_{i}{ }^{-}\right) / 2$ is the average magnitude of the proton polarization, $A_{p}$ is the instrumental analyzing power.

$$
R=\left[\left(N_{L}{ }^{+} N_{R}-\right) /\left(N_{L}-k_{R}+\right)\right]^{1 / 2} \text {, }
$$

and $\mathrm{N}^{+}\left[\mathrm{N}^{+}\right]$is the number of neutrons that scatter to the left $\left(=0^{\circ} \pm 45^{\circ}\right)$ [right $\left.\left(\$ 180^{\circ} \pm 45\right)\right]$ for positive incident proton polarization, etc. This definition of $D_{N N}\left(0^{\circ}\right)$ is largely independent of false asymmetries caused by instrumental ineffictencles and misalignments or unequal beam current for spin up and spin down. The product $\bar{p}_{j} A_{p}$, wich provides the absolute normalization of $D_{N N}$, was empirically determined by measuring $R$ for the $0^{+} \rightarrow 0^{+}{ }^{14} \mathrm{C}(p, n)^{14} \mathrm{~N}(2.31 \mathrm{MeV})$ isobaric analog state (IAS) transitio. for which $D_{M N}\left(0^{\circ}\right) \equiv 1$.

Values of $D_{N N}\left(0^{\circ}\right)$ determined in this work are given in Table 2.8. Significantly, the values of $D_{N N}\left(0^{\circ}\right)$ for the pure GT transition are evenly distributed around the expected value of $-1 / 3$.

The average for six strong transtetions from $6 \mathrm{~L} t$, ${ }_{12} \mathrm{C},{ }^{13} \mathrm{C}$, and ${ }^{14} \mathrm{C}$ is $\mathrm{D}_{\mathrm{NN}}\left(0^{\circ}\right)=-0.32 \pm 0.05$.
Table 2.8. Iransverse spin transfer coefficient $D_{n, 1}\left(0^{\circ}\right)$ for $(p, n)$ reactions at $160 \mathrm{meV}$. The quoted uncertainties are absolute and arise from the statistical uncertainty in the yields of double-scattered meutrons for each transition plus an uncertainty of about 67 oring to the

\begin{tabular}{|c|c|c|c|}
\hline Reaction & $E_{x}(\mathrm{HeV})$ & $D_{\min }\left(0^{\circ}\right)$ & $\begin{array}{c}\text { Transition } \\
\text { type }\end{array}$ \\
\hline $4 i(p, n) G \mathrm{Be}$ & 0.0 & $-0.37 \pm 0.04$ & GT \\
\hline${ }^{7} \mathrm{Li}(p, n)^{7} \mathrm{Be}$ & $0.0 \pm 0.43$ & $-0.28 \pm 0.06$ & $\mathbf{G T}+\mathbf{F}$ \\
\hline${ }^{12} c(p, n)^{12 n}$ & 0.0 & $-0.24 \pm 0.03$ & GT \\
\hline${ }^{13} C(0, n){ }^{13}{ }_{N}$ & 0.0 & $0.05 \pm 0.06$ & $\mathbf{G T}+\mathbf{F}$ \\
\hline • & 3.51 & $-0.33 \pm 0.05$ & GT \\
\hline - & 15.1 & $-0.36 \pm 0.08$ & GT \\
\hline${ }^{14} C(0, n)^{14 n}$ & 0.0 & $-0.29 \pm 0.17$ & GT \\
\hline$\bullet$ & 2.31 & 1.0 & $F$ \\
\hline - & 3.95 & $-0.29 \pm 0.02$ & GT \\
\hline - & 13.72 & $-0.33 \pm 0.04$ & GT \\
\hline${ }^{90} \mathrm{Zr}(0, n)^{90} \mathrm{Nb}$ & $4.3-6.3$ & $0.24 \div 0.17$ & $\begin{array}{l}F+G T \\
\text { background }\end{array}$ \\
\hline$\omega$ & $6.4-13.1$ & $-0.28 \pm 0.08$ & $1^{+}$) (Giant \\
\hline 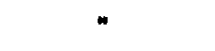 & $15.0-25.0$ & $-0.17 \div 0.09$ & $?$ \\
\hline
\end{tabular}
statistical uncertainty in the yields for the ${ }^{14} \mathrm{C}(p, n)^{14} \mathrm{~W}(2.31 \mathrm{HeV})$ calibration reaction.

Large systematic deviations fros this value would be indicative of unexpectedly large $L=2$ (or tensor) amplitudes at $0^{\circ}$ or failure of the assumed single-step direct-reaction mechanism.

1. Sumary of paper: Phys. Rev, Lett. 52 1960 (1984)

2. Ohfo University, Athens, $O H 45701$ and Indiana Unfversity, Bloomington, IN 45405.

3. Los Alamos National Laboratory, Los Alamos, N 87545.

4. University of Copenhagen, DK-2100 Copenhagen, Denmark.

5. Indiana University, Bloomington, IN 47405.

6. Universtty of Colorado, Boulder, CO 80309 .

7. Ohto University, Athens, OH 45701. 43210 .

9. G. Bertsch and I. Hamamoto, Phys, Rev, C 26. 1323 (1982). 
10. F. Osterfeld, Phys. Rew. C 26,762 (1922).

11. T. Izumto, Mucl. Phys. A335, 189 (1993).

12. 6. Bertsch, D. Cha, and H. Toki, Ptys.

Rev - C 24, 533 (1981).

13. 0. Scholten, 6. F. Bertsch and H. Toki, Phys. Rev. C 27, 2975 (1983).

14. C. D. Goodanan et al ., IEEE Trans. Mucl . Sci . 26, 2248 (1979).

15. R. A. Arndt and L. D. Roper, Scattering Aalyses Dial-in (ShID) Progra, phase shift solution FAB3, Virginia Polytechnic and State University, unpubl ished.

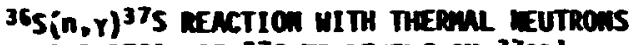 AD DECAY of $37 \mathrm{~s}$ TO LEVELS If ${ }^{37} \mathrm{Cl}$}
S. Raman
E. T. Urney ${ }^{3}$
W. Ratynski2
H. E. Bunker ${ }^{3}$
J. H. Starner ${ }^{3}$

The ${ }^{36} s(n, y)^{37} S$ reaction with theral neutrons has been studied utilizing a highly enriched ${ }^{36} \mathrm{~S}$ target. Fifteen $y$ rays were observed wich have been incorporated into a 37 s level schewe. The neutron separation energy of 375 was determined to be $4303.52 \pm 0.12 \mathrm{keV}$ and the thermal neutron capture cross section of 365 to be $230 \pm 20$ m. The subsequent $\mathrm{B}^{-}$decay of ${ }^{37} \mathrm{~S}$ to levels in ${ }^{37} \mathrm{Cl}$ has also been stlidied. Seven $y$ rays were observed which have led to an improved 375 decay scheme. (1984).

1. Abstract of paper: Phys. Rev. C 30, 26 2. Swierk. Po? and.

3. Los Alamos kational Laboratory, Los Alamos, N.M 87545 .

$$
\begin{aligned}
& { }^{36} j(d, p)^{37} \text { S AND } 36,36 S\left(d,{ }^{3} \mathrm{He}\right)^{33,35 p} \text { REACTIONSI } \\
& \begin{array}{ll}
\text { C. E. Thorn } 2 & \text { E. K. Warburton }{ }^{2} \\
\text { J. H. O1ness } & \text { S. Raman }
\end{array}
\end{aligned}
$$

The ${ }^{36} S(d, p)^{37} S$ reaction was studied at $E_{d}=25$ MeV with an enriched 365 target (81.12) and momentum analysis of the protons. Twenty-six groups were identified with levels in ${ }^{37} \mathrm{~S}$. Excitation energies were obtained with an uncertainty of $\$ 3 \mathrm{keV}$ and angular distributions yielded $l$ and $S$ values for the transfer reaction. Comparison is made to local systematics and the $k=1$ streng:h is also compared to recent $(n, y)$ results. The $34,36 \mathrm{~S}\left(d,{ }^{3} \mathrm{He}\right)^{33}, 3 \mathrm{sp}$ redctions were also studied. A mass excess for $35 \mathrm{p}$ of $-24854(5) \mathrm{kel}$ was obtained. (d, $\left.{ }^{3} \mathrm{He}\right)$ proton pickup st rength was observed to four states of $35 \mathrm{p}$ and seven states of 33p. These results are compared to predictions of Wildenthal.

1. Abstract of paper to be published. 11973.

\author{
DECAT Sange of licsa Feom \\ $\left(n, n^{\prime} y\right)$ ad $(n, y)$ results
Z. Gácsit ${ }^{2}$ J. $\mathrm{SI}^{2}$
J. L. Meil 2 E. T. Jurney ${ }^{3}$ S. Reman

A decay schene for IIGSn containing aproximately 190 rerays and 112 levels below $6.0 \mathrm{meV}$ hej been constructed by comining the results of I $\sin \left(n, n^{*} y\right)$ and $115 \sin (n, y)$ experients.

1. Nhstract of paper: Proc. Fifth Intern. Sym. Capture Gma-Ray Spectroscopy and Related Topics, Rnoxville, Tennessee, September 1984.

2. University of Rentucky, Lexington, Rentucky.

3. Los Alamos Mational Laboratory, Los AIamos, Mew Mexico.

\section{COSERYATION OF EXTREgELY LOA S-WATE

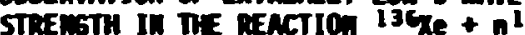
B. Fogelberg ${ }^{2}$
M. Mizunoto
J. Harvey ${ }^{3}$
S. Raman

The neutron cross section of 136xe has been investigated at the Dak Ridge Electron Linear Accelerator (ORELA). A sample of xenon gas. enriched to $93.6 \%$ in $135 \mathrm{Xe}$, was used as target. Measurements were made with an energy resolution of $0.1 \%$. The transmission data, wich were analyzed for neutron energies below $500 \mathrm{keV}$, show 35 resonances in this region. Only four very weak resonances were found to be possibly due to s-wave neutron interactions, giving an extremely Jow value for the s-wave strength function in the analyzed region $\left(S_{0}<1.0 \times 10^{-6}\right)$.

1. Summary of paper: Proc, Fifth Intern. Symp. Capture Ganma-Ray Spectroscopy and Related Topics, Knoxville, Tennessee, September 1984.

2. The Studsvik Science Research Laboratory, Nyköping, Sweden.

3. Engineering Physics and Mathematics Division.

4. JAERI, Tokai-Mura, Naka-gun, Ibaraki-ken, Japan.

\section{ELECTRIC DIPOLE TRANSITIONS FROM MEUTRON CAPTURE IN 167 ER RESOMANCES 1}

$$
\begin{aligned}
& \text { S. Kahane }{ }^{2} \text { G. G. Slaughter } \\
& \text { S. Raman C. Coceva }{ }^{3} \\
& \text { M. Stefanon }{ }^{3}
\end{aligned}
$$

Primary neutron capture $y$ rays have been studfed from 86 neutron resonances in $167 \mathrm{~F}$ - in the energy range 5-600 eV. The spins of these resonances were assigned as I=3 or 4 on the basis of the intensity ratios of suitable pairs of lowenergy $r$ rays. The measured intensities of the high-energy primary y rays have been converted to partial radiation widths and further analyzed to obtain average El gamma strength. Individual $r$ 
spectra from I=3 and 4 resonances have been suned separately after appropriate noral izations. The resulting average radiation widths (and hence the $y$-ray strength function) were in good agreenent with the Axel-Brink predictions based on a giant dipole resonance odel. These widths were also consistent with the assumtion that the rray strength function is independent of the initial spin. The partial El radiation widths were subjected to a distribution analysis, reveal ing that these widths had a slightly narromer distribution than that predicted by the Porter-Thomas law. The ratio of the average population of a particular lon-lying level from I=4 resonances to its population from $I=3$ resonances was found to be in good agreenent with a fire-step cascade model. The population of successive spins within a rotational band lay on seooth curves which, for different bands, were mearly parallel. A level schese for 16 Er has been constructed, and the neutron separation energy for this nucleus was deduced as $7771.6 \pm 1.0 \mathrm{KeV}$.

1. Abstract of published paper: Phys. ..ev. C 30,807 (1984).

2. Nuclear Research Centre-Negev, Beer-Sheva, Israel.

3. EMEA, CRE "Ezio Clementel," Bologna, Italy.

\section{LOCATION OF A DOORLAT STATE USING THE CHANREL $n+207 \mathrm{pb}$}
L. C. Dennis 2
S. Raman

The location of a doorway state in the $n+$ $207 \mathrm{pb}$ channel is established through a statistical analysis of the observed partial widths for gammarays and neutrons. Several statistical tests developed to help locate doorway states are presented. The statistical analysis focuses on the strong correlation between large partial widths in the two exit channels. Widths in both exit channels exhibit extromely large values in the energy region near $E_{n}=120 \mathrm{keV}$. This clustering of large widths, even when considered separately for each exit channel, is relatively unlikely to occur in a statistical sample. The strong correlation between channels decreases the likelihood for this clustering of large widths to occur in a statistical sample to less than 0.0003 .

1. Abstract of paper: Proc. Fifth Intern. Symp. Capture Ganma-Ray Spectroscopy and Related TopfCs, Knoxville, Tennessee, September 1984.

2. Florida State University, Tallahassee, Florida.

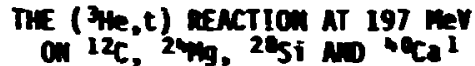
S. L. Tabor 2
A. Guternan ${ }^{3}$
G. Heuschaefer ${ }^{2}$
A. T. Collins"
J. A. Carr ${ }^{2}$
F. Petrovich2
D. L. Friesel ${ }^{5}$
C. Glover 5
c. C. Chang ${ }^{3}$
5. Y. van der Merf 6
S. Raan

Spectra have been measured for the (He,t) reaction on $12 \mathrm{C}$, $24 \mathrm{~kg}, 285 i$, and $40 \mathrm{Ca}$ at $\mathrm{E}_{1 \mathrm{ab}}=$ $197 \mathrm{HeV}$ and $\theta a b=15^{\circ}$. The analog of the giant dipole resonance (EOR) is strongly populated in the charge-exchange reaction for each of the targets used in this study. Differences between the spectral shape of the EDR known in $12 \mathrm{C}, 2 E_{5 i}$, $24 / \mathrm{hg}$, and $4 \mathrm{Ca}_{\mathrm{a}}$ from photonuclear work and their analogs in $12 \mathrm{k}, 2 \%, 24 \mathrm{Al}$, and $405 \mathrm{C}$ are discussed. Wo evidence is seen for the population of a conpact isovector giant quadrupole resonance (GQR) in any of these targets. Angular distributions have been measured at this energy for the $4{ }^{\circ} \mathrm{Ca}$ ( ${ }^{40} \mathrm{Ca}\left({ }^{3} \mathrm{He},{ }^{3} \mathrm{He}\right.$ ') $40 \mathrm{Ca}$ and ${ }^{40 \mathrm{Ca}}$ ( $\mathrm{He}, \mathrm{t}$ ) $40 \mathrm{Sc}$ react ions. The elastic ${ }^{3}$ He scattering from $4{ }^{\circ} \mathrm{Ca}$ is reasonably described by a volume Woods-Saxon optical potential that is very similar to one known from lowerenergy studies. Collecative DuA calculations using this potential reproduce the inelastic scattering to the 3 - and isoscalar COR states of $40 \mathrm{Ca}$ and an additional calculation using the GoldhaberTeller model roughly reproduces the measured charge-exchange angular distributions of the COR in ${ }^{40} \mathrm{Sc}$. Data for the inelastic-scattering and charge-exchange reactions to a few low-lying discrete states and the region including the $60 R$ in ass 40 have also been examined in DUA calculations using a microscopic double-folding model with a realistic effective interaction and particle-hole wave functions. Where possible these results are compared with results for other probes and rough consistency is founa. The decomposition of the strength observed in the GDR region via ( $\left.{ }^{3} \mathrm{He}, t\right)$ is considered in some detail and it is estimated that $30 \%$ of this strength is $\Delta S=1$.

1. Abstract of published paper: Nucl. Phys. 4422, 12 (1984).

2. Florida State University, Tallahassee, Florida.

3. University of Maryland, College Park, Maryland.

4. Brookhaven National Laboratory, Upton, New York.

5. Indiana University Cyclotron Facility, Bloomfington, Indiana.

6. Kernfysisch Versneller Institute Groningen, The Netherlands. 


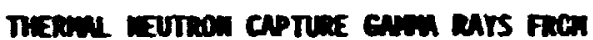
SURTR ISOTOPES: EXPERIFET MO THEORI I

$$
\begin{aligned}
& \text { 5. Rman J. C. Wells }{ }^{3} \\
& \text { R. F. Carltan }{ }^{2} \text { E. T. Jurney }
\end{aligned}
$$

We have carried out a systeatic investigation of y rays after theral neutron capture by al 1 stable suifur isotopes (325, 335, 345, and 365). The easurements were made at the internal target facility at the Los Almos Onega lest Reactor. We detected a larger muber of y rays: -100 in 335 , -270 in $345,-60$ in 355 , and -15 in 375 . Before developing detafled level schenes, we culled and then consolidated the existing informtion on energies and $\mathrm{J}$ values for levels of these nuclides. Based on the current data, wave constructed detailed decay schenes, wich imply that there are significant populations of 26 excited states in 335,70 states in 345,20 states in 355 , and 7 states in $37 \mathrm{~S}$. By checking the intensity balance for these levels and by comparing the total intensity of prianry transitions with the total intensity of secondary r rays feeding the ground state, we have demonstrated the relative completeness of these decay schewes. For strongly populated levels, the branching ratios based on the current measurewents are generally better than those avafiable fro previous measurements. In all four cases, a few primary dipole (E1) transitions account for a large fraction of the capture cross section for that particular nuclide. To understand and explain these transitions, wave outlined ab initio a theory of potential capture. Toward this end, we reviewed the theory relating off-resonance neutron capture to the optical-model capture. We studied a range of model-dependent effects (nature and magnitude of imaginary potential, surface diffuseness, etc.) on the potential capture cross section, and we have shown how experimental data may be analyzed using the expression for channel capture suitably modified by a factor that takes into account the model-dependent effects. The calculations of cross sections for most of the primary transitions in the sulfur isotopes are in good agreement with the data. Some discrepancies for weaker transitions can be explained well by an interfering compound-nucleus contribution to capture. This contribution is of the magnitude expected from statistical surveys of resonance capture data. Estimates of the cross section due to the valencecapture mechanism in s-wave resonances show that this cross section should dominate the more complicated compound-nucleus contribitions.

1. Abstract of paper to be published.

2. Middle Tennessee State University. Murfreesburo, TN 37132.

3. Tennessee Technological University, Cookeville, TN 38501.

4. Los Alamos National Laboratury, Los Alamos, NM 87545.

5. Atomic Energy Research Establishment, Harwell, England.

\section{The s- MU A-MUVE scatterims} of mavtrous on tra
J. A. Harvey
C. H. Johnson
R. F. Carlten'2
Boris Castel ${ }^{3}$

Heutron total cross section measurewents of "Coco up to $1.4 \mathrm{HeV}$ reported by Seibel et al." established the existence of one strong $d 5 / 2$ resonance at Q.956 $\mathrm{keV}$ and no s-mave resonances were found. Although several other small resonances were observed, their spins and parities could not be deternined. Divadeenam et al.s calculated the energies and strengths of possible $\mathbf{2}$-Ih states and predicted that the lacest 5 -wave resonance would be above $1.5 \mathrm{HeV}$ with the majority of the s-wave strength at 3 rev.

Neutron total cross section measurements of "Ca have been performed at ORELA over the energy interval from $10 \mathrm{keV}$ to 4 heV using a $200-m$ flight path. A $\mathrm{CaCO}_{3}$ saple $(1 / \mathrm{K}=126$ $\mathrm{D} / \mathrm{a}$ ) enriched to $968{ }^{48 \mathrm{Ca}}$ was used. Meutrons from the Ta target were used at higher energies while water coderated neutrons mere used beliow 500 keV. The enercy resolution $D / E$ was $-0.00025[1+25 \mathrm{E}]^{1 / 2}$ where $E$ is in $\mathrm{keV}$. The experimental data after subtracting the oxygen and carbon cross sections are show in figs. 2.24 and 2.25. In R-matrix analysis using the code SNAY' has been performed up to $4 \mathrm{MeV}$ to obtain resonance parameters and potential scattering phase shifts for $\ell=0,1$ and 2 wave resonances. The resulting fits to the total cross section are shown in Figs. 2.26 and 2.25.

Ho resonances could definitely be assigned to s-wave neutrons. The nonresonant cross section for low energy neutrons ( $400 \mathrm{keV}$ ) is very small $-0.5 \mathrm{~b}\left(R^{\prime} \sim 2.0 \mathrm{fm}\right)$. This can be described by scattering from a real Hoods-5axon potential with $r_{0}=1.21 \mathrm{fm}, a=0.66 \mathrm{fm}$ and $V_{0}=48 \mathrm{MeV}$.

Three large $d_{5 / 2}$ resonances (at $0.960,1.56$ and $1.78 \mathrm{MeV}$ ) mere identified up to $2 \mathrm{MeV}$ whose

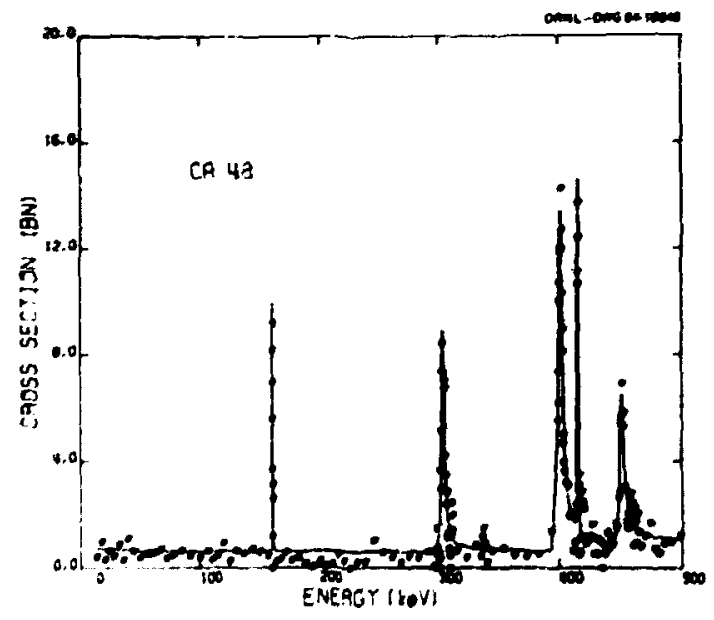

Fig. 2.24. Total cross section of ${ }^{4} \mathrm{Ca}$ obtained using water-moderated source. The curve is the R-matrix fit. 


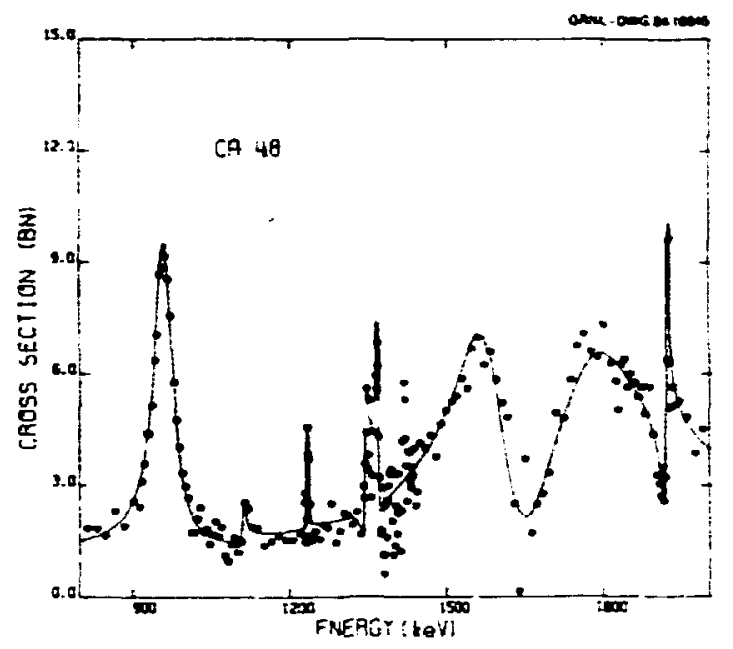

Fig. 2.25. Total cross section of ${ }^{48} \mathrm{Ca}$ obtained with neutrons from the Ta source.

combined strength (based on a radius of $5.27 \mathrm{fm}$ ) is -457 of the single particle limit. The $d 5 / ?$ neutron strength function from 0.8 to $2.0 \mathrm{MeV}$ is $14 \times 10^{-4}$ in conventional units. The $d_{3} / 2$ and $p$-wave strength functions are nearly 100 times smalier and the strave is approximately zero.

He have done a shell-model-in-the-cont inuum calculation 7 of the ${ }^{48} \mathrm{Ca}+n$ system. The $\mathrm{P}_{3 / 2}$ ground state aird the $P_{1} / 2$ first excited state at $2.03 \mathrm{HeV}$ of ${ }^{49} \mathrm{Ca}$ exhausts most of the p-strength in agreement with $(d, p)$ data and little remains at higher energies. The positive parity states are calculated as a combination of $199 / 2,2 d_{5 / 2}$ and $2 d_{3 / 2}$ S.P. States and $p_{3 / 2}$ and $p_{1} / 2$ neutrons coupled to the $3^{-}$collective state in ${ }^{9} \mathrm{Ca}$ at 4.51 MeV. No significant $\mathrm{J}=1 / 2^{+}$states lie in the energy region from 0 to $3 \mathrm{MeV}$ above the neutron separation energy and four $\mathrm{J}=5 / 2^{+}$states are predicted in this energy region. They represent a total of $60 \%$ of the available s.p. strength, fragmented by the particle-core interaction. The remainder of the strength is predicted to lie within the 3 to $5.5 \mathrm{MeV}$ energy region.

Division.

1. Engineering Physics and Mathematics

2. Middle Tennessee State University, Murf reesboro, IN 37132

3. Queen's University, Kingston, Canada K7L 3N6.

4. F. T. Seibel, E. G. Bilpuch and H. W. Newson, Annals of Phys. 69, 451 (1972).

5. M. Di vadeenam, $H, P$. Beres and $H, H$. Newson, Annals of Phys. 69, 428 (1972).

6. N. M. Larson and F. G. Perey, ORNL/TM-7485, 1980 and N. M. Larson, ORNL/TM-9179, 1984.

7. J. A. Harvey et al., Phys. Rev. C 28 , 24 (1983); R. F. Carlton et al., Phys. Rev. C 29, $1980($ ( 984$)$.

\section{COUPLED-CHAMIIEL OPIICAL WOEL FOR 0-0.5 HeV MEUTROWS In $60 \mathrm{Ni}$}

\author{
A. D. Mackellarl C. H. Johnson \\ R. R. Winters ${ }^{2}$
}

In a previous report ${ }^{3}$ averaged neutron scattering functions that had been deduced from high-resolution cross sectio.: measurements for various nuclei were compared to optical model potential (OMP) scattering functions for each partial wave. Assuming a spherical OMP a good fit to the average for 0-450 kell s-wave on $60^{\mathrm{Ni}}$ was achieved for a real well depth of $V_{0}=$ $48 \pm 5 \mathrm{MeV}$. (The model geometry was jnventional with $r_{0}=r_{0}=1.21 \mathrm{fm}, a_{0}=0.56 \mathrm{fm}$ and $a_{0}=0.48 \mathrm{fm} .9$ That value for $v_{0}$ is consistent with the kncm peak of the $351 / 2$ size resonances. However, since an unusually targe imaginary depth (namely $\omega_{0}=29 \pm 5 \mathrm{MeV}$ ) was required, it was concluded that a non-spherical model should be considered for this vibrational nucleus.

To include the vibrational effects we used the coupled-channel formalism 4 ,5 with the target channel being the $0^{+}$ground state and the $2^{+}$ state at $1.33 \mathrm{HeV}$ with $B=0.211$. (The 3 - state at $4.04 \mathrm{MeV}$ has negligible effects for $s$-wave neutrons.) Using the same geometry as for the spherical model we again fit the compound arid shape elastic cross sections ( $\sigma_{c}$ and $\sigma_{s e}$ ) by searching on $V_{0}$ and $W_{0}$ and found $V_{0}=50 \pm 5$ $\mathrm{MeV}, \mathrm{H}_{\mathrm{D}}=24 \pm 5 \mathrm{HeV}$. In other words, we found essentially the same well depths as for the spherical model.

Figures 2.26 and 2.?7 illustrate the effects of $V_{0}$ and $W_{0}$ in the two models. In both figures the abscissa is $V_{0}$ and the curves are calculated with various ido for $0.2-\mathrm{MeV}$ neutrons, i.e., at the mijpoint of the experimental region. For that energy the horizontal lines give the experimental $\sigma_{C}$ arid $\sigma_{\text {se }}$ with associated uncer tainties, as indicated by the shaded ar:-3:. A good fit requires that $W_{D}$ be chosen to fit both ${ }^{\circ} \mathrm{C}$ and $\sigma_{\mathrm{se}}$ for the same $V_{0}$.

In fig. 2.26 which is for the spherica: model, the observed ose restricts $V_{0}$ to anout $48 \mathrm{MeV}$. At that $v_{0}$ the $\sigma_{c}$ is at the peak of the $3 \mathrm{~s} 1 / 2$ resonance; thus, $W_{0}$ must be increased to abnut $29 \mathrm{MeV}$ to reduce the peak to the observed value. (A small $\mathrm{W}_{0}$ of about $0.5 \mathrm{MeV}$ would also $f$ it at $E_{n}=0.2 \mathrm{MeV}$ but not over the full $0-450$ keV region.)

In Fig. 2.27, which is for the vibrational model, the oc curves exhibit two additional resonances arising from particle-core couplings of the bound $2 \mathrm{~d}$ states with the $2^{+}$target state. The curve of ac for $W_{0}=1.5 \mathrm{MoV}$ illustrates that the particle-core resonances are moved upward from their unperturbed energies (arrows) whereas the 35 resonance is moved downward from its position in the spherical model. The solid points indicate the ficted $V_{0}$ and $H_{0}$ as stated above. The open symbols indicate an alternate solution with $W_{0}$ only $1.5 \mathrm{MeV}$ but with $V_{0}$ so large, namely $54.5 \mathrm{MeV}$, that the 35 size resnnance would nccur at $A=44$, far below the known peak near $A=55$. 
1. Consultant from thiversity of Kentucky, Lexington, äY 40506.

2. Consultant from Denison University, Granville, OH 43023.

3. A. D. Mackellar, B. Castel and C. H. Johnson, Physics Division Progress Report, ORNL-6004, 47 (1983).

4. T. Tamura, Rev. Mod. Phys. 37, 679 (1965). tion).

5. J. Rayral, ECIS-79 (private comunica-

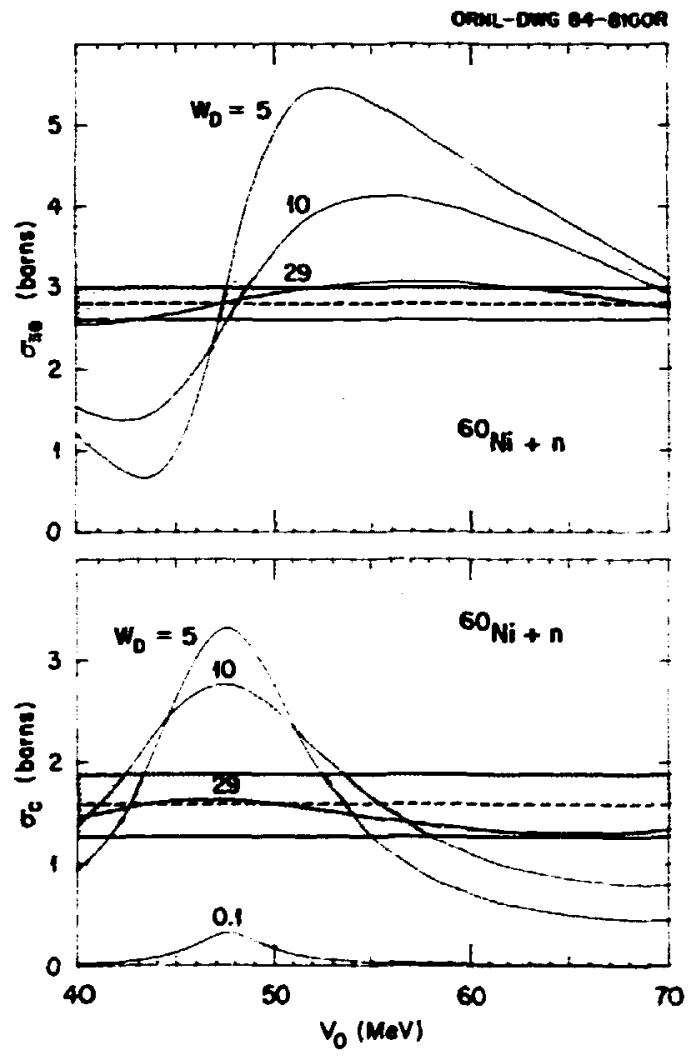

Fig. 2.26. Spherical OMP s-wave shape elastic and compound nuclear cross sections vs $V_{0}$ for various imayinary well depths at $E_{n}=200$ keV. The horizontal lines indicate the experimental values and the shaded areas represent the associated uncertainties.

OPTICAL WDOEL SCATTERIMG FUNCTIONS FOR 0-700 keV MEUTRONS on ${ }^{6} \mathrm{Kr}$
R. F. Carlton 1
J. A. Harvey 2
C. H. Johnson

At low neutron energies parameters for an optical model potential (OMP) can be deduced from high resolution scattering measurements by first parametrizing the data in terms of an $R$ matrix and then averaging those scattering func-

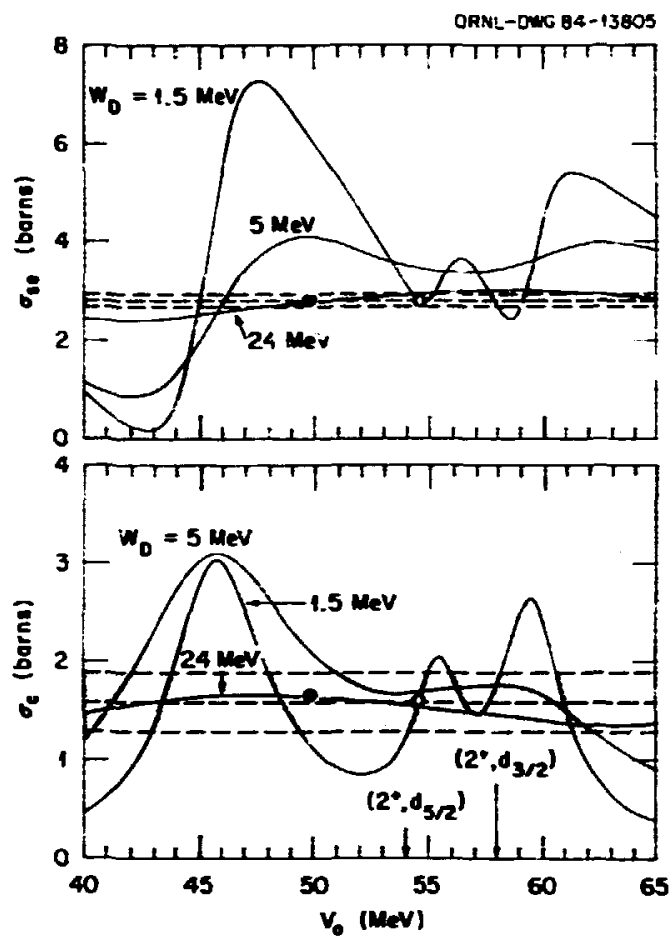

Fig. 2.27. Coupled channels orp s-wave shape elastic and compound muclear cross sections as for Fig. 2.26. THe $2^{+}(1.33 \mathrm{WV})$ target state is included with $B=0.211$. Arrows indicate unperturbed energies of the $\left[2^{+}, 2 d_{5 / 2}\right]$ and $\left[2^{+}\right.$, 2d3/2] particle-core states. Soliu points indicate the accepted model and the open points indicate a rejected solution.

tions for each partial wave in order to compare to the OAP. In the preceding raport ${ }^{3}$ such analyses were given for ${ }^{30} \mathrm{Si}, 32 \mathrm{~s}, 314 \mathrm{~s},{ }^{40 \mathrm{Ca}}$, and ${ }^{60 \mathrm{Ni}}$. Here we present new prel iminary analysis for $86 \mathrm{Kr}$. This mucleus has a closed neutron shell and it.s mass is just below the peak of the $3 p$ size resonance.

Raman et al ." measured the transmission of a 99.5\% enriched $86 \mathrm{Kr}$ sample using the $80-m$ flight path at the ORELA facility. We repeated the measurement with the resolution of the 200-m flight path and 7 nsec burst width. With this resolution the resonances are well resolved such that definitive assignments of the important sand p-wave resonances can be made for neutron energies up to about $400 \mathrm{keV}$. At higher energies the complicated structure makes assignments more difficult; nevertheless, our multilevel analysis is nearly complete for energies up to $700 \mathrm{keV}$.

In Fig. 2.28 the staircase plots show the cumulative sums of p-wave reduced widths for a 6.4-fm boundary radius. We spe that the sum for $P_{3 / 2}$ is about three times that for $P_{1 / 2}$ and that the average slope or strength function, $\tilde{s}$. $\left\langle\gamma^{2}\right\rangle / 0$, appears to be increasing in the region for $D_{3 / 2}$. These observations are consistent with a spin-orbit splitting of the 3p singleparticle resonance. In our final anaiysis we 


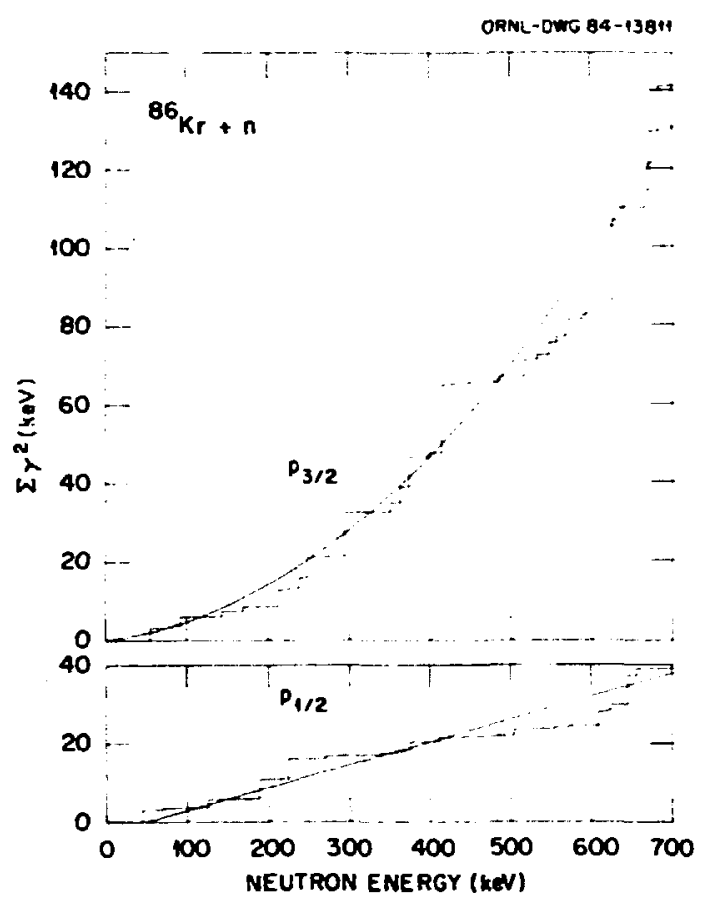

Fig. 2.28. Cumulative p-wave reduced widths.

will introduce an energy dependence for the $P_{3 / 2}$ strength function, but here we assume each strength function to be constant within the region. Thus, we find $\tilde{s}=0.022,0.058$ and 0.22 , respectively, for $s_{1 / 2}, p_{1} / 2$ and $p_{3 / 2}$.

of equal importance for an om analysis is the external R-function wich accounts for all leve:s outside the region from $E_{l}=0$ to $E_{u}=700 \mathrm{keV}$. For each partial wave it is parametrized by

$$
R^{\operatorname{ext}}(R)=\tilde{R}(E)-\tilde{s} \ln \left[\left(E_{U}-E\right) /\left(E-E_{\ell}\right)\right]
$$

where $\tilde{R}=a+b\left(E-E_{m}\right)$ and $E_{m}$ is the midpoint of the analysis. The fitted $(a, b)$ with $b$ in $\mathrm{MeV}^{-1}$ are $(-0.13,0.15),(0.60,0.14)$ and $(0.58,1.74)$ for $s_{1 / 2}, P_{1 / 2}$ and $P_{3 / 2}$.

From the parametrized scattering functions we determined an average for each partial wave by use of an analytical approximation 5 with an averaging width of $21=200 \mathrm{keV}$. Figure 2.29 presents the p-wave average in terms of compound and shape elastic cross sections, $q$ and se. The solid curves present the experimental values (divided by the statistical factors) and the dashed curves show the fit obtained by adjusting the well depths in an Oup of standard form with $r_{0}=r_{p}=1.21 \mathrm{fm}, a_{0}=0.66 \mathrm{fm}$ and $a_{0}=0.48$. fm. The fitted well depths are listed in the figure. We see that the imaginary well depths are essentially the same for both $p_{1 / 2}$ and $p_{3 / 2}$ and that the major part of the observed Jdependence in the $p$-wave cross sections is attributed to the real spin-orbit term. In other works, the spin-orbit splitting brings the $3 p_{3} / 2$ state down towards our region while pushing the $3 p_{1 / 2}$ state upward. The proximity
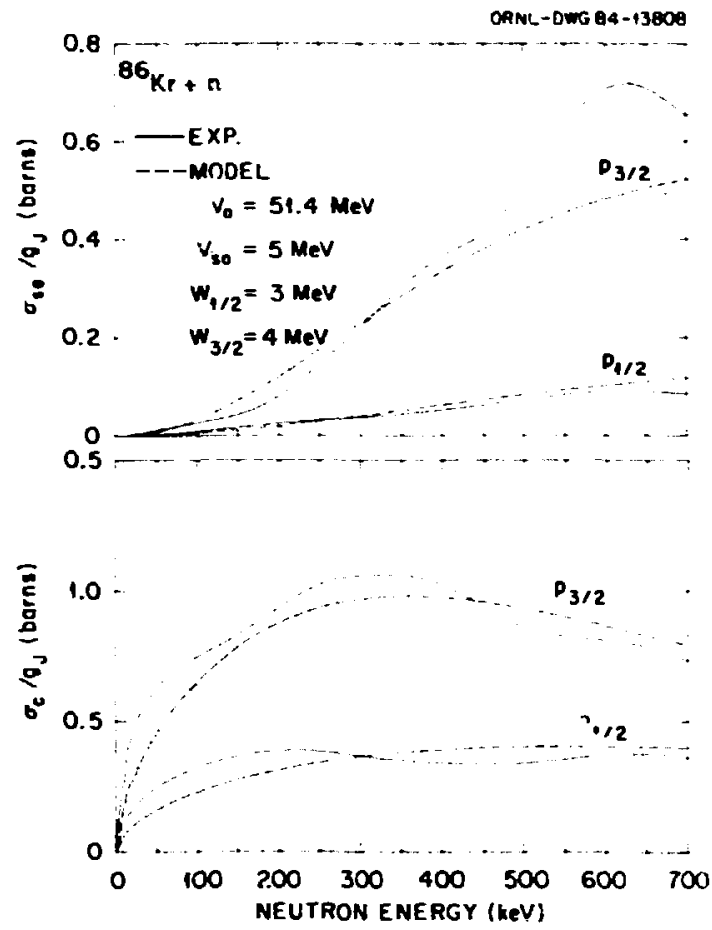

Fig. 2.29. Shape elastic and compound cross sections. Solid curves were deduced from the data by averaging the R-matrix scattering function with the averaging width $21=200 \mathrm{kev}$. Dashed curves are OMp fits with a given geometry and with the well depths adjusted as listed in the figure.

of the $3 p_{3 / 2}$ state may account for the rising strength function in -ig. 2.28 .

Table 2.9 lists the well depths deduced here for $86 \mathrm{Kr}$ along with those reported ${ }^{3}$ for the lighter nuclei. Some trends are apparent. For each nucleus the real well is deeper for p-waves than for 5-waves. Coupled channel calculations 5 suggest that most, but not all, of this $\ell$-dependence can be attributed to vibrational effects. For s-waves the smaller $V_{0}$ for $86 \mathrm{Kr}$ is consistent with a relatively large symmetry term for this nucleus. The fact that the imaginary well depths have large fluctuations indicates the effects of nuclear structure for each nucleus.

1. Middle Tennessee State University, Murfreesboro, TN 37132.

2. Engineering Physics and Mathematics Division.

3. C. H. Johnson et al... Physics Diviston Progress Report, ORNL-6004, 47 (1983).

4. S. Raman et al., Phys. Rev. C 28, 603 (1983).

5. C. H. Johnson et al., Phys. Rev. C 27, 1913 (1983)

6. A. O. Mackellar, B. Castel and C. H. Johnson, Physics Diviston Progress Report, ORNL-6004, 48 (1983). 
Table 2.9. Neutron optical model potential rell depths

\begin{tabular}{llcccc}
\hline Mucleus & 2 & $v_{n}$ & $v_{\text {so }}$ & $W_{D}$ \\
\hline${ }^{30} \mathrm{Si}$ & 0 & $44 \pm 1.7$ & & $4.5 \pm 1.5$ \\
& 1 & $62 \pm 2.5$ & 72 & $4.5 \pm 2.5$ \\
${ }^{325}$ & 0 & $51.5 \pm 0.4$ & & $6.0 \pm 3.0$ \\
& 1 & $61.4 \pm 1.1$ & $11 \pm 3$ & $2.7 \pm 1.5$ \\
${ }^{34 \mathrm{~S}}$ & 0 & $51.5 \pm 1.1$ & & $3.0 \pm 2.0$ \\
& 1 & $58.5 \pm 1.2$ & $6 \pm 3$ & $3.5 \pm 1.9$ \\
${ }^{40} \mathrm{Ca}$ & 0 & $53.5 \pm 1.0$ & & $5.7 \pm 0.3$ \\
& 1 & $56.1 \pm 1.0$ & $6 \pm 7$ & $0.6 \pm 0.3$ \\
${ }^{60} \mathrm{Ni}$ & 0 & $48 \pm 5$ & & $29 \pm 5$ \\
${ }^{86} \mathrm{Kr}$ & 0 & $48.5 \pm 1.0$ & & \\
& 1 & $51.4 \pm 1.0$ & $5 \pm 1$ & $3.5 \pm 1.0$ \\
\hline
\end{tabular}

assumed value.

MEASUREFENTS OF THE REUTRON TRANSHISSION AND CAPTURE CROSS SECTIONS IN 204pD!
D. J. Horen
J. A. Harvey ${ }^{2}$
R. L. Macklin 2
N. H. Nill 3

High resolution neutron transmission measurements have been performed on ${ }^{204} \mathrm{pb}$ in the energy interval $E=0.4-105 \mathrm{keV}$. The transmission data were analyzed using a multilevel R-matrix code to deduce resonance parameters. Previously obtained neutron capture data were reanalyzed in the interval 2.6-86 kev. Values of $g \Gamma_{n} \Gamma \downarrow \Gamma$ were determined from the capture data. For those resonances where $r_{n}$ could be determintd from the transmission data, the capture data were analyzed to extract $\Gamma_{\gamma}$. Our results yield an average capture for a stellar temperature $k T=30 \mathrm{keV}$ of $89.5 \pm 4.5 \mathrm{mb}$. The s-wave level density for $205 \mathrm{pb}$ corresponding to the neutron energy range investigated (1.e., E $-105 \mathrm{keV}$ ) relative to that for 20 Pb (which has about the same neutron separation energy) is greater by about a factor of 10 . The average s-wave strength function in this energy region is determined as $S_{0}=0.93 \times: 0^{-4}$. This is an order of magnitude greater than that for a similar energy region in $206 \mathrm{pb}+\mathrm{r}$. where a doorway is observed at $E \sim 500 \mathrm{keV}$. However, the strength function in the initial $E=0,-100 \mathrm{keV}$ in $20+\mathrm{pb}+\mathrm{n}$ is almost identical to the average value of that for $206 \mathrm{pb}+n$ then the averaging interval for the latter is taken as $E_{n}-0-1000 \mathrm{keV}$ (i.e.., over the doorway state). This suggests that the s-wave doorway state observed in the higher mass lead isotopes is completely mixed with "background" states in $205 \mathrm{pb}$, and most likely no intermediate structure will be observed in the $s$-wave strength fuiction for the $20+p b+n$ reaction.
1. Abstract of paper: Phys. Pev. C 29, 2126 (1984).

2. Engineering Physics and Mathematics Division.

3. Instrumentation and Controls Division.

\section{SPHERICAL OPTICAL MODEL DESCRIPTION OF 208pb+n SCATIERIMG REAR THEESHOLD}
D. J. Horen
C. H. Johnson

The spherical optical model potential (OMp) provides a good macroscopic description of nuclear scattering for energies for which the compound system can be treated statistically. Ti:a scattering of low energy neutrons by $20 \mathrm{pp}$ near threshold where the level density is quite small obviously does not satisfy these conditions. For example, there are only three $1 / 2^{+}$ resonances in the energy interval $E_{n}=0-1005$ $k e V$, and almost all of the $s$-wave strength is contained in a single resonance at $506 \mathrm{keV}$. Hence, it is clear that this energy regime is non-statistical and that standard averaging procedures would not be appropriate. However, it is still useful to attempt some sort of average description even at these energies in order to make comparisons to OMP predictions and, thereby, to relate to the OMP ana'yses wich are made for higher energy data. I

As distinguished from higher energy studies (i.e., $E_{n} 23,2 V$ ), the low energy region mich we study with ORELA is dominated by resonance structure. The data can be analyzed in terms of partial waves from which one can deduce information pertaining to possiule $l$-dependency of the spherical CAP. This is in contrast to work at hígher energies where one is constricted to investigations of the average potentials.

We have analyzed our $208 \mathrm{pb}+n$ data utilizing the R-matrix formalism and deduced a parameter set that reprofucies the differential elastic and total cross sections in the energy interval $F_{n}=40-1005 \mathrm{keV}$. The scattering function for each partial wave can be expressed in the form

$$
S(E)=e^{-2 i \phi(E)} \frac{1+i P(E) R(E)}{1-i P(E) R(E)} .
$$

where and $P$ are the hardsphere phase shift and penetrability, respectively. $R$ is the $R$ function which has been parametrized in terms of poles as

$$
R(E)=\operatorname{Rext}(E)+\left[\frac{\gamma^{2}}{E \lambda-E} .\right.
$$

The function Rext (E) can be parametrized in a variety of ways, and we have chosen to do so by use of a distribution of poles outside of the region analyzed. For our choice of boundary radius, $R_{0}^{e x}$ (E) (f.e., for s-waves) is a smoothly varying function whose magnitude is small in our energy interval of andysis. Together with the hardsphere phase shift, it 
determines the cross section which one would have in the absence of the observed resonances. This cross section is almost equivalent to the elastic cross section that one would calculate from a real potential. We find that the depth of the real well of a Hood-Saxon potential that fits the cross section calculated by setting the resonance part of the $R$-matrix to zero is very close to that required to yield the experimentally observed binding ener $: y$ of the $4 s_{1 / 2}$ neutron in $209 \mathrm{pb}$.

We can write the OMP $S$-matrix in the same form as that for the R-matrix as

$$
\operatorname{SOMP}^{\operatorname{OM}}(E)=\mathrm{e}^{-2 i \phi(E)} \frac{1+i P(E)[\bar{R}(E)+i \operatorname{ms}(E)]}{1-i P(E)[\bar{R}(E)+i \pi s(E)]} .
$$

For a real well (i.e., $M \equiv 0), S(E) \equiv 0$. Hence, we can see that what was done above is to assume that if we had averaged the experimentally determined S-matrix the resonance would not contribute significantly to the determination of $R(\bar{E})$. We have performed a numerical average ${ }^{2}$ of $S(E)_{\exp }$ and find this to be essentially so. Hence, we feel that our determination of the value of the real potential near threshold for a spherical potential model has validity.

The determination of the imaginary potential is more questionable. He have approached this i. two ways. One is to ignore the fact that there are too few s-wave resonances to allow a reliable average and to perform a numerical calculation with a large averaging interval. The other is to consider the trend of the data for other isctopes of lead. From earlier works, ${ }^{3}$ it has been shown that s-wave strengths in $206,207 \mathrm{pb}+n$ al so have doorway structure in the energy interval $E_{n}=0-1000 \mathrm{keV}$. Recently, it has been found ${ }^{4}$ that the s-wave strength in $204 \mathrm{pb}+\mathrm{n}$ is much more fragmented, and has a value in the interval $E_{n}=0-100 \mathrm{keV}$ that is essentially equal to that for the other lead isotopes when one averages the latter over a $1000 \mathrm{keV}$ interval. This has been interpreted ${ }^{4}$ as indicating that the compound sy stem $204 p b+n$ already is essentially statistical, and that the strength in the $s$-wave doorway states in $206,207,20 \mathrm{Bpb}$ is more or less uniformly distributed in $204 \mathrm{pb}+\mathrm{n}$. On this bas is we would expect that if we uniformly distribute the strengths in $206,207,208 \mathrm{pb}$ we would find OMP parameters for all of the lead isotopes to be similar (except for an A dependence) as one might expect for a global model. The value of 5 obtained by averaging is not too different from that determined from a uniform distribution.

The values of the real, $V_{0}$, and imaginary, Wo. spherical OMP that we deduce with the geometry given by Finlay et al.1 are shown in Fig. 2.30. The values of $V_{0}$ determined from our partial t-wave analyses are in good agreement with the value of the average $v_{0}$ extrapolated downward from fits to higher energy data. Our indicated values for $N_{0}$ should be considered as tentative, with large error bars. Further consideration must be given to investigating the meaning of methods to deduce these. However, it is interesting to note that our tentative values at least appear reasonable. Since our inter-

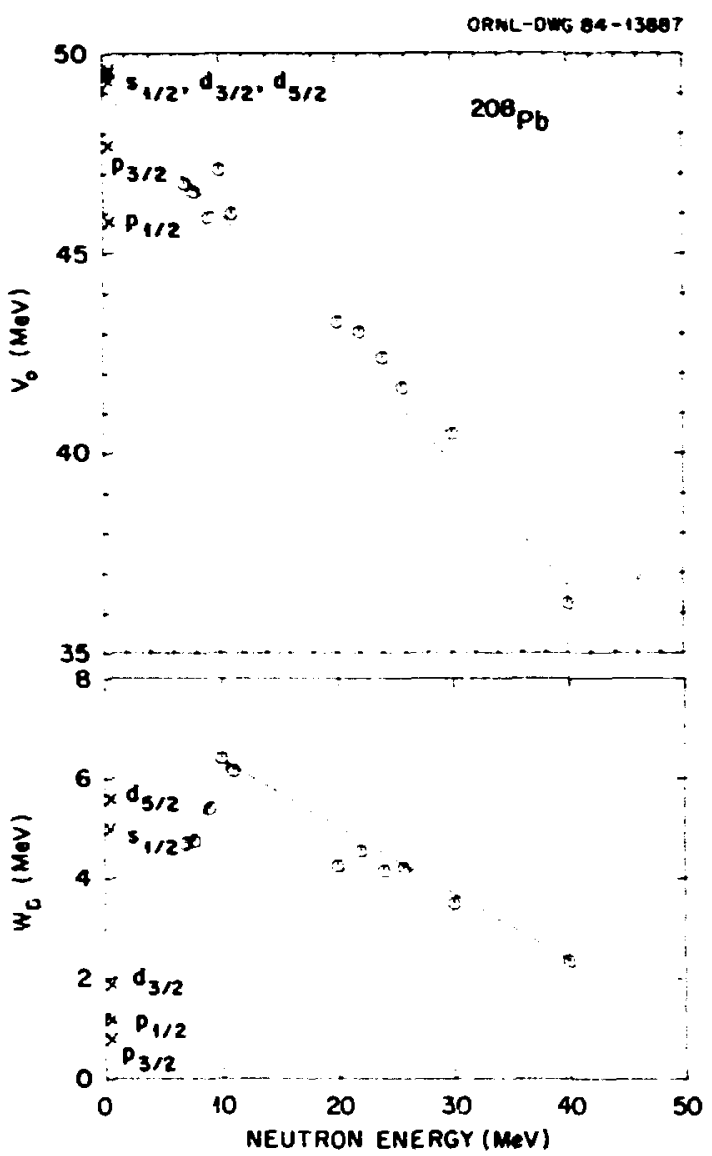

Fig. 2.30. Plots of the real, $V_{0}$, and imaginary. Ho, values for a spherical optical model description of $20 \mathrm{Bpb}+n$ scattering. The $x^{\prime}$ s have been deduced from this work. The remaining points are from Ref. 1.

mediate structure can be explained in terms of a real resonance (i.e.., particle-core excitation) within our region of analysis, it is clear that a spherical optical model description must contain a non-vanishing $H_{D}$. In another contribu$t$ ion we discuss a description of $208 \mathrm{pb}+n$ scattering in the s-wave channel in terms of a coupled-channel formalism with real potentials.

1. See for example, R. W. Finlay et al., Phys. Rev, C 30, 796 (1984)

?. C. H. Johnson et al., Phys. Rev. C 27, 1913 (1983).

3. D. J. Horen, J. A. Harvey and N, H. Hill, Phys. Rev. Lett. 38, 1344(1977); Phys. Rev. C 18, 722 (1978); 20, 478 (1979): 24, 1961 (1981). 4. D. J. Horen et al., Phys. Rev. C 29, 2126 (1984). 
COUPLED-CHNMEL CALCULATION OF S-MAVE CROSS SECTIOW FOR 200pb+n: $E_{n}=40-1005 \mathrm{keV}$
A. D. Mackell lar'
D. J. Horen
C. H. Johnson

The 5-wave channel for $208 \mathrm{pb}+n$ scattering in the energy range $E_{n}=40-1005 \mathrm{keV}$ is essentially describab? $e$ in an R-matrix analys is with

$$
R(E)=R_{\text {ext }}(E)+\frac{Y^{2}}{\left(E_{\lambda}-E\right)}
$$

where the resonance energy is $506 \mathrm{keV}$. Here we show th:t this intermediate structure can be reproduced using a coupled-channel calculation 2 with a simple particle-vibrator model.3

Experimentally it is found that the $45_{1 / 2}$ single particle state lies at an excitation energy of $2.03 \mathrm{MeV}$ in $20 \% \mathrm{pb}$. The neutron separation energy is $3.94 \mathrm{MeV}$ (which cor responds to the binding energy of the $299 / 2$ ground state). The number of $1 / 2^{+}$states that can be formed by particle-hole or particle-core excitations and wich lie in the energy interval $E_{n}=0-1000 \mathrm{keV}$ is very limited. One such state can be formed by coupling the $29 g / 2$ single particke to the $4^{+}$core excitation (i.e., 4.32 $\mathrm{HeV}$ in $208 \mathrm{pb}$ ). Our initial calculations are being done with the coupled-channe? code ECIS. " The coupling equations are of the torm

$$
\begin{aligned}
& {\left[\nabla_{\rho_{n}}^{2}-\frac{i n\left(\ell_{n}+1\right)}{p_{n}^{2}}-\frac{V_{d i a g}}{E_{n}}-1\right] R_{J_{n}} \ell_{n} j_{n}(r)} \\
& =\frac{1}{E_{n}} \sum_{n^{\prime} \ell_{r^{\prime}} \cdot j_{n}{ }^{\prime}}\left\langle\left( Y_{\ell_{n} j_{n}}(x) \phi_{n}\left|v_{c p l}\right|\right.\right. \\
& \left(Y_{\ell_{n} j_{n}}(x) P_{n^{\prime}}\right) J M^{>} R_{J_{n}} R_{n^{\prime} j_{n^{\prime}}}(r),
\end{aligned}
$$

where $p_{n}=k_{N} r, k_{N}$ is the projectile wave number, and $E_{n}=E-K_{w}$. The scattered neutron has an energy. $E_{n}$, given by the incident energy minus the excitation energy of the core state. Tne central potential is purely real and of thit Wood-Saxon form. The coupling potential can be expressed in terms of derivatives of a HoodSaxon form and amplitudes for excitation of phonon modes of the target nucleus. Our preliminary calculations have used values of $V_{d i a g}$ that result in binding the $451 / 2$ and $299 / 2$ single-particle states at their experimentally observed values. We used a value $\beta_{4}=0.07$ for the $4^{+}$core state.

In Fig. 2.31 we compare the coupled-channel calculated cross section with that calculated using our experimentally determined $s$-wave $R$ matrix. This preliminary comparison is quit. good. The position of the resonance is close to that observed experimentally, but the width is somewhat broader. In this calculation, the resonance is shifted upward only about $130 \mathrm{keV}$ from the sum of the experimentally observed (unperturbed values) $299 / 2$ single particle state and $4^{+}$core excitation. This results partly because the coupl:is is weak and partly because the values of $V_{d i a c}$ sed are those that yield the observed rather . han the unperturbed binding

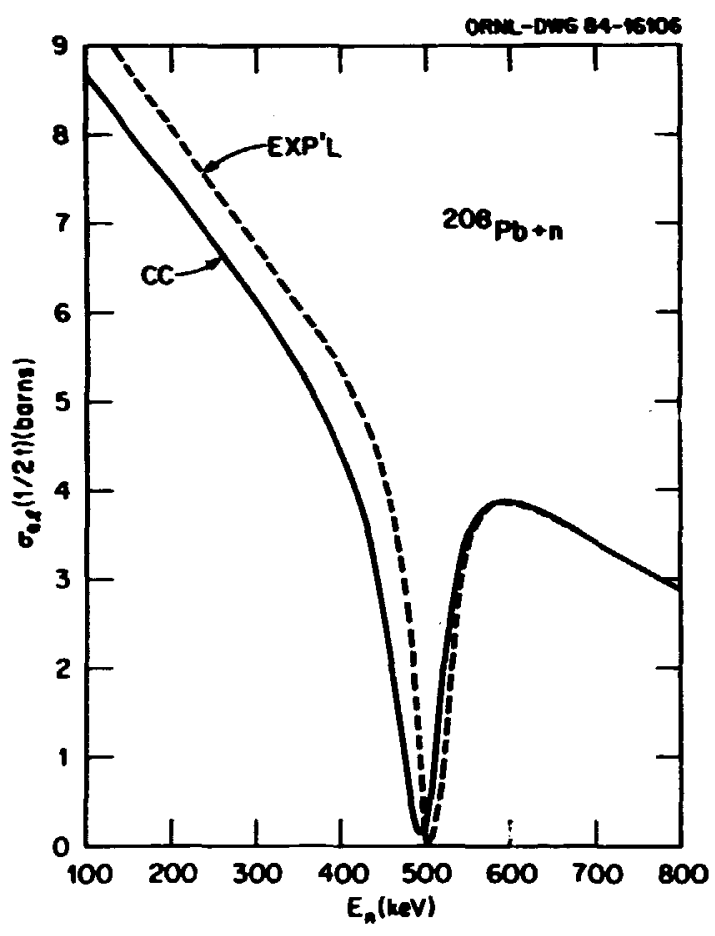

Fig. 2.31. Comparison of the experimental and coupled-channel calculated 5 -wave cross sections for $208 \mathrm{pb}+\mathrm{n}$.

energies for the single particle states. A more correct calculation would utilize $V_{\text {diag }}$ wich give the unperturbed single particle binding energies, and then reproduce the distribution of single particle strength in the bound as well as the unbound region. Such an investigation is in progress.

1. Consuitant from University of Kentucky, Lexington, KY 40506.

2. T. Tamura, Rev. Mod. Phys. 37,679 (1965).

3. A. Landa and G. E. Brown, Nucl. Phys. 75, 344 (1966).

4. J. Raynal (unpublished).

\section{ELASTIC AND INELASTIC SCATTERING OF} 180 FROM 28 ST AT $19.5 \mathrm{MeV} / \mathrm{amu}$
B. L. Burks
J. L. C. Ford, Jr. 1
D. J. Horen
E. E. Gross
R. L. Auble
D. C. Hensley
F. E. Bertrand
R. O. Sayer ${ }^{2}$
I. L. Blankenship
D. Shapira
T. P. Sjoreen ${ }^{3}$

To date no global optical-model parametrization has been developed to adequately describe heavy-ion elastic scattering data for a large range of projectile energies and target. projectile combinations. Therefore, it is 
necessary to measure elastic scattering data for inconing and outgoing heavy-ion reaction channels in order to determine reasonable opticalcodel potentials for use in direct reaction calculations such as DMBA. As a complementary measurewent to a study ${ }^{4}$ of reactions initiated by a 354-MeV 180 bean incident upon a ${ }^{28} \mathrm{Si}$ target we have beasured cross sections for ${ }^{28}$ Si $\left({ }^{18} 0,180\right)^{28}$ Si elastic scattering and also inelastic scattering to the lowest $2^{+}$states in both the target and projectile. ${ }^{28} \mathrm{Si}(180,180)^{28} \mathrm{Si} *\left[1.78 \mathrm{meV}, 2^{+}\right]$and ${ }^{28} \mathrm{Si}\left(18^{0}, 180^{-}\left[1.98 \mathrm{HeV}, 2^{+}\right]\right)^{28} \mathrm{Si}$, respectively.

The data described in this report were obtained using the coupled tandem and cyclotron machines and the broad range spectrograph (BRS) at HIRF. Particle identification and focal plane position mere determined using a multiparameter focal plane detector system wich has been described el sewhere. 5 Scattering data were measured for a laboratory -igle range of $2^{\circ}$ tc $15^{\circ}$, with a solid angle of $4.581 \mathrm{~ms} r$ corresponding to an angular acceptance of $\pm 2.0^{\circ}$ horizontal and $\pm 1.88^{\circ}$ vertical at each magnetic field setting. Five magnetic field settings mere required to provide ample overlap of scattering angle bite detected on the focal plane. The scattering yield has been analyzed by binning in 0.2 degree intervals, equivalent to approximately 0.33 degrees in the center of mass reference frame. Elastic scattering cross sections nave been determined for an angular range of $3.5^{\circ}$ to $20.9^{\circ} \mathrm{C} . \mathrm{m}$. ., as shown in Fig. 2.32 . Inelastic scattering cross sections were determined for excitation of the 1.78-MeV state in ${ }^{28} \mathrm{Si}$ from $3.5^{\circ}$ to $22.2^{\circ} \mathrm{C} . \mathrm{m}$. , and for excitation of the $1.98-\mathrm{MeV}$ state in $180^{\circ}$ from $3.5^{\circ}$ to $16.0^{\circ}$ c.m., as shown in Fig. 2.33.

An optical model fit to the elastic scattering data has been completed. The code PTOLEMY 6 was used to perform the optical model calculations and also automatic parameter searches for $x^{2}$ minimization. Two sets of optical-model potentfils were adopted as starting values for ritting the data; both taken from analysis of ${ }^{5} \cdot 0+28$ si elastic scattering data.7,8 Both parameter sets were derived from analysis of differential cross section angular distributions measured at 11 energies between 33 MeV and $215 \mathrm{MeV}$. The two parameter sets are sumarized in Table 2.10 along with the parameters resulting from an optical model fit to the $180+28$ si data presented here.

The potential labeled E18 15 an energyindependent Woods-Saxon optical potential reported by Cramer et al.7 The potential labeled A-type in Table 2.10 also uses a Woors-Saxon potential but includes a linear energy dependence of the diffuseness of the imaginary part of the potential. This potential along with a discussion of vartous other potential shapes was reported by Satchler. 8 Both starting parameter sets predicted angular distributions that were out of phase with the data. However, the shallow potential of Craner et al. more nearly reproduced the amplitude of oscillation observed in $\mathrm{Fig} .2 .32$ for the angular distribution of the cross section plotted as a ratio to the Rutherford scattering cross section. Although both parameter sets were

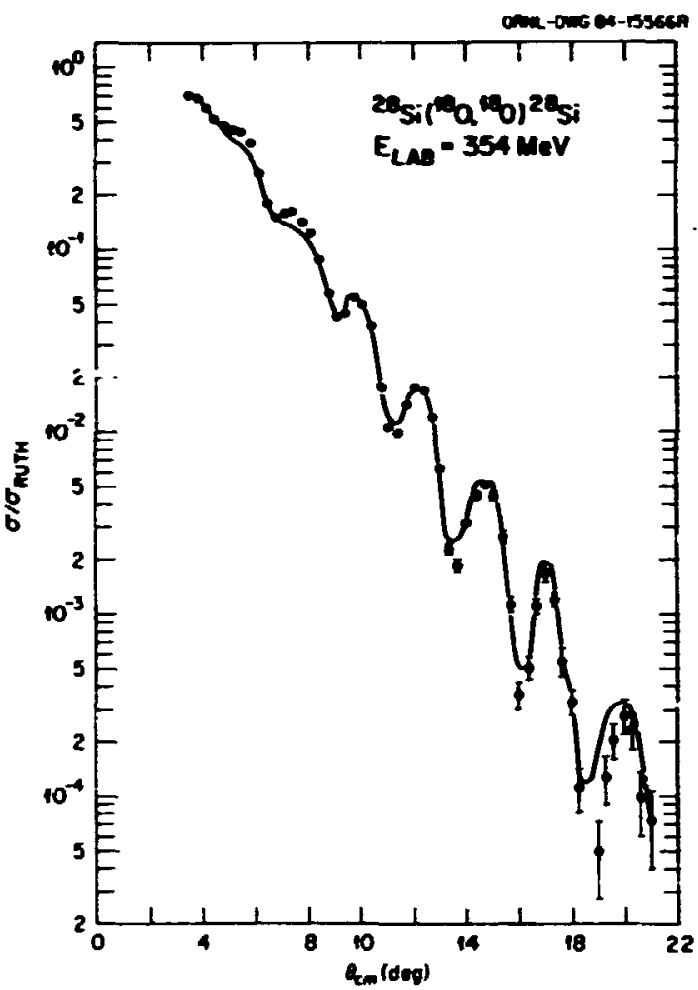

Fig. 2.32. Differential cross section data for $190+28$ Si elastic scattering at $354 \mathrm{MeV}$. The solid cirve is the best optical-model fit to the data generated using the parameters labeled E-type given in Table 2.10.

searched for an optimum $f: t$ to the data, it was found that the phase ar.j amplitude of oscillation of the data plotted in Fig. 2.32 could be reproduced more readily by modification of the shallow potential. The optical-model parameters which gave the best fit to the data are listed in Table 2.10, labeled E-type potential. Since the best fit was obtained by increasing the real well depth and decreasing the real well radius, the next step in this analysis is to explore the ambiguity in tnese parameters to see if a parameter set more closely resembling that of Cramer et al. could equally well reproduce the data.

A coupled-channels analysis of the elastic and inelastic scattering data shown in Figs. 2.32 and 2.33 is in progress using the E-type potential of Table 2.10 as a starting value. Intial calculations indicate that the best fit to the data will require significant reduction of the imaginary well depth so that the final parameter set will resemble the El8 potential of Cramer et al. even more r.losily. Although the coupled-channels analysis is still in progress, the calculations indicate that deformation parameters, $B_{2}$, required to $\mathrm{ft}$ the inelastic scattering cross sections are consistent with the values obtained by light ion scattering. 
1. Deceased.

2. Computing and Telecomunications Division, ORK.

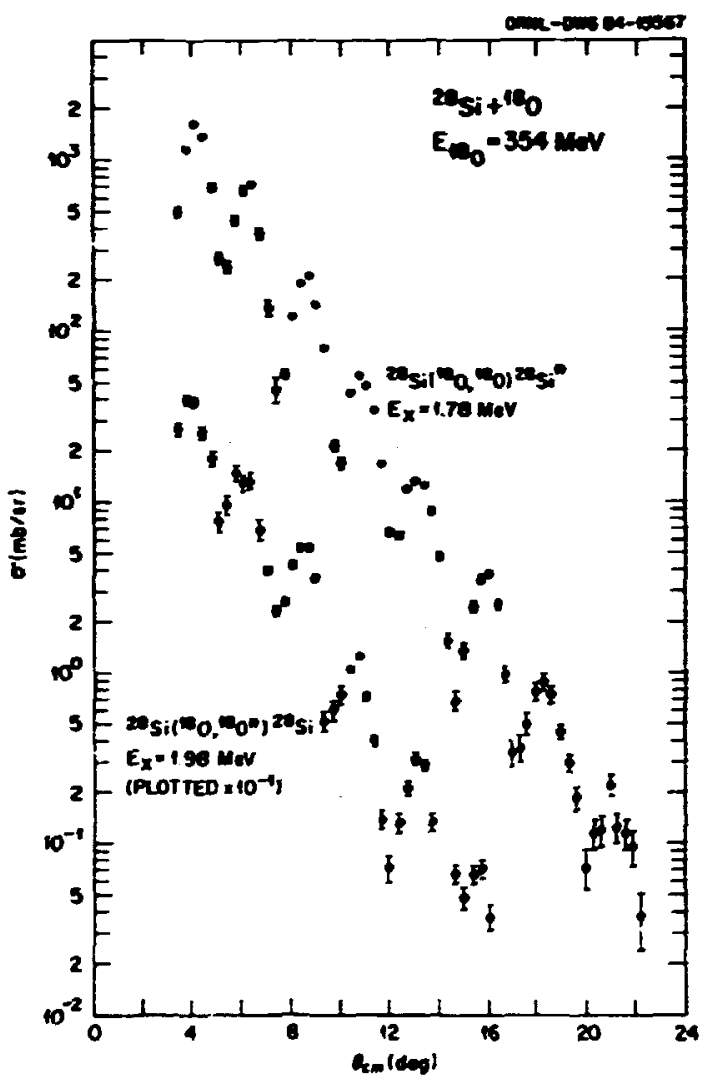

Fig. 2.33. Differential cross section ata for $180+205 i$ inelastic scattering at $354-\mathrm{HeV}$ populating the $1.78 \mathrm{MeV}$ state in $2 \mathrm{BSi}_{\mathrm{S}}$ and the $1.98 \mathrm{MeV}$ state in 180 . The latter angular distribution was mitiplied by a factor of 0.1 before plotting.
3. Solid State Division, CRM.

4. J. L. C. Ford, Jr. et al .., Oan-6004, p.147 (1983) inpubltshed. T. P. Sjoreen et al., ORK-6004, p. 148 (1983) unpubl ished. H. :. Hynes et al.., mucl. Instrum. Methods Phys. Res. 2224, 89 (1984). T. P. Sjoreen et al ., Mucl. Instrum. Methods Phys. Res. M224, 421 (1984).

5. 8. L. Burts et al .. "Spectroscopy of $27 \mathrm{Al}$ via the 28 si( $180,1 \%$ ) 27 Al Reaction." this report.

6. H. H. Macfarlane, S. C. Pieper, and $H$. Rhoades-Brom, private comunication, coaputer code PTOLEMT.

7. J. 6. Craner et al., Phys. Rev. C 14, $2158(1976)$.

8. 6. R. Satchler, Nucl. Phys. 1279, 493 (1977).

\section{SPECTROSCOPY of 27Al VIA ThE 2est $(10,1 \%)^{27} \mathrm{Al}$ IEACTICH}
B. L. Burts
J. L. C. Ford, Jr. 1
D. J. Horen
E. E. Gross
R. L. Auble
D. C. Hensley
F. E. Bertrand
R. O. Sayer 2
J. L. Bl ankenship
D. Shapira
T. P. Sjoreen ${ }^{3}$

During a recent experiment studying the $285 i\left({ }^{18} 0,18_{F}\right){ }^{28}$ Al sing! e charge exchange reaction, data were also obtained for the proton pickup reaction ${ }^{28} \mathrm{Si}(180,19 \%)^{27} \mathrm{Al}$. A $354 \mathrm{MeV}$ 180 bean was provided by coupled operation of the tandem and cyclotron machines at the Holifield Heavy Ion Research Facility (HHIRF) with a typical bean intensity of 3 to 4 pna incident on a $117 \mathrm{wg} / \mathrm{cm}^{2}$ self-supporting $28 \mathrm{Si}$ target. The reaction products were detected in the focal plane detector system of the broad range spectrograph (BRS).

At the beam energy used in this study, 19.5 MeV/anu, the proton pickup reaction channel is much stronger than the charge exchange reaction channel. Because of the $34 \mathrm{~cm}$ active length of the vertical drift chamer in the focal plane detector system of the BRS, position and particle-identification information were

Table 2.10. Cptical model potential parameters deduced from $160+20$ si elastic scattering data and used as starting values in the present analysis of $18_{0}+28$ st elastic and inelastic scattering. The E-type potential is the result of an optical-model fit to the $180+285 i$ elastic scattering data in fig. 2.32 .

\begin{tabular}{|c|c|c|c|c|c|c|c|}
\hline Potential & $\begin{array}{c}V \\
(\mathrm{MeV})\end{array}$ & $\begin{array}{c}r_{0} \\
\left(f_{m i}\right)\end{array}$ & $\begin{array}{c}a_{0} \\
(f m)\end{array}$ & $\begin{array}{c}\text { Ho }_{\text {(volume }} \\
\text { (MeV) }\end{array}$ & $\begin{array}{c}r I \\
(f m)\end{array}$ & aI & $\begin{array}{l}\text { rCoul } \\
(\mathrm{fm})\end{array}$ \\
\hline$E 18^{7}$ & 10.0 & 1.35 & 0.618 & 23.4 & 1.23 & 0.552 & 1.0 \\
\hline A-type 8 & 100.0 & 0.967 & 0.745 & 44.1 & 1.073 & 1.009 & 1.3 \\
\hline E-type & 15.0 & 1.30 & 0.710 & 42.0 & 1.19 & 0.707 & 1.0 \\
\hline
\end{tabular}


obtained simultaneously for excitation energy ranges of approximately 0 to $70 \mathrm{meV}$ in 27Al and 0 to $50 \mathrm{MeV}$ in $2 \mathrm{Al}_{\mathrm{Al}}$. Other reaction products were also observed but will not be discussed in this report, see Fig. 2.34.

Figure 2.35 shows a focal plane position spectru for the ${ }^{28} \mathrm{Si}(180,197) 27 \mathrm{Al}$ reaction measured at a laboratory angle of $2.1^{\circ}$. Prominent peaks corresponding to states in $27 \mathrm{Al}$ or I9f are labeled. The typical position resolution was approximately 1 mat, equivalent to an energy resolution of about $220 \mathrm{keV}$ Fwh. The data have been analyzed in 0.2 degree birs for laboratory scattering angles from 2 to 8 degrees. Differential cross sections have been determined for many of the states labeled in Fig. 2.35. Further peak-fitting is under way for the remaining states. The resulting angular distributions will be compared to heavy-ion transfer reaction calculations performed using the code PTOLEMY.

1. Deceased.

z. Computing and Telecomunications Division, ORNL.

3. Solid State Division, ORML.

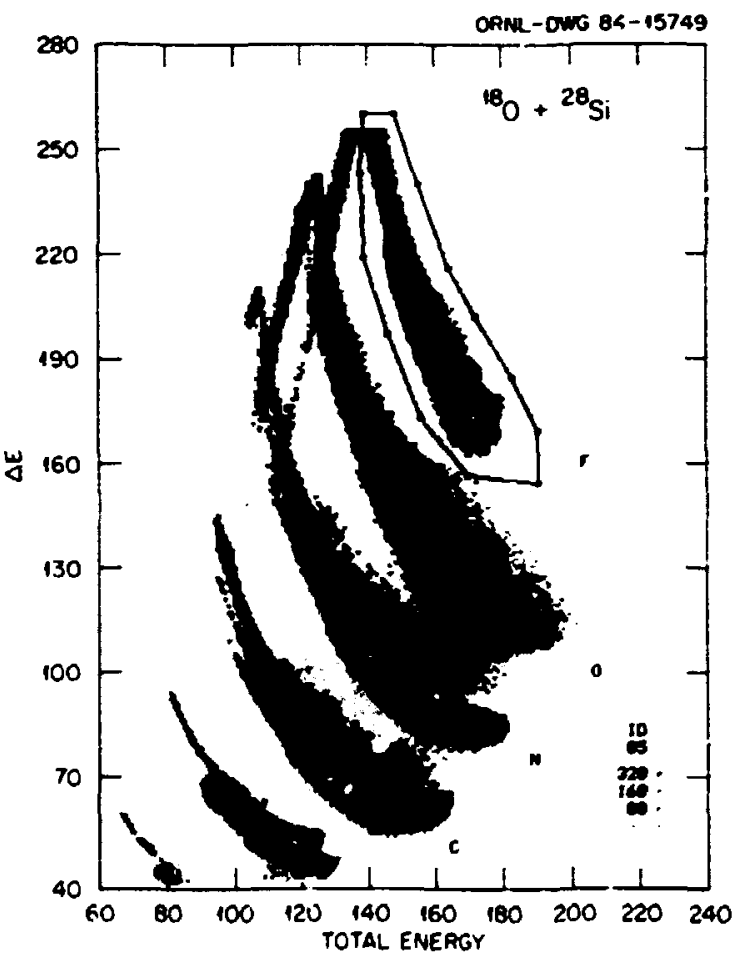

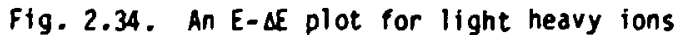
emitted from the $180+285 t$ reaction. The solid Iine enclosting the $Z$. 9 distribution indicates the particle identffication gate used to separate flourine ( $F$ ) nuclei from other reaction products.

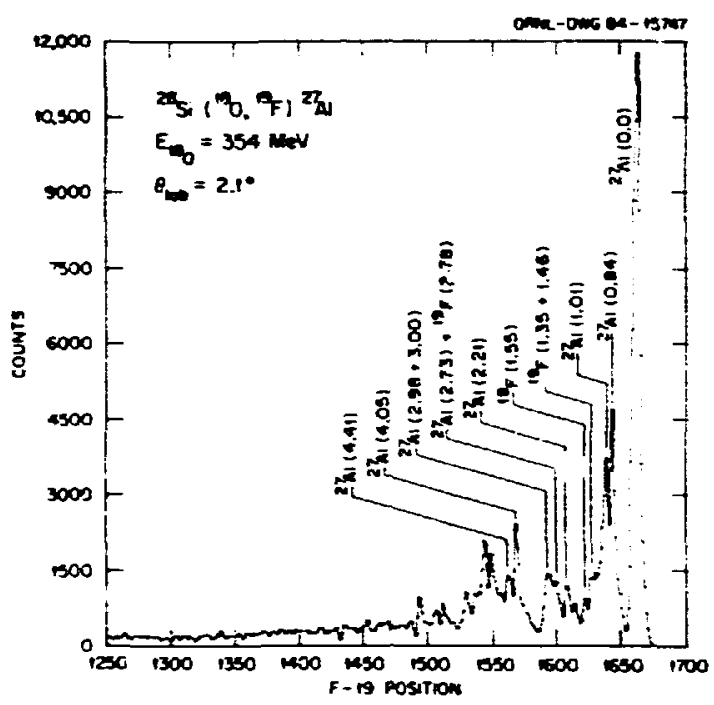

Fig. 2.35. A focal plane position spectrum for the ${ }^{28} \mathrm{Si}(180,19 \mathrm{~F}){ }^{27} \mathrm{Al}$ reaction at of ab $=$ $2.1^{\circ}$. Prominent peaks corresponding to states in $27 \mathrm{Al}$ or ${ }^{19} \mathrm{~F}$ are labeled with excitation energies in KeV.

\section{IMCLUSIVE STUDIES OF HEAVY PROOUCTS FROH $285 i+12$ C IWDUCED REACTIONS WITH SIMGLE UNIT MUS MD CHARGE IDERTIFICATION}
D. Shapira
M. Beckerman
J. Gomez cel Campo B. A. Harmon S. T. Thornton l

Studies of orbiting and deep inelastic reactions with ${ }^{28} \mathrm{Si}+{ }^{12} \mathrm{C}$ and other light systems have provided evidence for the presence of an upper limit to the orbital angular momentum stared in the dinuclear complex formed in deep inelastic collisions. 2 The value of this angular momentum limit can be related to a limit on the angular momentum in the entrance channel (with specific assumptions on the nature of nucleus-nucleus friction). In accordance with the model for nucleus-nucleus fusion put forth by R. Bass, it is expected that any limit on the formation of a dinuclear configurition will also prevent the fusion of the nuclei involvad. He have therefore decided to investigate these effects and to demonstrate the presence of angular momentum limitations on nucleus-nucleus fusion in the $28 \mathrm{~S} i+12 \mathrm{C}$ system.

The effects of reaching an absolute limit on the angular momentum leading to fusion are twofold. (1) The magnitude of the cross section for complete fusion of projectile and target should become inversely proportional to the center-of-mass bombarding energy at energtes beyond that for wich this limit has been reached (see Ftg. 2.36). (2) In addition, since complete angular momentum transfer to the composite system is no longer possible, we expect to observe the onset of processes where only part of the angular (and therefore linear) 


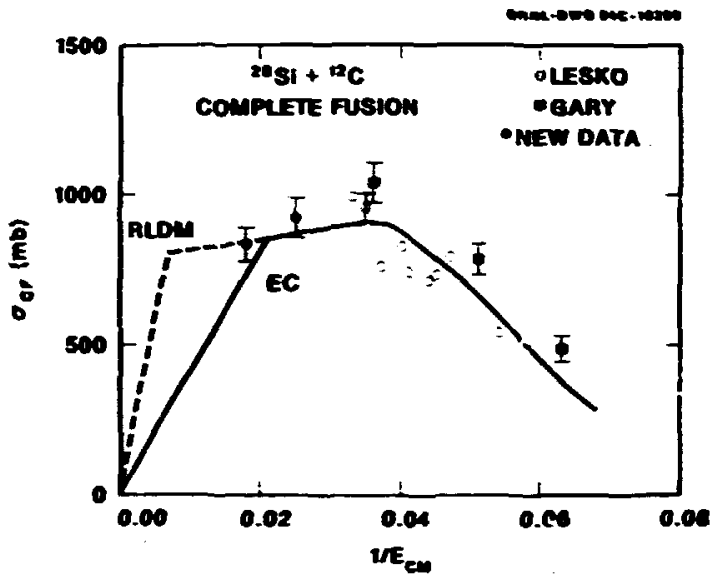

Fig. 2.36. Predictions for complete fusion of $205 i+{ }^{12 C}$ as a function of incident innterof $\rightarrow$ ass energy. The data shown as ojen circles and cross marks are from refs. 5 anc. 4 and the sol id circles present new data acquired by the authors of this report.

monentum of the projectile is transferred to the target (e.g., incomplete fusior.\}. The predicted cross section for fusion of ${ }^{28} \mathrm{Si}+{ }^{2} \mathrm{C}$ is shom in Fig. 2.36. This prediction is made in the spirit of the Bass model for nucleus-nucleus fusion but with a readjustment of the global parameters quoted by Bass 3 to fit fusion data measured at low energy. 4,5 The angular momentum limits are the values (E.C.) derived from the deep inelastic orbiting data ${ }^{2}$ and the higher cutoff value corresponds to an assumed rotating liquid drop limit (RLOM).

It becomes increasingly complicated to identify evaporation residues from the collisions of light heavy-ions, particularly at higher energy. The data analysis involves careful studies of the energy and velocity spectra of heavy products and the identification of yields from processes there complete momentum transfer has occurred. Complete identification (mass and charge) of the products facilitates such analysis and renders it less anoiguous. It is also expec:ed that the predominant incomplete fusion process in this system (28si beam with o target fragment) will be a process which is even nore forward peaked than the complete fusion - evaporation yield.

We report here on measurements of reaction products from the bombardment of a $12 \mathrm{C}$ target by a 28 si beam. Angular distributions for all products were measured at angles ranging from 2 degrees to 13 degrees. The mass and charge of the products were identified by measuring their energy and time-of-flight as well as energy loss in a gas-filled ionization chamber. The setup used in the experiment is shown in Fig. 2.37. Angular distributions of the evaporation residues, measured at 130 and 180 Mev bombaraing energies, are shown in Fig. 2.38 . The fusion cross sections obtained from integrating the angular distributions shown in Fig. 2.38 are

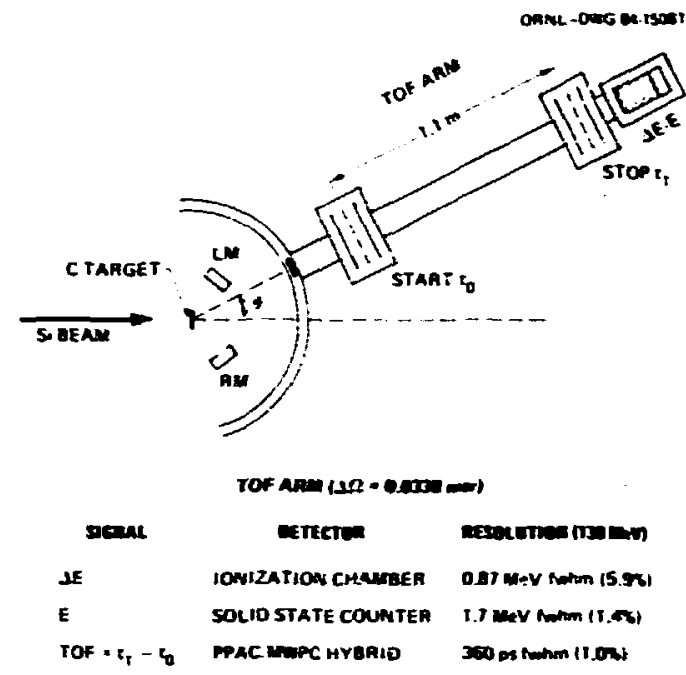

Fig. 2.37. The experimental setup for time of flight, energy loss and total energy masurements reported here.

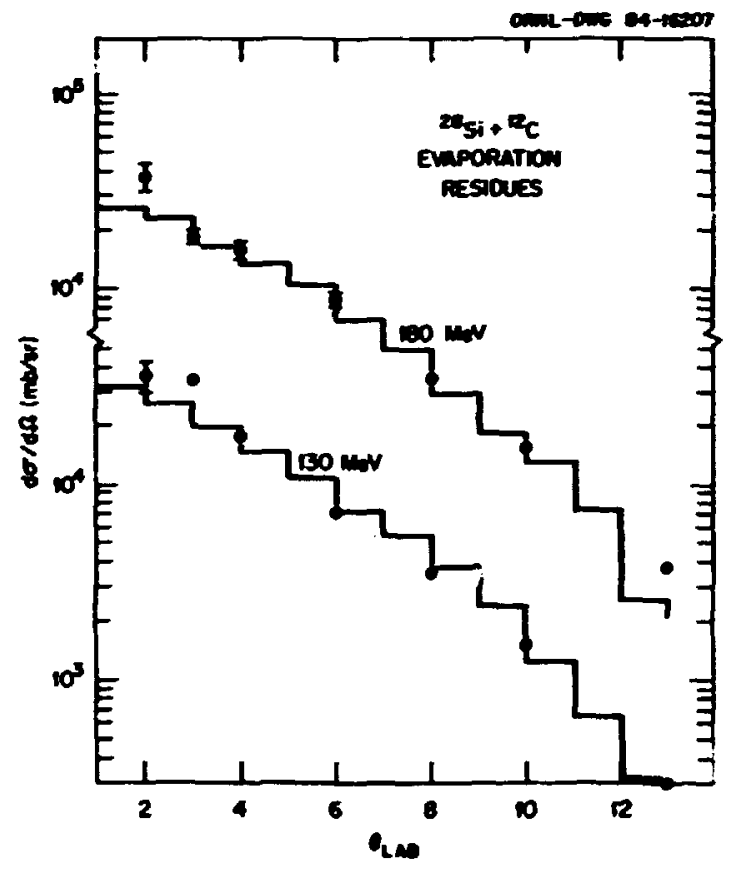

Fig 2.38. Angular distribution of evaporation residues fro collisions of $28 \mathrm{si}$ and ${ }^{12} \mathrm{C}$ nuclei. The histograns are angular distributions predicted by the Monte Carlo evaporation code PACE (6).

shown (solid circles) in Fig. 2.36. Similar data at higher energies are clearly needed and we plan such measurements using a $260 \mathrm{MeV} 285 \mathrm{Si}$ beam from the HHIRF facility.

The velocity spectra shown in figs. 2.39 and 2.40 serve to highlight the contribution of 

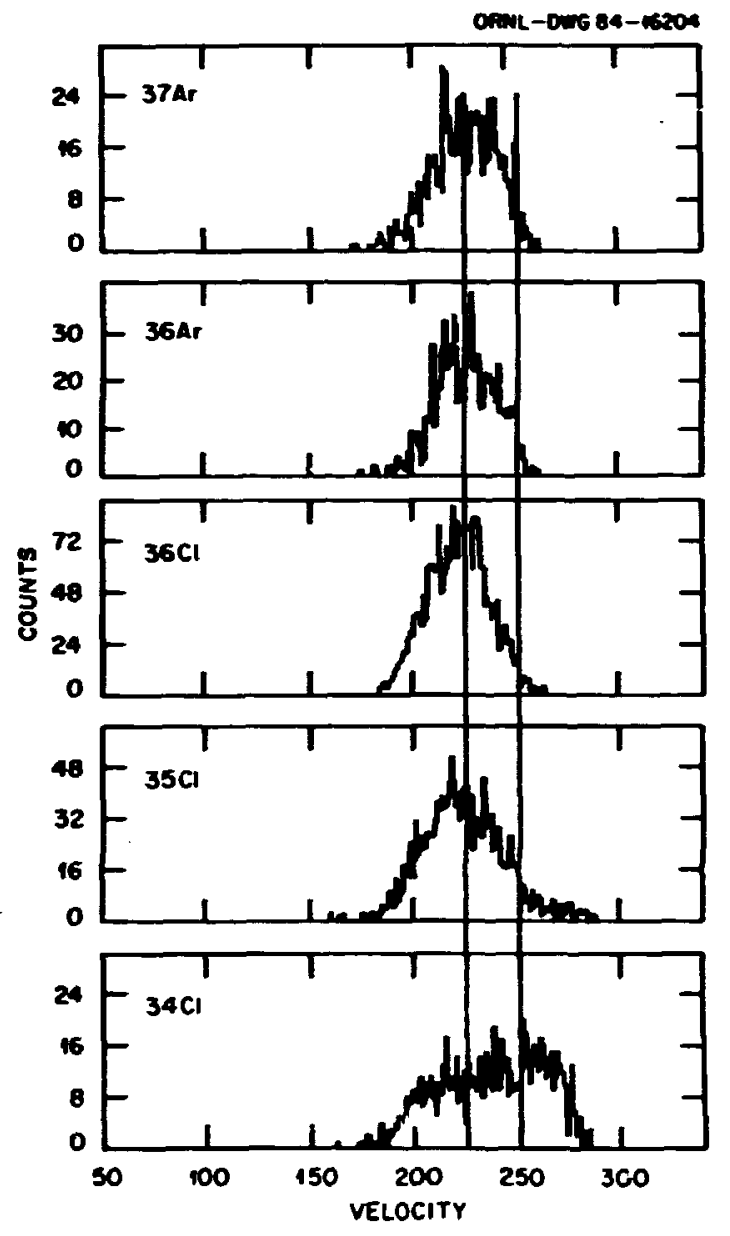

Fig 2.39. Velocity spectra for different evaporation residues assured at a laboratory angle of two degrees.

yields from the process of incomplete fusion of 28 si with ${ }^{12} \mathrm{C}$. The predominant incomplete fusion expected is that of $28 \mathrm{si}$ with a Be fragment from the target. This results in higher velocities. Figure 2.39 shows the velocity spectra of $35,36 \mathrm{Cl}$ and $36,37 \mathrm{Ar}$ which are not likely to have contributions from an ${ }^{36}$ Ar compound nucleus (28si $+{ }_{88}^{8}$ ). ${ }^{34} \mathrm{Cl}$ on the other hand shows some contributions from this process as do lighter elements. In fig. 2.40 we see the evolution of the ${ }^{34} \mathrm{Cl}$ spectrum over the angular range of $2^{\circ}$ to $6^{\circ}$ as a shift from the velocity of incomplete fusion to that of products irom complete fusion.

1. University of Virginia, Charloctesville, VA 22901.

2. D. Shaptra et al., Phys. Rev. Lett. 53, 1634 (1984).

3. R. Bass, Nuclear Rea:tions Between Heavy lons, Springer vertag (1980).

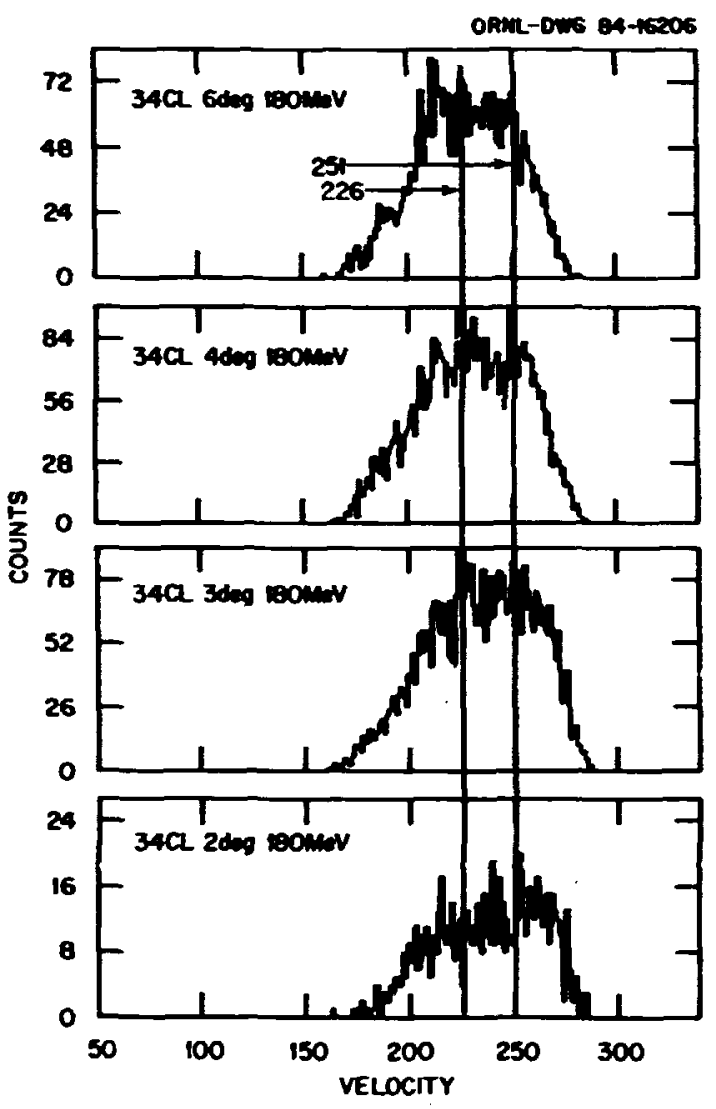

Fig 2.40. Velocity spectra for ${ }^{35} \mathrm{Cl}_{17}$ measured at four angles $\left(2^{\circ}, 3^{\circ}, 4^{\circ}, 6^{\circ}\right.$, and $\left.8^{\circ}\right)$.

4. S. Gary el al., Phys. Rev. C 25, 1877 (1982).

5. K. T. Lesko et al., Phys. Rev, C 25, 872 (1982).

6. A. Gavron, Phys, Rev. C 21, 230 (1980).

TWOFOLO ANO THREEFOLD COINCIDENCE MEASUREMENTS OF COMPLEX FRAETENT ENISSION IN $265 i+12 C$ COLLISIONS

$$
\begin{aligned}
& \text { D. Shapira R. Novotnyl } \\
& \text { S. T. Thornton }{ }^{2}
\end{aligned}
$$

$190 \mathrm{MeV}{ }^{28}{ }^{8} \mathrm{Si}$ ions from the Heidelberg MPI Tandem + Linac Accelerdtor Complex were :sed to bombard natural carbon targets. Complex fragments $(2>2)$ enitted in these colltstons were identified in an array of five large volume tontzation chambers (see Fig. 2.41). Each detector measures energy $E_{\text {p }}$ linear momentum p and the charge 2 of an entering fragnent.

This study is afmed at establishing the nature of the yield of recotling target-like nuclei. Inclusive energy spectra and angular distributions of these target-like recnils were 


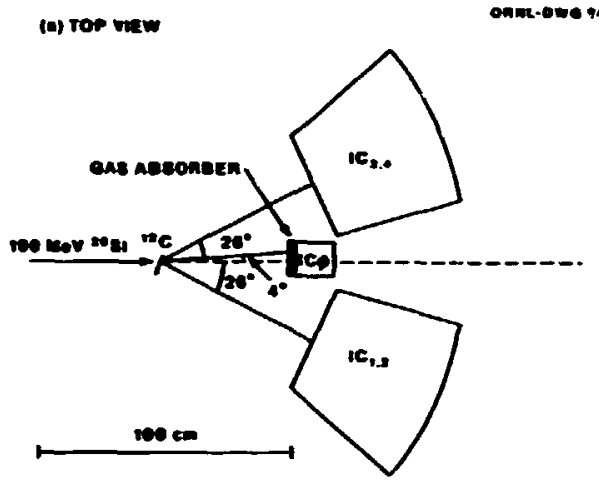

(D) nnowr vew

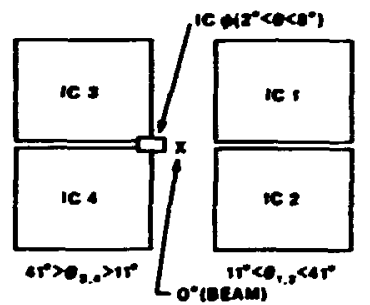

Fig. 2.4i. Experimental arrangement of the three detectors: (a) top view, (b) front view.

studied extensively, over a wide range of boonbarding energies in a series of measurements that confirmed the predominance of orbiting in collisions between light heavy nuclei. 3

In particular, the continuum spectra of recoiling carbon nuclei of the type shown in Fig. 2.42 could originate in two different processes:

$$
\begin{aligned}
& \text { (1) }{ }^{28} \mathrm{Si}+{ }^{22} \mathrm{C}+\left({ }^{28} \mathrm{Si}^{*}\right)+{ }^{2} \mathrm{C}^{*} \text {. } \\
& 2{ }^{4} \mathrm{Mg} *+{ }^{4} \mathrm{He}+12 \mathrm{C} \\
& \text { (2) }{ }^{28} \mathrm{Si}+12 \mathrm{C}+2{ }^{4} \mathrm{Mg}+\left(16 \mathrm{Og}^{*}\right)+ \\
& 2 \mathrm{Mg}^{*}+{ }^{4} \mathrm{He}+12 \mathrm{C}^{*}
\end{aligned}
$$

Twofold coincidence measurements were afmed at separating these two most ?ikely sources for carbon production and determining their relative contribution to the observed $12 \mathrm{C}$ yfeld. In both cases the most likely end products of this chain is a combination of ${ }^{4} \mathrm{He},{ }^{12} \mathrm{C}$, and $24 \mathrm{~kg}$ nuclei.

The experiment was arranged in such a way t.hat the ionization chamber placed at small angles had an absorber placed in front that served to keep any forward scattered beam particles and evaporation residues from reaching the active detector volume. ${ }^{3}$ This enabled us to do the experiment with 100 na of $2 \mathrm{Asi}\left(14^{+}\right)$but had the disadvantage inat particles with $Z>14$ could not reach the detector at all and only particles with $Z<10$ could be detected over a sufficiently large range of kinetic energtes.

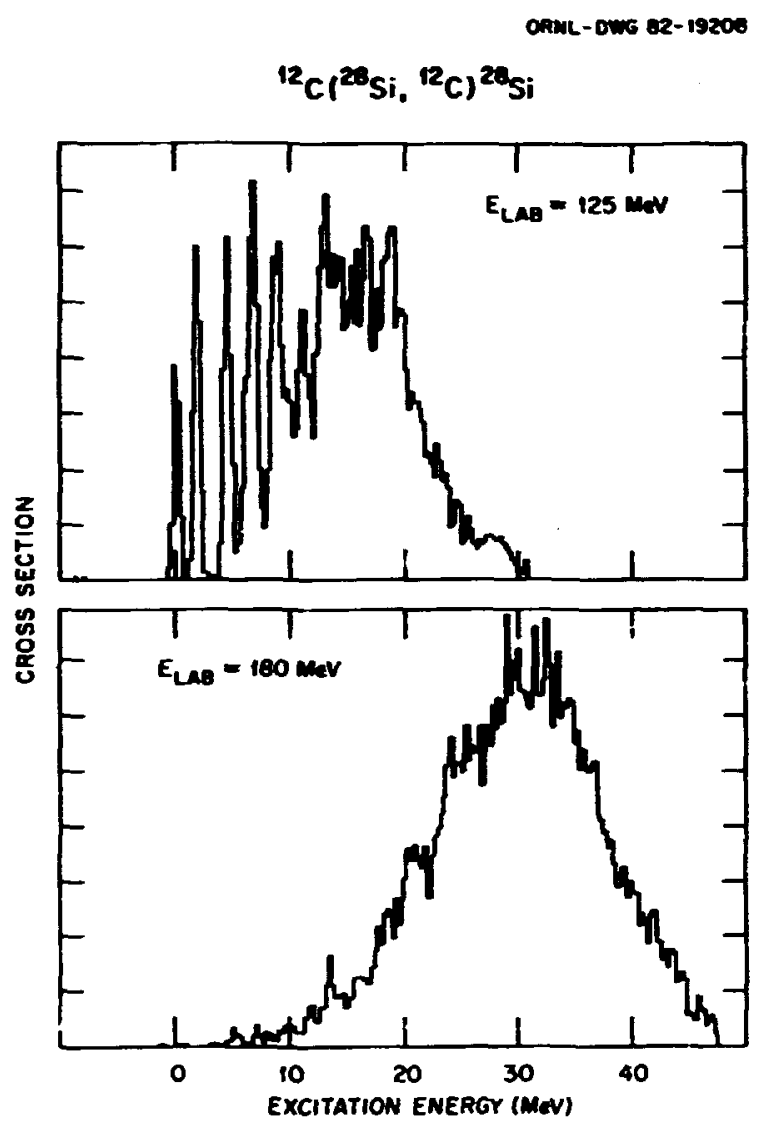

fig. 2.42. Energy spectrum of recoiling $12 \mathrm{C}$ nuclei from ${ }^{28} \mathrm{Si}+\mathrm{I}_{\mathrm{C}} \mathrm{C}$ induced reactions.

The bottom part of Fig. 2.43 shows the distribution of missing charge $\Delta Z=20-20-21,2$ for all two-body coincidences between ICO (ionization chamber at forward angles) and $I_{1} C_{1,2}$. Clearly the most dominant coincidence channel is that with $20+21,2=18$ (two units of charge missing). The top part of the same figure shows the distribution of all those events with $20+2:, 2=18$. The majority of these events have carbon and magnesium detected in coincidence. The low yield of ions with $20>9$ in ICO is due to the high energy threshold for the heavier nuclei caused oy the absorber placed in front of that counter.

Since also have the information on angle and energy of the detected nucleus, and assuning $A=2 \star Z$ also their approximate masses, we are able to calculate their momenta and from these deduce the totdl momentum of the missing particle(s). It is then possible to transform. event by event, the momentum of this missing particle to the center-of-mass system. The twodimensional distrioution of the momenta attributed to the missing particle(s) is show on Fig. 2.44. This distribution serves as a clear indication that the dominant process producing carbon fons is the first charn indicated since the higher density of "He momenta point in the direction of the recotiling $20 \mathrm{Si}$. Monte Carlo 


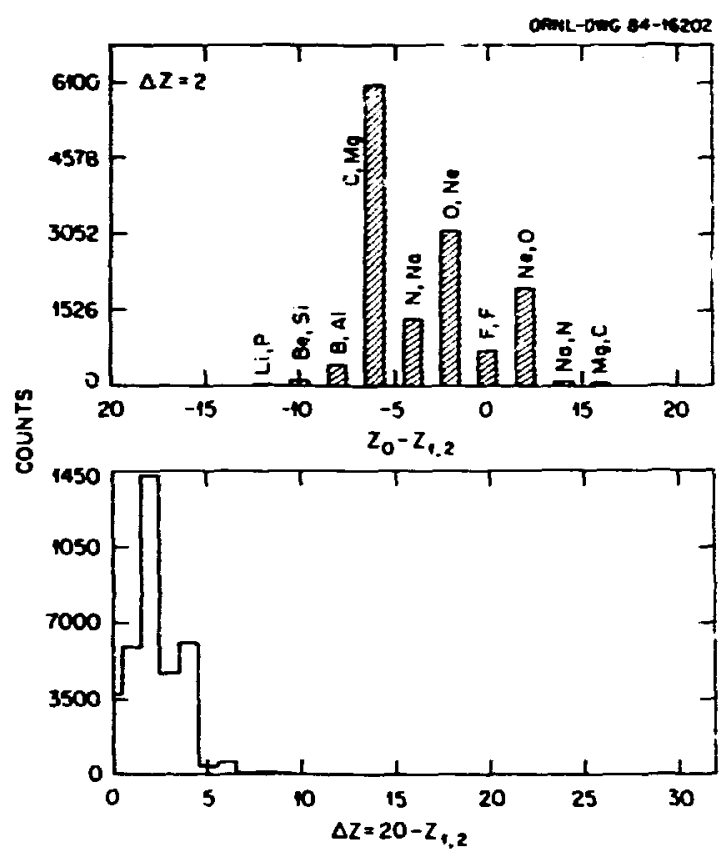

Fig. 2.43. Fracment abundance for two-body coincidences from ${ }^{28} \mathrm{Si}+{ }_{12 \mathrm{C}} \mathrm{colll}$ isions.

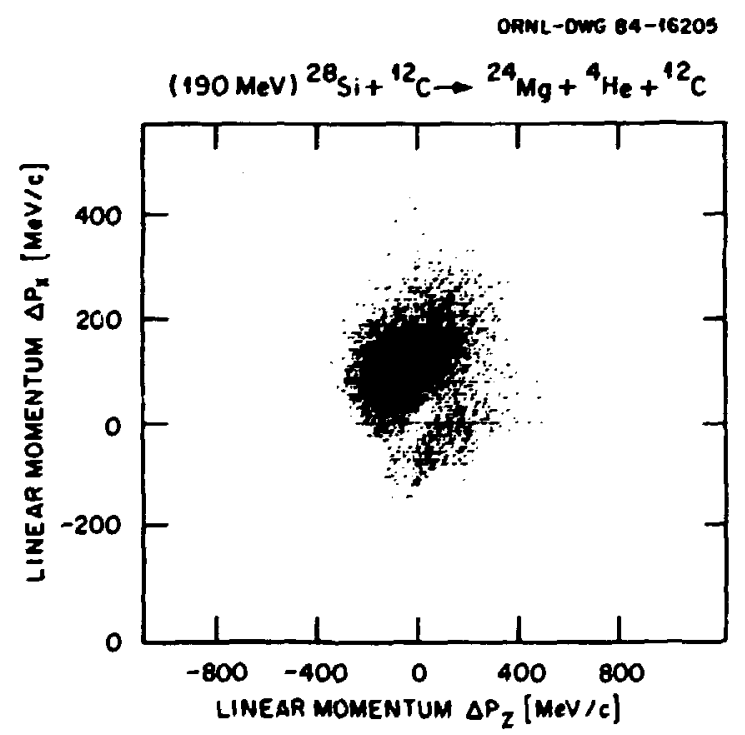

Fig. 2.44. Momentum of missing particle: components projected in the reaction plane.

simulation that take into account accual geometry and particle threshold will be employed in further ongoing quantitative analysis of these data.

1. II. Phystcal Institute, Universtty of Giessen, Giessen, West Germany.
2. Physics Departeent, University of Virginia, Charlottesville, VA 22901.

3. Phys. Lett. 1148, 111 (1982).

\section{MSS AND CHARGE FLOU OURIIS ORBITIMS OF LIGHT-KEAVY WUCLEI: STUDY OF THE 2ESI + IWI STSTEM}
D. Shapira
P. H. Stelson
J. Gomez del Campo
B. A. Harnon $^{3}$
J. L. C. Ford, Jr. 1
R. A. Parks ${ }^{3}$
B. Shivakunar ${ }^{2}$
S. T. Thernton

In a series of experiments performed at ORML and elsewhere it has been demonstrated that colliding light heavy nuclei are likely to form a long-lived rotating dinuclear systew which does not fuse. 4 The cross section for this process is tens of millibarns, a sutstantial magnitude for nuclear processes. "he fact that such a process requiree a precarious balance between attractive and repulsive forces in a nucleus-nucleus collision makes the existence and our understanding of this process important.

We focused on the study of backscattering of ${ }^{12} \mathrm{C}$ from ${ }^{28} \mathrm{Si}$. Very large cross sections for emission of B, C, $N$ and 0 isotopes to backward angles were measured. These particles emitted to the backward hemisphere mere concentrated mostly at large negative $Q$ value (corresponding to high excitation of the end fragments). The most probable kir.etic energy (the peak of the $Q$ value distribution) does not vary with angle of emission (completely relaxed), it does vary linearly, however, as a function of bombarding energy. It was aiso found that this particle yield at backward angle has a $1 / \sin \theta$ dependence in the center of mass system. These results indicate that the two colliding nuclef $\left({ }^{2} \mathrm{C}\right.$ and 285i) have formed a rotating dinuclear system. This complex subsequently decays with isotropic probability $d \sigma / d \theta=\sin \theta * d \sigma / d \Omega=$ const) and the kinetic energy of the enitted fragments equals the energy stored in the rotating complex and does not depend on the angle of enission in agreement with the observed behavior.

A question that comes immediately to mind is how long is long-lived? This question cannot be answered directly but it can be answered relative to certafn processes. For example the fsotropic angular distribution requires rotation of at least $180^{\circ}$ (that makes "long" about $10^{-21}$ sec!). We know that the dinuclear complex lives long enough for some mass and charge transfer to occur as well as complete equilibration of the energy degree of freedom, f.e.. complete damping of the kinetic energy in the entrance channel has occurred and most of the excitation for a given exit channel is in the heavy fragment" (equal temperature). One could next ask the question: "Since we do have mass and charge flow, was there enough $t$ tme for these degrees of freedom to equilfbrate?" Figure 2.45 points nut the inadequacy of the ${ }^{12 C}+285 i$ system for studying this question. The $12 \mathrm{C}+28 \mathrm{St}$ channel is bound to dominate under any assumption since this is the entraice channel as well as

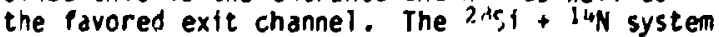




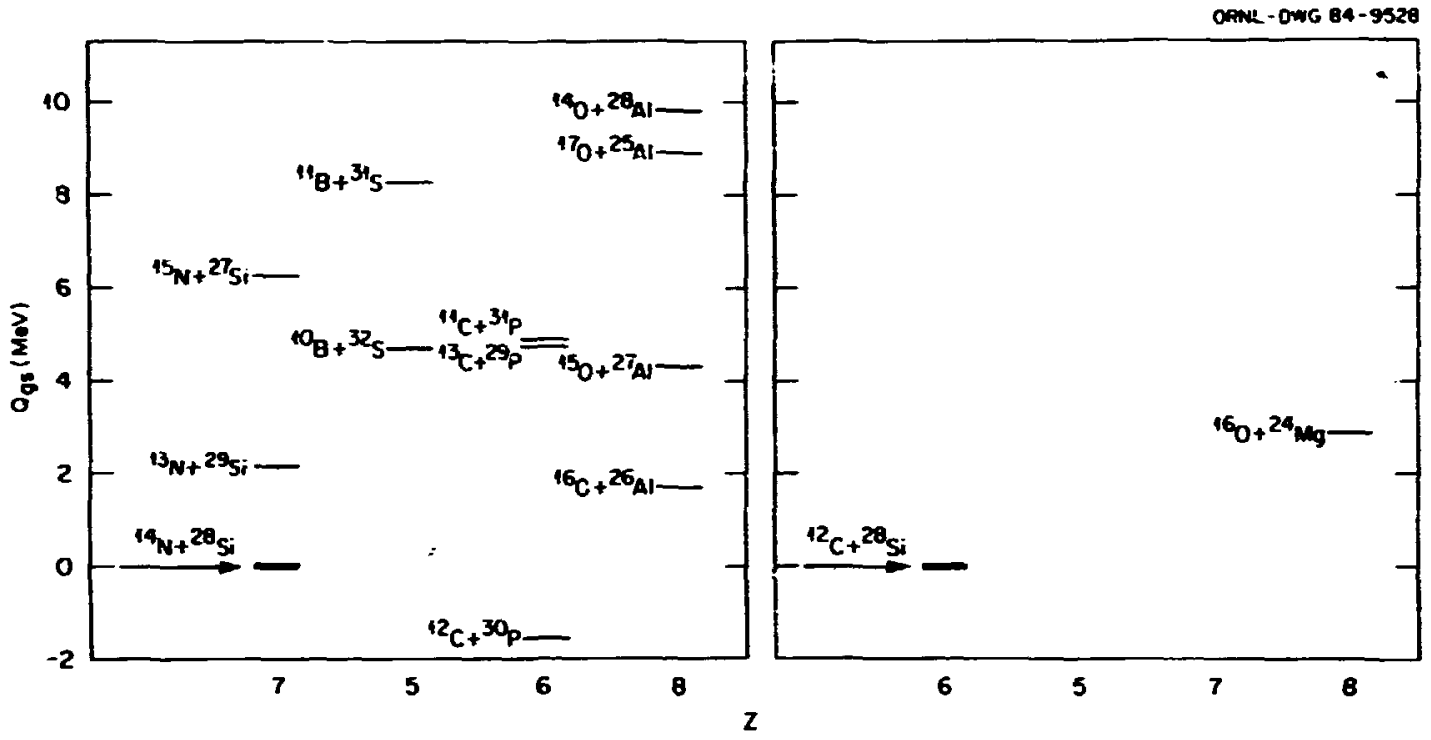

Fig. 2.45. Shifted energy level diagrams of different channels (partitions) of the ${ }^{12} \mathrm{C}+{ }^{28} \mathrm{Si}$ (right side) and $14 N+{ }^{28}$ si systems.

show on the left side of Fig. 2.45 is much better suited for answering this question. While the ${ }^{28} \mathrm{Si}+14 \mathrm{~N}$ exit channel would be favored with a fast process, the $12 \mathrm{C}+{ }^{30 \mathrm{p}}$ channel would dominate if equilibrium emission took place. In order to bypass some of the difficulties enccuntered in backscattering experiments, all the measurements discussed here were done at forward angles, reversing the role of target and projectile. $12 \mathrm{C}$ (or $14 \mathrm{~N}$ in the present experiment) were bombarded with energetic ${ }^{28}$ si beams and a study of the target-like nuclei, recoiling forward, was carried out. Naturai nitrogen yas ( $99 \%$ 14W) was used in the HHIRF supersonic gas jet target 5 to produce a $15 \mathrm{mg} / \mathrm{cm}^{2}$ thick i4N target. This target was bombarded with highly collimated (1/4 $\left.\mathrm{mm}^{2}\right)$ $5 \mathrm{pnA} 150 \mathrm{MeV} 28 \mathrm{Si}$ beam. The products were momentum analyzed in the spectrograph and identiffed ( $\triangle E, E)$ in an ionization chamber. A twodimensional energy vs. energy-loss map showing all the detected target-like products is shown in Fig. 2.46. The three other maps display focal plane position (momentum) vs, energy and show the mass and charge state distribution of the products. The projected energy spectra for products with $Z=6,7$ and 8 are shown in Fig. 2.47. These spectra are clearly dominated hy $12 C$, 1 in and 160 (parts $b, C$ and $d$ of Fig. 2.46). Measurements at other angles have shown that the centroids of these entergy spectra are unaltered and, as Fig. 2.48 shows, the emission probcbility for these products $(d \sigma / d \theta)$ remains constant with angle. Therefore, these products come, in all kelihood, from the same orbiting process previously observed in ${ }^{28} \mathrm{Si}+{ }^{2} \mathrm{C}$ and other systems. 6 The new information from these dats is also obvious: a brief examination of the data in Figs. 2.46 and 2.47 shows that the dominant yield is in the $12 C+30 p$ channel and not in the ${ }^{14} N+{ }^{29} 5 i$ entrance-like channel. $150 \mathrm{mev} 205 \mathrm{~g}=5$

OAML- Dons e4-15079

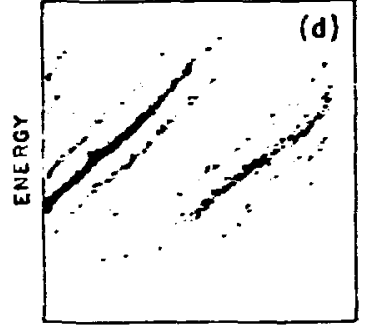

focal plane position

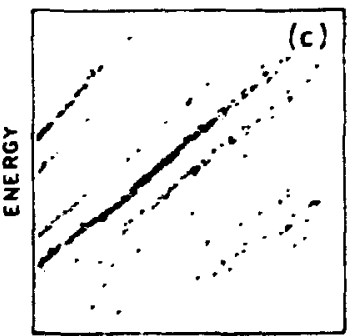

focal plane position

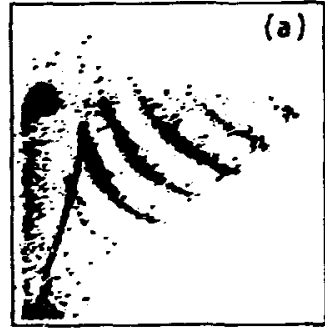

ENERGY LOSS

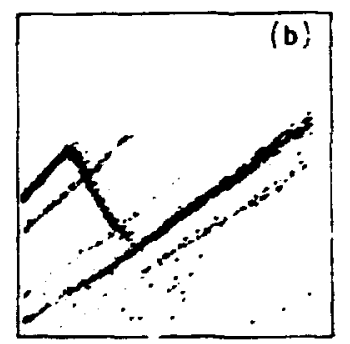

focal Plane position
Fig. 2.46. (a) $\Delta E$ vs, ? plot for target-like products reaching the counter: $(b, c, d)$ Momentum (focal plane position) vs, energy plots for $Z=8,7$ and 6 , respectively. These maps separate products according to charge state and mass.

The process therefore lasts long enough for energy, mass and charge flow to reach equilibrium. This still does not put an absolute time limit on the process but it is likely 
ORes- $-00084-1507 \theta$

$150 \mathrm{MeV} 2551+14 \mathrm{~N}$ $\theta=5^{\circ}$

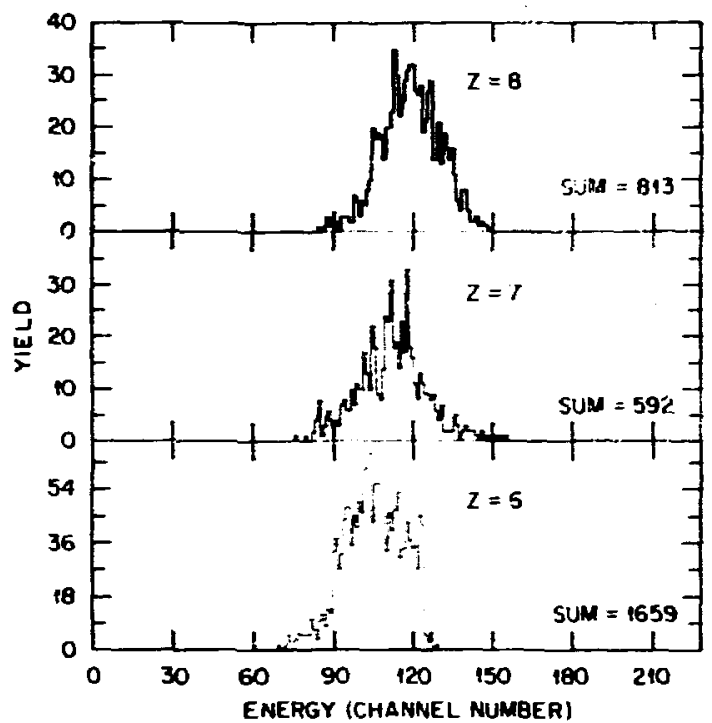

Fig. 2.47. Energy spectra for the carbon, nitrogen and oxygen ions seen in map (a) of Fig. 2.46.

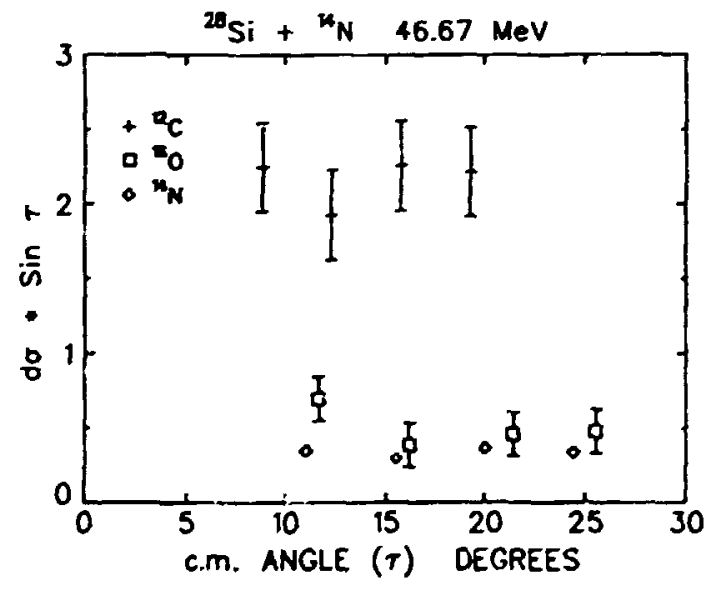

Fig. 2.48. Emission probability as a function of angle.

that more than the time needed for one rotation is involved. We are now actively engaged in calculations that assume equiliorium partitioning of the products and shall find out how these quantitative predictions compare with the observed data.

1. Deceased.

2. Permanent address: A. W. Wriyht Nuclear Structure Laboratory, Yale University. New Haven, CT 06520.
3. Physics Deparment, University of Virginia, Charlottesville, VA 22901.

4. D. Shapira et al ...Phys. Lett. 1148, 111 (1982).

5. D. Shapira et al., to be published in Nucl. Instrum. Methods.

6. D. Shapira at at to the published.

CRYSTAL BLOCXING MEASUREMERTS FOR THE IGO + Ge AND 2AST + Ge SYSTENS
J. jocaez del Canps
i. A. Biggerstaff
R. Ribas 1
C. D. Moak
D. Shapira
P. D. Miller

N.: Neskovic 2

The crystal tiocking technicue has been used successfully at tolftr in the study of reaction mechanisas for tie $160+12 \mathrm{C}$ reaction. ${ }^{3,4}$ In this report sew reasurements are described for the $160+G e$ and $285 i$ + Ge reactions, that extend the technique to heavier systems.

In order to successfully utilize the crystal blocking technique to measure nuclear reaction times it is crucial to establish a zero time calibration curve. This is usually done through a careful study of the elastic scattering crystal blocking angular distribution. It is also important to have predictions of the blocking distribution wich are based on theoretical calculations requiring a detailed knowledge of the properties of the crystal. All of the measurements on discussed here were done along the Ge $\mathrm{Cl10}$, axis, and the interaction potential used in the blocking distribution calculation was that of Doyle and Turner. 5 Thermal fluctuations re taken into account using an anplitude of 0.085 a (see review of Ref. 6). Corrections due to crystal thickness and eneray loss in the crystal were included in a manner similar to that used in the $160+12 C$ study. ${ }^{3}$ The thickness of the Ge crystal was 10 un.

The data for the elastic scattering blocking distribution of 160 on Ge 21102 at $: 00 \mathrm{MeV}$ bombarding energy are shown as crosses in Fig. $2.49(\mathrm{a})$. The detection angle was $22.5^{\circ}$ and the rest of the experimental setup was the same as for the :5n + ${ }^{2} \mathrm{C}$ study. ${ }^{3}$ Two theoretical curver :re shown in Fig. $2.49(a)$. The calculation show: : the iight curve $\left(\sigma=0^{\circ}\right)$ includes the effects of dechanneling and thermal fluctuations, wut nevertheless fails to reproduce the data. Since elastic scattering is a fast process ( $T<10^{-21} \mathrm{sec}$ ), the discrepancy between the light curve and the data can be interpreted only as effects due to crystal imperfections which are not included in the $\sigma=0^{\circ}$ curve. As ciscussed in Ref. 3, these effects can be evaluated by introducing a Gaussian smear (of

standard deviation o) to the theoretical distribution. good fit to the data on Fig. 2.49(a) is obtained with $=0.08^{\circ}$ (hedvy curve). In order to check the consistency of these procedures, another blocking experiment was performed using * 120-MeV I 0 (collimated to $2 \mathrm{~mm}$ ) from ORIC. The same Ge crystal was used and the results for the elastic scattering (circles) at $\theta$. $10^{n}$ are shown in Ftg. 2.49(b). Fxcellent 


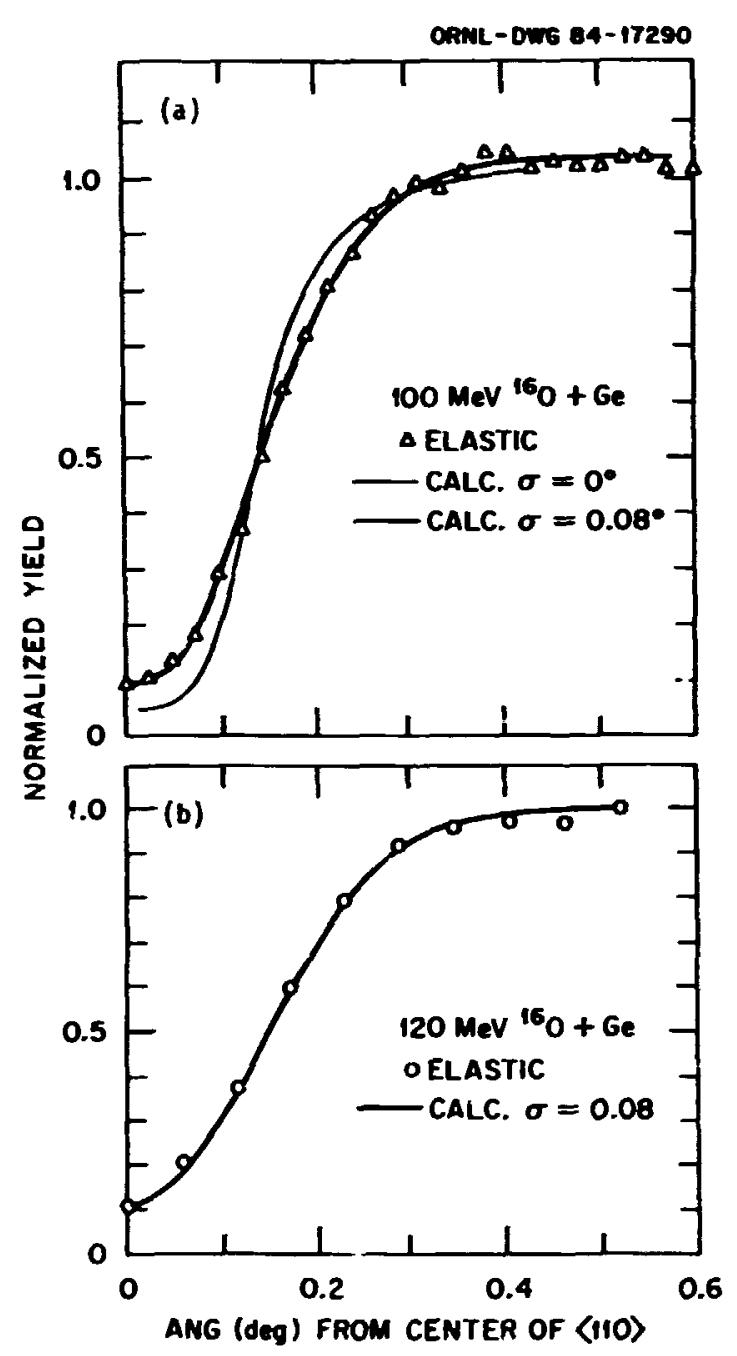

Fig. 2.49. (a) Blocking distribution for $100 \mathrm{meV} 160+\langle 110\rangle \mathrm{Ge}$. The effect of crystal imperfections is represented by $\sigma=0 . \mathrm{c}^{\circ}$. (b) Same as (a) for $120 \mathrm{MeV} 160$.

agreement with the data is obtained with a calculation using $\sigma=0.08^{\circ}$, the same value as for 100 MeV.

Products fram the $160+$ Ge reaction were detected for $Z=9$ to $Z=2$ using a $E-E$ position sensitive telescope. ${ }^{3}$ The analysis of the $Z=6$ to $Z=8$ reaction products is given el sentere" and confirms the ided that these nuclei are produced as the result of direct (fast) processes. However, detailed analysis of the enteted alpha particles shows significant time-delay effects. Figure 2.50(a) shows an energy spectrum of the alpha particles enitted at $22^{\circ}$ for the $120-$ MeV $160+$ Ge reaction. This spectrum was obtained with a solid state counter telescope $(E-2000 \mathrm{~m}, \mathrm{AE}=300 \mathrm{~m})$. The solito line, normalized to the data at the maximum, is the result of a complete fusion calculation from the computer code LILITA' and shows that nearly
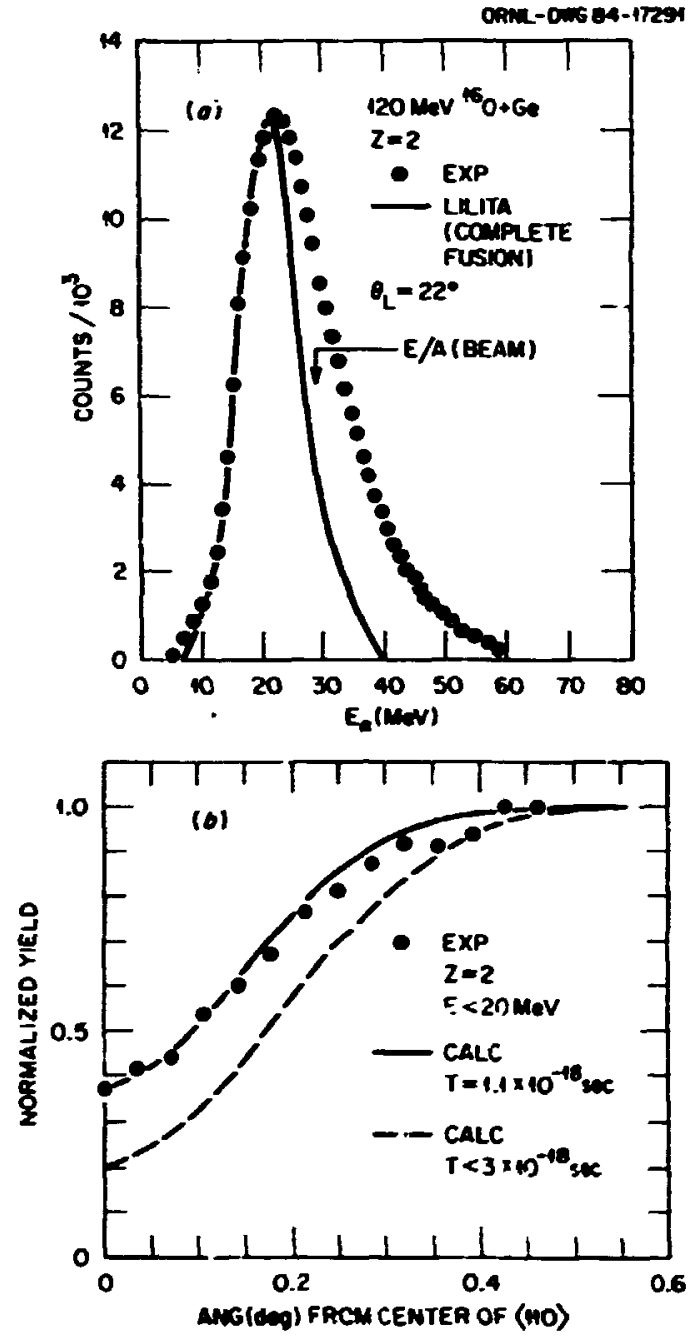

Fig. 2.50. (a) Energy spectru (points) for the = particles emitted in the 160 + Ge reaction. (b) Blocking angular distribution.

$50 \%$ of the alpha particles can be accounted for by a compound nucleus process. Figure $2.50(\mathrm{~b})$ shows the "blocking curve" for alpha particles below 20 Me'l enitted from the $160+$ Ge reaction. The 20 MeV energy linit assures that most of the alpha particles are from compound nucless eaission. Under the assumption of compound nucleus enission, the recoil velocities were calculated and the extracted decay time (for multiple particle exission) was $1.1 \times 10^{-17} \mathrm{sec}$ (solid line). The dashed curve in Fig. 2.50(b) is the lower linit for the tiae that can be meascred with the present Ge crystal for the compound emission process. The resule of $1.1 \times 10^{-17}$ sec agrees well with a Monte Carlo caiculation of multiparticle equilibrium emission wich yielos $2 \times 10^{-1} \mathrm{sec}$.

The 28 si + Ge reaction was usej to study de-excitation times for deep inelastic colli. sions (DIC). Usualiy for OIC reactions tha only 
reference to time is the reaction time (primary time) wich is the time needed for formation and separation of the dinuclear con. lex after large amounts of $p$ ergy dissipation and mass exchange have ociurred. Although considerable time delays are expected for DIC, most experimental and theoretical estimates predict values $T$ G $10^{-20} \mathrm{sec}^{8}$ too fast to be measured Jirectly. Nevertheless, the blocking technique will be sensitive to tha secondary ime, which reflects the time delay of the particle decay process (whether in equilibrium or not) of the primary reaction fragments. It is in this regard that the blocking measurement can provide unique information for the DIC process. Coincidence experiments between the enitted light particles and fragments have been carried out in the past t) study the equilibration effects of energy and mass transfer, but this is the first experiment in which a direct measurement of the secondary time is attempted.

The ${ }^{28} \mathrm{Si}+\mathrm{Ge}$ reaction was studied by bombarding the same Ge crystal used previously with a $28 \mathrm{Si}$ beam of $220 \mathrm{MeV}$ extracted from the HHIRF tandem. The detector was placed at an angle of $16.2^{\circ}$, and energy spectra and blocking anyular distributions were measured for nuclear reaction fragmerits of $Z=16$ to $Z=6$. The analysis of the erergy spectra show large e rgy aissipation $(Q=-, 0$ to $-100 \mathrm{MeV}$ ) which is -ypical for DIC reactions in this mass region. We also observe a small quasi-elastic component for $Z=12$ and $Z=13$ fragments. The resu's for the blocking angu? ar distributions are shown on Fig. 2.51 for some $u^{f}$ the DIC fragments. The

OAML-DW6 84-17292

BLOCKING DISTRIEUTIONS $220 \mathrm{MeV}{ }^{28} \mathrm{Si}+\mathrm{Ge}$

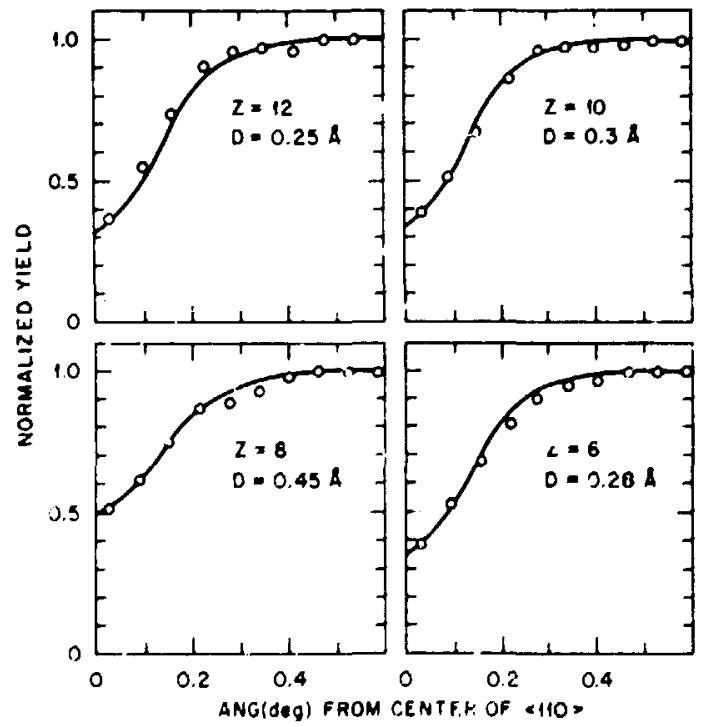

:ig. 2.51. Blocking angular distribution for biC fragmencs resulting from 220) $2 \mathrm{AS}_{\mathrm{S}}+\mathrm{Ge}$. The fie crystal is the same as for thre datd on figs. 1 and $?$. theoretical fits (solid iines) were calculated using the value $\sigma=0.08^{\circ}$ (see Fig. 2.49) and a value for the recoil distance $O(A)$ was indicated for each rase. The fact that $D \neq 0$ for all cases given in Fig. 2.51 indicates that sizable time-aclay effects are present in the DIC reaction. In ordor to extract the de-excitation time, $\left.\delta,\left(\delta=0 / v_{k} .{ }^{n} n\right]_{a b}\right)$ the recoil velocities $V_{R}$ have to be determined. There are two values of $V_{R}$ possible for a DIC reaction, depending on whether the time cielay responsible for the observed displacement, $D$, takes place during the collision (primary time) or is due to the decay of the fully accelerated fragments (secondary time). For the firit case $V_{R}=V_{r}, m$, and the extracted times will te $-10^{-17}$ sec, too long to be consistent with previous experimental observations. The second assumption, commonly used in the analysis of fragment-particle correlation data, is that the fragments decay after scission, in wich case the recuil velocities are those of the primary fragments (about $0.1 \mathrm{c}$ for the present experiment). These velocities have been determined by a Monte Carlo calculation in a way similar to that of Ref. 9, and the resulting $\delta$ values are given in Fig. $2.52(a)$. On Fig. $2.52(b)$ we show the differential cross sections (points) measured at $10^{\circ} .2^{\circ}$ and the result (histogram) of the Monte Carlo simuiation (the primary distribution is a Gaussian centered at $Z=14$ and $\sigma^{2}=21$. The main conclusion to

2ANL-0.0064--17233

220 MeV ${ }^{28} \mathrm{~S}_{\mathrm{S}} \cdot \mathrm{Ge}$

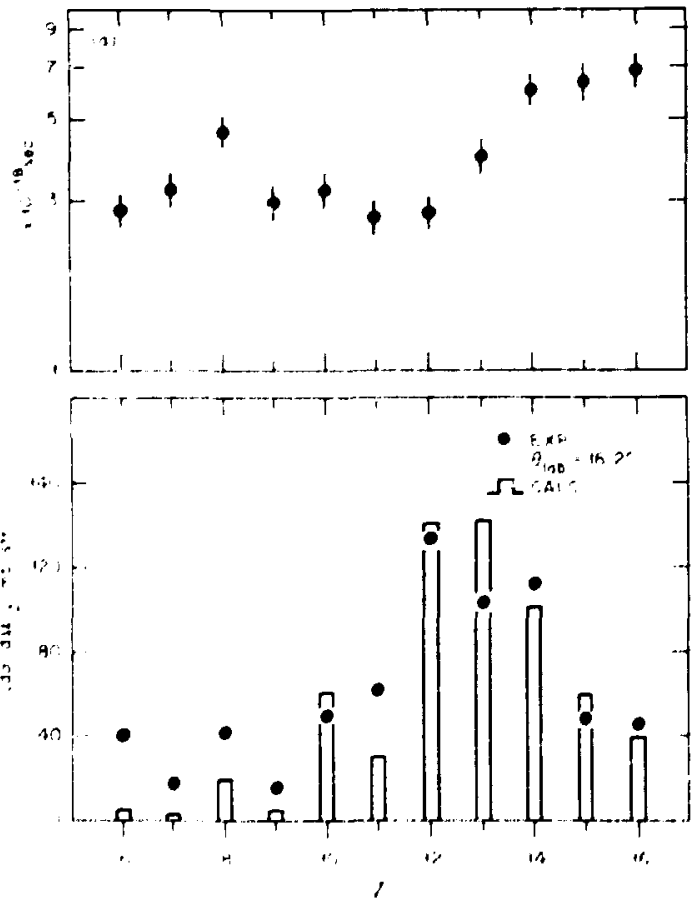

Fig. 2.52. (a) Results of the deexcitation time $\delta$ for DIC fragments. (b) Medsured and predicted cross sections for DIC of 2 Asi t ce. 
be drawn from Fig. 2.52 is that for all $Z$ fragments the time delay is large and this result can be interpreted only as due to a multiparticle evaporation process similar to the one observed for the evaporation residues of $160+12 \mathrm{C}$ (Ref. 3 ). Of course the data of Fig. 2.52 needs a good theoretical calculation that could explain both the $\delta z$ and $(d / d s) z$ distributions. Further analysis of these effects are in progress.

1. On leave from Instituto de Fisica University of Sao Paulo, Brazil.

2. On leave from Boris Kidric Institute, Belgrade, Yugosl avia.

3. J. Gomez del Campo et al., Phys. Rev. Lett. 51, 4J1 (1983).

4. J. Gomez del Campo et al.. Notas de fisica, Vol. 6, No. 1, 115 (1983). Proceedings of the 6th Oaxtepec Symposium on Nuclear Physics.

5. P. A. Doyle and P. S. Turner, Acta Crystal logr., Sec. 124, 390 (1968).

6. D. S. Gemmel l, Rev. Mod. Phys. 46, 129 (1974).

7. J. Gonez vel Campo and R. G. Stokstad, ORNL Report TM-7295 (1981).

8. R. A. Dayras et al., Phys. Rev. C 22, 1485 (1980)

9. J. Gomez dei Campo, Proc. Symposium on Heavy-Ion Physics, edited by J. Barrette and P. D. Bound, BNL-5!115, 93 (1979).

REMOVING SURFACE ABSORPTION FROH THE OPTICAL MODEL: CCA FITS TO 60NT + 6 NNI $^{2}$ ELASTIC NID INELASTIC DATA

\section{K. A. Erb M. J. Rhoades-Brown}

The imaginary component (W) of the optical notential is intended to account, in an average way, for the absorption of flux into channels that are not treated explfittly in a given model calculation. Conceptually, the short-range portion of $W$ correspends to compound nucleus formation, wille the longerrange pieces reflect reactions that are localized in the nuclear surface. It has long been hoped that in a sufficiently complete model calculation, wifch treated all ine important direct reaction channels explicitly, the need for optfcal model surface absorption could be eliminated. If such a situation could be realized, without sacrificing quantitative reproduction of the measured yields, a major source of ambiguity would be removed. Then, models for the real part of the optical potential for transition densities, and for direct r.actions in general, could be teste.. cleanly.

We have noted previously ${ }^{2}$ that our measurements of yields from boNf + hoNf and ${ }^{b O N i}+{ }^{b} \mathrm{be}_{\mathrm{F}}$ interactic s determined that the $2^{+}$- fnelastic cross sections nearly exhaust the total reaction cross sectton. He arqued? that the couplfi,g evidenced by these magn!tudes implies the existence of strong non-local effects which are difficult to mimic in standard onechannel oodels, and which thus could account for the failure of simle penetrability calculations to reproduce the $\mathrm{Mi}+\mathrm{Ni}$ fusion data. ${ }^{4}$ Rhoades-Brown and Braun-Munzinger subsejuently reported a coupled-criannel calculations that used a short-range imainary plus stanjard real potential, with explicit $2^{+}$and $3^{-}$inelastic coupling to achieve improved agreement witl. a portion of the fusion data. Such a calculation of fusion, although conceptually appealing, makes sense only if fits to elastic and inelastic yields can be achieved simultaneously.

We have recently succeeded in fitting our $60 \mathrm{Ni}+{ }^{60} \mathrm{Ni}$ elastic and inelastic data reasonably weil using an optical potential with $H=0$ in the surface region. The results are shown in Fig. 2.53. The coupled-channels progran, Ptoleay, was used, with measured $B(E 2)$ and $B(E 3)$ values and a standard real potential. The scattering calculation was insensitive to the details of $W$ in the interior region. This work raises the possibility of fitting quasielastic and fusion data simultaneously. NY.

I. Present address: SUNY at Stony Brook,

2. K. A. Erb, in Resonances in Heavy Ion Reactions (Springer-Verlag, 1982), p. 204.

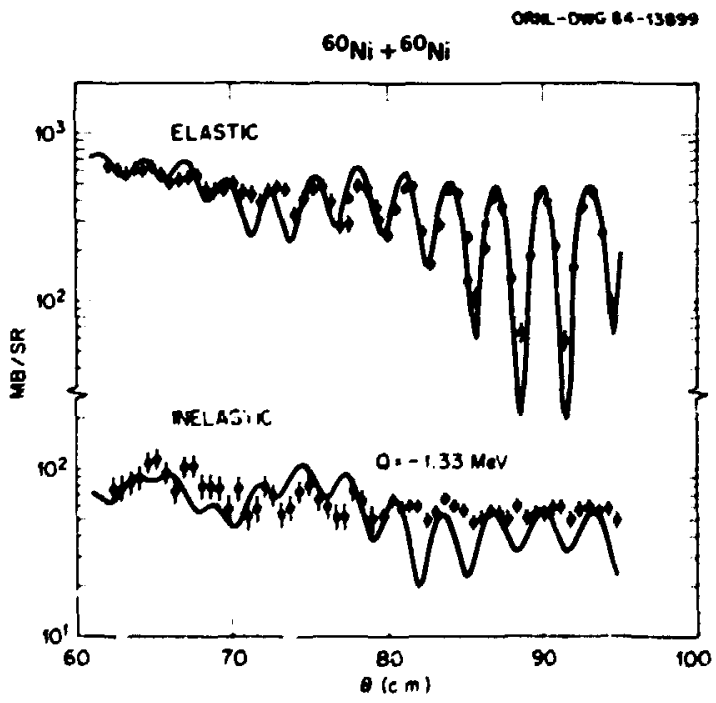

Fig. 2.53. Measured and calculated (CCA, Ptolemy) elastic and inelastic yields for nnNi + boNi collistone. of $E(1 a b)=228 \mathrm{meV}$. The lowest $2^{+}$and $3^{-}$levels of ho $\mathrm{Nf}$ were couplca with measured $B(E \lambda)$ values. The real potential was a Woods-Saxon with $V=73$ $\mathrm{MeV}, r_{0}=1.177 \mathrm{fm}, d=0.67 \mathrm{fm}$, while the imaninary potentfal was a Hoods-Saxon squared with parameters $W=10 \mathrm{MeV}, r_{0}^{\prime}=1.0 \mathrm{fm}$, $a^{\prime}=0.4 \mathrm{fm}$. 
3. Physics Division Progress Report for Period Ending September 30, 1983, ORNL-6004 (1983), P. 42.

4. M. Beckerman, et al., Phys. Rev. Lett. 45, 1472 (1980).

5. M. J. Rhoades-Brown and P. BraunMunzinger, Phys. Rev. Lett. 1368, 19 (1984).

\section{FUSION CROSS SECTIONS FOR BEANS OF $46,50 \mathrm{Ti}$ ON TARGETS $90 \mathrm{Zr}$ ND $93 \mathrm{~Kb}$}
P. H. Stelson
M. Beckerman:
H. J. Kim
U. Shapira

R. L. Robinson

The ${ }^{50} \mathrm{Ti}$ nucleus has a closed neutrun shell of 28 , and as a result, its first $2^{+}$state has an energy of $1.55 \mathrm{MeV}$ and $\beta_{2}=0.173$. On the other hand, ${ }^{46} \mathrm{Ti}$ (four neutron holes away from $n=2 B$ ) has a $2^{+}$state at $0.89 \mathrm{MeV}$ and $a \beta_{2}=$ 0.314 . Theoretical est imates 2,3 of subbarrier fusion cross sections based on the couplings to inelastic channels suggest a factor of 20 difference in subbarrier fusion cross sections then beams of ${ }^{46} \mathrm{Ti}$ and $50 \mathrm{Ti}$ are incident on targets such as ${ }^{90} \mathrm{Zr}$ or ${ }^{93} \mathrm{Nb}$. We used the velo:ity filter to measure these cross sections. The analysis of the data has not yet been completed. Preliminary analysis confirms the theoretical prediction. At energies well telow the barrier $\left(E_{B}-E_{C M}=12 \mathrm{MeV}\right)$, the ratio of the fusion cross sections, ${ }^{46} \mathrm{Ti}+{ }^{93} \mathrm{Nb} / 50 \mathrm{Ti}+93 \mathrm{Nb}$, is approximately a factor of 30 .

1. Guest scient ist from Massachusetts Institute of Technology, Cambridge, Massachusetts.

2. C. H. Dasso, S. Landune, and A. Winther, Nucl. Phys. A405, 837 (1983).

3. R. A. Broglia et al.. Phys. Lett. 1338, 34 (1983).

\section{IMELASTIC SCATTERING OF 28ST OM 208pb}

$$
\begin{array}{ll}
\text { D. C. Hensley F. E. Bertrand } \\
\text { J. R. Beene }
\end{array}
$$$$
\text { G. Vourvopoulos! }
$$

Heavy ion inelastic scattering has been shown to be a useful tool in providing collective nuclear structure properties. 2,3 Analyses using the coupled-channel rotational model formalism can provide information on deformation parameters $B_{2}$ and $B_{4}$, static quadripole moments of $2^{+}$ states, triaxial shape parameters, and the relative phases of matrix elements.

Previous heavy ion studies based their results on the analysis of elastic scattering data and data for the excitation of the first $2^{+}$ collective state. It has been shown in the scattering of $20 \mathrm{Ne}^{2}$ and $28 \mathrm{Si}, 4$ however, that the $2^{+}$angular distribution in the region of the grazing angle is very sensit, ve to the addition of $B_{4}$ deformation. Furthermore, for the case of
$285 i$ it was found that a range of values for the hexadecapole deformation parameter $B_{4}$ gave excellent fits to both the elastic and $2^{+}$inelastic anguiar distributions. From an experimental point of vicw, due to the existence of overlapping groups of states in the vicinity of the excitation of the $4^{+}$state, it has not been possible to extract reliable values for the $4^{+}$ cross section for the cases studied.

During a short run with the HIRF Tanden in July 1984, we were able to demonstrate that this difficulty can be overcome if the Spin Spectrometer is operated in coincidence with position-sensitive charged particle detectors. In studying the reaction $208 \mathrm{pb}\left({ }^{28} \mathrm{Si}, 28 \mathrm{Si}^{ \pm}\right)$, we identif'ed the $26{ }^{\circ} i 4^{+}$state by its gama-ray cascade $4^{+}+2^{+}+0^{+}$. At the same time, the gana-ray angular correlations for both the $2^{+}$ and $4^{+}$states were measured. Preliminary analysis of the data indicates that we will be able to map out the angular distribution for the $4^{+}$ state within one or two shifts of beam time without requiring excessive rates in either the Spin Spectrometer detectors or in the position sensitive detectors. Because several reaction channels have larger cross sections than does the inelastic scattering to the $4^{+}$state, we found that charged particle resolution on the order of $0.5 \mathrm{MeV}$, in addition to the resolutio. of the Spin Spectrometer, will be required to separate unambiguously the various reactions.

1. Hestern Kentucky University, Bowling Green, KY 42101 .

2. E. E. Gross et al., Phys Rev. C 17, 1665 (1978).

3. E. E. Gross et al., Nucl. Phys. 1401, 362 (1983).

4. J. J. Kolata et al., Phys. Rev. C 30, 125 (1984).

\section{MASSES OF ${ }^{77} \mathrm{Kr}$ AND ${ }^{75} \mathrm{Kr}$}

$$
\begin{array}{ll}
\text { D. M. Moltz' } & \text { R. E. Tribble } \\
\text { K. S. Toth } & \text { C. A. Gagliardi? } \\
\text { J. P. Sullivan? } & \text { F. T. Avignone, III }
\end{array}
$$

Direct mass measurements ${ }^{3}$ have recently been made for most of the known rubidium fsotopes. To supplement and complement these data, we have been involved in a systematic program to determine masses of extremely neutron-defictent rubidium and kryoton nuclides via $\beta$-endpoint measurements ${ }^{4}$ and transfer reactions ${ }^{5}$ induced by 1 ight tons. Herefn we report our latest work, f.e. the determination of the ${ }^{77} \mathrm{Kr}$ and $75 \mathrm{Kr}$ masses by medns of the $80 \mathrm{Kr}\left({ }^{3} \mathrm{He},{ }^{6} \mathrm{Ke}\right)$ and ${ }^{7}{ }^{3} \mathrm{Kr}\left({ }^{3} \mathrm{He},{ }^{6} \mathrm{He}\right)$ react ions, respectively. Helfum-3 particles from the Texas asm Uisiversity $224 \mathrm{-cm}$ cyclctron were allowed to impinge upon an tsotopically enriched gas target statically pressurized in a cell to $\sim 50$ Torr. React fon products were detected at the focal plane of an Enge spltt-pole spectrograph with a single-wine proportional $\triangle E$ counter and a $1.0 \mathrm{~cm}$ $\times 5.0 \mathrm{~cm} \times 600 \mathrm{um}$ siliticon surface barrter $E$ 
detector. Particle time-of-flight information was also obtained relative to the cyclotron if signal.

A 70-MeV bombarding energy and a scattering angle of $O_{f}=7.25^{\circ}$ were chosen by comparison with prior ${ }^{6}{ }^{3} \mathrm{He},{ }^{6} \mathrm{He}$ ) studies. The spectrograph was calibrated by ${ }^{6} \mathrm{He}^{2+}$ particles from the ${ }^{18} \mathrm{O}\left({ }^{3} \mathrm{He},{ }^{6} \mathrm{He}\right){ }^{150}$ reaction. The existence of two prominent 150 excited states at approximately the same $Q$-value as predicted for the ground state of ${ }^{75} \mathrm{Kr}$ made this an ideal calibrant. After calibration, successive enriched isotope samples of $82 \mathrm{Kr},{ }^{80} \mathrm{Kr}$, and ${ }^{78} \mathrm{Kr}$ were introduced into the gas cell and bombarded by $21.8,30.0$, and $34.0 \mathrm{mC}$ of ${ }^{3} \mathrm{He}^{2+}$ beam, respect ively. The ${ }^{82} \mathrm{Kr}\left({ }^{3} \mathrm{He},{ }^{6} \mathrm{He}\right)$ reaction was $\mathrm{v}^{-e d}$ to masure the mass excess of ${ }^{79} \mathrm{Xr}$, a mucleus wose known to $8.6 \mathrm{keV}$, to demonstrate the validity of botr our experimental technique and data analys is procedure.

Table 2.11 sumarizes our mass excesses and compares then with values found in the compilation of Wapstra and Bos. 7 One sees that the two values for ${ }^{79} \mathrm{Kr}$ are in excellent agreement. The ${ }^{75} \mathrm{Kr}$ mass was previously unknown (the literature value given in Table 2.11 is deduced from systematics). There is a discrepancy between our " $\mathrm{Mr}$ mass and the adopted number based" on the precise ${ }^{1} \mathrm{Br}$ mass and the $B$-decay energy of ${ }^{77} \mathrm{Kr}(\operatorname{Ref}, 8)$. It would appear that the B-decay scheme of ${ }^{77} \mathrm{Kr}$ is not well understond.

We are also in the process of completing the analysis of the ${ }^{75} \mathrm{Rb}$ positron endpoint spectrum. with the newly determined ${ }^{75} \mathrm{Kr}$ mass this endpoint will provide us with the 75 Rb mass. Overali then, our investigation will have yielded masses for $75,76,77_{\mathrm{Rb}}$ and $, ., 75,76,77_{\mathrm{Kr}}$. We plan to compare these measurements with mass formula predictions. SC.

1. Iniversity of South Carolind, Columbia, TX.

2. Texas AsM University, College Station,

3. M. Epherre et al., Phys. Rev. C19, 1504 (1979); G. Audi et al., Nucl. Ph.s, A378, 443 (1932).

4. D. M. Moltz, K. S. Toth, F. T. Avignone, 111, H. Nama, 8. G. Ritchte and 8. 0. Kern, Phys. Lett. 1138, 16 (1982).
5. D. M. Moltz, K. S. Toth, R. E. Tribble, R. E. Neese and J. P. Sullivan, Phys. Rev. C26, 1914 (i382).

6. R. C. Pardo et al., Phys. Rev. C18, 1249 (1978).

7. A. H. Mapstra and K. Bos, At. Data Mucl. Data Tables 19175 (1977).

8. S. Thulin, Ark. Fysik 9, 137 (1955).

\section{DELAYED PROTON DECAY OF 1450y MD OF THE IEN ISOTOPE $151 \mathrm{Vb}$}
K. S. Toth
D. M. Moltz ${ }^{2}$
F. T. Avignone ${ }^{1}$
R. S. Moorel

We recently completed a study ${ }^{3}$ of the $B$ delaye 1 proton spectra of $147 \mathrm{Dy}$ and the hitherto unknow isotope ${ }^{149} \mathrm{Er}$. The purpose was twofold: (1) to provide decay energy data for comparison with the predictions of available ass formlae, and, (2) to identify $149 \mathrm{Er}$ so that its B-decay properties cuuld be studied in detail to obtain information conceming the single-neutron and single-proton levels in ${ }^{149} \mathrm{Er}(N=81)$ and $149 \mathrm{Ho}$ $(N=82)$, respectively.

The investigation has now been expanded to include a search for the delayed-proton branch of $1450 y$ and an attempt to identify $151 \mathrm{Yb}$ the next $N=81$, ever -2 , isotone above $149 \mathrm{Er}$. As before, thin ta. gets were bombarded with heavy ions accelerated in the Lawrence Berkeley Laboratory 88-inch cyclotron. A heliur: gas-jet apparatiss was used to transport radioactive products to a shielded area and assayed with a Si-particle telescope and a Ge detector. The telescope, consisting of a 20-um $\triangle E$ detector combined with a 300-um E detector, was necesary for the selective detection of low-energy protons in the presence of intense $a$ radiation and a profusion of a particles emitted in the decay of nearby nuclides. The Ge detector was of the $\gamma-x$ variety, suitable for detecting both lowand high-energy photons. Events registered in each detector were tagged with a time signal for half-life information.

In a series of $12 \mathrm{C}+142 \mathrm{Nd}$ bombardments (from 135 to $195 \mathrm{MeV}$ ) we looked for delayed protons from the $B$ decay of $145 \mathrm{Dy}$, an isotope mose half-life has been reported to be $18 \pm 3 \mathrm{sec}$ (Ref. 4) and $13.6 \pm 1.0 \mathrm{sec}$ (Ref. 5). Yields

Table 2.11. Summary of ma. measurements (all values are in MeV)

\begin{tabular}{lllr}
\hline & $7{ }^{9} \mathrm{Kr}$ & $77 \mathrm{Kr}$ & $75 \mathrm{Kr}$ \\
\hline Reaction 0-value & & & -12.970 \\
Mass Excess & -8.977 & -10.646 & $-64.231(16)$ \\
Literature (Ref. 7) & $-74.441(31)$ & $-70.155(25)$ & $-70.231(30)$ \\
\hline
\end{tabular}


were measured for $y$ rays belonging to $146 \mathrm{Dy}$, $146 \mathrm{~Tb}, 145 \mathrm{Dy}, 145 \mathrm{~Tb}$, and $144 \mathrm{~Tb}$. An energy of - $188 \mathrm{MeV}$ was then selected to emphasize the $1{ }^{2}$ Nd $\left({ }^{12} \mathrm{C}, 9 n\right)$ reaction. Figure 2.54 shows the accumulated proton spectrum. We assign these B-delayed protons to 145Dy on the basis of excitation function data and on the fact that they decayed with a $(15 \pm 4)$-sec half-life. High-energy endpoints of delayed-proton spectra are fixed by differences between the electroncapture (EC) decay energies of the parents and the proton binding energies in the corresponding EC daughters. Tine predicted $Q_{E C}-B_{p}$ value from the 1971 Atonic Mass Evaluation for $1450 y$ is $5.70 \mathrm{MeV}$. (Note the EC decay energy for $145 \mathrm{Dy}$ is not given in Ref. 6; based on decay energies for nearby isotopes we estimated it to be 7.24 MeV.) This prediction is consistent with our data (Fig. 2.54).

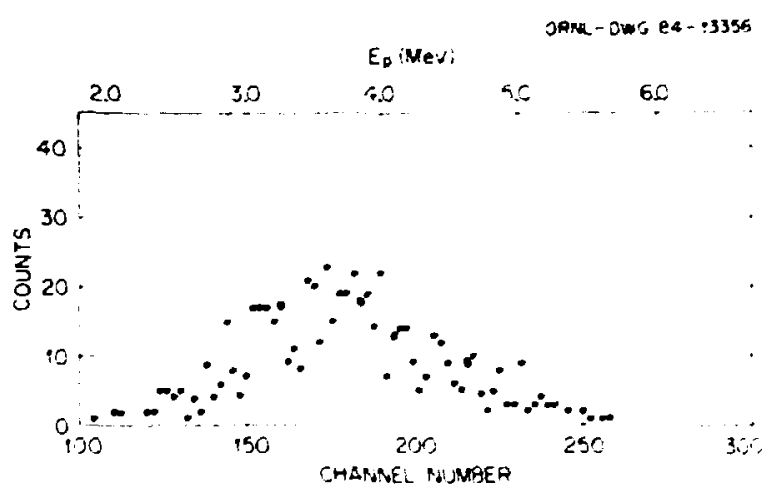

Fig. 2.54. Delayed-proton spect rum observed in 188-MeV $12 \mathrm{C}$ bombardments of $142 \mathrm{Nd}$; it is assigned to the $B$-decay of ${ }^{165} \mathrm{Dy}$.

In the attempt to identify $151 \mathrm{yb}$, proton and $r$-ray spectra were investigated in $160+1445 \mathrm{~m}$ irradiations. Yields as a function of incident energy for $y$ rays known to follow the $B$ decays of $152 \mathrm{Yb}$, $152 \mathrm{Tm}$, and $156 \mathrm{Tm}$ were used to select the peak of the $(160,9 n)$ excitation function. Prelfminary andyses indicate the existence of a delayed-proton emitter with a half-life of $\sim 0.5 \mathrm{sec}$ wich we tentatively assign to the new isotope $151 \mathrm{Yb}$. SC.

1. University of South Carolina, Columbia, CA.

2. Lawrence Berkeley Lahoratory, Berkeley,

3. K. S. Toth et al., Phys. Rev. C30, 712 (1984).

4. G. D. Alkhazor et al., Z. Phys. A305, 185 (1982).

5. E. Nolte et al,, Z. Phys, A306, 223 (1982).

6. A. H. Hapstra and K. Bos, At. Data Nucl. Data Tables 19. 175 (1977).
SIMGLE-MEUTRON ND SIMGLE-PROTON STATES IN $149 \mathrm{Er}$ NWO $149 \mathrm{Ho}$
Y. A. Ellis-Akovali

R. S. Moore!
K. S. Toth
D. M. Moltz

R. L. Mlekodajz

D. C. Sousa'
The investigation of levels in nuclei with single particles (or holes) plus closed-shell configurations is of considerable interest since it provides data for comparison with calculations based on Hartree-Fock and independentparticle models. These models have sets of phenomenological parametr is which can be improved by being fit to experimental results. The goal is to arrive at a universal description of single-particle properties throughout the periodic table.

He have been involved in studying decay properties of short-lived, proton-rich isotopes near the 82-neutron shell to obtain structure information for even- $Z$ nuclei with $N=81$ and $N=83$, and, for add $-Z$ muclei with $N=82$. Of relevance to the present investigation was our study of $1 \rightarrow 70 y^{\text {III }}$ and $1+70 y 9$ decays wherein we identified the $s_{1 / 2}, d_{3} / 2$, and $h_{11 / 2}$ nectron states in ${ }^{147} \mathrm{Dy}^{4}$ and, the $s_{1 / 2}, d_{3 / 2}, d_{5 / 2}$, and $97 / 2$ proton states in $147 \mathrm{~Tb}^{5}$ The immediate interest was to extend these single-particle level systematics to ${ }^{149} \mathrm{Er}$ and $14{ }^{14} \mathrm{Ho}$ and a search was begun for the unknown muclide ${ }^{149} \mathrm{El}$. In a series of $12 \mathrm{C}+{ }^{14+} \mathrm{Sm}$ bombardments we ident ified ${ }^{6} 149 \mathrm{Er}$ via its a-delayed-proton activity to be a $(9 \pm 1)-s e c$ isotope. With this half-Iffe information in hand we now have investigated its a-decay properties.

As in the earlier study, ${ }^{5} 149 \mathrm{Er}$ was produced in the $144 \mathrm{gm}(12 \mathrm{C}, 7 \mathrm{n})$ reaction. Irradiations were made with $135-\mathrm{MeV} 12 \mathrm{C}$ ions from the Holifield Heavy Ion Research Facility tandem accelerator. The target was a $2.1-\mathrm{mg} / \mathrm{cm}^{2}$ thick samarium metal foil enriched in $144 \mathrm{Sm}$ to $96.5 \%$. A helium gas-jet apparatus thermalized products recoiling out of the target and transported them to a counting station for assay with $r$ - and $x$-ray detectors. Singles and coincidence data were laken simultaneously.

Transitions were assinned to ${ }^{169} \mathrm{Er}$ decay on the basis of measured half-lives, $y$-ray coincidence relationships, and energies of $x x$ rays observed in coincidence with the $r$ rays. The $14^{9} \mathrm{Erm}$ and $1.3 \mathrm{Erg}$ decay schemes were found to be simflar to those of $1.70 y^{m}$ and $1470 y 9$ (see Refs. 4 and 5). A cascade of three transicions (111.2, 343.9, and $435.9 \mathrm{keV}$ ) is assigned to follow 1 " $\mathrm{Er}$ a decay; they depopulate and connect the following single-proton states in $14{ }^{3} \mathrm{Ho}_{\mathrm{O}} 37 / 2 \rightarrow d_{5 / 2} \cdot d_{1 / 2} \cdot s_{1 / 2}$. Two $\mathrm{Y}$ rays (111.0 and $630.5 \mathrm{keV}$ ) in coincidence with one another are ascribed to $1{ }^{1}{ }^{3} \mathrm{Er}^{\mathrm{m}}$; this isomeric deexcitation connects the $h_{11} /, d_{1} / 2$, and $s_{1} /$; single-neutron levels in $1109 \mathrm{E}$.

The order of the single-proton states in 1.7Th and in $11{ }^{1} \mathrm{Ho}$ is very different from those in odd $-Z N=82$ nuclef with $Z$ \& 63 where the $97 / 2$ and $\pi_{1} / 2$ orbitals are below the $5: / 2, \pi_{1} / 2$ and $h_{1} / / 2$ states. One must remember, however, that the $77 / 2$ orbital is a hole state for $Z, 59$ and 
that the $d_{5} / 2$ orbita? becomes a hole state in $147 \mathrm{~Tb}$. With this in mind, we show in Fig. 2.55 the proton level systematics for $N=82$ isotones. Hole states are indicated as having negative energies with the ${ }^{147} \mathrm{~Tb}$ and $149 \mathrm{HO}_{\mathrm{O}} \mathrm{s}_{1 / 2}$ orbitals shown as being close to the $h_{11 / 2}$ ground states. One then sees that the ${ }^{147} \mathrm{~Tb}$ and ${ }^{149} \mathrm{Ho}$ levels are not discontinuous with respect to the energies of the same orbitals in the lower- $Z$ isotones. We noted in Ref. 5 that the two hole states appeared to be wore tightiy bound in $147 \mathrm{~Tb}$. How that the investigation has been extended to $149 \mathrm{Ho}$, the extra binding in $147 \mathrm{~Tb}$ does seen to exist due to the influence of the $Z=64$ subshell on the quasiparticle energies.

Excitation energies of the $h_{11 / 2}, d_{3} / 2$, and $s_{1 / 2}$ neutron states in $H=81$ isotones are shown in Fig. 2.56. One sees that the $149 \mathrm{Er}$ level data fit the systematics for the muclei with lower atomic numbers. Overall our results for $1{ }^{47} \mathrm{Oy}$ and ${ }^{149} \mathrm{Er}$ clearly establish the trend predicted by Silverberg. ${ }^{7}$ His calculations showed that the $h_{11 / 2}-d_{3 / 2}$ splitting with increasing $Z$ would first increase, reach a maximum at around ${ }^{139} \mathrm{Ce}$, and then begin to decrease. Included in Fig. 2.56 are the $M 4$ transition rates, expressed in Heisskopf units, where the isomeric decay branchings art known. He uti?fized the constancy of these transition rates for $Z>58$ (also predicted in Ref. $T$ ) and estimated the isomeric branch for $149 \mathrm{Er}$ to be 2.7\%. Hote that while $h_{1 !} /$. Tevel in 131 Sn has been located $^{A}$ the $M 4$ transition has not been observed. By using an extrapolated Heisskopf factor of 7.5 units we calculated the isomeric decay branch to be $0.057 \%$, or only $0.0087 \%$ for the unobserved 241.8-keV photon.

He plan to extend this investigation to levels in $151 \mathrm{Yb}$ and $15.1 \mathrm{Tm}$. Preliminary data9 from delayed proton studies indicate that $151 \mathrm{Yb}$ has a half-life of $=0.5 \mathrm{sec}$.
SC.

i. University of South Carolina, Columbia,

2. UNISOR, Oak Ridge, Tennessee.

3. Eastern Kentucky University, Richnond, KY.

4. K. S. Toth, et ai.., Phys. Letters 568, 29 (1975)

5. K. S. Toth, et al., Phys. Rev. C25, 667 (1982).

(1984)

K. S. Toth, et al., Phys. Rev. C30, 112

7. L. Silverberg, Mucl. Phys. 60, 483 (1964).

8. B. Fogelberg and J. Bloaquist, Phys.

Lett. 1378, 20 (1984).

S. K. S. Toth, et al., contribution submitted to the 7th International Conference on Atomic Masses and Fundamental Constants. Darmstadt, Mest Germany, September 3-7, 1984; to be published in conference proceedings.

INITIAL RESULTS WITH THE USE OF THE UNISOR GAS-JET ION SOURCE; ELECTRON-CAPTURE DECAY PROPERTIES OF $154 \mathrm{Tm}$
K. S. Toth
H. K. Carter ${ }^{2}$
D. M. Moltzi
E. H. Spejewski2
R. L. Mlekodaj
C. R. Bingham

The first on-line test of the coupled heliumjet UNISOR separator system was done. Overall, the progress that had been accomplished was very encouraging. There was no problem with the high-voltage transition through the punping line. The ion-source filament operated throughout the testing without failure. Targets witnstood $\sim 150$ pnA of 160 beam and the fourposition target wheel made changing targets a

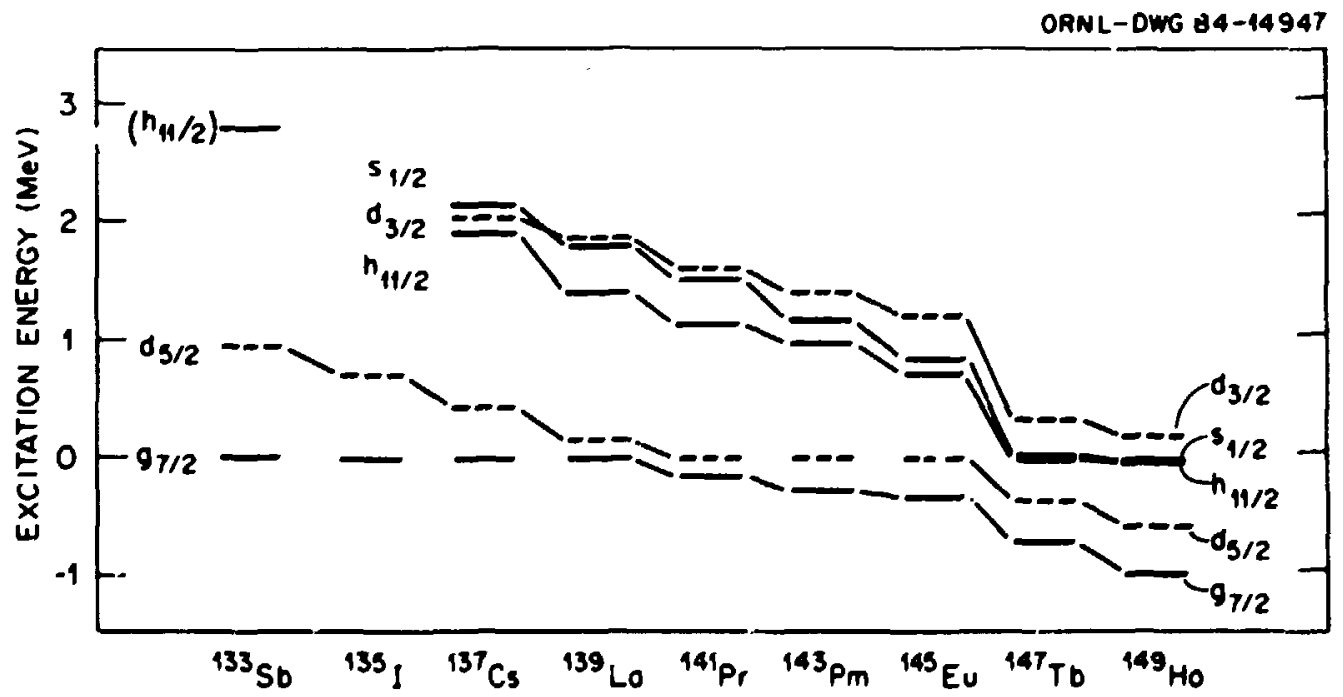

Fig. 2.55. Energy syslematics of single-proton states in $N=82$, odd $-Z$ isotones. Hole states are indicated as having negative energies. 


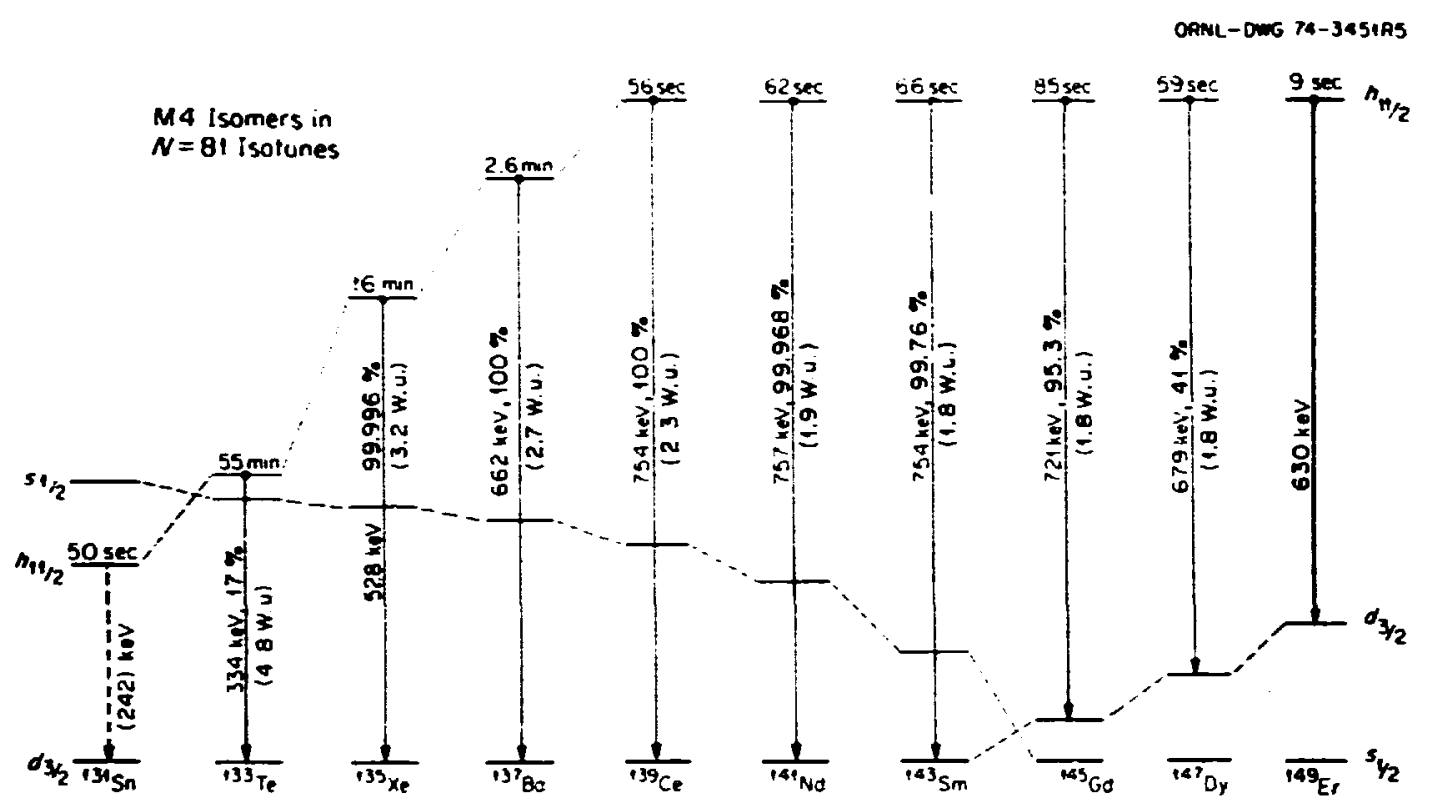

Fig. 2.56. Energy systematics of single-neutron states in $N=81$, even- $Z$ isotones. Included are the rates, expressed in Weisskopf units, for the $M 4$ transitions connecting the $n_{11 / 2}$ and $d_{3 / 2} l e v e l s$.

trivial operation. One major problem encountered was poor mass resolution which caused significant cross-contamination in the separator beams. This was almost certainly due to a combination of the ac heating of the filament and to the larqe output current (mostly helium) of the ion source. A ripple, and sometimes a serious overload, in the separator high-voltage sisply resulted. The test run involved 160 bombardments of $92 \mathrm{Mo}$, nat $\mathrm{Ag}$, [142 $\mathrm{Nd}$, and $1.45 \mathrm{~m}$. Based on estimated production cross sections, the total efficiencies were in the range of 0.01-0.04\% for non-alkali metals and 4\% for cesium. There was no dependence of the yield on half-life down to 460 msec.

The hollow-cathode ion source was modified to operate with a dc filament so as to remove the ripple on the separator power supply. This modiffcation was tested in an experiment in which l'is sim was bombarded with itho ions to search for the $B$-decay branches of the wellknown a emitters, $154 \mathrm{Yb}\left(T_{1 / 2}=400 \mathrm{msec}\right)$ and ${ }^{154} \mathrm{Tm}\left(T_{1 / 2}=3.4 \mathrm{sec}\right)$. The change made the helium-jet system much more manageable. The separator bedm stability durily this run was exceptional. Gama rays from $[S \rightarrow 4 b$ a decay were not observeJ; the isotope's a/total branching ratio must therefore be ciose to unity. The $8^{+}+6^{+}+4^{+} \cdot 2^{+}+0^{+}$trancitions in $154 \mathrm{Er}$, the $154 \mathrm{Tm}$ a-deca, daughter were seen, however. ${ }^{24} \mathrm{Tm}$ B-decay scheme is being put together, following which the nuclide's a/total branch will be determined.

These initial on-line tests indicate: (1) The efficienctes are about a factor of three lower than had bean expected. (2) improvements to the system should first focus on the ton source.
(3) The ion source hold-up time is substantially less than those of the standard UNISOR ion sources and experiments irvolving isotopes with half-lives much less than a second can now be done. SC.

1. University of South Carolina, Columbia,

2. UNISOR, Dak Ridge Associated Universities.

3. Iniversity of Tennesseo, Knuxville, IN.

EVIDENCE FROM \& DECAY THAT $Z$ \& 82 IS MOT MGIC FDR LIGHT LEAD ISOTOPES!

K. S. Toth

Y.A. Fllis-Akovali

C. R. Ringham 2

D. M. Moltz $z^{3}$

H. K. Carter"

R. L. Mlekodaj"

E. H. Spejewski

D. C. Sousa 5

Alpha-decay transttions between ground states of doubly-even nuclei are taken to represent unhindered dt-jys. Reduced widths for these swave transtitions behave in a reguler fashion as a function of both neutron and tomic number. They are largest for nuclei two or four particles beyond a closed shell (with sharp minima at the she11) and they then decresie as the next closure is approached. The s-kidve widths for 196Pb, 15FPb, 190Pb, and 192Pb, hiruever, have been reported ${ }^{6}$ to behave anomalously, f.e... they purportedly increase hy a factor of 30 between 
186po $(N=104)$ and $192 p \mathrm{~b}(x=110)$ instead of decreasing as one nears $N=126$.

The [electron-capture (EC) + $B^{+}$] strengths were deduced in Ref. 6 from $k \times$-ray intensities. A nuber of corrections are involved in such deterninations. We undertook the investigation of the $\left(E C+\theta^{+}\right)$decay schenes of these neutrondeficient lead isotopes, in conjunction with studies of their a-decay properties, to obtain more reliable a-branching ratios. Results for $192 \mathrm{pb}$, 190Pb, and 184pb have been sumarized? earlier.

More recently we produced $105 \mathrm{pb}$ in the $1600 y(325,6 n)$ reaction by bombarding a (2.y2 $-\mathrm{mg} / \mathrm{cm}^{2}-$ thick) dysprosium metal foil enriched in $1600 y$ to 78.95 with 200 - 1 teV 325 ions from the Holifield Heavy Ion Researih Facility tandew accelerator. Reaction products mere mass-separated with the UISOR on-line isotope separator. Our $y$-ray and a-particle data yieid a $106 \mathrm{pb}$ a branching of $100 \mathrm{~b}$, a value mich is wuch larger than the 4.83 branch used by Hornshoj et al. 6 in their analysis of a widths.

In considering a-decay rates we consider them within the theoretical formal ism developed by Rasmussen" wherein decay probabilities are represented by a reduced width, $\delta^{2}$. Decas energies, half-lives, and a branches are needed to compute the reduced widths. In comparing these quant ities for 186,188,190,:92pb as obtained in our current investigations with earlier data, large discrepancies were found, not only for the $186 \mathrm{~Pb}$ branching ratio, but for the $18 \mathrm{BPb}$ and $190 \mathrm{pb}$ a branches as well. As d result, the corresponding widths are increased by factors of 32,7 , and 4.5 . Our width for $192 \mathrm{pb}$, on the other hand, is less than the value based on tlie earlier data because the nuclide's half-life is 3.5 min, rather than 2.3 min used in Ref. 6 .

Figure 2.57 shows s-wave reduced widths for nuclei with $Z$ from 78 to 100 plotted as a func$t$ ton of $N$. One sees the regularity of the reduced widths as a function of neutron number

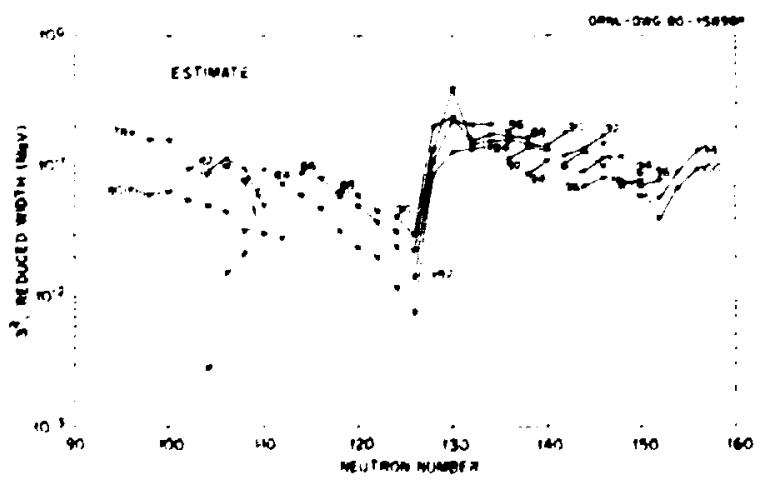

Fig. 2.57. Reduced widths for s-wave a transactions plotted as a function of $N$ for isotopes wth 2 from 78 to 100 . The dashed line connects widths for $186,188,190,192 \mathrm{~Pb}$ calculated from earlier data. Open points for $Z=82$ connected by the full ine, as widths for $18,, 18 \mathrm{~A}, 190,192 \mathrm{~Pb}$ calculated from our experimental results. with the extremely sharp break at $N=126$. This discontinuity has been shom to be a shell structure effect. A less pronounced miniman is seen at the subshell closure at $N=152$. The lead anomaly 6 is indicated by the dashed line wich connects the 186, 180, 190, 1924b widths calculated from earlier data. The widths for $186,108,190,192 \mathrm{pb}$, computed from our data are shom $b_{j}$ the open points. It is clear that they have a dependence on W wich is sinilar to that observed for other eienents. However, these new data indicate neutron deficient lead isotopes to be less hindered toward a decay than mercury isotopes, contrary to the expectation of a shell effect at $Z=82$.

Our results seen to be related to the disappearance of the $Z=82$ gap in the vicinity of $N=114$ mich has been predicteds on the basis of Hartree-Fock-Bogoliubor calculations of proton single-particle energies, and, to the existence of varying shapes in mercury and platinum isotopes in this ass region. In $182,184,186,180 \mathrm{Hg}$ it has been shom $10^{\circ}$ that wel! defermed prolate bands cross the slightly oblate (c -0.1$)$ ground-state bands. Plat inum nuclei with $A<190$, on the ocher hand, are believed It to be prolate in their ground states. If one supposes that 186,180,196,194p al so have slightly oblate ground states then a transitions from oblate mercury to prolate platinum isotopes would be expected to be hindered mereas lead a decays, wich to not involve shape changes between the parent and daugnter nuclei, would not be. (Low-lying $0^{+}$excited states in 192,194,196, 198pb have been described 12 by oblate two-particle, two-hole configurations; their ground states, howevor, have been assumed to be spherical.) Further detailed calculations of a-decay rates incorporating shape changes are needed. If such theoretical results do indeed agree with our experimental observations, then the study of a-decay rates may prove to be a useful tool to oeduce information concerning nuclear shapes.

\section{Summary of a paper: Phys. Rey. Lett.}

53. 1623 (1384).

2. University of Fennessee, Xnoxville, TK. SC.

3. University of Sccith Carolina, Columbla,

4. JMISOR, Oak Ridge, TN.

KY.

6. P. Hornshoj et al.. Nucl. Phys. 1230, 365 (1974).

7. K. S. Toth et al.. Proc, of the 4th

International Conference of Nuclei far From Stability, Helsingar, Denmark, 7-13 June 1981, D. 174. CERN Repurt 81-09 (July 1981).

8. J. 0. Rasmussen, Phys. Rev. 113, 1593 (1959;.

9. R. A. Sorersen, D. 498 in Proceedings 4th International Lonference on $\mathrm{NL} \cdot \mathrm{lel}_{\mathrm{e}}$ Far From Stabiltty, CERN 81.09 (1981); Nucl. Phys. A120, 221 ('984).

10. R. V. F. Janssens et al., Phys. Lett. 1318, 35 (1983); W. C. Ma et al., Phy5. Lett. 1398, 276 (1984), and references therein. 
11. M. Finger et al.. Mucl. Phys. N188, 369 (1972) : F. R. My et al.. Phys. Lett. 68B, 113 (1971): J. Saurage-Letessier et al.., Kucl. Phys. A370, 231 (1981); B. Merlo-Ponorsta, Z. Phys. 1293, 9 (1979).

12. P. Yan Duppen et al., Phys. Rev. Lett. 52, 1974 (1984).

\section{BETA-OELAYED PROTON RCTIVITIES: ${ }^{1630 y}$ ND ${ }^{149} \mathrm{Er}^{2}$}
X. S. Toth
D. M. Holtz ${ }^{2}$
Y. A. Ellis-Akovali
E. C. Schloemer
F. T. Avignone, III ${ }^{2}$
M. D. Cable

The a-delayed proton spectra of 14 ? Dy and the hitherto unknown isotope, I+ "Er, were investigated at the Lawrence Berkeley Laboratory 88-inch cyclotron in $: 2 C$ bombardaents of $I+2$ nd and $14+5$ Sen, respectively. A helium gas-jet system was used to transport product nuclei to a shielded area where they were assayed with a $\triangle E-E$ particle telescope and a $G e y-x$ detector. The ${ }^{12} C+162$ ind proton data isee Fig. 2.58 (a)] confirm preliminary results of Klepper et al.- that the : 10 Dy delayed-proton spectrum has a high-energy cutoff at about $4.5 \mathrm{MeV}$ and is dominated by distinct peaks below $4 \mathrm{HeV}$ in excitation. The proton spectrum follawing $1{ }^{9} \mathrm{Er}$ decay [see fig. 2. 3is (b)] his a half-life of $9 \pm 1 \mathrm{sec}$ and extends ron ahout 2.0 to $6.5 \mathrm{MeV}$; it has less of the inirp stricture obseryed for $\therefore \rightarrow$ Dy.

The intrinsic struccure of delayed-proton spectra that accompany heavy mass precursors usually is no: resolved due to the large density of states in tha excitation energy range fed by

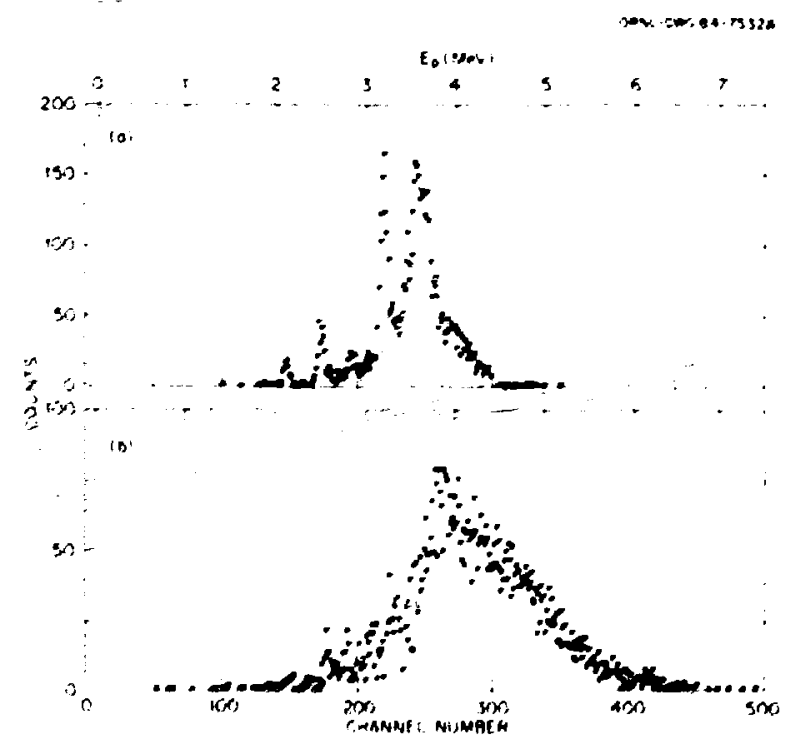

Fig. 2.58. nelayph-nroton spectra ohserved in $12 \mathrm{C}+142 \mathrm{Nd}[\mathrm{part}$ (a)] and $1 \mathrm{C}, 11 . . \mathrm{Sm}$ [part (b) 1 irradiations made at an incident eneray of $\sim 135$ Me; they are assignad to li" Dy and $109 \mathrm{Er}$, respectively. the a decay. In Fig. 2.58 (a), however, the peaks have full widths at half $-20 x i m$ wich are on the order of the $\triangle E-E$ detertor resolution, i.e., - 60 keV. The indication is that the i470y a decay is sampling either selected 107 Tb states or else an energy region in $147 \mathrm{~Tb}$ were the level density is not higi. The reader is reminded that $14{ }^{4} \mathrm{~Tb}$ consists of a single proton coupled to the doubly-closed core of I"6 Gd (N $=92$ and $Z=64$ ). In Fig. 2.58 (b), wile there are peaks in the lower half of the spectrun, the structure is much less distinct than in fig. 2.58 (a). This could be due to a larger level density in $149 \mathrm{Ho}$, a nucleus stil: with 32 meutrons but now having three protons beyond $Z=64$.

High-energy endpoints of delayed-proton spectra are fixed by differences between the electron-capture (EC) decay energies of the parents and the proton binding energies in the corresponding EC daughters. Predicted $Q_{E C}-B_{p}$ values from the 1977 Atomic Mass Evaluations for $1470 y$ and $b+9 \mathrm{Er}$ are 4.42 and 6.44 HeV, respectively. These predictions are consistent with our data, see Figs. 2.58 (a) and (b).

1. Sumary of paper: Phys. Rev. C30, 712 (1984). SC.

?. University of South Carolina, Columbia, CA.

3. Lawrence Berkeley Laboratory, Berkeley,

4. 0. Klepper et al., 2. Phys. A305, 125 (1982).

5. A. H. Wapstra and $K$. Boz, At. Data Nucl. Data Tables 19, 175 (1977).

GAwA-RAY DECAY PATHUAYS OF A COAPOUND DUCLEUS WITH HIGH AMGULAR MOIENTUM AND HIGH EXCITATION ENERG I
!. รั. Lee
M. L. Haibert
i. Batetasin
J. R, Reene
it. Kim
N. R. Johnson
R. O Sayer
F. K. McGowan
H. T. Milner
D. G. Sarantites ${ }^{2}$$$
\text { M. P. Fewell's }
$$

The r-rays emitted from a high-spin compound nuileus consist mainly of many stretched iow multipoiarity $(\lambda=1,2)$ transitions thich remove most of the angular momentum of the nucleus. The starting point of this $Y$-ray cascade is usually referred to a, the entry point wich is distributed over $d$ wise region in the (E*,I) plane centered at about une neutron binding energy above the yrast line." Initially, the $y$-ray decay follows a path through regions of high level density and this leads to a large number of possible decay pathways resulting in al unresolved cont inuum of $y$-rays. Near the end of the continuum cascade. all the pathways converge into the yrast band. A few bands just above the yrast band (c I Mej). as well as the yrast band itself, usually can be onserved os discrete y-ray lines. 
Knowledge of the decay route from the entry states to the yrast line is necessary in order to extract inforation on nuclear structure fros the associated continum $y$-ray spectrum. Imis route deteraines the region in the $(E, I)$ plare from mere the $r$-rays are enitted, al lowing us to associate the masured nuclear properties with the proper nuclear spin and excitation energies (temperature). So far, there is no direct deteraination of the decay pathways from the entry states to the yrast states. Models for the decay pathways have been proposed 5-8 based on indirect information such as yraststate side-feeding intensities, r-ray wltiplicity distributions, and continuu $y$-ray spectra. Therefore, an experimental deternination of the decay pathways is not only necessary for an unterstanding of the continum $r$-ray results but it is also necessary if we are to mke any eaningful comparison of these high-spin properties with theoretical calculations.

In the present experiment the entry states in $13^{\circ} \mathrm{Ce}$ were populated in the reactions of 144-HeV 345 with 100\%. The spin spectrometer was triggered with two se counters. The continuum spectra (from the Mal detectors) coincident with 130 Ce discrete lines (from the Ge detectors) were constructed as a function of total pulse height, $H$, and fold, $k$. These were then unfoljed to remove the detector response and trigger bias. The spectra from each $(H, k)$ bin represent all the r-rays eaitted in the cascade from a given entry point to the ground state, but the order of eaission of these r-rays cannot be determined directly from a spectrum.

The decay pathway can be determined, however. by using a differential bethod as described in the following. Assume that from a state $(E, M)$. the nucleus aits a $y$ ray with energy $E_{y}$ and ends in a state $\left(E-E_{r}, M-1\right)$. From the state $\left(E-E_{Y} M-1\right)$, the rest of the cascade is identical $t r$ che cascade with entry point (E-E $M-1)$. Therefore, if we subtract the spectrum with entry point (E-E, $M-1)$ from the spectrum with entry point $(E, M)$, we will cbtain a difference spectrum with only one $r$ ray of energy $E_{Y}$. On the other hand, the spectra from entry point $\left(E_{2}, M-1\right)$ with $E_{2} * E-E_{1}$ is different from the spectrum of $(E-E, M-1)$. Thus, in the difference spectrum there is no complete cancellation of $r$ rays. In fact, the difference spectrum will contain negative counts in some channels. although the net counts still correspond to one $y$ ray. Therefore, the methor of deterwining the decay pathway is to subtract from the spectrum with $\left(H_{1}, k\right)$ the spectra with $\left(H_{2}, k-1\right)$ for all possible $\mathrm{H}_{2}$ values. The difference spectrum with the least amount of negative counts gives the $\mathrm{H}_{2}$ value to wich the state $\left(H_{i}, k\right)$ will most Iirely decay. To measure the negative counts in the spectrum, we can define a parameter $F$ as $F=\left|C_{j} / \int\right| C_{i} \mid$, were $C_{i}$ is the counts at channel i. For spectra with only positive counts, $F=1$, wereas spectra contzining some negat ive counts will have $f$ smaller than 1.

The difference spectra obtained are shown in Fig. 2.59. These are the differences of the $k=22, H^{*}=7.5 \mathrm{MeV}$ spectrum ( $H^{*}$ is the excitation energy above the yrast line) and the $k$. $21, H^{*}=3,6,9$, and 17. MeV spectra. We see

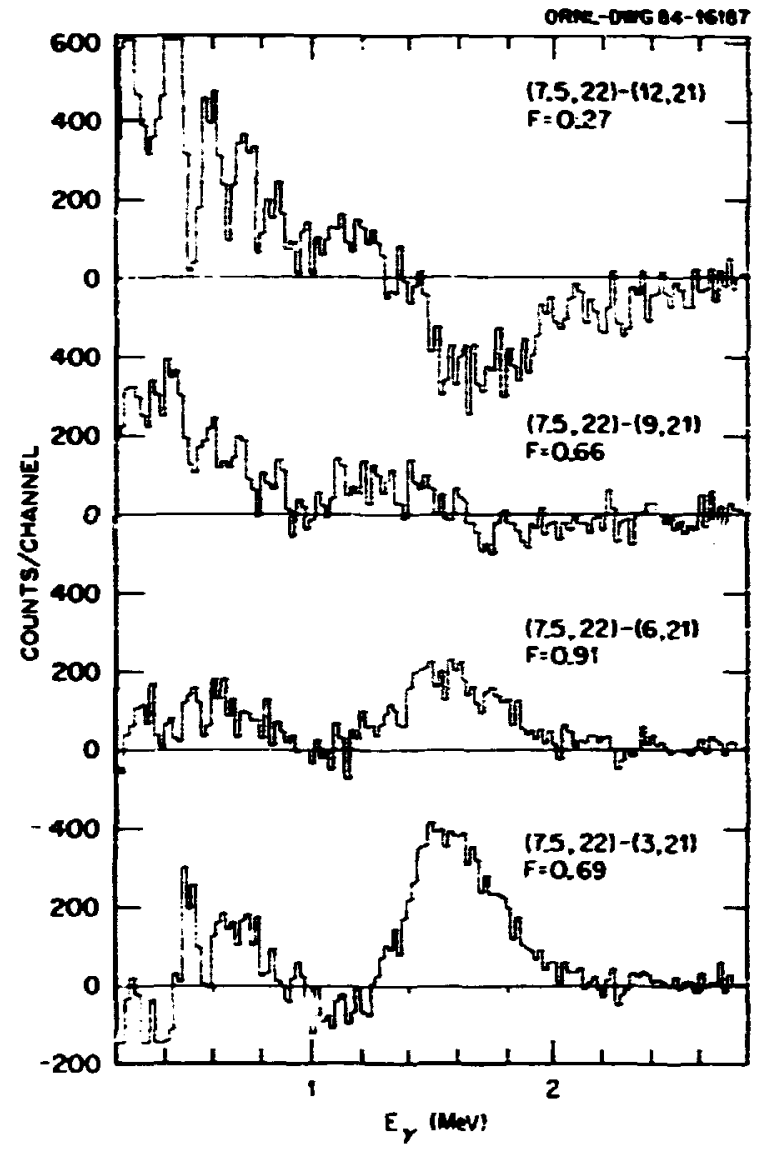

Fig. 2.59. Difference spsectra of $k=22$, $H^{*}=7.5 \mathrm{MeV}$ and $\mathrm{k}=21$.

clearly that only the mitrle spectrum, i.e., $\left(H^{*}=1.5 \mathrm{MeV}, k=22\right)-\left(H^{*}=6 \mathrm{MeV}, k=21\right)$ had mostly positive counts. Inis indicates that the most probable path from $H^{*}=7.5 \mathrm{MeV}$ and $k=22$ is through states with almost the same $H^{*}$ value. Similar features are seen for the decay of $\left(\mu^{*}=13.5 \mathrm{MeV}, k=22\right)$ states. The $F$ vaives from such difference spectra have been calculated and they are shown in fig. 2.60 for the decay of the $k=22, H^{*}=7.5 \mathrm{FeV}$ region. These results track the decay path fron $k=22$ to $k=14$. The peak of the $F$ value distribution is at about the same energy above the yrast line. This indicates that nuclei mosi likely decay along bands parallel to the yrast line.

The atnve results show that the decay pathways in the high spin and high excltation energy regions are mainly parallel to the yrast line, contrary to early predictions that nuclei with high excitation energy wil decay toward the yrast line through statistical (mainly EI) $y$-decay. This dominance of collective E2 decay in the high-spin regions was in fact suggested recently by Newton. 9 This is due to the increase of the $\gamma$-ray energy for rotational collective E2 transitions as a function of spin which can be shown to vary approximately as with the iffth power of $(4 !-2)$. The present resijits 


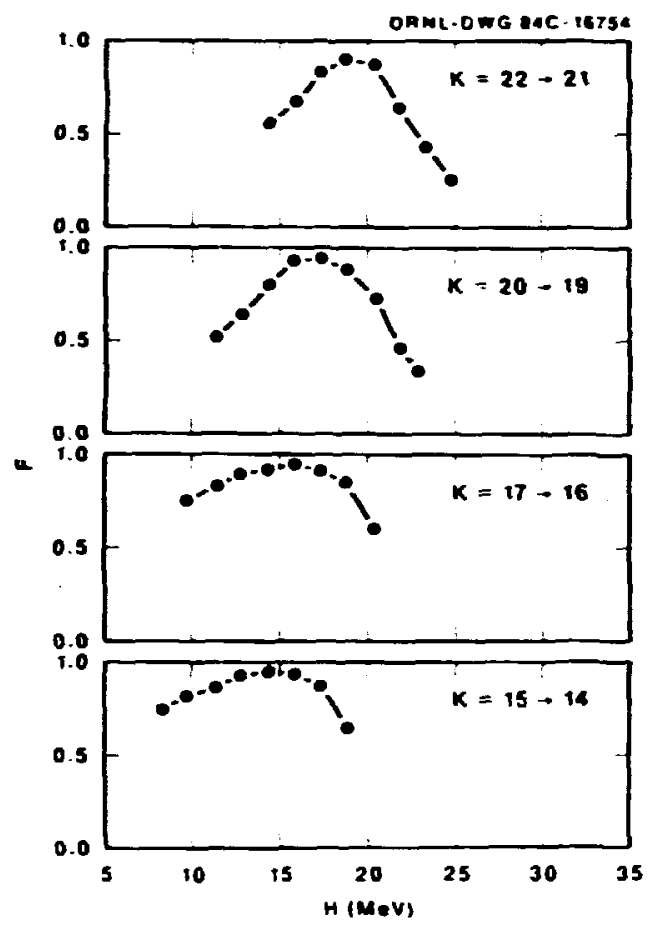

Fig. 2.60. The distribution of decay probability $F$ in excitation energy as function of $k$.

also show that the nuclear deformation and collective motion still exist at high excitation energy, and therefore the nuclear shell effects are not completely attenuated at these excitations.

Since the continuum $y$-rays are emitted from regions with a broad distribution in excitation energy rather than from a narrow region near the yrast line, it is mandatory that we measure both the spin and the excitation energy of the continuum r-rays. In the cases where only the multiplicity (spin) is used for selection, one should recognize that the spin-dependent nuclear properties are averaged over a wide region of excitation energy. On the other hand, when the total y-ray energy is used in selecting spectra for study, it selects an unknown mixture of soin, excitation energy and decay pathways. Therefore, the interpretation of such data can be highly ambiguous.

1. Sumiary of paper to be published.

2. Washington Universtty, St. Louts, MO.

3. Australian Natforal University, Canberra, Australía.

4. D.G. Sarantites et al., Phys. Lett. 1158,441 (1982).

5. J.0. Newton et al., Nucl. Phys. A141, $631(1970)$.

6. E.J. Liotta and R.A. Sorensen, Nucl. Phys. A297, 162 (1978).

7. M. Wakat and A. Faessler, Nucl. Phys. A307, 307 (1978).
8. G. Leander, Y.S. Chen and B.S. Milsson, Phys. Scr. 24, 164 (1981).

9. J.0. Mewrton. Phys. Scr. 24, 83 (1981).

\section{NH IMUESTIGATION OF THE COLLECTIYITY IN THE TRAST BAN OF $15{ }^{\circ} \mathrm{Er}$ BT LIFETIIE MEASUREREMTSI}
H. Oshima2
N. R. Johnson
F. K. Heliowan
I. Y. Lee
C. Baktash
R. V. Ribas 4
Y. Schutz ${ }^{3}$
J. C. Mells's

Both experimental and theoretical investigations of deformed rare-earth muclei have shown that the interplay between collective rotations and single-particle excitations deteraines the structure of these nuclei at high spins. From the macroscopic point of view, the Coriolis force wich comes into play at high rotational frequencies causes alignment of the singleparticle angular momenta along the axis of the collective rotation. Such an alignment of single particles will result in a deformation change from prolate to triaxial or oblate shapes. Furthermore, centrifugd l force can cause stretching of the nucleus. The E2 matrix elements along the yrast line should reflect such deformation changes. Indeed, recent lifetime measurements in the s-bands of $156-158$ Dy (Ref. 6) and 159-163 Yb (Refs. 7,8), wich lie near the $N=90$ region, indicate a significant reduction in the $E 2$ matrix elements and suggest that these nuclef undergo a deformation change from prolate to triaxial shape at high angular momentum. In order to extend our understanding of the evolving pattern of shapes and the forces that determine these deformation changes in sof $t$ transitional nuclei (near $N=90$ ), we have carried out lifetime measurements on all states in the yrast sequence of ${ }^{158} \mathrm{Er}$ from $\mathrm{I}^{2}=2^{+}$ through $I=24^{+}$.

Lifetimes of these high spin states in ${ }^{158} \mathrm{Er}$ have been measured by means of the recolldistance method. Excited $15 \mathrm{~A}$ Er nuclet were produced via the reaction $128 \mathrm{Te}\left({ }^{34} 5,4 n\right)^{158} \mathrm{Er}$ at a bombarding energy of $155 \mathrm{MeV}$. Deexcitation $y$ rays were detected in a Compton-suppressed Ge detector positioned at $0^{\circ}$ and gated by five germanium detectors at $90^{\circ}$ to the incident beam. The recoil-distance device used in the experiment has been described ${ }^{9}$ prevtously. Coinctdence counts were recorded event by event on magnetic tape for fourteen recofl-flight distances ranging from $20 \mathrm{um}$ to $2.515 \mathrm{~mm}$. In addition, a lead-backed target was used to get the spectrum at zero separation.

from the coincidence data, three types of decay curves were obtained for each state: (1) one from the total projected coincidence spectra; (2) one from the spectra generited by gating on all transitions below the state of interest; and (3) one from the spectrd gated by the next transition above the state of interest. A few comments abuut these three types of spectra are in order. Total projected coincidence spectra provide data of improved statistical quality for weak transitions, but can lead 
to difficulty in fitting the decay curve due to added complexity in treating side feeding. $A$ decay curve obtained from the sumed spectra gated on transitions below the state of interest includes essentiaily the same fraction of side feeding as the one obtained from "singles" spectra. The ajor difference is that the gated spectra contain fewer $y$ rays, thus aking the analysis simpler and more reliable. In principle, the third type of decay curve produces by far the simplest case for extraction of lifetimes since the data are unencumbered with the problems of sidefeeding into the state of interest. This approach is limited only by the statistical quality of the data that can be collected for high-spin states. In all of these coincidence spectra, events of low $r$-ray witfplicity mich correspond to Coulonb excitation or residual decay are considerably reduced.

The three types of decay curves were obtained for transitions from the $2^{t}$ through $24^{+}$nember of the yrast band. Lifetimes were extracted by a computer code 10 wich applies all of the usual corrections to the lata and handles the problea of feeding by direct sclution of the Bateman equations.

A summary of 1 ifet imes for members of the yrast sequence is given in Table 2.12. The lifet imes in the $t$ able are weighted averages of values extracted from the different t;ipes of analyses described above. Reduced transition probabilities were extracted from the lifetimes, and transition quadrupole moments, $q_{t}$, were calculated according to the expression

$$
B(E 2 ; I+1-2)=\frac{5}{16 \pi}<1200 \mid I-20>{ }^{2} Q_{t}{ }^{2} \text {. }
$$

In Fig. 2.61, the $Q_{t}$ values are plotted as a function of spin. In this figure two important features are conspicuous: The $0_{t}$ values of the g-band appear to increase with spin up to $8^{+}$. and, after the backbending which occurs at about $14^{+}$, the at values become smaller compared with the g-band and even decrease at lifgher spins.

It is interesting that many nuclef lying at or near the 90-neutron transitional boundary show, at least qualitatively, simflar behavior.

Table 2.12. Lifetimes of yrast states in $158 \mathrm{Er}$

\begin{tabular}{lccll}
\hline$I^{\pi}$ & $E_{Y}(\mathrm{keV})$ & $r(p S)$ & $B(E 2 ; 1+I-2)$ & $Q_{t}(e b)$ \\
\hline 2 & 192.1 & $37 !(37)$ & $0.65(6)$ & $5.7(3)$ \\
4 & 334.8 & $19.2(18)$ & $0.96(9)$ & $5.82(27)$ \\
6 & 442.8 & $3.7(4)$ & $1.27(14)$ & $6.37(34)$ \\
8 & 522.7 & $1.33(26)$ & $1.55(30)$ & $6.9(7)$ \\
10 & 578.8 & $1.13(45)$ & $1.10(44)$ & $5.7(11)$ \\
12 & 607.9 & $1.2(6)$ & $0.81(41)$ & $4.9(12)$ \\
14 & 509.5 & $3.1(4)$ & $0.76(10)$ & $4.7(3)$ \\
16 & 472.6 & $3.48(24)$ & $0.97(7)$ & $5.26(18)$ \\
18 & 566.0 & $1.82(13)$ & $0.76(5)$ & $4.64(17)$ \\
20 & 650.4 & $1.16(7)$ & $0.57(3)$ & $4.01(12)$ \\
22 & 740.1 & $0.96(17)$ & $0.38(7)$ & $3.3(3)$ \\
24 & 805.7 & $0.43(14)$ & $0.56(18)$ & $4.0(6)$ \\
\hline
\end{tabular}

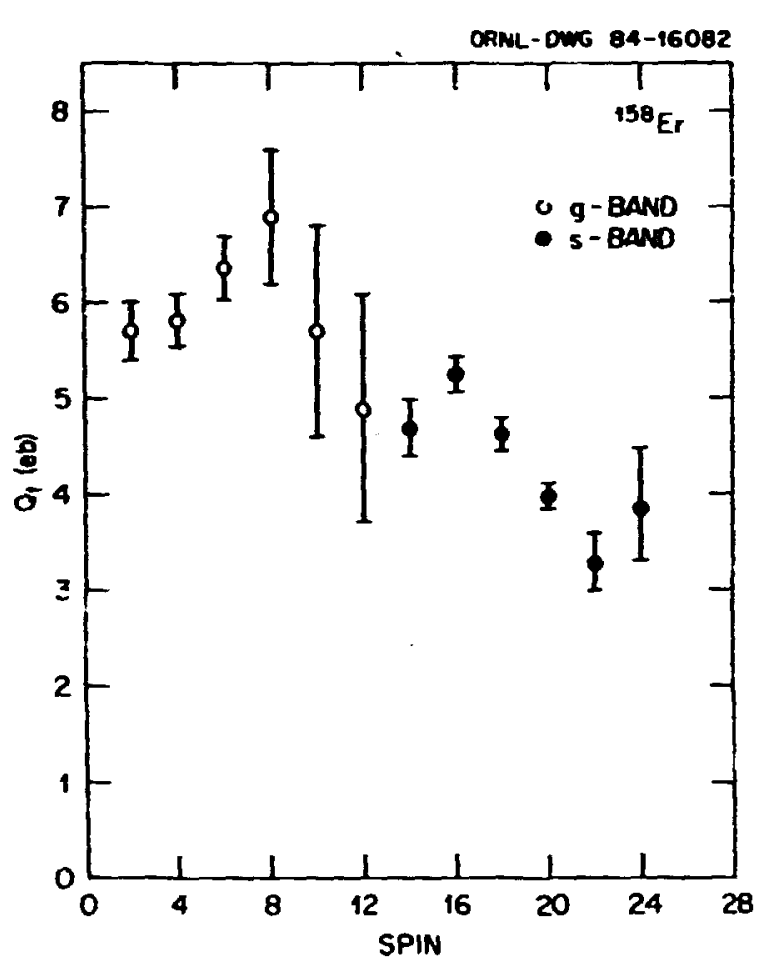

Fig. 2.61. Transition quadrupole moments of yrast states in $158 \mathrm{Er}$ plotted as a function of spin.

Several theoretical calculations have been performed in order to understand these phenomena. Recently. a group at Lund has reported preliminary results 11 of some self-consistent cranked Hartree-Fock-Bogolfubov (HFB) calculations of the shape of the ${ }^{158} \mathrm{Er}$ as a function of angular momentum. They find that, in the g-band, variation of $Y$ is rather small (within $2^{\circ}$ ) while $\varepsilon_{2}$ shows a steady increase, ranging from 0.20 at ground state to 0.24 at $10^{+}$. Thts result accounts qualftatively for our observed behavior except for the $10^{+}$state, where we see significant reduction in the collectivity. Furthermore, in a similar calculatian for the s-band in $160 \mathrm{Yb}$ it has been shown 12 +tat $160 \mathrm{Yb}$ undergoes a triaxial deformation at high spins. The similar tendency of the experimental $Q_{t}$ values were observed in $158 \mathrm{Er}$ and $t \mathrm{t}$ could be attributed to the polarizing effect on the core caused by the high-i aligned quasiparticles.

1. Summary of paper to be published.

2. Dn leave from Japan Atomic Energy Research Institute, Tokaf, Ibaraki 319-11, Japan.

3. Present address: University of Sao Paulo, Sao Paulo, Brazil.

4. On leave from CRN, Strasbourg, France.

5. Tennessee Technological Untversity, Cookevtlle, IN. 
(1984).

6. H. Enling, et al.. Mucl. Phys. A419, 187

7. M. R. Johnson, Proc. of 1982 Ins International Symposium on Dymanics of huclear

Collective Hotion, ed. by $K$. Ogama and $K$.

Tanabe (Inst. for Nuclear Study, University of

Tokyo, 1982) p. 144.

8. H. P. Fevell, et al., Proc., Conf. $\alpha$ : Kigh Angular Monentum Properties of Ruclei, Oak Ridge. Tennessee, Nov. 1982, Vol. 4 of Muclear Science Research Conference Series. ed. by $N$. R. Johnson (Harwood Acaderwic Publishers, New York, 1983) p. 69.

9. N. R. Johnson, et al., Physics Division Progress Report for Period Ending June 30, 1981, ORM-5787 (1981).

10. J. C. Mells and H. P. Fewell, to be published.

11. R. Bengtsson and S. Aberg, private cominication.

12. R. Bengtsson, et al., Mucl. Phys. A405, 221 (1985).

GUASIVIBRATIONAL BANDS RT MIGH SPINS IH 15OYb'
C. Baktash
Y. Schutz 2
1.. Courtney 4
I. Y. Lee
A. 1. Larabee 4
F. K. MeGowan
L. L. Riedinger 4
N. R. Johnson
A. W. Sunyar 5
M. R. Hohnson
D. C. Hensley
E. der Mateosians
O. C. Kistners
D. G. Sarantites 6
M. P. Fawall ${ }^{3}$
1. Ragnarsson 7

Rare-earth nuclei with neutron numbers $N \leq 0$ offer a unique opportunity to study spin induced nuclear shape transitions. Being situated between the nearly doubly magic $146 \mathrm{Gd}$ "core" at one end, and the well-deformed nuclei at the other, they span a variety of shapr:s which range from moderately oblate to prolate. In particular, the transitional suclei $(N=88)$ which have soft potential energy surfaces are predicted to experience large changes in their shape parameters as the angular momentum is increased and high-j quasiparticles are excited. Study of these shape transitions and the associated excitation modes provides valuable insight into the questicn of the interplay between collective and single-particle degrees of freedom in nuclear systems.

To investigate these effects. we have studied the high spin states in $158 \mathrm{yb}$, which were populated using $20 \mathrm{Ne}(\mathrm{E}=115 \mathrm{MeV}$ ) and $64 \mathrm{Ni}$ (E $=285$ MeV) beams. The $20 \mathrm{Ne}$ run utilized an array of six Ge counters along with two $25 \mathrm{~cm} \times 25 \mathrm{im} \mathrm{Nal}$ crystals which served as a total energy spectrometer. More than 100 million events, defined by colncidence firing of both Nal cetectors and a minimum of two Ge counters, were collected in this mode. The hind run was carried out in the Spin Spectrometer by replacing six of the Nal elements with Ge detectors. By demanding a coincidence firing of a minimum of two Ge and 22 Nal detectors, collection of those events with rich multiplicitiy content was emphasized. Off line analysis of these data started with separate gain matching of all Ge and hal detestors and removal of the neutron-induced signals. the corrected data - - then scanned to generate several Er-Er at-ices that were gated with various two-dimensional cuts in the tctal pulse-height vs coincidence fold map to enhance the An-channel. A recently developed background subtraction technique was used to remove nearly 90\% of the Compton events present in these mtrices before generating gated coincidence spectra.

On the basis of the $\gamma-\gamma$ coincidence data from these runs, established the positive- and negative-parity yrast states up to $I x=\left(40^{+}\right)$ and $\left(31^{-}\right)$, respect ively (Fig. 2.62). Figure 2.63 compares the kinematical monts of inertia, $g(1)$, of these two bands with similar bands in 160\% (full and open circles, respectively). A noteworthy feature is the

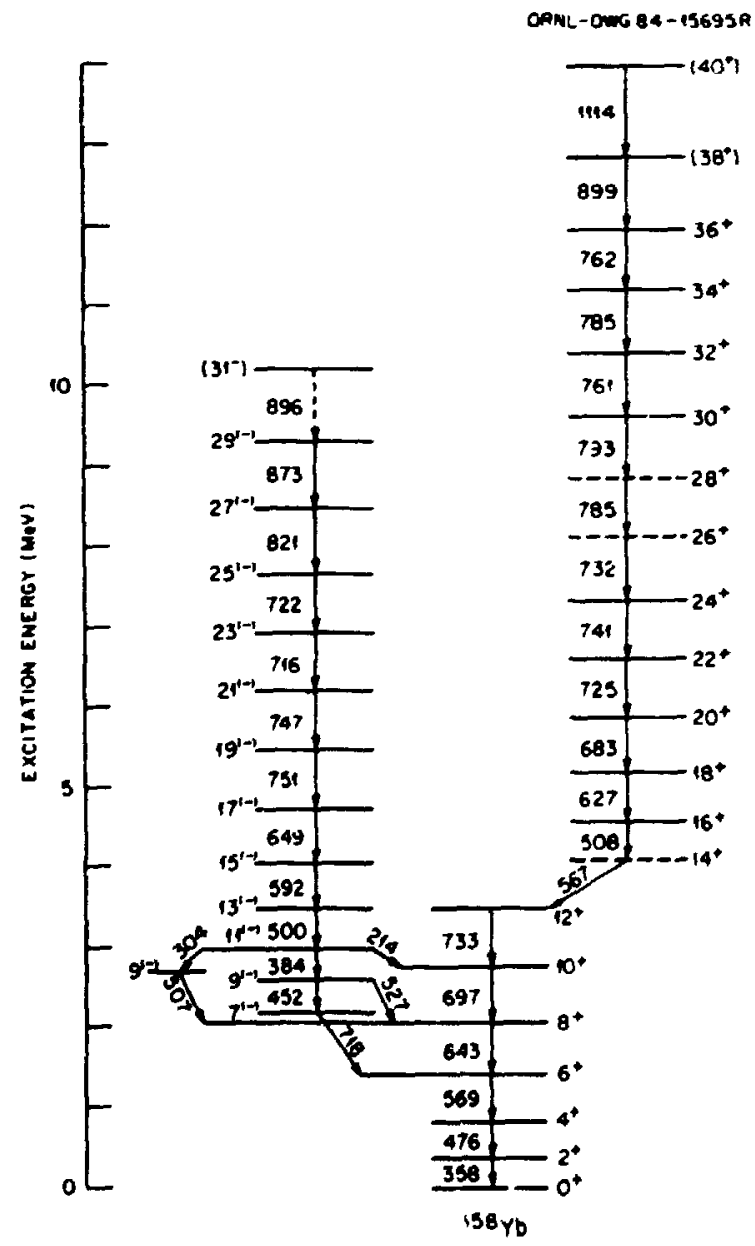

Fig. 2.62. Partial decay scheme for L'A $^{19} \mathrm{Yb}$. Gammd ray energies are gf ven in keV. Dashed horizontal line: inficate uncertain ordering of the transition of $r$ rays. Parentheses indicate uncertain spin-party assignment. 


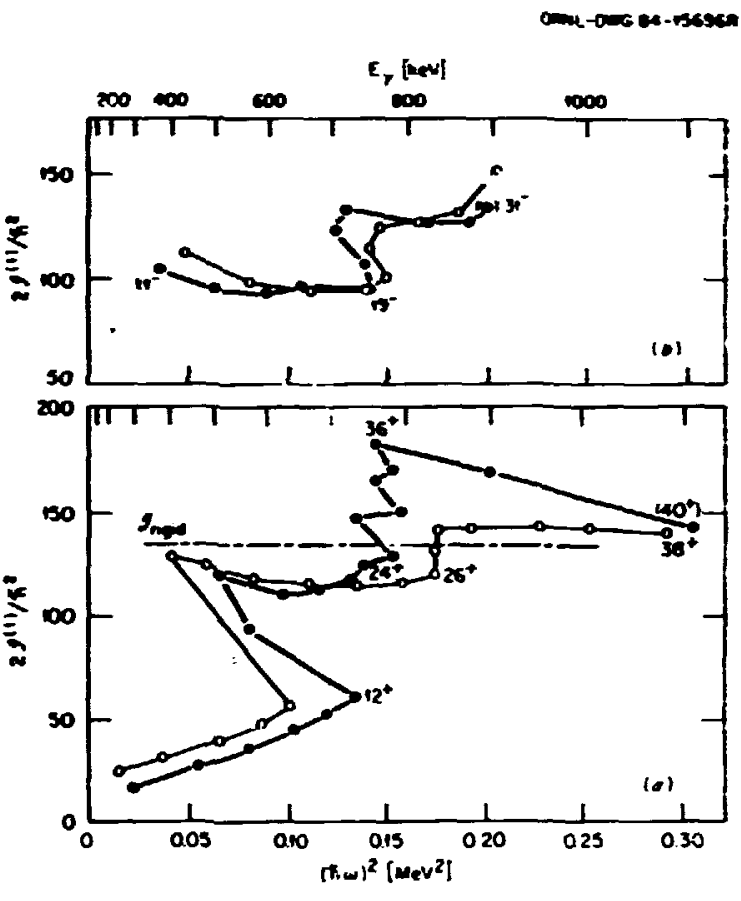

Fig. 2.63. Plots of moments of inertio $2 . F(1) / \hbar^{2}$ vs $\left(\hbar_{\omega}\right)^{2}$ for (a) positive bands, and (b) negative parity bands in $158 \mathrm{yb}$ (full. circles) and $16 \pi \mathrm{yb}$ (open crosses).

contrasting behavior of the two bands in $158 \mathrm{yb}$. In the case of the negative-parity band (Fig. 2.64), wich consists of $i_{13 / 2} h_{g / 2}$ neutron orbitals, observe its first band crossing at an angular frequency of $\hbar_{\omega}=0.36 \mathrm{MeV}$, close to that of the $160 \mathrm{yb}$. Both nuclei exhibit an upbending in the frequency range of $\kappa_{\omega}=0.44$ $\mathrm{MeV}$, wich has been shom to be due to Coriolis alignment of an $h_{f} / 2$ proton-pair in $N=90$ isotopes. The overafl'behavior of this band is not very different from other odd-spin odd-parity bands in the neighboring collective nuclei.

The low and medium spin states in the positive parity band show a similar collective Dehavior up to spin 24. At higher spins. however, this band shows an excitation pattern which is quite different from that of the negative partity band or other positive-parity bands in neighboring nuclei. In particular, the stretching oi $g(1)$ resumes past spin 20 and assumes an extreme form, namely, a vertical ascent, after the second discontinuity at spin $I=24$. The resulting moment of inertia exceeds th: rigid rotor value by nearly $40 \%$. Such a lirge.$(1)$ has not been observed previously art is a consequence of the quasivibrational excl:ation mode. (The energies of the $r$ rays deexc'ting the states with spin $24<I<36$ remain withif, a $130-790 \mathrm{keV}$ interval.) Rotational alignmelt effects cannot explain this sharp rise in $\mathscr{g}(1)$, nor the near constancy of the gamma ray energies. (Aligrant of proton $h_{I / / 2}$ is expected to occur at higher rotatictid frequencies, and that of the $\mathrm{h}_{\mathrm{g} / 2}$ neutron orbital can at best account for only half of the oterved gain in alignwent.) A likely explanation is that the nuclear shape gradually evolves frow being prolate at I" $=24^{+}$to oblate at $I^{\pi}=36^{+}$. Indeed, on the basis of cranked shell andel calculations, Ragnarsson et al. Mave recently suggested that these states belong to an yrast band which continuously miniaizes its energy by adjusting its shape paraneters and acquiring larger $y$ values, were $r$ is the asymetry paraeter. This band finally terminates on the oblate axis upon reaching spin 36 , wich is obtained by aligning the angular mamentim of its valence particies along the oblate axis. Although such terminating bands have long observed in very light muclei, the positive parity band in $15 \mathrm{yb}$ is the first evidence for the existence or such bands in a heary nucieus.

1. Sumary of paper to be publisned.

2. On leave froa CRN, Strasbourg. France.

3. The Australian Mational University, Canberra, Australia.

4. University of Tennessee, inoxville, TN.

5. Brookhaven Mational Laboratory, Upton, NY.

6. Washington University, St. Louis, $\mathbf{m}$.

7. Lund Institute of Technology, Lund, Sweden.

\section{COULOAB EXCITATION OF $160 \mathrm{DY}$ MITH LEAD IONS}
R. V. Ribas '
N. $k$. Johnson
I. Y. Lee
L. L. Riedinger ${ }^{3}$
D. R. Haenni 2
R. M. Diamond"
F. S. Stephens" H. Kluge"

Coulomb excitation of $1600 y$ was carried out with a $20 \mathrm{Apb}$ bean from the Lawrence Berkeley super HILAC. This was done in order to study the changing collectivity at high spins by measuring the reduced transition probabilities between states in the yrast sequence. Gamma rays emitted following Coulomb excitation were detected by two Ge(Lf) detectors in uninciden ie with scattered projectiles and recjiling nuclei. These particles were detected in a patr of twodimensional position-sensitive parallel-plate avalanche detectors. The target was $0.5 \mathrm{mg} / \mathrm{cm}^{2}$ (99\% enriched) 1600 ; deposited onto a $0.7 \mathrm{mg} / \mathrm{cm}^{2}$ $\mathrm{Ni}$ foil.

The Coulomb Excitation Data Analysis Code (GOSIA) otidincd from D. Clines was used to determine the set of matrix elements that best account for the experimental $y$-ray ytelds by means of a ieast squares fitting. Etght sets of data, corresponding to four aifferent regions of the scattering angles for two bombarding energies ( 942 and $1035 \mathrm{MeV}$ ) ware used in order to obtain unambiguously the complete 5 st of transition matrix plements up to spin $20^{*}$. The inftial set of matrix elements used in the 
fitting were those of the symatric rigid roter model. Since the $B$-band and the $K=2$ and 4 band heads in $160 \mathrm{~b}$, have rather high excitation energies ( $1.5 \mathrm{maV}$ ) and since no tra isitions associated with these bands were oiserved in the spectra, only the g-band, the $r$-band and the s-band mere included in the level scheme. The s-band was assumed to have the same intrirsic quadrupole mont as the $g$-band, and intertand matrix elements were taken only at the crossing point. The diagoral elements mere kept fixed during the analysis and were calculated $j$ using an intrinsic quadrupole wont that reproduces the $2^{+}$- $0^{*}$ transition probability. This vilue, wich is weil known from previous measurem nts, was allowed to vary only in a narrow range around the knom value.

The reduced transition probabilities obtained from the present experiment are shom in Table 2.13. As seen, these results are consistent with the previous Coutomb excitation data of Sayer, et al.7 at lower spins, with the exception of the $8^{+}+6^{+}$transition, where the present result is $\sim 152$ higher than that of Ref. 7 .

We have used our $B(E 2)$ values in Table 2.13 to calculate trensition quadrupole moments $\left(Q_{t}\right)$ according to the following expression

$B(E 2 ; I+I-2)=\frac{5}{16 \pi}<I 200 \mid I-20>2 Q_{t}{ }^{2}$.

Table 2.13. Reduced E2 transitfon probabilitfes for iranstitions in $1600 y$.

\begin{tabular}{|c|c|c|}
\hline Transitfon & $\begin{array}{l}\text { Present } \\
\text { hesults }\end{array}$ & Sayer, et al. 7 \\
\hline $4+2$ & $\begin{array}{r}3.33_{-0.05}^{+0.10} \\
-0.00\end{array}$ & $3.53 \div-.18$ \\
\hline $6+4$ & $\begin{array}{r}3.91+0.3 \\
-0.2\end{array}$ & $4.00 \pm 0.10$ \\
\hline $8+6$ & $\begin{array}{r}5.85+0.3 \\
-0.2\end{array}$ & $5.16 \pm 0.16$ \\
\hline $10 \rightarrow 8$ & $\begin{array}{r}7.30+0.8 \\
-0.6\end{array}$ & $6.81 \pm 0.34$ \\
\hline $12+10$ & $7.33^{+0.6}=0.2$ & $8.2 \pm 1.8$ \\
\hline $14+12$ & $\begin{array}{r}7.90+0.6 \\
-0.4\end{array}$ & \\
\hline $16+14$ & $5.59_{-0.1}^{+0.2}$ & \\
\hline $18+16$ & $7.70_{-1.0}^{+1.5}$ & \\
\hline $20+18$ & $\begin{array}{r}8.73+2.4 \\
-2.0\end{array}$ & \\
\hline
\end{tabular}

These values along with those for $1560 \mathrm{y}$ and 15.0y from Enling et al. B are shom in Fig. 2.64. The same pattern observed for the spin dependence of the reduced transition probabilities for the g-band in the lighter $156,15 \% \mathrm{~b}$ isotope: is also present in $1600 \mathrm{y}$. The $B(E 2)$ value for the $10^{+}+8^{+}$transition is about $40 \%$ above the rotational model prediction and corresponds to a transition quadrupol went of $\sim 8.6 \mathrm{eb}$, still larger than the axim. for the less deformed $15 \mathrm{Dy}$ as seen ir. Fig. 3.64. Althougin the error extracted for two transition probabilities in the s-band $\left(20^{+}+18^{+}\right.$and $\left.18^{+}+16^{+}\right)$are quite large, the data in rig. 2.64 do not seem to indicate evidence for a decreasing collectivity in these higher-spin miners of the rotationaligned band as is observed for $156,158 \mathrm{Dy}$.

1. Un leave from University of Sao Paulo, Brazfl. (Fellow of Fundacás de Amparo à Pesquisa de Sao Paulo.)

2. Present address: Texas MuM University, College Station, Texas.

3. University of Tentressee, Knoxville, Tennessee.

4. Lawrence Berkeley Laboratory, Berkeley. California.

5. D. Cline, private commication; for details of the code, see T. Czosnyka et al., Publ. of University of Rochester, UR-NSRL 263 (to appear in Nucl. Phys.)

6. D. C. Lupiparo, R. L. Rasera and M. E. Caspari, Nucl. Phys. A178, 577 (1972).

7. R. 0. Sayer et al.. Phys. Rev. C9, 1103 (1974). (1984).

8. H. Eniting, et al., Nucl. Phys. M19, 187

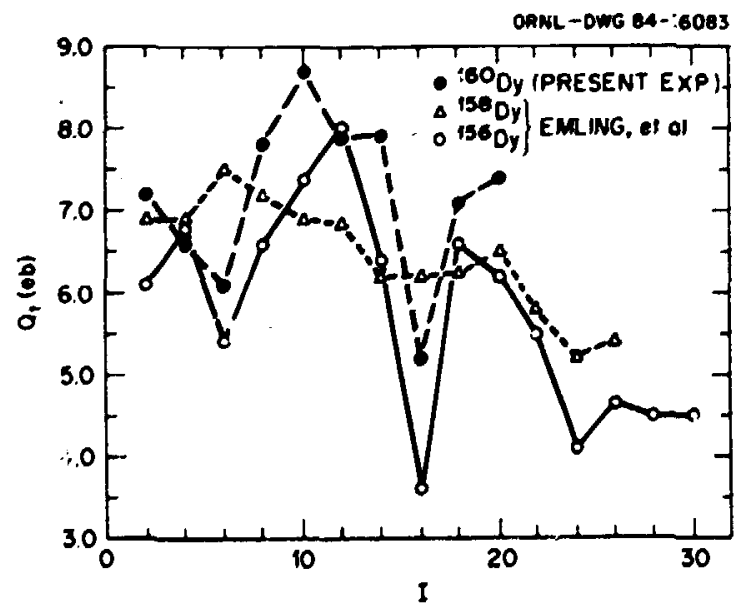

F19. 2.64. Plot of transition quadrupole moments $\left(Q_{t}\right)$ for states in $156,158,1600 y$. 


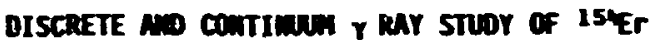
AT HIEH SPIISI
C. Baktash
I. Y. Lee
M. R. Johnson
O. C. Kistner 2
D. Horn' ${ }^{2}$
C. Y. Chen 2
J. X. Saladin ${ }^{3}$
D. 6. Sarantites"
A. J. Larabee 5
T. Schutz 6
H. Oshima
E. der Mateosian 2
A. W. Sunyar ${ }^{2}$
C. J. Lister ${ }^{2}$
0. Dietzsch"
T. Serov"
K. Honkanen"

Study of the interplay between the macroscopic and microscopic effects in muclei as a function of angular mentum has become the focus of nuclear structure studies in recent years. Theoretically, cranked shell model calculations by several groups predict shape transition patterns in the light rare-earth. nuclei wich depend sensitively on the neutron numer, 7,8 For example, the Er isotopes with II $\leq 86$ are predicted to have nearly spherical shapes at low spins and gradually become collate at higher angular womenta (Fig. 2.65). In contrast, for neutron number $M>88$, the equilibrium shapes are expected to change from prolate at spins I $\leq 40$ to triaxial at $I \approx 50$. of particular interest is the predicted transition to superprolate $(B=0.6$ ) shapes for $: 8-82$ isotopes at very high spins. Such large deformations correspond to a major-to-ainor axis ratio of nearly 2:1 and have been observed only in the fission isomers. Experimental verification of these predictions provides a very stringent test of the validity of these calculations, wich have been very successful in describing the nuclear behavior at high angular comenta. To investigate these predicted spin induced shape changes in :54Er, we used the:
${ }^{124}$ Te $\left(3^{4} \mathrm{~S}, 4 \mathrm{n}\right)$ reaction to populate the higt spin states in this mucleus. The 345 beam (i70 reV) as obtained from the double tandea facility at Brookhaven Mational Laboratory. The experibentai setup consisted of six $6 e$ and eleven MaI detectors wich served as a cultiplicity filter. Four of the Ge detectors were surrounded by annular Mal Comton-suppression shields wich gave a suppression factor of better than 2.5 for the 6ceo source. By demanding a coincidence of at least two be and two Mal detectors, high wltiplicity events were. emphasized. Meariy 140 million such events were collected on an event-by-event basis.

In the off-line analysis, the gains of all te detectors are equalized and the fa-fe coincidence data were scanned to generate an $\mathrm{Er}_{\mathbf{\gamma}} \mathrm{E}_{\mathbf{\gamma}}$ matrix. This matrix contained nearly 12 willion photo peak-rhoto peak counts before symetrization, of mich aproximately 406 belong to 154Er. These data formed the basis for a revised and expended decay scheme of-IStEr shom in Fig. 2.66. Angular correlation inforation were used to deduce the witfpolarity of the newly found transitions. The noteworthy features of the decay scheme include:

(1) The level schere shows a shell model structure up to the maximum observed spin of 38.

(2) All of the expected optimal states generated by the aligmment of the valence nucleons around the oblate symetry axis are clearly established in the decay scheme (bold horizontal lines in Fig. 2.66). The configurations of these states $\left(11^{-}, 21^{-}, 27^{-}, 33^{-}, 36^{+}\right)$ are indicated in this figure.

(3) Existence of high energy y rays reeding the highest optial sate, $I^{*}=36^{+}$, reflects the fact that above this state core excitation becomes necessary. Consistent with this picture

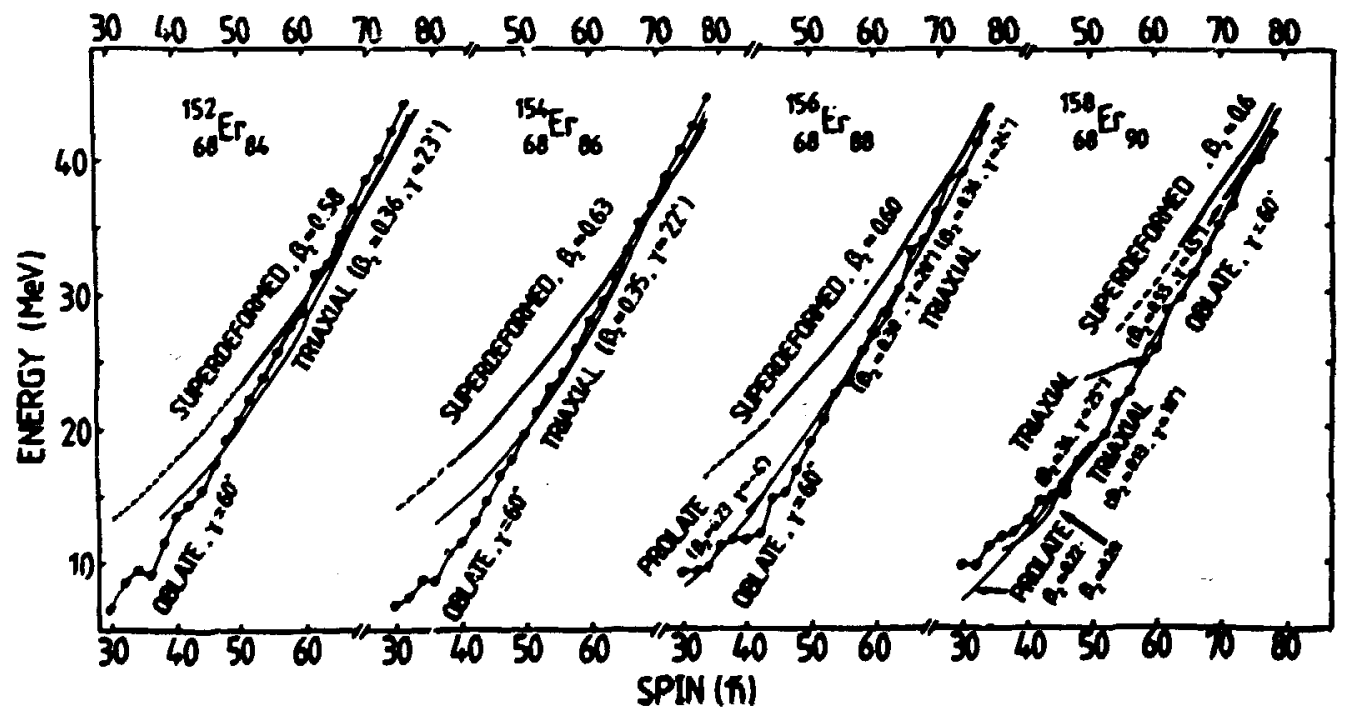

Fig. 2.65. Predicted equilibrium shapes is a function of anoular momentum and excltation energy for erbium-1 sotopes (Ref. 8). 
onar-0ureseseses

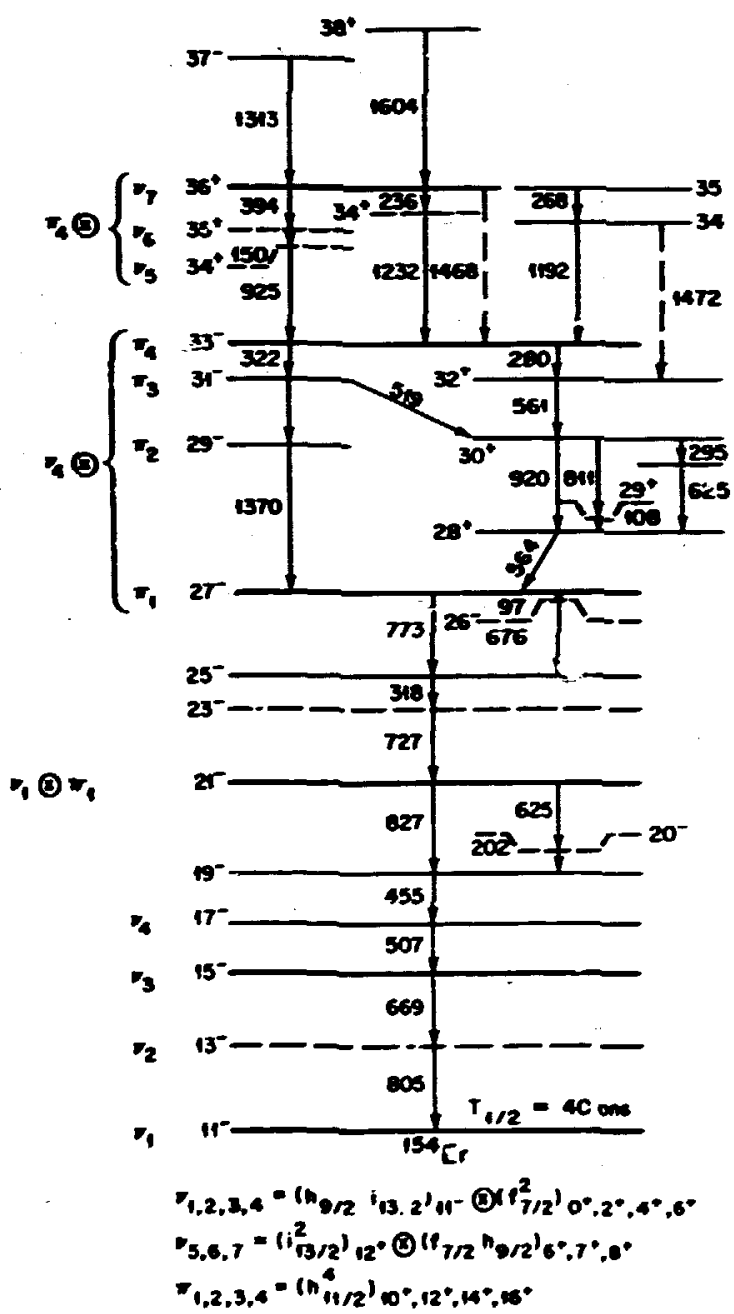

Fig. 2.65. Partial deray scheme of 15bEr showing levels above the $I \pi=11$ - isomeric state. Dasned horizontal lines indicate uncertainty in the level ordering. Neutron (v) and proton ( $\pi$ ) configurations are indicated for several states.

is the fragmentation of the feeding into this state that was experimentally observed.

(4) The level spacings of states with $I^{\prime \prime}=27^{-}, 29^{\circ}, 31^{\circ}$, and $33^{-}$closely resemble those of the $\mathrm{i}^{\prime \prime}=\mathrm{i}^{\prime} \mathrm{t}, 12+, 14+$, and $15+$, states in 1500y formed by $\left(n^{4} 11 / 2\right)$ prot on-excitation. Also, the $1467 \mathrm{keV} Y$ ray betwee: $I^{\pi}=36^{+}$and 33- optimal states is very siaflar to the octupole transition that convects the corresponding opt.final states in $153 \mathrm{Ho}$. They both arise from $f_{13 / 2}+f_{7 / 2}$ neutron transition, and reflect the exctication energy of the $t_{1} / \mathrm{s}$ neutron orbtial.

The above obsarvations are in good agreerent with theoretical predictions that roncollec:ive excitations dominate the yrast structure of .54 Er below spin 50. To verify the predicted onset of collectiv:ty at higher spins, we have searched for the ricige-valley structure in the unfolded $\mathrm{Er}_{\mathrm{T}} \mathrm{EY}$ correlation maps. Such a structure has been coserved in 1520y,' and interpreted to arfse fro collective rotation of a superdeformed shape. We have found no evidence for such structures in the $E_{\gamma}=1000-1400 \mathrm{kel}$ range, where a strong quadrupole structure has been observed in the energy spoctru. This inplies that contrary to the $152 \mathrm{Dy}$ cose, either the collective bands in $154 \mathrm{Er}$ are not fed even at the higlest angular momentun populated in this reaction (60.). or the finterband tiansitions divert the population way from these bands. Either possibility is in conflict with the theoreticai predictions.

1. Sumary of a paper to be published. aY.

2. Brookhaven Mational Laboratory. Upton,

3. University of Pittsburgh.

4. Washington University, St. Louis, mo.

5. University of Tennessee, Rnoxvilie, $T$.

6. On leare from CRH. Strasboug. France.

7. I. Ragnarsson et al., Mucl. Phys. A347, $287(1980)$.

8. J. Dudek. Private Comunfication.

9. B. M. Nyako et al., Phys. Rev. Lett. 52. 507 (1984).

\section{Tb raneA RAY SPECTROSCOPY AS a FINCTION OF SPIII AD TEPERATURE ?}
V. Schutz ${ }^{2}$
C. Baktash
I. Y. Lee
F. K. MeGowan
N. R. Johnson
D. G. Sarantites"

\author{
M. L. Halbert \\ D. C. Hensley \\ L. Courtney \\ A. J. Larabee ${ }^{3}$ \\ L. L. Riedinger
}

Discrete-line spectroscopy in ${ }^{15 \mathrm{~B}} \mathrm{~b}$ suggests coexistence of two different band structures at high spins. Unile the energies of the pr.itiveparity band up to $I=24$ and the negative-parity band up to its ximum observed spin of I = 31 show collective rotational patterns, y rays deexciling states with $I^{\prime \prime}=24^{+}-36^{+}$have nearly equal energies. The latter behavior is interpreted as due to termination of a band wherein the nusleus continuously adjusts its shape parameters wile gradua! ly evolving toward an oblate shape. To ascertain wich band structure dominates above the yrast line and beyond spin 40, we have examined the continuum y ray data. The experimental setup consisted of six Ge detectors placed in the Spin Spectrometer as described in Ref. 5. Following removal of neution signals on an event-by-event basis, all singles spectra were corrected for double-hit events and unfolded using measured response functions of the individual $\mathrm{MaI}$ elements. The response functions also were used to effect the transformation of total pulse-height (H) into excitation energy (E), and coincidence fold $(X)$ to multiplicity $(M)$. The use of the Spin 
Spectrameter allows a detailed characterization of the quasicontinuar $y$ rays. In the following we shall exwine the evolution of these contimun $r$ ray spectra with spin and excitation energy. their muttpolarity composition, and their E $-E_{Y}$ correlation patterns.

Spin and Te-perature Dependeoce. Singles Y ray spectra were generated hy gating simultaneousiy on the strong-discrete transitions in 158\%, ana various $(H, K)$ curts. Typical spectra for two $h$ and two $E$ values are shom in Ffg. 2.67 (a) and (b). The marrou peat around $E_{Y}=$ 750 kel contains minly yrast transitions deexciting states in the $I=26-36$ spin range. The higher energy edge of this peak falls rapidly and does not wove noticeably with increases in the alti-plicity or the excitation energy. Instezd, a second and wech broader structure gradually develops around 1.15 elel at higher mitiplicity and excitotion energies. The nuwer of transi-tions in the lawer energy part of the spectru increases also with increasing witiplicity but decreases with increasing excitation energy. This behavior indicates that the low-eneruy transitions and the transitions in the high-energy bup originate from different regions of the $(E, I)$ map and $w y$ be due to different odes of excitation of the mucleus.
Mitfpolarity Becomposition. The angular distribution has been measured at seven angles using single bal detectors of the spin spectroeter. A smple $A_{2} / A_{0}$ spectro obtained from a Legendre polymowials alysis is displayed in Fig. 2.67 (c) for $k=29$. The decoposition of the eneray spectru into stretched anadrupoles and stretched dipoles is show in Fig. 2.67(d). One oiserves a strong quadrupole peak at 750 kel on top of a veaker dipole perk and a second quadrupole bup centered at 1.15 MeV. The intensity of these three structures increase with increasing mitiplicity. The higher energy quadrupole bup can be interpreted in terns of a collective rotation of the deformed mucleus and $w y$ indicate the onset of a triaxial deforantion of $158 \mathrm{~m}$ at high spin and terperature.

Correlation Patterns. Two-dimensional EY-EY coincidence spectra mave been constructed for different $(H, K)$ bias using all possible coincidences between wy two InI datectors in the Spin Spectrometer. A two-dtimessiomal unfolding method was used to yeld photo-peak-photopeak coincidence speccra. Fig. 2.68 displays such a spectrum corresponding to the difference between two $(H, K)$ bins for a constant $H$. The spectru is dominated by coincidences with transitions in the $750 \mathrm{keV}$ energy region. On? recognizes the

ORNL-DWG 34-15693
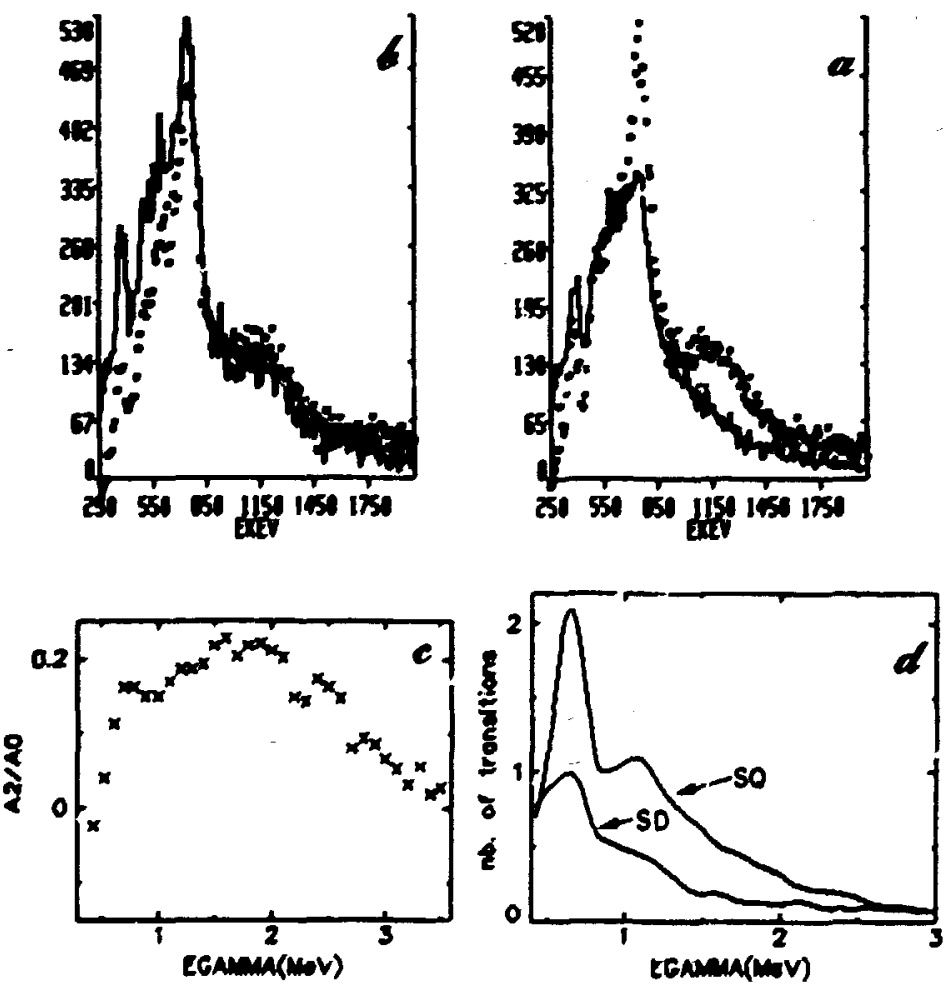

Fig. 2.67. Lnfolded energy spectra for: (a) $M=23$, E $=3.2 \mathrm{MeV}$ (full line); $M=31, E=32 \mathrm{MeV}$ (square). (b) $M=31, E=3.2 \mathrm{KeV}$ (Pull line): $M=31, E=9.4 \mathrm{MeV}$ (square). (c) Angular diseribution coefficient for $M=29$. (d) Stretched dipole and stret,ched quadrupo'e spectra for $M=29$. 


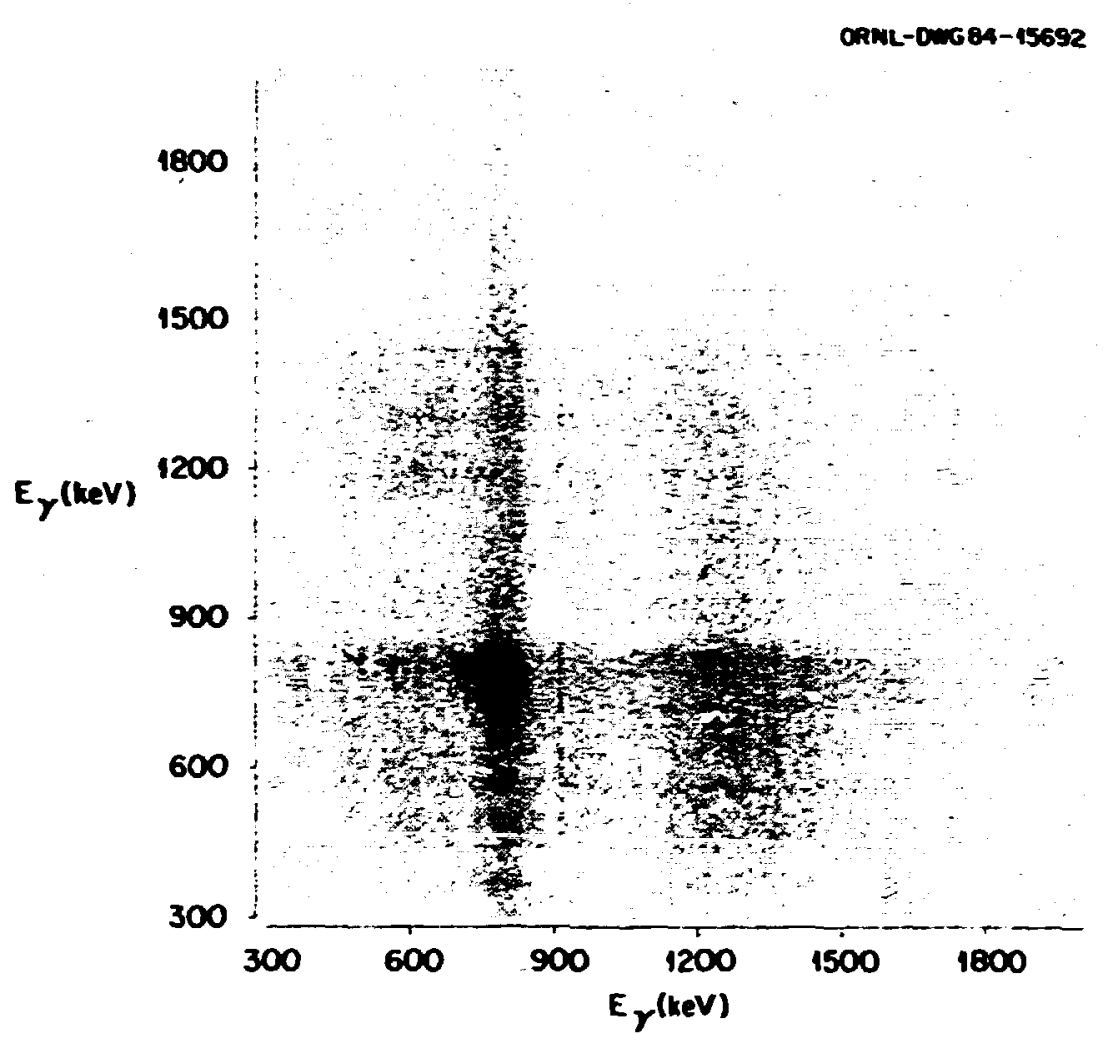

Fig. 2.68. Unfolded coincidence-spectrum corresponding to the difference of "wo ( $H, k)$ binds: (12 MeV, 31) - (12 MeV, 23).

coincidence pattern of the discrete yrast tránsitions with the first backbending at $570 \mathrm{keV}$. Above $E_{Y}=850 \mathrm{keV}$ one observes a cut-off of the valley-ridge structure, wich indicates absence of collective rotational bands beyond an angular frequency of $F_{\mathrm{u}}=0.45 \mathrm{MeV}$. The quadrupole bump observed at I.15- HeV in the single energy spectra originates ainly from coincidences with the 750 keV transitions; only $20 \%$ of the transitions in the $1.15 \mathrm{meV}$ region are in coincidence with transitions from the same energy region. This fact indicates that only a fere transitions decay along rotational bands buflt on possible triaxial deformations which anight exist at high spin and tencerature.

Using the code GNALE, we are now carrying out simulations of the decxcitation pattern of hioh spin-high energy states in 158yb. Inis will aid in the interpretation of the origin of vartous components in the energy spectrum, especfally the ?ow energy dipole component.

1. Sumary of paper tn be published.

2. On leave from CPN, Strasbourg. France.

3. University of Tennessee, Knoxville, TN.

4. Washingt on University, St. Louis, in.

5. C. Baktash et al. "Quasivibrational

Bands at High Spins in isoyb", this section.
E2 ND E4 OETERHIMTIOUS IN $233,234,235,230_{\mathrm{U}}$
J. D. Zuabro²
E. B. Shera ${ }^{3}$
Y. Tanakat
R. A. Kaumann 2
C. E. Bermis, Jr.
M. Y. Hoehn
H. Reuter ${ }^{3}$
R. M. Steffen

Precise intrinsic quadrupole, $\phi_{0}$, wnd hexadecapole moments, $H_{0}$, of $233,234,235,238 U$ have been determined from monic $K, 1, M$, and $M$ $X$ rays. The charge distribution parameters a:e lisied in Table 7.14 , together with the derived intrinsic moments. For $233,235 \mathrm{U}$, seven E2 matrix elements mere independentiy determined. These E2 matrix elements are in good agreem." with the adiabatic rotational model; this agreement is further improved if a correction for $\Delta x$ - I band mixing is included, as indicated in Table 2.15. The measured hexadecapole moments are in good ayreement with shell-correction calculations and Hartree-Fock calculations, as shown in Fig. 2.69.

1. Summary of paper: Phys, Rev. Lett. 53, 1888 (1984).

2. Princeton University, Princeton, 'iew Jersey.

3. Los Alamos National Laboratory, Los Alamos, Hew Mexico. 
Table 2.14. Uranius charge parameters. The perameters $c, a$, and $\left\langle r^{2}\right\rangle^{1 / 2}$ are given in units of $f_{0} ; b_{b}$ in units of $e_{b}$ and $H_{0}$ in units of $e^{2}$.

\begin{tabular}{|c|c|c|c|c|c|}
\hline & Mucleus & $233 v$ & $-\mathbf{v}$ & $235 v$ & 230u \\
\hline \multirow[t]{7}{*}{ This work } & $c^{*}$ & $6.9518(16)$ & $6.9703(13)$ & 6.9859 (17) & 7.0110 (12) \\
\hline & $a^{*}$ & $0.5125(12)$ & $0.5009(10)$ & $0.5029(13)$ & $0.5046(9)$ \\
\hline & $\theta_{0} t$ & $10.29 \div(59)$ & $10.610(57)$ & $10.630(59)$ & $11.188(56)$ \\
\hline & $H_{0} t$ & $2.55(30)$ & $2.49(14)$ & $? .64(10)$ & $2.28(11)$ \\
\hline & $\beta_{2}$ & $0.2431(4 v)$ & 0.2507 (18) & 0.2485 (13) & $0.2653(14)$ \\
\hline & $\beta_{4} *$ & 0.091 (15) & $0.0843(71)$ & $0.0913(45)$ & 0.0572 (49) \\
\hline & $\left\langle r^{2}\right\rangle \mid / 2 t$ & $5.8158(66)$ & 5.8289 (31) & 5.8343 (28) & 5.8604 (23) \\
\hline
\end{tabular}

Hodel-dependent analysis (statistical-uncertainties only).

$\mathrm{T}_{0}$ and $\mathrm{H}_{0}$ include 0.57 and $2.0 z$ model uncertainty, respectivaly.

Table 2.15. Spectroscopic quadripole moments and $8(E 2)$ values for $233,235 \mathrm{U}$.

Spectroscopic and intrinsic ( $q$ and $Q$, rispectively) are given in units of $b$, and the $B(E 2)$ values are given in $(e b)^{2}$. Errors do not include model uncertainties.

\begin{tabular}{|c|c|c|c|c|c|c|c|}
\hline & \multicolumn{3}{|c|}{${ }^{233} U(I=5 / 2)$} & \multicolumn{4}{|c|}{$235 \mathrm{U}(I=7 / 2)$} \\
\hline & \multirow{2}{*}{$\begin{array}{l}\text { Present } \\
\text { experifment }\end{array}$} & \multicolumn{2}{|r|}{ Theory } & \multirow{2}{*}{$\begin{array}{l}\text { Fresent } \\
\text { experiment }\end{array}$} & \multicolumn{3}{|c|}{ Theory } \\
\hline & & $\begin{array}{l}\text { Adiabatic } \\
\text { rotation }\end{array}$ & $\begin{array}{c}\text { Rotation } \\
+\Delta K=1 \text { mixing }\end{array}$ & & $\begin{array}{l}\text { Adiabatic } \\
\text { rotation }\end{array}$ & $+\Delta X^{R C}$ & $\begin{array}{l}\text { Rotation } \\
=1 \text { mixing }\end{array}$ \\
\hline$q(I)$ & $3.663(8)$ & 3.677 & 3.666 & $4.936(6)$ & 4.955 & & 1739 \\
\hline$B(E 2 ; I+I+I)$ & $5.041(16)$ & 5.020 & 5.034 & $4.834(16)$ & 4.757 & & 4.816 \\
\hline$q(I+1)$ & $0.642(30)$ & 0.686 & 0.649 & $1.870(30)$ & 1.93: & & 1.851 \\
\hline$B(E 2 ; I+1+2)$ & $1.756(26)$ & 1.757 & 1.781 & $1.189(41)$ & 1.223 & & 1.267 \\
\hline$B(E 2 ; \quad I+1+1+2)$ & $3.969(37)$ & 3.993 & 3.973 & $4.653(73)$ & 4.611 & & 4.613 \\
\hline$B(E) ; 1+1+1+3)$ & $2.729(41)$ & 2.683 & 2.720 & $2.120(51)$ & 2.117 & & 2.193 \\
\hline$B(E 2 ; I+2+I+3)$ & $2.974(60)$ & 2.948 & 2.305 & $3.778(96)$ & 3.842 & & 3.789 \\
\hline $\mathrm{O}_{20}$ & & 10.295 & (12) 10.303 (13) & & 10.619 & 10) 1 & $10.651(12)$ \\
\hline 5 & & 0.0 & $-1.4(6) \times 10^{-3}$ & & 0.0 & & $-2.5(5) \times 10^{-3}$ \\
\hline$x^{2} / D F$ & & 1.5 & 0.5 & & 6.4 & & 1.6 \\
\hline
\end{tabular}


4. Purdue University, Mest Lafayette. Indiana.

5. C. E. Bemis, Jr., et al., Pt.js. Rer. C 8, 1466 (1973).

6. D. A. Close, J. J. Mlanify, and J. P. Davidson, Phys. Rev. C 17, 1433 (1978).

7. H. Brack et al., Mucl. Phys. 1234, 185 (1974).

B. J. Libert and P. Quentin, Phys. Rev. C 25, 571 (1982).

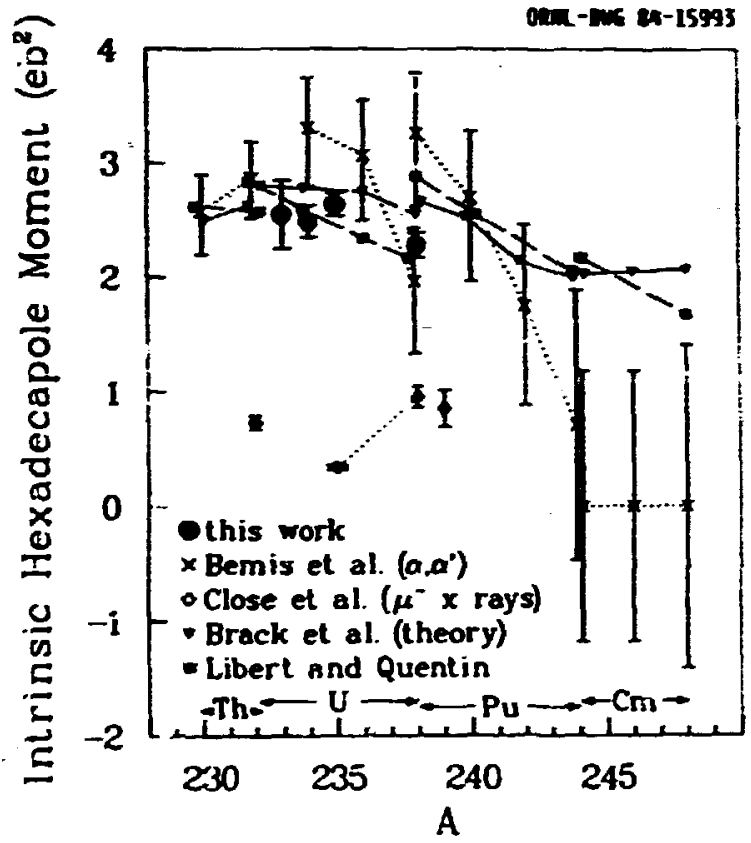

Fig. 2.69. The intrinsic hexadecapole moment vs. A, for thorium, uranium, plutonium, and curium isotopes. The legend indicates the sources of the data plotted. Values for isotopes of the same element are connected by dashed or solid lines. (Bemis et al.. Ref. 5; Close et al.., Ref. 6; Brack et al., Ref. 7; Libert and Quent in, Ref. 8 ).

\section{LICETIMES OF HIGH-SPIN STATES IN I62Yb}
F. K. McGowan
I. Y. Lee
N. R. Johnson
C. Baktash
Y. Schutz'
A. J. Larabee ${ }^{2}$
J. C. Nellis 3

The interplay of collective rotations and single-particle excitations determines the structure of neutron-defictent rare-earth nuclei at high spins. It is generally thought that mony nuclet wich have prolate shapes in their ground states will have a tendency to move toward oblate shapes at higher spins in part due to the alignment of angular momentum along the rotation axis. The discontinuities observed in the yrast sequence of even-A nuclel near $N=90$ around spins of 14 or 16 are mainly due to the aligrment of an $i_{13 / 2}$ meutron pair. These changes in the single-particle structure are expected to influence also the collective properties of the nucleus. The aligrant of the nucleon orbits and its polarizing effect on a soft melear cere should result in a deformation change and in particular in a ioss of the axial sy etry of the mucleus. Aditionia! deforation changes ay be caused by the centrifugal force acting in fast rotating nuclef. Therefore. E2 mitrix elewents along the yrast line should provide information on the two parameters $c_{2}$ and $y$ describing the quadrupole deformation of a nucleus as a function of rotational frequency.

To search for possible shape changes, we have masured the lifetimes of high-spin states in $162 \mathrm{Bb}$ oy eans of the recoil-distance ethod. The nuclei were populated by the reaction IIfcd (50 $\mathrm{Ti}, 4 \mathrm{n}$ ) at a center-of-ass energy of 150 HeV. The recoil distance boice was resigned to fit in the annular cpening of a $25-\mathrm{cn}$ and $25-\mathrm{ca}$ Wal crystal wich acts as a total-enargy filter and thus provides some selectivity of reaction c'annel. The recoil velocity $v=? .177 \pm 0.048$ $\mu \mathrm{m} / \mathrm{ps}$ ( $\mathrm{v} / \mathrm{c}=2.394 \mathrm{x}$ ). Spectrd were obtained at 13 recoil distances ranging from 16 to $5000 \mathrm{~mm}$. The com-plexity of the $y$ spectra limits the number of useful decay curves extracted from the data. For example, the $y$ spectra of $161-163$ Yo contain -150 Y rays or 300 peaks (shifteo + unshifted). In addition the-e are y rays from the $(n, n y)$ reaction on th. materials in the recoil distance device $(F, M a I, A I, F e, M i)$. For instance, the $439.7 \mathrm{key}$ y ray from $23 \mathrm{Ma}$ $(n, n)$ matches the $10^{+} \rightarrow 8^{+}$transition of 439.2 keV in $152 \mathrm{Yb}$. Four nultiplets (Y rays of equai energy) exist in $162 \mathrm{Yb}$. Also, the upbend in the continuation of the ground-s-ate band at the $18^{+}, 16^{+}$, and $14^{t}$ states gives rise to 3 transitions of nearly equal energy.

The time dependence of any decay curve is determined by the lifetime of the initial state of the observed transition and by the time structure of the cascades feeding into this initial state. The feering of a par'icular level, in general, proceeds vfa experimentally observed disciete transitions. However, a fraction of the intensity is fer intr the level through many complex paths (side feeding). The side feeding from the quasi-ganma continuum into a particular level was modeled either by the decay of two states which share the feeding intensity or by the time distribistion of a rotational cascade with parameters based on an effective moment of inertia, an average transition moment $Q_{t}$, and an average $y$-multiplicity (number of steps in the feeder cascade).

The transition quadrupole moments $Q_{t}$, extracted from the reducer transition probabilities along the yrast line

$$
B(E 2 ; 1+1-2)=\frac{5}{16 \pi}<1200 \mid 1-20>2 Q_{t}^{2} \text {. }
$$

are plotted versus the rotational frequency which is obtained from kio. Ey/2 for $1+1-2$ transitions of $K$ : 0 bands. Although the uncertainties are large, the dats shown in Fig. 2.70 for the ground band of $162 \mathrm{Yb}$ are not inconsistent with an enhancement of collectivity for rotational frequencies $k_{w}<0.27 \mathrm{MeV}$. The $a_{t}$ for 


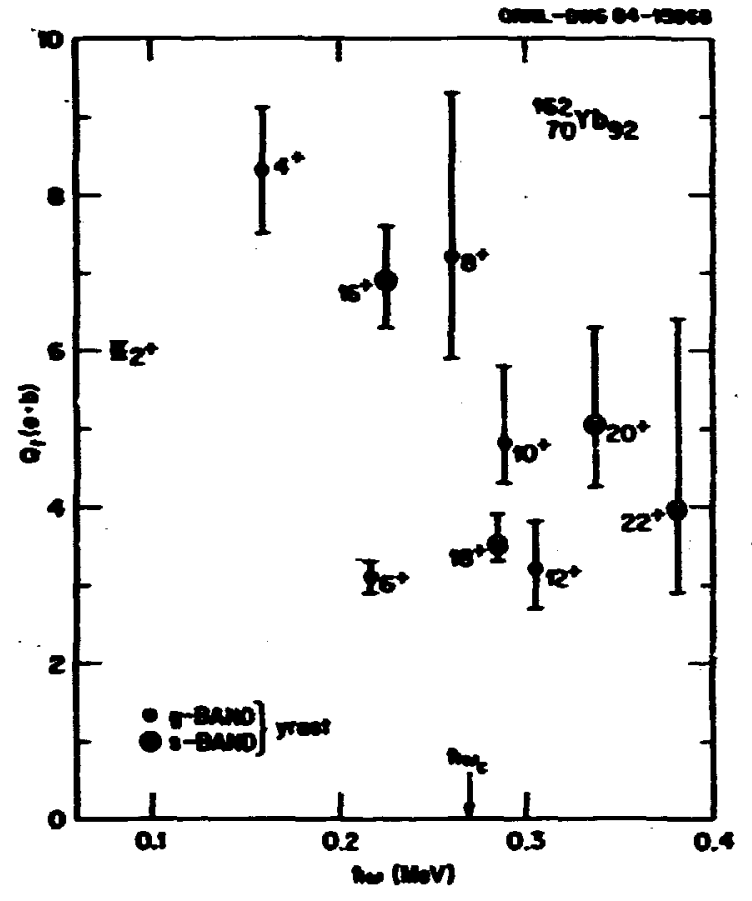

Fig. 2.70. Transition quadrupole ments of some yrast states of $162 \mathrm{Vb}$ as a function of the rotational frequency.

the $6^{+}$state is anomalously small and difficult to understand (the $6^{+}+4^{+}$transition in 156 y was observed to be low). Bocher et al." publislied $Q_{t}$ values for the $2^{+}, 4^{+}, 6^{+}$, and $8^{+}$states of 6 $e-b$ based on a :ery limited knowledge of the level scheme ${ }^{\prime} 162 \mathrm{Yb}$. An analysts of our data with this level scheme yields $Q_{t}$ values in agreement with values given by sochev et al.., but the quality of fit is poor because of the intensity imbalance of the intraband transitions I $\rightarrow$ I-2.

Above $K_{\omega}=0.27 \mathrm{MeV}$, the croseing frequency at wich a pair of $1_{13 / 2}$ quasineutrons align, there appears to be a significant decrease of $Q_{t}$ to a value of $4.5 \mathrm{e} \cdot b$ in both the $g$ - and 5 -bands. The missing intertand yrast transition $14^{+}+12^{+}$ implies a very mak interaction strength beiween the 0-quasiparticle g-band and the 2-quasiparticle s-band. One might consider the yrast band as the continuation of the g-band. However, its g-band character does not prevail: instead, upbending occurs at $K_{w}=0.31 \mathrm{MeV}$. The extracted aligned spin $f(\omega)$ is about an compared to $10 K$ at the first backbend. This upbending could be attributed to the alignment of a second less favored $i_{13 / 2}$ guast neutron pair.

The level structure in $162 \mathrm{Vb}$ is very simtlar to $1550 \mathrm{y}$. 1.e., backbend at $H_{\omega}=0.29 \mathrm{MeV}$ followed by an upbend at $n_{1 s}=0.34 \mathrm{MeV}$. Enling et al.5 at GSI have already published an extensive set of quadrupole transition moments $Q_{t}$ between states mose structure is influenced mainiy by quasiparticle excitations. Therefore. it is of interest to compre the linited results fron i62 of th those from ischy. Delow the first backbend in 1SC fres 6 to $7.5 \mathrm{e}-b$ with increasing otational frequency. The $q_{t}$ values are clearly retarded at ho > 0.29 hel compared with $9.7 .5 \mathrm{ed}$ reached in the g-band just belout the onset of quasiparticle aligument. The orerall trend of $Q_{t}$ from the less extensive resuits of 1620 with The is similar to the results from $15 \mathrm{CDy}$. The loss of collectivity could indicate aither a decrease of $c_{2}$ defornation or a positive $y$ deformation or a cobination of both. Selfconsistent tor calculations by Bengtsson et al.6for the $\Pi=90$ nucleus $16 \%$ suggest potentialenergy surfaces wich are very fiat in the vicinity of the winimu. Therefore, aligned quasiparticles woving in oblate orbitals can easily polarize the ruclear core, i.e., change the wass distribution in the equatorial plame of the prolate corf jielding a triaxial shape. However, the ofserved reduction of collectivity at the larger rotational frequencies iplies a greater degree of triaxiality than recent calcuIations predict.

1. On leave from CRH, Strasbourg, France.

2. Iniversity of Tennessee, Koxoxille, Th.

3. Tennessee Technological Unfversity, Cookerflle, $\mathrm{T}$.

4. B. Bochev et al., Mucl. Phys. 1267, 344 (1976).

5. H. Eml ing et al., Mucl. Myss. M419, 187 (1984).

6. R. Hengtsson et al., Nucl. Phys. M05. 221 (1983).

\section{SPIN ND TEPEDATURE OEPENDEMCE OF THE MLTIPOLAITY OF THE r-RAY CONTIMUM}
D. G. Sarantites!
J. J. Gaardhoje 2
F. A. Dilmanianl
J. D. Garrett 2
M. Rajagopalan I
S. Pontoppidan 2
M. Jyaskelyinen 1,3
P. J. Mol ans
H. Puchta 1,4
Th. Lindblad 6
R. Moodward 1
J. R. Beene
$\mathrm{K}$. Honkanen $\mathrm{I}$
T. Semkow 1
B. Herskind 2
M. L. Halbert
D. C. Hensley
G. B. Hagemann 2
G. Sletton'
I. Y. Lee
H. T. Mtlner
F. Plastl

\section{J. Hattula ${ }^{j}$}

Previous studies of the ultipolarity of the y-ray continuum from rotational nuclel, based either on angular distributions or on internal. conversion coeffict: ints, have led to confliteting results. In particular, sone data on internal conversion 7 indicate that the high-energy continuum ts purely EI milie results obtained at the Holifield laboratory ${ }^{6}$ show that there are significant Ml or E2 cuntributions. We present here preliminary results from the Spin Spectrometer in mich r-ray angular distributions were obtained with respect to the spin direction of the enftting nucleus. 
The ethod of determining the spin arignar:t was first used in Ref. 9, and ay be outlined briefly as follows. The r-ray cascade fra decay of rotational muclei is dominated by stretched E2 transitions (i.e.. $\Delta I=-2$ i wich have a toroidal intensity pattern about tne axis of the enitting mucleus. by searching ior the plane of axim $r$-ray intensity, a computer progra can deduce the most probable cirection of the spin al ignment for each event. Applying this method to a sequence of events, the program builds up the angular distribution of the $Y$ rays as a function of $B$, the angle between the cbserved $r$ ray and the nuclear spin.

The data presented here are from reactions of 230-HeV SoTi on 1245n, one of 49 targets bombarded in a survey experiment. I0, II The compound nucleus is 174hf and the high-spin entry states selected here belong mainly to $274 \mathrm{ff}$. Siaflar data have been obtained for other targets of the survey.

The full analysis involved several steps before the angular distributions could be generated. First, 15 regions of $(h, k)$ space were defined $(H=$ sum of haI pulse height, $k=$ mber of responding detectors per event). These correspond to average $r$-ray multiplicities $M_{y}=16,20,23,27$, and 30 , and to three cuts in excitation energy above the yrast line. Next, the raw NaI spectra for each $(H, k)$ rayion were unfolded (i.e.., corrected for, detector response, including scattering and suming iffects, on the basis of measurements with $r$-ray sources). The lower part of Figs. 2.71 and 2.72 give examples of $E_{y}$ spectra for low and high excitation in the $H_{y}=27$ region.

The spectrus in Fig. 2.71 shows a sharp drop in intensity at $E_{Y}-1.3 \mathrm{MeV}$, the upper edge of the so-called yrast bump known to consist of stretched E2 transitions. At higher $E_{\gamma}$, the characteristic statistical spectrim can be observed. It can be represented by $\mathrm{H}_{Y}=\mathrm{CE}_{Y}{ }^{3}$ $\exp (-E / T)$, where $T$ is the nuclear temperature. The three cuts in excitation energy chosen for this analysis correspond to $T=0.4,0.7$, and $1.0 \mathrm{MeV}$. For the $T=1.0$ cut (Fig. 2.72), the edge of the yrast bump occurs at higher $E_{Y}$, as expacted for a rotational nucleus. However, an unexpected feature is the slow approach to the $E_{Y}{ }^{3} \exp (-E, T)$ shape seen will above the yrast bump. The excess transitions in the intermediate region amount to 0.5 to 1.5 per cascade.

The next step was to crcate angular distributions, as outilined above, for the $80-k e V E_{Y}$ bins shown in Figs. 2.71 and 2.72. (At nigh $E_{Y}$ adjacent bins were combined to maintain reasonable statistics.) Examples for two $E_{Y}$ bins are shown in Fig. 2.73 for the highest region in $\left(T, M_{Y}\right)$ space.

The angular distribution for stretched dipoles ( $A J$ - -1 ) is readily distinguishable from that for stretched quadrupoles $(\Delta I=-2)$, allowing the quadrupole fractfons shown in the upper part of Figs. 2.71 and 2.72 to be deduced. The yrast bumn, as expected, is $100 x$ quadrupole. At low $E$. there is a dipole component that we have reported earlier 12 in the light $Y b$

isotopes. A rew feature is that the quadrupoleifke angular distribution extends beyond the upper edge of the yrast bump and seems to be orm-0us 84-:.1214

$174 \mathrm{Hf}$

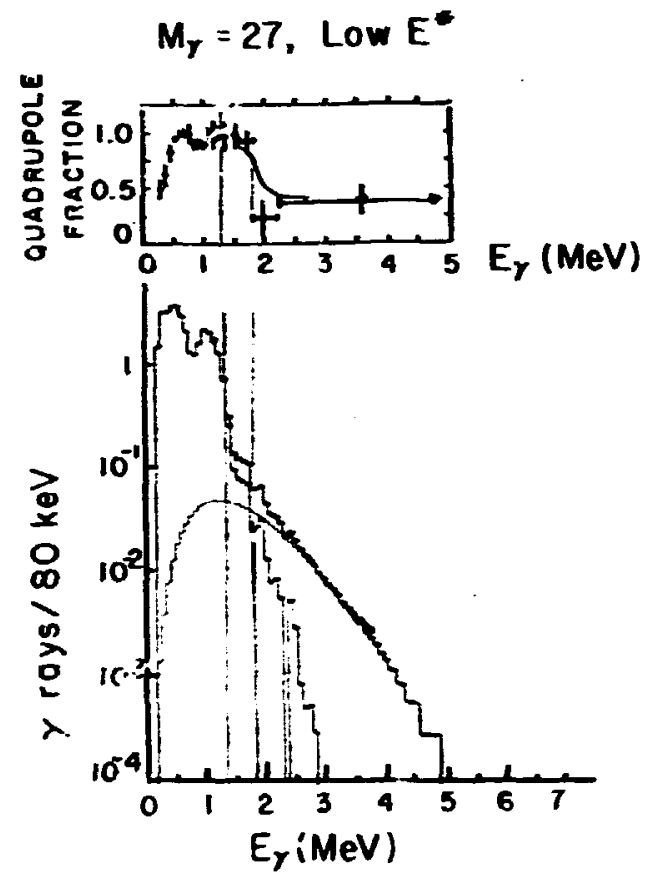

Fig. 2.71. Energy spectrua (lower part) and quadrupole fraction (upper part) as a function. of $E_{Y}$ for the region with average $M_{Y}=27$ and low excitation. The yrast bump ends at $1.3 \mathrm{MeV}$ but the stretched quadrinole-like. character continues to about $1.6 \mathrm{MeV}$. The curve with the broad peak at about $1 \mathrm{MeV}$ is a fit to the expresstion $\mathrm{CE}_{\gamma}{ }^{3} \exp \left(-\mathrm{E}_{\gamma} / T\right)$ with $\mathrm{T}=0.4 \mathrm{MeV}$.

associated with the excess yield noted in Fig. 2.72. Unstretched dipoles ( $\Delta I=0)$ have an angular distribution similar to that of stretched quadrupoles. They can be distinguished by the sign of the A4 coefficient, but $A^{4}$ is not jefined adequately with the present data. If the excess transitions were due to collective quadrupoles, their yield and energy would increase with I for a given T; the data do not show this feature. The other possibility, that they are unstretched dipoles, is consister.t with our conversion-electron results. 8 The two pieces of data taken together indicate that the excess yield centered at $E_{Y} \sim 2.2 \mathrm{MeV}$ is due to unstretched $M I$ transitions. It is interesting to note that Chen and Leander 13 have predicted the existence of $\Delta I=0 \mathrm{Ml}$ transitions at $2.3 \mathrm{MeV}$ in similar nuclef for bands with small signature splitting.

1. Washington Unfversity. St. Louis, 10 63130.

2. Niels Bohr Institute, Copenhagen, Cenmark.

3. Present address: University of Jyvöskyly, Finl and. 
4. Present address: Sektion Physik,

Universitst Winchen, 8046 Garching, West Germiny.

5. Liverpool University, Liverpool, U.K.

6. Research Institute for Physics,

Stockholn, Smeden.

7. S. J. Feenstra et al., Phys. 803, 183 (1979).

8. L. Mesterberg et al., Phys. Rev. Lett. 41, 96 (1978).

9. F. A. Dilmanian et al., Phys. Rev. Lett. 49. 1909 (1982).

10. D. 6. Sarantites et al., ORR-6004, 110 (1983).

11. J. J. Gaardhsje et a1., Phys. Scripta T5, 178 (1983).

12. M. Jytiskel ginen et al., Phys. Rev. Lett. 49. 1387 (1982); Phys. Lett. 1198, 65 (1982).

13. Y. S. Chen and G. A. Leander, Proc. Conf. on High Angular Momentum Properties of Muclei. ed. N. R. Johnson, pp. 327-338 (1982).

ORIL-OWG B4-17213

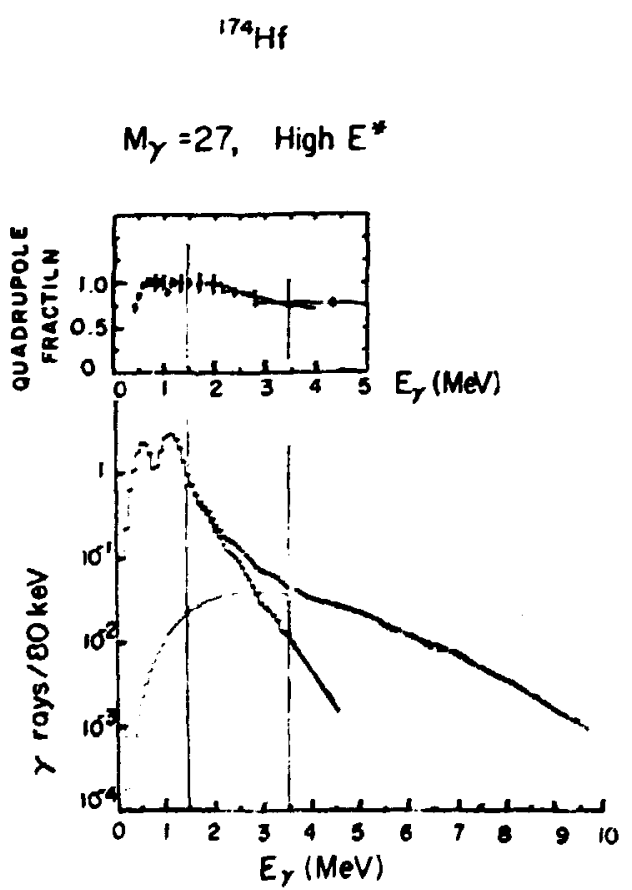

Ffg. 2.72. Same as Fig. 2.71 for highexcitation region $(T=1.0 \mathrm{MeV}$ ). The upper edge of the yrast bump occurs at E $\sim 1.4 \mathrm{MeV}$, but the quadrupole-like character of the radiation persists to consfderably higher Ey than in Fig. 2.71. The excess yield of $r$ rays above the yrast bump that is not accounted for by the $E_{\gamma}{ }^{3}(-E Y / T)$ shape is larger than in Fig. 2.71.
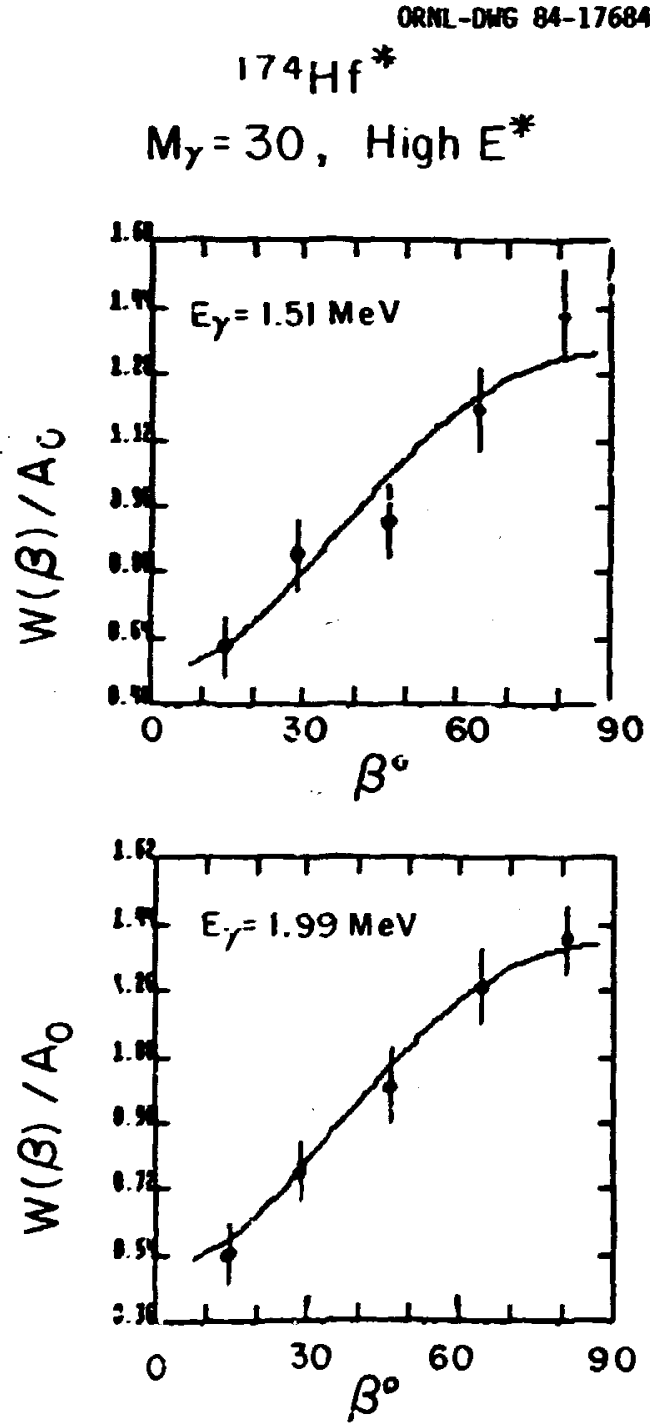

Fig. 2.73. Gamma-ray angular distributions with respect to the spin direction of the emitting nucleus for the $(H, K)$ region corresponding to $T=1.0 \mathrm{MeV}, M_{Y}=30$. Data are shown for two bins of y-ray energy.

\section{REACTION RECHANISH FOR ONE NO THO PARTICLE TRANSFER HITH VERY HEAVY IONS}
M. H. Guidry
S. Juutinen 1
X. T. Liul
C. R. Bingham!
A. J. Larabee
L. L. Riedinger!
L. H. Coureneyl
C. Baktash
I. Y. Lee

M. L. Halbert

M. P. Fewell

D. Cline 2

B. Kot Iinskt2

A. BEk I I n 2

D. Sarantites ${ }^{3}$

T. M. Senk ow ${ }^{3}$

K. Honkanen ${ }^{3}$

M. Rajagopalen 3

It has been suggested that transfer reactfons with very heavy fons (A > 40) witht hold 
considerable proaise as a tool fr.r scudying nuclear structure. 4,5 Resolution of the closely spaced collectire states excited by heavy ions is achieved most easily using high resolution Ge $r$-ray detectors to observe the deexcitation gawa rays in coincidence with the scattered ions. To reconstruct the initial distribution one needs the r-ray feeding patteru. The nonyrast portion of the transfer population will decay to the yrast. line by enission of a quasicontinuum cascade of $Y$ rays, with indiviaual transitions difficult to resolve. Both the population of discrete states on or near the yrast line, and the population of the quasicontinuum present unique opportunities to study nuclear structure. However, to fully exploit these opportunities it is necessary to separate these two portions of the population. To do so requires both tot:il energy and angular moment um information for the states populated.

Here, we report on the use of the ORNL Spin Spectrometer 6 to measure total $\gamma$-ray energies and multiplicitios for heavy-ion transfer reartions populating high spin states in the reaction $161 \mathrm{Dy}$ (58 $\left.\mathrm{Mi},{ }^{59} \mathrm{Mi}\right) 160 \mathrm{Dy}$, with the $E_{\text {iab }}=$ 270 MoV $S_{\theta} \theta_{\text {Ni }}$ beams (giving $\theta_{L A B} \sim 130^{\circ}$ ) produced by the Hollified Heavy Ion Research Facility.

The experimental procedure involves detection of $Y$ rays by $G e$ detectors in coincidence with scattered beam-like and projectile-like fragments wich are observed using large solid angle pesition-sensitive parallel-plate avalanche detectors. Each particle-particle-r coincidence trigge.'s the Spin Sper.trometer, wich is used to determine the total energy and mitiplicity of the resulting $r$-ray cascade in the standard manner. 6

Figure 2.14 shows the total energymultipificity $(E, M)$ spectrum gated on the strong ground-band transitions in $1600 \mathrm{D}$. In addition, we show as a dashed line the lowest contour only for the corresponding inelastic excitation of $16^{1} \mathrm{Dy}$. The $(E, M)$ distribution is clearly quite different for the two reactions.

The yrast line sketched in Fig. 2.74 is that for $1600 y$. It represents the total energy and multiplicity expected if the yrast line of $160 \mathrm{D}^{\prime}$ and ground state of ${ }^{59} \mathrm{Ni}$ were populated in the transfer and is deduced fram the known energies in the $1600 y$ yrast band by assuming that the transitions are stretched E2's near the yrast line, and that the spin spectrometer multiplic1 ty is corrected to account for the trigger $Y$ ray, and the mostly internally converted $2^{+} \rightarrow 0^{+}$transition. In addition, $\sim 400 \mathrm{KeV}$ is added to the total energy to account for these missing r-rays. This prescription is consistent when checked against the expected results for the Coulomb excitation reaction ${ }^{16}$ ? Dy ${ }^{6 \mathrm{H}} \mathrm{Ni}$, GRNI') I $62 D y$, and is expected to be most relfable near the bottom of the distribution in Ffg. 2.74. The narrow width of the distribution of total energy with multiplicity leaves littie room for statistical transitions. Therefore, the prescription relating multiplicity to spin for the yrast line may be applifed to the whole distribution of Fig. 2.74 with an error not much beyond the resolution in 4 (typically 1-2 units). With this assumption. Fig. 2.74 becomes a map of the cross section for populating a

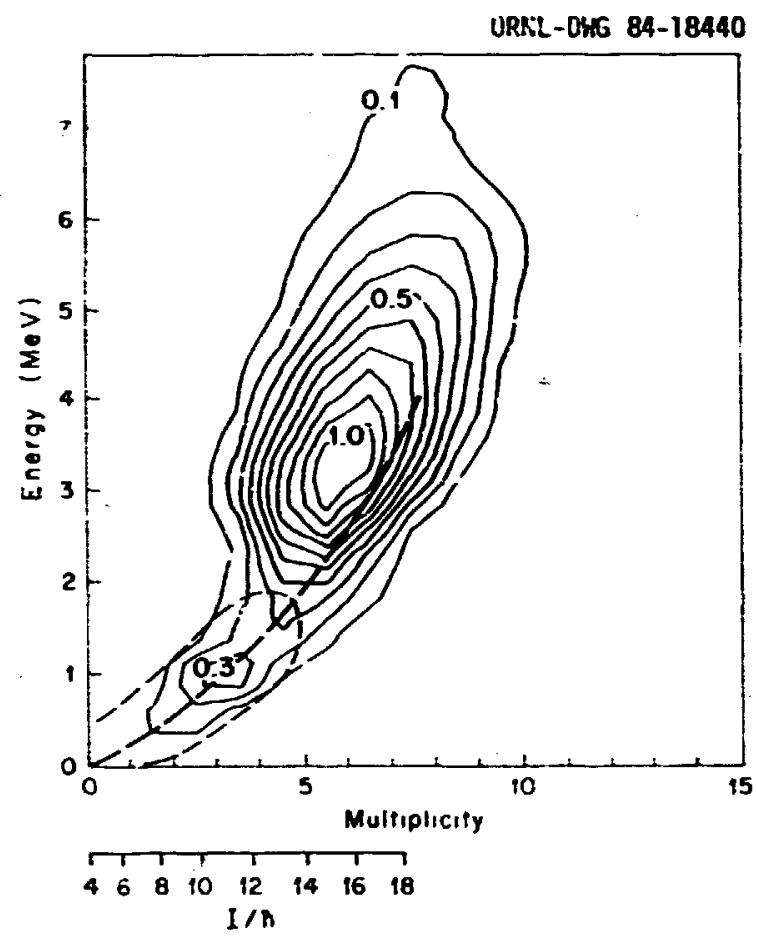

Fig. 2.74. The total energy - multiplicity $(E, M)$ distribution for the transfer reaction

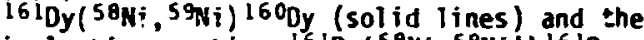
inelastic reaction $16 \mathrm{IDy}\left({ }^{58} \mathrm{Ni}, 5 \mathrm{ANi}^{\circ}\right) 16 \mathrm{I}_{\mathrm{Dy}}$ (dashed line, outer contour only). The distributions are gated on the strong ground band transitions in $16^{\circ} \mathrm{Dy}$ and $16 \mathrm{IDy}$ respectively, as observed by the Ge detectors. The heavy dashed 1 ine represents the $1600 y$ yrast 1 ine. Events below it reflect finite resolution. The estimated resolution in $E$ and $M$ is about $40 \%$.

given differential element of total energy and angular momentum in the internal structure of the ${ }^{59} \mathrm{Ni}$ and ${ }^{160} \mathrm{Dy}$ proouct nuclei.

Figure 2.75 shows a cranked shell model calculation? for bands near the yrast line in I6nDy. The average alignment is $-6 \mathrm{~h}$ for the low lyin t bands in this region. A theoretical model for transfer to the quasicontinuum will be discussed separately. ${ }^{8}$ Essentially kinematic effects associated with 0-windows, L-windows, and binding energies of the transferred particles conspire in the reaction $16 \mathrm{IDy}_{\mathrm{D}}\left(5_{\mathrm{N}} \mathrm{N1}, \mathrm{5}^{5} \mathrm{N1}\right)$ $16^{\circ}$ Dy to 1 imit strong transfer to a band of states lying within 1-2 MeV of the yrast line (inset to Fig. 2.75). The dominant factor in this case is that excitation above the yrast line requires the removal of more tightly bound particles from $1610 y$. Ihis strongly suppresses the form factor for transfer greater than 1-2 MeV above the yrast line, unless particles are transferred between heated nuclei with particles exclted before transfer to less tighly bound states. The population pattern seen in Fig. 2.74 provides direct evidence for a cold mechanism giving large cross sections for 
ORIL-DIG 84-18441

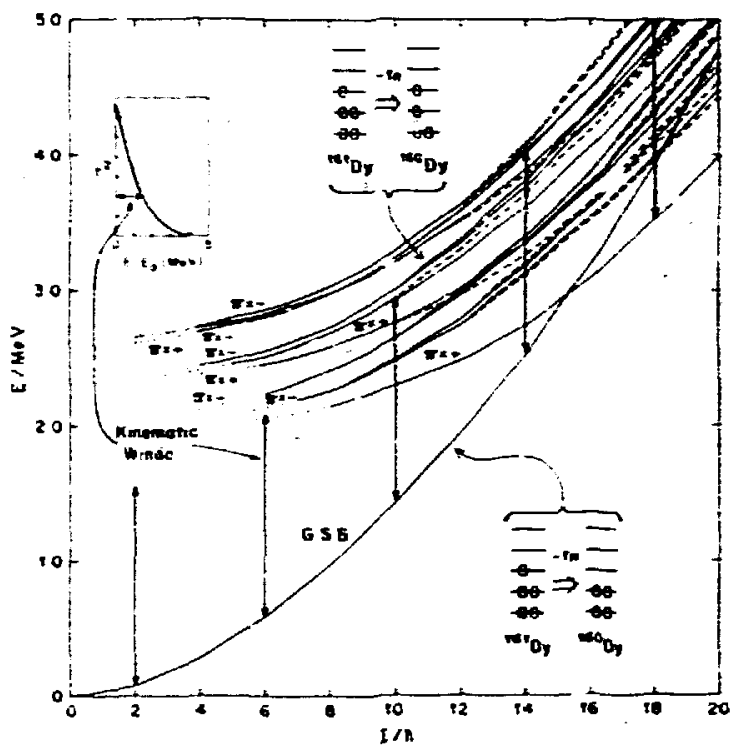

Fig. 2.75. Low-lying bands in 1600y calculated using the cranked shell model with parameters reflecting the standard systematics in this region. The kinematic window discussed in the text is indicated by the inset ind the arrows, and strongly suppresses transfer to states ly in: outside the window. The other insets indicate schematically the kind of picr'p process populating the bands.

transfer between very heavy ion; if ground-state Q-values are chosen properly. This cold transfer mechanism is obviously significant for using these reactions as spectr scopic tools, and for a general understanding of heavy-ion reartion mechanisms. In addition, it raises interesting pussibilities for populating states and nuclef inaccessible to other react fons.

The qualitative and even quantf tat fue features of the population distribution are easily understood. Because of the pairing gap, at low angular monenta $(I<10)$ only the ground band, and possibly a few collective bands. lie in the kinematic window. The population in the lower maximum of Fig. 2.74 prfinarfly represents di rect population of the ground band. The width of the lower portion of the distribution is conifstent with the inherent total energy resolution of the experiment. As the angular momentum is increased the 2-quasiparticle states come down relative to the yrast 1 ifne. Above I $\sim 10$ the 2-quasiparticle bands begin tu fall within the kinemstic window, and the sudden brodening of the total energy distribution represents the kinematic accessibility of those non-yrast bands. The peak in this non-yrast population lies $\sim 500-1000 \mathrm{keV}$ above the yrast line at I 14-16. This is where, natvely, one would expect the aligned bands to be strongly populated, that is, 8-10 units of collective angular momentum (peak of the lower mintmum) plus $\sim 6$ units of aligned angular momentum gives the angular momentus of the uper maximan, and the excitation abore yrast coincides quantitatively with the avel density of aligned band's in Fig. 2.75, wighted by a kinematic factor decreasing approximately exponentially with energy above the jrast line. This qualitative discussion neglects the differences anong spectroscopic factors for different aligned bands. This should be included to provide more stringent tests of high-spin models.

This analysis assumes that the ourat of collective angular momentu is the sane for transfer to the ground and aligned bands. This is not generally crue, the to the angle dependent form factor discussed theoretically in Refs. 4 and 5, and for wich we provide experimental evidence below. However, it should be a good assuaption for the average properties of a group of bands.

These simple considerations account for all the basic features of the quasicont imum spectra in a natural may. This inplies that the data of Fig. 2.74 are related directly to the density of 2-quasiparticle states as function of energy and angular momentum. and provides clear evidence fur a gap between the ground and excited 2-quasiparticle states wich decreases with angular monentum.

1. University of Tennessee, Knoxville, $M$.

2. University of Rochester, Rochester, $\boldsymbol{W}$.

3. Mast ington University, St. Lovis, $M$.

4. M. H. Guidry, T. L. Kichols, R. E. lleese, J. O. Rasimussen, L. F. Oliviera, and

R. Donangelo, Mucl. Phys. A361, 275 (1581).

5. C. H. Dasso, T. Dossing, S. Landowne, R. A. Broglia, and A. Winther, Mucl. Phys.

A389, 191 (1982).

6. M. Jäskel Jifnen, et al., Nucl. Inst. and Meth. 204, 385 (1983).

7. R. Bengtsson and S. Fravendorf, Mucl. Phys. 1327, 139 (1979).

8. M. M. Guidry, G. Leander and M. Kincatd, to be publisned.

\section{CROSS SECTION FOR POPULATION OF HIGH SPIN STATES} IN TRANSFER REACTIONS
M. H. Guidry
S. Juut inen
X. T. Lful
C. R. Bingham!
A. J. Larabee
L. L. Riedinger
L. H. Courtney
C. Baktash
I. Y. Lee
M. L. Halbert
M. P. Fewell
D. Citne 2
B. Kot IInskt ${ }^{2}$
A. Bäklin ${ }^{2}$
D. Saraneftes 3
T. H. Senkow
K. Honkanen ${ }^{3}$
M. Rajagopalen ${ }^{3}$

We report on the use of the ORNL Spin Spectrometer to measure total $r-1$ ay energies and multiplicities for heavy-ion transfer reactfons populating high spin states in the reac-

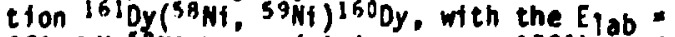
$270 \mathrm{MeV} 5 \mathrm{~S}_{\mathrm{N} f}$ beams (giving $\mathrm{G}_{\mathrm{LAB}} \sim 130^{\circ}$ ) produced 
by the Holified Heary Ion Research Facility. Relating the total $\gamma$-ray energies and altiplicities to the enercy and angular momeris of the states populated allowed separation if the direct population of the yrast "ne from that feeding the yrast line by quasicontinesua transitions. Moreover, cross sections for oneparticle transfer to yrast high-spin states and the near-yrast quasicontinuul were deternined.

The experimencal procedure invel ves detection of $r$ rays by 6 e detectors in coincidence with scittered bean-like and projectile-like fragments wich are observed using large solid angle position-sensitive parallel-plate avalarche detectors. Each pari icie-porticle-r coincidence triggers the Spin Spectrometer, wich is used to determine the total energy and mitiplicity of the resulting $\gamma$-ray cascade in the standard manner. ${ }^{4}$

Discrete 1 ines up to the $18^{+}+16^{+}$grast transition are seen for the reaction product $1600 y$. Peak intensities range from $~ 200$ counts for the $19^{+}+16^{+}$, to $\sim 30,000$ counts for the $4^{+}+2^{+}$transition. No strong channels other than inelastic scattering and i-neutron pickup are seen, and the spectrum quality is comparable to that for sub-barrier reactions. In a separate contribution in this report we snow the tota! energy-iultiplicity $(E, M)$ spec $t$ rum yated on the strong ground-band transitions in $160 \mathrm{Dy}$. There, the general features of the quasicontinuum transfer population are discussed. In this contribution we discuss the population of the discrete states.

For the discrete yrast states obsorved in the transfer spectrum it is possible to be more specific than in the case of the quasicontinuum. By gating in the (E, M) plane to exclude the feeding from the quasicontinuum, a Ge spectrum may se constructed for direct population of the yrast states. The cross sections for di rect population of individual ; rast states may be deduced from this Ge spectrum by correcting for feeding. The results are shown in Fij. 2.16 , along with the corresponding cross sections for the inelastic scattering reaction ${ }^{1620 y}\left({ }^{58} \mathrm{Ni}\right.$. $58_{11}$ ') $1620 y$, mich should be quite similar to that expected for the inelastic reaction ${ }^{1600 y}$ $(58 \% 1,58 \mathrm{NI})^{1600 \mathrm{Dy}}$. No spectal provision was made in the experiment to detect the low energy and highly converted $2^{+}+0^{+}$transition, so only states with $I^{\pi}>4^{+}$are shown. Note that because the total energy resolution is several hundred $\mathrm{keV}$, and the multiplicity resolution is. 1-2 units, such $(E, M)$ gating also might include some non-yrast states. However, a detalled comparisol of the yrast gate in the transfer data to the corresponding gate in the inelastic scattering (assumed mostly yrast) shows that up to $I \sim 12^{+}$the transfer yrast gate contains lass non-yrast population than the experimental uncertainty in the popilation. This concluston is buttre'ssed by the absence uf any strong peaks representin decay or non-yrast to yrast states. Within the yrast gate below spin 12 the level densities should be suffictently low that, if non-yrast states are populated, the deexcitation $\gamma$-rays should be resolved. Above $12^{+}$the statistics are not good enough to rigorously exclude non-yrast contributions from the cross
ORAL-DWG 84-18442

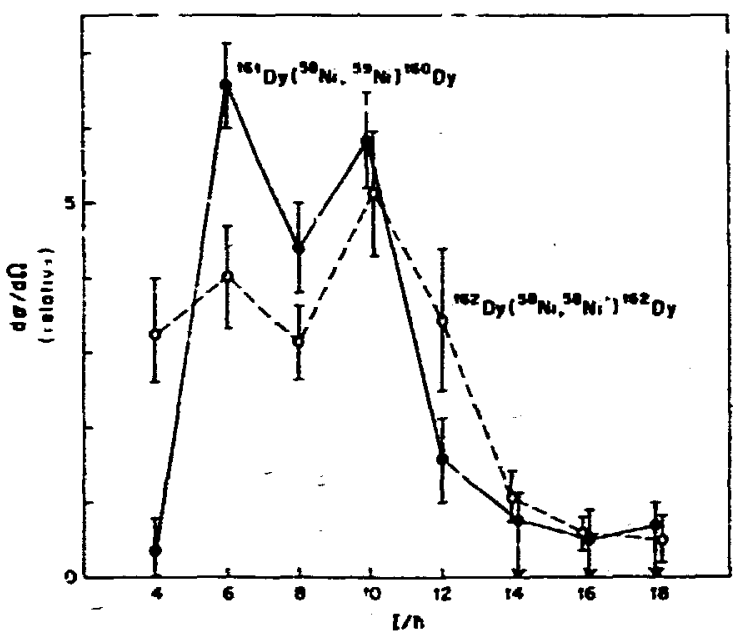

Fig. 2.76. Differential cross sections (arbitrary scale) at the grizing angle for popu1 ation of ground band states in the transfer raiction $16 \mathrm{DDy}$ (58Wi,59i)160Dy (solid) and the inelastic scattering reaction I620y (58Ni, 5ENi')!62Dy (dashed).

sections, meaning that the small cross sections for direct population of the $14^{+}-18^{+}$actually represent upper $1 \mathrm{f}$ ifits.

The most striking feature of the transfer cross sections is the dramatic suppression of the $4^{+}$state relative to that seen in inelastic scattering. Such a minimum is too Heep to ie explained by the standard interferences giving the oscillating cross sectio.:s for inelastic scattering, but has a simple explanation in terms of the geometrical surface form factors for transfer discussed in Refs. 5, 6. Popula$t$ ton of the ground band of $1600 y$ requires pickup of the $[642] 5 / 2^{+}$neutron from 161 Dy if we assume a Ni isson model. For maximum transfer from this orbit, the combination of damping bj the imaginary potential, barrier penetration effects, and distribution of the single particle wavefunction on the surface, favors a collision between the heavy ions with approximately a $45^{\circ}$ angle between the symmetry axis of the rotor and the line joining the fons at closest approach. This condition favors high collective angular momentum transfer. 5 This is precisely what is suggested in Fig. 2.76, and is the mos: likely explanation for the extremely low $4^{+}$cross section.

1. University of Tennessee, Knoxville, TN.

2. University of Rochister, Rochester, NY.

3. Mashington University, St.. Louts, Mo.

4. M. Jayskelaginen, et al., Nucl. Inst. an ! lieth. 204, 385 (1983).

5. M. H. Gutdry. T. I.. Nichols, R. E. Neese, J. O. Rasmussen, L. F. Oitviera, and

R. Donange10, Nucl. Phys, A361, 275 (1981). 
6. C. H. Dasso, T. Dossing, S. Landowne, R. A. Broglia, and A. Winther, Nucl. Phys. A389, 191 (1982).

MUULAR FIENTM TRASFER IN VERY HEAVY ION BIRECT REACTIONS
A. H. Guidryl R. K. Kincaid R. D.olangelo 2

One- and two-particle transfer reactions with very heavy ions ( $A>40$ ) provide a unique oppoitunity to obtain information about single particle wavefunctions and particle-particle correlations for states which are highly collectively excited. The results of several recent experiments ${ }^{3-6}$ indicate that large cross sections are being observed for the direct, cold population of collective states in such transfer reactions. Given that these experiments are feasible, it becomes important to realiscically estimate how wich rollective excitation can be present in such systems when the transfer takes place, since the more collective the excitation at the time of transfer, the more sensitive the data is Iikely io be to the interplay of collective, single-particle, and pairing trodes.

He tave specifically addressed the case of rotational excitation to deterwine the ampunt of angular somentum in the region of the classical turning point, where the transfer is most likely. The reaction is viewed as a process in whir'. there is strong collective inelastic excitation of the entrance channel as the nuclei approach each other, transfer of one or two particles near the distance of closest approach. and strong inelastic excitation in the exit channel as the ions recede from each other. ${ }^{\text {? }}$ Although the nuclei may be highly collectively excited at the time of transfer, experimental data near the barrier ${ }^{3}$ suggest thew to be cold (near the yrast line). We presume that for collisions near the barrier it is necessary to describe the inelastic excitation in entrance and exit channels by coupled channels methods, while the transfer itself might be subject to a low order perturbation approach. For present purposes, however, a purely classical calculation may be used to provide a good estimation of the angular momentum transfer near the classical turnfing point (close agreement of predictions by classical and quantum mechinical coupled channels calculation has been varified for a representative test case.)

Exact classical trajectories for head-on collisions were calculated using a complex Woods-Saxun potential wich is a function of the radial separation of the ions $r$ and the angle $x$ between the syminetry axis of the rotor and the line of centers between the ions. For the Coulomb excitation, well-known parameters from the literature were used. A series of inelastic scattering experiments using $40 \mathrm{Ar}$ on rare-earth targets 8 was used to determine the nuclear potential parameters. The energies for the colliston were chosen to bring the fons to a nearest radial separation of $\sim 1.5\left(A_{1}\right) / 3+$ $\left.A_{2}{ }^{1 / 3}\right) \mathrm{fm}$, where the data indicate the transfer cross sections are largest.
Figure 2.77 shows calculations of the collective angular omente in the classical rotor near the turning point, weighted by the transfer form factor. for several heavy ions on a representative rare earth target and a representative

ORNL-DIS 84-18443

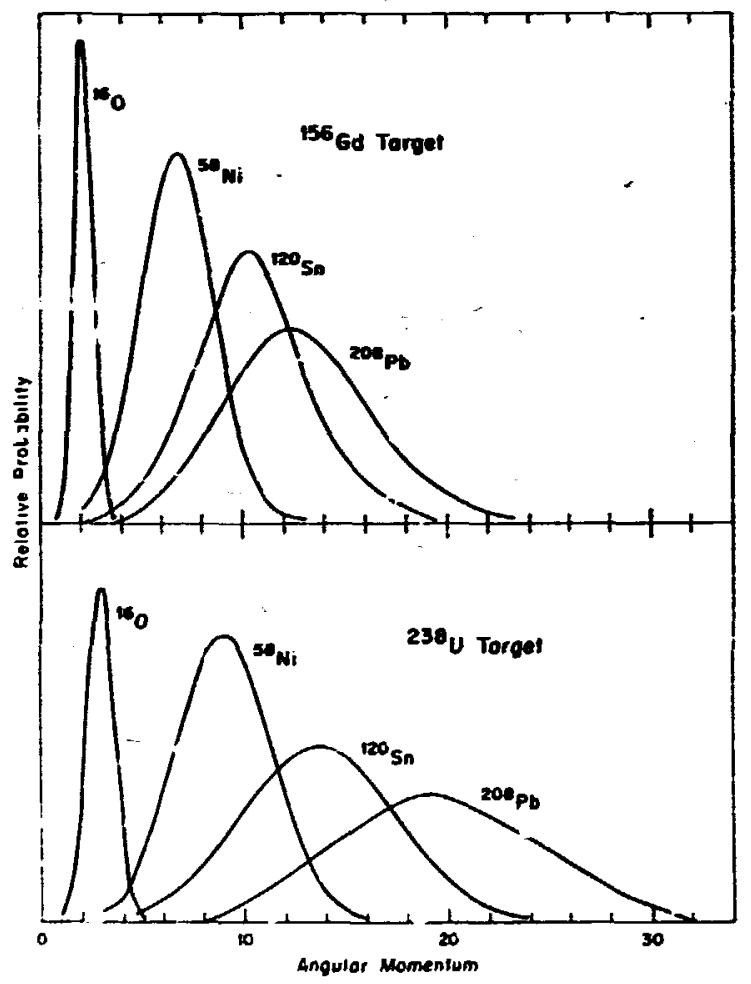

Fig. 2.17. Most likely collective angular momentum when particles are transferred for some representative heavy ion systems.

actinide target. The calculation assumes the transfer probability to be proportional to the square of a form factor, dropping exponentially tn ore-half of its value at the turning point when the ions are I for outside the turning point. Whereas with oxygen ions we may expect rather small angular momentum transfer, with Sn or $\mathrm{Pb}$ the transfer between states with collective angular momenta in the range $I=10-25 \mathrm{~h}$ appears quite feasible. These calculations consider only the collective excitation of angular momentum. The transferred particle(s) may also carry angular momentum, wich would further increase the angular monentum populated direct iy by the transfer. Since large nuclear structure modification by collective angular momentum in this angular momentum regime is expected, these reactions hold considerable promise as a speceroscopic tool.

1. University of Tennessee, Knoxville, TN.

2. Ntels Bohr Institute, Copenhagen, Denmark. 
3. M. M. Guid et al to to be published.

4. P. A. Butler, et al., private comunication and to be published.

5. A. Machiavelli, et al.. private comonication and to be published.

6. C. Lauterbach, et al., private comanication and to oe published.

7. H. W. Guidry, et al., "hucl. Phys A361, 275 (1981).

8. R. E. Neese, et al ., Phys. Lett. 858, 201 (1979); R. E. Neese, Ph.D. Thesis, University of Tennessee, 1981 (unpublished).

9. M. W. Guidry, et al., Nucl. Phys. M30, 485 (1984).

\section{A MOOEL FOR HEAT ION TRAMFER

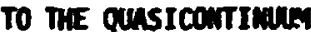

\author{
M. M. Guidry l R. H. Kincaid \\ 6. A. Leander ${ }^{2}$
}

In recent experimerts we have observed large cross sections populating the region within a few MeV of the yrast line and extending up to I 15-20 by heavy ion transfer reactions on rareearth nuclei. ${ }^{3}$ Since little direct information exists on the structure for muclei in this regime of temperature and angular momentum, a theoretical understanding of the data being obtained could provide insight into the way nuclei accomodate internal excitation energy and angular momentum. Such information is significant in its own right, but also is important for a istafled understanding of more complicated processes such as deep inelastic collifsions, since such reactions may proceed through an initial doorway involving energy transfer by inelastic and few-particle transfer mechanisms.

As a first approximation we might separate the reaction mechanism into that part responsible for the angular momentum, and that part giving rise to finternal excitation (heating). Then, since such a separation is surely unrealistic, we may consider the interplay of these effects in the overall reaction mechanism in further work. The beginnings of such an approach are outlined in this report.

We first assume that, for the strongly deformed nuclei and very heavy fons under consideration, the dominant transfer mechanisa involves strong inelastic excttation up to the turning point region, transfer of a particle or twr and strong inelastic excftation as the nuclef recede from each other.

With these assumptions, the average angular momentum transfer may be described by inelastic excitation in the entrance and exit channel o? :-u with a spread in angular momentum transferred by the particle. The latter can be est1mated :rom the distribution of single-particle angular momenta avallable in the excitation regi on or interest (e.g. using the Cranked Shell Mode1), and by imposing an L-matching window for the particle transfer. Analytical rormulae have been given for such L-windows, and methou: for calculating the inelastic excitation have been amply described in the literature.
Here we ignore the angular monentum transferred by the particles and concentrate on the factors governing the tcidi energy of the states populated in the transfer reactions. The yrast lines are taken to define zero temperature for the system and excitation energy is measured from there. To avoid unnecessary confusion, it is assumed for the reaainder of the discussion that the reaction involves transfer of one nucleon from a deformed target mucleus to a spherical projectile, and that the spherical projectile accepts the transferred particle in its ground state. All excitations ay then be discussed relative to the yrasi line of the deformed mucleus.

Then, the probability for the transfer reaction to populate an energy interval lying a distance $\delta$ abovi the yrast line way be expected to depend on three important factors: (1) An optinu Q-idindow resulting from momentum atching considerations. For neutron transfer the Q-window is approximately a Gaussian centered at a distance from the yrast line given by the ground-state Q-value. Analytical formulas have been obtained for the width. For the heary nuclei we consider here, the Gaussian 0-window has a full width of $\sim 5 \mathrm{FeV}$. (2) A radial form factor $f(r)$ which decreases for higher excitation $\delta$ above the yrast line as a penalty fur transferring more and more tightly bound particles. The square of the form factor ay be approximated by

$$
f^{2}(r) \sim \exp \left[-2 \sqrt{\left.2 \pi n^{\prime} f^{2}(S+\delta) r\right]}\right.
$$

where $S$ is the separation energy for a particle, and $\delta$ is the excitation energy relative to the yrast line. Unless the Q-window is shifted far above or below the yrast line, the form factor dependence on excitation energy tends to dominate the Q-window factor. (3) A strength function describing the density of states with suitable structure to be populated in the transfer reaction. This function will depend on energy and angular momentum.

Figure 2.78 compares the square of the product of the Q-window and radial form factors for both the one-particle transfer 161 Oy $\left({ }^{58} \mathrm{Ni}{ }^{59} \mathrm{Ni}\right) 160 \mathrm{Dy}$ and the two-particle transfer $1{ }^{2} \mathrm{Oy}\left({ }^{5 \mathrm{~B}_{\mathrm{Ni}}}, 6 \mathrm{O}_{\mathrm{Ni}}\right)^{160 \mathrm{Dy} \text {. The oifference }}$ between the two primarily reflects a higherlying 0 -window in the 2 -particle transfer reaction. The data ${ }^{3}$ indicate that states further from the yrast line are populated in the reac-

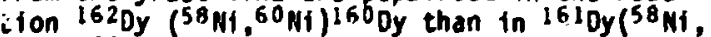
INf) $1600 y$. This would be expected from purely Xinematic considerations (Fig. 2.78).

Although only a rather idealized model has been outlined here, the kinemattcal factors (1) and (c) should be amenable to precise calculation in a more realistic model. Comparison of the expected $k$ inematical weighting to the measured distribution of the transfer population might then allow extraction of strength functions as a function of both energy and angular momentum of the states populated in the transfer reaction.

1. Untversity of Tennessee, Xnoxville, TN. 
2. UnISOR, Oak Ridge Associated Universities.

3. M. U. Guidry, et al., separate coutribu$t$ tions to this progress report, and to be published.

Con-Dir 84-18444

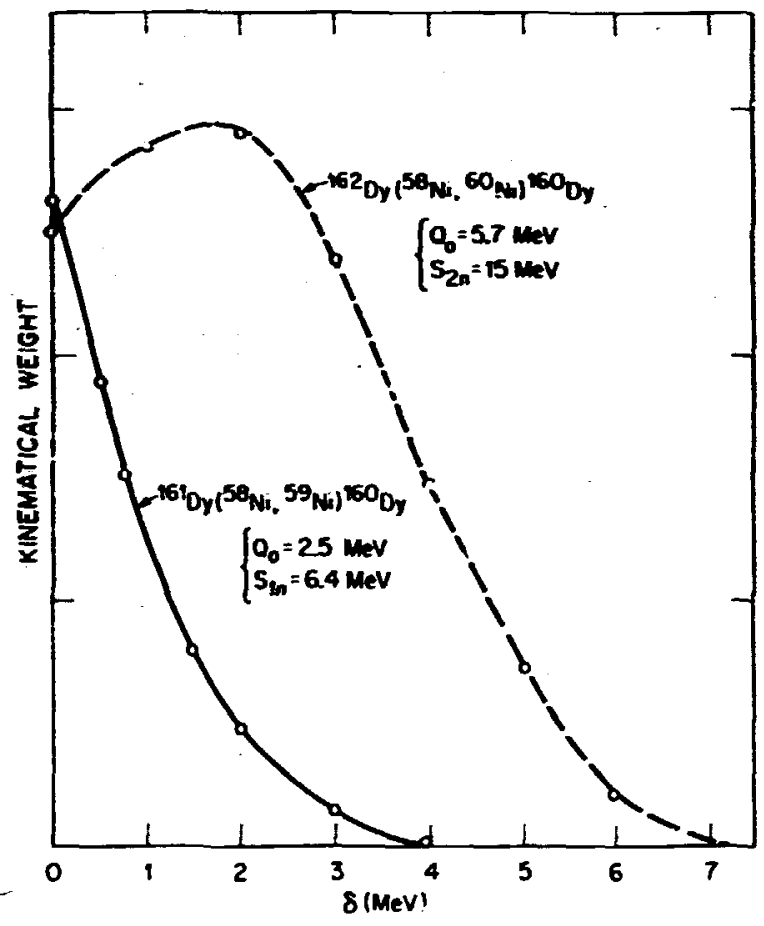

Fig. 2.78. Comparison of the square of the product of the Q-window and radial form factors for both the one-particle transfer 161Dy( 58Wi, 59Ni) 1600y and the two-particle transfer $1620 \mathrm{y}$ ( $58 \mathrm{Ni}, 60 \mathrm{Wi}) 160 \mathrm{Dy}$.

\section{FISSION FRAGMENT AMGULAR DISTRIBUTIONS} FROM 12C-ADD 160-INDUCED REACTIONS 1
A. Gavron ${ }^{2}$
K. Eskola4
P. Eskola ${ }^{3}$
A. J. Sierk ${ }^{2}$
M. M. Fowler ${ }^{2}$
J. Bofssevain2
H. Ohn
J. B. Wilhelmy ${ }^{2}$
H. C. Britt ${ }^{2}$
S. Wald

R. L. Ferguson

The study of fisston fragment angular distributions from heavy-ion-induced fission has been a very active field of investigatfu.: juring the last two years.7-16 The interest ha been $k$ indled in part by the observations that for very heavy fisstonting systems, the fisston antsotropy is, as expected, consistent int th compact saddle-point shapes provided that light heavy fons (e.g., 160$)$ are used to induce fitssion, but that wen heavier projectiles (e.g., 325) are used, the anisotropy is larger than expected, indicating that the fissioning systems are unexpectedly elongated at the time that the angular distribution is determined. Back et al. S have suggested that the explanation lies in extra push effects and that the increased anisotropy constitutes a signature for "quasi fission.

We have made fission fragment ingular distribution masurements? 8 for 160 -induced reactions on 1420, 170 Er, 1920s, and 238 $\mathrm{U}$ and for ${ }_{12} \mathrm{C}$-induced reactions on 174 $\mathrm{b}$, 198 $\mathrm{Pt}$, and $230 \mathrm{U}$. The oxygen energies were 140, 250, and $315 \mathrm{hel}$. and the carton energies mere 95, 188, and 291 MeV. The standard transition-state odel, together with the saddle-point moments of inertia from the rotating rinite-range odel (RFRA), 7,8 was used to analyze the angular distritutions. The muber of particles (neutrons) eaitted before fission plays an important role in this analysis, and since there is considerable uncertainty associated with this question, the data were analyzed under ton extreme assumptions: (1) all neutrons are exitted prior to fission, and (2) no neutrons are emitted before fission. In cases of agreement petween theory and experiment, the measured values are expected to fall between the two calculated eitremes. This was- in fact, found to be the case for all reactions, except those involving 238U targets, as can be seen in Fig. 2.79. From this and from an examination of other data, it was concluded that the transition-state model (together with RFRH saddle-point shapes) is valid only when the fission barrier is higher than the temperature at the saddle point for a significant number of the partial waves that contribute to fission.

1. Sumary of paper: Phys. Rev. Lett. 52 , 589 (1984).

2. Los Alamos Mationa i Laboratory, Los Alamos, Mew Mexico.

3. Present address: Theoretical Physics Department, Uhiversity of Helsinki, Helsinki, Finland.

4. Present address: Physics Department, University of thelsinki, Helsinki, Finland.

5. Chemistry Department, Mainz University, Mainz, Hest Germany.

6. Lawrence Berkeley Laboratory, Berkeley, California.

7. A. Gavron et al., submitted to Physical Review $C$.

8. A. Gavron et al., Phys. Rev. Lett. 52, 589 (1984).

9. B. B. Back et al., Phys, Rev. Lett. 50 , 818 (1983). (1983). (1983)

11. H. Rossner et al., Phys. Rev. C 27, 2666

12. L. C. Vaz and J. M. Alexander, Phys. Rep. 97, 1 (1983).

13. L. C. Vaz and J. M. Alexander, Z. Phys. A312, 163 (1983).

14. M. B. Tsang et al., Phys. Lett. 1298, 18 (1983).

15. M. B. Tsang et al.., Phys. Rev, C 28, 747 (1983).

16. P. 0. Bond, Phys. Rev, Lett. 52, 414 $(1984)$. 

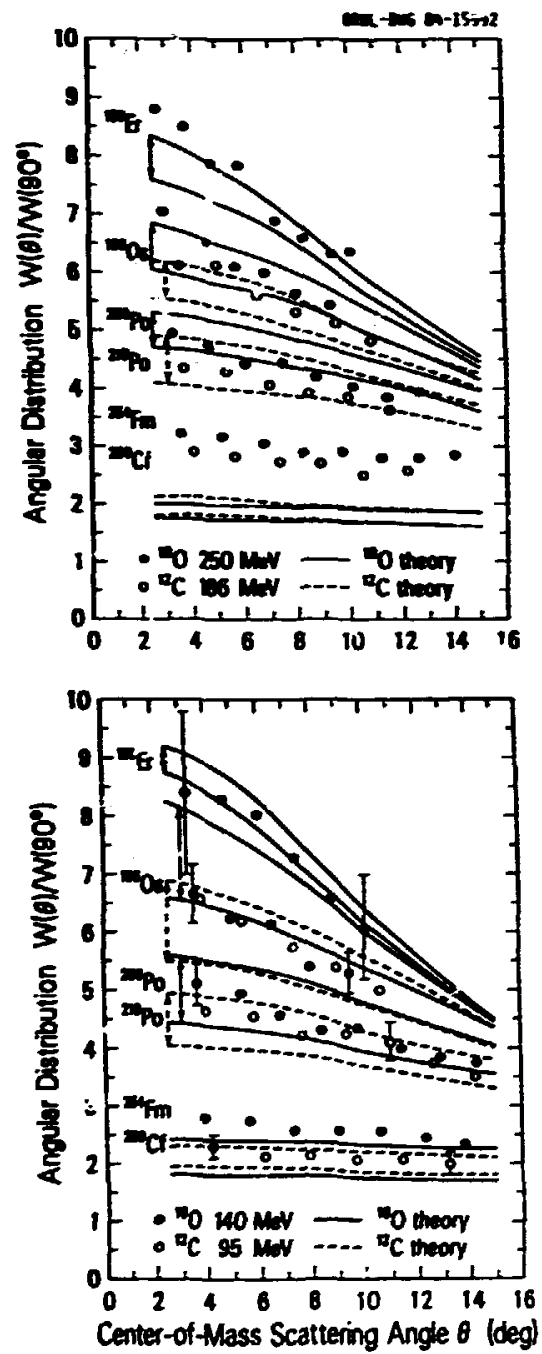

Fig. 2.79. Experimental results of anisotropy in fission-fragment yields. The curves correspond to calculations for each set of data under two extreme assumptions (see text). The full curves are calculations for 160-induced reactions and the dashed curves are calculations for 12C-induced reactions.

COINCIDENCE REASUREMENTS BETWEEN EVAPORATION RESIDUES AND LIGHT PARTICLES PRODUCED IN $160+40 \mathrm{Ca}$ AND $28 \mathrm{Si}+{ }^{40} \mathrm{Ca}$ REACTIONS
C. Maguired
2. Kuil
W. C. Mal
S. Robinson!
D. Watson!
G. Hord ${ }^{1}$
H. Ikezoe 2
D. G. Kovar
G. Rosner ${ }^{2}$
G. Stephans 2
E. L.igricht ${ }^{2}$
B. Hilkins 2

T. C. Awes

G. R. Young

The experimental evidence that preequilibrium or incomplete fuston processes occur in light and medium weight heavy-ton-induced reactions at hi her bombarding energies ( $E_{1 a b}>6-3 \mathrm{MeV} / \mathrm{am}$ ) cores from iie observation of energetic nonstatistical light particles in coincidence with fusionl ike products 3,4 and the observation that full linear momentum has not been transfe:red to the compound nucleus (i.e.., the evaporation residues show a velocity deficit ${ }^{5}$ and the fission fragments too large a folding angle ${ }^{6}$ ?. At present the details of the reaction mechanism giving rise to the incomplete fusion process are not well understood, and progress has been hindered by the experimental tifficulties in quantitatively distinguishing between the contributions frow incomplete and complete fusion to the evaporation residue (fission fragment) yields. In the present study the velocity (energy) spectra of evaporation residues and coincident light particles are measured with the mtivation of better establishing the kinematics and, hopefully, better understanding the reaction echanisa. Two projectiles, ${ }^{160}$ and 28Si, were used to investigate the projectile dependence.

Coincidence measurements of evaforation residues with light particles ! $1<A<6$ ) were performed for the reactions $160+40 \mathrm{Ca}$ and ${ }^{28} \mathrm{Si}+{ }^{40} \mathrm{Ca}$ with beans of $\left.160 / \mathrm{E}_{\mathrm{lab}}=157 \mathrm{MeV}\right)$ and ${ }^{28} \mathrm{Si}\left(E_{\mathrm{lab}}=252 \mathrm{MeV}\right.$ ) obtained from the Argonne Mational Laboratory Supercondicting Linac. The masses of the evapnration residues produced at $\theta_{1}$ ab $=9^{\circ}$ were identified, and their velocity spectra were measured in a channel plate start detector/Si stop detector time-of flight system with a flight path of $\sim 1.2 \mathrm{~m}$. in example of the mass resolution obtained is shown in Fig. 2.80. The light particles were identified and their energy spectra mere measured in six triple-Si detector-telescopes located at $\theta_{\mathrm{ab}}=+59^{\circ},+28^{\circ},-15^{\circ},-25^{\circ},-40^{\circ}$, and $-70^{\circ}$, where the negative angles are on the opposite side of the beam from the time-of-flight detector.

The results of the measurements are presently in the process of being analyzed. The preliminary results from the $160+{ }^{40} \mathrm{Ca}$ measurement show that the singles evaporation residues (40 6 A < 52) have velocity centroids $9.4 \%$ lower than expected for complete fusion, consistent with the previously reported results of Chan et al. ${ }^{7}$ The coincidence measurements show that the foward-angle alpha particles in coincidence with the evaporation residues have energy spectra with a significant high-energy (-bean velocity) component (see fig. 2.81), while no such component is apparent in the coincidence proton spectra. The dependence of the evaporation residue velocity spectra and light-particle energy spectra with coincidence angle are not well reproduced by LILITA calculations for complete fusion, and indicate the presence of significant contributions from a reaction process consistent with the kinematics for fusion of $12 \mathrm{C}+40 \mathrm{Ca}$ [assuming $60 \mathrm{U}+40 \mathrm{Ca} \rightarrow 12 \mathrm{C}+40 \mathrm{Ca}+\mathrm{a}$ (beam velocity)]. More complete ca'culations are under way to further substantiate the interpretation suggested by these preliminary calculations.

1. Vanderbilt University, Mashville, 
2. Argonne Rational Laboratory, Argonna, Illinois.

3. P. Conthier et al., Phys. Rev. Lett. 44, 1387 (1980).

4. J. Hilczynski et al.. Phuss. Lett. 808, 41 (1979).

5. H. Morgenstern et al., Phys. Lett. 1138 , 463 (1982).

6. B. Bark et al., Phys. Rev. C 22, 1927 (1980).

7. Y. Chan et al., Phys. Rev. C 27, 44T (1983).

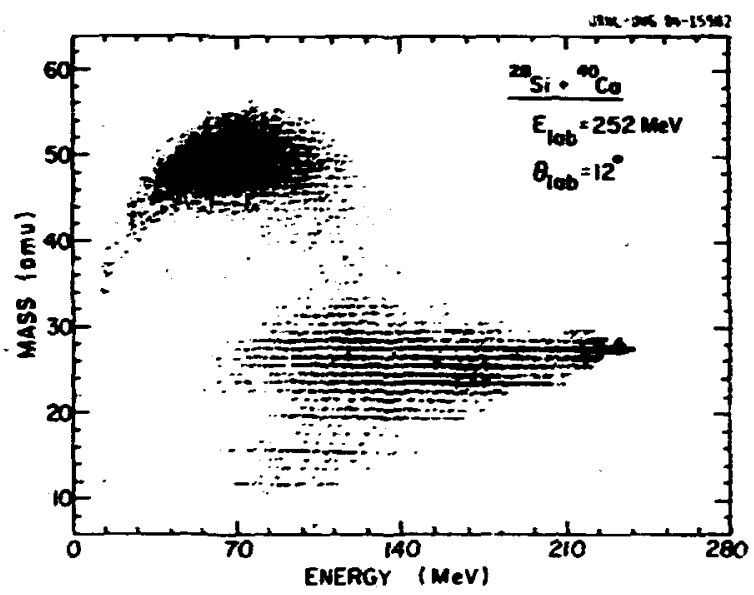

Fig. 2.80. Example of rass resolution attained in the ${ }^{28} \mathrm{Si}+{ }^{40} \mathrm{Ca}$ reaction at $\mathrm{E}_{1 \mathrm{ab}}=252 \mathrm{MeV}$.

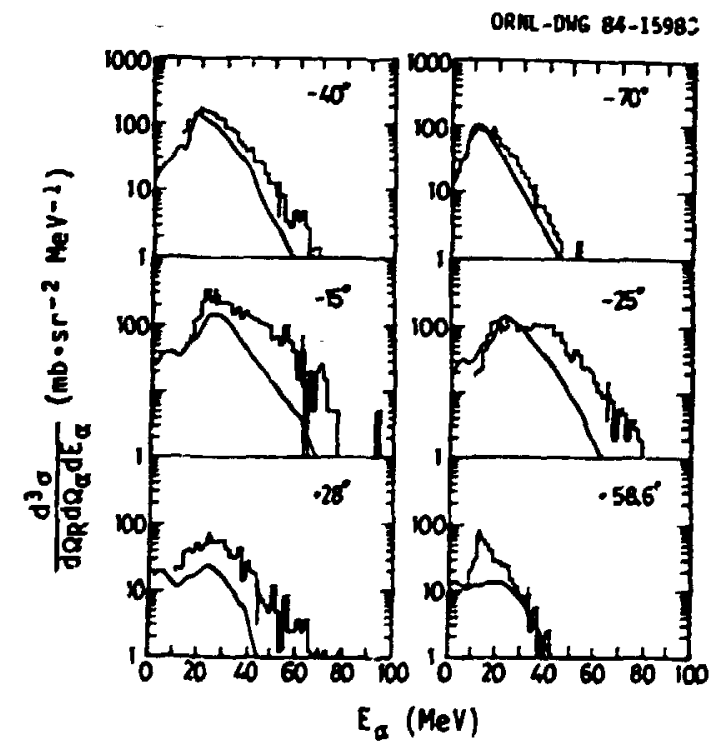

Fig. 2.81. Evaporation restdues (mass $>40$ ) observed at $9^{\circ}$ in the reactions of $15 \%$-MeV 160 with ${ }^{40} \mathrm{Ca}$. Error Dars represent the statistical errors.
REUTROA EIISSIOU IN IMELASTIC REACTIONS $12 \mathrm{C}+15 \mathrm{gd}$ ab $20 \mathrm{he}+150 \mathrm{nd}$
6. A. Petitti
A. Gavren 2
F. E. Cbenshain
J. R. Beene
F. Plasil
B. Cheyris ${ }^{3}$
G. R. Young
R. L. Ferguson
A. Jaskelainent
C. . Maguire 5

Spectra and ancular oistributions of neutrons in coincidence with light fraguents produced in inelastic collisions of $12 \mathrm{C}$ on $158 \mathrm{Gd}$ at $192 \mathrm{meV}$ and of $20 \mathrm{ke}-150 \mathrm{Nd}$ at 176 and $239 \mathrm{MeV}$ have been measured. The data were analyzed to determine if the neutron spectra couid be explained entirely on the bosis of adission from the projectilelike frayments (PLF) and targetlike fragments (ILF) after the fragments have opached the asymtotic velocity due to their mtual Coulaw repulsion or wether there is evidence for some kind of nonequilioriur emission mechanism. No evidence for nonequil i ibl ium neution emission is found for the lle + Md reaction at either eneryy. For the $C+$ Gd reaction, a sall fraction of neutrons (5x) are dee to nonequilibrium emission.

For the $12 \mathrm{C}$ : $158 \mathrm{G}$ - reaction, the seall nonequilibrium component. appears to be concentrated on the opposite side of the bean from the detector telescope used to measure the energy and charge state of the PLF, although a sali component, hidden by the large nubbers of neutrons emitted by the PLF and kinematically focused in its direction of travel, by bresent on the same side of the beam.

Some of the result: of the analysis are given in Figs. 2.82 and 2.83, wich show the neutron er.ergy spectra recorded by the neutron cetec$t$, irs on the opposite side of tne bean from the telescope. In both figures the upperwost sper:trum is closest to the bean $\left(15^{\circ}\right)$, and the botton spectrum is at the largest angle $\left(140^{\circ}\right)$. The data points (triangles) are shown along with the results of a procedure in wich the events recorded in two reference neutron detectors were transformed into the other neutron detectors. One of the reference detectors was located at a back angle and was assumed to detect only neutrons emitted by the TLF. The other detector was directly behind the fragment telescope and was assumed to detect only neutrons emitted by the PLF. The results of the transformation are shown as dots in the figures. As can be seen in the figures, for the Me + Md reaction the transformed spectra agree well wth the data, wereas for the $C+$ id reaction, the data points at forward angles and high neutron energies lie well above the transformed spactra, indicating the presence of nonequilfbrium entssion in this reaction.

1. Georgia State Universicy, At lanta, Georgid.

2. Los Alamos National Laboratory, Los Alamos. New Mexico.

3. Institut de Physique Nucleaire de Lyon, Villeurbanne, France. 
4. Hashington University, St. Lou is, Missuri.

5. Vanderbilt Ini versity, Mashville, Tennessee.

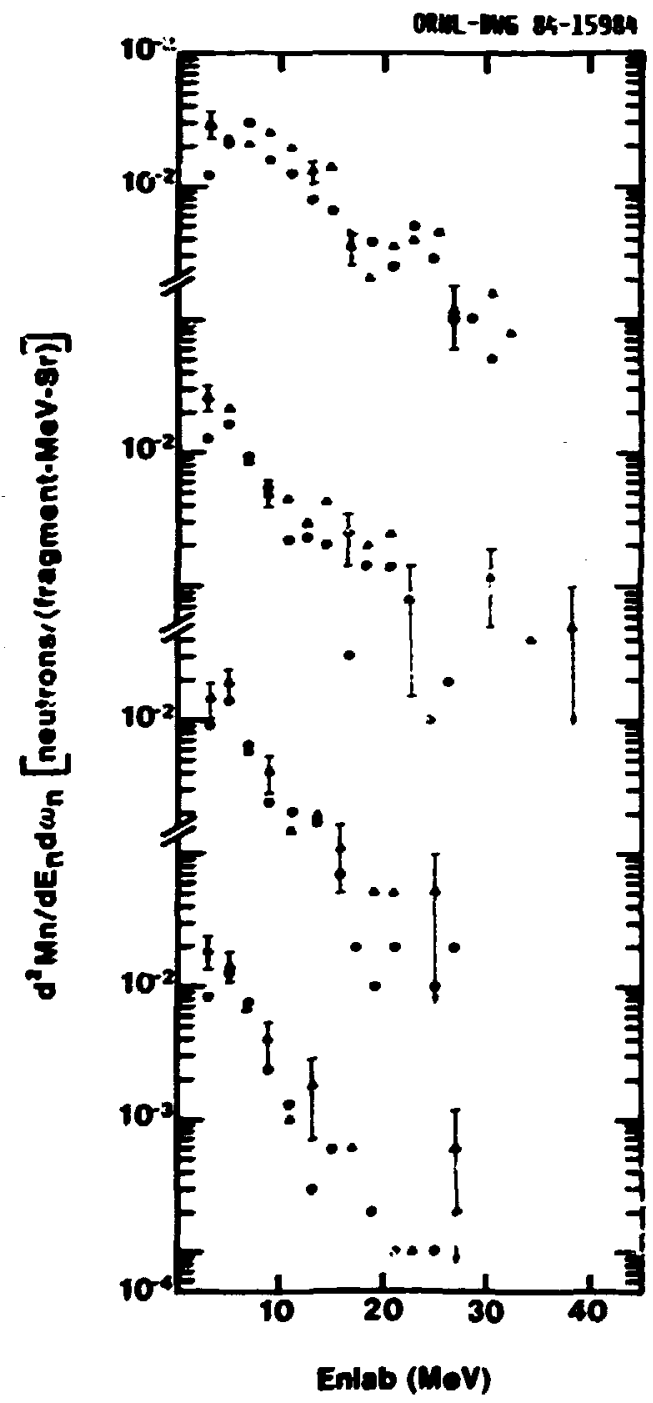

Fig. 2,82. Weutron energy spectra recorded in cetectors on the opposite side of the beam from the heavy-ton detector telescope in the reaction $20 \mathrm{Ne}+150 \mathrm{Nd}$ at $239 \mathrm{MeV}$. The data points ( 1 ) are shown along with the results of the transformed spectra $(\bullet)$ described in the text.

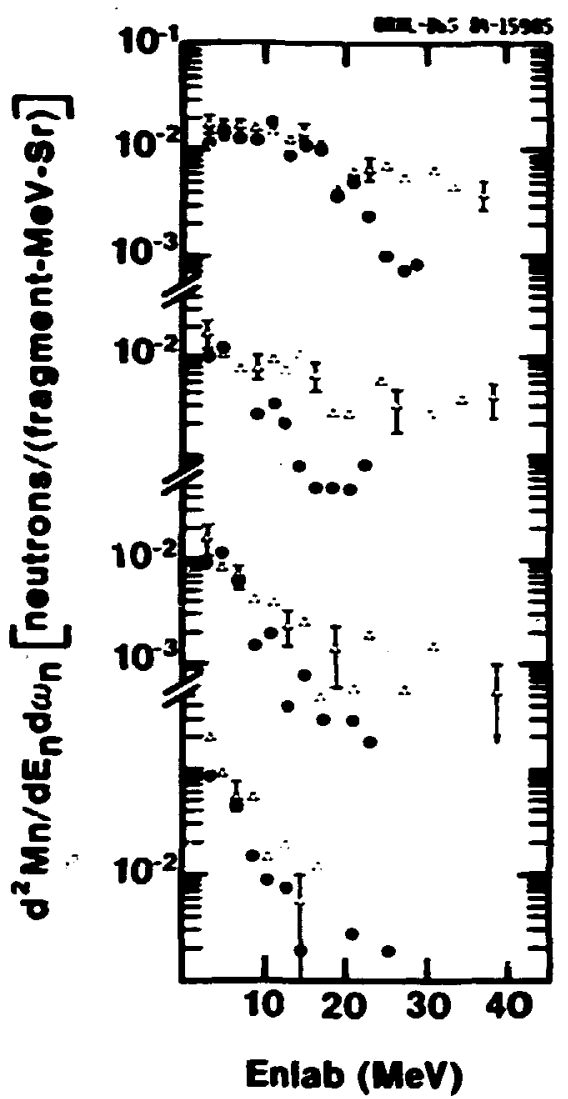

Fig. 2.83. Same as Fig. 2.82, but for the $12 \mathrm{C}+158$ fid reaction at $192 \mathrm{MeV}$.

AZIMUTHAL CORRELATIONS BETMEEN LIGHT PARTICLES EMITTED IN 160-IDOUCEO REATTIONS

ON ${ }^{12 C}$ And 197 Au AT 400 MeV
M. B. Tsangl
G. R. Young
H. G. Lynch1
T. C. Awes
C. B. Cinttwood 1
R. L. Ferguson
D. J. Fields 1
F. E. Obenshain
D. R. Kleschl
F. Plasil
C. K. Gelbke
R. L. Robinson

For intermediate-energy nuclear collisions, particle entssion prior to the attatnment of full statistical equilibrium of the entting nucleus is expected to provide information about the early stages of the reaction. The global trends of single-particle inclustve cross section: can be rather we!l described in terms of the concept of loc.al statist.tral equtlibrtum. $2-4$ 
Recent results from two-proton corielation measurements at sallil relative wome-ta are consistent with the ewission of energetic light particles from localized region of high excitation.S Additional inforntion about the dynarical and geanetrical aspects of the reaction may be ubtained from investigations of light-particle correlations. In order to search for such dynam al correlations and to assess :ne importance if phase-space constraints for sall nuclear systews, we have measured aziwhal angular correlations between energetic light particle; anitted in 160 -induced reactions on a light $\left({ }^{12} \mathrm{C}\right)$ and a heavy $(197 \mathrm{Au})$ target at an incident energy of $\mathbf{4 0 0} \mathrm{MeV}$.

Prescaled singles and coincident light particles $(0, d, t)$ were detected using seven telescopes with solid angles between 13 and $40 \mathrm{~ms}$. Three of these telescipes mere mounted at the polar angles, masurea with respect to the bean axis, of $\theta=40^{\circ}, 70^{\circ}$, and $130^{\circ}$ and the azimuthal angle of $0^{\circ}$. The remaining four telescopes mere positioned at the polar angles of $\theta=40^{\circ}, 70^{\circ}, 130^{\circ}$, and $160^{\circ}$; their azimuthal angle was varied between $50^{\circ}$ and $130^{\circ}$.

The inclusive energy spectra of protons, deuterons, and tritons detected at $\theta=40^{\circ}$ and $70^{\circ}$ have been measured. For reactions induced on $197 \mathrm{Au}$, estimated upper limits for emission from the compound nucleu* were determined by assuming that the energy spectra measured at $\theta=160^{\circ}$ are entirely due to isotropic evaporation from the compound nucleus. The energy spectra at $45^{\circ}$ and $70^{\circ}$ are dominated by noncompound emission processes. For reactions on $12 \mathrm{C}$, on the other hand, the shapes of the energy spectra are consistent with evaporation from the compound nucleus.

In cider to reduce systematic errors, the azimithal correlation is defined by the ratio of the coincidence cross section divided by the singles cross sections $\sigma_{x y} / \sigma_{x} \sigma_{y}$. A low-energy threshold of $36 \mathrm{MeV}$ was applifed in computing the cross section. For reactions or, $197 \mathrm{Au}$, that threshold strongly reduces contributions from compound nucleus decay. Figure 2.84 shows the azimuthal correlations of two coincident light particles emitted at $\theta=40^{\circ}$ and $70^{\circ}$. For reactions on ${ }^{22} \mathrm{C}$, there $i c$, a clear enhancement for the emission of two cotncident if ght particles to opposite sides of the beam axis, Fig. 2.84a. These carrelations be understood in terms of the phase-space constraints imposed by momentum conservation.6 To illustrate the effect of mumentum conservation, we performed schematis calculations ${ }^{6}$ for source of $A_{s}=28$ nucleons and temperature $T=7.1 \mathrm{MeV}$ moving with the velocity of the compound nucleus, $v_{0}=0.13 \mathrm{c}$. To conjerve total linear momentum, in these calculations the entire residual source is assumed to recotl after the emisston of the first prticl. These schematic calculations reproduce the iverall trends of the data rather well, indicating that the preferential emission of coincident light particles to opposite sides of the beam axts may be explained in terms o." the phase-space constraints imposed by momentum conservation on fintte nuclear systems.

Entirely different azimuthal correlations are observed for reactions on $197 \mathrm{Au}$, Fig. 2.84b.

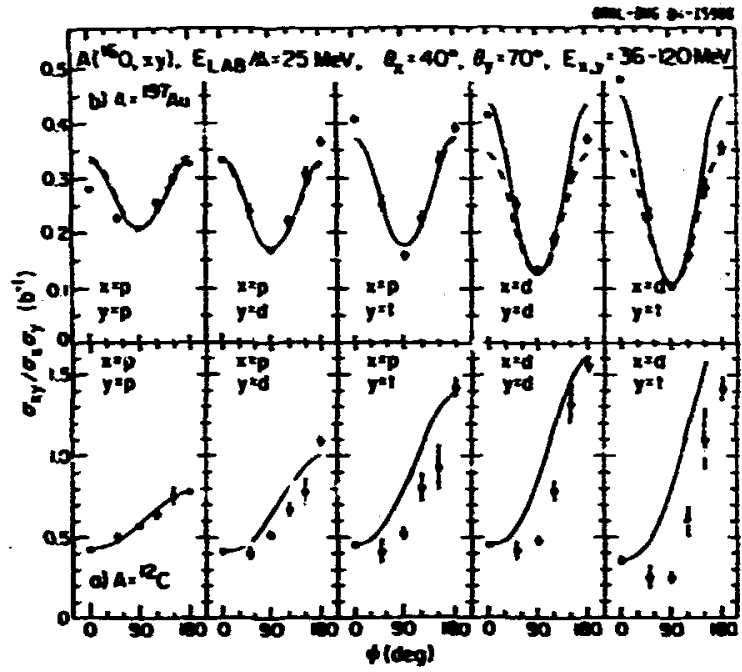

Fig. 2.84. Azimuthal angular correlations between coincident light particles enitted at $\theta=40^{\circ}$ and $70^{\circ}$ with respect to the bean axis for 160 -induced reactions on $12 \mathrm{C}$ ( $a$, lower part) and ${ }^{197} \mathrm{Au}$ (b, upper part) at 400 -heV incident e iergy. A low-energy threshold of $36 \mathrm{MeV}$ was applied. See text for explanation of solid and dashed curves.

These correlations are nearly left-right symmetric about the beam axis and exhibit a characteristic " $V$ " shape corresponding to the preferential emission of energetic light particles in a plane wich contains the bean axis. Close inspection of the coincidence cross sections at $\phi_{x}=0^{\circ}$ and $180^{\circ}$ shows a saall enhancement for the emission of coincident protons to opposite sides of the beam axis. Coinctident deuterons and tritons, on the other hanu, are preferentially emitted to the same side of the beam axis.

The preferential emission of coinctdent light particles in a plane containing the beam axts is consistent with the recent observation' that noncompound light particles are preferericially exitted in the entrance-channel scattering plane (defined as the plane wich contains ihe beam axis and wich is perpendicular to tile semiclassical orbital angular momentum vector for the relative motion between the projectile and target nuclei). These observations could be described by assuming the superposition of a collective motion in the reaction plane on the random motion of the individual mucleons.

The calculations with the rotating hot source parameterization are shown by the solid curves in Fig. 2.B4D; they were performed with the parameters $T=5.6 \mathrm{MeV}, V_{0}=0.09 \mathrm{c}, E_{c}=10$ $\mathrm{MeV}$, and $R_{w}=0.1 \mathrm{c}$. The calculations for two sideward moving sources are shown as dashed curves in Fig. 2.84a; they were performed with the parameters $T=6 \mathrm{MeV}, \mathrm{V}_{0}=0,12 \mathrm{C}, \mathrm{E}_{\mathrm{c}}=$ $10 \mathrm{MeV}$, and $\theta_{0} \cdot 35^{\circ}$. Both of these schematic calculations can reproduce the overall trends of the azimuthal correlations rather well. 
Except at very forward angles, the eaission of energetic light particles is largely associated with fusionlike reactions 7,8 in mich the ajor part of the projectile is absorbed by the target nucleus. Therefore, the above paraneterizations mst not be interpreted in terms of the eaission from one or two hot sources wich exist separately fror the composite system. In particular, the two--ource parameterization should not be interpreted in terns of the sequential decay of excited projectile and target residues. We introduce these parameterizations solely to illustrate the effects wich may arise fron the superpositions of random and ordered velocity components. The relative success of the two parameterizations my be understood in terms of the relative importance of these two velocity components. The randow component decreases for heavier particles, but the ordered velocity component remains constant. As a consequence, havier particles are more sensitive to the collective motion of the exitting systew. Both of these rather simple parameterfizations describe the qualitative trends of the data, but they are not unique, and similar ayreement may be obtained by other models wich superimpose collective and statistical velocity components.

To provide a quantitative comparison of tie cross sections corresponding to the emission of coincident light particles to the same $\left(0_{x}=0^{\circ}\right)$ and to opposite $\left(\phi_{x}=180^{\circ}\right)$ sides of the beam axis, Fig. 2.85 shows the ratios of these coincidence cross sections, $\sigma_{x y}\left(\phi_{x}=180^{\circ}\right) / \sigma_{x y}\left(\phi_{x}=\right.$ $\left.0^{\circ}\right)$. For reactions on $197_{A u}$ this ratio decreases with increasing mass of the two coincident light particles, in contrast to the strong increase measured for reactions on $12 \mathrm{C}$. The dot-dashed and dashed lines in the figure illustrate the effects due to momentum conservation for the case that the momentum of the emitted particle is shared by $A_{s}=28$ and $A_{s}=40,60$, 90, and 213 nucleois, respectively. (The number $A_{s}$ should not be ijentified with the number of "participant" nucleons since monentum may also be transferred to the cold spectator matter.) For these calculations, particles were assumed to be emitted with Maxwellian distributions corresponding to a temperature of $T=7.1 \mathrm{MeV}$ and initial mean velocity parallel to the beam axis of $v_{0}=0.13 c$ (dot-dashed line) and $v_{0}=$ $0.11 \mathrm{c}$ (dashed lines). After the emission of a particle of mass number $A_{x}$ and velocity $v_{x}$, the mean velocity of the second particle was assumed to be changed by $i \vec{v}_{0}=A_{x} \vec{v}_{x} /\left(A_{s}-A_{x}\right)$. (The parameters for the source consisting of $A_{S}=28$ nucleons are identical with the parameters used for the calculations shown in Fig. 2.84a.) Qualitatively, the ratios $\sigma_{x y}(\$=$ $\left.180^{\circ}\right) / \sigma_{x y}\left(\phi=0^{\circ}\right)$ mitht be explatined in terms of the competing effects caused by shadowing -11 and momentum conservation, although different interpretations may be possible. If preequilibrium emission originates from a localized region of high excitation, 5 absorption or rescattering by the adjazent spectator nuclear matter will enhance emission to the same side of the beam axis. Momentum conservation, on the other hand, will favor emission to opposite sides of the bean axts. Whether coincident light particles

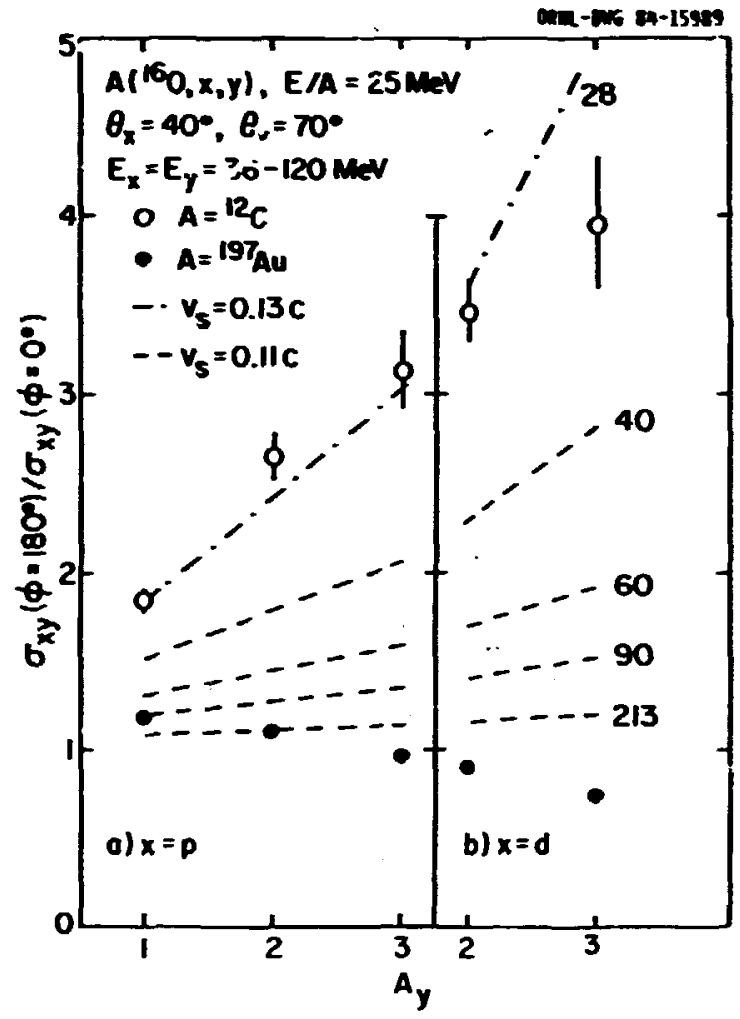

Fig. 2.85. Ratio of cross sections corresponding to emission of coincident light particles to opposite sides and to the same side "the beam axis for $16 \mathrm{~J}$-induced reactions on ${ }^{12} \mathrm{C}$ (open points) and $197 \mathrm{Au}$ (full points) at $400 \mathrm{MeV}$ incident energy. The dashed and dot-dashed curves illustrate the effects of monentum conservation for systems with finite number of nucleons. The calculations are explained in the text.

are preferentially enitted to the same or to opposite sides of the beam axis will depend on the relative magnitude of these two opjosing effects. Absorptive effects are expected to be more pronounced for the emission of composite light particles than for the emisston of nucleons. This is in qualitative agreement with the trends measured for reactions on $197 \mathrm{Au}$ were it is observed that coincident protons have a slight preference to emerge at opposite sides of the beam axis, whereas coincident inmposite light particles have a silight preference to emerge at the same side of the beam axis.

1. National Superconducting Cyclotron Facility, Michigan State University, East Lansing, Michigan.

2. T. C. Awes et al., Phys, Lett. 1038, 417 (1981).

3. T. C. Awes et al., Phys. Rev. C 25, 2361 $(1982)$.

4. G. D. Westfall et al., Phys. Lett. 116B, $118(1982)$. 
5. H. 6. Lynch et al., Phys. Rev. Lett. 51, 1850 (1983).

6. W. 6. Lynch et al., Phys. Lett. 1068, 274 (1982).

7. M. B. Tsang et al.., Phys. Rev. Lett. 52 , 1964 (1984). (1981).

. T. C. Aures et al., Phys. Rev. C 24, 89

9. P. A. Eottschalk and M. Mestron, Rucl. Phys. A314, 232 (1979). (1980).

11. X. A. Friedman, Phys. Rev. C 29, 139 (1984).

FIMUL-STATE IMTERACTIOUS

BETUEEA MOCOMPOUND LIGHT PARTICLES

FOR 160-IIDUCED REACTIONS

ON 197 Au RT E/and $=25$ ReV

$\begin{array}{ll}\text { C. B. Chitmoodl } & \text { M. B. Tsangl } \\ \text { J. Aichelin' } & \text { J. C. Shillcock } \\ \text { D. H. Boal } & \text { T. C. Awes } \\ \text { G. Bertschl } & \text { R. L. Ferguson } \\ \text { D. J. Fields } & \text { F. E. Obenshain } \\ \text { C. K. Gelbkel } & \text { F. Plasil } \\ \text { H. G. Lynchl } & \text { R. L. Robinson }\end{array}$

G. R. Young

Two-particle correlations at small relative momenta may contain information about the spacetime characteristics of the enitting source because of their sensitivity to final-state interactions ${ }^{3}$ and quantum statistical ef fects. 4,5 Previous investigations of the space-time tharacteristics of highly excited nuclear systems were based on analyses of two-pion ${ }^{6-9}$ and twoproton 10=12 correlations. In the only case which allows a direct comparison, 9,10 different source dimensions mere extracted from the two measurements. The interpretation of lightparticle correlations may be complicated by sensitivities to ensemble averaging, ${ }^{13}$ reaction dynamics, 13 momentum conservation, $I 1$ and sequential decay of particle unbound resonances.14-16 Since correlations between different light particles are expected to exhibit different sensitivities to these effects, we have measured correlations between noncompound protons, deuterons, and tritons emitted in 160 -induced reactions on $197 \mathrm{Au}$ at $E / \mathrm{ann}=25 \mathrm{MeV}$. The observed two-deuteron correlations, for example, cannot be interpreted in terms of the decay of particle unstable resonances.

The experiment was performed at the Holifield Heavy Ion Research Facility. A oold target of $9.7 \mathrm{mg} / \mathrm{cm}^{2}$ was bombarded with 160 fons of 400-HeV incident energy. Small-angle correlations between coincident light particles were measu -ed with six $\Delta E-E$ telescopes consisting of silicon $\triangle E$ and $\mathrm{MaI}(\mathrm{Tl}) \mathrm{E}$ detectors. The detectors were mounted in a closely packed hexagonal array that was centered at the scattering angle of $15^{\circ}$. Each telescope subtended a solid angle of $7.6 \mathrm{msr}$; the angular resolution and angular separation between adjacent telescopes were $1.6^{\circ}$ and $5.1^{\circ}$, respectively. For a quantitative presentation of the data, we define the correlation function, $R\left(\vec{p}_{1}, \vec{p}_{2}\right)_{1}$ in terrs of the singles cross sections, $\vec{\sigma}\left(\vec{p}_{1}\right), \sigma\left(\vec{p}_{2}\right)$, and the coincidence cross section, $\sigma\left(\vec{p}_{1}, \vec{p}_{2}\right)$,

$$
\sigma\left(\vec{p}_{1}, \vec{p}_{2}\right)=\sigma_{0} \sigma\left(\vec{p}_{1}\right) \sigma\left(\vec{p}_{2}\right)\left[1+R\left(\vec{p}_{1}, \vec{p}_{2}\right)\right] .
$$

where $\vec{p}_{1}$ and $\vec{p}_{2}$ denote the womenta of particles 1 and 2 . The normalization constant, $\sigma_{g}$ (used for all correlations shown in Fig. 2.86), was determined previously 11 by requiring the twoproton correlation function to vanish for Sufficiently large relative womenta at wich final-state interactions are negligible. The experimental correlation functions show in Fig. 2.86 were obtained by inserting the weasured cross sections into Eq. (1) and by summing both sides of the equation over all energies and angles corresponding to a given pomentum of relative motion, $\Delta p=\mu\left|\vec{p}_{1} / m_{1}-\vec{p}_{2} / m_{2}\right|$, where $m_{1}$ and $m_{2}$ denote the masses of particles 1 and 2 and $\mu$ is the reduced mass. This procedure corresponds to a significant averaging process and tends to reduce the measured correlation function.11

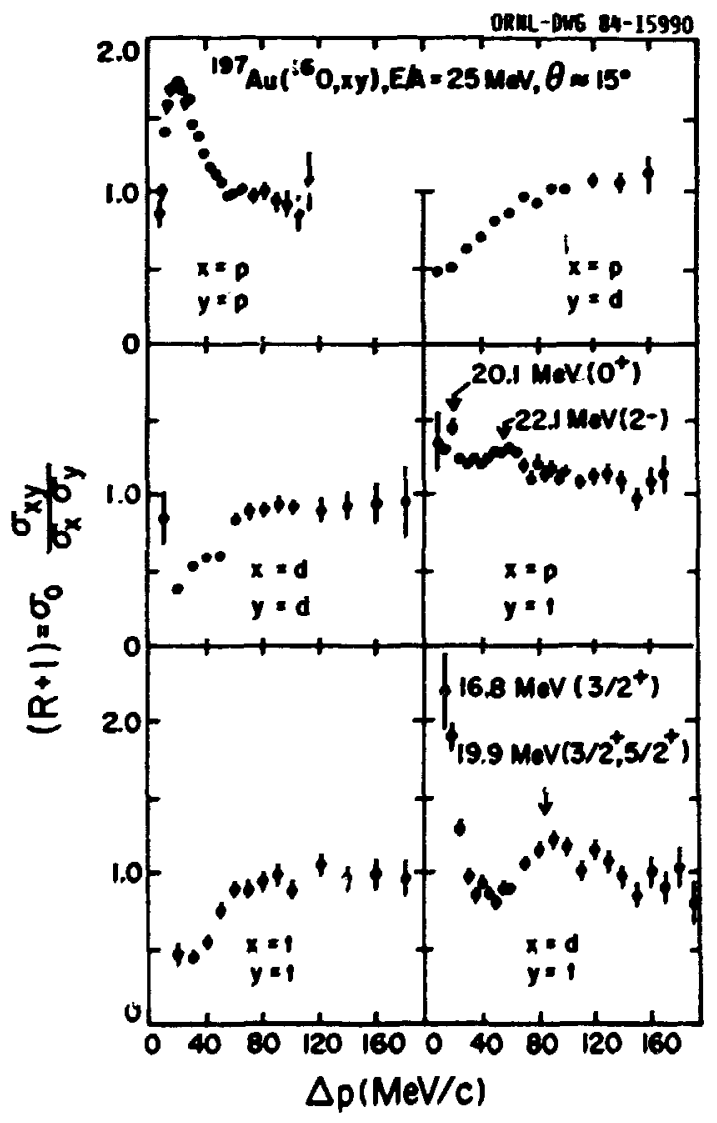

Fig, 2.86. Experimental correlation functions, $1+R(\Delta 0)$. plotted as a function of the momentum of relative motion. The locations of several known particle unstable resonances are indicated by arrows. The errors are purely statistical. 
The tm-proton correlations have been discussed previously. II Because of the dominance of the attractive 5 -wave interaction, correlations arising from the emission and decay of unbound 2 He nuclei can be very similar to those caused by final-state interactions between protons randonly enitted from a source of small space-time extent. ${ }^{4-16}$ More generally, lightparticle correlations resulting from final-state interactions should be more pronounced for systems with sharp resonances. The locations of several known particle unbound states of "He and $5 \mathrm{He}$, decaying into $p+t$ and $d+t$, respective?y, are indicated by arrows in Fig- 2.86. The enhanced correlations at these locations may be interpreted in terms of final-state interactions or, alternatively, in terws of enission of particle unstable nuclei. However, the strong suppression of the $p+d, d+d$, and $t+t$ coincidences at small relative momenta cannot be interpreted in terns of the emission and decay of particle unstable nuclei.

In order to illustrate that nonresonant final-state interactions between composite light particles contain useful information about the space-time characteristics of the emitting system, we have extended the treatment of hadron interferometry previously applied to two-proton ${ }^{3}$ and two-pion 4,5 correlations to two-deuteron and two-triton correlations. In previous investigations, the emitting region was issumed to be of Gaussian form in space and time, characterized by the parameters $r_{0}$ and $\tau$, respectively. In order to reduce the ambiguities resulting from the unknown value of $\tau$ and to obtain an upper limit for $r_{0}$, we used $\tau=0$ corresponding to a source of negligible lifet ime.

Correlations calculated for $r_{0}=8 \mathrm{fm}$ are shown in Fig. 2.87a. The attractive potential set (RG) predicts enhanced correlations at $\Delta p=$ $30 \mathrm{MeV} / \mathrm{C}$. This enhancement becomes more pionounced as $r_{0}$ is decreased. In contrast, the repulsive poteritial set (RM) predicts a suppression of the correlation function at these values of IP. The data clearly favor the phase shifts extracted by the coupled-channels $R$-matrix method. (r.or comparison we also show the correlations predicted for the case of pure Coulamb interaction between the two deuterons; see dotdashed curve.) In order to illustrate the sensitivity of the calculations to the source radius, we show two calculations for the repulsive potential set (RM) corresponding to $r_{0}-8$ and $4 \mathrm{fm}$, respectively. Although the two-deuteron correlations appear to be less sensitive to the source dimensions than twoproton correlations, they indicate a large source radius of $r_{0}-8 \mathrm{fm}$, compared to the values $r_{0} \leqslant 4$ fri extracted from two-proton correlations. 11

For large source dimensions, the neglect of the short-range nuclear interaction might be $d$ satisfactory approximation. Because of difficulties in ostaining appropriate $t-t$ phase shifts, we have calculated two-tition correlations only for the simplifying cose of pure Coulomb interaction. In Fig. 2.87b, these calculations are compared to the measured twotriton correlations. Although we cannot make a definitive statement, the size of the emitting

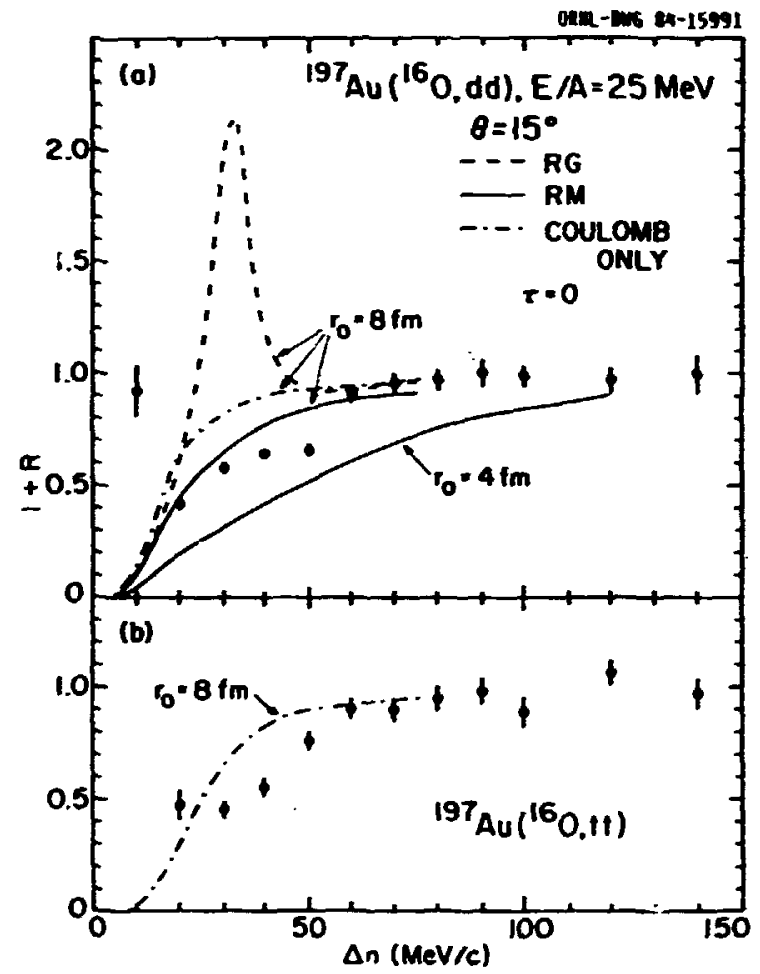

Fig. 2.87. (a) Comparison of d-d correlation function data with calculations based on phase shifts of Refs. 17 (RG) and 18 (RH). The dotdashed curve neglects the nuclear part of the potential. (D) Comparison of $t-t$ correlation function data with a calculation using only the Coulomb part of the potential. The radius parameter $r_{0}$ is indicated in the figure. The experimental correlation functions were normalized to $R(\Delta p)=0$ at large relative momenta.

region appears to be large, just as observed for the two-deuteron correlations.

The difference between source sizes extracted from the analyses of the two-proton and the two-deuteron and two-triton correlations could indicate uncertainties in the interpretation of these correlations. Future investigations should clarify this point. However, larger source radii for composite particles might not be unreasonable. In terms of a thermal model. final-state interactions were suggested 19 to keep light iragments close to equilibrium for a longer period of time than nucleoris. The freeze-out densities for nucleons and light frayments were estimated ${ }^{19}$ to be of the order of $0.5 p_{0}$ and $0.05 p_{0}$, respectively. While the corresponding change in scale of $101 / 3=2.15$ should not be directly equated with the approximate factor of 2 obtained from the analysis of the correlation measurements ( $r_{0} \leq 4 \mathrm{fm}$ for $p-p$, $r_{0}-8 \mathrm{fm}$ for $d-d$ and $\left.t-t\right)$, the sicale change is suggestive.

Significant correlations, with difierent. yualitative shapes, were measured for all combinations of $p, d$, and $t$ for 160 -induced reactions on 197Au at $400 \mathrm{MeV}$. Our analysis of the 
two-deuteron correlations favors phase shifts obtained from the R-matrix approach, consistent with a repulsive interaction between two deuterons at small relative womenta. The enitting source radii extracted frum two-deuteron and two-triton correlations appear to be roughly twice as large as the ones obtained from two-proton correlations. This presents an intriguing puzzle wich wust be addressed by reaction models of mucleon and light-fragment production.

1. Mational Superconducting Cyclotron Facility, Michigan State University. East Lansing, Michigan.

2. Siman Fraser University, Bumaby, B.C., Canada.

3. S. E. Koonin, Phys. Lett. 708, 43 (1977). 4. G. I. Kopylor and I. M. Podgoretskii, Sov. J. Mucl. Phys. 18, 336 (1974); G. I.

Kopylor, Phys. Lett. 503, 472 (1974).

5. F. B. Yano and S. E. Koonin, Phys. Lett. 788, 556 (1978).

6. S. Y. Fung et al.. Phys. Rev. Lett. 41. $1592(1978)$. (1983).

. D. Beavis et al.. Phys. Rev. C 27, 910

(1983).

8. 0. Beavis et al., Phys. Rev. C 28, 2561

(1984).

A. Lajc et a! ., Phys. Rev. C 29, 2173

10. F. Zarbaksh et al., Phys. Rev. Lett, 16, 1268 (1981).

11. H. G. Lynch et al., Phys. Rev. Lett. 51, 1850 (1983).

12. H. A. Gustafsson et al., Phy5. Rev. Lett. 53, 544 (1984).

13. M. Gyulassy, Phys. Rev. Lett. 48, 454 (1982).

14. M. A. Bernstein et al., Phys. Rev. C 29. 132 (1984;), and to be published.

15. P. D. Bond and R. J. de Meijer, Phys. Rev. Lett. 52, 2301 (1984).

16. H. G. Lynch et al.. Phys. Rev. Lett. 52, 2302 (1984).

17. F. S. Chwieroth et al., Nucl. Phys. A189, 1 (1972).

18. G. M. Hale and B. C. Dodder, Few-Body Problems in Physics, ed. B. Zeitnitz, Elsevier. Amsterdam, Vol. II, D. 433.

19. D. H. Boal, Phys. Rev, C 30, 749 (1984).

STRUCTURE IN THE ASYWETRIC REACTION $56 \mathrm{Fe}+238 \mathrm{U}$ AT $15 \mathrm{NeV} / \mathrm{amy}$
A. Heston-Dankes
V. E. Viola, Jr.2
A. Mignerey 1
C. Merouane
S. Bradley!
K. Kutatkowski ${ }^{2}$
H. Brever
D. Benton
F. E. Obenshain
R. L. Ferguson

One of the goals of this experiment was the search for structure in the kinetic energy spectrs of the Fe-like reaction products. Structure has previously been observed for the symetric reaction $56 \mathrm{Fe}+56 \mathrm{Fe}$ in this energy range, 3 and recent results from the group of Frascaria et al. show clear structures excited in asy etric reactions of $36 \mathrm{Ar}$ and $20 \mathrm{Ne}$ beaws with $208 \mathrm{~Pb}$ targets. ${ }^{4}$ It has been suggested that these structures arise from excitation of the target mucleus. This interpretatioi. is able to explain the relatively narrow widths observed at high excitation energy.

The $15-\mathrm{HeV} / \mathrm{am} 56 \mathrm{Fe}$ bean from the HIRF was used to study the reaction ${ }^{56} \mathrm{Fe}+238 \mathrm{U}$. The projectilelike products mere measured with discrete charge and ass resolution by mans of the time-of-flight cechnique. The start and stop detectors were of the Breskin design and were manufactured by the Oak Ridge detector laboratory under the dirertion of J. Blankenship. The stop detector was a large $x-y$ position-sensitive device wich permitted position correction of time-of-flight and energy signals. The energy anc charge of the fragments were determined by a large-area segmented $\triangle E-E$ ion chaber. The 150-torr pressure of $\mathrm{CF}_{\text {, provided sufficiént }}$ stopping power to feasure all projectilelike fragments down to $Z \sim 10$. The best time resolution achieved by the double Breskin system was $\sim 300$ ps. This necessitated using a 2.5-m flight path to obtain a mass resolution of $\sim 0.8$ mass units for the elastically scattered ${ }^{56} \mathrm{Fe}$. The energy resolution from the Snell chamber was I\% for elastically scattered ${ }^{56} \mathrm{Fe}$ at $5^{\circ}$.

At the time of this report, only charge data are available from the ${ }^{56} \mathrm{Fe}+238$ ij reaction. Results from the previously studied $56 \mathrm{Fe}+56 \mathrm{Fe}$ system ${ }^{3}$ indicate that the $Z=27$ (cobalt) spectrum shows qualitatively the same structure as the isotopically separated ${ }^{57}$ Co spectrum. This is because the dominant cobalt isotope is $A=57$. Separation into individual isotopes serves to sharpen structure al ready evident in the inclusive sper.trum. Figure 2.88 shows the low excitation energy (high total kinetic energy) region of the cobalt total kinetic energy spectrum obtained from $15-$ HeV/amu ${ }^{56} \mathrm{Fe}$ on $238 \mathrm{U}$. Shown for compartson in Fig. 2.89 are the results obtained at $14.6 \mathrm{KeV} / \mathrm{am}$ for the symetric reaction $56 \mathrm{Fe}+56 \mathrm{Fe}$. The distinct structures in the cobalt spectrum from the $238 \mathrm{U}$ target are in qualitative ayreement with those obtained for asymmetric reactions with $208 \mathrm{~Pb}$ targets. 4 The peak corresponding to a total kinetic energy loss, Eloss, of $\sim 25 \mathrm{MeV}$ probably comes from the direct one-protu.i transfer and can be compared to the most prominent peak in Fig. 2.89. The next major rise at $E_{10 s s}=50-60$ is probably the sequertial one-particle evaporation, primarily from ${ }^{58} \mathrm{Co}_{0}$ neutron ission. This is consistent with the second major peak cisserved in the ${ }^{56} \mathrm{Fe}+56 \mathrm{Fe}$ reaction at E;oss $\sim$ $50 \mathrm{MeV}$. However, for the $23 \mathrm{BU}$ reaction there appears to be a substructure with possible peaks at $E_{\text {loss }} \sim 50$ and $65 \mathrm{MeV}$ superimposed on the broader underlying structure.

The overwheiming conclusion from all evaporation calculations is that it is difficult to produce or preserve structure at large energy losses. The widths of narrow peaks in primary spectra are broadened by the assumptions of isotroptc particle emission and of distributions in excitation energy division. Hence, when clear structure is observed at large energy 


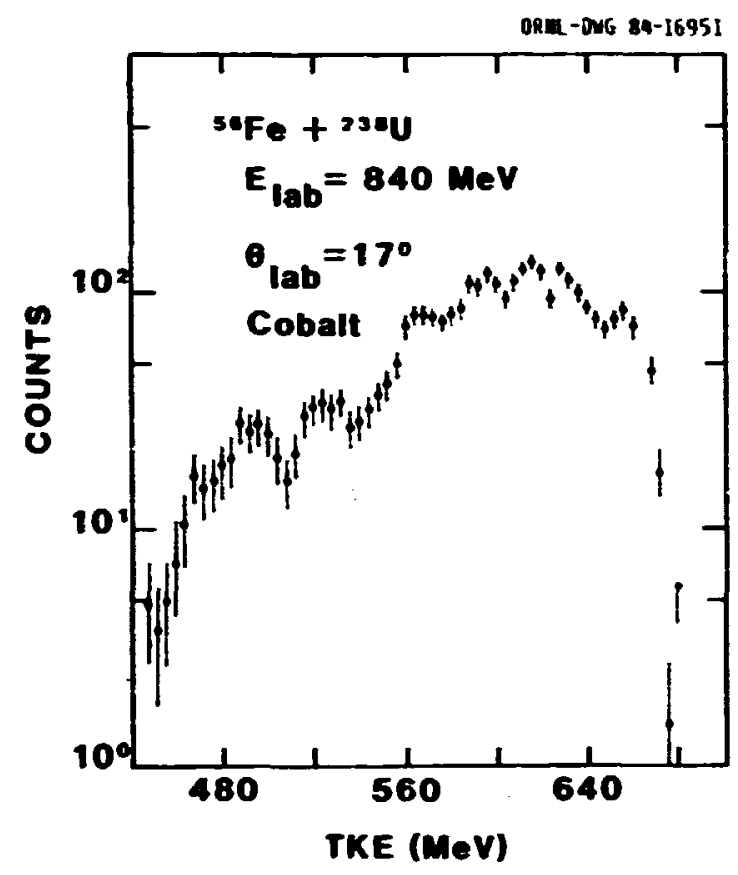

Fig. 2.88. Total kinetic energy spectrum of the cooalt product $(Z=27)$ measured at a laboratory angle of $17^{\circ}$ in the reaction of B40-MeV ${ }^{56} \mathrm{Fe}$ with ${ }^{238} \mathrm{U}$.

losses, processes other than evaporation would appear to be responsible. In the ${ }^{56} \mathrm{Fe}+218 \mathrm{U}$ reaction, four additional peaks may be identified, centered at $E_{\text {loss }}=85,115,155$, and 190 MeV. As energy loss increases, the width and energy spacing of the structures also increase. This trend is the same as that observed in the reaction of $11-\mathrm{MeV} / \mathrm{amu}{ }^{36} \mathrm{Ar}$ on $208 \mathrm{~Pb}$ (Ref. 4). The explanation of target excitations producing the structure is attractive since the cobalt product remains relatively cold and sequential particle enission will not wash out any structure produced in the primary reaction.

Attempts have been made to deduce excitation energy sharing in asymetric damped reactions by fitting the shape of secondary energy spectra using excitation energy division as a parameter.5 The underlying envelope of yield is surely fed by sequential decay, wich gives this method some vallitty. However, care must be taken to recognize the possibility of other mechanisms, even at high energy losses. which could make deconvolution of the spectra difficult.

This is the first time our group has identffied structure in an asymetric system. Severd : ctors are probably responsible for this. The increased bombdrding energy may have increased the cross section for these processes or decreased that for the competing damped reactton mechanism, which dominates the enerzy spectra at lower bombarding energtes. Also, the increase in soltd angle subtended by the timeof-flight. system, due io the use of larye-area
ORML-2N6 84-16952

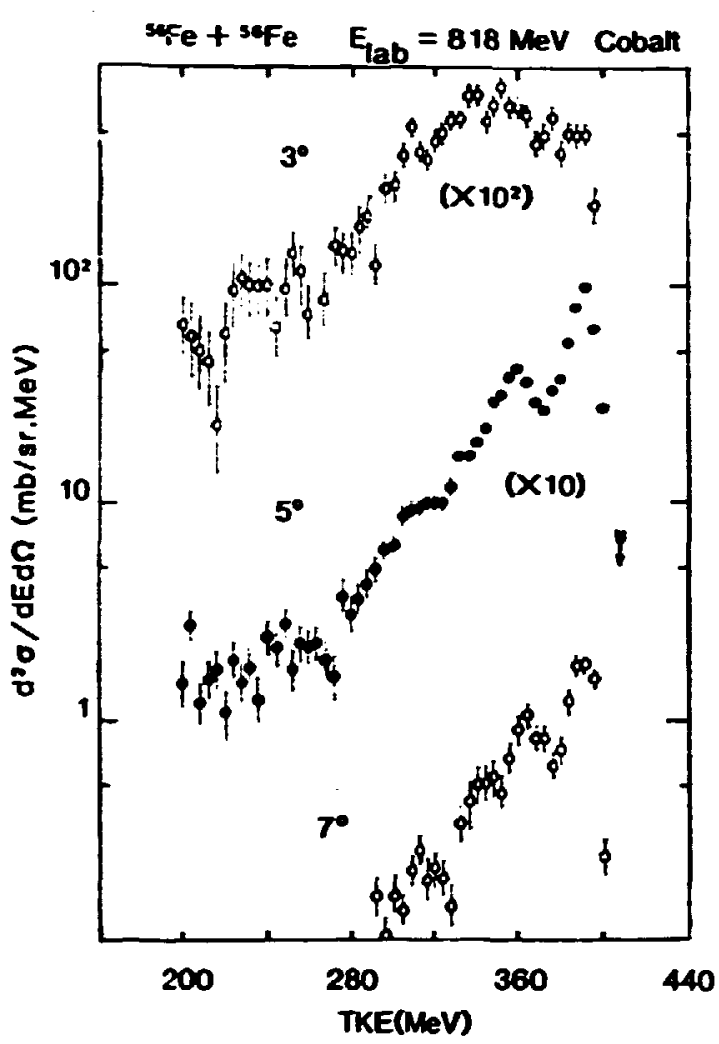

Fig. 2.89. Total kinetic spectra of the cobalt products $(Z=27)$ easured at laboratory angles of $3^{\circ}, 5^{\circ}$, and $7^{\circ}$ in the reaction of B18-HeV $56 \mathrm{Fe}$ with $56 \mathrm{fe}$.

Breskin detectors, made study of the ${ }^{56} \mathrm{Fe}+238 \mathrm{U}$ reaction experimentally feasible.

1. University of Maryland, College Park, Maryland.

2. Indiana University, Bloomington, Indiana.

3. A. Hignerey et al., Proc. Horkshop on Nuclear Dynamics III, Copper Mountain, Coln., March 1984, p. 76.

4. Ph. Chomaz et al., preprint (1984).

5. H. Sohlbach et al. Proc. Horkshop on Nuclear Dynamics III, Copper Mountain, Colo., March 1984, p. 72.

\section{INCLUSIVE CROSS SECTIONS FOR $\pi^{\circ}$ EMISSION} IN 25-MeV/anu MEAVY-ION REACTIONS
P. Braun-Munzinger
R. Freifelderl
F. E. Obenshain
J. Stachel!
F. Plasil
G. R. Young

We have jtudted production of neutral pi mesons using heavy ton beams at energtes per 
nucleon well below the value required for free mucleons to interact and produce a pion. The experiment used the $25 \mathrm{HeV} / \mathrm{amu} 160$ bean from the ORNL Holifield complex to bombard $27_{A I}$ and nat targets. The purpose of the experiment was, after es-dblishing a nonzero value for the pionproduction cross section at such low bonbarding energies, to obtain energy distribution, angular distribution, and target mass-dependence data which wight allow one to learn wat mechanisa is responsible for concentrating so wch of the kinetic energy of the projectile into creation and ejection of one outgoing particle. Theorists have provided a range of explanations from "ordinary" (involving coupling of relative and Fermi momenid of individual mucleons to give energies in excess of the nucleon-nucleon threshold) tc "exotic" (involving very shortrange clustaring of mucleons to form a mssive hard scattering center, or involving a nuclear counterpart to the familiar electronagnetic breasstrahiung emission of photons). One of the fascinations of such measurements is the concentration of over half of the center-of-mass energy to produce a pion $\left(m_{-} 0=135 \mathrm{NeV} / \mathrm{c}^{2}\right.$ $\left.\mathrm{m}_{ \pm} \pm=139 \mathrm{MeV} / \mathrm{c}^{2}\right)$. While the emission of ve:y energetic nucleons is familiar from the observation of nucleons in the high-energy region of evaporation spectra from compound or composite systems of nuclei, the particles enitted were present in the entrance channel. If emission of a quark-antiquark pair (i.e., meson, here a pion) requires large momentua transfer in the scattering of the constituents, one wishes to inguire as to wiat constitutes such scattering, or tho the participants are, in such low-energy experiments.

The experimental method used in the present work consisted of detecting the two energetic photons enitted in decay of $a \pi^{2}$, in an array of Cerenkov detectors. A ${ }^{\circ}$ decays 98.83 of the time into two equal energy (in the $x^{0}$ rest frame) photons with a lifetine of $8.3 \times 10^{-17}$ secords. The photons enitted in the decay have energies of the order of $70 \mathrm{MeV}$ or Varger. increasing with increasing ${ }^{0}$ kinetic energy. The photons tend to cluster about a laboratory opening angle $\theta_{12}=2 \cos ^{-1}\left(\beta_{1}\right)$, where $\beta_{k}$. $v_{\pi}, i c$. Low-energy or nearly stopped $x^{0}$ 's decay into two correlated gama rays wich are near $180^{\circ}$ separation in the laboratory system. This makes the $\pi^{\circ}$ 's easier to identify than charged pions. At Iow energies the charged-pion ! ifetime approaches the proper lifetifie of $26 \mathrm{~ns}$, and consequently, the pions decay before detection.

Lead-glass Cerenkov shower counters were used to detect the decay photons. These detectors are large rectangular bloc'.s of optical glass lodded with $255 \%$ by meight lead oxtde. Cerenkor light emitted by electrons and positrons as they pass through the glass is detected with photomultiplier tubes attached to the glass blocks. The setectors used were arranged as 20 pairs of detectors, with each telescope placed either $15^{\circ}$ above or below a plane containing the beam, at $30^{\circ}$ intervals in polar angle. The beam pipe occupted the $0^{\circ}$ and $180^{\circ}$ positions. The front, or converter, block of each telescope was a $10 \mathrm{~cm} \times 10 \mathrm{~cm} \times 5 \mathrm{~cm}$ thick bloct of FZ-type glass riewed on a side edge $(20 \mathrm{cs} \times 5 \mathrm{~cm}$ ) by a fast 5-ca, 14-stage phototube. The rear, or absorber, hlock of each telescope was a $15 \mathrm{~cm} x$ $15 \mathrm{a} \times 34$ a thick block of SF 5 glass riewed frca the rear $15 \mathrm{~cm} \times 15 \mathrm{~cm}$ face by a $12.5-\mathrm{cm}$, 10-stage phototube. Pulse heights and time of arrival relative to the cyclotros. $P F$ for all good events mere recorded on manetic tape and analyzed of line.

A Monte Carlo code was used to simulate $x^{0}$ decay in the laboratory for kinetic energies between 0 and $110 \mathrm{Mel}$ and for all emission angles (polar and azimuthal). The geomet,ic acceptance of the detector array was $2.9 \%$ if $4 \pi$ and decreased with increasing $x$ kinetic energy to a value of $0.3 \%$ at $T_{\pi} \sim 100 \mathrm{HeV}$. The detection efficiency of the converters was taken from the measurements of $C$. Michel.2

Neutral pions were tagged by calculating the invariant mass of a detected photon pair and searchini for events here this clustered about $135.6 \mathrm{MeV}$, the $x^{0}$ rest mass. A scatter plot of invariant mass vs. detector pair opening angle shows a distinct cluster of events is found near $135 \mathrm{HeV}$ for $\theta_{12}>90^{\circ}$. A projection onto the invariant ass axis is given in Fig. 2.90. By recasting the invariant mass equation in the form

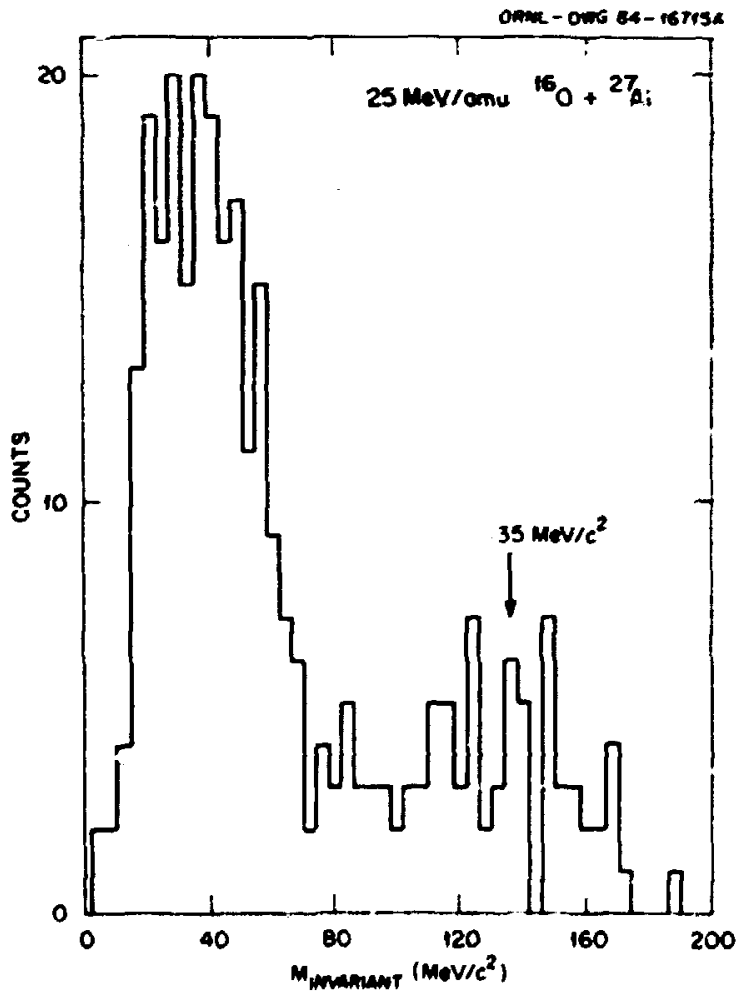

Fia. 2.90. Invariant mass spectrum of twopruton event: produced in reactions of $5-\mathrm{MeV} / \mathrm{am}$ lho, $27 \mathrm{hl}$. 


$$
T_{x}=m_{x} c^{2} \frac{2}{\left(1-\cos \theta_{12}\right)\left(1-x^{2}\right)}-1 \text {. }
$$

where $X=\left(E_{\gamma_{1}}-E_{\gamma_{2}}\right) /\left(E_{\gamma_{1}}+E_{\gamma_{2}}\right)$, the kinetic energy $T_{x}$ can be obtained. The resulting energy and angular distributions for the Al target are shown in Fig. 2.91.

The difficult background arises from penetrating cosmic-ray muons and products of cosmicray hadron showers. Since energetic muons do not interact (except by electroweak processes, wich have very small cross sections) in the glass, they penetrate the array in a more or.
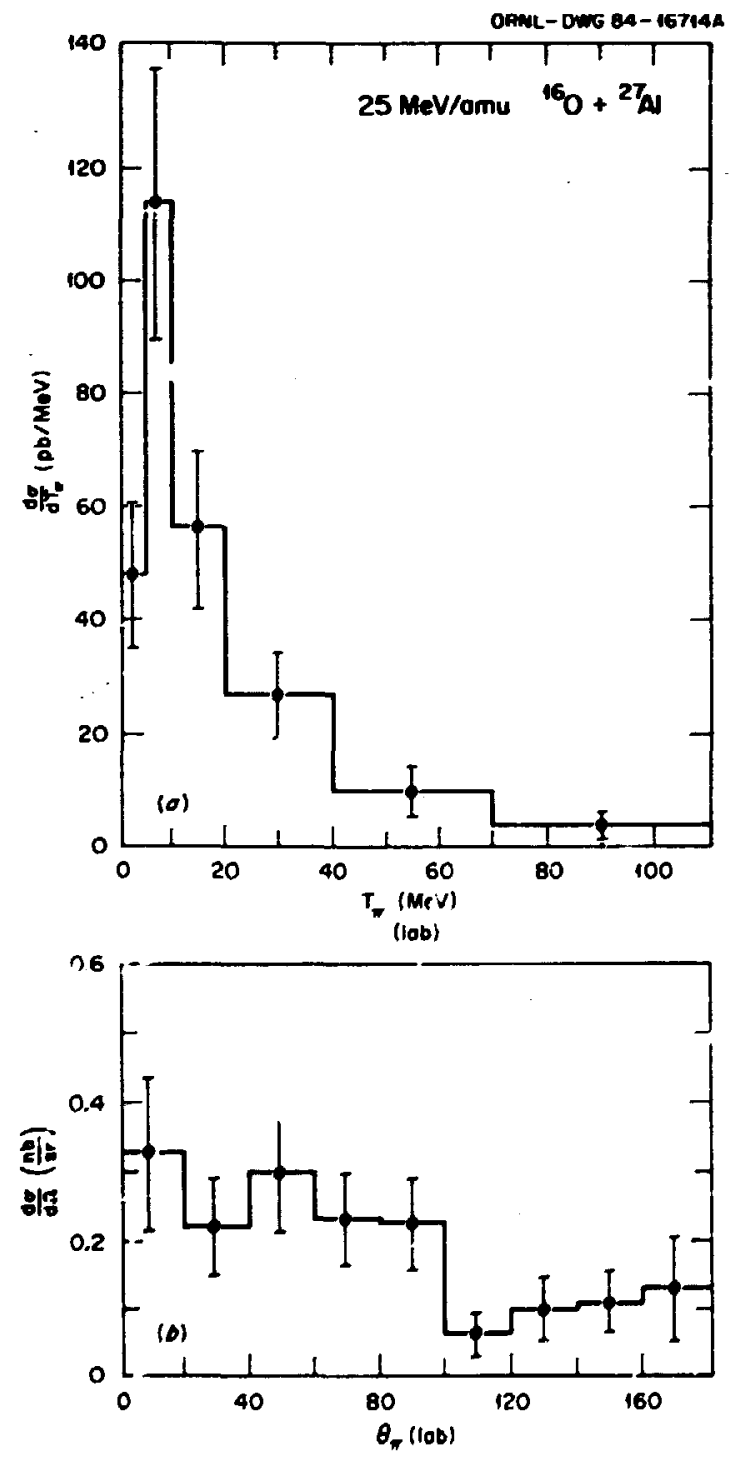

Fig. 2,91. (a) Laboratory kinetic energy spectrum of $\pi^{0}$ 's produced in reactions of $25-\mathrm{MeV} / \mathrm{amu} 160+27 \mathrm{Al}$. (b) Laboratory angular distribution of $\pi^{0}$ 's produced in the same reaction. less straight ?ine, and thus tend to be concentrated near the horizontal plane. Showers and muons are suppressed by the invariant mass cut, a prompt cut on the event time relative to the beam Rf timing, and by requiring a mitiplicity of exactly four hit counters. This last requirement was checked to cause only an $\sim 12$ loss in real $\pi^{0}$ events (due to, for example, random coincidences between $a \pi^{0}$ and beam-related $\gamma$ background).

Even with these precautions, for $\theta_{12}<110^{\circ}$ a large contamination of cosmic rays in the invariant mass spectrum is found. To tag these cosmic rays, 2.5-cm-thick plastic paddles were placed in front of telescopes to veto cosmic rays hitting the arrays by detecting their Cerenkov light. As plastic has a 48-cm radiation length, only $<4 \%$ of the photons convert in the paddle, resulting in small efficiency loss but a very large reduction in cosmic-ray background for $\theta_{12}<120^{\circ}$.

An integrated $x^{0}$ r viluction cross section of $5.9 \pm 2.4 \mathrm{nb}$ is found for the reaction 25-HeV/anu $160+A l$. Scaling according to our earlier prescription ${ }^{3}$ to the value for the $12 C+12 C$ systen, a cross section of $2.8 \mathrm{nb}$ resuits, wich is an order or magnitude less than the result at $35 \mathrm{MeV} / \mathrm{amu}$ (Ref. 3). It was ;ointed out earlier" that "Fermi-gas" type production models 5 fail to reproduce the observed cross sections by three orders of magnitude at $35 \mathrm{MeV} / \mathrm{amu}$; this failure is expected to be much worse at $25 \mathrm{MeV} / \mathrm{amu}$.

Howev: $r$, a mode ${ }^{6}$ based on cooperative action of several target and projectile nucleons ("clusters") yields fair agreement with production cross sections and spect.ral shapes for the 35-MeV/amu results. When the curve of cross section vs. beam energy for $12 \mathrm{C}+12 \mathrm{C}$ is extrapolated to $25 \mathrm{MeV} / \mathrm{amu}$, it appears to give agreement within a fartor of 2 with the present total cross section result. It will be interesting to comp are our spectral shapes with the predictions of this model when these calculations become available for our case. The energy distributions are concentrated at lower $k$ inetic energies than the $35-\mathrm{MeV} / \mathrm{amu}$ data and have an exponential slope of $\sim 17.5 \mathrm{MeV}$. The angular distributions are somewhat forward peaked, as are those for the intermediate-energy $\left(24<T_{\pi}<80 \mathrm{MeV}\right.$ ) pions observed at $35 \mathrm{MeV} / \mathrm{amu}$. Unlike the latter re-rits, the present results do not show an obvious decrease at $\theta=90^{\circ}$.

A completely different model based on pionic bremsstrahlung ${ }^{7}$ predicts a $\pi^{0}$ cross section for ${ }^{12} \mathrm{C}+{ }^{12} \mathrm{C}$ at $25 \mathrm{MeV} / \mathrm{amu}$ of $2.5 \mathrm{nb}$, similar to the observed value. This model has provided good agreement with energy and angular distribu$t$ ions measured for ${ }^{12} \mathrm{C}+{ }^{2} \mathrm{C}$ at $60-85 \mathrm{MeV} / \mathrm{amu}$. Predictions for these quantities for asymetric systems such as ours are eagerly awatted.

1. State Unfuersity of New York, Stony Brook, New York.

2. C. Michel, Saclay (to be published).

3. P. Braun-Munzinger et al., Phys. Rev. Lett. 52, 255 (1984). 
4. R. Shyan and J. Knoll, Phys. Lett. 1368, 221 (1984).

5. 6. Bertsch, Phys. Rev. C 15, 713 (1977). 6. R. Shyan and J. Knoll, Mucl. Phys. A426, 606 (1984).

7. D. Yasak et al.. Phys. Lett. 938, 243 (1980).

\section{TETEPHITHTIO OF THE REST FRIME FOR IENR-THRESHOLD $x^{\circ}$ EMISSIO IV REAT-IOH RERTIO'S}
J. Stachel 1
P. Braun-Munzinger
T. C. Ares
P. d Youngl
F. E. Obenshain
P. Paull
F. Plasil
6. R. Young

Pion production in heavy-ion collisions at energies below the free mucleon-nucleon threshold, i.e., at energies where the pion mass exceeds the kinetic energy of a projectile nucleon in the zero momentum frame, has been discussed for a long time in terms of probing the intrinsic Fermi notion in the projectile and target nuclei. However, as the beam energy is lowered, this process gets more and more unlikely, and below a certain energy it should not be observed anymore. In a sharp cut-off Fermigas model, this occurs ${ }^{2}$ at $E_{\text {ab }}=54 \mathrm{MeV} / \mathrm{amu}$. However, as long as the total center-of-mass energy exceeds the pion mass, pions still can be produced by a cooperative sharing of the bean energy of several (or all) projectile mucleons. The experiments presented here are meant to extend the experimental information into that kinematic domain. ${ }^{3}$ The production of a pion of 100-HeV kinetic energy with a 35-MeV/anu in $\mathrm{N}$ beam requires Fermi momenta as high as $\sim 380 \mathrm{MeV} / \mathrm{c}$ or, alternatively, $60 \%$ of the total bean energy. The information from the present experiments, combined with the results of previous expe.iments at higher $\$$-am energies of $44 \mathrm{MeV} / \mathrm{amu}$ and 60-84 MeV/amu .efs, 4 and 5), enables us to determine wich of the alternative production mechanisms is dominant at a given bean energy.

In the experiments presonted here, neutral pions have been detected through their predominant fast decay into two high-energy $r$ rays. These two $y$ rays mere observed in coincidence in an annular array (coplanar with the beam axis) of lead glass Cerenkov detector telescopes. Each telescope consists of a converter and an absorber section with respective depths of 1.6 and 15 radiation lengths (for details see Ref. 3). The measurement of the two $r$ energies and angles (given by the openting angles of the separate telescopes) allows a reconstruction of the pion invariant mass, its kinetic energy, and emission angle.

In a first experiment, ${ }^{3}$ data from ten of these telescopes, wich covered a solid angle of about $8.5 \%$ of $4 \pi$, were combined. A 35-MeV/anu $14 \mathrm{~N}$ beam of the $K=500$ superconducting cyclotron at Michigan State University was user to bombard targets of natural $\mathrm{Al}$. Ni, and $\mathrm{W}$. In the present experiment, using the same beam and the Ni target, we aimed at obtaininn "e, - detailed information on one particular targat and combined data from 20 Cerenkov telescopes. Because of the finer granularity of this setup. the $r$-ray angular resolution inproved from $24^{\circ}$ to $17^{\circ}$, thus providing a significantly better pion angle and kinetic energy resolution. Ine solid angle covered by this setup was somewhat smaller (about $6 \%$ of $4 \mathrm{k}$ ).

The probability that the two decay $r$ rays of a pion entered our detectors is obtained from Honte Carlo simulations wich take into account the pion decay kinematics. The response of the converter sections to 125 -ivel electrons has been measured to be 75\%. This provides an upper limit for the response to $Y$ rays. The energy dependence of this response is obtained from Monte Carlo sialuatiuns ${ }^{6}$ that include the conversion of a $r$ ray into an electromagnetic shower, the subsequent Cerenkov light enission and collection probabili.j, and the photumitiplier response. The resulting detection probability increases monotonically with increasing $r$ energy until it levels off at $\sim 150$ MeV at $68 \%$, in oood agreenent with our measured value.

The very intense background of beaicorrelated r radiation and neutrons and the background due to cosmic rays is effectively reduced by (1) our coincidence technique. Which requires a four-fold trigger of two absorber signals. together with the corresponding two converter signals; (2) the $\sim 2-n s$ time resolution of the setup, wich allows the application of narrow gates on the various detector-detector and detector-beam time differences; (3) a software threshold on low $\gamma$ energies (30-40 $\mathrm{HeV}$ ) and small opening angles between the two $r$ rays (a minimum is kinematically determined by the pion kinetic energy); and (4) a gate on the pion invariant mass. The effects of the different cuts on the data were investigated by Monte Carlo sirulations, and if necessary, corrections were applied to the results.

Figure 2.92 shows the experimental invariant mass spectrum. It exhibits a clear peak around the $\pi$ invariant mass of $135 \mathrm{MeV}$. Approximately 900 pions were detected in a beam time of one week. Also displayed in Fig. 2.92 is the resui. of a Monte Carlo simulation. Its gond agreement with the data shows that effects of the geometry, the $r$-ray energy and angular resolution, and of the various cuts on the data mere treated properly. An effective background suppression is al so apparent.

There is one piece of experimental information that can provide information on the velocity of the source that enits the pions. Figure 2.93 shows a plot of the double differential cross section versus the rapidity $y$ and the momentum perpendicular to the beam di rection $P_{\perp}$ for the present experiment at $35 \mathrm{MeV} / \mathrm{amw}$. It can be seen that the experimental cross sections are symmetric around $y=0.01 \pm 0.05$. Wich indicates that the pion source is moving very slowly. Thts again is a strong hint that single nucleon-nucleon collisions are not the origin of the presently abserved pion cross sections. For this process a soul e veloctty of holf the beam rapidity (labeled is $1 / 2 \mathrm{p}$ in Fig. 2.93) is expected, in clear contradiction to the experimental results. On the other hand, pion 


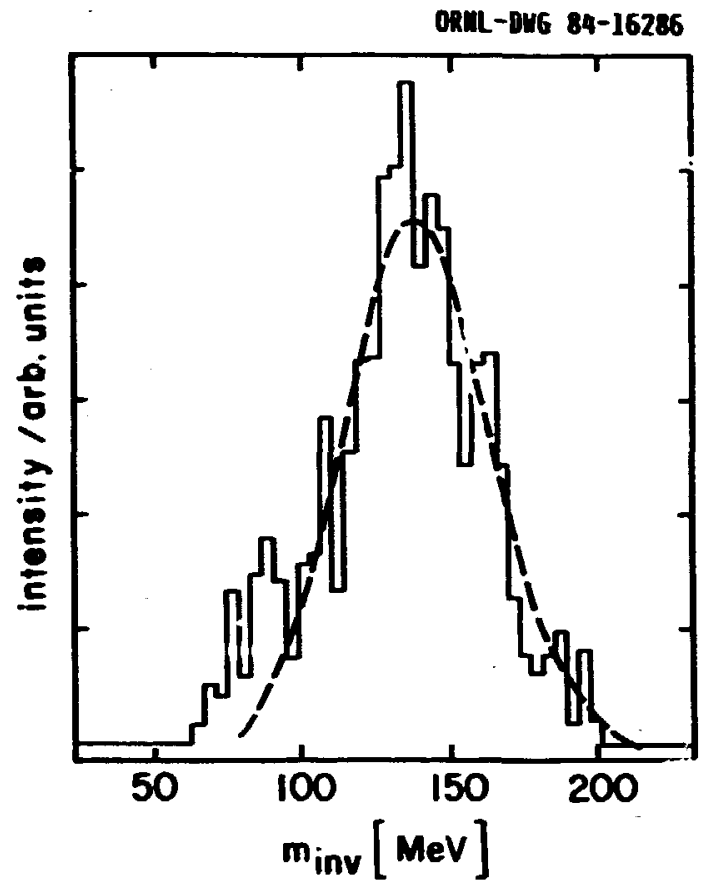

Fig. 2.92. Experimental invariant ass spectrum for 35-MeV/anu ${ }^{14} \mathrm{~N}$ on $\mathrm{Ni}$, together with the result of a Monte Carlo simulation (dashed line).

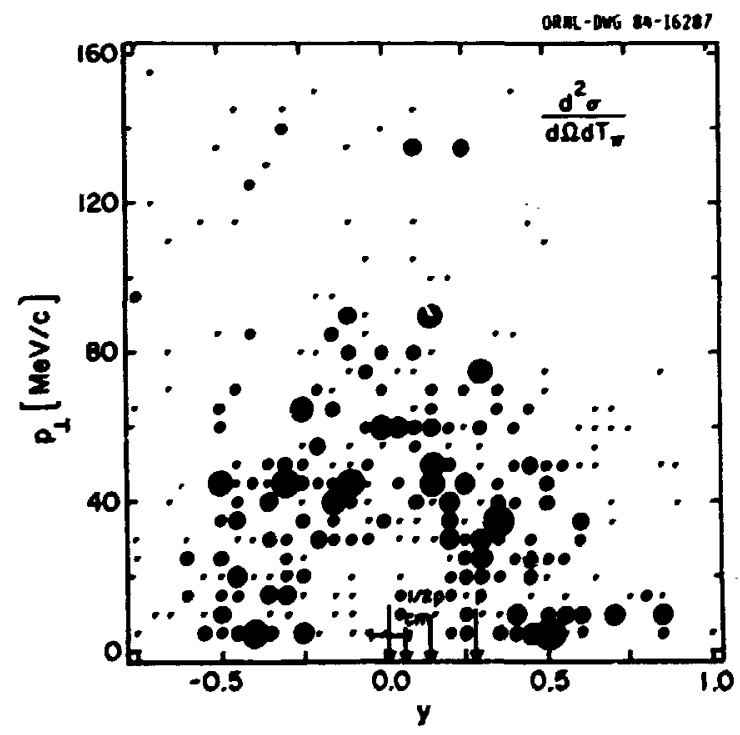

Fig. 2.93. Double differential cross sections $d^{2} \sigma / d a d T_{7}$ versus rapidity, $y=1 / 2 \log \left[\left(E+p_{1}\right) /\right.$ $\left.\left(E-P_{1}\right)\right\}$, and momentum perpendicular to the beam axis $\mathrm{P}_{\perp}$ for $35-\mathrm{MeV} / \mathrm{amu}{ }^{14} \mathrm{~N}+\mathrm{Ni}$. The arrows indicate the projectile rapidity, half the projectile rapidity, the rapidity of the $14 \mathrm{~N}+\mathrm{Ni}$ center-of-mass system, and the centrotd of the experimental cross sections, $\langle y\rangle=$ $0.01 \pm 0.05$. emission from the $M+M i$ composite system is compatible with the data. Monte Carlo simulations indicate, however, that pion reabsorotion effects play an important role. For exaple. for a pion source located near the edge of the combined $\mathrm{A}+\mathrm{Mi}$ systen, corresponding to the complete stopping of the projectile, a considerable reduction in the apparent source velocity is expected, we to these effects. The low average rapidities are in accordance with similar informations for the asynetric systens $12 C+23 A_{U}$ and $12 C+56 \mathrm{Mi}$ at $E_{l a b}=60-84$ MeV/and, where the pion sourise also ws found to wove significantly wre slowly than the nucleonnucleon center-of-mass system. This source velocity can be in ${ }^{2}$-rpreted in terms of the size of the system emitting the pions. At 60-84 $\mathrm{HeV} / \mathrm{am}$ it has been concluded 5,7 that if the wole projectile nucleus is active, then the system involves 14-15\% of the $U$ mucleus and 34-412 of the $\mathrm{Mi}$.

The experimental data collected so far can be interpreted as displaying a transition in the pion production mechanis from mucleon-nucleo, collisions at high Dean energies, with increasing importance of high Fermi womenta as the energy is lowered, to a cooperative sharing of the beaw energy of several projectile mucleons, e.g., via multiple quantum mechanical of $f$-shell collisions, and finally towards a collective production mechanism dominated by the mean field.

1. State University of New York, Stony Brook, New York.

2. G. Bertsch, Phys. Rev. C 15, 713 (1977).

3. P. Braun Hunzinger et al.., Phys. Rev.

Lett. 52, 255 (1984).

4. H. Heckwolf et al., Z. Phys. A315, 243 (1984).

5. H. Noll et al., Phys. Rev. Lett. 52, 1284 (1984).

6. C. Michel, to be published.

7. E. Grosse, Proc. VI High Energy Heavy Ion Study, Berkeley (1983).

\section{HEAVY-ION-INDUCED FISSION AT ENERGIES UP TO $20 \mathrm{MeV} / \mathrm{amu}$}
T. C. Awes
M. M. Fowler'
J. Boissevain'
F. E. Obenshain
H. C. Britt
H. Ohm"
K. Eskola 2
P. Eskola ${ }^{3}$
F. Plasil
J. B. Withel nol
R. L. Ferguson
G. R. Youny

During the pas year we have published our extensive studies of angular-momentum-dependent fission barriers.5,6 This work, carried out at energies up to $10 \mathrm{MeV} / \mathrm{amu}$, led to the conclusion that calculated barriers from the rotatingfintte-range model (RFRM) ${ }^{7}$ adequately describe the measurements over a large range of excitation energy, angular momentum, and mass of the fissioning system. The statistical model. together (in some cases) with calculated bass 
fusion cross section, was used in the data analysis. Encouraged by our success, we extended our fission studies into the energy range between 10 and $20 \mathrm{MeV} / \mathrm{amu}$. To the extent to which it was possible, we have studied the save compound (or composite) systews, i.e.. $158 \mathrm{Er},{ }^{186} 05$, and $208,210 \mathrm{Po}$. In addition, we made measurements on a $238 \mathrm{U}$ target. The target and projectile combinations were as follows: ${ }_{12} \mathrm{C}$ on $174 \mathrm{Yb}, 198 \mathrm{Pt}$ and $238 \mathrm{U}$; 160 on $142 \mathrm{Wd}$, $170 \mathrm{Er}$. 1920s; and $238 \mathrm{U}$; $32 \mathrm{~S}$ on $126 \mathrm{Te}, 144 \mathrm{Nd}$, and 230 $\mathrm{U}$; and $58 \mathrm{Hi}$ on ${ }^{96} \mathrm{Zr}$, ${ }^{116} \mathrm{Cd}$, and ${ }^{23} \mathrm{~B}_{\mathrm{U}}$. The energies ranged from 95 to $291 \mathrm{MeV}$ for ${ }^{12} \mathrm{C}, 140$ to $315 \mathrm{MeV}$ for 160,350 to $700 \mathrm{MeV}$ for $32 \mathrm{~s}$, and

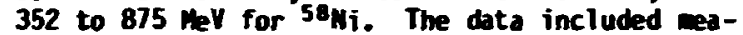
surenents of angles and velocities of coincident fission fragments, from wich the linear anentum transfer to the fissioning system could be deduced. Fission following incomplete womentus transfer was found to be substantial only in reactions on 2380 targets. In our cross section analysis, we have included only events with conplete and nearly complete momentum transfer.

We had expected at these higher energies to observe limitations resulting from various dynamical processes such as incomplete fusion and/or extra push effects. This, indeed, turned out to be the case, since it was no longer possible to describe the measured cross sections in terms of statistical model calculations with RFRH fission barriers and with Bass model fusion cross sections. It was expected that in reactions with $12 \mathrm{C}$ and $16^{\mathrm{O}}$ ions, incomplete fusion would result in lower observed fission cross sections, except in the case of the $238 \mathrm{~J}$ target, where even partially fused systems are highly fissile. Results of $12 \mathrm{C}$ and 160 bombardments of $238 \mathrm{U}$ are shown in $\mathrm{Fig}$. 2.94a for the near full momentum transfer component, together with calculations using the Bass model. On the basis of the good agreement between theory and experiment, we conclude that the Bass model constitutes a reasonable parametrization of fusion (complete and incomplete) in this energy range. This situation did not prevail in the case of reactions of ${ }^{32} \mathrm{~S}$ and $58 \mathrm{Ni}$ with $23 \mathrm{BU}$, as can be seen from Fig. 2.94b. The discrepancy between the Bass model results and the measured fission cross sections for the near full momentum transfer component can be attributed to higher Coulomb repu" $s, i$ between target and projectile nuclei, resuiting in the need for an additional energy (extra push) to achieve fusion.9

In Fig. $2.94 \mathrm{c}$ we shuw the fission excitation functions for the $160+142$ Nd and the $12 C+174 Y_{b}$ systems, together with statistical model calculations in which the Bass model fustion cross sections and the RFRM fission barriers have been incorporated. It can be seen that, at the highest energfes, calculated cross sections are three to five times higher than measured cross sections, wich saturate at a level consistent with a maximum angular momentum of the fissioning system of about $65 \mathrm{~h}$. This observed saturation in the fission excitation function may, presumably, be attributed to incomplete fusion, and the implication is that collisions with partial waves beyond $\sim 65 \hbar$ involve processes that result in residual nuclei with reduced angular momenta. While incomplete
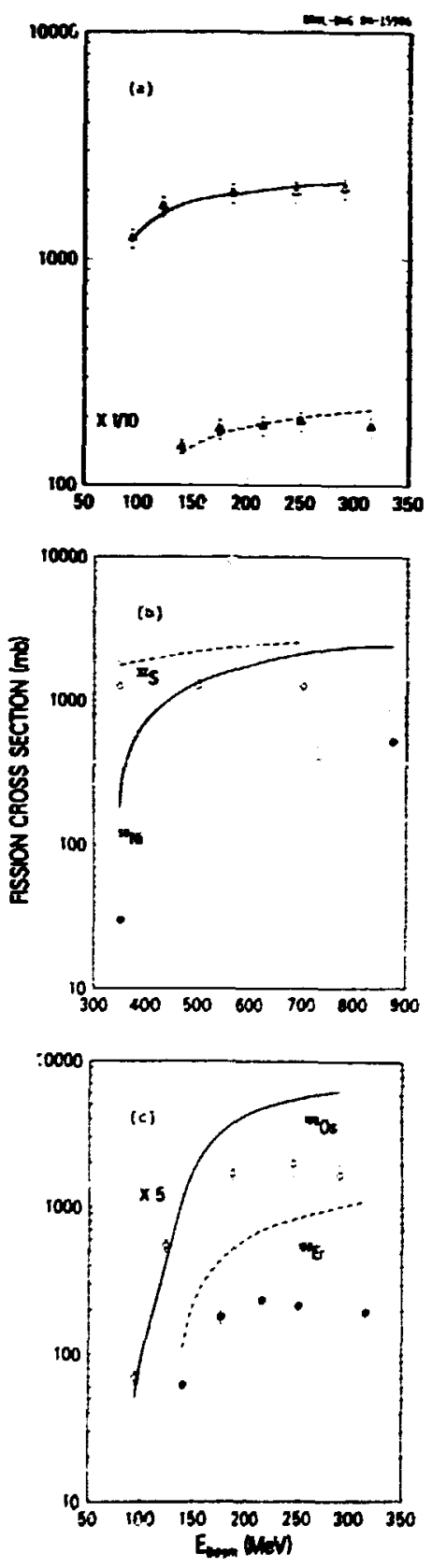

Fig. 2,94. Fission cross sections for reactions of $23 \mathrm{~B}_{\mathrm{U}}$ wth $12 \mathrm{C}$ (open triangies), 160 (closed triangles). 32 S (open circles), and $3 B_{N f}$ (closed cfrcles) are shown in (a) and (b). In (c), cross sections are shown for the $160+142$ Nd (open circles) and the 12C + 174 Yb (closed circles) systems. The solid and dashed lines depict Bass fusion cross sections in (a) and (b) and statistical model calculations using the Bass mortel in (c).

fusion is the most likely explanation, our results cannot be accounted for quant ftatively on the basis of the Wilczynski model. 10 This is because partial waves of only up to $60 \mathrm{~h}$ (for 
$16 \mathrm{~J}+142 \mathrm{Nd}$ at $315 \mathrm{MeV}$ ) are expect od to contribute to complete fusion, 10 wich wo account for only $10 \%$ of our observed fission cross section. Furthermore, based on our masured momentun transfer distributions, fission following incomplete fusion does not contribute significantly to the fission cross sections in the ${ }^{158} \mathrm{Er}$ and 2860 os cases. Thus, it appears that fusion with marly full mentum transfer wust be taking place with partial waves above the limit predicted by the Uilczynski sum-rule model. 10

We way proceed with a schematic description of the impli-ations of our findings for the incomplete usion process by restricting ourselves to, ituations in wich alpha particles (or larger nuclei) escape absorption by the target, and by assuming that the angular momentum imparted to the residual system scales with the mass of the fraction of the projectile absorbed. As was stated above, for the $160+142$ ind reaction, complete fusion, accompanied by some fission, accounts for partial waves up to $65 \mathrm{~h}$. The first alpha particle emitted in the incomplete fusion process will leave the residual systew with $75 \%$ of the incoming angular momentum, and since the nucleus does not decay predominantly by fission, this angular momentum must be $\$ 65 \mathrm{~h}$. Thus, the partial waves associated with single alpha-particle emission must lie between 65 and about $87 \mathrm{~h}$ (i.e., 65/0.75 h). In addition, the Bass modri, which describes the $238 \mathrm{U}$ data adequately, predicts that partial waves of up to $105 \mathrm{~h}$ (at $315 \mathrm{MeV}$ ) contribute to fusionlike reactions. If this is the case, we must also attribute the reactions in this angular momentum window (between $\sim 87 \hbar$ ard $\sim 105 \hbar$ ) to situations that leave the residuai nucleus with an angular momentum of $<65 \mathrm{~h}$. The emission of ${ }^{8} \mathrm{Be}$ (two alpha particles) is consistent with the above requirement. Obviously, further experiments are necessary to clarify the picture. In particular, measurements of evaporation residue products, both inclusive and in coincidence with alpha particles, are needed to confirm the above conjecture.

For systems with high values of the $Z_{p} Z_{t}$ product, we expect that an additional energy (extra push) ${ }^{9}$ is needed for fusion to t.ake place. It was already indicated in $\mathrm{Fi}_{s} .2 .94 \mathrm{~b}$ that this effect is likely to play a role in our reactions with $32 \mathrm{~S}$ and $58 \mathrm{Ni}$ projectiles. However, even for react !ons with 160 fons, at energies above about $120 \mathrm{MeV}$, the highest partial Wuves involve ancular momenta that are sufficient to increase tile effective fissility to the point where extra push' effects are expected. Calculaticns with the parameters of Bjornholm and Swiatecki' (with the exception of our use of $a=10$, which is within their limits of uncertainty) are compared with measurements in Fig. 2.95. For the 160 -induced react: or (Fig. 2.95a), agreement between experiment. . theory at the higher energtes is much better than that wich was obtatned with the Bass model. except for the cose of $23 \mathrm{BU}$. We cinclude that, even with 160 tons, there is some evidence
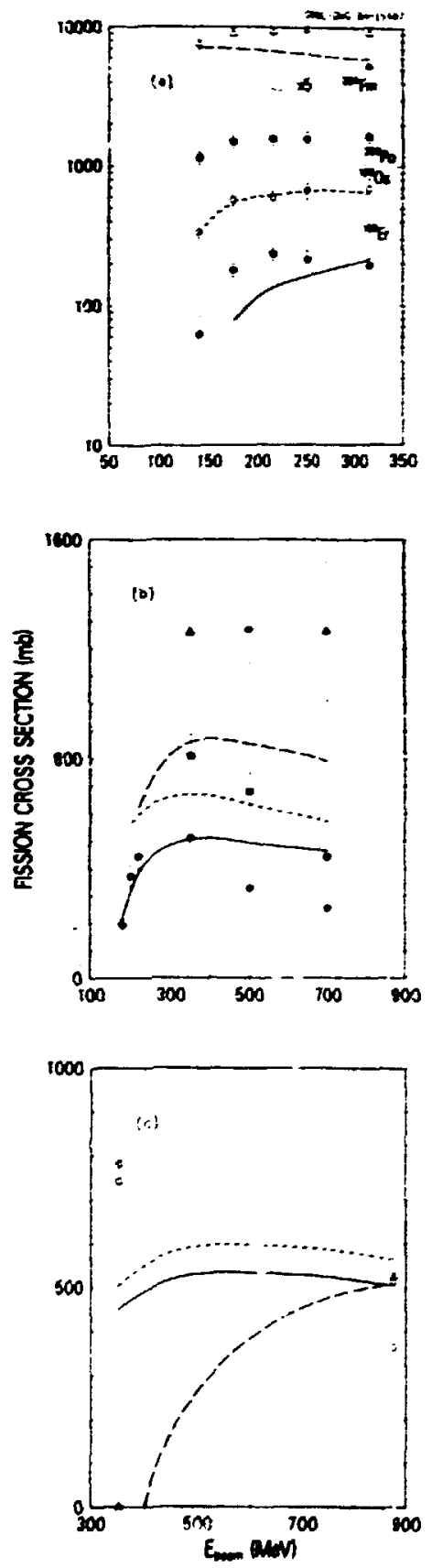

$F: g .2,95$, Comparison of measured fission cross sections with extra push model cross sections (curves). In (a), reactions with 160 are shown, and the labels indicate the composite systems. In (b), $32 \mathrm{~S}$-induced reactions on $126 \mathrm{Te}$ (circles, solid curve), I4tind (squares, shortdashed line), and $238 \mathrm{U}$ (triangles, long-dashed line) are shown. Reactions of $58 \mathrm{Ni}$ with $96 \% \mathrm{r}$ (circles, solto line), wth ${ }^{116} \mathrm{Cd}$ (square: short-dashed line), and 238u (triangles, longdashed line) are shown in (c). 
for a dymanical linitation to the fusion process and that the extra 1 -sh odel 9 determines the highest partial waves for mich complete fusion takes place.

In Figs. 2.95b and 2.95c similar comparisons are shown for reactions with $32 \mathrm{~S}$ and $58 \mathrm{Ni}$. There does not appear to be a consistent quantitative agreement between experiment and theory, although it is clear that some kind of dynanical limitation plays a role in the cases that we have studjed. Best agreement was found in the case of Sthi-induced reactions at the highest energy (875 MeV). in addition, a clear cut illustration of the extra push threshold effect is provided by the $352-H e l$ S6Hi +2300 result, were our measured fission cross section is $0_{-0}^{+50} \rightarrow$ and were a threshold of $460 \mathrm{MeV}$ is predicted by the extra push rodel.

1. Los Alamos Mational Laboratory, Los Al anos, New Mexico.

2. Present address: Physics Department, Uni versity of Helsinki, Helsinki, finland. 3. Present address: Theoretical Physics Department, University of Helsinki, Helsinki, Finland.

4. Chemistry Department, Mainz University, Mainz, West Gerwany.

5. J. van der Plicht et al.., Phys, Rev. C 28, 2022 (1983).

6. F. Plasil et al., Phys. Rev. C 29, 1145 (1984).

7. H. 6. Mustafa, P. A. Baisden, and H. Chandra, Phys. Rev. C 25, 2524 (1982); A. J. Sierk, unpublished results. (1977).

8. R. Bass, Phys. Rev. Lett. 39, 265

9. H. J. Swiatecki, Hucl. Phys. A376, 275 (1982); S. Bjornholm and H. J. Swiatecki, Nucl. Phys. A391, 471 (1982).

10. J. Wilczynski et al., Phys. Rev. Lett. 45, $606(1980)$.

\section{MUCLEUS-MUCLEUS REACTIONS AT EMERGIES UP TO 200 GeV.aAn - PLANS FOR AM EXPERIIENT AT CERM}
T. C. Awes
C. Baktash
J. R. Beene
E. E. Gross
R. L. Ferguson
T. A. Gabriel 1
J. W. Johnson
I. Y. Lee
F. E. Cbenshain
F. Plasil

$$
\text { G. R. Young }
$$

In early 1984 a jor commitment was made to participate in the PS 190 collaboration at CERM. This new initiative is expected to be an important research activity of the Physics Division during the next three to four years. The CERN experiment PS 190 is an approved experiment to be run in late 1986 and again in parly 1987.

The Gesell Ischaft fUr Schwerionenforschung (GSI), Darmstadt, Hest liermany, and the Lawrence Berkeley Laboratory (LBL), Berkeley, California, will provide the means necessary to accelerate 160 ions in the PS and SPS accelerators at CEKN by building an ECR source and an RFQ injector. The 160 energies will be $800 \mathrm{GeV}$ and $3200 \mathrm{GeV}$. It is possible that muclei in the energy range between 10 and 50 teV-an will also be available.

Currently, the PS 190 collaboration consists of two totally independent expericents that have recently beer assigned separate SPS experimert nubers. The first, in 37, is centered about a streamer chaber, with $R$. Stock as spokesman. The second, in BO, wates use of the GSI/LBL plastic ball, and H. H. Cutbrod of BSI is the spokesman. onkl is associated with the ill 80 collaboration. Other institutional mers of this collaboration, in addition to $6 \mathrm{SI}$, LBL, and ORul, are the lniversities of Lund, Sweden, and of inster. dest Cermany. The experiment is to be performed in the mest experimental hall of the SPS. The author list of this contribution to the Physics Division Progress Report includes only the ORIL menbers of the $M$ collaboration, since the list of all collaborators from the various institutions is rot as yet final.

The plastic ball, currently located at LBL, will be med to CERh. It will be suitable only for the study of the target rapidity region, including: (1) cetermination of matter fiow from a global analysis approach: (2) determination of the extent of spectator dray from determinations of the average value of $P_{1}$ in this rapidity region: (3) determinations of pertinent temperatures from inclusive energy spectra; (4) determinations of entrops from d/p ratios; and (5) determinations of source sizes from twoparticle correlations. More pertinent to the type of physics to be addressed at SPS energies is the mid-rapidity region, encompassing most of the participant region as well as the region in which the effects of the quark-gluon plasma may manifest themselves. A key question to be considered here is the deternination of conditions under wich mximu baryon density buildup can be attained (stopping versus transparency). cinergy flow questions, as determined from ylobel analysis, also play a major role in this region. In recent cosmic-ray data, unexplained fluctuations in pseudo-rapidity were observed. Search for such effects can also be carried out. The observation of direct gamma production may be one of the signatures of the quark-gluon plasma. Thus, the measurement of these ganna rays and their identification in the presence of a large background of gammas from $\pi^{0}$ decay are an taportant aspect of the experiment.

As was stated above, the plastic ball is adequate to cover the target rapidity region. For the wid-rapidity reglon. three new devices are needed: (1) a $6-\mathrm{m}^{2}$ array nf sampling calorimeters, each calortmeter consisting of a hadronic and an electromagnetic portion, (2) a multiplicity array of about $10^{4}$ cells, and (3) a finely segmented 1000-element lead glass electromagnetic calorimeter capable of distinguishing between intrinsic gamas and the decay of neutral piuns. Finally, the projectile rapidity (trigger) devices will consist of a "bull's-eye" scintillator to determine the projectile charge and a urantum calorimeter to determine the projectile energy. The responsibility for the various devices was assigned as follows: plastic ball, LBL/GSI; multiplictty array, GSI; 
6- ${ }^{2}$ array of calorimeters, 0Ru; electromagnetic, $r / \pi^{\circ}$ calorimeter, University of muster; "bull's-eye" scintillator, LBL/GSI; and zerodegree calorimeter, Onln. Work is in progress at CRML to design and build the two types of calorimeters listed above. The calorimeters are funded jointly by LBL and by CAML.

The mid-rapidity calorineters are based on the design of Fibjan, Millis, and their coworkers at CERK. Like the CERN calorimeters, the ORHL/LBL version consists of modular stacks, each covering an area of $20 \mathrm{~cm}$ by $120 \mathrm{~cm}$. The echanical design is such as to allow the stacks to be closely packed with a minimum of-dead space between them. Each stack consists of six 20-ce by 20-cm towers; each tower is read out separately. Again, as in the CERH design, each tower consists of an electromagnetic section in wich primarily $\pi^{0}$, gama rays, and electrons are detected, followed by a hadronic section for the detection of charged pions, protons, neutrons and other hadrons. While both the electromagnetic and the hadronic sections of the CERH calorimeters contain uranium, because of funding and procurement constraints, the ORML/LBL design will consist of a lead/plastic electromagnetic section and a stainless steel/plastic hadronic section. Twenty-five stacks cotering $6 \mathrm{~m}^{2}$, with a total of 150 towers of $20 \mathrm{~cm}$ by $20 \mathrm{~cm}$, are to be built. The high-granularity GSI charged-particle multiplicity array will be placed in front of the calorimeters.

The crucial parameters in calorimeter design are the $e / p$ ratio !the ratio of the average effective energy deposited in the plastic by an electron to the average effective energy deposited in the plastic by a proton of the same energy) and the energy resolution, $\sigma / E$, expressed as a percentage of $E$. It is desirable for the $e / p$ value to be as close to 1.0 as possible, and $\sigma / E$ to be as $\ln$ as possible. For a calorimeter consisting entirely of interleaved sheets of iron and plastic, typical e/p and $\sigma / E$ values are 14 and $25 \%$, respectively, at $E=5 \mathrm{GeV}$. For a uranium/plastic calorimeter, the corresponding values are about 1.1 and about 15\%. Extensive Monte Carlo simulation calculations were performed to optimize the ORML/LBL mid-rapidity calorimeter design, with regard to both $e / p, \sigma / E$, and overall containment. It was decided that the length of the lead/plastic electromagnetic section will be 15 radiation lengths and that the hadronic section will be sufficiently long to contain $96 \%$ of th: energy of a 50-GeV proton. Both of these lengths are considerably greater than those of the CERN calorimeters. For each stack, the electromagnetic section will thus consist of 27 lead sandwiches (consisting of $1-\pi \mathrm{mm} A l+3-\mathrm{mm}$ $\mathrm{Pb}+1-\mathrm{mm} \mathrm{Al})$, interleaved with 27 plastic sheets ( $3 \mathrm{~mm}$ thick). The length of the section $1321.6 \mathrm{~cm}$, and the weight is $2811 \mathrm{~kg}$. The hadronic section will consist of 122 stainless steel plates of 8-mm thickness, interleaverl with 122 scintillator plates of $3-\operatorname{mm}$ thickness, The length of this section is $134 \mathrm{~cm}$, and the weight is $1947 \mathrm{~kg}$. The overall length of each stack will thus be $156 \mathrm{~cm}$, and the overall weight will be $2227 \mathrm{~kg}$. The calculated e/p ratio for the above design is 1.5 at $5 \mathrm{GeV}$. However, by balancing the electromagnetic and hadronic signals, the effective $e / p$ values are 0.91 at $1 \mathrm{GeV}$ and 1.01 at $5 \mathrm{GeV}$. The calculated resolution values are $26 \%$ at $1 \mathrm{GeV}$ and $17 \%$ at $5 \mathrm{GeV}$. Thus, the overall performance of our design is expected to be only marginally worse than that of a uranium calorimeter.

Since the energy resolution requirements for the zero-degree trigger calorineter are higher than those of the mid-rapidity calorimeters, and since only me has to be built, it was decided to build it entirely from a uraniua/plastic cunbination. Monte Carlo calculations mere performed for a 70-cm by 70-cm array of 10-ca by 10-can cells, with an electromagnetic section consisting of 32 uranium sheets (each $0.2 \mathrm{~cm}$ thick) interleaved with 32 plastic sheets (each $0.3 \mathrm{~cm}$ thick) and with a hadronic sect ion consisting of 275 uranium/plastic cells with the same dimensions as those of the electromagnetic section, except that the uranium sheets will be $0.3 \mathrm{ca}$ thick. The output events were analyzed to determine the ef fects of calorimeter size on resolution and on containment. $30 \mathrm{~cm} \times 30 \mathrm{~cm}$, $50 \mathrm{~cm} \times 50 \mathrm{~cm}$, and $70 \mathrm{~cm} \times 70 \mathrm{~cm}$ calorimeters were considered. The performance of the $50 \mathrm{~cm} x$ $50 \mathrm{~cm}$ calorimeter was deened to be marginal. For example, its containment for a 50-GeV proton was 97: of the containment of a $70 \mathrm{~cm} \times 70 \mathrm{~cm}$ device. The resolution was also degraded. It was thus decided to build a $60 \mathrm{~cm} \times 60 \mathrm{~cm}$ zerodegree calorineter.

Both the mid-rapidity calorimeters and the zero-degree calorimeters will be built during 1985 and will be available for tests and calibrations in mid-1986.

1. Engineering Physics and Mathematics Division.

\section{PERFORMAICE OF RECOIL MASS SEPARATOR}
H. J. Kim
C. E. Bemis, Jr.
P. H. Stelson

The ability of the recoil mass separator (RMS) to perform the separation of evaporation residues has been studied in detail for a number of target-projectile combinations. $0^{\circ}$ excitation functions and angular distributions of evaporation residues induced by fustion of $u, F$, and $T i$ beams with $T\}, Z r, T a$, and $W$ targets near and below the Coulomb barrier were investigated. Calibrations of the RMS acceptance were made using both the $180^{\circ}$ backscattering of light-ion projectiles and alpha particles from a $244 \mathrm{~cm}$ source. The acceptance was established as a function of ion velocity and charge to obtain absolute fusion cross sections. The fraction of residues collected by the RMS at $U^{\circ}$ ranges from less than 16 for such asymmetric systems as $0+W$ to more than $30 \%$ for symmetric systems $\left(\mathrm{e.g.} . \mathrm{TH}_{\mathrm{H}}+\mathrm{Ti}\right)$. Although with lower collection effictencies, the residues from asymmetric sys.tems are separated much more cleanly from the energy degraded incident particles. Because 
observed results depend on kinematics of particular reaction as mell as on the quality of incident beam, deducing a sieple figure of merit regarding the filtering power of the Rats is not uniquely possible. But, our study indirates that fusion cross sections down to the few hundred microbarn level should be obtainable for wost target-projectile combinations uxing the Rus. Preparations are in progress to study nuclei far frae stability, wich are generally produced with very seall cross section in fusion reactions, by observing decay of separated residues or coincidences between prompt gan rays and the residues.

As an example of our calioration experiments and tests, wention tere our experiments with monoenerget ic 20epb ions, wich served as a codel for our evaporation residue experiments for the $160+182,184,186 y$ Po compound systems. Monoenergetic $208 \mathrm{~Pb}$ ions in the specific energy range 25-40 keV per and were generated from $180^{\circ}$ Rutherford backscattering of 25 - to 40 - HeV $12 \mathrm{C}$ ions from $208 \mathrm{~Pb}$. Incident bean fluence was simitaneously determined using a monitor detector at $\sim 30^{\circ}$ with a well-defined solid angle. The velocity acceptance window, charge-state acceptance window, and absolute efficiency of the RuS as a function of $\mathrm{Pb}$ ion energy was determined. The backscatter kinemati-s determine a unique energy for $208 \mathrm{~Pb}$, with a nearly flat angular distribution across the ans aperture when the Rus is at $0^{\circ}$. Our calibrations indicate that for $35 \mathrm{keV}$ per anu, PD recoils from 160 on $W$, the charge-state acceptance is $-20 \%$; velocity acceptance is $\sim 60 \%$; and the angular distribusion acceptance is $24 \%$. For this very asymmetric projectile $(160)$, target $(182,184,186 \mathrm{H})$ system, the relatively broad angular distribution is clearly the limiting RMS efficiency factor. The overall efficiency is $0.5 \%$ in these cases. The kinematically reversed conditions, $H$ projectile +0 target, would dramatically improve the efficiency.

\section{DEVELOPNENT OF A POLARIZED EU TARGET}
B. Shivakumar
C. E. Bemis, Jr.
J. R. Beene
D. Shapira

As part of the development of a polarized Eu target, the gas jet apparatus' has been modified to facilitate operation at high temperatures. The existing nozzle assembly has been replaced by one capable of being heated to $900^{\circ} \mathrm{C}$. The assembly is connected to a high temperature feedthrough which takes the gas line at high temperature from a regton of high vacuum to a regior at atmospheric pressure. A similar feedt: rough then connects to the oven/se-der assemt ly hich has also been designed to operate up to $900^{\circ} \mathrm{C}$. The oven/seeder contains two reservoirs, one for heating $\varepsilon_{u}$ and the other for heating the Ar carrier gas. The heating of the two reservolrs is done in an Ar atmosphere. The temperature of the apparatus is controlled at the Ar and Eu reservoirs and at the nozzle. An Ar gas jet has been operated successfully at $850^{\circ} \mathrm{C}$. The apparatus is currently being setup to test the seeding of EU atoms into the Ar jet. Weasurewents will be made of the Eu target thickness (using elastic scattering of an $1 \overline{0}$ beam) at temeratures around $800^{\circ} \mathrm{C}$. Once the target has been optinized, we intend to proceed with the optical puping of the Eu atoms in the jet and the full implementation of the polarized target facility.

1. J. L. C. Ford, Jr et al ., Phys. Div. Prog. Rep. Sept. 30, 1983, ORI -6004, p. 153 (1983).

2. B. Shivakumar et al.. Phys. Div. Prog. Rep. Sept 30, 1983, ond-6004, p. 154 (1983).

\section{A LOEARITITIC TOTM EVET COUIER SYSTEN}

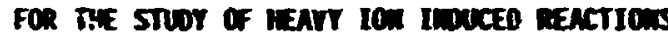

$$
\begin{aligned}
& \text { D. Shapira J. L. Blankenship' } \\
& \text { B. L. Burks }
\end{aligned}
$$

The products from a reaction induced by E.zergetic heavy fons are in general highly excited. The particles that are eventually detected are fragments that have cooled down by enission of $\gamma$ radiation, neutrons, protons, alpha particles or heavier muclei.

A measurement of observables associated with the primary reaction products requires kinematic reconstruction of the initial event based on simultaneous detection of all (or most of) the decay products and their residues. While this approsch is typical in the study of high energy nuclear colitisions, it has usually been bypassed in low energy nuclear physics. At bombarding energies below a few MeV/anu, excitation of the primary outgoing fragments is 1 imited and very few products emerge. Furtherwore, the cross sections for the processes studied are quite large in gener?1, so that limited measurenent of a few final products and some correlation studies usually suffice. At energies above 10 MeV/anu one expects that processes where the two outgoing products are highly excited will dominate. Also, processes where the incoming projectile or target nuclet themselves break up become important. As the excitation energy of the products increases and as fragmentation becomes more probable, the number of final products emerging from the colliston increases rapidly. Extraction of the observables associated with the primary process from measurement of individual products then becomes increasingly dependent on confecture and modeling of the reaction process.

He propose to build a multiparticle counter syster that will measure a large fraction of the products emerging from a collistion between energetic heavy ions. Practicality dictates that such a device cannot address all reactions but it should address a wide range c. nuclear physics phenomena.

We will liait the study to systems of nuclet lighter than $A \sim 100$. Heavier systems decay predominantly via $Y$ and neutron emission or fisstion, and the $4 \pi y$-ray spectrometer addresses 
similar inclusive measurements for these heavy systens. For the lighter systens the principal decay wode is light particle enission: neutron, proton and a particles; the enission of $r$ rays and symetric fission, although present, are not dominant for this mass range.

The detector we plan to build will be designed primarily to detect the residue of a fast moving heavy fragment and all, or most, of the light particles eitted from it.

Figure $\mathbf{2 . 9 6}$ is a simulated density contour for alpha particles enitted in the forward hemi-

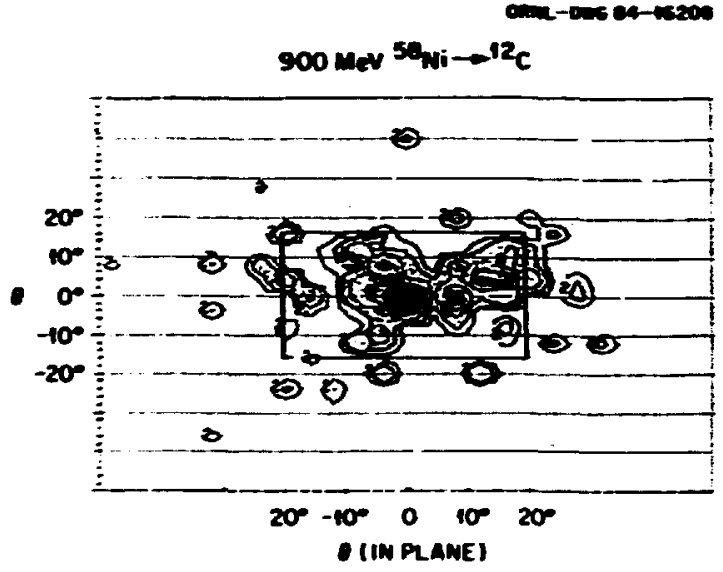

Fig. 2.96. Density contours for a particles eaitted in correlation with scandium nuclei detected at $9=4^{\circ}$ and $\phi=180^{\circ}$. sphere from collisions of 890 MeV 59 wi particles with $12 c$ muclei. The density contour shows only alpha particles that energe in coincidence with a ${ }^{2} \mathrm{Sc}_{21}$ ion detected at $\mathrm{B}=-4^{\circ} \phi=180^{\circ}$ (in plane). There is a strong concentration of a particles in the direction of the enitted SC fragent. The a particles evaporated from a large, fast-moving fragment are eaitted, woving approxiately in the sane direction as the heavy fragnent because their nost probable velocity in the rest frame of the enitting nucleus is swall (only few $M e V / a m$ ). With a counter systen that covers the approximate area shown in the box on Fig. 2.96 one can capture, therefore, wost of the enitted light particles together with the heavy residue.

The counter system we are proposing is shown, scheatically, in Fig. 2.97. The front portion consists of a large area, gas-filled ionization chamer. The 30-ca-deep ionization chmber will stop the heavy residue and provide an event trigger. The detector iill yield energy ( $\mathrm{C} 1 \mathrm{x}$ resolution), energy loss ( $-2 x$ resolution) and spatial position $(x, y)$ signals. The mitielectrode design will perwit identification of heavy ions over a wide dynanic range (better than 1:20 in ion ranges) but iight particles will deposit a negligible acount of energy in the gas counter. An array of 96 scintillation counter telescopes arranged in a $6 \times 16$ array behind the gas crunter will yield the spatial position $\left(2.4^{\circ} \times 2.4^{\circ}\right.$ granularity), energy and identity of light particles. Each element of the scintillation counter array will inciude a 1-mthick of and an approxiately 10-co-thick E detector. Charged light particles will be identified by the AE-E technique and this design

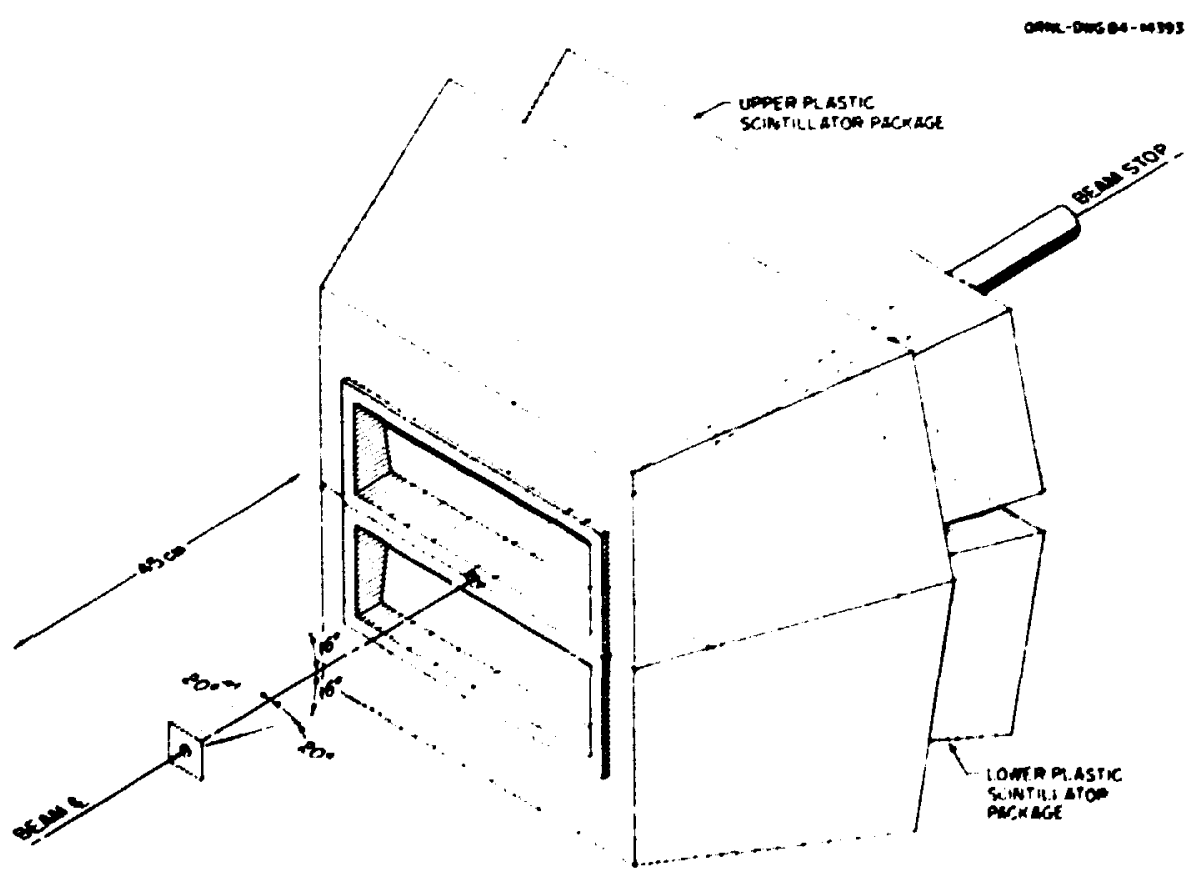

Fig. 2.97. Schcmatic drawing of the klLl detector. 
should identify protons vitn energies ranging

fro 10 to $140 \mathrm{rer}$ and alphe particles with energies ranging from 30 to $\mathbf{4 0 0} \mathrm{MeV}$. The neutrons will leave a signal only in the thicker scintillator. Crude ToF information will be used to get the neutron energy $(-10-15 \pi$ resolution).

The ionization counters straddle the reaction plane and are organized in two separate wolcmes. Micy can be operated at different pressures and can thus detect and identify two fragents for an asymmetric mass split. Eacl. half of the detector has a split anode structure that allows the sinultaneous study of two heavy fragnents. one in each half. Provision is also ade for the bean to exit the chaber and be stopsed domstrean far from any of the light-ion detectors.

1. Instrumentation and Controls Division, anin.

\section{LAXE IOAIZATIO OETECTOAS FO: GEAN IOU RERCTIOUS}

J. L. Blankenshipl F. E. Obenshain A. H. Snell

Several design changes for this time-offlight (TOF) facility were reported in the previous progress report. 2 Additional cesign andifications to the $80-\mathrm{ca}$ ion chaber have been mode. Curved anodes and Frisch grid mere used in the original design to provide maxim rigidity, and therefore minimua microphonics. However, the Frisch grid curvature introduces a variation in the electric field strength fro center to outside extremes of the drift space. wich causes variations in the drift velocity and drift time as a function of out-of-plane angle.

The new design. shown in Fig. 2.98 incorporates flat aluminum plates for the anodes. guard bars on the sides for field grading and electrostatic shielding from housing ground, anc a flat Frisch grid structure. Two grid structures have been iabricated. The first design eaploys stainless steel wire of 280- dianeter in woven wire mesh spaced $8 \times 8 \mathrm{~cm}^{2}$ and provides good shielding and fair reach-throughfield charge collection behavior. The second design consists of a plane of phosphor bronze wire of 114-in dianeter spaced $1.59 \mathrm{~mm}$ on center. This secand design has not been tested in the $80-\mathrm{cm}$ fon chamber, but has been used witt success in the 40-cm ion chamber used in the broad range spectrometer.

1. Instrumentation and Controls Division. ORM.

2. ORNL Progress Repor: for period ending September 30, 1983, ORNL-6304, D. 152.

Fig. 2.93. The electrode asse-bly of the 80-ca-deep ion chamber. The four planar anodes are shom at the top, and the Frisch orid is below the anode asse-bly. Field shaping is facilitated by the guard bars wich are on each side and uniformly spaced from the Frisch grid to the cathode at the bottom.

\section{DESIEM OF AN EUADRATION RESIOUES DETECTOR}

$$
\text { J. L. Blankenshipl F. E. Oenshain }
$$

A gas-filled detector systea for measuring AE, E, G, and b. and velocity of evaporation residues has been designed and is shom in Fig. 2.99. Velocity is deternined by measuring

ORIh Photo 6148-84

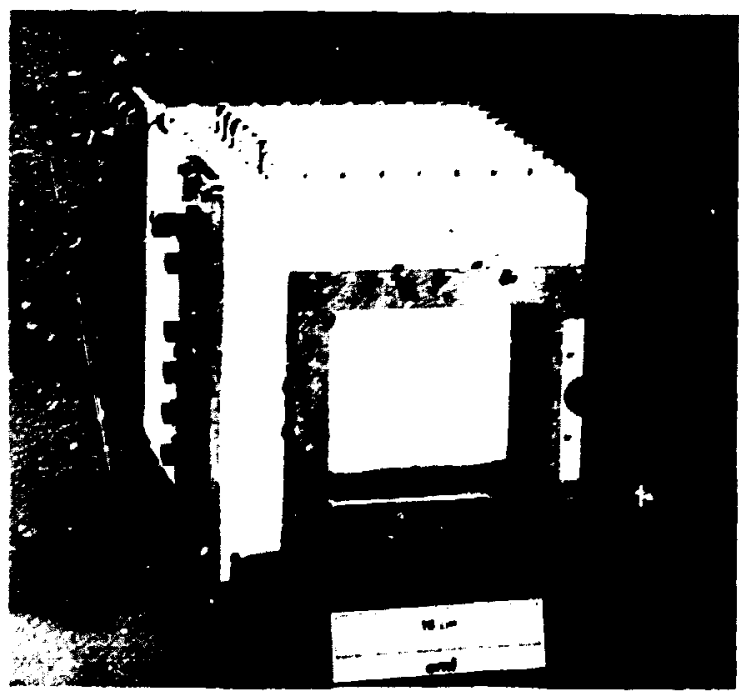

Fig. 2.99. Front view of evaporation residue detector showing gas inlet and out let. 
the time of flight over a $20-\mathrm{Cm}$ path using Breskin-type altiwire, position-sensitive proportional counters as start and stop detectors. The entrance window is ade of $-55 \mathrm{ug} / \mathrm{cm}^{2}$ polypropylene and has an $8.5 \times 10.5 \mathrm{~cm}^{2}$ cpening. The start detector has an $8 \times 10 \mathrm{~cm}^{2}$ sensitive area, and the cathodes are constructed of 90 percent transparency, $45 \times 45$ lines/inch nickel mesh.

A conventional, Frisch grid ionization chaber is located between the start and stop detectors, in the same gas volume. The anode is 8-co-long and is a segment of a circular band whose inner and outer radii are selected to atch the target-to-anode distances. This design provides a af signal mich is independent of in-plane angles. The stop detector has aluinu-striped cathodes for $\theta$ and readout and has an $8 \times 14-\mathrm{Cr}^{2}$ working area. The detector systen is designed to fit inside the 1.6-m chamber against the outside wll.

At the rear of the detector, a $1-m$-thick sheet of $\mathrm{KE}-102$ plastic scintillator, enclosed in a light pipe and pressure bearing window. provides a measure of the residual energy. The chaber design incorporates a senicircular segment of a $1.9 \mathrm{~cm}$ i.d. pipe in the sidewall to permit the closest possible approach to the bean center line. After the detector design has been demonstrated as satisfactory, a second detector. which is a mirror image of the first, will be built. This detector pair will then span the bean. antir.

1. Instrumentation and Controls Division,

\section{AFTERPULSES FROA RESIDUA GASES IM} PHOTOULLTIPLIER TUBES
N. H. Hill
J. A. Harvey ${ }^{2}$
D. J. Horen
C. H. Johnson

At the ORELA time-of-flight facility we have extended the energy for neutron detection down to about $10 \mathrm{keV}$ for liquid and plastic scintillators by accepting pulses corresponding to a few photoelectrons. At such low levels there is a background of afterpulses wich originate either in the scinefilator or in the photomultiplier, a 5-inch RCA-8854 PMT, but appear several usec after the earlier detection of a neutron or y-ray.

In our previous study ${ }^{3}$ of the afterpulsing phenowenon we cosserved pulses originating from electron-atom collisions in various residual gases in the PMT and we made the conventional" assumption that most of that ionization occurred in the large region between cathode and first dynode. However, our subsequent calculations on that assumption gave spectral shapes inconsistent with those observed. Therefore, we have extended ti.e measurements in an effort to find the points of origtn of most of the ions.

For this study we irradiated various new RCA8854 tubes with light pulses from a Monsanto
MV52 green LEV. Each pulse ejected about 600 electrons from the cathode: Afterpulse time spectra were recorded in I-nsec channels out to 6 usec and in 25-nsec channels at later times. Spectra were recorded with a mitistop per start-time digitizer with a 1.1-usec deadtine and corrected for deadtice losses. Figure 2.100 shows for ane tube the spectrum in units of afterpulses per photoelectron per usec. He attribute the narrower peaks to backstreaming ions from localized surfaces and the broader peaks to ions from residual gases. Figure 2.101

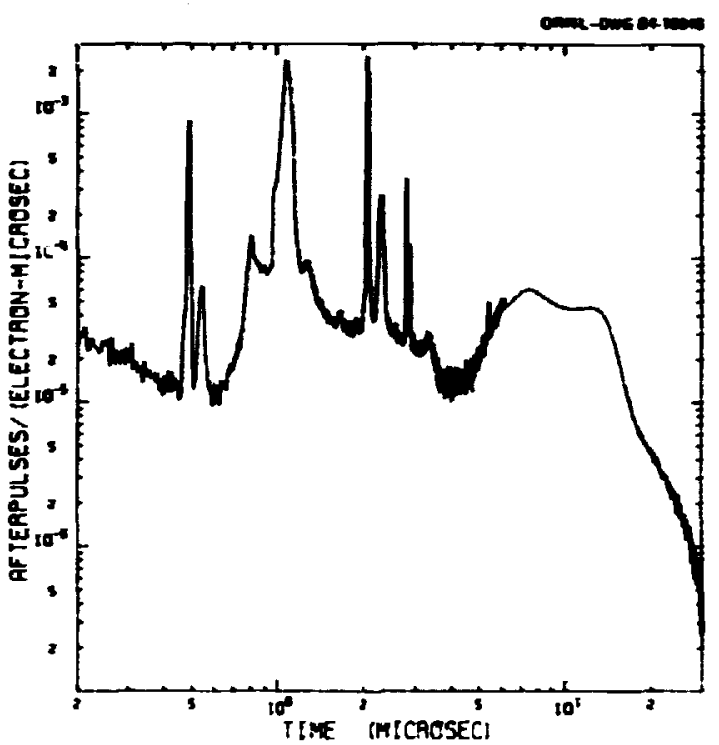

Fig. 2.100. Afterpulse spectrum for an RCA-8854 photomultiplier tube.

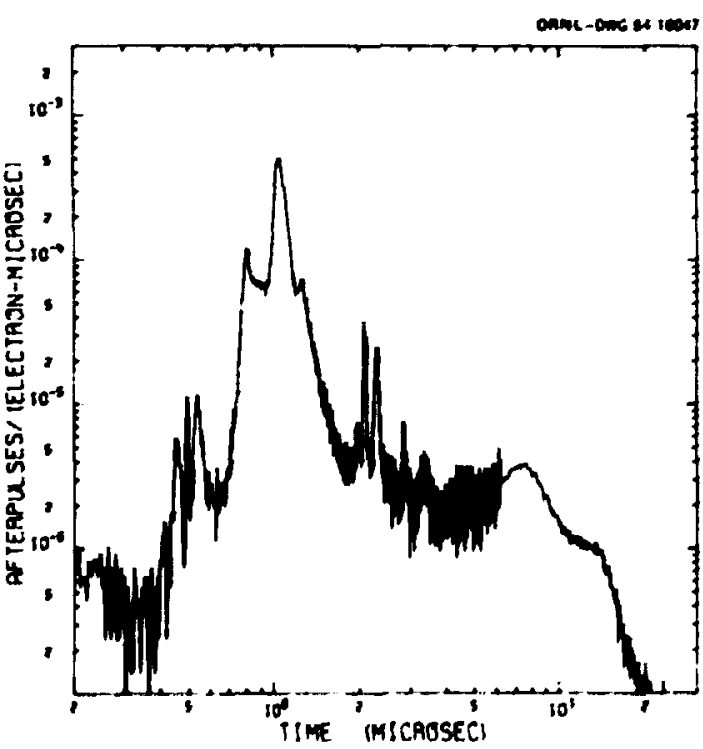

Fig. 2.101. Afterpulse spectrum for the PMT of Fig. 2.100 with the and dynode gated off during the initial pulse. 
Shows the spectrum for this tube with the and dynode gated off during the initial pulse. Gting elininates most ion formation in the dynode region. A comparison with Fig. 2.100 shows that most fons in the ungated ade origi nate in the dynode region. The exception is the "pecestal" between 0.8 and 1.2 usec. In fact, our calculations predicted this "pedestal" arises from the residual the gas between cathode and first dynode.

Also observed is a pronounced peak on top of the pedestal at 1.1 usec. Previously ${ }^{3}$ me assumed this peak also arises from fons in the cathode-dynode region but now we conclude that it arfses from sonization of the the gas in the region between the 1st and and or 3rd dynodes. It is wore intense because the lst dynode has high gain. In an auxiliary experiment we ceasured the spectrun for an RCA-4522 RMT, wich does not have the high gain drnode, and we found a mich smiller peak relative to the pedestal. To further check this conclusion on the origin of the pedestal-pesk we varied the But grid voltage relative to the cathode and first dynode. As expected, a change in voltage such as to decrease grid-to-cathode acceleration but to increase extraction from the dynodes resulted in a later pedestal but earlier peak.

In addition to the 1.1-usec Het peak, Figs. 2.100 and 2.101 also show broad spectra of pulses the to ions originating somxthere in the dynode region. If such a PMT is used with a scintillator for neutron detection, little can be done to eliminate the afterpulses from a detected neutron. However, when used at a factlity such as CRELA, the serious afterpulses frow the r-burst can be elfininated by gating of the drnode during the burst. Further improvement can be obtained by the use of 2 mI's in fast cofincidence on the sase scintillator.

1. Instrumentation and Controls Division.

2. Engineering Physics and Whthentics Divisics.

3. N. W. Hill et al., Physics Division Progress Report, Cank-6004, 155 (1983).

4. G. A. Horton, H. M. Sith, and $R$. Wasserman, IEEE Trans. on Rucl. Sci. 15-14, 43 (1967).

\section{PREPLATIOU OF ThEeETS FOR MULENR PHTSICS RESENRCH}

$$
\text { D. H. Golbrath F. X. MeGowan }
$$

The preparation of targets for nuclear physics research by the SPUTTEReELL (focused ton bea sputtering systea) has continued to be extrenely useft:. Thin targets from meturials with very high melting points, such as refiactory metals, are easily produced. Self-suppoting targets of Si as thin as $30 \mathrm{mg} / \mathrm{cm}^{2}$ have been uroduced. Sfince fon bean sputtering is a "cool" process, thin targets of Po evaporated on carbon foll backings have been covered with $5 \mu \mathrm{g} / \mathrm{cm}^{2}$ carbon by sputtering successfully.

Table 2.16 contains examples of targets prepared by ion beaw sputtering and by evaporation.

Tasie 2.16. Tarjets for nuclear physics research

\begin{tabular}{|c|c|c|c|c|}
\hline Element & Weight & Backing & Method & Remarks \\
\hline $\begin{array}{l}5 i \\
74 \mathrm{Ge} \\
90 \mathrm{Zr} \\
93 \mathrm{Mb} \\
181 \mathrm{Ta} \\
238 \mathrm{U} \\
27 \mathrm{Al} \\
\mathrm{Ag} \\
\mathrm{In}_{124 \mathrm{Sn}} \\
128 \mathrm{Te} \\
\text { Sb } \\
197 \mathrm{Au} \\
197 \mathrm{Au} \\
197 \mathrm{Au}\end{array}$ & $\begin{array}{l}30-120 \mu g / \mathrm{cm}^{2} \\
500 \mu \mathrm{\mu g} / \mathrm{cm}^{2} \\
50-150 \mathrm{\mu g} / \mathrm{cm}^{2} \\
50-150 \mathrm{\mu g} / \mathrm{cm}^{2} \\
50-15 \mu \mathrm{\mu g} / \mathrm{cm}^{2} \\
1 \mathrm{mg} / \mathrm{cm}^{2} \\
30-50 \mu \mathrm{\mu g} / \mathrm{cm}^{2} \\
1 \mathrm{mg} / \mathrm{cm}^{2} \\
160 \mathrm{\mu g} / \mathrm{cm}^{2} \\
1.0-1.5 \mathrm{mg} / \mathrm{cm}^{2} \\
0.8 \mathrm{mg} / \mathrm{cm}^{2} \\
1 \mathrm{mg} / \mathrm{cm}^{2} \\
150 \mathrm{\mu g} / \mathrm{cm}^{2} \\
110 \mathrm{\mu g} / \mathrm{cm}^{2} \\
40 \mathrm{\mu g} / \mathrm{cm}^{2}\end{array}$ & 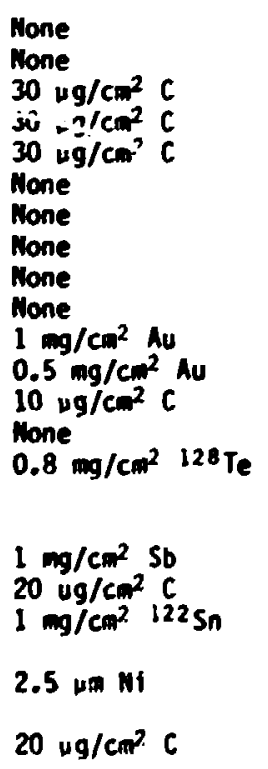 & $\begin{array}{l}\text { Sputtering } \\
\text { Sputtering } \\
\text { Sputtering } \\
\text { Sputtering } \\
\text { Sputtering } \\
\text { Sputtering } \\
\text { Evaporation } \\
\text { Evaporation } \\
\text { Evaporation } \\
\text { Evaporation } \\
\text { Evaporation } \\
\text { Evaporation } \\
\text { Evaporation } \\
\text { Evaporation } \\
\text { Evaporation }\end{array}$ & $\begin{array}{l}\text { Recoil distance device } \\
\text { Line shaped target } \\
\text { Covered to contain } 128 \text { Te } \\
\text { target in recoil distance } \\
\text { device } \\
\text { Covered to contain So } \\
\text { Recoil ass spectrometer } \\
\text { Pb-backed target for } \\
\text { r-ray spectroscopy } \\
\text { Stoppers for recoil } \\
\text { distance device }\end{array}$ \\
\hline
\end{tabular}


Many productions of detector windows and planes for avalanche counters were done. Carbon stripper foils for ORIC and tandem are now being próuced routinely.

\section{SPECIN EWHACEETTS TO THE EVET HNDLE}

$$
\text { D. C. Hensley }
$$

The Event Handler 1,2 is a programable cumac based processor with auxiliary crate controller capability and is the major control device for data acquisition at the HIRF. Because of the success of this device in maging ine frint-end data acquisition electronics, special emphasis has been placed on making it as general as possible without degrading its basic perforance.

One particular problea to be faced in data acquisition is wat to do with 24 bit devices winen the principle data strean is only 16 bits wide. CANAC supports 24 bit devices, principally scalers, but most data acquisition is based on 16 bits due to the 32 bit structure of typical data acquisition computers. The Eve.it Handler already had special instructions for setting the top bits of a 16 bit word (useful when 11 and 12 bit ADCs are used), but it lacked any general way to transait the ful! 24 bits from the CAMAC dataway over a 16 bit path.

The following enhancements inelp to overcome this difficul:y. It has always been possible to transnit the :6 bottom hits of a CaraC word, but it is now possible to transmit the 8 top bits of the sane CAMAC word. This byte is piaced in the position of the $a$ botton bits (the a top bits are set to zero) of the lo bits of the transaitted word. This top byte can be transmitted at any time, either before or after the 2 bottom bytes are transwitted. If the upper byte needs to be comunicated over the carac dataway, but in the position of the lowest byte, this can be une by first writing the top byte of the Event Handler CAMAC data register (COR) into the 2 lower bytes of the sare register lessentially shifting the top byte duwn 2 bytes!. Then the newly formed CDR can be written out over the CAMAC dataway to michever module requires it Since this operation destroys the original 2 lower bytes, one would presumably transfer these bytes first.

The handling of the Event Handler Save Register was made more symutric. Mot only can one store a 24 bit CakAC data mord in this register, but now one can either transmit the 16 bottow bits or move them to the COR. This latter capability allows one to move a 16 bit word from one crate to another, woving it through the Event Handier.

Provisiors have been ade for enhancing the speed of the Event handler - special "hooks" have been provided wich allow an external device to specify the unconditional pranch address in a branch operation. A particular external device would be one wich could look ahead to deternine wich of many possible routines needed jervicing next.

In order to protect the integrity of the final data strean, the Event Handler has been changed so that it will wait forever to transait a word into a full FIFu. Formerly it would wait about 4 ins before it would proceed to its next operation:. While it is recomended that the Event Handler not be used in this way, in the event that a fIFO should manage to become totally full, a requested transaission will not get lost.

Finally, the auxiliary crate controller part of the Event Handler, wich previously could generate normal CAMAC cycles or "short" CAMAC cycles (terminated at the end of timing strobe 5i), can now generate even shorter cycles in which tiaing strobe $\mathrm{SI}$ is not generatec on the CAMAC dataway.

In addition to their use at the HIRF, Event Handlers are being implemented at the University of Notre Dane in conjunction with a project at Argonne National Laboratory and at Texas $A$ \& $M$ University. Ise of the device is also being considered for projects at ANL. Princeton, Chalk River, and Kansas State.

1. D. C. Hensley, IEEE Trans. Nucl, ici. NS-26, 710 (1979).

2. D. C. Hensley, IEEE Trans. Nucl. SCi. WS-26. 4454 (1979). 


\section{THE UNISOR PROGRAM}

The University Isotope Separator - Oak Ridge is a consortiv of eleven acadeaic institutions, Dak Ridge Associated Universities and Oak Ridge Mational Laboratory. It operates an isotope separator installed on-line to the accelerators within the Holifield Heavy Ion Research Facility. The accouncs mich follow describe work at the UMISOR facility or work associated with UAISOR research perforined principally by investigators outside of the Physics Division. Research and developient perforned at UISOR principally by Physics Division staff menbers are included in the Muclear Physics section of this report.

In addition, theoretical research perforaed by Uitson staff and collaborators is included in the Theoretical Physics section.

\section{THE WISOR MASS SEPARATOR}

$\begin{array}{ll}\text { R. L. MekodajI } & \text { H. K. CarterI } \\ \text { E. H. Spejewskil } & \text { J. D. Cole }\end{array}$

The vacuum system of the mass separator has been completely converted to turbomolecular pums in order to provide a relatively of l-free vacuum. Mis change was implemented to cut down on problems caused by deposits of cracked diffusion pump oil on lenses, insulators and other critical surfares. This changeover was coupled with a complete cleaning and realignment of all internal surfaces.

In order to accommodate a rearrangement of the experimental space in the south addition of Building 6000 , the high-voltage rack with all of the ion scurce supplies has been relocated in a new dehumidified room constructed above the of $\mathrm{f}$ line ion source area. The control console and the power distribution cabinet also are in new positions.

In order to facilitate the suning of beams down the sensitive laser facility, several new alds have been built into the separator ionoptical system. A new horizontal and vertical deflection system has been installed at the exit of the first lens box to precisely align the bean entering the second lens box. This el iminates steering introduced when the bean is not correctly centered. A vertical deflection adjustment has been butlt into the $30^{\circ}$ deflectors to correct any vertical error at that Doint.

In addition, new faraday cup located on a moveable carriage in the focal plane allows the monitoring of a beas at any position along the focal plane and has been useful for continuously mont toring one bean mile transmitting another mass through one of the heam ifnes.

Several raw developments are helping to carry out the defocussing of the tandem beams intch is necessary to avofd the target-destroying tight pocus atedinable Pron the candem. Highly damsge-resistant phosphors of $\mathrm{Al}_{2} \mathrm{O}$ have been produced and installed in the UNISOR beam handling box as well as a ewo-dimensional beam scanner wich is integrated into the MHIRF beamscanning system. These aids. llong with opera- tor experience, enable uniform defocussing of the bean spot up to the optimun size of about 8 diameter mile retaining greater than 905 of available beas intensity.

The last section of beas line to UNISOR from the 1.6-m scattering thamer to the He-Jet box has now been upgraded to the new all stainlesssteel contruction and should result in much improved vacuum quality in that region.

A new, faster stepping-wot or controller and a higher torque motor have been 1. stalled on one of the UNISOR tape transport systems. With this system and a simple loop of tape, a source can be moved to a counting station in ahout 0.15 sec. Because of built-in acceleration and deceleration, longer distances can be covered in proportionately shorter times.

\section{UNISOR, Oak Ridge Associated} Universities.

2. Louisiana State University; present address: Idaho hational Engineering Laboratory. Idaho Fallis, Idaho.

\section{MUCLEAR ORIEMTATION FACILITY}
E. H. Spejewskil
J. H. Lami iton 3
F. T. Avi inone, III
K. S. Kranet

Funding has been obtained for a nuclear orfentation facility to be installed in the UNISO? laboratory. It will consist of a helfum dilution refriverator and superconducting magnet tn provide orientation at temperatures $=10 \mathrm{mK}$. The final design of the system is nearing completion. It is expected that a purchase order will be placed for the major equipment early in 1985, with construction of the separator beam-transport, and suaport mechanisims inttidteu thereafter.

This facility will allow the measurement of angular distributions of nuclear radiations and, there'ore, detemination of multipole mixing ratios of the radiations and of spin-parizy cianges between the nuclear levels. A nuclear magnetic resonance capabtlitey will be added to allow the measurement of the nuclear g-factor of 
ground and isomeric states, and thus a direct determination of spin.

1. UnISOR, Dak Ridge Associated Iniversities.

2. University of South Carolina, Columbia, South Carolina.

3. Vanderbilt University, Mashville, Tennessee.

4. Oregon State University, Corvallis, Oregon.

\section{SEARCH FOR OEFORUATION IN THE VERY LIGHT SIMRIUA ISOTOPES}
R. L. Mekodaj'
P. Sermes 2
G. A. Leanderi
R. N. Fink2
R. A. Braga2 3. D. Kern ${ }^{3}$
K. S. Toth

The possibility of a new, region of stable nuclear deformation around the neutron-deficient Sm isotopes has recently been discussed in the literature. 4 These calculations of intrinsic equilibrium deformations indicate that these nuclei should be rotational at $A \sim 135$. The determination of the rotational properties of this region, as well as the characteristics of the adjacent "ransition region coupled with th: possibility of observing beta-delayed proto. emission, pramises a ric area for many future experiments.

Recent on-line ion source test runs have shown that Eu and $\mathrm{Sm}$ isotopes around this region can be made in good yields using a $35 \mathrm{Cl}$ or $32 \mathrm{~S}$ beam on a natural Ag target. A.11 known gama lines from the decay of $\mathrm{Sm}$ isotopes from mass 135 to 140 and Eu isotopes of mass 139 and 140 were observed in a few minutes running time at each mass. Longer experiments are being planned.

1. ORAU, Oak Ridge Associated Universities.

2. School of Chemistry, Georgia Institute of Technology, Atlanta, Georgia.

3. Department of Physics and Astronomy, University of Kentucky, Lexington, Kentucky.

4. G.A. leander and P. Móller, Phys. Lett. 110B (1982) 7.

THE DECAY OF MASS-SEPARATED $191 \mathrm{mHg}$ AND $1919 \mathrm{Hg}$ TO 291 AI AND ODD-MASS MU SYSTEMATICS

$$
\begin{aligned}
& \text { J. L. Woodl E. F. Zganjar } \\
& \text { C. D. Papanico.opulost E. Van Halle }
\end{aligned}
$$

In the studies of the $191 \mathrm{mHg}+191 \mathrm{Au}$ and $1919 \mathrm{Hg}$ + 191 Au decay schemes at INISOR and at KOOL/LISOL (see the report here on dynamical supersymmetries in 191Au) we have obtatned very detafled information on the structure of $191 \mathrm{Au}$. In particular, we belifeve the $191 \mathrm{mHg}\left(\mathrm{N}=13 / 2^{+}\right)$ and $1919 \mathrm{Hg}\left(\mathrm{J}^{\prime}=3 / 2^{-}\right)$decays, together, populate all states of spin $1 / 2=17 / 2$ below $1 \mathrm{MeV}$.
This, in combination with the unique level spin and transition mitipolarity assignments made possible by the nuclear orientation data, provides an exacting test of the scheme of systematics that we have proposed $5,6,7$ for the oddmass Au isotopes. This is a crucial step in the chain of level scheme studies that we have made to probe the structure of muclei far from a stability in the Pt. Au and Hg isotopes. Without the use of systematics, elucidating nuclear structure far from a stability is, in our opinion, iepossible. These data are in the process of beins analyzed.

1. School of Physics, Georgia Institute of Technology.

2. Louisiana State University.

3. Catholic Unifersity of Louvain, Louvain, Belgium.

4. UNISOR, Oak Ridge Associated Universities.

5. E.F. Zganjar, et al., Phys. Lett. 588, 159 (1975).

6. E.F. Zganjar, in Future Directions in Studies of Muclei Far From Stability, ed. J.H. Hamilton, et al. (North Holland, Amsterdam, 1980), ค. 49-62.

7. J.L. Hood, ibid., p. 37.

DYMAMICAL SUPERSYIMETRIES AND THE POSITIVE-PARITY STATES IN $191 \mathrm{AW}$
J. L. Hood
L. Vanneste 6
C. n. Papanícolopulos $\$$
E. Van Halle
E. F. Zganjar2
J. D. Cole $\mathrm{C}^{3}$
M. Huyse
K. S. Krane4
A. V. Ramayya?
R. L. Mlekodaj5
J. H. Hamilton?
1. Vervier 8

It has been suggested that dynamical supersymmetries may be present in the spectra of complex nuclei.9 The particularly simple supersymmetric structure of Ref. 9 is generated by a single particle with $j=3 / 2$ and $L=0,2$ bosons with $0(6)$ symmetry. The nuclide $191 \mathrm{Au}$. wich has a $j=3 / 2$ ground state and lies in a region of even-even nuclei with $0(6)$ symmetry, 10 is under study to determine whetrer its lowlying spectrum can be described by this classification scheme. This is an extension of the study of 193 Au described in Ref. 11.

High statistical quality $Y_{-}^{-t}$ data for the $191 \mathrm{mHg}+191 \mathrm{Au}$ and $1919 \mathrm{Hg}+191$ Au decays, taken at UNISOR, have been analyzed and a level scheme for $191 \mathrm{Au}$ has been constructed. The next step is the assignment of level spins and transition multipularities to the scheme. This is being done using low-temperature nuclear orientation data taken at the K.)OL facilfty operated on-line to the Leuven-isotope-Separator-On-Line (LISOL) at the CYCLONE Cyclotron in Louvain-la-Neuve, Belgium. These data are being analyzed.

1. School of Physics, Georgta Institute of Technology,

2. Loustsiana State University. 
3. Louisiana State Uhiversity; present

address: Idaho Vational Engineering Laboratory, Idaho Falls, Idaho.

4. Oregon State Universfty, Corvallis, Oregon.

5. UNISOR, Oak Ridge Associated

Universities.

6. Catholic University of Louvain, Louvain, Belgium.

7. Vanderbilt University, Mashville, Tennessee.

8. University of Louvain-la-lleuve, LouvainIa-Meuve, Belgiun.

9. F. Iachello, Phys. Rev. Lett, 4, 772 (1980).

10. R.F. Casten and J. Cizenski. Mucl. Phys. 1309, 477 (1978)

11. J.L - lood, Phys. Rer. C24, 1788 (1981).

\section{EROUID STATE SHAPE ND CROSSING OF IEAR} SPHERICAL AN DEFOAED PADS IN $182 \mathrm{hgl}$
W. C. Ma2
K. Zhao'
A. V. Ramayya 2
J. D. Cole
J. H. Hamil ton ${ }^{2}$
E. F. Zganjar ${ }^{3}$
S. J. Robinson ${ }^{2}$
E. H. Spejewski4 M. E. Barclay 2

The energy levels of $182 \mathrm{Hg}$ have been identified for the first time through comparison of in-beam studies of the reactions $156,154 \mathrm{Gd}(32 \mathrm{~S}, 4 \pi) 184,182 \mathrm{Hg}$ and $y$-X-ray coincidences. Levels up to $12^{+}$in $182 \mathrm{Hg}$ were established from $r-r$ coincidences and singles measurements. The data establish that the ground state shape is near spherical, and that the ground band is crossed by a mell-deformed band at $4^{+}$. In contrast to IBA model predictions that the deformed band will rise in energy in $182 \mathrm{Hg}$ compared to $184 \mathrm{Hg}$, the energies of the deformed levels in $182 \mathrm{Hg}$ continue to drop.

1. Abstract of paper: Phys. Lett. 1398 (1984) 276

2. Vanderbilt University, Mashville, Tennessee.

3. Louistaas State University, Baton Rouge, Loutstana.

4. UNISOR, Oak Ridge As:;octated Universities.

\section{SHAPE COEXISTENCE In 187AU AND 285 AU}
E. F. Iganjar
M. A. Grinim
J. L. Llod2
H. K. Carterh

We are exploring the possibility that the low-energy structure of 187 Au and 185 Au contains four families of states, via:

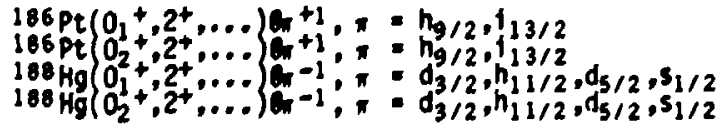

This invoives andiysis of $\gamma-\gamma-t$ and $e-\gamma-t$ data for the decays of $187 \mathrm{mHg}\left(\mathrm{J}=13 / 2^{+}\right)$, $187 \mathrm{gHo}\left(j=3 / 2^{-}\right), 185 \mathrm{mHg}\left(j \pi=13 / 2^{+}\right)$and, $185 \mathrm{gHa}\left(J=1 / 2^{-}\right)$.
The level density in $187 \mathrm{Au}$ at low energy is very high, resulting in an extremely high density of lines in the $y$-ray and conversionelectron spectra. We have developed techniques of running coincidence gates to elucidate the $187 \mathrm{~m}, 9 \mathrm{Hg}$ decay schenes and to extract $y$-ray and conversion-electron line intensities for closely spaced iultiplets. The technique, as applied to the determination of at values, is shom schemtically in Fig. 3.1. In addition, to check that mitiplet structure has not been missed, we are in the process of exhaustively extracting absolute coincidence intensities for both $r-y$ and e-r data. So far, we have established 48 excited states below $1150 \mathrm{keV}$ in $187 \mathrm{Au}$ (cf. 27 excited states reported in Ref. 5). Further, we have been able to establish a number of EO+H1 (+E2) transitions mich interpret to connect the 186Pt $\left(0^{+}\right) \triangle \mathrm{h}$, and $186 \mathrm{pt}\left(\mathrm{O}^{+}\right)$ - ho bands. These are lifsted in Table 3.1 . In particular, transitions of 270.71 and 272.19 keV should be noted. These transitions form a ultiplet of five lines, the other three having energies of $271.0,271.47$ and $271.67 \mathrm{keV.5}$ This quintuplet is clearly established by the coincidence data (which shows the 271.47 and $271.0 \mathrm{keV}$ lines to be in coincidence with lines at 298.57 and $299.4 \mathrm{keV}$, respectively--other coincident lines and the running gate technique separate these pairs) 6 We have levels mich are candidates for $\left.{ }^{188} \mathrm{HgjO}^{+}\right) \mathrm{x}^{-1}$ band(s). Further analysis of the $187 \mathrm{~h}, 9 \mathrm{Hg}+187 \mathrm{Au}$ decay data are necessary before the picture is complete, but al ready the scheme that we have constructed points to the (co)existence (in a geometrica) picture) of four different "shapes" in $187 \mathrm{Au}$.

The complexity of $187 \mathrm{Au}$ suggests that the data we obtained on the $185 \mathrm{~m}, 9 \mathrm{Hg}+185 \mathrm{Au}$ decays are inadequate for completely elucidating the $185 \mathrm{Au}$ level scheme. This is partfcularly signt ficant since it has been reported that very converted transitions in $165 \mathrm{Au}$ have $M$ multi polarity (with abnormal penetration factors).

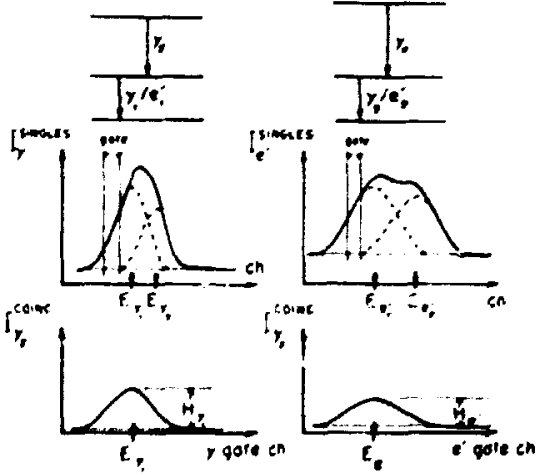

Fig. 3.1. A schematic description of tile technique used to determine a values for closely spaced doublets (or mittiplets). A coinctdence gate is advanced across the doublet and, for example, the "colncidence response" of $r_{2}$ to $r_{1}$ and $e_{1}$ ( $H_{r_{1}}$ and $H_{e_{1}}^{-}$, respectively) is extracted from $r-\gamma$ and e-r colncidence data. respectively. $a_{4}$ is determined from $\mathrm{H}_{e_{1}} / \mathrm{H}_{\gamma_{j}}$ with corfection for detection efficiency for $r_{1}, e_{3}$ and normalization using $a_{k}$ 's (determined in this manner) for transitions of known multipolarity. 
Table 3.1. Transitions and their ak values and $Y$ Fi.tipalarities, between

states of the same spin in the two $\mathrm{hg/2}$ bands in $187 \mathrm{Au}$.

\begin{tabular}{|c|c|c|c|c|}
\hline$J_{i} \mathbf{T}+J_{f}{ }^{\pi}$ & $E_{\text {trans }}$ (keV) & ak expt & $a_{k}(\mathrm{HI})^{\text {th }}$ & $Y$-ault. \\
\hline $\begin{array}{r}1 / 2^{-}+1 / 2^{-} \\
3 / 2^{-}+3 / 2^{-} \\
5 / 2^{-}+5 / 2^{-} \\
1 / 2^{-}+1 / 2^{-} \\
9 / 2^{-}+9 / 2^{-} \\
11 / 2^{-}+11 / 2^{-} \\
13 / 2^{-}+13 / 2^{-}\end{array}$ & $\begin{array}{c}270.71 \\
\text { not found } \\
284.35 \\
272.19 \\
322.92 \\
332.42 \\
388.19\end{array}$ & $\begin{array}{l}1.15^{25} \\
- \\
0.2911 \\
0.8119 \\
0.30^{4} \\
0.22^{8} \\
0.52^{7}\end{array}$ & $\begin{array}{l}0.372 \\
- \\
0.329 \\
0.372 \\
0.233 \\
0.215 \\
0.142\end{array}$ & $\begin{array}{l}E 0+\ldots \\
- \\
M 1+E 2 \\
E 0+\ldots \\
E 0+\ldots \\
M 1 \\
E O+\ldots\end{array}$ \\
\hline
\end{tabular}

We plan a restudy of these decays to resolve this abnormality and to ascertain if $185 \mathrm{Au}$ also exhibits four "shapes".

1. Louisiana State University, Baton Rouge, Louisiana.

2. Georgia Institute of Technology, Atlanta, Georgia.

3. Georgia Institute of Technology. Atlanta, Georgia; present address University of

Loutsville, Louisville, Kentucky.

4. UNISOR, Dak Ridge Associated Universities.

5. C. Bourgeois, et al.. Mucl. Phys. A295, 424 (1978).

6. E.F. Zganjar, et al., in Proc. 4th Int Conf. on Nuclei Far From Stability. CERN 81-09, p. 630 .

7. C. Bourgeois, et al.. Mucl. Phys. A386, (1982) 308.

\section{A MEW CLASS OF LON-ENERGY STRUCTURE AT CLOSED} SHELLS: LEVELS IN $187-191$ TI
E. F. Zganjar
L. L. Riedinger5
J. D. Cole
C. R. Binghams
J. L. Wood ${ }^{3}$
G. M. Gowdy
G. Campbeils
E. H. Spejewski6

The mechantsm of pair excitation across a shell gap to produce shape coexistence now appears to be fairly well established, 7 Recent evidence 8 for low-lying $0^{+}$states in $192-198 \mathrm{~Pb}$ indicates that the excitations are due to a proton $2 p-2 h$ configuration. Since the $\pi(2 p-2 h)$ excitation in the neutron-deficient even-A $P b$ isotopes occurs at low energy, one expects to see a new class of low-energy structure in the neutron-defictent $\mathrm{TI}, \mathrm{BI}$ and odd-Pb isotopns. In particular, levels due to the coupling $\pi-1 ;(2 p-2 h)$ should appear at low energy in the neutron-defictent odd-mass $I 1$ isotopes.

A major puzzle in our studies of excited states in 189.191 Tl has been the observation of a considerable number of levels "extra" to those expected from systematic trends in the heavier odd-mass $\mathrm{Tl}$ isotopes. He are investigating the possibility that these extra states art due to the couplings $\pi^{-1} \pi(2 p-2 h), \pi=s_{1} / 2, d_{3 / 2}$. This has necessitated a restudy of the $191 \mathrm{mpb}+191 \mathrm{Tl}$ and $193 \mathrm{mpb}+193 \mathrm{Tl}$ decay schemes.
These data are in the process of being analyzed. There is also evidence" for some "extra" states in $187 \mathrm{Tl}$. An attempt to make a detailed study of the 187 $\mathrm{aPb}+187 \mathrm{Tl}$ decay scheme was unsuccessful, protably because of a long hold-up time in the catcher of the target/ion source (the half life of $187 \mathrm{mpb}$ is $18 \mathrm{~s}$ ).

Cur interpretation of the lowest-lying extra states in $187,189,191 \mathrm{Tl}$ is shown in Fig. 3.2.

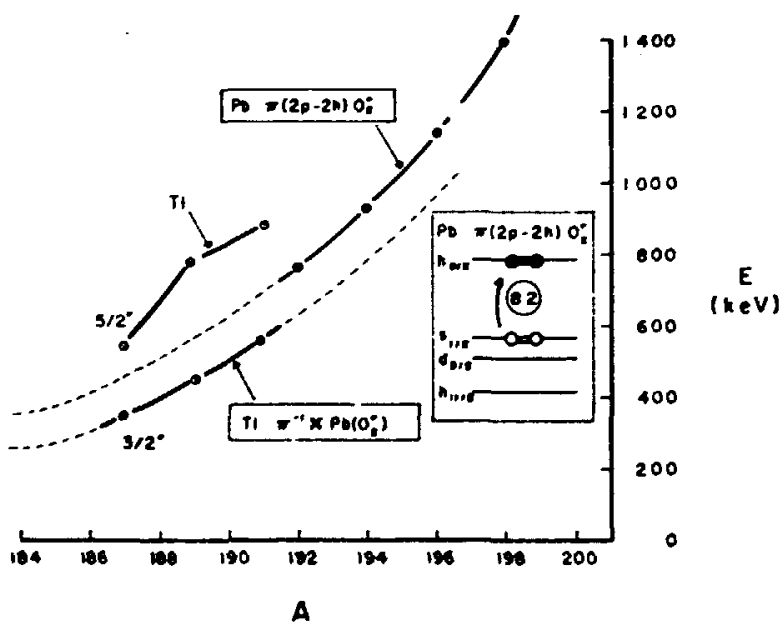

Fig. 3.2. The $\mathrm{O}_{2}{ }^{+}$energies in $192-198 \mathrm{pb}$ from Ref. 8, and the new low-lying states in 187-191 T1. The $\mathrm{O}^{+}$states in the $\mathrm{Pb}$ isotopes have been argued ${ }^{2}$ to be due to the $\pi(2 p-2 h)$ configuration. Based on the present systematics. it is suggested that these new low-lying bands in 187-19ITl are due to the $\pi^{-1} \mathrm{~Pb}\left(\mathrm{O}_{+}^{+}\right)$configuration. The pair excitation leading to the $\mathrm{Pb}\left(\mathrm{O}_{3}+\right)$ core state results in a lowering of the Fermi energy from $s_{1 / 2}$ to $d_{3 / 2}$ (in a deformed field, this corresponds to a lowering from $1 / 2^{+}$ 400+ to $\left.3 / 2^{+} 402 t\right)$ : this is consistent with the tentative spin assignment.

The task for the inmediate future is to identify the corresponding states in 19371 and to make rellable spin-parity assignments.

1. Loutstana State University, Baton Rouge, Louistana. 
2. Louisiana State University; present address: Idaho Hational Engineering Laboratory, Idaho Falls. Idaho.

3. Ceorgia Institute of Technology, Atlanta, Ceorgía.

4. 6SI Darmstadt; present address: SCGE Co.. Colubia, South Carolina.

5. University of Tennessee, Knoxville, Tennessee.

6. INISOR, Oak Ridge Associated Universities.

7. K. Heyde et al ., Phys. Repts, 102, 291 (1983).

8. P. Van Duppen et al.. Phys. Rev. Lett. 52. 1974 (1984).

9. P. Misaelides et a1.. Z. Phys. A3)1, 199 (1981).

MEASUREEEMT OF THE ISOTOPE SHIFTS ND MPERAILE STRUCTURES OF 192mT AD $201 \mathrm{~T}$ RT THE UNISOR LASER FACILITY
J. A. Bounds I
R. L. Mlekodaj2
H. K. Carter 2
E. H. Spejewski2
C. R. Binghanl
H. M. Fairbank, Jr. ${ }^{3}$
P. Juncar

The laser facility on line to the UnISOR separator has successfully measured the atomic hyperfine structure of an on-line produced isotope, $192 \mathrm{Tl}$. Using the laser facility essentially as reported previously, 5 the first online data are shown in Fig. 3.3.

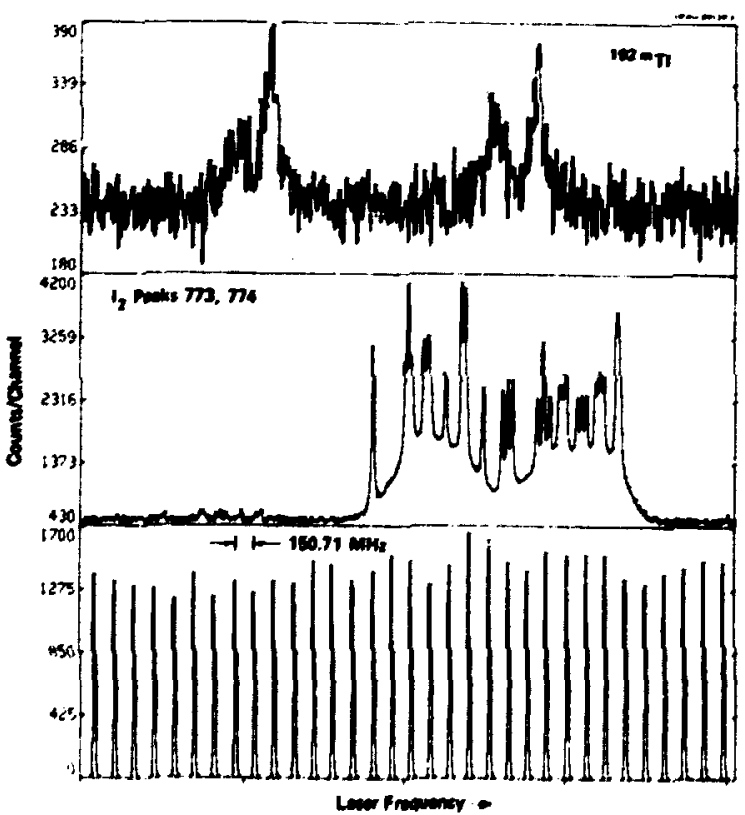

Fig. 3.3. Atomic Hyperfine Structure of $192 \mathrm{Tl}$ obtained in 45 minutes at a rate of $1 \times 10^{5}$ atoms/sec. The middle section is a portion of the saturated absorption spectrum of $I_{2}$ and the botton section shows irequency markers.
The difficulties reported last year, i.e., th deposition on the fiber optic detector, high PuT background due to radioactivity, and low ion bean transinission through the laser line, have been resolved. The lia deposition proble was solved by increasing the separation between the charge exchange cell and the fiber optic detector. The resulting increase in optical pumping is preverted by introducing a manetic field in the space which Zeenan shifts the levels out of resonance until the atom reaches the detection region. The PAT background was reduced by shielding of the bean collitators and the laser line transwission ws improved by realigrment of ion optics and more careful control of ion source parameters. With the new experimental conditions, the efficiency of the detection systew ms carefully studied using the medical isotope $201 \mathrm{Tl}$ in an off-line mode. Using standard gama-ray spectroscopy, we could accurately deterwine the $\mathrm{II}$ bean intensity through the laser line. From these data determined our syste detection efficiency to be $9 \times 10^{-4}$. in good agreement with our value of $6 \times 10^{-4}$ obtained last years by neutral current measurewent techniques. These data enabled us to obtain an inproved isotope shift and hyperfine structure for $201 \mathrm{Tl}$ as shomn ip. Tables 3.2 and 3.3.

With the fimproved laser systen, the fimproved target/ion source arrangement and improved beams from the tanden accelerator beans of approximately 105 atoms/sec of $192 \mathrm{il}$ were obtained through the laser line. The his spectrum of $192 \mathrm{Tl}$ shown in Fig. 3.3 was obtained ir. 45 minutes at an average bean intensity of $1 \times 10^{5}$ atoms/sec. Our preliminary analysis of these data and ganma-ray spectra taken simultaneousiy suggest we have observed the hfs of the high spin state of $192 \mathrm{TI}$. The initial results are given in Tables 3.2 and 3.3 .

1. University of Tennessee, Knoxville, Tennessee.

2. UNISOR, Dak Ridge Associated Universities.

3. Colorado State University, Ft. Collins, Colorado.

4. Laborstoire Aime Cotton, Orsay, France.

5. H.K. Carter et al.. Physics Division, Prog, Rept. ORNL-6004 (1983), p. 169.

\section{MEW METHOD TO MEASURE RELATIVISTIC DOPPLER} SHIFT: FIRST RESULTS ND A PROPOSALI

$$
\begin{array}{ll}
\text { P. Juncar2 } & \text { C.R. Binghanth } \\
\text { H.K. Carter3 } & \text { D.J. Peggt } \\
\text { R.L. Mekodaj3 } & \text { J.D. Coles } \\
\text { J.A. Bounds4 }
\end{array}
$$

Itilizing a newly derived relatívistic relation, $\sigma_{0}=\sqrt{\sigma+\sigma_{-}}$, the collinear-laser/fast-atom beams technique has been used to measure the relativistic Doppler effect to in accuracy comparable to the best previous experiments ( 5 parts in $10^{3}$ ). Improved calibrati in data and a similar technique, but employin; two 
Table 3.2. Measured hFs level splitting in the II 535.2 . transition (in $\mathrm{MHz}$ ).

\begin{tabular}{|c|c|c|c|c|c|c|}
\hline \multirow[t]{2}{*}{$n$} & \multicolumn{3}{|c|}{$\Delta v\left(62 p_{3 / 2}\right)$} & \multicolumn{3}{|c|}{$\Delta v\left(72 S_{1 / 2}\right)$} \\
\hline & this work & others & method & this work & others & ethod \\
\hline 205 & $529.3(1.6)$ & $530.076546(3)^{a}$ & ABRR & $12292.4(8.3)$ & $12291(15)^{C}$ & cptical \\
\hline 203 & $524.6(2.0)$ & $524.059953(3)^{a}$ & ABRR & $12179.7(5.5)$ & $12184(15)^{C}$ & optical \\
\hline 201 & $510.0(10.0)$ & $510(120)^{b}$ & optical & $12045.3(9.4)$ & $12052(120)^{b}$ & optical \\
\hline 192 & - & - & - & $2071(65)$ & - & optical \\
\hline
\end{tabular}

af.R. Petersen, H.6. Palmer, J.H. Shirley, Bull. M. Phys. Soc. 13, 1674 (1968).

DR.J. Hull and H.H. Stroke, J. Opt. Soc. A. 51, 1203 (1961).

CA.I. Odintsor, Opt. and Spec. IX, 2, 75 (1960).

Table 3.3. Isotope shifts of the 535.2 m line in $201,203,205 \mathrm{Tl}$ (in $\mathrm{GHz}$ ).

\begin{tabular}{llll}
\hline & this work & others \\
\hline$\Delta(203-205)$ & $-1.758(18)$ & $-1.766(21)^{\mathrm{a}}$ \\
$\Delta(201-205)$ & $-3.537(15)$ & $-3.72(12)^{\mathrm{b}}$ \\
$\Delta(192-205)$ & $-10.75(25)$ & - \\
\hline
\end{tabular}

A. 1. Odintsov, Opt. and Spec, IX, 2, 75 (1960).

bR.J. Hull and H.H. Strake, J. Opt. Soc. Am. 51, 1203 (1961).

lasers, is proposed wich should improve the test of time dilation by at least another factor of ten.

1. Abstract of paper submitted to Phys. Rev. Letters.

2. University of Tennessee and Laboratoire Ainte cotton, Orsay, Franr,e.
3. UNISOR, Oak Ridge Assoclated Universitfes.

4. University of Tennessee, Knoxville, Tennessee.

5. Louisiana State University. Baton Rouge, Loutstana; present address: Idaho Nattonal Engineering Laboratory, Idaho Falls, Idaho. 


\section{EXPERIMENTAL ATOMIC PHYSICS}

The experfmental atomic physics progran within the physics divisfon is carried out by two groups, mose reports will be given in this section. Work of the accelerator atcic physics group is centered around the 6.5-II EN tanden accelerator; consequently, wost of its research is concermed with atomic processes occurring to, or initiated by, feu Mel/am heavy ions. Other activities of this group include higher energy experiments at the Holifield Heavy Ion Research Facflity (hinRF), studies of electron and positron channeling radiation, and collaborative experiments at other institutions. The second experimental group concerns itself with lower energy atouic collision physics in support of the Fusfon Energy Progra. During the past year, the new Electron Cycletron Resonance Source has been completed, and some of the first data from this facility will be presented here. In addition to these two actirities in experimental atonic physics. other chapters of this report will describe progress in theoretical atouic physics, experimental plased diagnestic development, and atouic data center compilation activities.

\section{CORRELATED TI ELCTRON EFFECTS II HIGHY CHAREED IOH-ATOM COLLISIOUS}
S. Datz
M. Frost ${ }^{1}$
C. Bottcher
P. Hvelplund
L. H. Andersen 1
H. Knudsen 1

We have studied the fate of electrons released in collisions between highly charged Auq+ ions (20 MeV) and He atons and find that the large transfer ionization (TI) cross section observed can be accounted for by transfer of two electrons to a highly correlated state on the Au projectile followed by the loss of one electron to the continuum. Autoionization lines are also observed, but they are attributable to electron transfer accompanied by core excitation (TE).

In these experiments, we collide Auq+ at 100 keV/u (i.e., at $2 v_{0}=$ orbital velocity of the He electrons) with He atoms and measure the spectrum of ejected electrons moving in the rest frame of the Au ton projectile, in coincidence with the charge state of the emergent Au fon.

Figure 4.1 shows electron spectra obtained with Aulst ions. The singles spectrum at the top of the figure displays two features: a peak at $54 \mathrm{eV}$ made up of electrons wth veloc, ities corresponding to zero velocity in the rest frame of the 20-MeV Au fons and a symmetric set of small peaks with corresponding low energy autofontzing electrons leaving the Au core in the forward and backward laboratory directions.

From the coincidence spectra, it can be seen that the observed autotonizing electrons are all associated with Au $15+$ and hence with transfer plus excitation (TE), i.e.,

$$
\begin{gathered}
\mathrm{Au}^{15+}+\mathrm{He}+\left[\mathrm{Au}^{14+}\right]^{+*}+\mathrm{He}^{+} \\
\mathrm{Au}^{15+}+\mathrm{e}
\end{gathered}
$$

Transfer ionization is defined as the loss of - larger number of electrons from the target

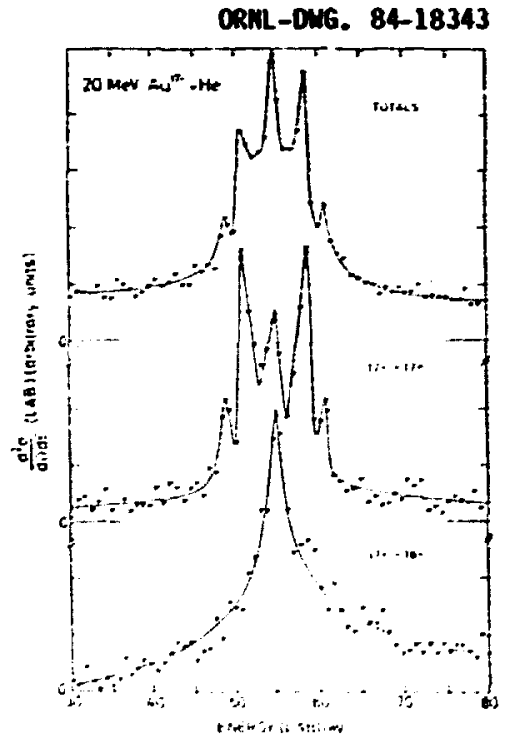

Fig. 4.1. Double-differential cross section in the laboratory frame as a function of electron energy.

than are transfered to the projectile. In the case of a helfum target

$$
\mathrm{Au}^{\mathrm{S}}+\mathrm{He}+\mathrm{Au}^{14+}+\mathrm{He}^{++}+\mathrm{e}
$$

the occurrence of a free electron in coincidence with a bound state capture implies the formation of $\mathrm{He}^{+t}$. The 'ectrum of these electrons is shown in the bottom portion of fig. 4.1. In a separate experiment, we showed that the yield of these electrons corresponds to a formation cross section of $6 \times 10^{-16} \mathrm{~cm}^{2}$, a number equal to the entife measured II cross section. 
Wuch can be learned from a study of the detailed shape of this spectrua, and to do this we tra.usform coordinates from laboratory system to the rest frame of the ion (fig. 4.2). The upper portion showing the singles spectrum is dominated by electron capture to the cont inuun. as evidenced by the asymetric distribution with enhanced density in the backward hewisphere ("negative energy"). The $15+14$ TI coincidence spectrum is seen to be synuetric, a shape characteristic of electrons which have been released from the pro-jectile, otherwise known as "electron Inss to the continuum" (ELC).

ORNL-0.16. 84-18344

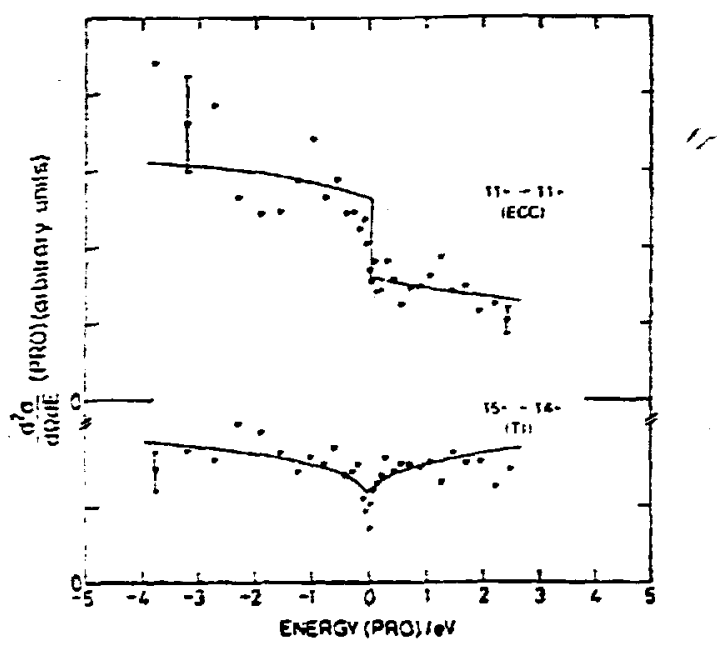

Fig. 4.2. Double-differential cross section in the projectile rest frame as a function of electron energy. Negative energfes correspond to enission in the backward hemisphere.

The flatness of the distribution suggests a simflarity to the process of fonization near threshold $(e, 2 e)$ wherein two electrons share the total available energy in a so-called Mannier state. The electrons dispose themselves on opposite sides of the nucleus and move apart along a line. ${ }^{2}$ In our case, we use a twodiuensional model to obtain an insight into the four-body coulomb problem, i.e., two electrons in the field of two nuclef. To fllustrate, we consider the potential surface for two electrons confined to the line of joinfing nuclef of charges $2+$ and $16+$ separated by a distance 10 ap. In Fig. 4.3 is plotted a contour map of this surface in the plane generated by the coordinates of the two electrons relative to the helfum nucleus; this represents a natural generalization of the hyperspherfcal plane used in discussions of threshold phenomeria.

The quadrant IV contains the saddle point $x$ associated with single-election capture, but two-electron capture would more likely proceed $v$ fa the ridge $R$ over the pass $P$ in quadrant $I$. In the latter case, the electrons would

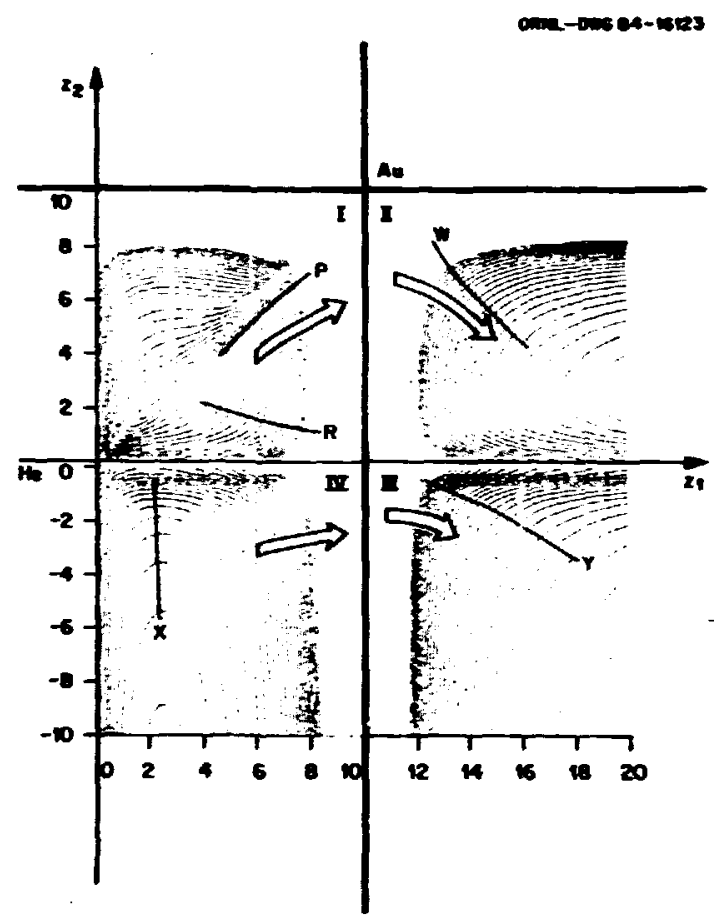

Fig. 4.3. Potential energy contours at 0.3 a.u. (8.1 eV) intervals for two electrons in the field of charges $2+$ and $16+$ separated by 10 a 0 . The coordinates $z_{1}$ and $z_{2}$ represent distances of the electrons from the $2+$ nucleus. Thus, the points lie" represents both electrons on the He nucleus and $\mathrm{Au}^{\prime}$ both electrons on the Au nucleus. The quadrants correspond to the linear configurations: I(He-e-e-Au), II (He-e-Au-e), III(e-He-Au-e), and IV(e-He-e-Au).

Dlausibly end up on the ridge W (quadrant II) which is the Wannier ridge associated with an almost isolated Au nucleus. On these grounds, we can conjecture that the energy distribution of cusp electrons should be predicted by Hannier theory wich should apply whether both electrons are free or bound. It should be emphasized that this model, as yet, contains no dynamics, i.e., quasiadiabatic motion on the potential surface is assumed. The feasibility of numerical solutions to the model is betng assessed.

1. Physics Institute, University of Aarhus, DK-8000, Aarhus C, Denmerk.

2. C. Bottcher et al., this report.

\section{ELECTRON AME POSITRON CHANNELING RAOIATIOH}
S. Datz
B. L. Berman
R. I Pantel $?^{2}$
J. l phart

The study of channeling radiation has now proceeded past the point of quantitative prnof 
of principal. In the case of sipple single canponent crystals, higher-lying line energies ay now be predicted to within a percent using Hart ree-Fock atcaic wave functions (for spherically symetric atoms) with proper planer or axial averaging in a eny-bea calculation.

\section{Ditrond}

Perhaps the best crystal with which to study channeling radiation is dianond, because of its high Debye temperature and its low $Z$. Indeed, the first measurement of channeling radiation from diamond showed remarkably sharp structure.

Our recent results for the (110) channelingradiation spectra at three incident electronbean energies are shom in Fig. 4,4, together with the many-bea calculation of transition energies and strengths from the "standard" Hartree-Fock potential. It can be seen that there is agreement between experifient and theory, although there is a slight tendency for the calculated transition energies to lie higher than the experimental spectral peaks (this is attributable to sifiple kinematic effects from multiple scattering). For the (111) plane, however, this tenden:y is much exaggerated, as shown in Fig. 4.5. Both a (III) potential obtained from $x$-ray-diffraction data and an empirical potential fitted to the data are shallower than the standard potential and both fit the data better. We believe the fact that significant charge in the diamond crystal is distributed along the (111) valence-bonding

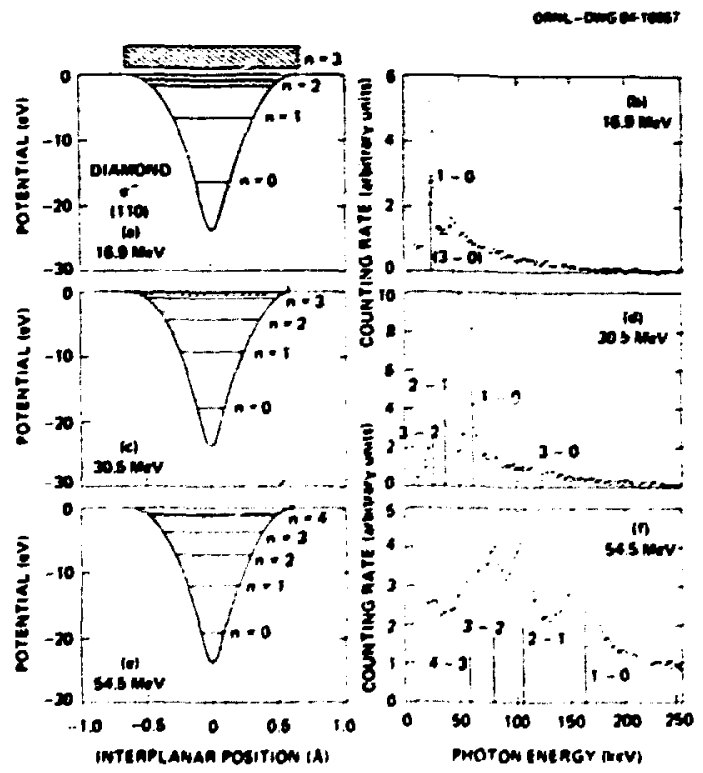

Fig. 4.4. The (110) potential and eigen. values [parts (a). (c), and (e)] and channelingradtation spectra [farts (b), (d), and (f)] for 16.9-MeV, 30.5-MeV, and 54.5-MeV electrons, respectively, channeled in diamond. Note the increase with electron-beam energy of the line energies and linewidths.
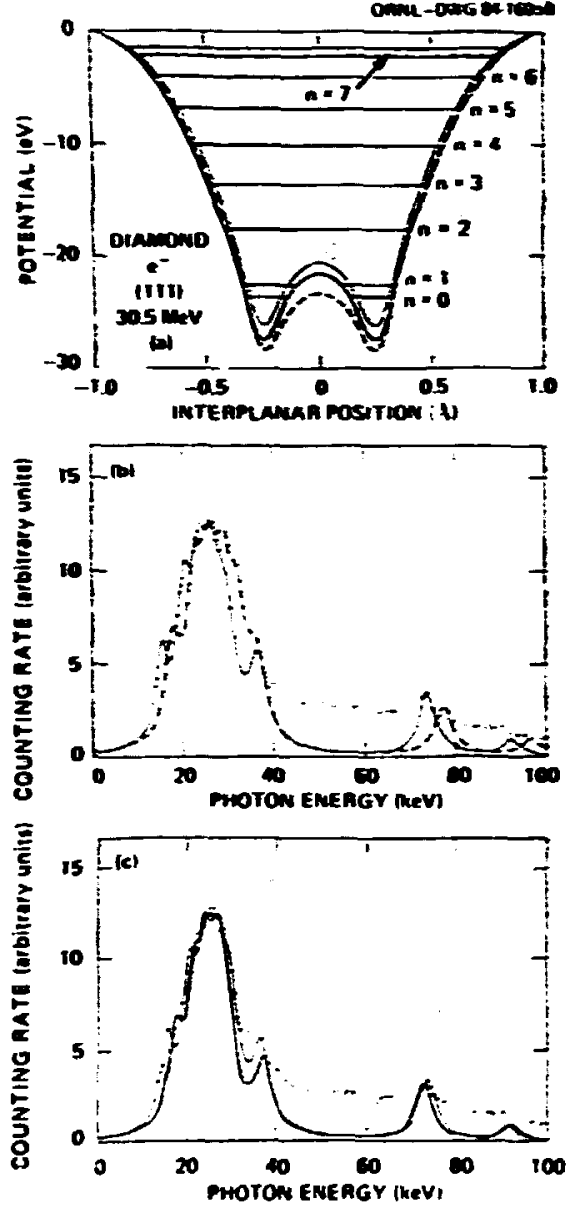

Fig. 4.5. (a) (111) potentials for disuond: the "standard" many-beam potential dashed curve); a potential based upon x-ray-diffraction data (iight solid curve); and an empirical potential based upon the best fit to the 30.5-MeV data (heavy solid curve), together with the corresponding eigenvalues. (b) The (1II) spectrum for 30.5-MeV electrons, together with the calculated spectra obtained from the standard (dashed curve) and $x$-ray-diffraction (light solid curve) potentials. (c) The same spectrum, together with the spectra calculated from the $x$-ray-diffraction (1 ight solid curve) and eapirical (heavy solid curve) potentials.

direction alters the (111) interplanar potential as shown th the figure. In fact, the data shown in Fig. 4.5 can serve as a quantitetive measure of this asymmetric charge distribution.

\section{Platelet Defects in Diamond}

Platelets in diamond [nitrogen mono- or di-layers preclpitated along the (100) planes] influence the channeling radiation dramaticaliy. In partlcular, they cause an energy shift in the (100) spectral ilnes, as shown in Fig. 4.6. We have shown recently that these lineshifts can be accounted for by equating the lattice distortion 

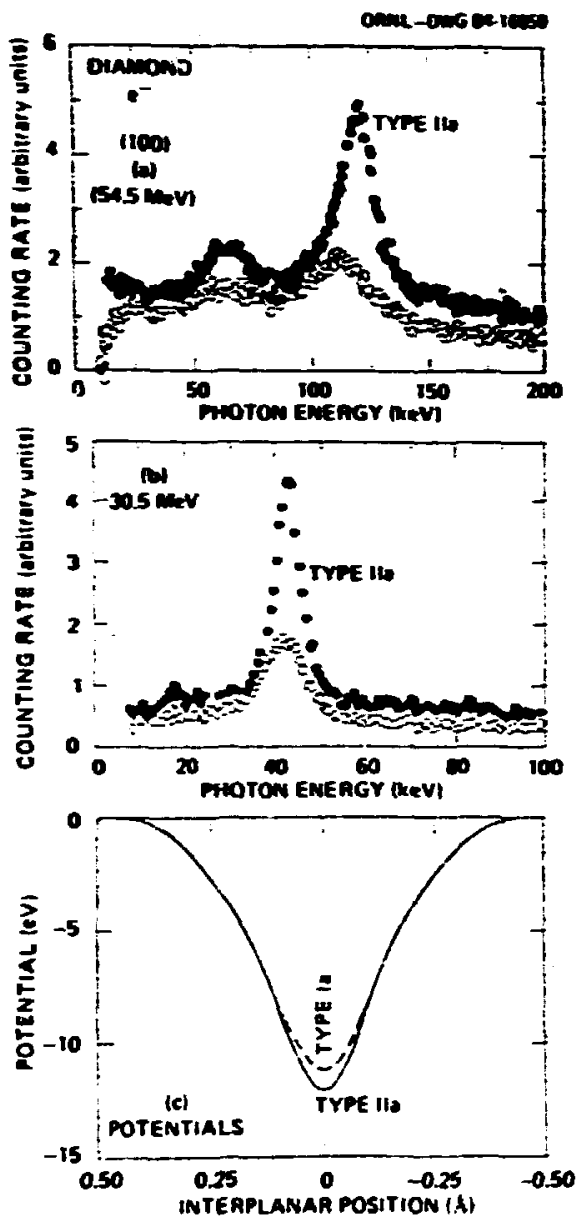

Fig. 4.6. (100) channeling-radiation spectra from both Type-Ia (with platelets; open data points) and Type-IIa (without platelets; closed data points) diamonds for (a) 54.5-MeV and (b) 30.5-Mey incident electrons. Mote in particular the energy shift for the $I \rightarrow 0$ transition. (c) (100) potentials for diamond: the one wich uses the accepted value of 0.040 \& for the thermalvibration amplitude, as is appropriate for the Type-IIa di amond (solid curve) and the one witch uses the value of $0.055 \mathrm{~A}$, which arises from the best fit to the data for the Type-Ia diamond (dashed curve).

caused by the platelets with a large average thermal-vibration amplitude perpendicular to the (100) planes and hence to the platelets. The (100) potential so altered is also shown in Fig. 4.6. These results also serve to show that channeling radiation can be used as a diagnostic probe of impurities and defects in crystals.

\section{Temperature Effects}

The bottom of the planar or axial averaged potential is affected by lattice vibrations. This is taken into account by introducing a Debye-Waller factor into the potential. Larger vibrational aplitude raises the botton of the potential and shifts the eigenstates upuard, with the lowest state being most affected and the upper states hardly af fected at all.

As the crystal temperature is decreased, one expects a lowering of the rell bottom and an increase in the energy of photons arising from transitions to lower states. This is demonstrated in fig. 4.7 where spectra of radiation from 54-meV electrons channeled in the (100) and (110) planes of Si are showa for two teaperatures. The temperature dejendence of the transition energies for the lomest-lying energy levels (the 1 - 0 transitions), show in Fig. 4.8, is sensitively dependent on the Debye temperature. These data consistent ly yield a value of the Debye temperature of silicen of $495 \pm$

$10 \mathrm{~K}$ in agreewent with shell wodel calculations, but in sharp disagreement with the value of $543 \mathrm{~K}$ obtained from previous $x$-ray diffraction studies. The cause of this discrepancy is not yet understood.

\section{Isotope Effects: Lif and Lfo}

Our recent results for Lill and Lid show two interesting effects for these lowest $-Z$ crystals.
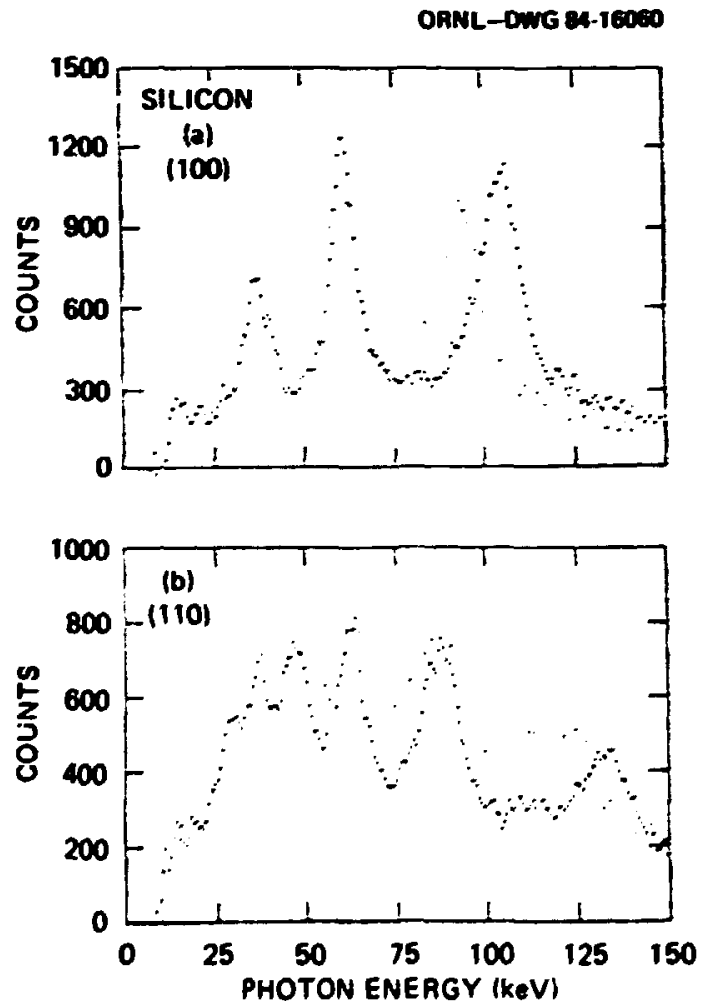

Ffg. 4.7. Superposed channeling-radiation spectra for 54.5-MeV electrons tncident along the (a) (100) and (b) (100) planes of silicon for two different temperatures: (a) $-190^{\circ} \mathrm{C}$ (heavy data points) and $+5^{\circ} \mathrm{C}$ (light data potnts): (b) $-180^{\circ} \mathrm{C}$ (heavy data points) and $+7^{\circ} \mathrm{C}$ (11ght data points). Note the large energy shifts of the 1 - 0 transitions. 
Cart-oms eq-1Cist

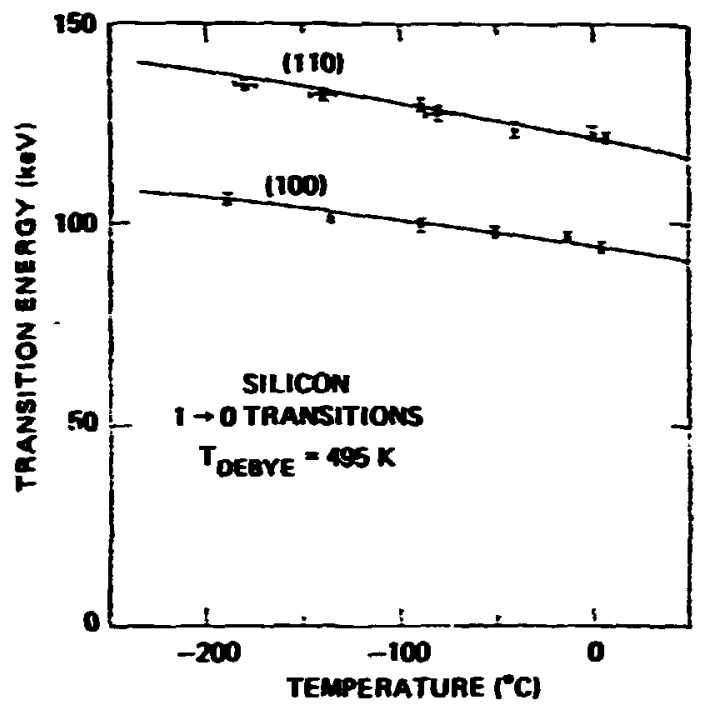

Fig. 4.8. Teaperature dependence of the channeling-radiation transition energies for the 1 - O transitions of 54.5-HeV electrons channeled along the (100) and (110) planes in silicon. The fits to these data (solid curves) yield a consistent value of $495 \mathrm{X}$ for the Debye temperature of silicon.

One is the largest disagreement to date between measured and calculated transition energies, as show, for example, in Fig. 4.9 for the case of 54.5-MeV positrons and electrons incident along the (100) and (110) planes of LiH. The other can be seen in Fig. 4.10, where (110) channel-

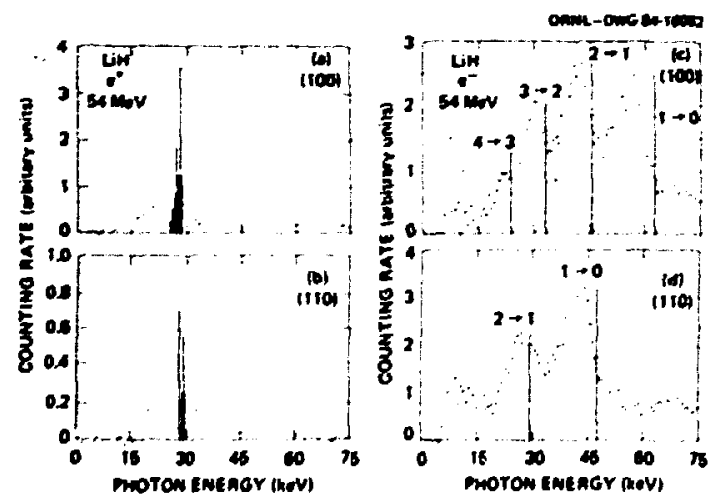

Fig. 4.9. Channeling-radiation spectra for LiH, both for 54.5-Mk positrons incident along the (a) (100) and (b) (110) planes and for 54.5-MeV electrons incident along the (c) (100) and (d) (110) planes, together with the calculated transtition energies and strengths (vertical lines) obtained from the standard many-beam theorettcal treatment. Note the very large discrepanctes between experiment and theory.

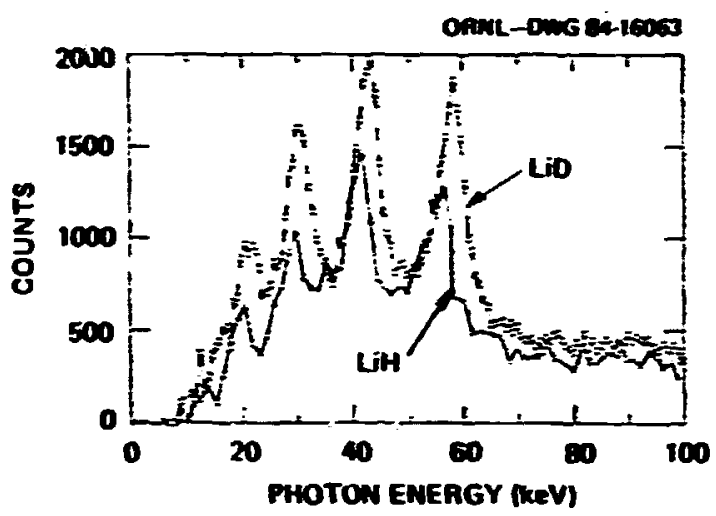

Fig. 4.10. Superposed channeling-radiation spectra for 54.5 int electron incident along the (110) planes of LiH (triangles) and LiD (squares). Wote that the ito spectrum resembles a spectrun that one night exf at to obtain from the cooled Lill crystal.

Ing radiation spectra from Lik and lio (both obtained at room teeperature) are superposed.

In general, the Lid il..es Ife at higher energies than the LiH lines and reflect the saliler vibrational aplitudes of the $D$ ions in the lattice. as one would expect from the fact that deuterons are heavier than protons. This would cause LiD to appear as a low-temerature version of LiH. The line energies are listed in Table 4.1. The largest effect is the 103 shift seen in the $1+0$ transition in the (110) plane. This plane has the most meakly bound states and the effect of lattice vibrations on the bottom of the potential well is most significant. The thermal vibration amplitudes required to fit the data are totally at variance with those listed in Crystallographic Data but lie close to those recently caculated by D. Mosteller. $^{3}$

1. Department If Physics, Lawrence Livermore Hat ional Laboratory. Livermore, CA 94550.

Table 4.1. Channeling radiation line energies (keV) for LiH and LiD crystals

\begin{tabular}{|c|c|c|c|}
\hline & & LIH & LiD \\
\hline$(100)$ & $\begin{array}{l}1 \rightarrow 0 \\
2 \rightarrow 1 \\
3 \rightarrow 2 \\
4: 3\end{array}$ & $\begin{array}{l}57.0 \pm 0.6 \\
41.8 \pm 0.4 \\
29.6 \pm 0.4 \\
21.1 \pm 0.5\end{array}$ & $\begin{array}{l}58.3 \pm 0.4 \\
43.6 \pm 0.3 \\
29.3 \pm 0.3 \\
21.2 \pm 0.4\end{array}$ \\
\hline$\langle 110\rangle$ & $\begin{array}{l}1 \rightarrow 0 \\
2 \rightarrow 1\end{array}$ & $\begin{array}{l}41.3 \pm 0.5 \\
26.3 \pm 0.5\end{array}$ & $\begin{array}{l}45.3 \pm 0.4 \\
28.1 \div 0.3\end{array}$ \\
\hline (111) & $\begin{array}{l}1: 0 \\
2: 1 \\
3: 2\end{array}$ & $\begin{array}{l}52.0 \pm 0.4 \\
36.5 \pm 0.4 \\
26.0 \div 0.4\end{array}$ & $\begin{array}{l}52.8 \pm 0.4 \\
37.4 \pm 0.3 \\
25.1 \pm 0.3\end{array}$ \\
\hline
\end{tabular}


2. Department of Electrical Engineering. Stanford University, Stanford, ca 94305.

3. Solid State Division, Oath, private connications.

\section{RESOMNT COHEREDT EXCITATIO OF REAT IOMS} morins In CRYSTRLline curmas

C. D. Moak

J. A. Biggerstaff

0. H. Crawford

S. Datz

P. F. Dittner
J. Gomez del Capo
H. F. Krause
P. D. Miller
P. L. Pepmiller
M. D. Brom?

Figure 4.11 illustrates the phenomenon called resonant coherent excitation. In this exaple, a fast one-electron ion is moving in a crystalline channel. The ion is shown moving nearest the aiddle string of atoms wich lies just above the ion path; the smaller black circles illustrate another string outside the plane of the figure. The six atoms schentically represented by the large black circles are closest to the ion path and they give rise to an electric field which acts upon the ion as shown by the sine wave. The space variation of this field is, for the ion, a time-varying field of frequency $v=$ $v / d$ where $v$ is the ion velocity and $d$ is the distance between the atoms. The field is never a pur? sine wave and its wave-shape depends sensitivily upon the ion path, the type of crystal lattice and the particular channel axis being used $n$ that lattice. In general, the field is rich $n$ harmonics of the fundamental string frequenc, and, as shown in the figure, a more apprcpriate expression is $v=K v / d$ where $K$ is an 1 iteger. Resonant excitation of the projectile can occur whenever one of these frequencies happens to match the frequency of some transition $(\Delta E)_{i j}$ where $i$ and $j$ denote the initial and final state of the system being excited. In Fig. 4.11, we have a one-electron ion and the transition is from the hydrogenic ground state to the first excited state. of course, $\triangle E$ could have been the energy for nuclear excitation of the first excited level of a bare nucleus. In the one-electron case, the numerical example is carried further for the one-electron ion $\mathrm{C}^{5+}$ in the <100) axis of a gold crystal, and it can be seen that the fundamental frequency occurs for
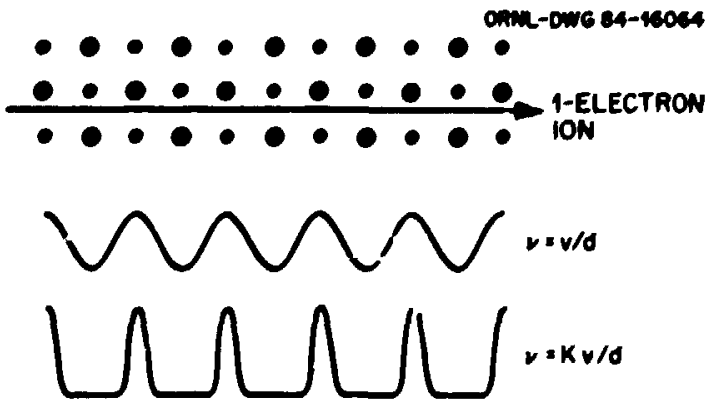

Fig. 4.11. Conditions for resonant coherent excitation of channeled ions. an ion energy of $6.8 \mathrm{meV} / \mathrm{am}$ and the second harmonic occurs at 1/4 as much energy (half the velocity). The minerical exaples are helpful for scaling to other ions 'with different energy levels. Once the ion is in an excited state, its electron is less tightly bound and it moves in a larger region around the nucleus; thus, it is more likely to be ionized. Unen the ion velocity is tuned to a resonance, there is a drop in the population of the one-electron fraction and a peak in the bare-ion fraction. Detailed features of these resonances can be seen in Refs. 3-7.

An additional complication is that sone of the oscillating fields are along the ion path and some field components are perpendicular to the ion path; generally, there is a wixture. Longitudinal oscillating fields are superimposed on the steady braking field wich is also longitudinal, and the result is that resonances with longitudinal fields are split by a Stark ef fect; transversely oscillating fields do not produce such splittings. All of these phenomena have been seen and they are illustrated in the references. ${ }^{3-7}$ An example of resonant coherent excitation with a Stark mixed level is shown in Fig. 4.12. The level positions deduced from the resonances observed, as in Fig. 4.12, have been compared with theory ${ }^{4}$ and the results are not very satisfactory as shown in Fig. 4.13; similar results are shown for $\mathrm{H}^{6+}$ in Fig. 4.14. Here, open circles are theoretical predictions and experimental points are full circles. For odd narmonics ( $K=$ ? for example), the fields are mostly transverse, no splitting is found and theory and experiment are in excellent agreement. For even harmonics, splittings are predicted and found but although the $2 s p z$ component occur at the expected energy, the 2sp component is shifted in energy with respect to the expected position. Certain ions at higher energies from the Holifield accelerator might provide the data to explain the difference. As shown in Fig. 4.12, the resonances are not sharp enough to provide very high accuracy in the

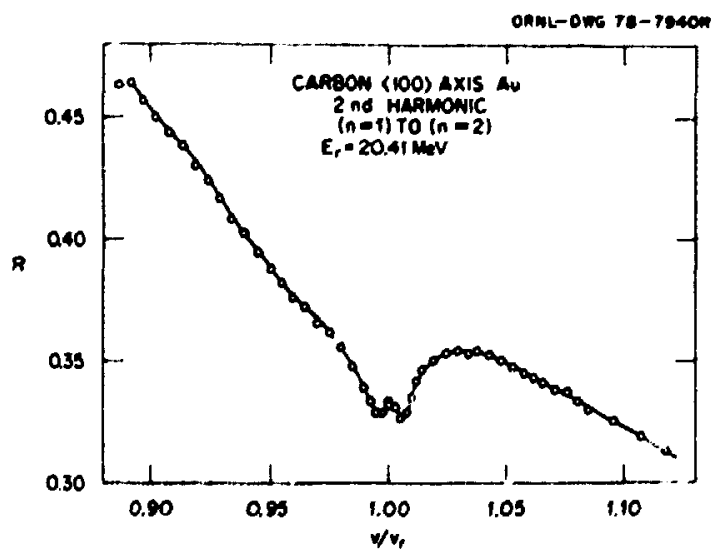

Fig. 4.12. Ratio of counts, $R, C^{5+} /\left(C^{5+}+\right.$ $\left.\mathrm{C}^{6+}\right)$ as a function of velocity divided by the resonant velocity for the vacuum state. 


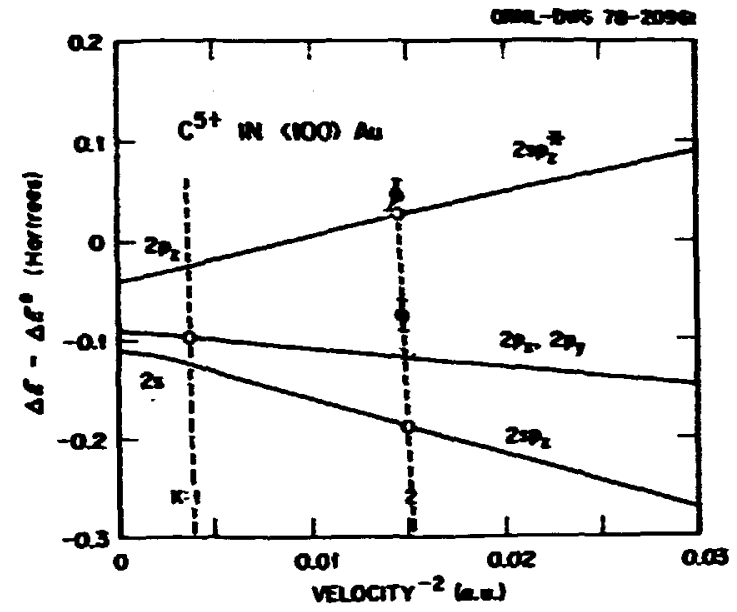

Fig. 4.13. Velocity dependence of energy shife from vacuum levels for $\mathrm{C}^{5+}$. The open circles are expected energy shifts from theory. and solid circles are experimentally observed shifts.

estimate of the Stark level splittings. One reason is that the ion energy changes as it slows down inside the crystal; this means that the ion cannot remain at resonance throughout the crystal regardless of the bombarding energy setting. To avoid smearing the resonance, $\Delta v / v_{0}$ should be as small as possible, where $\Delta v / v_{0}$ is the percentage velocity change in passing through the crystal. In Tables 4.2 and 4.3 , we show some cases witch fllustrate the improved resolution to be expected using the Holifield accelerator. The tables fllustrate, for our thinnest crystal, the energy loss in the $\langle 100\rangle$ channel expressed as a fraction of the resonance

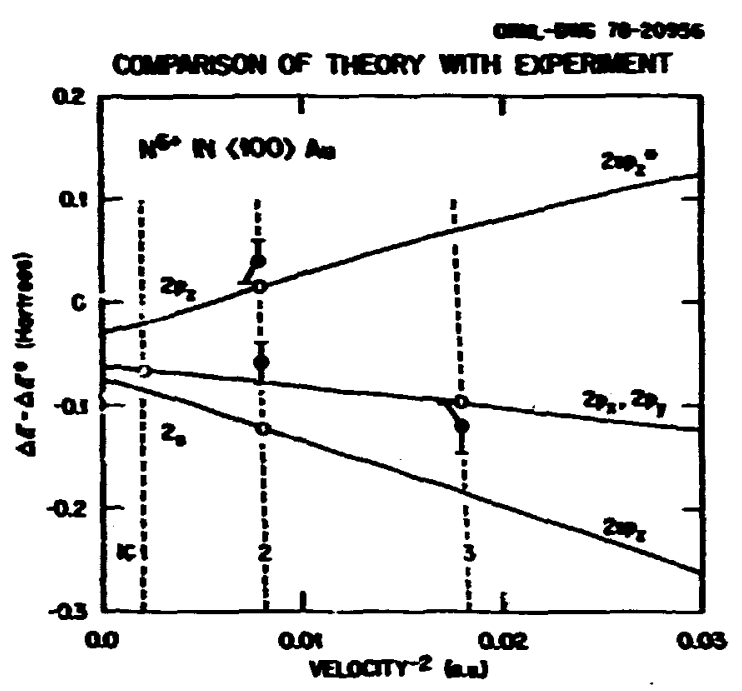

Fig. 4.14. Velocity dependence of energy shift from vacuu levels for $h^{5+}$. The open circles are expected energy shifts from theory, and solid circles are experimentally observed shifts.

energy and the fractional velocity change in passing through the crystal for $K=1$, the fundamental frequency (odd harmonic, predominantly transverse oscillating fields). For $\mathrm{C}^{5+}$ we see that $\Delta v / v_{0}$ is 0.551 . In Fig. 4.12 we see that $0.551 \%$ (or 0.00551 in abscissa units) is enough to linit the resolutic... Another example, ${ }^{6+}$, has a $\Delta v / v_{0}$ of $0.277 \%$ and, as we can see in Fig. 4.15 , the resonances show up more clearly. The next improverients would come with $0^{+}+$and $F^{8+}$. The $K=2$ resonances for $0^{7+}$ and $F^{b+}$ would ife at $86.0 \mathrm{MeV}$ (tandem terminal voltage $10.7 \mathrm{w}$ )

Table 4.2. Resonance parameters for one-electron tons using the fundamental frequency $(K=1)$ for ions traversing the $\langle 100\rangle$ channel in a Au crystal 1470 A thick. The resonant energy is given by $E_{0}=0.00524 Z_{1}^{4}$ in MeV.

\begin{tabular}{|c|c|c|c|c|c|c|c|c|}
\hline & $Z$ & MeV/amu & Eo, MeV & $V(M V)$ & $\begin{array}{c}\text { Random } \\
\text { Loss } \Delta E \\
\text { MeV }\end{array}$ & L/2AE/E & $\Delta v / v_{0}$ & $\operatorname{MPC}^{+}$ \\
\hline$B e^{3+}$ & 4 & 1.34 & 12.1 & 3.02 (EN) & 0.240 & $0.99 \%$ & $0.50 \%$ & \\
\hline$B^{4+}$ & 5 & 3.28 & 32.8 & 6.56 (EN) & 0.260 & $0.4 \pi \%$ & $0.20 \%$ & \\
\hline$c^{5+}$ & 6 & 6.79 & 81.6 & $13.59(H, T)$ & 0.257 & $0.16 \%$ & $0.08 \%$ & $6^{+}$ \\
\hline$n^{6+}$ & 7 & 12.58 & 176.2 & $2 \pi .17(H, T)$ & 0.235 & $0.07 \%$ & $0.03 \%$ & $7^{+}$ \\
\hline $07+$ & 8 & 21.47 & 343.4 & (42.92) Coupled & 0.210 & $0.03 \%$ & $0.02 \%$ & $8^{+}$ \\
\hline
\end{tabular}

TMPC: Most Probable Charge 
Table 4.3. Resonance parameters for one-electron fons using the second harmonic frequency $(K=2)$ for fons traversing the ciod chanacl in a An crystal 1470 \& thick. The resonant energy is given by $E_{0}=0.00131 Z_{l}$ in mer.

\begin{tabular}{|c|c|c|c|c|c|c|c|c|}
\hline & 2 & nev/ans & $E_{0}$. MeV & $V(m)$ & $\begin{array}{c}\text { Randon } \\
\text { Loss aE } \\
\text { hel }\end{array}$ & $1 / 2 \Delta E / E_{0}$ & $\Delta v / v_{0}$ & mpt \\
\hline$c^{5+}$ & 6 & 1.70 & 20.4 & $3.40 \mathrm{EN}$ & 0.449 & 1.106 & $0.55 \%$ & $5.5^{+}$ \\
\hline$n^{6+}$ & 7 & 3.15 & 44.0 & $6.29 \mathrm{En}$ & 0.469 & 0.568 & 0.288 & $6.5^{+}$ \\
\hline $0^{7+}$ & 8 & $3 . .1$ & 85.9 & $10.74(H, T)$ & 0.505 & $0.29 \%$ & $0.15:$ & $7.7^{+}$ \\
\hline$F^{8+}$ & 9 & 8.60 & 163.3 & $18.14(H, T)$ & 0.487 & 0.158 & 0.083 & $8.8^{+}$ \\
\hline$n e^{9+}$ & 10 & 13.10 & --- & -- & $\cdots$ & -- & -- & Becoming \\
\hline $\mathrm{Na} \mathrm{LH}^{\mathrm{H}}$ & 11 & 19.18 & 41.2 & (40.11) Coupled & 0.428 & $0.05 \%$ & 0.027 & wre \\
\hline Mgl1+ & 12 & 27.17 & 652.0 & (54.34) coupled & 0.396 & $0.03 x$ & 0.022 & totally \\
\hline A) $: 2+$ & 13 & 37.42 & 1010.4 & $-\infty$ & --- & -- & $\cdots$ & stripped \\
\hline Sill+ & 14 & 50.33 & 1409.4 & $\ldots$ & $\cdots$ & $\ldots$ & $-\infty$ & + \\
\hline
\end{tabular}

tmPC: Most Probable Charge

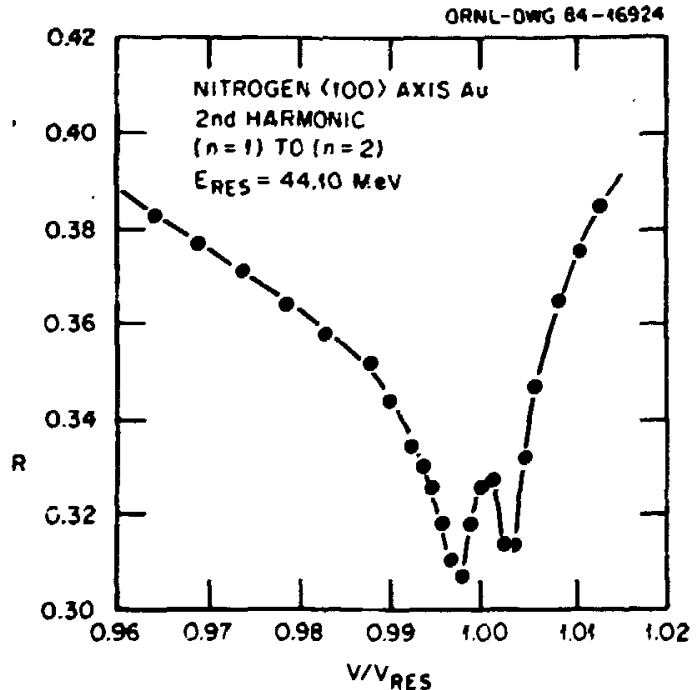

Fig. 4.15. Ratio of counts, $R, \mathrm{~K}^{6+} /\left(\mathrm{K}^{6++}+\right.$ $N^{7+1}$ as a function of velocity divided by the resonant velocity of the vacuum state.

and $163.6 \mathrm{MeV}$ (tandem terminal voltage $18.1 \mathrm{MV}$ ), respectively.

These latter two experiments have just been completed. The anticipated sharpening is, indeed, observed but the Stark split double -peak structure is entirely absent in both cases. In another experiment, we investigated the $K=1$ resonance in $\mathrm{C}^{5+}$ at $81.6 \mathrm{MeV}$. The depth is enormous, a factor of at least 4 below the secular trend, but the resonance is very broad. Perhaps this is a sigr of power broadening or saturation. In any case, these entirely new results require some time for interpretation.

1. Chemistry Division, ORNL.

2. Naval Surface Weapons Laboratory, Silver Spring, MD 20910.

3. S. Datz et al., Phys. Rev, Lett. 40, 843 (1978).

4. C. D. Moak et al., Phys. Rev. A19, 977 (1979).

5. 0. H. Crawford and R. H. Ritchie, Phys. Rev. 120,1848 (1979).

6. S. Oatz et al., Nucl. Instr. Meth. 170, $15(1980)$.

7. C. D. Moak et al.. Nucl. Instrum, and Methods 194, 327 (1982).

ION-ION COLLISION STUDY USING THE FOLDEO BEAN APPARATUS

$$
\text { H. J. Kim }
$$

We have observed the formation of $\mathrm{Ar}^{4+}$ from $A r^{3+}+A r^{3+}$ collitsions and $\mathrm{H}^{4+}$ from $\mathrm{N}^{3+}+\mathrm{N}^{3+}$ collifions at $C-M$ energies of $120 \mathrm{keV}$ using the 
folded been apparatus. Although we were successful in separating sigal events fro backgrownds, wuch work rewins to be done before absolute charge-changing cross sections for ionion collisions can be reliably and routinely deduced from observed results. Making reasonable assumtions and using the previously neasured $\mathrm{Ar}^{3+} \mathrm{Ar} \rightarrow \mathrm{Ar}^{\mathrm{b}+}$ cross sections to calibrate our bean overlap and target length, we obtained approximate cross sections for the miticharged ion collis ins, i.e..

$$
\begin{aligned}
& \sigma\left(A r^{3+}+A r^{3+}+A^{4+}\right)-5 \times 10^{-16} \mathrm{~cm}^{2} \\
& \sigma\left(A^{3+}+R^{3+}+A^{+}\right) \sim 1 \times 10^{-16} \mathrm{~cm}^{2} .
\end{aligned}
$$

These numbers appear large but they are commensurate with 5 fo promotion for the Ar ${ }^{3+}$ and curve cross ing for $\mathrm{A}^{3+}$ case.

Recently, our ain eifort has been directed toward rendering the results obtained with the folded bean apparatus wore amenable to absolute cross section determinations by making the separation of reaction products from the tail of the main bean cleaner and by siaplifying the selective detection of reaction products.

\section{DIELECTRONIC RECONBIMTION: A STATUS REPORT}

$\begin{array}{ll}\text { P. F. Dittner } & \text { P. D. Miller } \\ \text { S. Datz } & \text { C. D. Moak } \\ \text { C. M. Foul } & \text { P. L. Pepmiller }\end{array}$

Following the publication of our measurements [Ref, 2] on the dielectronic recombination (DR) of $\mathrm{B}^{2+}$ and $\mathrm{C}^{3+}$, we modified the analysis section of our merged bean apparatus. Previous'y the initial charge state $(q+)$ beam and th: $(q-1)^{+}$ beam mere magnetically deflected thr ugh $\sim 6^{\circ}$ resulting in a motional onf $\sim 40,000 \mathrm{volts} / \mathrm{cm}$. This high field had the disadvantage that it caused recombined $(q-1)^{+}$ions in high $n$ states to be Stark-stripped back to $q^{+}$, thus resulting in a loss of DR signal, e.g., for $\mathrm{C}^{3+}$ all $n>27$ were lost. The charge state analysis is now done by electrostatic deflection (E $\sim 4000 \mathrm{~V} / \mathrm{cm}$ ) through $\sim 0.6^{\circ}$ (see Fig. 4.16) resulting in a larger signal, e.g., for $\mathrm{C}^{3+}$, all $n^{>} 45$ are lost. In addition, the flight distance between the interaction region and the charge state analyzer was decreased from $2.5 \mathrm{~m}$ to $25 \mathrm{~cm}$, but the hoped for decrease in noise due to charge-

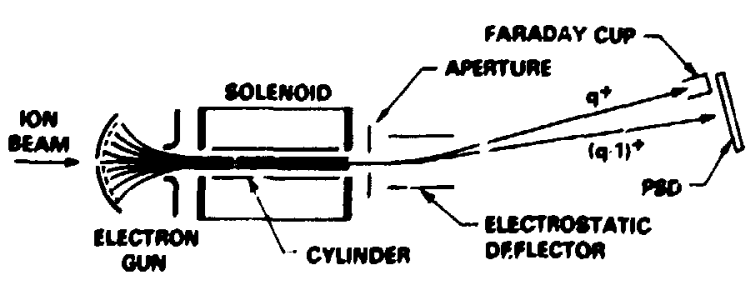

Fig. 4,16, Schematic diagram of merged beam apparatus. changing collisions with the background gas was not realized. We think that the cominant part of the nolse arises from slit edge scattering and has only a anc dependence on the flight distance. Wevertheless, the overall signal-tonoise ratio was improved by a factor of -2 for the $\mathrm{C}^{3+}$ on experiment.

In order to decrease the uncertainty in the background subtraction needed to extract the on rate from the total rate of $(9-1)^{+}$ions, we now modulate the electron energy at each ion energy (Ei) step. ${ }^{3}$ This sodulated electron energy method (nkER) works as follows: (1) The electron gun is operated with a fixed cathode voltage, $-V_{C}$. (2) The relative energy $\left(E_{r}\right)$ is varied. by stepping through a range of $E_{i}$ 's fro D to $E_{r}$ above the Rydberg linit of the DR resonance energy ( $E_{S}$ ). (3) An $\sim 10$ Hz square-wave codulated voltage having two values, 0 and $V_{T}$, is applied to the cylinder surrounding the electron bean. (4) The events $\left((q-1)^{+}\right.$ions) accumulated when $V_{T}$ is $0 n, H_{T}$, are back jround and are subtracted from the numer of events, $M_{0}$, accuinlated then the cylinder voltage equals zero. Ideally, the events, due to $O R, n_{5}$, should be given by $N_{s}=N_{0}-N_{T}$. However, dt $E_{r}>E_{s}$, where $H_{s}=0$, we found that $N_{0} \neq H_{T}$ because when the electron velocity is changed the outgassing (due to the electron beam) changes and, in turn, the number of noise events changes. In view of the aforementioned, our procedure for extracting $M_{s}$ is to construct a smoothed $E_{i}$ dependent $N_{T}$ (from the $N_{T}$ data), multiply it by a constant a determined by insisting that $N_{s}=N_{0}-a H_{T^{\prime}}=0$ when $E_{r}>\varepsilon_{s}$, and continuing the subtraction where $0<E_{r}<E_{s}$ to yield $H_{s}=H_{0}-\alpha N^{\prime}$. Current integration of the initial $q+$ bean gives the number of $q+$ ions, $N_{j}$, and the ratio $R_{s}=H_{s} / N_{j}$ and, as neretofore, [Ref. 3].

$$
R_{s}=\frac{p_{e} L}{v_{i}} \int f\left(v_{r}\right) v_{r} \sigma d v_{r}
$$

where $\rho_{e}$ is the electron density, $L$ is the length of the interaction region, $v_{j}$ is the ion velocity, $v_{r}$ is the relative velocity, o is the DR cross section, and $f\left(v_{r}\right)$ is the $v_{r}$ distribution due to the velocity distribution of the electron beam.

Using the MEEM, we have measured $R_{s}$ over a wide range of $E_{1}\left(0<E_{r}<25 \mathrm{eV}\right)$ for the Li-. like ion, $\mathrm{C}^{3+}$ and $0^{5+}$, and for the Na-like lons $\mathrm{p}^{4+}, \mathrm{s}^{5+}$, and $\mathrm{Cl}^{6+}$ having the resonant transition $2 s-2 p$ and $3 s-3 p$, respectively. According to theory, the DR cross se:tion of these ions (except $\mathrm{C}^{3+}$ ) has, as its most prominent feature, a very narrow pedk at $E_{s}$ and, if $f\left(v_{r}\right)$ were narrow, Eq. (1) predicts large values of $R_{s}$ only near $E_{r}=E_{s}$. Our measurements show non-zero values of $R_{s}$ over a wide range of $E_{r}$, peaking a little below $E_{s}$ and a highly asymmetric (higher yield towards lower $E_{r}$ ) shape. We belfeve that our electron beam has a broad energy (hence velocity) distribution which we are currently trying to determine from our data and the theoretical values of $\sigma$. 
1. Department of Physics, University of Delaware, ilewark, DE 19711.

2. P. F. Dittner et al., Phys. Rev. Lett. 51,31 (1983).

3. For the former experimental procedure, see pp. 188-192 in Physics Division Progress Report, ORKL-6004 (1983).

\section{CHARGE TRNISFER TO mUTICHAREED RECOIL IOHS} IN A PEWIING TRAPI
D. A. Churcii ${ }^{2}$
R. A. Kenefick ${ }^{2}$
H. S. Burns ${ }^{2}$
C. S. $0^{3}$
R. Holmes ${ }^{3}$
S. Huldt

\begin{tabular}{|c|}
\hline $\begin{array}{l}\text { S. Berry } \\
\text { M. Breinig } \\
\text { S. B. Elstons } \\
\text { J.-P. Rozet } \\
\text { I. A. Sellin } \\
\text { D. Taylor }\end{array}$ \\
\hline
\end{tabular}

Low-energy neon recoil ions with charges rangirg from $+2 e$ to $+8 e$ were produced by impact of 35-MeV Cl ions foil stripped to a mean charge near lle. The recoil ions (3e $<q<6 e$ ) were confined for times of 100 msec to several seconds in a .enning trap. Charge transfer from neon atoms to the fons reduced the stored ion number exponentially with storage time. Measurements of the stared ion number versus time, following de'lection of the fast lonizing bean, yielded storage time constants, which were measured at several pressures. Pressures were determined with a calibrated nude ion gauge, and with a quadrupole residual gas analyzer. Flow of gas through the vacuum system to the piunp required additiunal calibration of pressure gradients. Using the target gas density obtained from the pressure measurements, and the storage time constant, rate coefficients for low energy electron transfer were calculated for each neon charge state. The mean energies of the ions were estimated by reducing the confining axial dc potential of the Penning trap. and observing the fon number stored versus trap depth. It was found that, above a certain depth, the number of stored ions maximized. Below this depth, the fon number for a fixed storage time was reduced due to loss from the trap of the highest energy ions. From the measurement, a mean ion collifsion energy of $2 q \mathrm{eV}$ was obtained.

Work continued on charge transfer from water vapor to the neon ion charge state. The chofce of water vapor as a target arose from theoretical predictions about the charge and temperature dependence of the rate coefficient, plus the ubiquitous presence of water vapor in most vacuum systems. The principal difficulty with these measurements, as it was for neon, lies in the target density determination. Due to the polar nature of the $\mathrm{H}_{2} \mathrm{O}$ molecule, the surface attracting force, plus dissociation of the molecule on the surfaces, can lead to errors in partial pressure determinations. Final pressure calibration measurements based on known singlycharged fon rate coefficients are now underway. of course, since the measurements must be made in target gas mixtures, the neon ion rate co- efficients are required to obtain the final results.

Following the successful demonstration of recoil ion storage in the Penning trap, work has been initiated on the use of a radio-frequency trap. This trap docs not utilize a manetic field, but the low measured energy of the recolls in the Penning trap implies that the magnetic field, used for strong racial ion confinement, is not essential. The absence of a magnetic field has two important implications: higher precision in spectroscopic measurements can be obtained, and the experimental configuration is open to permit easy introduction of atomic or laser beams. Of course, the operation of the rf trap is more involved, and the device is not a replacement of the Penning trap for collision measurements. Following initial tests using electron impact ion production, the $r f$ trap has been mounted on the bean line in series with the Penning trap, so that simultaneous operation is feasible in principle. It is expected that the $m / q$ resolution of the Penning trap is superior, so this can be used to identify ions in cases of uncertainty in the rf crap.

1. D. A. Church et al., Phys. Rev. Lett. 51 , 636 (1983).

2. Department of Physics, Texas Agy

University, College Station, TX 17943.

3. Department of Physics, Unfversity of Tennessee, Knoxville, TN 37996.

4. Department of Physics, University of Lund, Lund, Sweden.

5. Adjunct staff member from the University of Tennessee, Knoxville. TN 37996.

THE EFFECT OF TRAMSVERSE CORRELATIONS IH ION-CHANELLING IN VERY THIR CRYSTALS: EXPERIMENTAL ND THEORETICAL RESULTS
H. F. Krause
S. Datz
P. F. Dittner
P. D. Miller
1. Comez del Campo
C. D. Moak
N. Neskovi $\bar{c}^{1}$
P. L. Pepmiller

Considerable effort has been directed toward a detafled understanding of the transmitted angular distribution of fons channeled along the axial directions of single crystals. ${ }^{2}$ When the profectile energy is low and/or when the crystal is thick enough to cause significant multfplescattering effects, the transitted beam becomes concentrated along the chosen axis with lower intensity arms extending radially outward along the principal planar directions. Under these condftions, the characteristic star pattern observed is well understood, and it provides a useful aid in deterinining the crystal orientation in channeling experiments. For a very thin crystal and a swift projectile, the pactern of transmitted particles is somewhat more complex and not so well understood. Under these conditions, the projectile scatters as if tt has undergone a single collision with the strings of 
atons adjacent to the axial channel. Previous experimental single-collision investigations of the axial channeling pattern have been directed toward understanding the angular distributions produced wen the projectile direction deviates from the crystal's axial direction. Systenatic singl=-collision studies have not been performed when the projectile and axial directions are aligned. We have performed such studies in an attemt to observe theoretically predicted rainbow scattering effects ${ }^{3}$ in single-coilision channeling patterns. Dur results, consisting of noth measured and theoretical angular distributions, suggest that the predicted rainbow efie occurs in channeling.

Swift protons (3-7 MeV) produced by the EM tandem Van de Graaff accelerator were narrowly collimated (angular divergence $<0.012^{\circ}$ Fwhi) before illuinating a sall portion of a very thin silicon single crystal. The crystal thickness was $1400 k$ in the $\langle 100\rangle$ direction: this thickness corresponds to an atomic string length of about 520 atoms along the $\langle 110\rangle$ axis. AxialIy channeled particles that emerged from the crystal were jetected by a two-dimensional po: * tion sensitive proportional counter. ${ }^{4}$ The overall detector angular resolution measured in the horizontal and vertical laboratory directions was $0.012^{\circ}$ and $0.008^{\circ}$ (FWHM), respectively. Detector spatial resolution and the angular extent of the illuminated target as viewed from the detector contributed about equally to the overall angular resolution.

The experimental 20 angular distribution for 7-MeV protons channeled in the (110) axtal direction of silicon is shown in Fig. 4.17. Particle intensity cuts through the distribution at several levels (percentage of the peak count) show that the particle intensity decreases monotonically and very rapidly from that in the undeflected bean direction. Fig. 4.17 al so shows the angular locations of the last atom in each atomic string adjacent to the channel as viewed from the center of the axial channel at a longitudinal location halfway through the crystal (i.e., the location of the center-of-mass for each atomic string). The narrow [100] and wide [111] planar channels that intersect at the $\langle 110\rangle$ axis are also shown. Under our experimental conditions, we see that (a) arms in the angular distribution appear to point toward the nearest atomic strings (evidence of a transverse correlation effect) and (b) the scattered protons avoid the wide [111] planar channels. It is clear that 7-MeV protons do not undergo significant multiple scattering in such a short channel length. Multiply scattered projectiles would statistically fill availabl: phase space in planar channels, giving rise to a large number of trajec-tories in the [111] planar directions and a comparatively smaller number of trajectories in the $[100]$ planar directions. More importantly, we show that the binary collision regime was reached to good approximation at $7 \mathrm{MeV}$ by comparing the measured angular distri. bution (Fig. 4.17) to one obtained in a binary collision Monte Carlo trajectory calculation shown in Fig. 4.18.

In the Monte Carlo simulation, each profectile entered the axial channel at a randomily

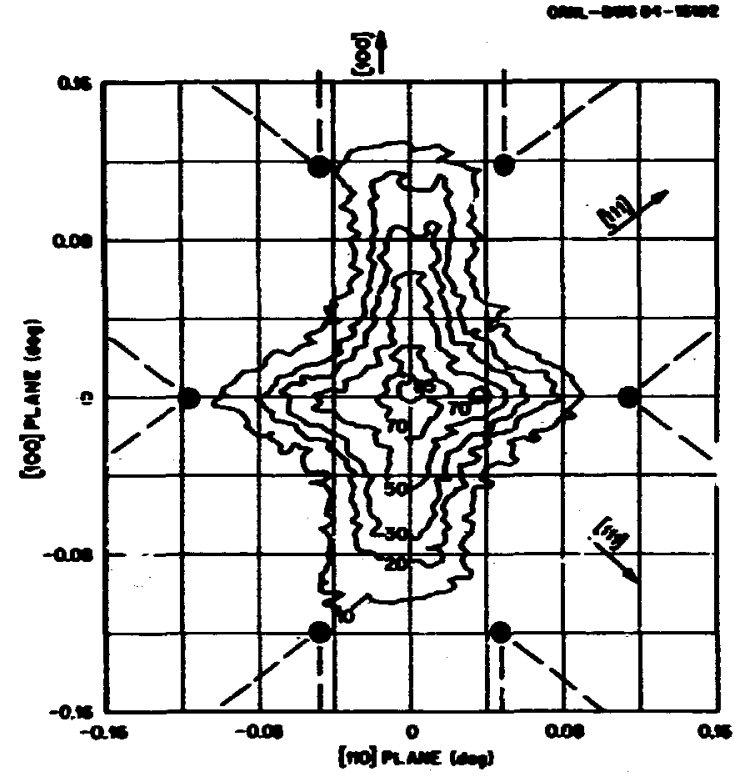

Fig. 4.17. Measured angular distribution for 7-MeV nrotons axially channeled along the 〈IIO) axis of a silicon crystal (thickness $=2000 \mathrm{~A}$ ). Particle intensity contours at 10, 20, 30, 50, 70 , and 85 percent of the peak intensity are show. The transverse location of atoaic strings and the [100], [111] planar channeling directions are identified (see text).

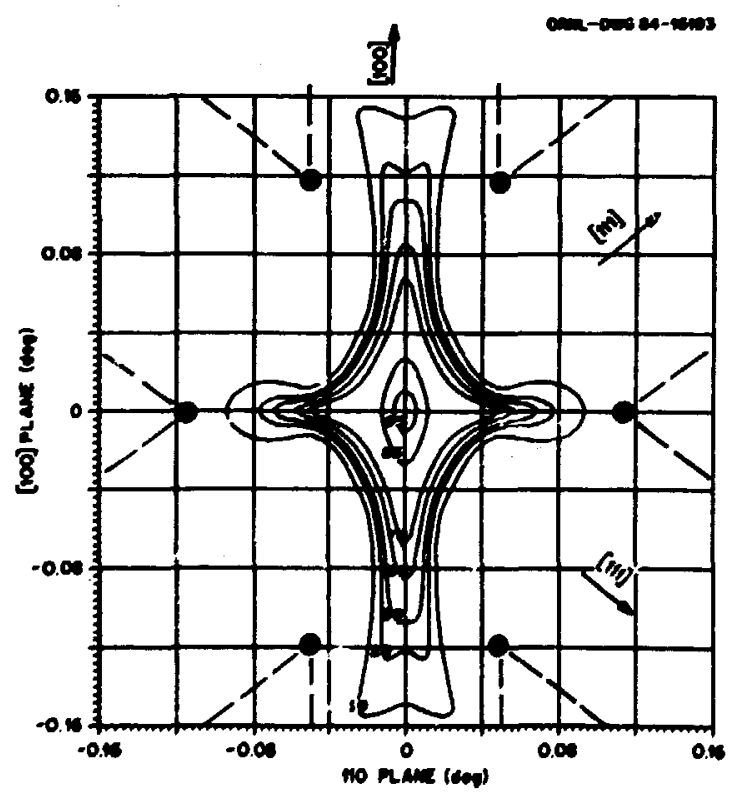

Fig. 4,18, Calculated angular distribution for 7-MeV protons axially channeled along the <110) axis of a silicon crystal (thickness. 2000 A). Particle intensity contours at 10, 20, $30,50,70,85$, and 95 percent of the peak intensity are shown. The transverse location of atomic strings and the [100], [111] planar channeling directions are identified. 
selected location traveling parallel to the axial direction (no beam divergence). The ionic projectile inter-acted with each atonic string adjacent to the channel through a transverse electric field that was calculated using the Linthard potential.5 The Lindhard potential constant used $(C=4,00)$ gives rise to a trassverse electric field that is in exce!'ent agreement with that given by the Holier. potential over the impact paraneter range of interest

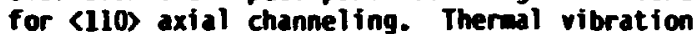
effects were simulated. The transverse monentu imparted to the projectile in a binary collision with each string was assumed to occur at the string's center-of-mass. The net transverse momentum imparted to the projectile from all strings determined the overall deflection. The deflection of each particle in the horizorital and vertical di rections were graded using a bin size $\left(0.002^{\circ}\right)$ wuch smaller than the measured experfimental resolution, and these results were later averaged over the experimentally deterained angular resolution functions (see fig. 4.18).

The measured angular distribution for 7-MeV protons (Fig. 4.17) is in excell lent agreement with the binary collision simulation calculated at the same energy (Fig. 4.18). The unaveraged Monte Carlo results (angular resolution = $0.002^{\circ}$ ) shoved a well resolved ridge (maximm) whose shape is similar to the $20 \%$ contour shown in Fig. 4.18 and a ninimum halfway between the ridge and the unde-flected bear position (height about $50 \%$ of the ridge height in a vertical cut across the [110] planar direction). The ridge has been shown analytically to be the consequence of rainbow scattering within the crystal. ${ }^{3}$ From the similarity of measurements and the averaged Monte Carlo results, we conclude that the experimental angular resolution was insufficient to resolve the predicted rainbow scattering ridge. An angular distribution measurement at $7 \mathrm{MeV}$ in the $\langle 100\rangle$ axial channel (not shown) appears to conftrm this conclusion. Here, the averaged Monte Carlo calculation indicated that four maxima should be observed at about $0.03^{\circ}$ in the difection of the closest atomic strings (different from 〈110〉 axial symmetry). Two maxima in the correct location were observed. Slight misalignment of the crystal and the beam in one direction is probably the reason for incomplete resolution of the peaks in the $\langle 100\rangle$ rainbow ridge.

1. Borts Kidrić Institute of Huclear

Sciences, Belgrade, Yugoslavia.

2. D. S. Gemmel, Rev. Mod, Phys, 48, 129 (1984).

3. N. Heskovíc pp. 203-206 in Physics

Diviston Progress Report, ORNL-6004 (1983).

4. R. H. Feartck et al., summary of paper to be published.

5. J. Lindhard, Mat. Fys, Medd, Dan. Vid. Selsk., 34, No. 14 (1965).
AMNLOUS FEM-FREE PATHS OF COMYOY EIECTROMS PROOUCED BY 25 a.u. HIGILY STRIPPED HI IONS IN C ND AI TAREETS
C. Bottcher
R. Latz $z^{2}$
D. Hof $\operatorname{annn}^{2}$
4. Burkhard ${ }^{2}$
H. J. Frischkorn ${ }^{2}$
P. Koschar ${ }^{2}$
S. D. Berry ${ }^{3}$
R.-0. Groeneveld ${ }^{2}$
H. Breinig
I. A. Sellin'

Considerable evidence has accumulated over the past decade suggesting that unbound electrons associated with the passage of fast ions through foils y be correlated with their parent ions over distances an order of agnitude larger than the wean free path for free electrons of the same velocity.

We have measured the yields of convoy electrons associated with $\mathrm{Mi}^{24+}$ and $\mathrm{Mi}^{26+}$ traversing polycrystalline r. and Al foils of thickness $3-500 \mathrm{\mu g} / \mathrm{cm}^{2}$ at inergies of $15.2 \mathrm{MeV} / \mathrm{anu}$. These foils are thin enough that mean free paths of a few hundred $A$ may be inferred from a growth curve, such as that shown in Fig. 4.19. The mean free path for convoy electrons is $2000 \pm$ 200 in contrast to the free electron value of $\sim 100 \wedge .1$

We have sought to explain this effect in terms of Couionb focusing. As the electron moves through the foll, it is subjected to repeated scatterings, but between scatterings it is pulled back towards the parent ion by Coulomb attraction. A very simple model of the competition between scattering and foccusing suggests that for ionic velocities $\mathrm{z}_{\mathrm{q}} \mathrm{l} / 2$ Bohr velocities, an enhancement of the mean free path by a factor of $\sim 10$ is $r$ usonable.

It is possfble to write down a Boltzmann equation wich describes the random walk of the electron under repeated scatterings in the presence of a Coulomb field. If this equation can be solved numerically without too drastic

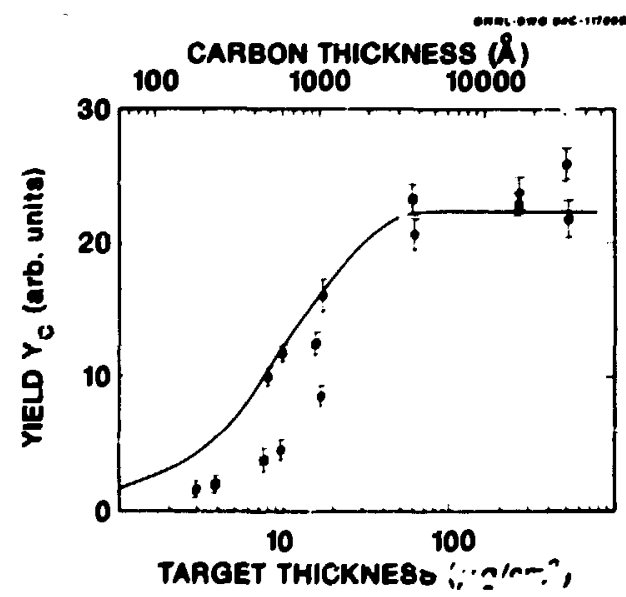

Fig. 4.19. Convoy electron yield versus tar get thickness. The open ctrcles and squares refer to $\mathrm{N1}^{24+}$ ions at $15.2 \mathrm{MeV} / \mathrm{amu}$ in $\mathrm{Al}$ and $\mathrm{C}$ fotls, and the closed circles to $\mathrm{NI}^{26+}$ in $\mathrm{Al}$. The full line is a fit to the data of the form: $1-\exp (-x / \lambda)$. 
approximation, it my be possible to put the heuristic model on a firm footing.

1. Sumary of paper submitted to the Symposiun on the Physics of Electron Ejection in Ion-Atom and Ion-Solid Interactions held at the Institute of Physics, University of Aarhus in Denmark, June 29-30, 1984.

2. Universität Frankfurt $M, 06$ Frankfurt an Main. Federal Republic of Cermany.

3. Department of Physics, University of Tennessee, Knoxville, IH 37996.

4. Mdjunct staff rember fran the University of Tennessee, Knoxvilie, i 37996.

THE MECATIVE IOW SOHRCE IEST FACILITY AS A MEGATIVE ION ATOHIC PAYSICS RESEARCH FACILITY

$$
\text { G. D. Alton T. J. Krale }
$$

We have made major modifications to the Hegative Ion Source Test Facility ${ }^{2}$ during the fiscal year in order to perform neqative ion source research and the proposed $\mathrm{Be}^{-}$and $\mathrm{Ca}^{-}$experiments. The Negative Ion Source Test Facility, shown schematically in Fig. 4.20, is a miltiplepurpose facility with emphasis placed on extending our knowledge of methods and mechanisms involved in the generation of negative fons and of the structure and properties of these ions.

Figure 4.20 illustrates the basic components which make up the facility. The facility can be operated for the generation and acceleration of

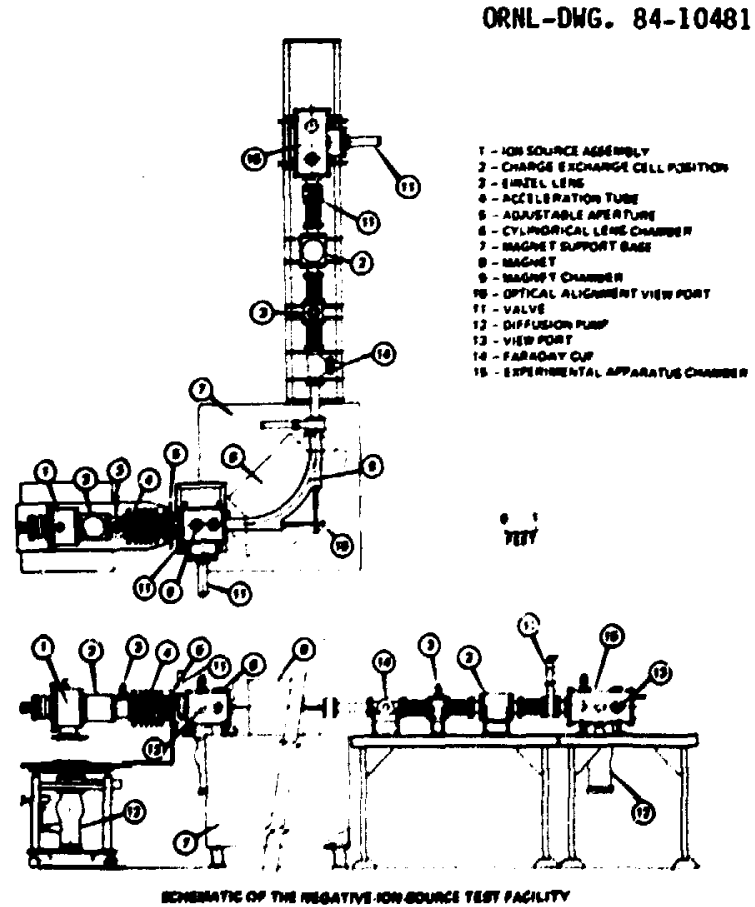

Fig. 4.20. The modified negative ion source test facility. positive ions wich can be double chargeexchanged to produce negative ions prior to or following momentun analysis. In the former case, positive ions produced in a hollow cathode positive source are accelerated to znergies up to 30 keV for singly charged particles. These are then focused through a recirculating Li charge-exchange cell where they are converted to negative ions through double charge exchange. Negative ions, thus created, are post-accelererated by the accelerator tube assembly and injected into the analyzing monet at energies up to 150 keV. Post-momentu analysis chargeexchange can also be effected by reversing the polarities of the ion source housing and magnet, and focusing the analyzed positive fon bean through the Li charge-exchange cell situated inmediately before the experimental chuber. In this way, positive ion beam energies up to 150 keV for singly charged particles can be obtained. The experimental arrangenent should permit observation of very short-lived negative ion states.

The facility can also be equipped with direct extraction-type negative ion sources for producing extensive numbers of high-intensity negative ion beans. Specifically, we have fabricated, installed, and tested a universal charge-exchange negative ion source which is presently being used to cenerate positive and negative ions. 3 The charge-exchange source consists of a positive ion hollow cathode source, ion extraction electrode system, einzel lens, and recirculating type $L i$ charge-exchange ceil. Hegative ions can be formed in either of two cells located prior to or following agnetic analysis. The post-analysis charge-exchange cell is located in close proxinity to the experimental chamber so that we can study metastably bound negative ions with lifetimes as short as a few tens of nanoseconds.

In addition to the previously described modifications to the facility, we have converted the magnetic mass analysis system so that circularly symmetric beams can be formed at the plane of analysis. (This modification was necessary in order to optimally tiansport the fon bean to our experimental chamber.) We have, as well, fabricated and installed beam transport components (lenses, magnetic steerers, valves, drift tubes, and apertures), a postanalysis charge-exchange cell and an experimental chamber which houses a specially designed electrostatic deflection system, Faraday cups, and electrostatic energy analyzer for performing the intended research.

In addition to the rather extensive changes in the Negative Ion Source Test Facility, we have converted our data acquisition system from analog to digital. The data acquisition system, shown schematically in Fig. 4.21, centers around a LeCroy 3500c CAMAC based data acquistition and control microcomputer and/or mult fchannel analyzer system. The system has avaflable $64 \mathrm{kB}$ of RAM and $30 \mathrm{kB}$ of PROM fimmare for data acquisttion, manipulation, and experimental apparatus control. The data acquistition time for accumu. lation in a particular channel is determined by monitoring the transmitted fon beam in the Faraday cup after the electrostatic analyzer and 


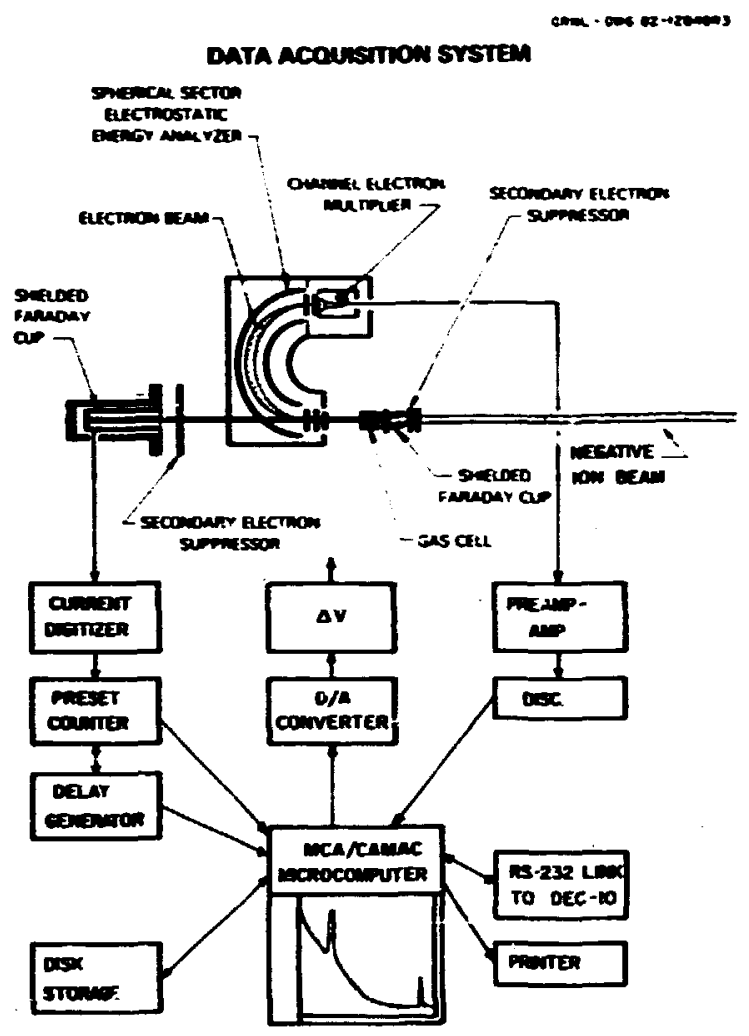

Fig. 4:21. A schematic diagram of the data acquisition system for measuring auto- and collisional-detachment properties of negative ions.

digitizing the analog signal which is fed into a preset scaler. Aftior accumulation of a predetermined amount of ciarge, a signal from the preset scaler is used to inhibit further data acquisition, to store acquired data, and to advance a multichannel scaling unit to the next channel where the prucess is repeated. Stgnals derived from the CAMAC based multichannel scaling unit are used to advance a locally constructed external DAC which drives a bipolar operational amplifier used to change the voltage on the electrostatic energy analyzer. The electron signal, which is transmitted through the spectrometer, is detected with a conventional channel electron multiplier/ampliffer circuit and fed into a CAMAC based $A D C$ where tit is stored in the memory of the multichannel analyzer. A typical electron energy spectrum ootained by this method is shown in this section of this report.

1. Postdoctoral Fellowship through Oak Ridge Associated Universities with the Physics and Health and Safety Research Divistons, ORNL.

2. G. D. Alton et al.. D. 192 in Physics Division Progress Report, ORNL-5025 (1974).

3. G. D. Alton et al.. D. 14 in Physics Divtsion Progress Report, ORHL-6004 (1983).
PROCRESS TOWARO MEASUREIENT OF THE PROPERTIES OF METASTABLY BOUWD REEATIYE IOWS

\section{G. O. Alton T. J. Rvale R. H. Compton 2 D. J. Pegg ${ }^{3}$ J. S. Thompson}

The present report deals with progress made toward measurement of the auto- and cullisionaldetachment properties of negative ions of the Group IIA elements (Be- and $\mathrm{Ca}^{-}$) using electron spectroscopic techniques. The experimental techniques and procedures utilized in the present investigations are siniliar in manj respects to thuse cescribed in previous reports concerning $\mathrm{H}^{-}$and $\mathrm{He}-$ negative ions. 5,6 However, the present experiments are being performed at the llegative Ion Source Test facility 78 mich has been extensively modified in order to generate negative ions through charge exchange and perform ion source related experiments as well as interesting negative ion atomic physics experiments. In addition to the rather extensive changes in the llegative Ion Source Test Facility, we have converted our data acquisition system from analog to digital. The data acquisition systen centers around a LeCroy 3500 C CAMAC based data acquisition and control microcomputer and/or mult ichannel analyzer system. The mortified facility, thus, is a multiple purpose facility wich can be used to extend our knowledge of methods and mechanisms involved in the generation of negative ions, as well as to study their interesting atomic structure properties. The reader is referred to the description given in this section of this report for more details on the facility.

A schematic drawing of the experimental apparatus is shown in Fig. 4.22. The postanalysis charge-exchange cell is located in close proximity to the experimental chamber so that we can study metastably bound negative ions with lifetimes as short as a few tens of nanoseconds. The negative, positive, and neutral components which leave the charge-exchange cell are separated by a computatiunally designed deflection system. After electrostatic deflection through $10^{\circ}$, the negative and positive beams are monitored in shielded and biased

and is ane

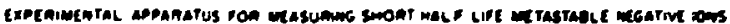
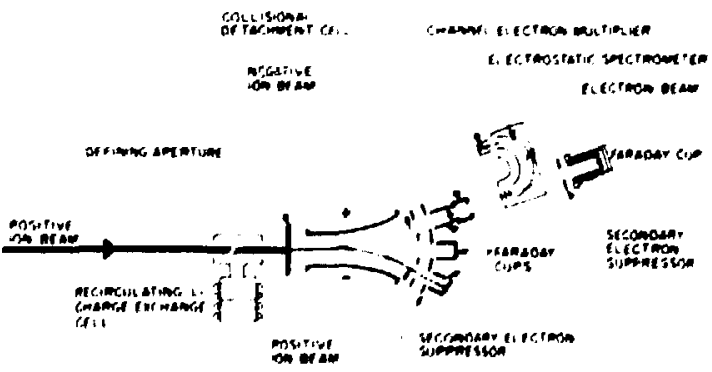

Fig. 4.22. Schematic drawing of the experimental arrangement for performing electron spectroscopic measurements of short half-life metastable negative ions. 
Faraday cups. A portion of the negative ion beaw is al lowed to pass through a small aperture located in the center of the first Faraday cup and enters a gas cell mich is used to collimate the bean and produce collisionally detached electrons wich are used to calibrate the energy scale of our aeasurements. The ion bean then passes through a spherical sector electrostatic andiyzer and is monitored in the rear Faraday cup. Detached electrons accomanying the ion bean are energy analyzed with the spherical sector electrostatic energy analyzer in the fixed-pass energy wode. In this mode of cperation, electrons moving slower than the pass eneriy of the spectrometer are accelerated, wile those woving faster than this pass energy are decelerated. In this wy, our spectroneter resolution function reasins approximately constant.

An energy spectru resulting from the autoand collisional-detachment of He- wich was produced by doubly charge exchange using the previously described experimental apparatus and the digital data acquisition system is show in Fic. 4.23. Our present efforts are concentrated on measurements of the production efficiencies as a prelude to performing spectroscopic measurements similiar to those described previously for $\mathrm{He}^{-}$wich are designed to ident ify energy levels of the auto-detaching states of Ca-. Calciua was chosen over beryllium as the element to study first because of the ease of generation of $\mathrm{Ca}^{+}$beams relative to those of $\mathrm{se}^{t}$. Experience with this ion will enable iaprovements to

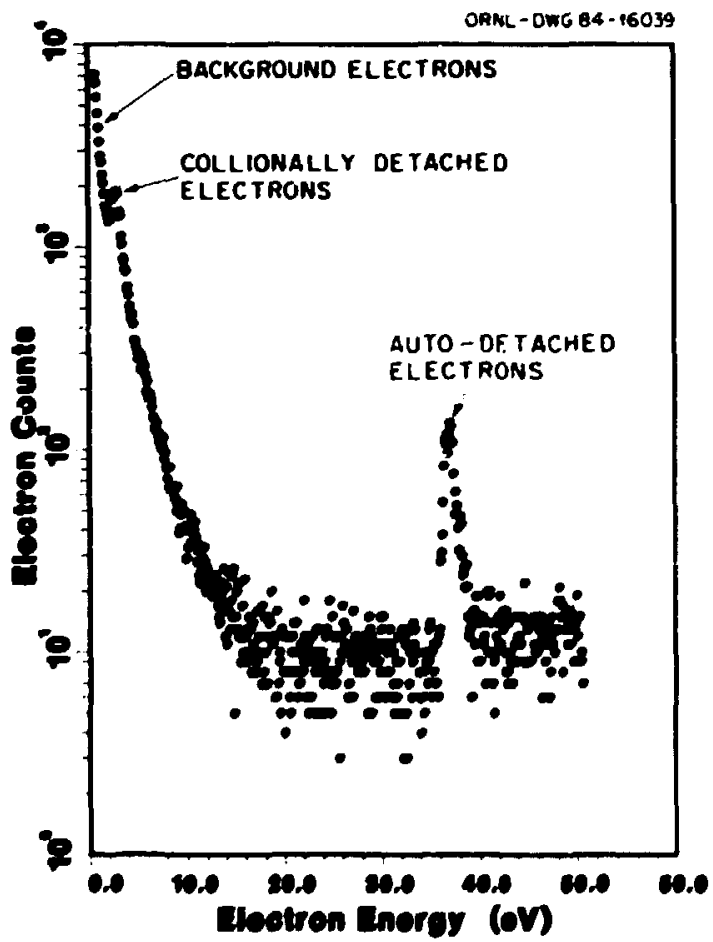

Fig. 4.23. Electron energy spectrum for metastable $\mathrm{He}$ - interaction with Ar. be made in the hollow-cathode source before studying Be-. (Beryllium is highly toxic and will require special handling during periods of source a intenance.) In fact, during preliminary studies of the $\mathrm{Ca}^{-}$ion, the hollow cathode source proved to be very unreliable with many internal electrical connections, filament, and oven related probleas. Substantial progress has been made in reducing the number of failures and, therefore, significantiy improving the reliability of the source. Extensive modifications to the ionization chamber and oven were required wich has led to a core reliable source. These modifications will be the subject of a forthcoming report.

Calculations by Bunge et al.9 predict that the Ca- ion exists in metastable $[A r]\left(454 p^{2}\right)^{4} p$ states mich are bound by $-0.55 \mathrm{eV}$ with respect to the metastable (4s4p) ${ }^{3} p^{0}$ state of the ca atom. The $J=5 / 2$ level of this state should, for example, autodetach via a forbidden process driven by magnetic interactions between the electrons in a manner analogous to the decay of the metastable quartet $J=5 / 2$ state of $\mathrm{He}^{-}$. The energy of these autodetached electrons should be $1.34 \mathrm{el}$ relative to the rest frame of the woving ion based on an electron affinity value of $0.55 \mathrm{eV}$. The Iffetimes of the three levels associated with the $\left(4 \$ 4 p^{2}\right)^{4} p$ state have not, as yet, been calculated. One can expect a differential metastability anong the levels as in the case of $\mathrm{He}^{-}$due to the different strengths of their couplings with doublets of the same configuration. In $\mathrm{He}^{-}$, the lifetimes are in the approximate range of $10^{-5}$ to $10^{-3}$ sec. For a heavier ion such as $\mathrm{Ca}^{-}$, one might expect stronger agnetic interactions between electrons and wich aight lead to a breakdown of LS coupling resulting in somewhat shorter lifetimes than in a light ion such as $\mathrm{He}^{-}$. This being the case, our present experimental arrangewent should permit observation of such short-lifed decaying states. For extremely long-lived states, however, another procedure may be necessary to determine the energy levels of $\mathrm{Ca}^{-}$. such as using laser photodetachnent techniques, or extending the decay length at our experimental chamber.

He have recently obtained prelifinary results on $\mathrm{Ca}^{-}$ions in the energy range from $60-120 \mathrm{keV}$ produced by sequential charge-exchange interactlons with Li vapor. At $120 \mathrm{keV}$, the laboratory frame energies at the collisional - and autodetachment peaks in $\mathrm{Ca}^{-}$are $1.6 \mathrm{eV}$ and $6.0 \mathrm{eV}$, respectively. We are cont inuing our work on Caby investigating the production process, methods of reducing the background, and the effects of stray magnetic fields on the intensity and resolution of low-energy electron peaks. The collifional - and auto-detachment spectroscopic portion of this work is expected to be completed in the near future, at which time we will direct our attention toward measurements of similtar properties of the Be- negative ion.

1. Postdoctoral Fellowship through Dak Ridge Associated Untversities with the Physics and Health and Safety Research Divistions, DRIIL. 
2. Health and Safety Research Division, ORRi.

3. Mdjunct staff memer fron tie University of Tennessee, Knoxville, Th 37996.

4. Graduate Student from the University of Temessee, Knoxville, In 37996.

5. G. D. Alton, R. H. Compton, and D. J. Peg9. Phys. Rer. A28, 1405 (1983).

6. G. D. Alton, R. H. Compton, and D. J. Peg9, sumary of paper subaitted to Physical Review A.

7. G. D. Alton et al..,p. 192 in Physics Division Progress Report, ORil-5025 (1974).

8. G. D. Alton et al.. p. 14 in Physics Division Progress Report, ORin-6004 (1983).

9. C. F. Bunge et al.. Mucl. Instr. Meth. 202, 299 (1982).

\section{SHAPE OF THE ELECTRON CAPTURE TO THE COMTImUN CUSPS FOR $\mathrm{H}_{3} \mathrm{H}_{2}$, MOM the TAREETS IN THE VELOCITY RAME 6.3 TO 18.0 a.U.}
S. D. Berry
G. A. Glass ${ }^{3}$
I. A. Sellint
K. -0 . Groeneveld 5
D. Hofmann 5
L. H. Andersen 6
S. B. Elston
P. Engar
H. Stolterfoht?
H. Schaidt-Böcking'
G. Nolte
G. Schivietz?
H. Breinig

The velocity spectrun of electrons ejected into the forward direction in energetic ion-atom collisions exhibits a cusp-shaped peak when the emergent electron velocity $\vec{v}_{e}$ matches that of the outgoing ion velocity $\vec{v}_{p}$ in both speed and direction. For projectile ions with few electrons, the electrons predominately arise from transfer of a target electron to a low-lying projectile-centered cont inuum state (ECC). whereas if there are many projectile electror,s, those electrons can be lost to similar low-lying continuum stat.es. This process is termed electron loss to the cont inuum (ELC). For several years, there has been much interest in both the experimental and theoretical aspects of this problem; $;, 9$ we are concerned here with only ECC.

In early theories, a first-order perturbative treatment of the charge-exchange anplitude was used to explain the ECC cusp, with the final electronic state described in terms cf a simple Coulomb wave centered on the projectile, leading to a cusp nearly symmetric in $\left(\vec{v}_{e}-\vec{v}_{p}\right)$. However, later experimental results for both heavy and light bare projectfles showed a skewness of the ECC peak shape toward lower electron velocities.

To account for this asymetry, the distortion of the outgoing electron by the interaction between it and the residual ton must be considered, but in a way which goes far beyond a simple postcollision interaction description. In 1978, Shakeshaft and Spruch (SS) included a second-order term in the Born expansion 10 to approximate this effect upon the E.CC shape while Chan and Eichler (CE) proposed that slightly extending the first Born approximation could account for the cusp skewness. II subsequent experiments 2,13 showed an asymmetry more simt.
Iar to that predicted by SS than that by CE, but the strong dependence of the asymmetry upon projectile $Z$ and velocity of the SS theory was not found. More recently, Jakubassa-Amundsen ${ }^{14}$ has calculated the shape of the ECC Cusp within the semiclassical impact approximation (SCIA) for the case of argon projectiles and helium targets; the resulting shape compares well with experiment.

Here, we present a sumary of results, obtained from accelerator runs at Brookhaven National Laboratory (BRL), Lawrence Berkeley Laboratory (LBL), Hahn-Heitner-Instutut (HI), and Oak Ridge Wtional Laboratory (ORHL), contained in our paper recently accepted for publication. 15 By using a general, model independent method of describing the cusp shape that explicitly takes into account the ef fect of a given experimental choice of analyzer geometry, resolution, and angular acceptance, we can coapare spectra from different experiments directly and extract the basic underlying shape of the ECC cusp.

To accurately describe that shape, the cross section was expanded over two of the projectile frame variables $v_{e}^{*}=\left|\vec{v}_{e}-\vec{v}_{p}\right|$ and $\theta_{e}$ (angle ith respect to the projectile direction). This expansion is then transformed to the laboratory frame and integrated over the velocity and angular acceptance of the spectrometer to yield:

$$
\begin{aligned}
Q\left(v_{e}, \theta_{e}\right)= & c \sum_{n, l} B_{n, l}\left(v_{p}\right) \times \int_{v_{e} Q_{e}}\left(v_{e}\right)^{2}\left(v_{e}^{\prime}\right)^{n-1} \\
& P_{l}\left(\cos \theta_{e}^{\prime}\right) s\left(v_{e}, Q_{e}\right) d v_{e}^{d Q} e^{d}
\end{aligned}
$$

Here $Q$ is the measured spectral function, $C$ is an overall amplitude, $B_{n, l}$ are the coefficients for each term. $P_{l}$ are Legendre polynomials, and $S$ is the function describing the acceptance of the particular spectrometer arrangement used.

By making some reasonable assumptions as to the functional form of $S$, we are able to perform the integration above and thereby make direct comparisons with the experimental data using standard fitting techniques. The results of these comparisons are values of the coefficients $B_{n}$, which, to the degree the assumptions in making this expansion are valid, are independent of the experimental arrangement. The term with $n=l=0$ is that commonly known as the "Dettmann" cusp. 16

A summary of the fit results is presented in Table II of Ref. 15, and a complete discussion of the results appears there. Here, we briefly review those conclusions.

The most striking feature of the fit results is the consistency of the value of the $B_{01} \mathrm{co}$ efficient ( $n=0, l=1$ ), which is also the major source of the asymetry found in the cusp shape at these energies. The value of this coefficient, relative to the leading term $B_{00}$, remains constant at $\sim .45$ for projectile $Z_{p}$ values between 6 and 18, projectile velocities between 6 and $18 \mathrm{a} .1 \mathrm{~b}$, and target $z_{t}$ values of 1 and 2. As noted by Macek et al. 17 the presence of this term implies the necesstiy of second Born terms in any theoretical treatment 
of the problem, as in the approach of Slitieshaft and Spruch. 10 However, the SS theory has. strong dependence on $Z_{p}, v_{p}$, and $Z_{t}$ not found here. The results of Jakubassa-A undsen 14 are very promising, but we presently lack a detailed understanding of the dependerce on $Z_{p}, V_{p}$, and $Z_{t}$,redicted by her approach.

Also evident from the fit resuits is the very swall contribution of terus with $l=2$. This suggests, at least for the combinations of $Z_{p}$, $v_{p}$, and $Z_{t}$ used here, that the expansion may be Ifaited to the lowest four terms $n=0,1$ and $l=0_{2} 1$.

In addition, the data collected using atonic hydrogen targets suggests that the shape is substantially similar to that found for helium targets.

Finally, we reproduce here in Fig. 4.24 some representative fit results. The top half of the drawing contains comparisons of the ernansion of the equation above with the data, and the bottom half of the figure contains the component parts of the fit (sumed over $n=0,1$ ) for $l$ values of 0,1 , and 2 (termed $S, P$, and 0 , respectively).

In sumary, we hope that by analyzing our data in a model independent, apparatus indepen-

OAnL-DWG AR. ICWO

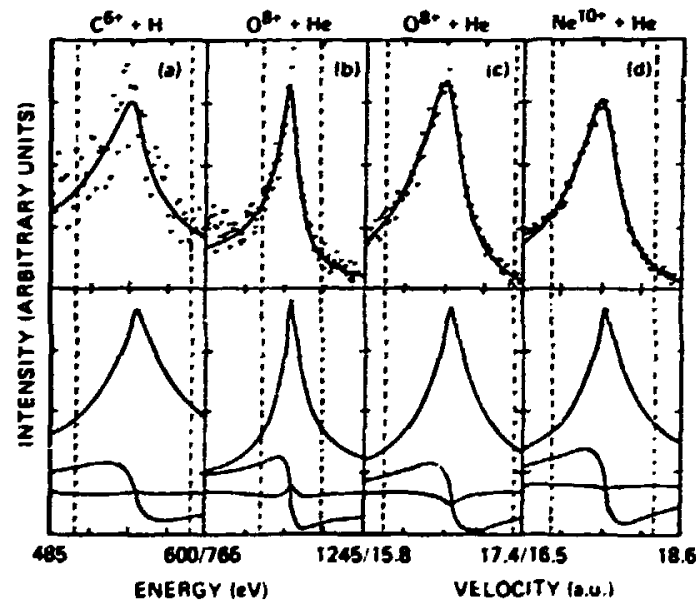

Fig. 4.24. The top row shows four comparisons between fitted spectra (solid lines) and data (dots) incorporating background subtraction where appropriate for: (a) 12-MeV bare carbon projectile, (b) 30-Mey bare oxygen projectiles. (c) 110-MeV bare oxygen projectiles, and (d) 155-MeV bare neon projectiles. The vertical dashed ifnes indicate equivalent arbitrary $(1 \pm .04) v_{p}$ limits for each of four spectra. The bottom row displays $S, P$, and $D$ components of the fitted function (sumed over both $n=0,1$ components) and the corresponding pitted function shown above. The botton spectra have been displayed with a s-functional line widtn, to remove the dependence of the cusp shape on the particular experfmental line width, allowing for a more direct comparison of the results. dent anner, we can provide insight into the ECC process at high velocities, and thereby stimulate further theoretical investigation.

1. Sumary of paper to be published in Physical Review A.

2. University of Tennessee, Knoxville, $T$ 37996.

3. Maval Surface Meapons, Silver Spring, w 20910.

4. Adjunct staff menber from the University of Tennessee, Inoxville, TH 37996.

5. Institut für Rernphysik, Universität Frankfurt, Nugust-Euler-Strasse, 06 Frankfurt an Min. Federal Republic of Cermeny.

6. Institute of Physics, Uaiversity of Aarhus, OK-8000, Aarhus C, Denmark.

7. Hahn-Heitner-Institut für Kernforschung, D 1000 Berlin 39. Federal Republic of Germany.

8. W. Meckbach and R. A. Baragiola, in Inelastic Ion-Source Collisions, edited by $M$. $M$. Tolk, J. C. Tully, W. Keiland, and C. M. White (Academic Press, Rew York, 1977) i, p. 283;

1. A. Sellin, J. Phys. (Paris) 40, Cl-255 (1979):

C. R. Yane, IEEE Trans. Mucl. Sci. IS-26, 1078 (1979) :

M. H. Lucas, Proceedings of the Workshop on Physics with fast nolecular-Ion beans. edited by D. S. Genell, Argonne Mational Laboratory Report Ho. AHL/PHY79-3, P. 291;

$V$. H. Ponce and $\mathrm{V}$. Meckbach, Coments At. Nol. Phys, 10, 231 (1981);

I. A. Sellin, Proceedings of the XII International Conference on the physics of Electron and Atomic Physics, edited by S. Datz

(hmsterdam, 1982):

K.-0. Groeneveld et al., Coments on Atamic and Molecular Phys. 14, 187 (1984).

9. M. Breinig et al., Phys. Rev. A25, 3015 (1982).

10. R. Shakeshaft and L. Spruch, Phys, Rev. Lett. 41, 1037 (I978).

1. F. T. Chan and J. Eichler, Phys. Rev. A20, 367 (1979); R. Shakeshaft and L. Spruch, ibid. 20, 376 (1979).

12. S. D. Berry et al.. IEEE Trans. Nucl. Scj. WS-30, 902 (1983).

13. M. Breinig et al., Phys. Rev. Lett. 45, $1689(1980)$.

14. D. H. Jakubassa-Anundsen, J. Phys. B16, $1767(1983)$

15. S. D. Berry et al., Phys, Rev. A (to be published.

16. K. Dettman, K. G. Harrison, and M. W. Lucas, J. Phys, BJ, 269 (1974).

17. M. H. Lucas et al., J. Phys. B13, 4833 $(1980)$;

J. Macek et al., Phys. Rev. Lett. 46, 1571 (1981).

\section{EN TANDEM OPERATIONS}

$$
\begin{aligned}
& \text { P. L. Pepmiller G. F. Mells } \\
& \text { P. D. Miller }
\end{aligned}
$$

The beginning of this report perfou found us in the midst of a major tank opening. During 
the sunuer of 1983, machine performance had degraded substantially so a decision was made to go in for jor maintenance. The acceleration colum was stripped of all nonessential pieces before doing a complete washdown with soap and water. After drying out, the graded resistor chain was reinstalled. Then we tried using a new belt aterial. Poly-C, instead of cur standard belt. This type of belt had been used previously in vertical machines with great success. It costs considerably less than the standard belt, does not produce dust, and can be bought as a long strip and assewbled in the machine. After installation and initial tensioning, we closed the machine and began voltage tests without tubes. He experienced tank sparks at voltages above $5 \mathrm{~mW}$, however. their frequency was no higher at $7.3 \mathrm{mr}$ than at $5 \mathrm{~m}$. After about 50 hours of voltage tests, we re-entered the achine for inspection and reinstallation of tubes. At this point, it was noticed that the Poly- $C$ belt had failed. There were multiple puncture holes through the belt, wich resulted in longitudinal splits. The causes of the spark holes are not clear at this time. Fortundely, we were able to borrow a standard belt from Kansas State University to replace ours. The tank opening was concluded with the installation of rebuilt tubes. The machine resumed normal operation in late December 1983. Since that time, it has logged over 1700 hours in support of basic atonic physics research. During the first five months of operation, there were numerous tank openings, mainly due to problems with belt guides. However, the generator has now oper3ted for four months without a failure. Major projects begun during this reporting period include installation of a new ion source, replacement of magnet power supplies, upgrading of various outdated or unreliable power supplies throughout the laboratory, and new operator's training guide and schedule.

\section{ATOMIC PHYSICS FOR FUSION FROGRAM}

\section{ECR MUTICHARGED ION SOURCE}

$$
\text { F. W. Meyer J. Johnsan W. Hale }
$$

Construction and installation of the Electron Cyclotron Resonance (ECR) multicharged ion source was completed on schedule early in FY 1984. Al though the ECR fon source was designed for operation at $10.6 \mathrm{GHz}$, initial source testing was carried out at $2.45 \mathrm{GHz}$ in order to gain experience in source operation while awating delivery of the $10.6 \mathrm{GHz}$ microwave anplifier scheduled for June 1984.

During the intilal testing phase at $2.45 \mathrm{GHz}$, $X$-ray measurements of the bremsistrahlung spectrum produced by the source provided evidence of ECR heating of electirons. A calibrated Si-Li $X-r$ ay detector indicated electron energies up to $160 \mathrm{keV}$. Following construction and installdtton of the beam line and charge analyzer in March 1984, source performance could be dssessed more directly by measuring charge state distributions of extracted beams. Even at the lower microwave frequency, fully stripped $C$ beans mere obtained, wich were used to initiate the first planned experiment involving the new source, total cross sections measurements of electron capture by fully stripped light ions incident on atomic hydrogen. Operation of the source at its design frequency of $10.6 \mathrm{CHz}$ started on schedule in sune 1984. Since that time efforts toward source optimization have been ongoing, in parallel with the above-mentioned cross section measurements.

The salient features of the ECR ion source, shown in Fig. 4.25, are sumarized in Table 4.4. The source consists of two stages, and is quite similar in size to MIMIMAFIOS. T The first stage, wich supplies plasma to the second stage to facilitate startup, is operated in overdense mode. 2 A helical slow-wave launcher ${ }^{3}$ is used to inject $2.45 \mathrm{GHz}$ microwaves into the first stage which is located in an axial magnetic field of 5-7 kG. PI asaa density is controlled by the microwave power level and gas pressure, which varies in the $10^{-3}-10^{-4}$ torr range. Since microwave absorption in the first stage is nonresonant, the magnetic field can be tuned to optimize second stage performance. The second stage is separated from the first stage by two stages of differential punping, which is sufficient to maintain ?ow $10^{-6}$ torr pressure in the second stage during source operation.

In the second stage, electrons confined in a minimum- 8 configuration are heated by resonant absorption of $10.6 \mathrm{GHz}$ microwaves that are injected radially immediately following the differential pumping section. The minianum-B structure is produced by a superposition of an axial mi rror field and a radial hexapole fieid. Three conventional water-cooled solenoids are used to establish the axial magnetic field. ${ }^{4}$ The hexapole field is produced by a compact assembly 4,5 of sincos permanent magnets positioned around the cylindrical vacuum wall of the second stage. Cooling of the permanent magnet assembly is achieved by water circulation through the voids created between the cylindrical vacuum wall and the duodecagon defined by the placement of the $\mathrm{SmCO}_{5}$ bars.

As regards the mechanical design, care was taken to ensure ease of assembly and access. The source divides frico three sections, each of which is separately supported by, and can ve rolled freely on, precision tracks. The three solenoidal field coils are supported by a sintlar track structure, and can be moved freely about to expose otherwise inaccessible source parts during source disassembly, or to change the axial mirror ratio in the secono stage wile the source is fillly assembled.

Ion extraction is accomplfshed by a threeelement extraction electrode, the first two elements of with can be biased independently for fon focussing and prevention of electron hackstreaming. The position of the anode and the extraction electrode, and the extraction gap itself can be varicd by the use of shims. An electrostatic unfootentifal lens operated in "accei" modef images the extracted beam onto the entrance slit of a stigmatic $90^{\circ}$ magnetic charge 
CRII. Photo 6434-84

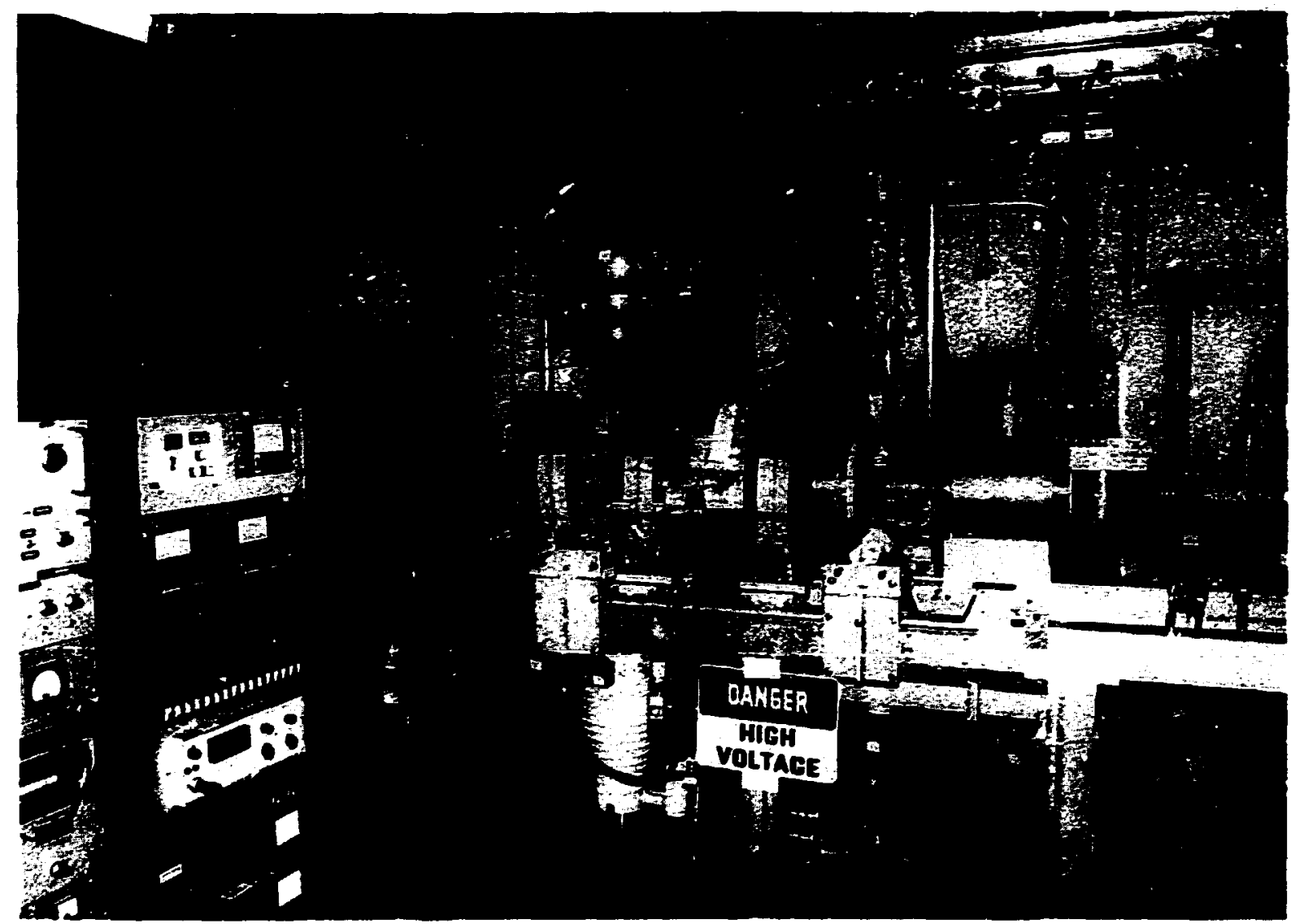

Fig. 4.25. The ORNL ECR mitficharged fon source.

analyzer having a $4 \mathrm{C}-\mathrm{cm}$ radius of curvature. The entrance sift assembly is located about $100 \mathrm{~cm}$ downstream of the source anode; object and image distances for the $90^{\circ}$ magnet are $80 \mathrm{~cm}$. Retractable Faraday cups located fmuediately after the entrance and exit slits are used to measure total extracted beam currents, and charge-selected beam intensfties, respectively. Both entrance and exft slit assemblies feature independently adjustable horizontal and vertical slit jaws, to which current can be measured, An electrostatic quadrupole lens located downstream of the exit slits can be used to transport the charge-selected beam to those experiments requiring maximum beam intensfties. Prior to telivery of the $10,6-\mathrm{GHz}$ microwave system, the ECR ion source was operated at an interim frequency of $2.45 \mathrm{GHz}$. In order to matntafn roughly the same ratio of hexapoleto-axtal magnetic field strength at the lower f requency, only 6 of the $12 \mathrm{SmCO}_{3}$ bars were used, reducing the strength of the hexapole by a factor of two. An additional $25 \%$ decrease in hexapole strength was obtained by shimining out the bars to thetr maximum radius determined by adequate clearance to the solenofdal field windings.
Operation of the source at the lower frequency was far from optimum, due matnly to poor coupling between the second stage plasma and the $2.45 \mathrm{GHz}$ microwaves. Due to their longer wavelerigth, only a few modes propagated in the second-stage cavity; microwave absorption seemed to occur preferentially on the microwave injection side of the second stage which is furthest removed from the extraction region. Even in this mode, the high-charge-state capability of the new source significantly exceeded that of the ORNL PIG ion source. Figure 4.26 shows a typical charge stage distribution for Ar source gas obtained at this frequency. For light ion production, heams up to fully stripped I $3 \mathrm{C}$ $\left(10^{-14} \mathrm{el}\right.$. A) and up to $\mathrm{H}-1 \mathrm{fke} 0\left(10^{-12} \mathrm{el}\right.$. A) were produced at the lower frequency.

Signiffcant improvement in source performance was obtafned, as expected, after installation of the $10.6 \mathrm{GHz}$ microwave system, both in terms of total extracted beam intensfty and mean charge state of the extracted beams. Mfcrowave absorp$t$ fon increased dramattcally, as evidenced by very low reflected power (typically less than 10\%) durfing source operat on. Dpt imum chargestate distributions were obtained for a secondstage $m$ irror ratio of about 1.6 , significantly 
Table 4.4 URML ECR source parameters

\section{Microwaves}

First stage $2.45 \mathrm{GHz}$ ( $300 \mathrm{Y} \max$ )

Second stage $10.6 \mathrm{GHz}$ (2.2 kH max)

$50-200 k$

$20-800 u$

Mgnetic Fields

Mirror ratio

Hexapole field at vacuum wall

Field in extraction plane

1.6

Field in first stage

Total solenoid power

$4.0 \mathrm{kG}$

$4.5 \mathrm{kG}$

$5-7 k 6$

$60 \mathrm{kH}$

Vacuum (operating condition)

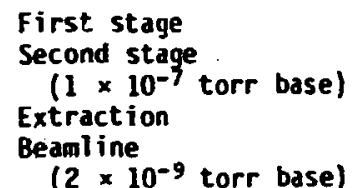

$10^{-3}-10^{-4} \mathrm{~T}$

$1-6 \times 10^{-6} \mathrm{~T}$

$1 \times 10^{-7} T$

$2 \times 10^{-8} T$

Dimensions

Solenoids ID

Solenoids $\mathrm{OD}$

$18 \mathrm{~cm}$

$40 \mathrm{~cm}$

Hexapole ID

$9.5 \mathrm{~cm}$

$33 \mathrm{~cm}$

$8.6 \mathrm{~cm}$

$0.5 \mathrm{~cm}$

$0.8 \mathrm{~cm}$

Anode aperture

Extraction aperture

$2.6 \mathrm{~cm}$ below the 2.1 mirror rutio attainable at maximum second-stage field coil separation. Figure 4.27 shows a measured charge state distribution obtained for As with the source operating at 10.6 GHz. As has been noted by other workers, 7,8 an admixture of $\mathrm{O}_{2}$ was found to significantly increase the Ar high-charge-state

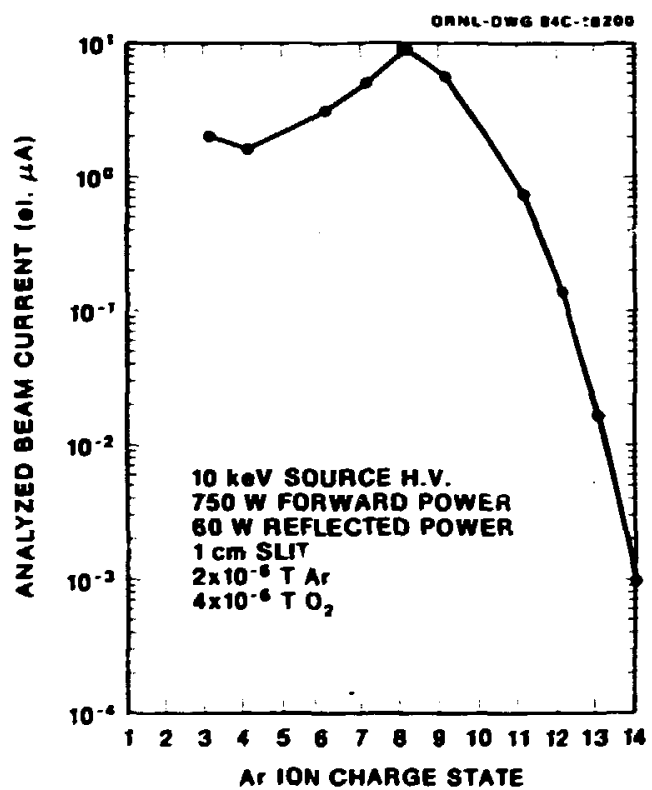

Fig. 4.27. Ar charge state distribution from ECR ion source - $10.6 \mathrm{GHz}$ source operation.

output of the source. Production of light ion beans has yet to be optimized. So far I I $\mathrm{C}$, I $\mathrm{SW}_{\mathrm{K}}$ $180,1 \%$, and $22 \%$ e beams have been obtained, with intensities varying from a few $\mu \mathrm{A}$ for charge states up to and including He-like, about 0.1-1 for H-like, and 1-50 nA for fully stripped ions.

1. R. Geller, B. Jacquot, and C. Jacquot, Proceedings of the 7 th Symposium (1983 Internationa I) on Jon Sources and Ion Assisted Technology - ISIAT 1983, p. 187, Kyoto, Japan.

2. G. Lisitano, $M$. Fontanesi, and $E$. Sindoni, Appl. Phys, Lett. 16, 122 (1970).

3. D. P. Grubb and T. Lovell, Rev. Sci. Inst. 49, 77 (1978).

4. Physics Divísion Progress Report, Dak Ridge National Laboratory report ORNL-6004, December 1983.

5. K. Halbach, Nucl. Inst. Meth, 169, 1 (1980).

6. Operation in "decel" mode is nr* posstble in the present application due to the favorable conditions for Penning discharge produced by the combination of ion source magnetic fringe field and lens electrostatic fíeld.
Fig. 4.26. Ar charge state distribution from ECR ion source - $2.45 \mathrm{GHz}$ source operation. 
7. H.-6, Hethers, ה. Euscher, R. Fiedler, and H. Krauss-Vogt, IKP - Annual Report 1983, KFA Jul ich.

8. D. J. Clark, Y. Jongen, and C. M. Lyneis, P. 133 in Proceedings of the 10th International Conference on Cyclotrans and heir tpplications, April 30-hay 3, 1984, East Lansing, MI, IEEE Conference Record 84CH1996-3 (1984).

\section{ELECTROM CAPTURE CROSS SECTION MEASUREMEMTS}
F. W. Heyer
C. C. Havener
A. M. Howald
R. A. Phaneuf

Heasurements of total electron capture cross sections for fully stripped and $H-l$ ike $C, N, 0$, $F$, and $\mathrm{ke}$ ions incident on $\mathrm{H}$ and $\mathrm{H}_{2}$ have veen performed in the energy range 0.2 to $10 \mathrm{keV} / \mathrm{amu}$ using ion beams produced by the new ECR aulticharged ion source. While straightforward experimentally, these measurements are of significant current interest both from a basic and applied scientific perspective, and also provide a convenient mechanism for exploring the range of operating parameters and capabilities of the new source.

The experiment enployed the ORML atonic hydrogen gas target, a directly heated tungsten tube in wich molecular hydrogen is thermaliy dissociated. A collimation section preceding the target limited the magnetically charge and1 yzed incident bean to a divergence of $\pm 1.7 \mathrm{mr}$, and 1 m cross section inside the target. Inmediately downstream of the collision target cell, charge analysis occurred in an electrostatic parallel-plate analyzer. A single CEM operated in pulse-counting mode was employed for particle detection. The electron-capture signal and primary beams were measured ilternately for a preselected number of cycles under con:puter contrul : the total electron capture cross sections were deduced from the fraction of ions which capture an electron at a known (calibrated) target thickness.

Figures 4.28-4.32 show the experimental electron capture cross section results obtained for the measured fully stripped and $\mathrm{H}-\mathrm{l}$ ike light fons incident on atomir. and molecular hydrogen. The error bars shown reflect random uncertainty in terms of reproducibility of the measurements at two standard deviations. Systematic uncer$t$ atnties are estimated to be $\$ 9 z$ for both the $H$ and $\mathrm{H}_{2}$ cross sections.

In the case of the fully stripped $C, N$, and 0 projectiles incident on $H$, the present measurements can be compared to theoretical calculations. Very good agreement is found between the present results for $\mathrm{C}^{6+}, \mathrm{N}^{7+}$, and $\mathrm{O}^{6+}$ incident and $H$, and the close-coupling calculations of Fritsch and Lint employing a modified atomicorbtcal (1.0) expansion. Intle falling systematically below the $A D$ calculations, the discrepancy is less than $20 \%$ at energies above $1.5 \mathrm{keV} / \mathrm{ams}$, and approaches $40 \mathrm{~h}$ at the lowest energies measured. For $\mathrm{C}^{6+}$ and $\mathrm{O}^{8+}$ incident on $H$, close coupling calculations by fireen et al.2 and Shipsey et al, 3 , respectively, employing a molecular-orbital (MO) expansion are also in
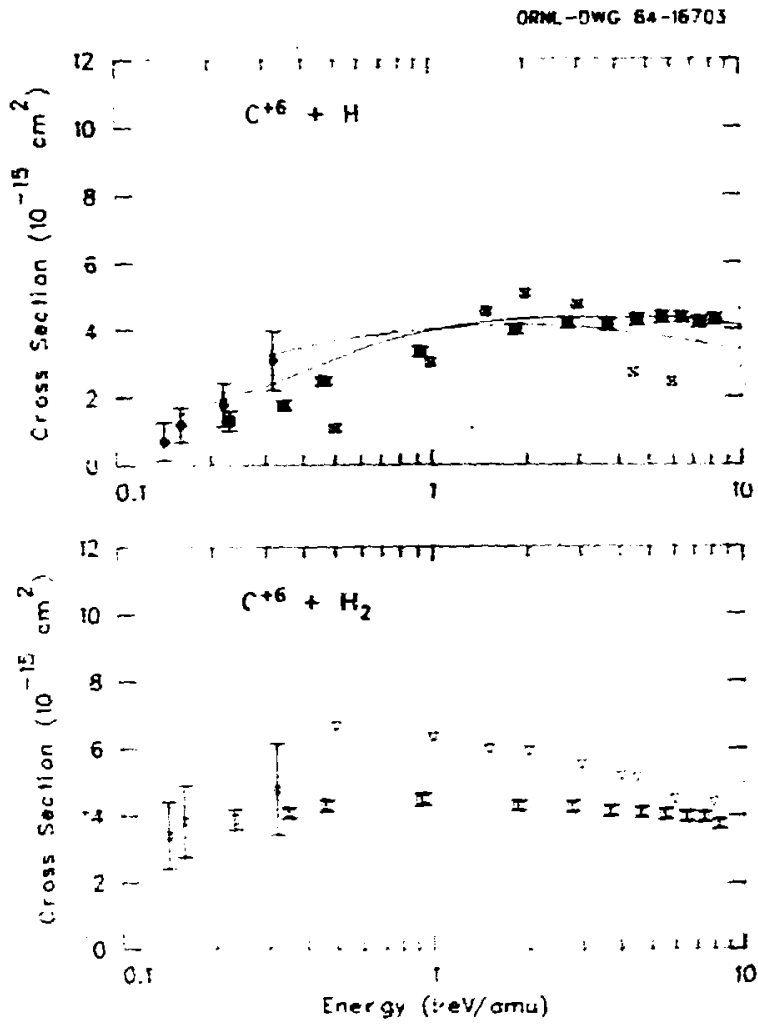

Fig. 4.28. Total el ectron capture cross sections for $\mathrm{C}^{6+}$ incident on $\mathrm{H}$ and $\mathrm{H}_{2}$ us energy; solid and open squares - present results for $H$ and $\mathrm{H}_{2}$ targets, respectively; solid and open diamonds - measurements of Phaneuf et al. (ref. 5); cross hatched boxes and inverted triangles measurements of Panov et al. (ref. 6). Curves represent theoretical results for $\mathrm{C}^{6+}+\mathrm{H}$. Solid line - AO calculation by Fritsch and Lin (ref. 1); dashed line - $m$ calculation by Green et al. (ref. 2); chain dashed curve - wittchannel Landau Lener calculation by Janev et al. (ref. 4).

reasonable accord with the present results. The $M n$ results lie systematically above the $10 \mathrm{cal}-$ cilations by about $10 \%$ above $1.0 \mathrm{keV} / \mathrm{and}$, and by as much as $40 \%$ at $0.2 \mathrm{keV} / \mathrm{amu}$.

An interesting comparison can be made between the total electron capture cross sections for fully stripped projactiles of nuclear charge?. and those for $\mathrm{H}$-itke projectilies of nuclear charge $Z+1$. It is observed that the cross sec. tions for the pairs of ions $C^{6+}$ and $\boldsymbol{W}^{++}, W^{+}$and $0^{7+}, 0^{8+}$ and $\mathrm{FBt}^{8+}$, and $\mathrm{F}^{9+}$ and the ${ }^{9+}$ are nearly identical over the entire energy range covered, indicating that the projectile charge is the prime determinint of the sntal electron capture cross section for these highly stripped systens. This finding is consistent with the theoretical results 2,3 that, for the fully stripped ions, electron capture occurs preferentialiy into high $n-l e v e l s(n>1)$. The presence of atightly 
$20 \times 4-0=084-85704$
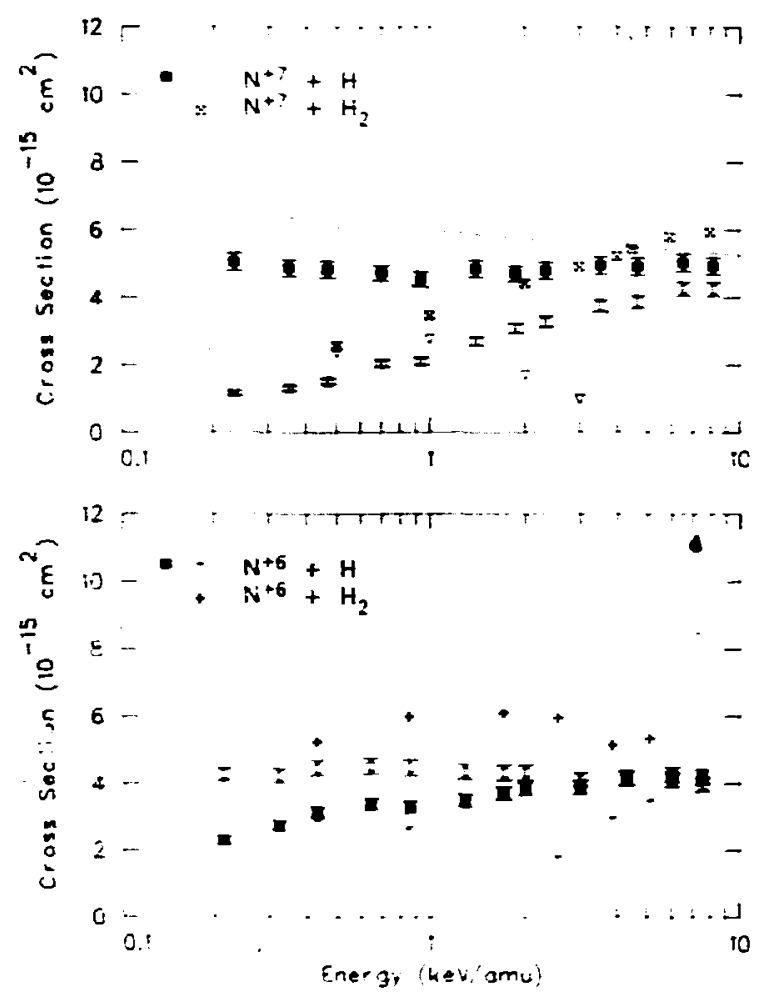

Fig. 4.29. Total electron capture cross sect ions for $\mathrm{N}^{7+}$ and $\mathrm{N}^{6+}$ incident on $\mathrm{H}_{\text {and }} \mathrm{H}_{2}$ vs energy; solid and open squares - present results; data points without error bars measurements by Panov et al (ref, 6): solid line - AO calculation by fritsch and Lin (ref. 1); dashed curve - multichannel Landau Zener calculation by Janev et al. (ref. 4).

bound electron in the ionic core should have only a minimat effect on the behavior of the electronic wavefunction of these highly excited states. The interesting question of how many electrons can be added to the core before an effect is observed in orms of the tatal electron capture cross section will be addressed in future experiments.

1. W. Fritsch and C. D. Lin, Phys, Rev. A 29, 3039 (1984).

2. T. A. Green, E. J. Shipsey, and J. C. Browne, Phys. Rev. A 25, 1364 (1982).

3. E. J. Shipsey, T. A. Green, and J. $C$. Browne, Phys. Rev. A 27, 821 (1983).

4. R. K. Janev, D, S, BelfC, and B, H. Braritsen, Phys. Rev. 2B, 1293 (1983).

5. R. A. Phaneut, I. Alvarez, F, H. Meyer, and D. H. Crandall, Phys. Rev. 26, 1892 (1982)

6. M. N. Panov, A, A, Basalev, and $K, 0$. Lozhk in, Phyz. Scr. T3, 124 (1983).
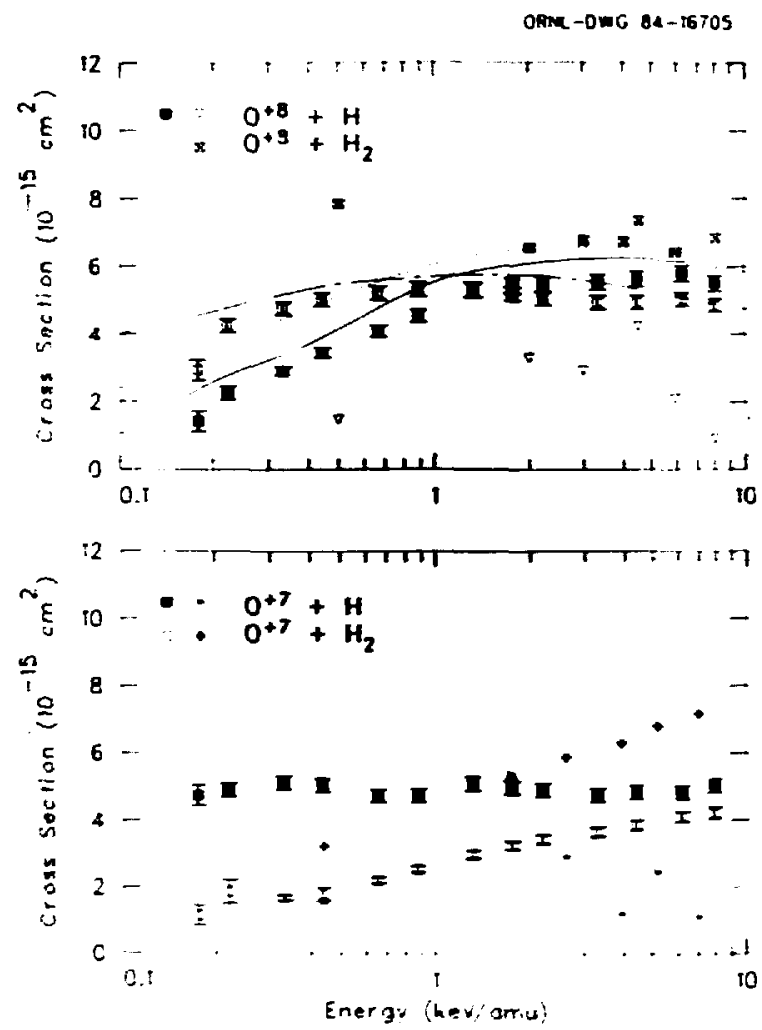

Fig. 4,30. Total electron capture cross sections for $0^{\text {t+ }}$ and $0^{7+}$ incident on $\mathrm{H}$ and $\mathrm{H}_{2}$ vs energy; solid and open squares - present results; data points without error bars measurements by Panov et al. (ref. 6) ; sol id 1 ine - 10 calculation by Fritsch and Lin (ref. 1): dashed line - mo calculation by Shipsey et al. (ref. 3); dashed curve - ma!:ichannel Landau Zener calculation by Janev et al. (ref. 4).

\section{IOH-RTOM MERGEO-BEANS EXPERIKENT}

$$
\begin{gathered}
\text { C. C. Havener } \\
\text { R. A. Doneuf }
\end{gathered}
$$

An ion-atom merged beaw experiment has been developed to measure the total electron capture cross sections for collisions of witicharged fons with $H$ at energies in the range $1-500$ a V/amu. Our afin is to extend to lower energies previous measurements made at GRML using a pulsed laser source, I At such low energies. theoretical models remain essentially untested. In some rultiply fonized systems, an orbiting mechanism is predicted to give very large electron capture cross sections with a $1 / v$ velocity dependence.

The current arrangement of the apparatus is shown in Fig. 4.33. A 2-5 ki, mass-andlyzed beam of $I F$ from a duoplasmatron ton source passes through the optical cavity of a 1,06-um No:YAG Iaser there up to I KH of ctrculating intracavity power is mointained. isp to is 
Oma-DMG 8*-16706
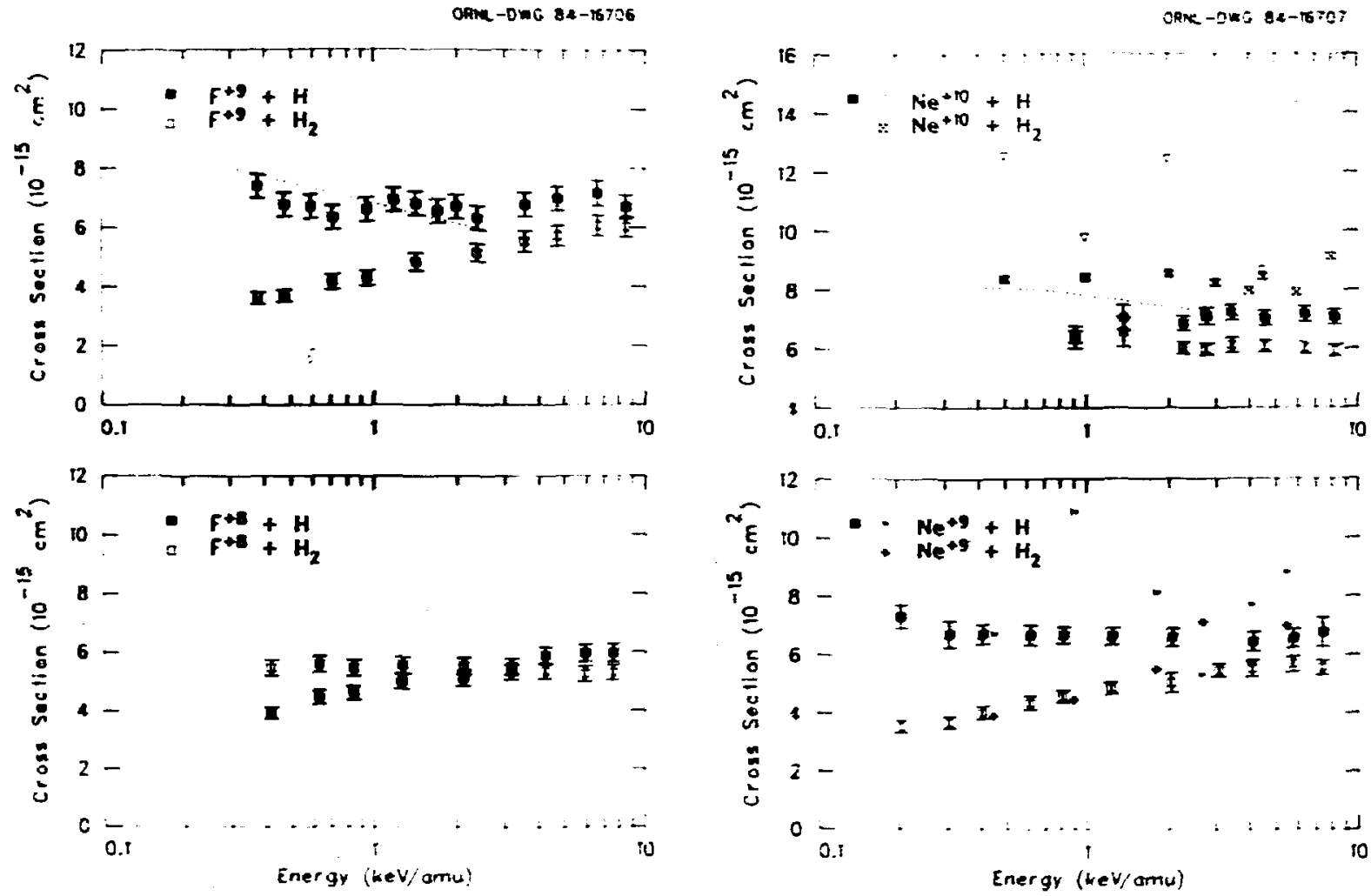

Fig. 4.31. Data points - present measurements of the total electron capture cross section for $F$ at and $F^{a t}$ incident on $H$ and $H_{2}$ vs emergy; dashed curve - multichannel Landau Zener calculation by Janev et al. (ref. 4).

Fig. 4.32. Solid and open squares - present measurements of the tot $\$ 1$ electron capture cross section for $\mathrm{He}^{\mathrm{lot}}$ and $\mathrm{Ke}^{9+}$ incident on $\mathrm{H}$ and $\mathrm{H}_{2}$ vs energy; data points wi thout error bar; measurements by Panov et al. (ref. 5): cashed curve - altichannel Landau Zener calculation by Janev et al. (ref. 4).

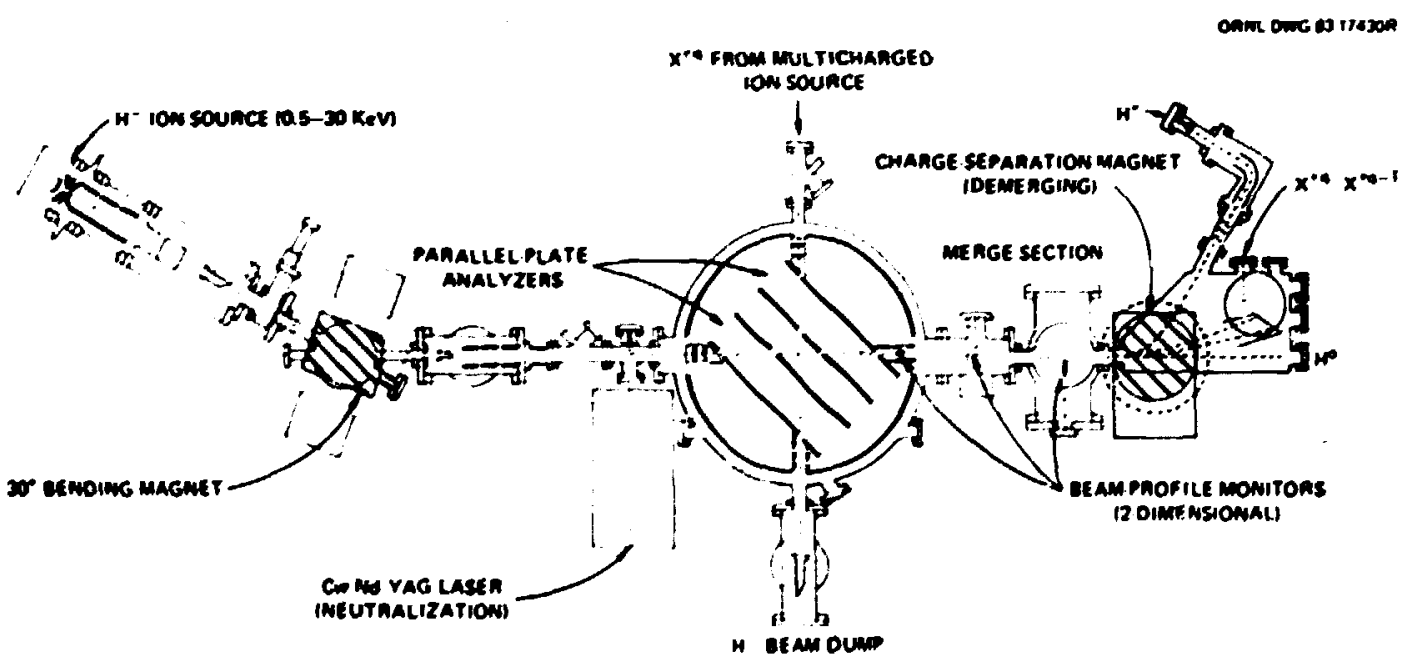

rON-ATOM MEACED-eEAMS EXrRAIMENT

Fig. 4.33. Schematic of the ton-ztom merged beam apparatus. 
photodetachuent has been achieved, producing a co! inated flux of up to $2 \times 10^{12}$ neutral $H$ per second. The undetached $H$ r beam is electrostat $i-$ cally separated from the neutrais and collected in a differentially pumped bean dump. The apparatus has been operated on-l ine using the DRin-PIG multicharged ion source, wich has produced a highly collimated $200 \mathrm{nA}$ beam of $\mathrm{N}^{3+}$ at $30 \mathrm{keV}$. This $\mathrm{N}^{3+}$ beam has been successfully merged with the neutral $H$ beam in the $70 \mathrm{~cm}$ ultrahigh vacuum merge path $\left(P=5 \times 10^{-10}\right.$ torr). A two-dimensional scan of the spatial beaw overlap at one position in the merge path, shown in Fig. 4.34, is generated by a commercial rotating-wire beam-profile monitor. Additional scans at different positions along the merged path i.ust be made before the spatial overlap over tire entire merged section can be accurately quant ified. The ions are then magnetically demerged, separating the $\mathrm{K}^{+}$from the $\mathrm{N}^{3+}, \mathrm{N}^{2+}$, and the neutrals. Signals in the $H^{+}$detector resulting from the bean-beam interaction have been observed above background by modulating both the $\mathrm{M}^{3+}$ and $\mathrm{H}^{\circ}$ beans. These beam-bean signals are only about $10 \mathrm{~Hz}$ compared to $8 \mathrm{kHz}$ due to the $H^{\circ}$ being stripped on background gas and : $\mathrm{kHz}_{2}$ due to photons emerging from the $\mathrm{Kq}^{+}$ Faraday cup.

ORNL-DWG 84-16165

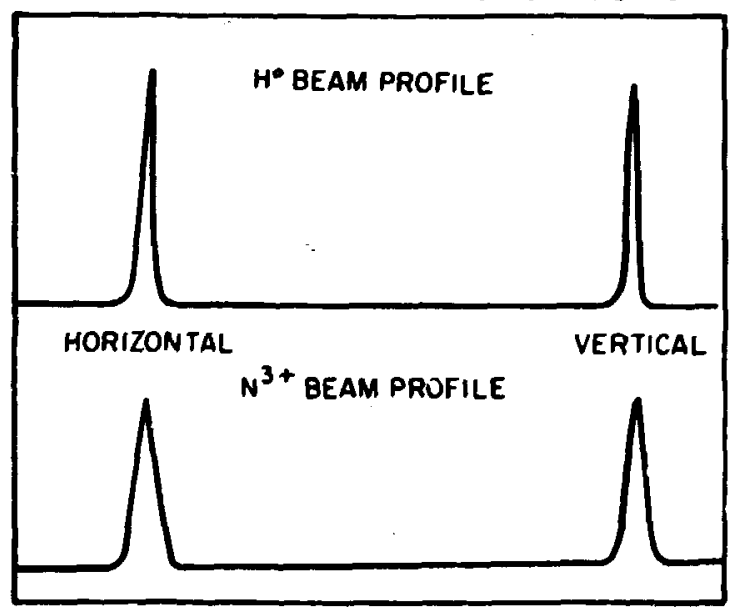

Fig. 4.34. The left and right portions of the upper trace show the respective horizontal and vertical profiles of a $20 \mathrm{nA} \mathrm{H}^{\circ}$ beam. The botton trace shows the profiles of a $120 \mathrm{nA} \mathrm{N}^{3+}$ beam and its relative position with the Ho beam.

Modifications are under way which are expected to significantly improve the signal-tonoise ratio. The gas load from the duoplasmatron fon source wili be further decoupled, reducing the pressure in the merge path, and therefore the $8 \mathrm{kHz}$ background signal due to stripping, by at least a factor of two. The $\mathrm{H}^{+}$ signal beam will be focused and electrostatically deflected out of the plane of magnetic dispersion, assuring detection of all the signal and eliainating the $1 \mathrm{kHz}$ background due to photons produced in the multicharged ion cup. With this decoupling of the $\mathrm{H}^{+}$detector from the photon background, only the $\mathrm{n}^{3+}$ beam need be chopped to separzte the signal from the background, thereby halving the time required to acquire reasonable statistics. Within a few months these modifications will be complete ano the apparatus will be reasy for more definitive cross-section measurements.

1. R. A. Phaneuf et al., Phys. Rev. A 26, 1892 (1982).

\section{ELECTROM-ILPACT IONIZATION OF MULTICHARED METALIC IONS: $\mathrm{Cu}^{2+}, \mathrm{Cu}^{3+}, \mathrm{Mf}^{3+}, \mathrm{Sb}^{3+}$}

D. C. Gregory

A. M. Howald

The present study is part of our continuing effort to provide accurate cross section data for metallic ions of interest in controlled thermonuclear fusion research.

Details of the electron-ion crossed-beams apparatus have been published, 1,2 Ion beams were obtained from the ORIL-PIG multicharged i on source. All measurements are independently absolute, and a typical absolute uncertainty at good confidence level (equivalent to two standard deviations for statistical uncertainties) near the peak cross section is $\pm 8 \%$. Typical relative uncertainties (due mainly to signal count ing statistics) are shown in the figures.

The cross section for ionization of $\mathrm{Cu}^{2+}$ (Fig. 4.35) is typical of the measurements for $\mathrm{Cu}$ and $\mathrm{Ni}$ reported here. The data are compared to predictior c of the three-parameter Lotz semiempirical formula ${ }^{3}$ and distorted-wave calculations. ${ }^{4}$ The latter provide a reasonable prediction for the single ionization cross sections for $\mathrm{Cu}^{2+}, \mathrm{Cu}^{3+}$, and $\mathrm{Ni}^{3+}$. In each case, the Lotz prediction for total ionization is somewhat higher than the measurement, even taking into account the expected effects of multiple ionization. At high energies, the two calculations converge with the data in each case. In the $\mathrm{Cu}^{2+}$ measurements, a significant contribution to the cross section is observed below the ground state ionization threshnid due to the presence of metastable ions in the beam. In fact, significantly different metastable contents were observed from day to day, and nearthreshold measurements for two ground state/ metastable ratios are plotted. In general, for ionization of $\mathrm{Cu}^{2+}, \mathrm{Cu}^{3+}$, and $\mathrm{Ni}^{3+}$ we concisde that distorted-wave direct ionization calculations give a reasonably accurate representation of single tonization. No large infirect ioniza$t$ ion contributions are observed. The threeparameter Lotz formula, although not as reliable as distorted-wave calculations, is observed to be well within its predicted factor-of-two accuracy in each case.

in contrast to the above examples, electron impact ionization of $\mathrm{Sb}^{3+}$ has a considerable 


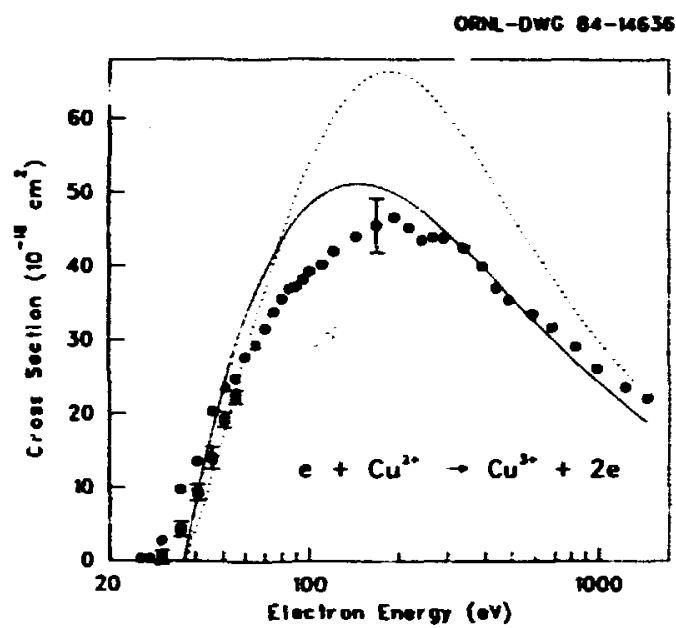

Fig. 4.35. Electron impact ionization of $\mathrm{Cu}^{2+}$. The circles and squares present nearthreshold data with ion beans having different metastable i i content. A typical absolute uncertainty ar good confidence level is plotted near $200 \mathrm{eV}$. The tweories are from the threeparaneter Lotz formula for $3 d$ ionization (dashed curve - ref. 3) and distorted wave calculations of Pindzola et al. (ref. 4).

contribution fron indirect effects (Fig. 4.36). The indirect ionization, contributing up to $45 \times 10^{-18} \mathrm{~cm}^{2}$ to the total cross section, is mainly due to non-dipole-allowed excitation of inner-shell electrons to autolonizing states. The cross section for this type of transition increases abruptly to a maximum at or just above

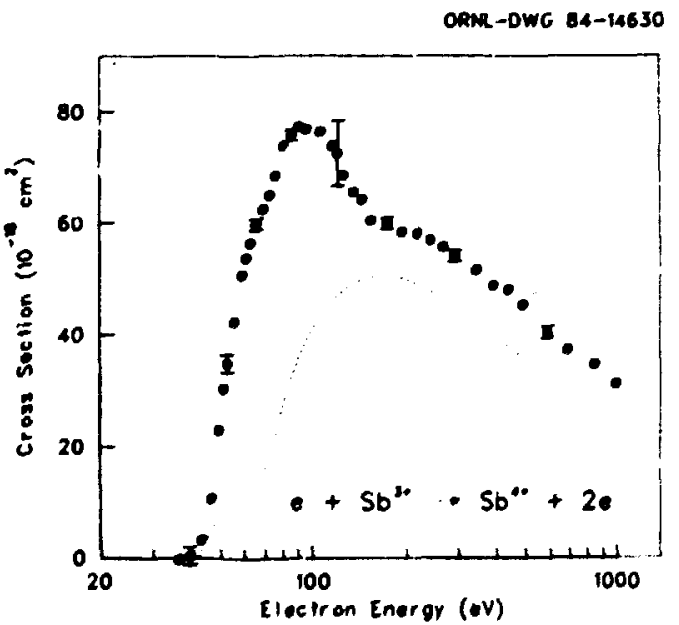

Fig. 4.36. Electron impact fonization of $\mathrm{Sb}^{3+}$. Typical relatfve uncertaintfes are plotted, and the absolute uncertainty at good confidence level is shown near $100 \mathrm{eV}$. The dashed curve is from the Lotzz formula for $4 d$ and ss fonfzation (ref, 3). its threshold energy but has a wuch faster falloff with increasing energy ( $\sigma$ a $/ / E$ ) than coes direct ionization ( $\sigma$ a $\operatorname{mE} / E$ ), so that its signature is a "hump" superimposed upon the direct cross section burve. This measurement was undertaken as a check of detailed distorted-wave indirect-ionization calculations wich predicted the observed feature. 5 Cood agreenent is found with the calculations and predictions of that stidy.

The examples discussed fllustrate two goals of our current research efforts: First, selected measurements are made to provide specific data requested by the fusion cominity for electron-impact ionization of alticharged ions. Second, cooperative studies involving experiment and detafled calculations provide our best means of understanding the relative imortances of the many possible mechanisus which can lead to ionization by electron impact.

1. D. H. Crandall, R. A. Phaneuf, and D. C. Gregory, Electron Impact Ionization of Multicharged Ions, CRliL/TL7020, Gak Ridge Matl. Lab.. Septeber 1979.

2. D. C. Gregory et al.. Electron I-pact Ionization of Multicharged Ions at ORNL,

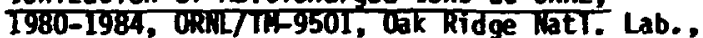
1985.

3. Wolfgang Lotz, Z. Physik 220, 466 (1969).

4. M. S. Pindzola et al., Survey of Experimental and Theoretical ETectron-Impact Ionization Cross Sections for Transition Metal Ions in Low Stages of Ionization, ORRL/TR-9436, Uak Ridge WatT. Lab., 1985

5. M. S. Pindzola, D. C. Griffin, and C. Bottcher, Phys. Rev. A 27, 2331 (1983).

SIMGLE, DOUBLE, AND TRIPLE ELECTRON-IMPACT IOUIZATION of $\mathrm{Xe}^{6+}$
A. M. Howald
D. H. Crandall
J. C. Gregory
R. A. Phaneuf

Electron impact ionization of $\chi e$ ions has been studied previousiy at Oak Ridge National Laboratory from several dffferent perspectives. Experimentally, the ORNL-PIG ion source and electron-ion crossed beans apparatus have been used to study single fonization of $x e$ fons from $\mathrm{Xe}^{2+}$ to $\mathrm{Xe}^{6+}$ (Ref. 1). and $\mathrm{Sb}^{3+}$ (Ref. 2), whtch is isoelectronic with $x e^{6+}$. The $x_{e}$ isonuclear sequence and $\mathrm{In}^{+}, \mathrm{SL}^{3+}, \mathrm{Xe}^{6+}$ isoelectronic sequence have also been studied theoretically, 1,3 and comparisons made with experiment.

During the past year, electron-fmpact foni$\mathrm{zation}$ of $\mathrm{Xe}^{6+}$ was studfed from yet another point of view. Measurements were made of the cross sectinns for double and triple ionization of this ion. The processes are

and

$$
\begin{aligned}
& e+x e^{6 t}+x e^{8 t}+3 e \\
& e+x e^{6 t}+x e^{9 t}+4 e
\end{aligned}
$$

and the cross sections are denoted by 068 and o6y, respectively. 
The results of these measurements are shown in figs. 4.38 and 4.39 , with the previousiy measured cross section ${ }^{4}$ for single ionization of $X^{6+}$ (denoted by $\sigma_{\in}$ ) shown in Fig. 4.37 for comparison. The peak of $\sigma_{67}$ is roughly an order of magnitude larger than the peak of $\mathbf{a 6 8}$, wich in turn is roughiy an order of magnitude larger than the peak of 069 . As expected, the successive ionization thresholds increase along this sequence.

The dashed line in Fig. 4.37 is an estimate of the cross section for direct single ionization of ie $^{6+}$ from the semiempirical formula of

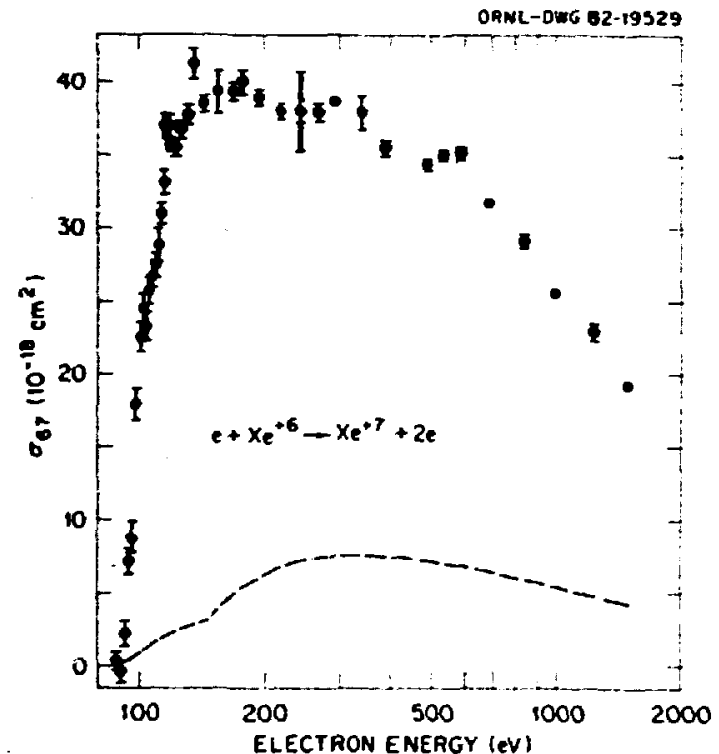

Fig. 4.37. Measured cross section for single fonization of $\mathrm{Xe}^{6+}$ by electron impact. The dashed line is the Lotz formula estimate for direct fonization of an outer shell 45 electron.

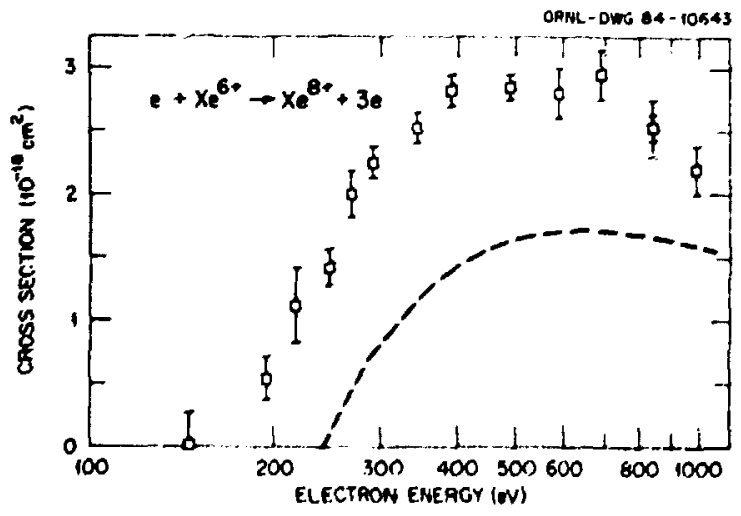

Ft3. 4.38. Double ionization of $\mathrm{Xe}^{6+}$ by electron impact. The dashed line is the Lotz formula estimate for direct single fonization of an inner shell 40 or $4 \mathrm{~s}$ electron.

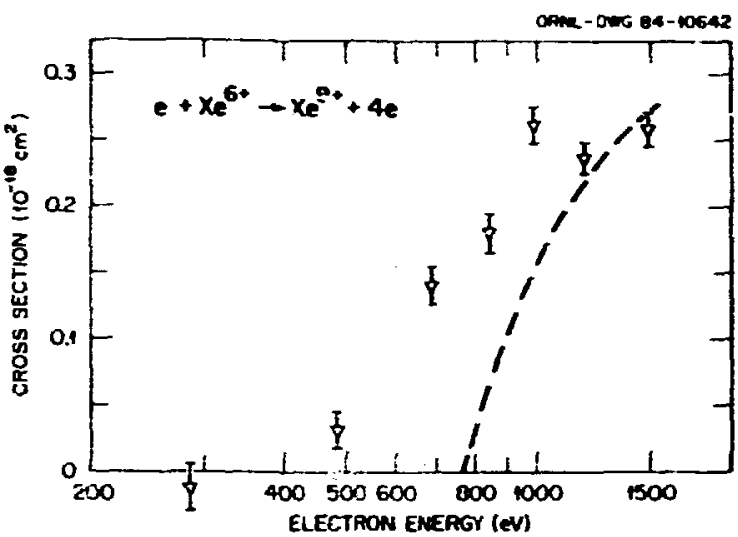

Fig. 4.39. Triple ionization of $x e^{6+}$ by electron impact. The dashed line is the Lotz formula estimate for direct single ionization of an inner shell $3 d$ electron.

Lotz. The actual cross section is much larger due to ionization by mechanisms other than direct ionization, especially electron-impact excitation of an inner-shell electron followed by autoionization.

Single direct ionization of an inner-shell electron of $x^{6+}$ can also lead to production of $\mathrm{Xe}^{\mathrm{B+}}$ or $\mathrm{Xe}^{9+}$ if the $\mathrm{Xe}^{7+}$ is produced in a multiply excited autoionizing level. For example, collision of an electron with $x e^{6+}\left(4 p^{6} 4 d^{10} s_{s}^{2}\right)$ can remoye in inner-shell up electron to form $\mathrm{Xe}^{2+}\left(4 p_{4 d}^{5} 0_{5 s^{2}}\right)$ which can subsequent ly autoi onize to form $x e^{8+}\left(4 p^{6} 4 d^{10}\right)$. A number of calculated eneray levels of various ionization stages of $X e$ are shown in Fig. 4.40 along with some autoionizing transitions.

In Fig. 4.38 the dashed line is the sum of the Lotz estimates for direct single ionization of $x^{6+} 4 p$ and 45 electrons. The resulting $x_{e} e^{6+}$ ions are in autoiontzing levels. Hence (assuming a branching ratio near unity for autoionization versus radiative stabilization), this gives an estimate of the double ionization cross $\mathrm{sec}$ tion due to the process of single direct ionization followed by autoionization. Similarly, the dashed line in Fig. 4.39 is the Lotz estimate for single direct removal of a 3 d electron from $\mathrm{Xe}^{6+}$. In this case the resulting excited $\mathrm{Xe}^{7+}$ has sufficient potential energy to doubly autoionize to form $\mathrm{Xe}^{9+}$.

The double ionization (Fig. 4.38) cross section calculated for this mechicnism is similar in magnitude to the measured cross section (within a factor of 2) but has a threshold value that is higher than the experimentally-observed ionization onset. For triple fonization (Fig. 4.39) the Lotz estimate of the cross section for the mechanism of single direct fonization followed by double autoionization also has the correct magnitude and also has a predicted threshold that is higher than observed.

Other higher-order mechanisms must be responsible for the observed non-zero cross sections below $240 \mathrm{eV}$ and $760 \mathrm{eV}$ in Figs. 4.38 and 4.39, 


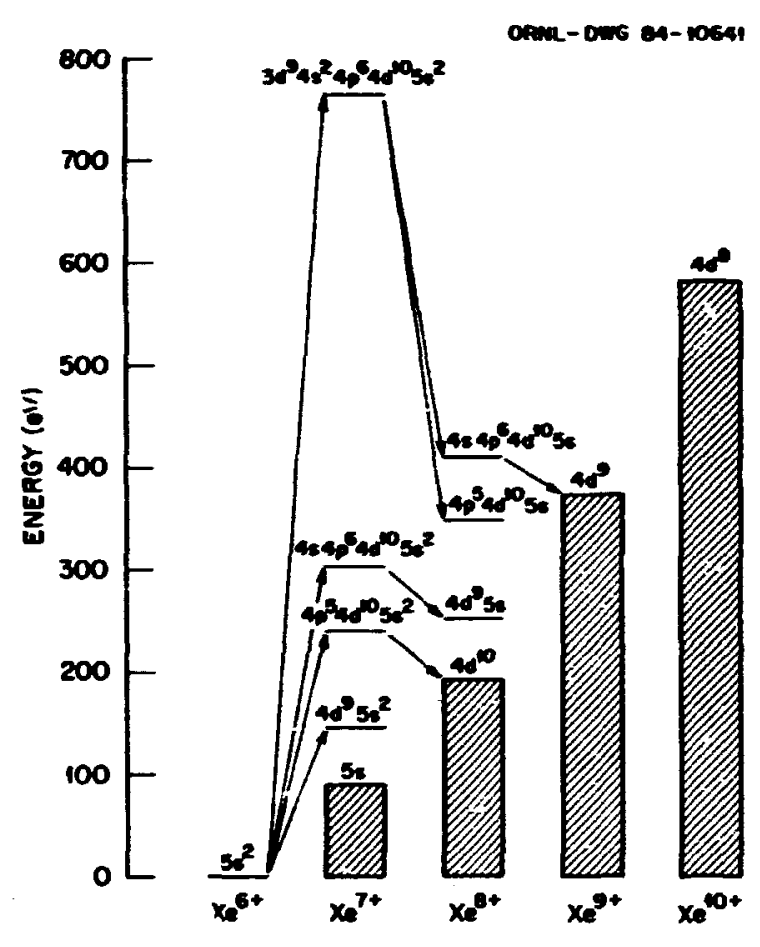

Fig. 4.40. Calcu? zted energy levels (f rom ref. 5) of $X e$ ions, showing some electron-impast and autoionizing transitions.

respectively. There are at least four possibilities: direct multiple ionization; electronixpact excitation followed ay double or triple autoionization; direct single ionization accompanied by the simultaneous excitation of a second electron; or multiple excitation in a single collision, followed by autofonization. Direct multiple ionization of $\mathrm{Xe}^{6+}$ is expected from the classical Binary Encrunter Approxima$t$ ton (BEA) model to be of ney?igible fmportance. The other three processes have not ceen studied in detail and are not yet well understood. Our experimental results offer intriguing evidence that one or more of them can play a significant role in electron-fmpact multiple ionization of multiply charged ions.

1. D. C. Griffin, C. Bottcl ar, 1. S. Pindzola, S. M. Younger, D. C. Gregory, and D. H. Crandall, Phys. Rev. A. 29, 1729 (1984). 2. D. C. Gregory and A. M. Howald (unpublished).

3. M. S. Pindzola, D. C. Griffin, and

C. Bottcher, Phys. Rev. A 27, 2331 (1983).

4. D. C. Gregory and D. H. Crandall, Phys. Rev. 27, 2338 (1983).

5. S. Pindzola, private communication (1984).
MEH ELECTRON-ION CROSSED-BENS APPARATUS

$$
\text { D. C. Gregory F. W. Meyer }
$$

Since 1977, electron-iapact ionizat ion cross section measurements have been completer at oam for some 44 different ions with initial charges ranging from +2 through +6 and spanning the periodic table from boron to tantalum. 1,2 These total cross section measurements for single, double, and triple ionization have all utilized the ORNL-PIG ion source and crossed ion-electron bews apparatus. 3,4 With the completion of the new ORML-ECR ion source, owr capabilities for producing miticharged ion beans now extend to considerably higher ionization stages. Since some aspects of the existing apparatus are inadequate for operation with more highly charged ions, the experiment has been redesigned.

Electrostatic separation of ion beams by charge state is difficult to achieve. In a crossed-beans ionization experiment, as little as 1 part in $10^{8}$ cross-talk between exit channels produces an intolerable background level. and separation becomes progressively more dif:ficult as the charge increases and the initialto-final charge ratio approaches unity. In addition, low-noise electrostatic analyzers mst usually be mechanically modified for each charge ratio under study. To overcome both of these difficulties, a metic spectrometer is being constructed for product charge-state analysis.

The modified apparatus is shown in Fig. 4.41. The ion-beam optics and ultra-high vacuum system will renain much the same as in the present arrangement through the interaction volume in the center of the electron gun. The combined beam containing the primary fons and furtherionized signal ions then enters a doublefocusing analyzing magnet which disperses the beam components by charge state. Signal ions are deflected through $90^{\circ}$ and focussed into a channel electron multiplier. The ion optics are designed such that the crc ;ed-beams interaction region is imaged onto the signal detector with unit magnification. The primary ion beam is trapped in one of two movable, guarded Faraday cups, depending on the initial-to-final charge ratio for that particular experiment. Deflection plates and einzel lenses are provided as diagnostic tools both in the main interaction chamber and imediately before the signal ion detector.

The apparatus is designed to allow measurements ranging from fnitial-to-final charge state ratfos of $4 / 5$ to $15 / 16$. Inttfal tests of the completed apparatus are planned for early 1985. The next phase in the development of the crossed-beams apparatus will involve redesign or replacement of the existfing magneticallyconffned electron gun to extend the available range for reliable measurements to electron energies greater than $1.5 \mathrm{keV}$.

1. D. H. Crandall, R. A. Phaneuf, and D. C. Gregory, Electron Impact Ionization of Multicharged lons, ORNL/TM-7020, ahk Ridge Mat 1. Cab., September 1979. 
ophe owg exteris

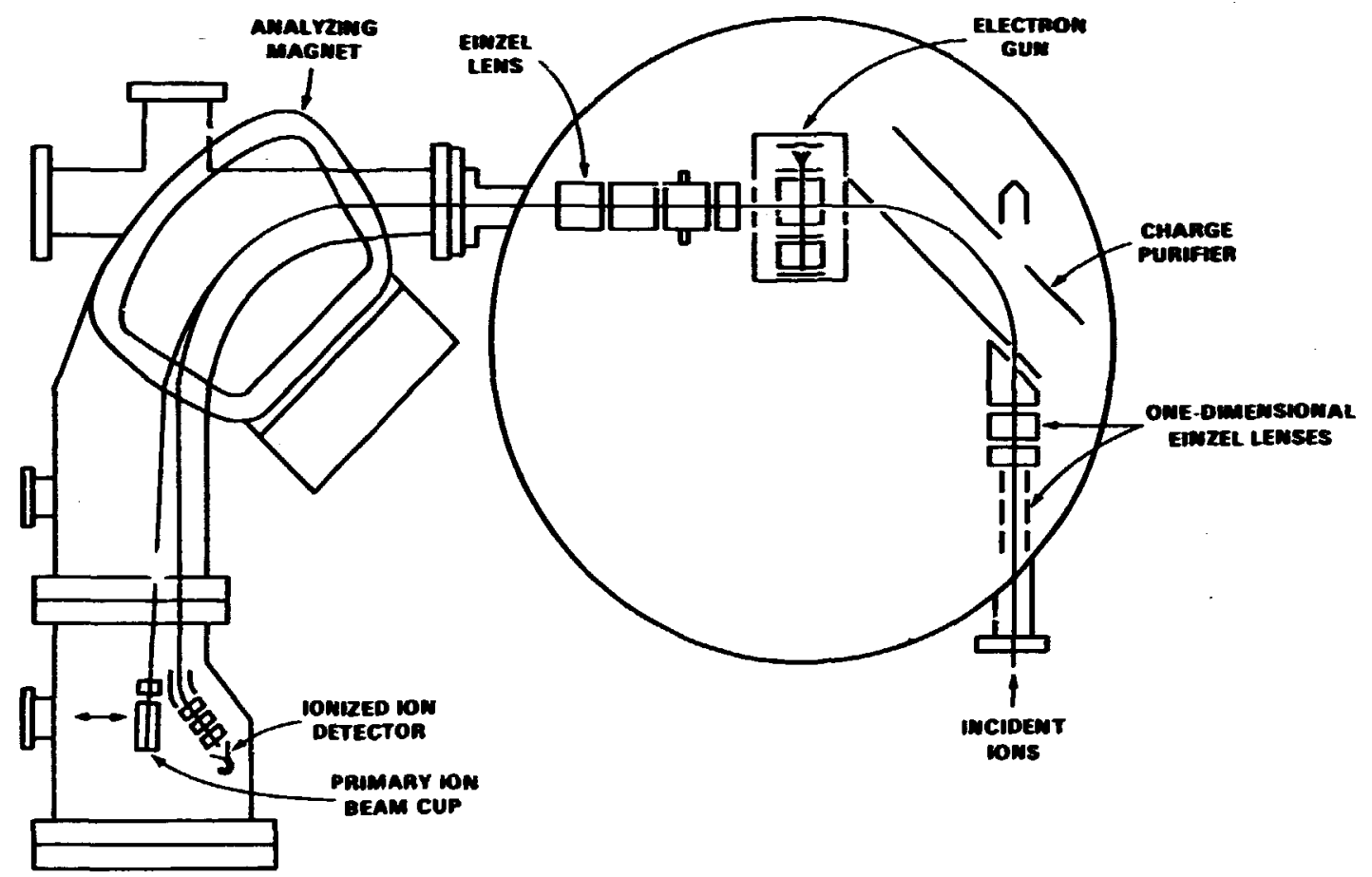

Fig. 4.41. New electron-ion crossed beams apparatus with post-collision charge-analyzer magnet. Measurements will include single and multiple ionization of a wide range of highly ionized $(6<q<15)$ target ions.

2. D. C. Gregory et al., Electron Impact Ionization of Multicharged Ions at ORNL, 1980-1984, ORNL/TM-9501, Oak Ridge KatT. Lah., T985.

3. D. C. Gregory, P. F. Dittner, and D. H. Crandal!, Phys. Rev. A. 27, 724 (1983); D. H. Crandail et al., Phys. Rev. A. 25, 143 (1982).

4 P. 0. Taylor et al., Rev. Sci. Instrum. 45, $588(1975)$.

\section{RADIONETRIC STANDARD}

FOR THE EXTREME ULTRAVIOLET

$$
\begin{aligned}
& \text { P. M. Griffin } \\
& \text { J. H. Johnson }
\end{aligned}
$$

A light source utilizing bremsstrahlung and transftion radiationd, 2 generated by keV electron impact on metals is being investigated. This low power, compact, simple lamp has potential as a secondary-standard radiometric souice for the soft $x-r a y$ (SXR) and extreme ultraviolet (EUV) spectral region. Its spectral radiance is being characterized by a few easily measurable and controllable electrical, mechanical, and chemical parameters.

The essentidl elements of the lanp are the straight edge of a 0.12 -mm-thick, ohmically heated, tungsten cathode and a flat metal anode.
A well-defined thin sheath of keV electrons is electrostatically drawn from the hot edge of the cathode located $\mathbf{0 . 1 8} \mathrm{mm}$ from and perpendicular to the anor.e target. At the intersection of the flat sheath of impinging electrons and the flat anode, a 6-mm-long, narrow. luminous 1 ine is generated.

An example of spectrometric measurements of radfation generated by the prototype source is shown in Fig. 4.42. It should be noted that at each spectroneter setting corresponding to a given value of $m \lambda$, several discrete portions of the output spectrum are simultaneously sanpled. The emissions from wave?ength intervais ( $\mathrm{Am \lambda} / \mathrm{n}$ ) A wide, centered at wavelengths $(m \lambda / n) A$, contribute to the measured signal at that setting. Relatively high spectral order-numbers $n$ must he considered since, for high 2 metals, bremsstrahlung and transition radiation intensities vary as $\lambda^{-3}$ and $\lambda^{-2}$ respectively. The number of spectral orders observed is limfted hy the product of the short wavelength cutoffs of the grating and detector efficiencies. The undulations in the data in Fig. 4.42 are accounted for in terms of the dropping-out rate of the highest integral spectral orders in proceeding to smaller values of $m i$.

As this source is operated, bremsstrahlung, generated as the penetrating electrons are decelerated or deflected below the surface of the target, is the principle light emitting 


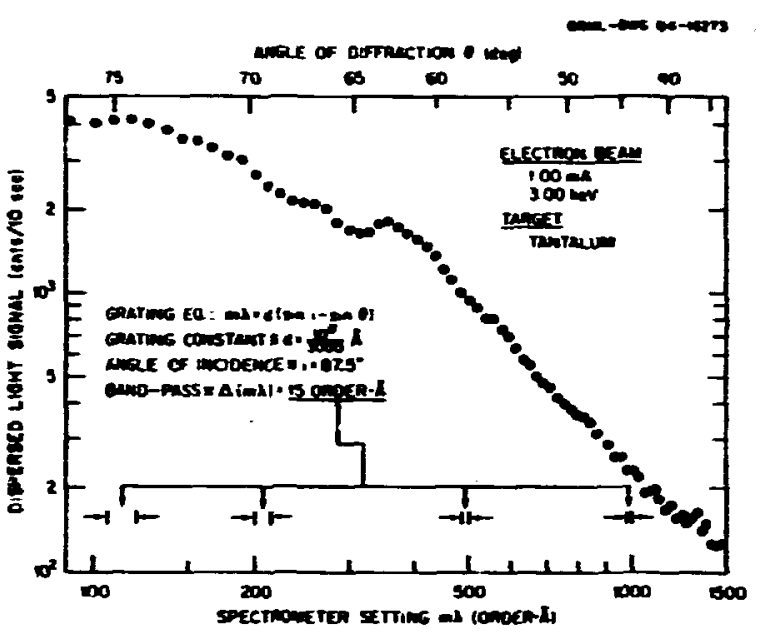

Fig. 4.42. Spectronetric measuresent of the SXR and EUV light generated by 1.00 af 3.00 kel electrons incident on a tantalum target in the prototype radionetric source. Data are from a step-wise scan with a 2.2 meter, grazingincidence spectrameter. The dispersed light was detected with a $\mathrm{ZnO}^{-\mathrm{TiO}_{2}}$ ceramic channel-electron-multiplier. The spectrometer entrance slit subtended a 0.C05 steradian sol id angle at the source.

mechanisn for the continuum shortward of $-450 \mathrm{~A}$. of particular interest is the lanp's scalable emission in the overlapping SXR and EUV spectral regforis as displayed in Fig. 4.43. The aforementioned sfectrometer was set to simultaneousiy sample the enissions from $7.5,3.8$, and $2.5 \mathrm{~A}$ intervals in the continuum observed at 90, 45, and $30 \mathrm{~A}$, respectively. As expected according to the theory of brensstrahlung, the combined signals scale with electron current at four representative electron energies. The utility of this lamp as a practical stardard is indicated in that statistfcally acceptable datd were obtained with only $10 \mathrm{sec}$ integration times and electron bean powers of only 2 to 15 watis.

At velengths longer than $450 \mathrm{~A}$, transition radiation should be the dominant light-emitting mechanism in the lamp. Phenmenologicaliy. tnis radiation is a consequence of the collapse of the dipole fiels, produced by the approaching electron and its positive image charge in the metalifc target, as the electron "transits" through the vacuum-target interface. The Fourier transform of the resulting electromagnetic pulse is a "white" frequency specirum. The optical constants of watever material exists at the surface characterize this radiation, rather than the bulk properties of the target. A reproducible surface is selfmaintained if the source is operated at an adequate electron-beam areal power-density. Investigations of the transition radiation component of the lamp's emission have been hampered by the overlapping of high spectral orders of shorter wavelength bremsstrahlung. Solutions to this problem are being sought through the use
OAne-0w6 24-45.44

SCALING OF GREMSSTRALUMG WITH ELECTRON CURAER:

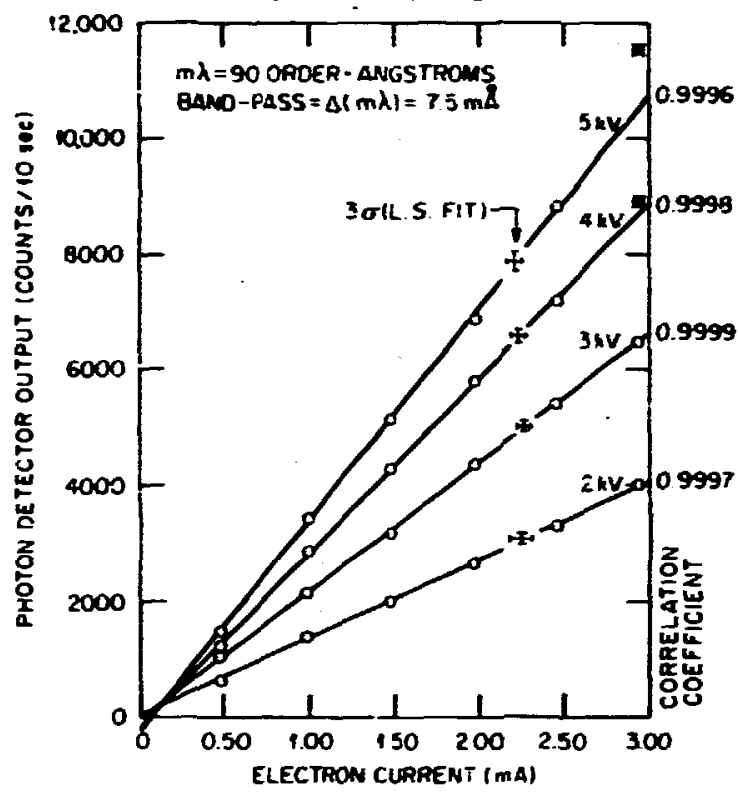

Fig. 4.43. Dependence of brensstrahlung signal strength on electron beam current for accelerating voltages of $2.00,3.00,4.00$, and $5.00 \mathrm{kV}$. The least-squares fits of the signals with current had standard deviations of 32,31 , 49, and 61 counts/10 sec, respectively. The $h$ ighest current measurements at 4.00 and $5.00 \mathrm{kV}$ were excluded in the fits since their deviations were greater than the $3 \sigma$ error-bars which are shown for each line.

of combinations of thin metal-foil spectral filters wich effect appropriate transmission "windows."

An improved model of the source and vacuum system have been designed and ouflt which should permit operation at electron energies greater than $10 \mathrm{keV}$. This will permit generation of transition radiation of much longer wavelength. In addition, with the resulting greater elestron penetration depths, the bremsstrahlung will be completely absorbed before it can energe from within the target to proceed to the spectrometer. This technique will permit, where applicable. less complicated procedures for studies of the transition radiation component of the l anp's emissions.

1. H. Ginzburg and I. Frank, J. Phys. (USSR) 9. 363 (1945).

2. H. Boersch and G. Sauerbrey, Proceedings of the International colloquium on optical propert es and tectronic structure of Vetals and Alloys, F. Abeles, editor, horth-holland Pubtishing $C_{0 .}$. Ansterdem (1966). 


\title{
5. THEORETICAL NUCLEAR PHYSICS
}

\author{
INTROOUCTION NAD OVERVIEM
}

The theoretical physics program in the Physics Division at ORNL involves research in both muclear and atomic physics. These programs benefit from interactions with the various experimental programs in the Division, and from access to good computing facilities as discussed briefly below and in the section on :HIRf facilities.

In muclear physics there is extensive activity in the fielis of direct nuclear reactions with light- and heavy-ion projectiles, the structure of muclei far from stability and et elevaied temperatures, and the microscopic and macroscopic descriptica of heavy-ion dynanics, including the behavior of nuclear molecules and "supernuclei." New research efforts in relativistic nuclear collisions and in the sturly of quark-gluon plasma have continued to grow this year.

The atomic thenry progran deals with a variety of ionization, multiple-vacar.cy production, and charge-exchange processes. Many of the problems are selected xecause of their relevarce to the magnetic fusion energy program. In addition, there is a joint atonic-nuclear theory effort to study positron production during the collision of two high- $Z$ numbers, i.e., lj+U.

As this repert is prepared, the theory program in the Physics Division is being significantly expanded. A new Distinguished Scientist program, sponsored jointly by the thiversity of Tennessee and ORML, has been initiated. Among the first appointments is G. F. Bertsch in theoretical physics. As a result of this appointment, Bertsch and an associated grouy of four theorists split their time between UT and ORNL. In addition, the State of Tennessee has established a significant budget to support the visits of outstanding scientists to the Joint Institute for Heavy Ion Research at ORNL. This budget should permit a significant improvement in the visitor program at ORNL. Finally, the Laboratcry warded a Wigner post-doctoral appointment to a theorist wo will work in the theory group nf the Physics Division. This significant expansion in the theory effort should be a stimulus to both the theory and experimental programs in the Physics Division.

The VAX-780/FPS-164 computer system has been in use for the whole report period. Dur experience continues to be that the FPS-164 performs at a rate comparable or superior to a contemporary matn frame computer such as the IBM-3033 or a $D C D-7600$ for the large, numerically intensive scientific calculations common to theoretical physics.

HEAVY-ION DYNAMICS

\section{FOLDING-MODEL AMALYSIS OF ELASTIC AMD IMELASTIC A-PARTICLE SCATTERIMG USING A DENSITY-DEPENDENT FORCE ${ }^{1}$}
A. M. Kobos ${ }^{2}$
R. Lindsay
B. A. Brown ${ }^{3}$
G. R. Satchler

A folding model with a density-dependent form of the sami-realistic Mar effective interaction was applied to a-particle scattering. A previous analysis of elastic scattering at 140 and $172 \mathrm{MeV}$ was applied to data at other energies from 2.5 to $120 \mathrm{MeV}$. The model was a'so extended to inelastic scattering, using hoth he collective model and a valence-plus-core-polarization model for the transition densities. The proton transition densities were normalized to measured $B$ (EL) values. When necessary, the neutron transition densities were rescaled to fit the $\left(a, a^{\prime}\right)$ data, providing a source of information on the neutron contributions. The neutron transition multipole moments thus obtained were compared to those derived from $\left(p, p^{\prime}\right)$ data at $800 \mathrm{MeV}$, as well as from other sources.

1. Ahstract of paper: Nucl. Phys. A425, 205 (1984).

2. Oxford Universicy, Oxford, England,

3. Michigan State Intversity, East Lansing, Michigan.

4. SERC Darestury Laboratory, Daresbury, Warrington, E.ngland. 


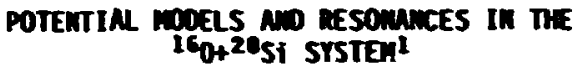
A. M. Kobos ${ }^{2}$
6. R. Satchler

Two recent papers have given optical potential descriptions for the elastic scattering of the 160+20si system at energies near the coulomb barrier, both of wich anifest partial wave resonances. Our comment ws that there is at least one other potential that reproduces the measurements but wich does not result in such resonances.

1. Abstract of paper: Phys. Rev. C 30, 403 (1984).

?. Oxford University, Oxford, Eng'and.

\section{A EOBNL OPTICAL POTETIA MFirsis of 160+28SI ELASTIC SCATIERIMSI}
A. H. Kobos 2
G. R. Satrhler

The differential cross sections for $160+205 i$ elastic scattering at many energies between 18 and $35 \mathrm{MeV}$ in the center of mass are described very well over the whole angular range up to $180^{\circ}$ by two optical potentials. One describes the data at twelve energies between 18 and 29 MeV and another one at two energies, 31.6 and $34.8 \mathrm{MeV}$. The two potent fals differ in parameter values, but in both cases the real part consists of a folded potential supplemented in the surface with a double-peaked and energyindependent attractive correction wich is parametrized as the derivatives of two WoodsSaxon form factors. The imaginary potential is a sum of Hoods-Saxon volume and surface terms, and its radius increases iinearly with energy. The excitation function for elastic scattering at $190^{\circ}$ is also reproduced 11 uf to about 32 MeV. The 5 -matrix elements generated by these potentials do not show any resonance features. The qualitative features of the scattering are discussed, especially in terms of the decnmposition of the scattering anplitude into inner-wave and barrier-wave components.

1. Abstract of paper: Nucl. Phys, M27, 589 (1984).

2. Oxford University, Oxford, England.

ONE EFFECT OF USING RELATIVISTIC KINEMTICS IN THE AMALYSIS OF HEAVY-ION ELASTIC SCATTERIMG

$$
\text { M. F.l-Azah Farid }{ }^{2} \text { G. R. Satchler }
$$

The ion-ion potential deruced from an optical model analysis of heavy-ion elastic scattering may be sensitive to whether the transformation from lahoratory to center-of amass system used is relativistic or not. For illustration, semiclassical description is used to give a simple estimate of this effect. and its dependence on energy and the masses of the muclei. For exanple, the change in potential strength is larger than 201 for ${ }^{\circ} \mathrm{Ar}$ scattering frum $20 \mathrm{spb}$ at an energy as low as 4 MeV per nucleon.

1. Mbstract of paper: Physics Letters B (in press).

2. Guest assignee from Department of Physics, Faculty of Science, Assiut University, Assiut, Egypt, 8/1/83-8/1/84.

\section{SOME OPTICAL HODE MULSES OF THE EASTIC SCATIERIIS of MAr AT 1760 ReVl}
M. El-Mab Farid²
G. R. Satchler

The elastic scattering cross sections for OAr on 60Ni, I20sn, and 208po at 1760 heV were reanalyzed using both Woods-Saxon and folding model potentials. The latter required renormal izing by factors $N<0.7$. This provides further evidence that the real potentials at this energy are suhstantially weaker than those obtained for otiner systems at lomer energies. This was confirmed by comparison with data for $40 \mathrm{Ar}+200 \mathrm{pb}$ at $236 \mathrm{HeV}$. The 176n-thel data appear to deteraine the values of $b, h$ real and iaginary potentials in the vicinity of a strong absorption region, nearly one ferai inside the rainbow radius.

1. Abstract of paper to be subaitted to Nuclear Physics A.

2. Guest assignee from Department of Physics, Faculty of Science, Assiut Vr,iversity. Assiut, Egypt, 8/1/83-8/1/84.

\section{FOLDIMG-MOOEL POTERTINS FOR HEAYY-IO SCATTERIMS USIMG A SEMIREALISTIC DENSITY-DEPEMEERT FORCE}
M. E.1-Azah Fariol
G. R. Satchier

The real part of the optical potential for heavy-ion scattering was calculated in a folding model using a density- and energy-jependent oeneralization, called DOM $3 Y$, of the HJY effective interaction. The interaction strength and density-dependence were calforated against a realistic Gmatrix. The resulting potentials must he reduced hy factors $N \sim 0.8$ in order to fit 'low'-energy scattering data $(-5$ to 15 weV per nuclean). High energy ( $44 \mathrm{MeV}$ per nucleon) - Ar data required somewhat mo reducition. N 0.65 . Indicating that the energy dependence incorporater into the 00 izy is insufficient. Further, the same DOM3Y interaction had heen shown previously ${ }^{2}$ to give a good account of aparticle scattering over the same range of energies per nucleon with a constant renormalization factor of $N=1.3$. Consequently, the DOH3Y cannot simult aneously represent heavy-ion and aparticle scattering within the usual folding model. 
1. Guest assignee from Department of Physics, Faculty of Science, Assiut University, Assiut, Egypt, 8/1/83-8/1/84. (1984).

\section{EFFECTS OF POTEMTIA VRIATIOONS ON ONE- DIREISIOMnL BABRIER RELETRATION AND FUSION CROSS SECTIOAS BELON THE COUnors BurRIER}
M. H. Shalabyl
G. R. Satchler

It is known ${ }^{2}$ that coupling to non-elastic channels has very important effects on the fusion cross sections ofus (E). helow the Coulomb bo-rier. calculated using the simple one-channel pi iential barrier-penetration motel. Howner, as a prelfminary to such calculations, it is also of interest to know what effects result from the andiguities in the one-channel potential itself. Measured fusion cross sections near the barrier determine the position, height, and curvature of the barrier, and hence its outer face. in a relatively unambiguous way, hut leave uncertain the inner face and thus the shape of the potential at smaller radii. We explored a range of attractive nuclear potentials, from shallow (19 Mel) to very deep ( $8000 \mathrm{MeV})$, wich gave the same barrier position, height, and curvature. There was very little effect on ofus(E) until the attractive potential became so weak that the total potential exhibited only a shallow 'pocket inside the barrier. This led to osciilations in ofus(E), which could be assceiated with quasi-bound states in the pocket, hut wich are not like the effects seen experimentally in systems like $\mathrm{Ni}+\mathrm{Ni}$.

More iaportant is the presence of a meak 'cail' of the absorptive potential under the harrier. The usual model assumes that ahsorption by direct (surface) reactions is taken intc account explizitly by the coupled channels, while the fusion, or compound nucleus formation. is represented by a short-ranged ahsorption inside the harrier region. In practice, such a clear-cut division is unlikely, and we can expect some residual absorptinn under the harrier itself; i.a.., the harrier becomes complex. Dur calculations show (hat a relatively snall imaginary cart can producy tramatic changes. Figure 5.1 shows an example for $32 \mathrm{~S}+25_{\mathrm{Mg}}$ using the same potential as Ref. ? hust with a Hoods-Saxon form for the imaginary part and its diffuseness increased to $0.535 \mathrm{fm}$. This results in an imaginary potential of $-79 \mathrm{keV}$ at $9.2 \mathrm{fm}$, the posi $t$ inn of the barrier peak, which itself is $2.8 \mathrm{MeV}$ high. The ofus at $F_{\mathrm{cm}}=24 \mathrm{MeV}$ is increased hy a factor of 75. However, the sirape of the ofus(E) curve seen in this figure is not like that seen experimentally, so possibly the hehavior of ufus(E) ahserved at very lon energies can he used to pist limits on the amount of ahsorption that is present. under the harrier.

1. rusest, assignee from Facult:y of Sctence, Atn Shams lintverstty, airo, F.gyot, 6/83-1.:83.
2. See, for example, M. Rhoades-Brom and P. Braun-Munzinger, Phys. Lett. 1368, 19 (1984).

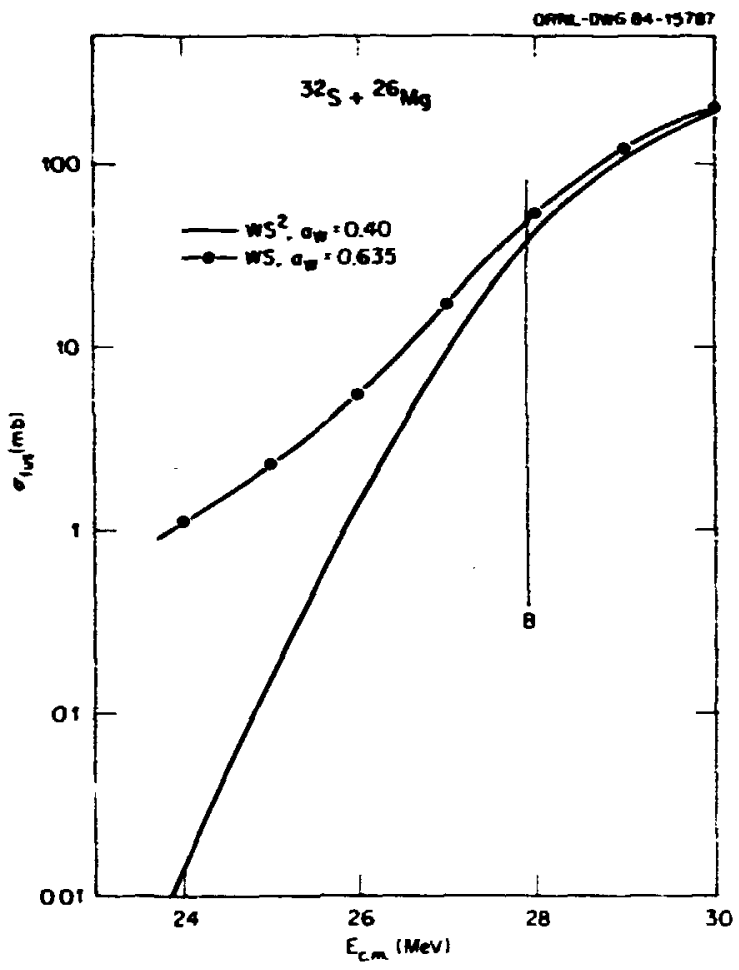

Fig. 5.1. Fusion cross section ofus(E) versus center-of-mass energy. The solid curve was calculated using the optical potential from Ref. 2. in which the imaginary part was the square of a Woods-Saxon form, with diffuseness ay $=0.4$ $\mathrm{fm}$. The broken curve used a similar potential except that the imaginary part had the WoodsSaxon form with ay $=0.635 \mathrm{fm}$. The height of the Coulomb barrier is indicated by $B$. The two curves coincide for energies ahove $33 \mathrm{MeV}$.

\section{COUPLEO ELASTIC ANO INELASTIC SCATTERING OF $160+28$ SI NEAR THE COULONB BARRIER, MITH A NEAKLY ABSORBING OPTICAL POTENTIAL}
M. M. Shalabyl
G. R. Satchler

The effects of using a weakly absorbing opt 1 cal potential on the elastic and inelastic scattering of $1 \mathrm{~h}_{\mathrm{O}}+{ }^{2 \mathrm{~B}} \mathrm{Si}$ near the Coulomb barrier were explored using moth the DNBh and the full coispled-channels treatment. Strong coupling ef rects are important. A fit to the inelastic Hatn at $\mathrm{E}_{\mathrm{cm}}$ - $21.1 \mathrm{Mel}$ appears to require a nuelear deformation length that is apprectably smaller than the Coulamb one.

1. ruest assignee from Faculty of Science, Ain Shams Inntversity, C,afro, E.gypt, 6/83-12/83. 
A DISPERSION RELATION MO THE ERERGY DEPERDENCE OF THE MEAYY-ION OPTICAL POTERTIRL

$$
\text { C. Mahaux }{ }^{1} \text { G. R. Satchler }
$$

The optical potential $U(E)=V(E)+i W(E)$ for the elastic scattering of two systems is, in general, complex and dependent on the energy $E$. Reaction theories imply : very general dispersion relation between the real and imaginary parts of the form

$$
V(E)=V_{0}-\frac{1}{T} P \int \frac{U\left(E^{\prime}\right)}{E-E^{\prime}} d E^{*} .
$$

where $P$ denotes the principal value and $V_{0}$ is independent of $E$. Consequent ly, if $H(E)$ varies rapidly with $E$ in some region, this equation implies that a corresponding rapid variation of $V(E)$ should be observed in the same region of $E$. Empirically, $W(E)$ for heavy :ons appears to depend only meakly upon $E$ for energies substantially above the Coulonb barrier. However, the magnitude of $W(E)$ should decrease rapidly as the barrier is approached because the numbe of ef fectively open nonelastic channels is reduced drastically. This should be reflected in a corresponding change in $V(E)$. Remembering that both $V$ and $W$ are attractive (negative), we see from Eq. (1) that V(E) should bacone more attractive as the Coulonb barrier is approached.

Such an effect has been observed at low ener gies for nucleon scattering, ant interpreted ${ }^{2}$ using Eq. (1). A similar behavior has now been seen $^{3}$ for $160+208 \mathrm{pb}$ and possibly ${ }^{4}$ for $160+$ Ni. A quantitative application of the dispersion relation (1) to these heavy-ion cases is underway.

Physically, $\Delta V(E)=V(E)-V_{0}$ arises from couplings to the nonelastic channels. If the dispersion relation can be used to reliably extrapolate $V(E)$ to lower energies, the important coupled-channels effects on sub-barrier heavyion fusion can be re-expressed in terms of the one-dimensional barrier penetration model. This is being explored.

1. Consultant from Iniversité de Liège, Liège, Belgium.

2. C. Mahaux and H. Ngô, Nucl. Phys. 1378, 205 (1982).

3. J. S. Lilley et al., Physics Letters, to be published.

4. G. R. Sächler, Phys. Lett. 588, 408 (1975).

\section{PATH INTEGRAL APPROACH TO MULTIOIMENSIONAL QUANTUN TUNNELING AND SUB-BARRIER FUSION}

$$
\text { A. B. Ralantekinl N. Taktgawa }{ }^{2}
$$

Recentiy, path integral approach to the coupled channel problem was formulated, transmission probablifties were wrked out for some simple cases, and the properties of the dissipation factor were studied in the adfabatic liait. ${ }^{3}$ We are now in the process of extending this wrk to include the effects of finite temperatures in the tunnelling process. In this context, calculations are being performed on temperature-dependent influence functionals for the three siaple cases stidied earlier, harmonic oscillators linearly or quadratically coupled to the translational motion and a systew with a finite nuber of equidistant energy levels linearly coupled to the translational motion. Also, a siaple many-body model to study microscopic effects is being developed.

1. Eugene P. Wigner Fellow.

2. Tohoku University, Sendai, Japan.

3. A. B. Balantekin and $N$. Takigawa, Annals of Physizs (in press).

\section{EFFECT OF DISSIPATION ON THE EIEENSOLUTIOAS REAR THE FISSION SADOLE POInI}

$$
\begin{aligned}
& \text { K. T. R. Davies } \\
& \text { A. J. Sierk } \\
& 2
\end{aligned}
$$

Two equivalent methods have been developed for solving the problean of small oscillations near equilibrium then dissipative forces are included in the dynamical equations of motion. One method relies on the Lagrangian formulation of the equations of motion, and the other method relies on the Hamiltonian formulation. The eigenvalues are, in general, complex, but for unstable or overdamped motion they are purely real. We have used the Lagrangian formulation to calculate the two lowest symmetric eigensolutions at the fission saddle point for nuclei with $Z^{2} / A$ ranging from 18.0 to 44.6 for ordinary two-body viscosfty, one-body wall-formula dissipation, and one-body wall-and-window dissipation. We find that two-body viscosity leads to relatively small changes in the directions of the efgenvectors, thereas one-body dissipation can lead to major changes in the directions of the eigenvectors. Also, with increasing dissipation, the fission eigenvalue $\lambda_{2}$ decreases in magnitude, and the stratching eigenvalue $\lambda_{4}$ changes from imaginary through complex to real and negative.

1. Summary of paper: Phys, Rev. C 28, 1181 (1983).

2. Los Alamos National Laboratory, Los Alamos, 87545 .

\section{STUDIES OF CONDITYONAL SADDLE POINT CONFIGURATIONS!}

$$
\text { K. T. R. navites A. J. Sterk }{ }^{2}
$$

We have developed a gereral method for determining an extremum on a potential energy surface subject to an arbitrary number of constraints. The baste equations are formulated using 
Lagrange min cipliers, and the extremun is obtained by iterating using a vector version of Newtor's method. We then specialize the problem to a single constraint wich can be, e.g., a multipo!e moment of the muclear shape or a parameter specifying the mass asymetry of the system. All of our calculations have been done for the constraint of mass asy metry wich wight typically represent the initial value of mass asyumetry of a particular heavy-ion reaction. Such calculations then give the conditional saddle points wich are trought to enable one to distinguish theoretically fast fission processes from true caspound nucleus formation. ${ }^{3-5}$ The advantage of the present method is that the conditional saddle points can be calculated when the mass asymmetry function is an arbitrary function of the general ized coordinates. This
should be contrasted with other studies $3-6$ in wich the mass asmmetry variable is one of the chosen generalized coordinates, in wich case the conditional saddle points can be determined by simply fixing the asymetry coordinate.

Our results have been obtained mainly with the liquid-drop model? surface energy. A study comparing the results using the liquid-drop model with those of the Yukawa-plus-exponential nuclear energy ${ }^{6}, 3$ shows that, for the latter. one obtains a larger neck for a given mass asymmetry. However, the main conclusions of our work do not depend on the type of macroscopic energy considered. In order to describe the nuclear shape which is assumed to be axially syametric, we use two different parametrizations: (i) a model consisting of sinoothly joined portions of three quadratic surfaces of revolution, $t 0$ and (ii) a Legendre polynomial expansion of the nuclear surface function." II

In Fig. 5.2 display various saddle points on $\sigma-r$ plots where $\sigma$ and $r$ represent respectively necking and separdtion degrees of freedom. By comparing the upper and lower parts of the figure, we that, for a given fissility $x$, as we increase the mass asymmetry $a$, the moments of the system effectively behave like those of

ighter nuclei. This occurs because, in a heavy-ion reaction appropriate to a given mass asymetry, the effective entrance-channel Coulomb repulsion is proportional to $Z_{1} \cdot Z_{2}$, where $Z_{1}$ and $Z_{2}$ are the projectile and target charges. Then, for a fixed total charge of the system, as a increases $Z_{1} \cdot Z_{2}$ decreases, giving rise to a less repulsive system.

In Fig. 5.3 we display the conditional saddle point energy as a function of mass asymmetry $a$, for various values of the ffssility $x$. The behavior of the energy depends upon whether the fissility is ahove or below the Businaro-Gallone point, ${ }^{12} x_{B G}=.396$. For $x<x_{B G}$ the energy always decreases monotonically with increasing $a$, On the other hand, for $x>x_{B G}$, as $x$ increases, the energy first increases until it reaches a maximum corresponding to the Busforaro-ijallone peaks, after wich it decreases. In the nelghborhond of $x=x_{B G}$, the saddle point energy with respect to $a$ is very flat due to the calescence at $a=0$ of the Bohr-Wheeler mass symmet,ric saddle family with the Businaro-Gallone mass asymetric saddle family. For $x=0$ the condi-

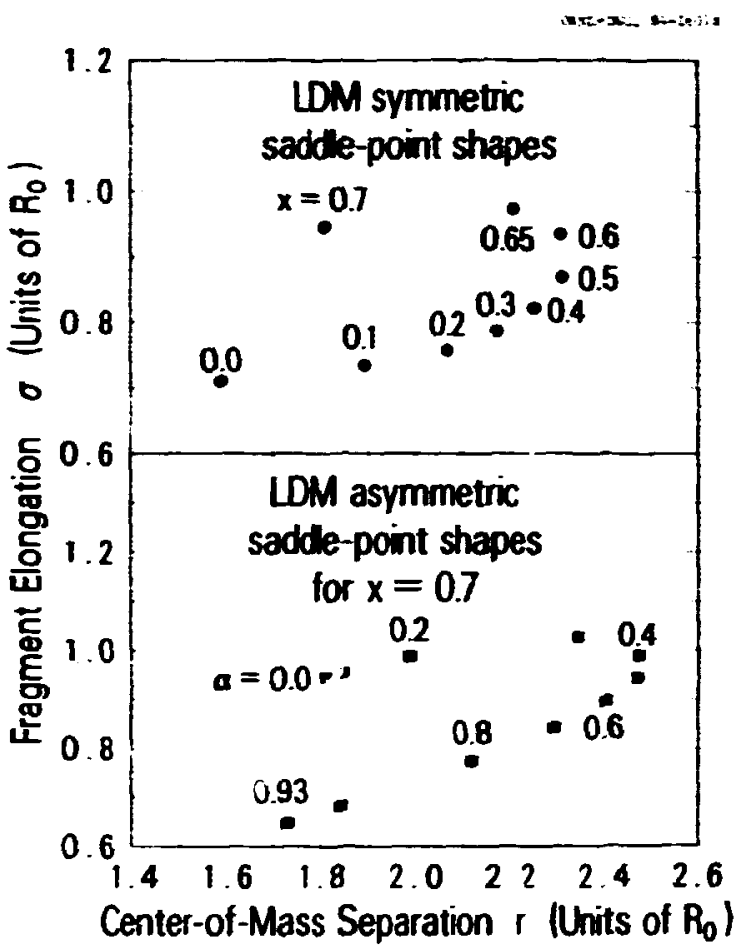

Fig. 5.2. Locations in o-r space of the saddle point.s of liquid-drop nuclei. The upper portion of the figure shows the positions of the Bohr-Wheeler family of symetric saddle points for selected values of the fissility parameter $x$. The lower portion shows the conditional saddle points as a function of constrained mass asymetry $a$ for $x=0.7$; the a values are in increments of 0.1 , except for the largest value $(\alpha=0.93)$.

tional saddle point is always the Bonr-Wheeler saddle, while for a approaching $l$ it corresponds to a single large sphere attacher to an infinitesimally small sphere.

1. Summary of paper to be submitted to Physical Review.

2. Los Alamos National Laboratory, Los Alamos, 87545 .

3. W. J. Swiatecki, Phys. Scr, 24, 113 (19R1).

4. W. 3. Swiatecki, Nucl. Phys, A376, 275 (1982).

5. S. Bjornholm and H. J. Swiatecki, Mucl.

Phys. A391, 471 (1982).

6. H. Feldmeter, p. 26 in Proceedings of the International Workshop $x$ on Gross Propettes of Nuclef and Nuclear Excitaltons, Hirschegg.

Austrfa, 1982, eत. hy H. FeTdmeier, Technische Hochschule, Darmst,adt, Report No.

ISSN-0720-8715, 1942.

7. W. D. Myers and W. J. Swiaceckt, Ark.

Phys, 36, 343 (1967). 
B. H. J. Trappe, J. R. Mix, and A. J. Sierk, Phys. Rev. Lett. 42, 215 (1979).

9. H. J. Krappe, J. R. Mix, and A. J. Sierk, Phys. Rew. C 20, 992 (1979).

10. J. R. Wix, huci. Mys. N30, 241 (1969).

11. S. Trentalange, S. E. Toconin, and A. J. Siert, Mrs. Dev. C Z2, 1159 (1900).

12. "I. L. Businaro and 5. Gallone, horo Cim. 1. 629 (1955); U. L. Dos inare and S. Gallane, heovo Cie. 1, 1277 (1955).

$\operatorname{lax}_{x \rightarrow-\operatorname{lom}}$

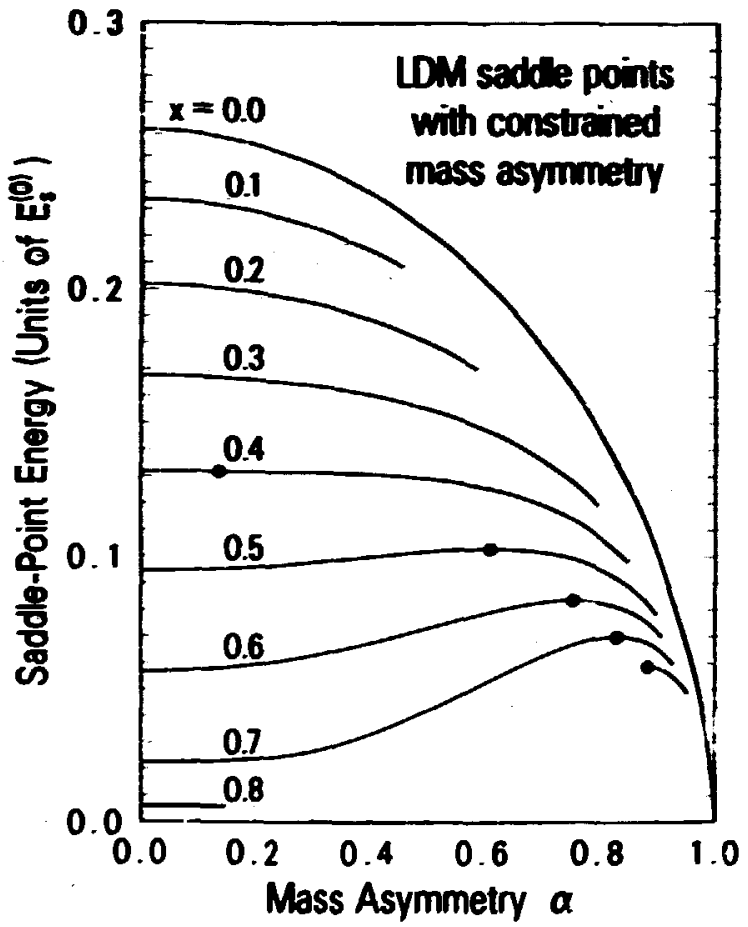

Fig. 5.3. Saddle-point energies as a function of constrained mass asymetry a for various values of the liquid-drop model fissilfty parameter $x$. The solid points correspond to the Businaro-Gallone family of asymmetric saddlepoint shapes with two unstable degrees of ireedom. The solid Iines terminate to the right at shapes with very small neck radif, beyond wich we cannot calculate. The curve for $x=0.8$ is not drawn where no constrained saddle point exists.

MACROSCOPIC ANO MICROSCOPIC FUSIOH STUDIES OF THE 208pb+58Fe REACTION
J. R. Hix $x^{1}$
A. J. Sierk 1
M. R. Strayer
K. T. R. Davies
A. K. Dhar ${ }^{2}$
A. C. Merchant 3

Macroscopic 4 and microscopic calculations are being done for the reaction $20 \mathrm{PPb}+{ }^{59} \mathrm{Fe}$ at fife bombirding energies corresponding tn cases wich have heen studied experimentaliy. ${ }^{5}$ in the macroscepic studies we examine the dependence upon anglar od eath $l$ of the asss transfer Afe to the lighter 50Fe moleus fro the bavfer 200 Po mucleus. Figare 5.4 displays Affe vs. I for the five energies considered, using towbody viscosity as the dissipation echantse. This figare shous that, for a given bubarding energy, the mass transfer is aero for angular momenta larger than a critical vilue at wich the muclei first come into contuct, at mich point it jups discontinuously in our calculations to a nonzero value. The mss transfer then increases with decreasiag angular minenta until values correspeading to a mass-synutric melear system are reached, belou wick sone oscillations eccur.

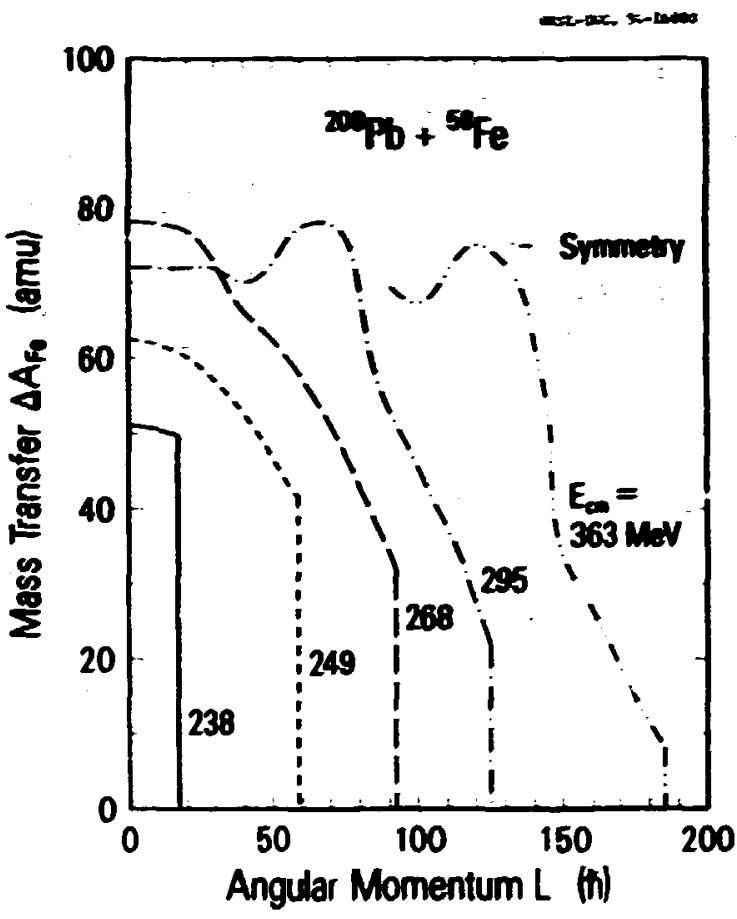

Fig. 5.4. Mass transfer $\triangle A F f e$ vs. angular momentum $L$ for the reaction $20 \mathrm{Bpb}+{ }^{5}$ bee. The calculations were done using two-hody viscosity with viscosity coefficient $\omega=0.02 \mathrm{TP}$.

In calcuiating the theoretical macroscopic capture cross section, we include all L values corresponding to mass transfers greater than 40 anu, and the results are displayed in Fig. 5.5. The error bars on the experimental points reflect the differences hetween two experimental procedures for calculating the cross section (one of mich is fientical to the theoretical method). Other than the lowest bombarding energy, our calculated curve for two-body vis. costiy lies substantially above the experimental pofnts, with the deviation increasing to almost a factor of 2 at, the highest bombarding energy. 


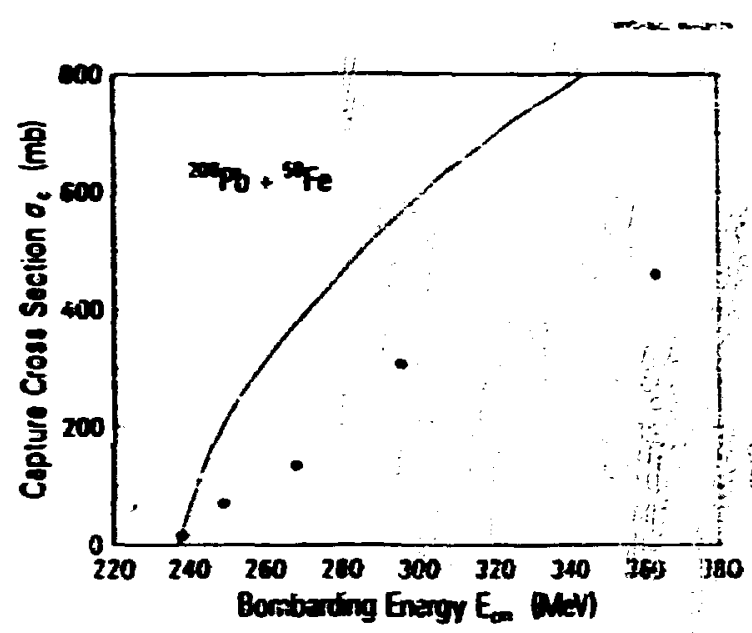

Fig. 5.5. Comparison of the experimental capture cross section with the theoretical results calculated for two-body viscosity, with viscosity coefficient $\nu=0.02 \mathrm{TP}$. The full curve smothly joins the theoretical points, while the full circles with error bars are the experimental results.

We regard this important discrepancy as experimentally demonstrating that two-body viscosity is not the complete dissipation mechanism in large-xiplitude collective muclear motion. additional calculations are being done using onebody visensity, taking into account the dissipation associated with a time rate of change of the mass asymetry degree of freedom in the completed wall-and-window formula.'

Some prel iminary time-dependent Hartree-fock (TDHF) calculations of this reaction have also been done. For example, studies using the Skyrme III effective two-body inter action give the following results for the capture cross sections

$$
\begin{aligned}
& { }_{{ }_{c}}\left(E_{c m}=238 \mathrm{meV}\right)=195 \pm 100 \mathrm{mb} \\
& { }_{c}\left(E_{\mathrm{cm}}=268\right)>649 \mathrm{mb} \\
& { }_{{ }_{c}}\left(E_{\mathrm{cn}}=363\right)=180 \pm 111 \mathrm{mb},
\end{aligned}
$$

where wave indicated the uncertainties in determining fuston. Comparing these microscopic cross sections with the macroscopfe results shown in $\mathrm{Fig} .5 .5$, we see that for $E_{C R}=238$ and $268 \mathrm{HeV}$ the TOHF ifmits are considerahly above the macroscopte curve. On the other hand, the TDHF cros: section for $E_{\text {rem }}=363 \mathrm{MeV}$ may not he very different irom the macroscopic value. In any case, the TDHF results st.rongly disagree with the experimental values, wich are ilways below the macroscopic curve in Fig.. .5 . However, wave previousiy tmonstrated that different effect fve two-body interactions can afve widely varying fusion results.", Therefore, additiond stiudfes are being done using the modified Skyme it interaction wich correctly reproduces nclear chapressibilities and fission byrier teifits. 19

$\frac{i}{4 i}$

i. Los bijeos tational Laborator, Los Aticios, Tres45.

2: Depertinet sf Mysics, McGill miversity. Mowtreal 0 ; HM, 2Fo Canda.

3. Mestent zuress: Centro Técnico Aecriespoxial, Inst ituto de Estudos Avancados, SEQ Parth, thazil.

i. A. if! six and A. J. Siert, (inwited puger) prissinted at the International Conference of Theirettical Approaches to Heary Ion Reaction Hechavitues - Paris, France, way 14-18, 1924.

5. Hock, T. T. Che, R. Qutourki, $A$. colbi i. Grosse, A. Olai, H. Sann; D. Schala, U. fucin, H. niller, S. Ejormbolm, H. Esbensen, M. Wolfi, i, and E. Morenzeni, Mucl. Phys. Ases, 334 (1982).

5. K. T. R. Davies, A. J. Siert, and J. R. Nix, Phys. Rev. C 13, 2385 (1976).

7. J. Randrup and $u$. J. Swiatecki, Lamrence Berkeley Laboratory preprint [BL-17273 (19A4).

B. R. T. R. Davies, R. R. S. Devi, and M. R. Strayer, Phys. Rev. C 24, 2576 (1981).

9. J. R. Maruhn, R. T. R. Davies, and H. R. Strajer, to be publ ished.

In. J. Bartel, P. Duentin, M. Brack, C. Guet, and H. B. Mikansson, Murl. Phys. B386. 79 (1982).

GEONETRY MU DTMMICS OF A ZERO-TEMPERATLRE, FERAI-GAS WOEL FOR PRE-EQUILIBRIUA EMISSION CE maEOns, MTH APPLICATION TO $16 \mathrm{O}+{ }_{3 \mathrm{mb}} \mathrm{AT} \mathrm{E}_{\mathrm{Lab}}=204 \mathrm{MeV}^{2}$
K. T. R. Davies
M. R. Strayer
B. Remaud ${ }^{2}$
Y. Raffray ${ }^{2}$
K. R. Sandya Devi ${ }^{3}$

A detailed motel for prompt, fa:: nucleon (pre-equilibrium) nucleon enission has been developed and analyzed. The hasic scattering behavior in the early stages of the collision is described by a classical traiectory calculation, with particle enission arising from a zerotemperature, Fermi-gas approximation. The emission of a nucleon wich has a veloctey component nomal to the reaction plane is taken into acccunt in the formalism. The refraction of the nucleon trajectories passing through the mean field has been studied and iffferent prescriptions for the ahsorption of nucleons have been carefully investigated. The method has

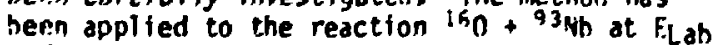
$=204 \mathrm{MeV}$, and various comparisone have been made with particle enission occurring in a recent DHF calculation. It is found that the calculated multiplicity is greatest for head-on collisions. In addition, inclusive and in-plane energy spectra and angular distributions have beon stiudied. The emission of forward-moving nucleons from the profectile (which pass through the target) takes place predominantiy in the reaction plane and the angular distribution is 
stroagly peaked, approxintely in the formard direction, with the energy spectru centered sbout the bean energy per mucleon.

1. Sunary of paper: Ann. Phys. 156, 68 (1984).

2. Institut de Physique, Université de tantes, 4072 Cedex, France.

3. Departwent of Physics, University of Arizona, Tucson, 12 85721.

\section{TIRE-DEPEMENT MRTAEE-FOCX STUDIES of the SEISITIVITY of DH TO The EFFECTIVE DT-BODY InTERACTION'}

$$
\begin{aligned}
& \text { J. A. Maruhn }{ }^{2} \text { R. T. R. Davies } \\
& \text { M. R. Sirayer }
\end{aligned}
$$

There has heen great "aterest in recent jears in studies of dynaical thresnolds wich govern fusion behavior in heavy-ion reactions. ${ }^{3-5}$ Two such thresholds can be identified. The first is the low-energy thresho'd which is associated with the phenowenon known in macroscopic studies as the extra push (or, in some cases, the extraextra push). th This threshoid can be characterized as follows. For head-on collisions of sufficiently light systems, fusion occurs for center-of-mass (CM) bombarding energies greater than or equal to the interaction barrier height. However, for a fixed cu energy, if one increases the repulsive potential energy of the systen by increasing the charnas of the target or projectile (or the orbital ongular momentum). fusion becomes less probable. Thus, e.g., for head-on collisions of a system exceeding a critical fissility, ${ }^{3-7}$ fusion will not occur unless the $\mathrm{CM}$ energy exceeds the barrier height by a certain threshold value known as the extra-push energy. This threshold occurs theoretically in both macroscopic ${ }^{3-7}$ and microscopic ${ }^{9}$ studies, and its existence is wil established experimentally. 5-7

The second type of dynamical threshold occurs only in TDHF calculations at relatively high energies, ${ }^{8}, 9$ where it is found that fusion diruptiy disappears for head-on collisions. This threshold is known as the TOHF angular moment un window. For energiks above this threshold, there exists a low-angular-momentum cutoff below which there is no fusion. This behavior is intimately associated with the transparency inherent in the mean-fjeld approximation and its existence is thought to be very questionable experimentally. 60 We have completed TDHF calculations primarlly devoted to the microscopic ana'ogue of the macroscopic extrapush threshold, although we have al so obtained some results for the window threshold.

TDHF studies of $86 \mathrm{Kr}+139$ La show that the extra-push fusion threshold is very sensitive to the two-hody effective interaction used. Figure 5.6 for head-on collisions. fllustrates how the dynamical hehavior depends upon the interaction. In this figure, display the rms radius of the total system as a function of time for Elan. $370 \mathrm{MeV}$ for the five Skyrme forces considered.

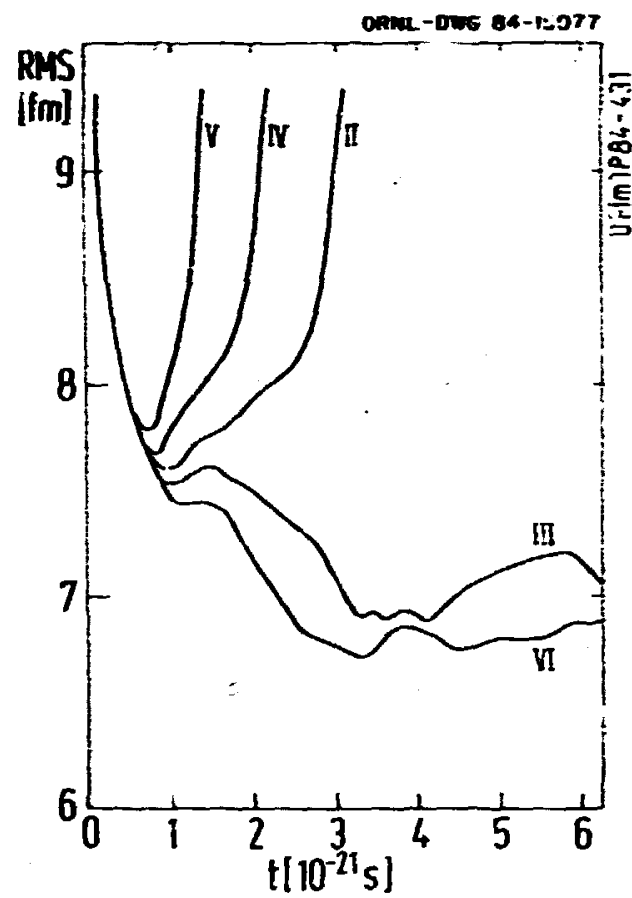

Fig. 5.6. The mos radius of the total systen as a function of time for head-on collisions of $86 \mathrm{Kr}+139 \mathrm{La}$ at $E_{\mathrm{Iab}}=370.0 \mathrm{MeV}$. The Roman numeral on earh curve labels the Skyrme force used.

The general behavior shown in this figure is similar to that obtained at other energies. The initial minimum of the rms radius depends strongly on the frorce. Then the smaller the value of this minimum, the more likely the system is to fuse. For Skyrme forces II, IV, and $V$ the initial coalesced system clearly reseparates, wile it is our interpretation that the reactions for Skyrme forces III and VI lead to true compound nucleus formation. Thus, the tendency to fuse decreases according to the following sequence of Skyrme forces: VI. III, II, IV, and $V$.

He sumartze the results for our fusion threshold studies in Fig. 5.7. The error bars in this figure show the uncertainties in determining the calculated thresholds. The calculations were performed in the lahoratory energy range from 370.C, to $830.0 \mathrm{MeV}$. The lower energy limit was cnosen hecause $i t$ is somewhat above the macroscopic interaction harrier, and we did not exceed the upper limft due to our concern both about numerical instahility and ahout possible important corrections to TDHF at higher energles. For skyrme forces III and VI, there is essentially no extra-push energy required since fuston occurs for all energies down to the interaction harrier. On the other hand. for Skyrme $V$, there is a iarge extra-push energy since the fusion threshold lies between 790.0 $\mathrm{MeV}$ and $830, \mathrm{MeV}$. This demonzerates that the ext,ra-push thresholds for dtfferent Skyrme 


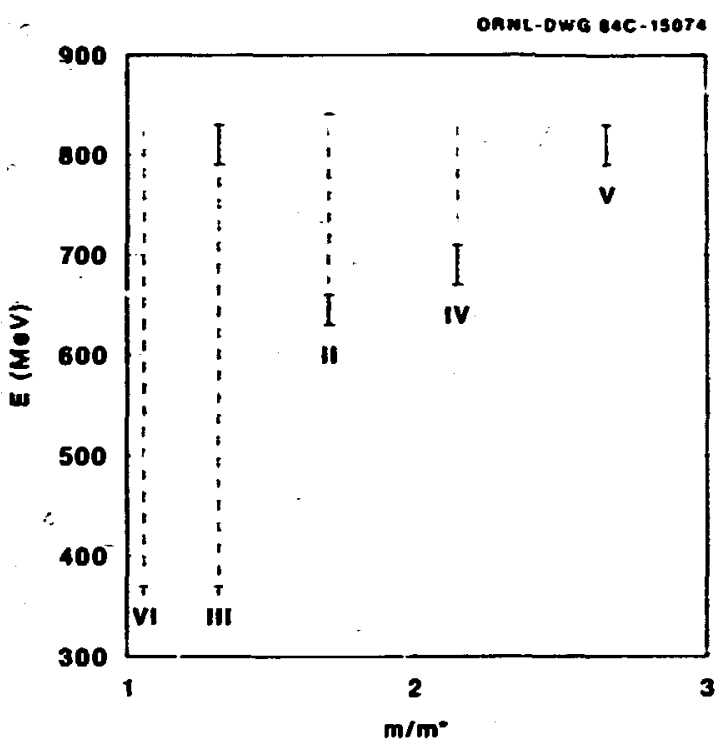

Fig. 5.7. Dynamical TOHF fusion thresholds for head-on collisions of ${ }^{86} \mathrm{Kr}+139 \mathrm{La}$. The laboratory energy thresholds for different Skyrme forces are plotted as a function of the inverse of the effective mass rat, $\mathrm{m} / \mathrm{m}^{*}$. The error bars indicate the uncertainties in determining the threshold. The lower error bars denote the extra-push fusion threshold, wile the upper error bars, when present, indicate the window threshold. The dashert lines joining the lower and inper error bars denote the fusion region. The ioman numerals label the Skyrme forces.

forces can differ by almost $500 \mathrm{MeV}$. Also, for Skyrme forces IV and $V$ ? after fusion occurs at lower energies, it continues un to $830.0 \mathrm{MeV}$, and no window threshold was determined in the energy range uncier consideration.

A1so, studies of both ${ }^{36} \mathrm{Kr}+139 \mathrm{La}$ and $15_{0}+$ 160 demonstrate that the relatively high-energy, angular momentum window threshold exhibits a pronounced force dependenc'e. In partícular, for $160+16_{0}$ the thresholds for skyrme II and IV differ by $20 \mathrm{MeV}$, with the threshold for Skyrme II only a few MeV below the energy usen in the experiment 10 which seemed to rule out the existence of the window. We suggest that it would be desirable to reanalyze the experimental and theoretical information, taxing finto account more carefully the force depentence of the window threshold.

1. Sumiary of paper to be submitted to Phyicical Review.

2. Consultant from University of Frankfurt, Frankfurt, Hest Germany.

3. J. R. Nix and A. J. Sterk, Phys. Rev. C 15, 2072 (1977).

4. W. J. Swiatecki, Phys. Seriptd 24, 113 (198!).
5. W. J. Smiatecki, Mucl. Phys. A376, 275 (1982).

6. S. Bjdrnholm and H. J. Suriatecki, Mucl. Phys. 1391, 471 (1982).

7. K. T. R. Davies, A. J. Sierk, and J. R. Mix, Phys. Rev. C28, 679 (1982). This paper contains additional theoretical and experimental refert.. - relevant to dynamical fusion threshol ds.

8. P. Bonche, K. T. R. Davies, B. Flanders, H. Flocärd, B. Gramaticos, S. E. Koonin, S. J. Krieger, and M. S. Weiss, Phys. Rev. C 20, 641 (1979).

9. K. T. R. Davies, K. R, S. Devi, and M. R. Strayer, Dhys. Rev. C 24, 2576 (1981).

10. A. Lazzarini, H. Doubre, K. T. Lesko, V. Metag, A. Scanster, R. Vandenbosch, and M. Merryfield, Phys. Rev. C 24, 309 (1981).

\section{TIRE-DEPEMENT HRRTREE-FOCK CALCULATIONS OF NUCLEAR MOLECULAR RESOWAMES 1}
M. R. Strayer
R. Y. Cusson 3
A. S. Imar ${ }^{2}$
P.-G. Reinhard ${ }^{4}$

He have performed time-dependent Hartree-Fock (TDHF) calculations at bombarding energies near the Coulonb barrier for " $\mathrm{He}+{ }^{14} \mathrm{C}$ systems. The collective oscillations of the nuclear density are followed in time and analyzed in terms of their classical quasiperiodic behavior. Using the density constrained Hartree-Fock method, we have also calculated the position of the DDHF collective path with respect to the multidimensioril energy surface of the compound nuclear system. In all of these cases the DHF path is found to oscillate about the shape isomeric minimum of the compounc system. The moments of the density are defined as

$$
\begin{aligned}
& M_{L I}(t)=\int d^{3} r r^{2} Y_{L O}(\hat{r}) p_{I}(\vec{r}, t) \\
& M_{L I}(\omega)=\int d t \exp (-i \omega t) M_{L I}(t)
\end{aligned}
$$

where the index I identifies the isoscaiar $(I=0)$ and isovector $(I=1)$ components. In $F ! g .5 .8$ some of these moments are shown for the ${ }^{4} \mathrm{He}+{ }^{14} \mathrm{C}$ system. Ne clearly ste an isoscalar octupole frequency at $4 \mathrm{MeV}$, two isoscalar quadrupole frequencies at 8 and $14 \mathrm{MeV}$, and an isovector dipole frequency at $4 \mathrm{MeV}$. The large deformations and characteristic slustering, observad in our calculations, suggest these collective states may correspond to molecular resonances ohserved in Ref. 5.

1. Summary of paper: Phys. Lett. 1358, 261 (1984).

2. Guest assignee from Wright Nuclear Structure Lahoratory, Vale University, New Haven, CT 06511.

3. Consultant from Duke University, Durham, NC 27706 .

4. Consultant from University of Friangen, Er!angen, West Germany.

5. M. Gal et al.., Phys, Rev, Lett, 50, 239 (1983). 


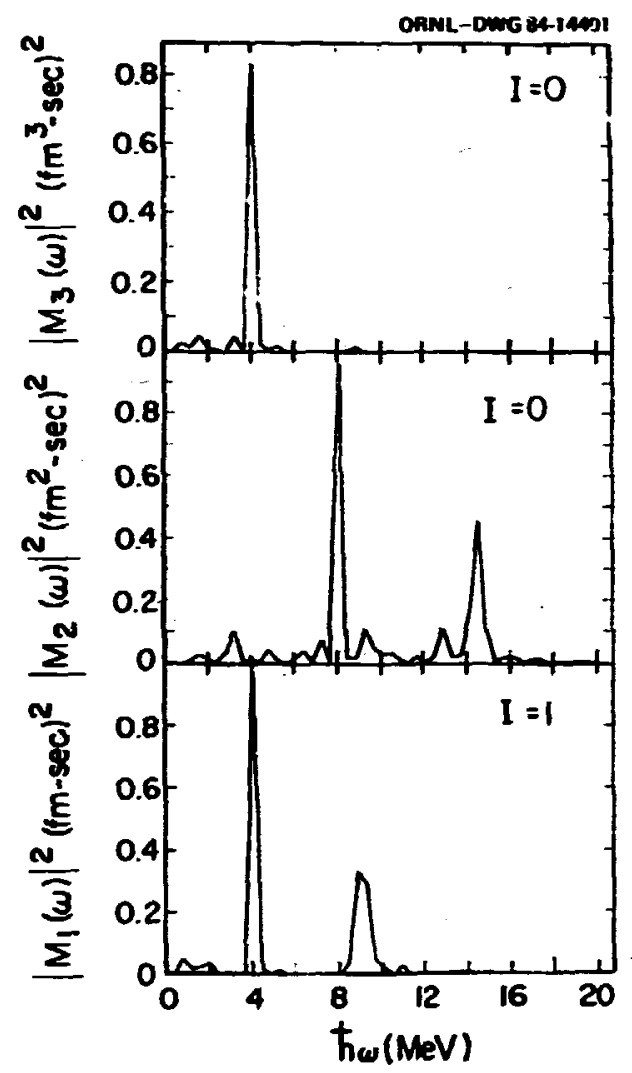

Fig. 5.8. The frequency dependence of the isoscalar quadrupole, isoscalar octupole, and the fsovector dipole moments for the 180 system.

\section{A TIME-DEPEMDENT IEAN-FIELD THEORY FOR PROMPT} MUCLEON EMISSIOW IH HEAYY-ION REACTIONS

$$
\text { A. S. Umar2 }{ }_{\text {D. J. Ernst }}^{3} \text {. Strayer }
$$

We have developed a time-dependent mean-field theory for the study or prompt nucleon emission in heavy-ion reactions. The model treats the relative motion of the fons clessically intle treating the internal excitations quantum mechanically. The motivation for the model comes from earlier time-dependent Hartree-Fock studies." Here, we study enission from one of the heavy ions inile consider the other as a structureless particle, located at a distance $R$ from the center of the enftting fon, producing a time-dependent field. This assimption is valid for mass asymmetric systens. Under thest conditions, the Hamiltonian for the system becomes

$$
H=H_{0}\left(\left\{r_{i}\right\}\right)+i_{k}+U\left(\vec{k},\left\{\dot{r}_{i}\right\}\right)
$$

where $H_{0}$ is the intrinstc Hamiltenian of the emitting fon and depends on the nucleon coordinates $\left\{r_{1}\right\}, r$ is the relative kinetic energy of the inns, and If is an external one-body field wich induces the coupling between the two fons. The nne-hory nature of if is an assumntion of our model. The warefunction associated with $H$ is taken to be a product form

$$
z\left(\left\{\vec{r}_{i} \mid, \vec{k}, t\right)=G(\vec{k}, t) \bullet\left(\left\{r_{j} \mid, t\right)\right.\right.
$$

were $\mathbf{G}$ describrs the motion of the structureless particle and is a time-dependent slater determinant for the mucleons in the emitting nucleus. Variation of the action with respect to $G^{ \pm}$and the single-particle states of yields coupled equations governing the relative motion and the intrinsic dymanies of the emitting nucleus, respectively. The expression for the invariant single cross, section is

$$
\frac{d^{3} d(1)}{d k^{3}}=\frac{1}{(2 \pi)^{2}} \sum_{\lambda=1}^{A} \int_{0}^{b_{\max }} d b b\left|c_{k \lambda}(t=-)\right|^{2}
$$

were b denotes the impact parameter, and $C_{k_{\lambda}}$ denotes the probability for occupying the continuma state $k$.

We have used as our external field a rank one separable potential having its range adjusted to contain the interaction within the strong absorption radius of the system and strength adjusted to the experimental altiplicity. We have studied neutron enission from the reaction $160+9{ }^{3} \mathrm{~Wb}$ at $E_{l_{a b}}=204 \mathrm{MeV} .5$ In Fig. $5.9 \mathrm{we}$

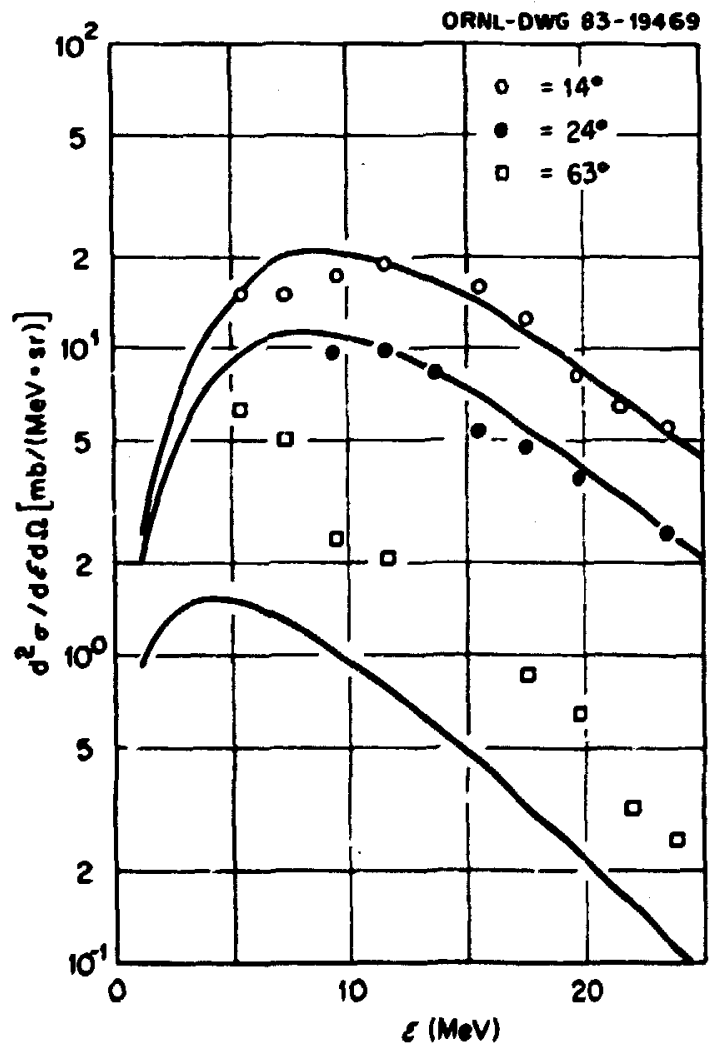

Fig, 5.9. Double differential cross sectiois as d function of laboratory energy for angles $14^{\circ}, 24^{\circ}$, and $63^{\circ}$. 
Show the double differential cross sections together with the experimental results. The discrepancy at large ang?es may be due to the exclusion of enission from ito.

1. Sumary of paper: Phys. Lett. 1408, 290 (1984).

2. Guest assignee fran Hright Nuclear Structure Laboratory, Yale University, New Haven, Ci O6511.

3. Consultant from Teras A \& M University, College Station, $D 77843$.

4. K. R. S. Devi et a.,., Phys. Rev. C 24, 2521 (1981).

5. A. Garron et al., Phys. Rev. Lett. 46, 8 (1981).

\section{H-N CORRELATIONS IN INCLUSIVE MOMEQUILIBRIM PARTICLE EMISSION ${ }^{2}$}

\author{
A. S. Unar ${ }^{2}$ M. R. Strayer$$
\text { D. J. Ernst }{ }^{3}
$$

Recent experiments ${ }^{4}$ have shown pronounced nucleon-nucleon correlations in inclusive particle enission fron heavy-ion reactions. Within tis framework of Koonin's formalism $\mathrm{m}^{5}$ the associated spatial localization calculated and interpreted as evidence for the formation of a "hot spot." We have recently developed a timedependent mean-field model for prompt nucleon emission in heavy-ion reactions. 6 In this model the two-nucleon cross section is given by

$$
\begin{aligned}
\frac{d^{6} d^{(2)}}{d^{3} k d^{3} q} & =\frac{1}{(2 \pi)^{5}} \int_{0}^{b_{\max }} d b \text { b }\left\{\sum_{\lambda, \nu=1}^{A}\left|c_{\lambda k}\right|^{2}\left|c_{\nu,+}\right|^{2}\right. \\
- & \left.\left|\sum_{\lambda=1}^{A} c_{\lambda k}^{*} c_{\lambda+\left.\right|^{*}}\right|^{2}\right\}
\end{aligned}
$$

where $b$ denotes the impact parameter, and $C_{k}$ denotes the probability for occupying the continuum state $k$.

Since are working with a mean-field model. it is important to understand the source of these corre'ations. Our wavefunction is, at all times, a siater deteminant. The ant isymetry of the wavefunction leads to the second term on the righthand side of Eq. (I). Dylromfcal long. range correlations are included in the timedependent field, and, as in time-dependent Hartree-fock theory, the short-range correlatfons serve to renormalize the interaction and produce a mean field. Within this model, we ignore the contribution of the particle exchange and polarization to the mean field and decomir.e it from the entrance channel configuratifons. The calculation of $\mathrm{Fq}$. (1) was done for the newtron emisston froin the sysiem $1 b_{n}+334 / 6$ at r. ab $=204 \mathrm{MeV}$. For this system we ftrst adfusted the paraineters of the externa' fiald to fit the singles neutron spectra. The details of the singles calculdtion are given in sef. 6 . We

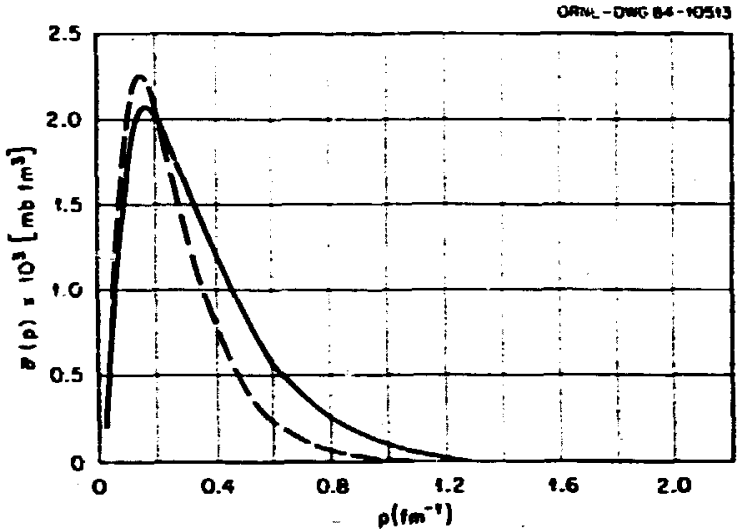

Fig. 5.10. Two-neutron cross section of Eq. (1) as a function of relative neutron momentum (solid line) and the same curve with the addition of final-state interaction (dashed curve). The dashed curve is divided by 100 .

then calculated the two-neutron cross section of Eq. (1) as a function of the rel ative neutron velocity as shown in Fig. 5.10 (solid line). The results show the characteristics of the twoparticle cross sections obtained for other systens. 5 A naive application of the uncertainty principle to the half-width of the two-neutron cross section of Fig. 5.10 yields a space localization of $2.5 \mathrm{fm}$, about the radius of the ${ }^{160}$ nucleus.

There is another type of correlation wich we treat using a final-state interaction model. When two nucleons are enitted in nearly the same direction, then they can interact substantially before they reach the detector. This two-body interaction is short range and is dominated by the deuteron pole. He have used the separable interaction of Yamaguchi as our residual interaction. The dashed line of Fig. 5.10 shows the efrect of this final-state interaction upon the two-neutron cross section. As we see, the effect of the final-state interaction leaves the moment um localization essentially unchanged.

1. Summary of pasers to be published in Physical Review $C$ and Proceedings of International Conference on Fuston Reactions below the Coulomb iarrier.

2. Fivest assignee from Wright Nuclear Structure Laboratory, Yale Unfversity, New Haven, C.T 06511 .

3. Consultant from Texas A \& M University, College Station, TX 77843.

4. W. F. Lynch et al.. Phys. Rev. Lett, 5!, 1850 (1983).

5. S. F. Konnin, Phys, Let.t. 70B, 43 (1977).

5. A. S. Imar, $M$. R. Strayer, and $0 . J$. Ernst, Dhys. Let.t. 1408, 290 (1984). 
CHAS In THE-DEPEWEAT HRTREE-FOCX COLISIOUS OF REAV IO.S'
M. R. Strayer
R. Y. Cusson ${ }^{3}$
A. S. Unar
P.-G. Reinhard"

Recently we performed time-dependent HartreeFock (TOHF) calcul ations, at bowbarding energies near the Coulanb harrier, for "He+ ${ }^{14} \mathrm{C}$, ${ }^{12} \mathrm{C}+{ }^{12} \mathrm{C}\left(\mathrm{O}^{+}\right)$, and $\mathrm{He}+{ }^{20}$ he systems. In particular, we exanined the collective oscillations of the muclear density in terms of its moments

$$
\begin{aligned}
& M_{L I}(t)=\int d^{3} r r^{2} i_{L 0}(\dot{r})_{D_{I}}(\vec{r}, t) \\
& M_{L I}(t)=\text { :dt } \exp (-i \omega t) M_{L I}(t)
\end{aligned}
$$

where the index I labels the isoscalar $(I=0)$ and isovector $(I=1)$ components. We have also cal' $\mathrm{J}$ lated the classical frequencies associated wit each of these col lective degrees of freedom. The position of the IOAF path, with respect to the uultidimensional energy surface of the com pound system, as also calculated. In all of these cases the IDHF path was found to oscillate about the stape isomeric minimum of the compound system.

Depending on the amplitude of these oscillations, the motion can be identified as being chaotic or quasiperiodic. For a more el aborate identification, we plot the Poincaré projects for the motion of the ${ }^{12} \mathrm{C}+{ }^{12} \mathrm{C}\left(\mathrm{O}^{+}\right)$system in Fig. 5.11. He see that both the isoscalar quadrupole and octupole modes seen to be filling most of
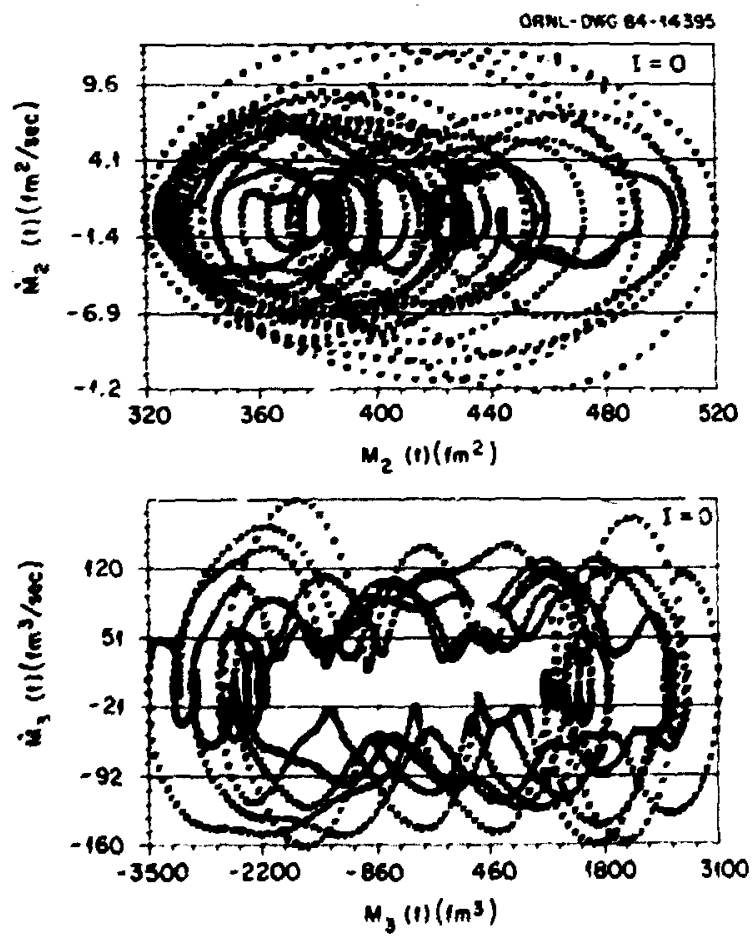

Fig. 5.11. Poincare phase space plots of $M_{L J}(t)$ vs. $A_{L J}(t)$ for the $1=0, L=2,3$ modes of the $24 \mathrm{Mg}$ system. the available phase space. The corresponding autocorrelation functions

$$
c_{\mathrm{LI}}(t)=\int \frac{d \omega}{2 \pi} \exp (i \omega t)\left|n_{L I}(\omega)\right|^{2}
$$

are sall for all of the relevant modes suggesting that the motion is closer to being chaotic rather than harmonic. In order to ieprove the periodicity of the TOHF results, we have "cooled" the system, thus bringing it closer to the shape iscmeric minims. The "cool ing" was done by perfonming a density-constrained Hartree-Fock calculation at a point on the THF path. This result was then used as the initial state for continuing the time evolution.

In Fig. 5.12 we show the time dependence of the moments for the "cooled" motion of the ${ }_{12} \mathrm{C}+{ }^{12} \mathrm{C}\left(\mathrm{O}^{+}\right)$system. The correlation functions associated with these ades are large, indicating a quasiperiodic behavior. These results are important from the point of view that the quasiperiodic motion represents a knom classical limit of quantum mechanics, wereas the chaotic motion does not. Figure 5.13 shows the classical frequencies for the "cooled" motion of

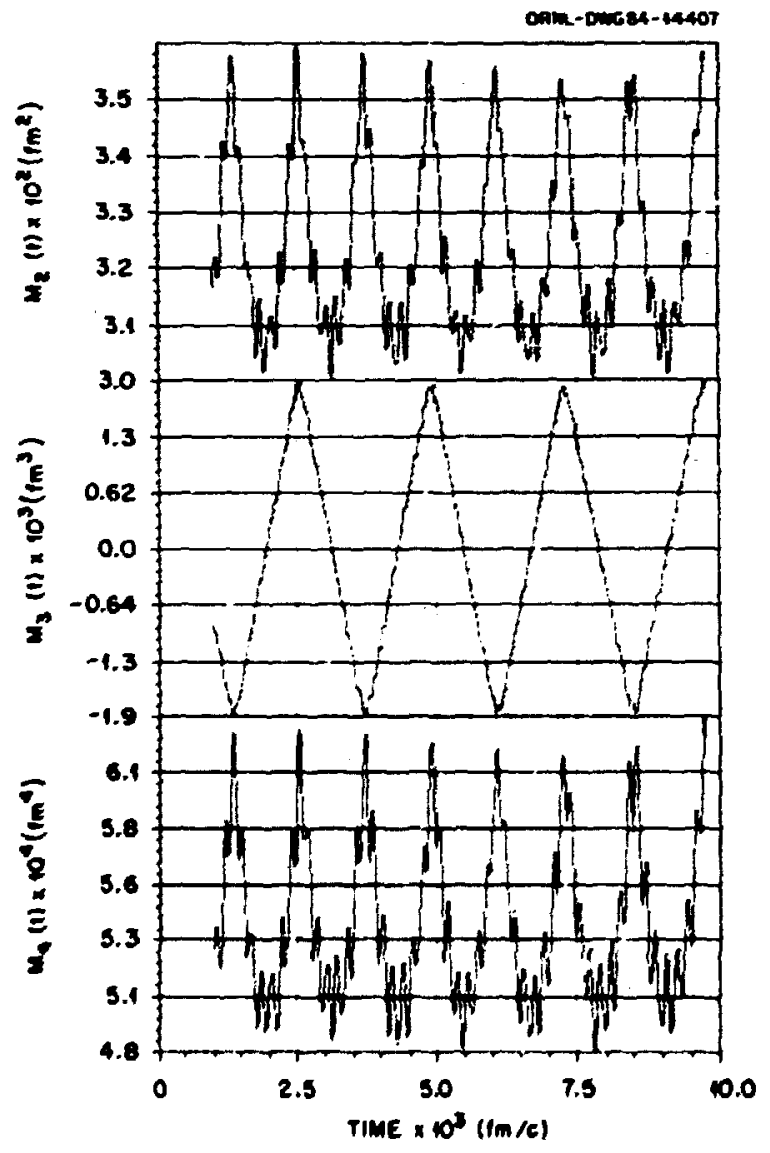

Fig. 5.12. Time dependence of the isoscalar quadrupole, octupole, and hexadecapole moments for the "cooled" modes of the ${ }^{24} \mathrm{Mg}$ system. 


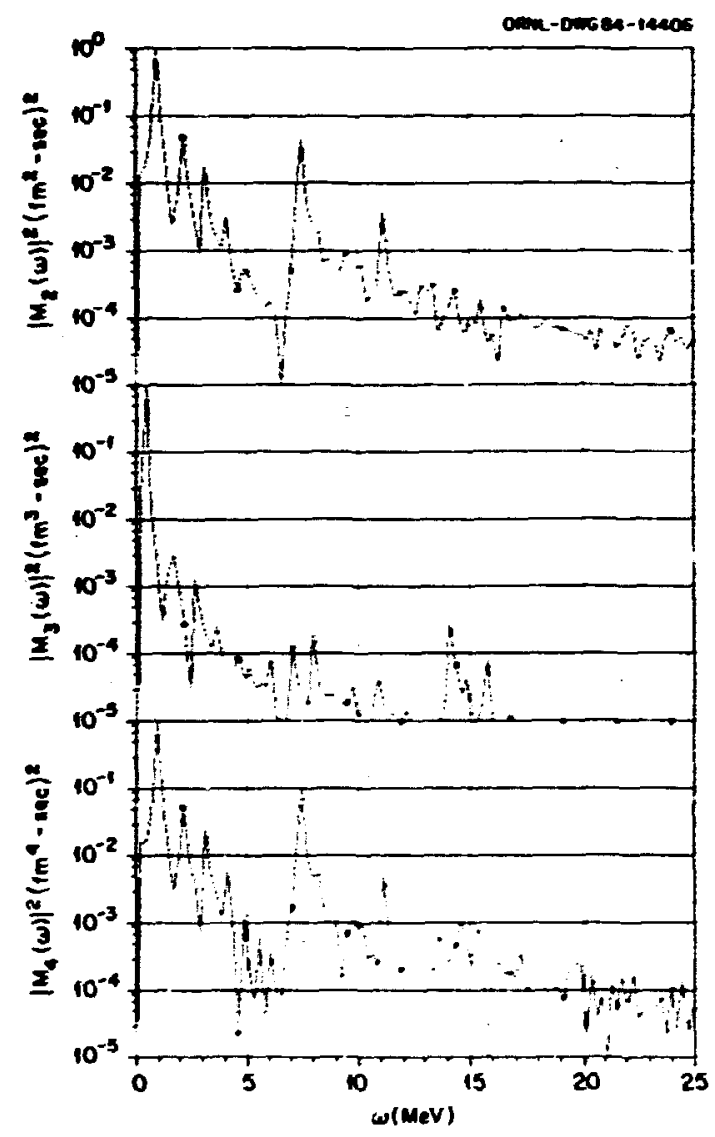

Fig. 5.13. The frequency depender.ce of the isoscalar quadrupole, octupole, and hexadecapole moments for the "cooled" modes of the $24 \mathrm{Mg}$ system.

the ${ }^{12} \mathrm{C}+{ }^{12} \mathrm{C}\left(\mathrm{O}^{+}\right)$systen. In this figure the first few hamonics are clearly visible suggesting that the motion is close to being harmonic.

1. Summary of paper to be publish ed in Proceedings of the International Confe-ence on Fusion Reactions Below the Coulonb Barifer, Cambridge, MA, June, 1984.

2. Guest assignee fron Wright Nuclear Structure Laboratory, Yale University, New Haven, C.T D6511.

3. Consultant frn:t Ouke Infversfly, Durham, NC. 27706.

4. Consiltant from Universfey of E.rlangen, Friangen, West Ciermany.

5. M. R. Strayer et. al.. Phys. L.et.t. 1358, $2.61(1984)$.

\section{REALFIELD CALCURATIONS OF FUUCTUIIOAS In MUCLER COLLISIONS}

\section{J. B. Marston 2 S. E. Koonin 3}

We apply the new variational principle of Balian and Veneroni to calculate the fluctuations in final fragment mass, charge, and kinetic energy for the systens $16_{0}+16_{0}\left(E_{\text {LAB }}=\right.$ $160 \mathrm{MeV})$ and ${ }^{40} \mathrm{Ca} \div{ }^{40} \mathrm{Ca}\left(E_{L A B}=278 \mathrm{HeV}\right)$. The calculated fluctuations are larger than conventional TOHF results and, in the latter case, are consistent with the experimental fragnent mass distribution.

1. Abstract of paper to be submitted for publication in Physical Review Letters.

2. Research Intern from California Institute of Technology, Pasajena, Ca 91125, sumer, 1984.

3. Consultant from California Institute of Technology, Pasadena, CA 91125.

\section{ROTRTING TOROIOAL WUEEI IN HEAVY-ION REACTIONS'}

Cheuk-Yin Hong

Recent experiemtnal measurements ${ }^{2}, 3$ on the correlation of the fragments in the bambardment of ${ }^{32} \mathrm{~S}$ and ${ }^{35} \mathrm{Cl}$ on ${ }^{58} \mathrm{Ni}$ at an energy of about 11 HeV per (projectile) nucleon reveal interesting three-fragment events. These events are characterized by: (1) a bonbarding energy threshold of slightly greater than $10 \mathrm{MeV}$ per nucleon. (2) a cross section of $150 \mathrm{mb}$ at about $11 \mathrm{MeV}$ per nucleon, (3) approximately equal fragments in the reaction plane with momentum vectors making an angle of about $120^{\circ}$ with respect to each other, (4) a value of $Q_{t}$, the difference of the estimated total final kinetic energy If of the three fragnents and the initial kinetic energy $T_{i}$ of about $-150 \mathrm{MeV}$, and (5) a detected multiplicity lower than that' for deep-inelastic collisions. The authors suggested that the scission configuration may be a closed configuration of oblate shape. We explore the possibility that. the pracess of a three-fragment. breakup observed in these reactions of ${ }^{35} \mathrm{Cl}$ and

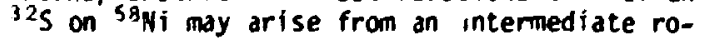
tating toroidal nucleus with $\ell \sim 130 h$. Tuanti. tative characteristies of the toroidal nuclei are presented and found to agree with those of the experiment. Further experimental investiga$t$ fon to search for a rotational symmetry of the three fragment events is suggested.

1. Sumary of paper to be published in Physical Review C.

2. n. Pelte and 1). Winkler, Nucl, Phys. 4423, 164 (1934).

3. U. Winkler, B. Weissman, M. Bühler, A. Corks, R. Novotny, and D. Pelte, Nucl. Phys. A425, 573 (1984). 


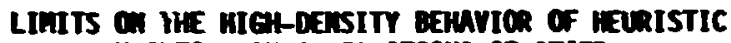
IMCLEAR MATTER EQUATIOHS OF STATE

\section{J. A. Mruhn'}

Both for hydrodynaic-model calculations and for sidiple analysis of experimental data in high-energy, heavy-ion coll isions, one has to eaploy a heuristic equation of state that is parametrized to reproduce some desirable properties near the ground state of muclear matter wile allowing flexibility at higher densities. In olmost all calculations to to now the finternal energy per nucleon as a function of density in and entropy s was set up as a sum of a zeroentropy contribution $u_{0}(n)$ and themal part $u_{t}$ that is assuned to be given by a Fenmi gas expression.

For the funition $u_{0}(n)$ the usual requirenents are those of correct ground-state binding energy $H_{0}\left(n_{0}\right)=B_{0}=-18$ Met; corresponding to a eini man in $t_{0}$, and a reasonable inconpressibility $\omega_{0}\left(n=n_{0}\right)=k / 9 n_{0}{ }^{2}$ with $X=200$ HeV.

The functions actually eaployed were either the quadratic equation of state

$$
u_{0}(n)=\frac{k}{18 n_{0}^{2}}\left(n-n_{0}\right)^{2}+s_{0}
$$

or the quasilinear one

$$
W_{0}(n)=\frac{K}{I C n n_{0}}\left(n-n_{0}\right)^{2}+B_{0} .
$$

Both of these, however, face some proilems at higher densities. As seen in Fig. 5.14, the speed of sound for the quadratic equation of state al ready exceeds the speed of light at about five times nomal nuclear density wile for the linearized one the compressional energy remains so small that in calculations it is very close to the ideal gas (i.e. $K=0$ ).

It was therefore thought necessary to develop a new family of equations of state with more realfstic properties at higher densities. After investigating a number of different functional forms, we propose the hyperbolic equation of state

$$
W_{0}(n)=B_{0}+a\left[\left(n-n_{0}\right)^{2} / n_{0}^{2}+\frac{a \mid a^{2}}{K^{2}}\right]^{1 / 2}-\frac{9 a^{2}}{K} .
$$

This reproduces all the ground-state properties and always approaches a sound speed equal to the speed of 1 fight at high densitfes.

The new parameter a characterfizes the slopc of $H_{0}(u)$ in the high-dersity limft; in fact

$$
H_{0}(n) \sim a \frac{n}{n_{0}} \text { for } n \cdots
$$

The curve for $H_{0}(n)$ interpolates between a parabola near no and a linear behavitgr at large $n$. Because the speed of sound is reiated to the curvature of this curve, it still exceeds the speed of light for intermediate va! uses of $n$ if a becomes larger than certain value depending on
ORML-0.56 84- 25756 $a=130$
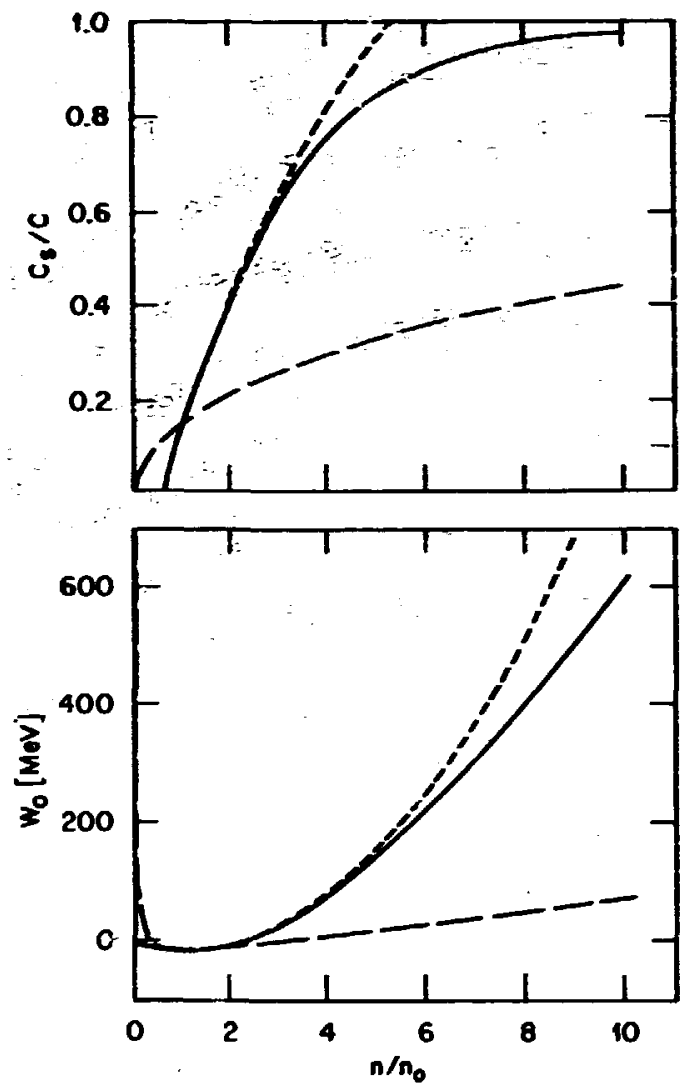

Fig. 5.14. Energy per nucleon and speed of sound in zero-temperature nuclear matter for the quadratic (short dashes), linearized (long dashes), and hyperbolic equation of state (full curve), the latter with $\alpha=130 \mathrm{MeV}$.

$K$. For $K=200 \mathrm{MeV}$, this value is $a=132.30$ MeV. This is not a fundamental limit on a; a different parametrization of $W_{0}$ might reduce the curvature in the transition region and thus allow s! ightly higher values of $\alpha$. h., iever, as seen in Fig. 5.14, for a close to this limiting value the resulting curve stays quite close to the quadratic equation of state up to almost $n$. 5 no. where it has to deviate to avold too large sound speens. For small values of $x$, one can go to arbitrarily soft equations of state, up to and below the linearized one.

What merges froin these considerations is that, even astde from the particular form of $H_{0}(n)$ proposed here, the parameter $a$, the asympsotic slope of $W_{0}(n)$ is probably a necessary ingredient of any such equation of state and will play a more important. pole than $k$ at the high densities.

1. Cunsultant from University of Frankfurt, Frankfurt, Hest iermany. 
DEISITY AS a constraimt an Excitation ERERGT In TH'T

\author{
R. Y. Cusson ${ }^{2}$ \\ P.-G. Reinhard ${ }^{3}$ \\ M. R. Strayer \\ J. A. Maruhn"
} H. Greiner 4

The time-dependent Hartree-Fock (TOHF) wethod aims at a mean-field description of the dymanics of a many-body systen. Nevertheless, a IDHF trajectory describes not only collective notion but also a great deal of internal excitation. It is of considerable theoretical and practical interest to have a method wich mould allow us to disentangle both contributions to the motion. The task is to find a fast iteration schene for constrained Hartree-Fock, in particula: for the case of multiple constraining conditions.

The Hartree-Fock state $\$$ is an antisymmetrized product of single-particle states $\left(\phi_{\alpha}\right)$. The most general gradient step can be witten as

$$
\left.\left.\left|\phi_{a}^{(n+n)}\right\rangle=0\left\{\mid \phi_{a}^{(n)}\right)-\tilde{j}\left(\hat{u}^{(n)}+\lambda^{(n)} \cdot \hat{\underline{p}}\right) \mid \phi_{a}^{(n)}\right)\right\}
$$

where $\hat{W}^{(n)}$ is the Hartree-Fock Hamiltonian to the $\{1+(n))\}$,

$$
\hat{w}^{(n)}=\hat{\mathrm{T}}+\operatorname{Tr}\{\hat{p}(n) \hat{v}\}
$$

and $\hat{T}$ is the kinetic energy, $\hat{v}$ is the interaction potential, and $\hat{p}(n)$ is the one-body density matrix

$$
\left.\rho^{-(n)}=\sum_{a} \mid \phi_{a}^{(n)}\right)\left(\phi_{a}^{(n)} \mid\right.
$$

The means Sran-Schmidt orthogonalization of the single-particle wave functions. The $\hat{D}$ is a damping operator to ensure convergency of the iteration where

$$
\langle\underline{\underline{Q}}\rangle^{(n)}=\sum_{a}\left(\phi_{a}^{(n)}|\underline{n}|_{a}^{(n)}\right)
$$

for î being a set. of one-body operators. The straight forwar 1 way to adjust the constraint is simply to project out of the step $\hat{H}(n)$ the $\mathrm{cm}$. ponent ding $\vec{i}$ (which would drive to change its expectation value).

At the start of an iteration sten we have now a set of xcrupled single-particle states $\{(n) ;\}$ and a constraining force $f(n)$. First, we perfonil a trial step with $\hat{b}(n)+s(n) \cdot n$. Since the ${ }_{1}(n)$ has not yet redchen the ireal valuse, this step will carse a change in 〈îे,

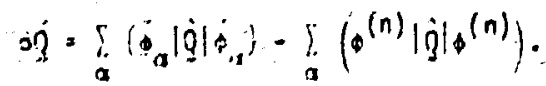

We correct. $x(n)$ t.o redice the change

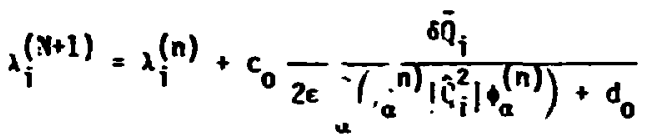

where $c_{0}$ and $d_{0}$ are muerical parameters to be adjusted such that optimal converging is achieved $\left(c_{0}-1\right.$ and $\left.d_{0}=0\right)$.

We study two 160 muclei in the Hartree-Fock ground state placed at a distance of $10 \mathrm{fm}$ and given an impact parameter zero.

For an initial energy of $1.56 \mathrm{MeV} /$ nucleon we are in a situation slightly above the Coulonb barrier. In Fig. 5.15 we show the results for that case. The distance of the ions as a function of time shows that the two nuclei come into contact and merge until a distance of $4.4 \mathrm{fm}$ is reached. Then they spring back, but they cannot escape because some relative energy is transferred to internal excitation and finally will flow into molecular vibrations. In order to give some iapression about the process, we have added a few contour plots of equidensity lines

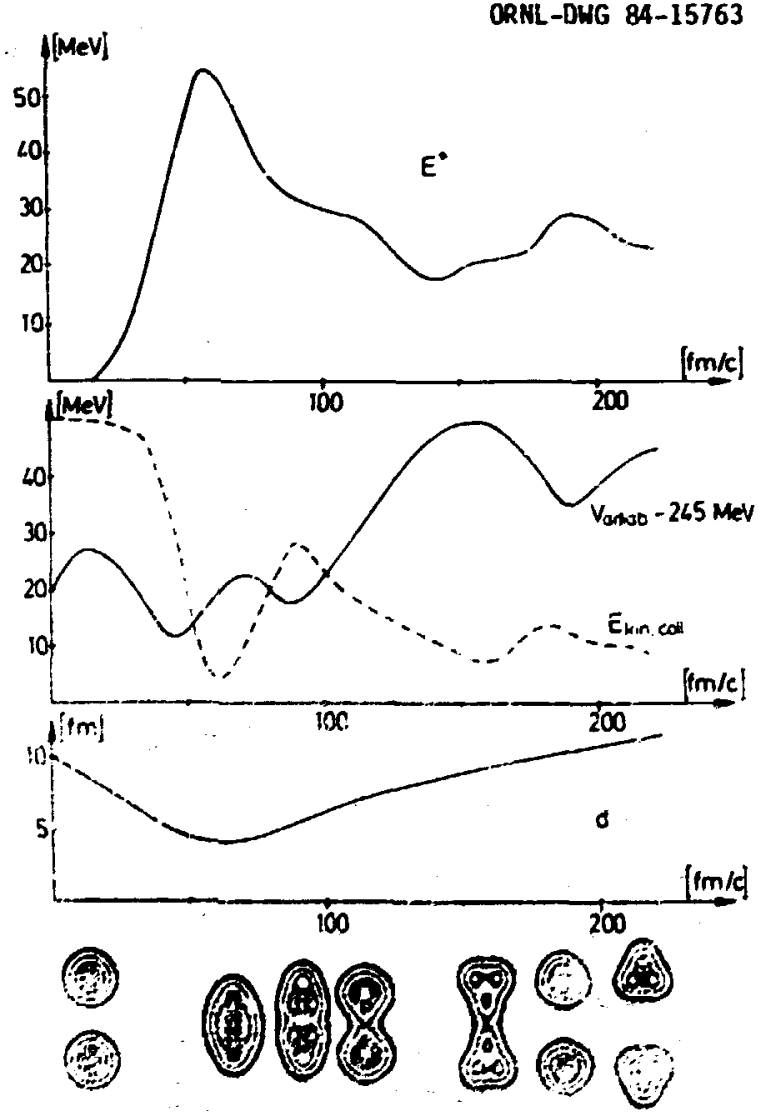

Fig. 5.15. Distance of the ions, adiabatic potenetal, inllactive kinatic energy and internal entruy as eiunetions of time for an initiali. zation with 1.56 MeV/nucleon. As an illisstration. A feiw contour plots of the enurfensity lines are givine. 
at some typical stages. This behavior is reflected al so by both collect ive energies, nanely the adiabatic potential and the collective kinetic energy. Initially, the systen needs some tiae to come across the coulowb barrier. Then; at $t=80 \mathrm{fil} / \mathrm{c}$, the mulear attraction sets on and accelerates the ions drastically. With lowering separation, a repulsive force builds up which, at $t=158 \mathrm{fw} / \mathrm{c}$, stops the relative motion and returns the process. Both the adiabatic potential and the collective kinetic energy are roughly symetrical about the turning point. However, the deviations from an ideal collective motion are visible, naely the maxima of $E_{k i n, c o l l}$ have unequal height, and these maxina do not precisely coincide with the winima of the adiabatic potential. This is due to the occurrence of strong internal excitations, as seen from the behavior of $E^{*}$.

In some sense, the internal energy behaves as expected; comparing the stages of sall orerlap initially and after the refiection, observe that an internal excitation of about 3 hey has been gathered during interaction time.

1. Summary of paper to be published in Zeitschrift fur Physik.

2. Consultant frem Ouke thiversity, Ourhaan, NC 27706.

3. Consultant from University of Erlangen, Erlangen, West Sermany.

4. Consultant from University of Frankfurt, Frankfurt, West Germany.

\section{RELATIYISTIC MEAN-FIELD DYMAICS APPLIED TO ${ }^{160+160}$ SCATTERING}

\author{
R. Y. Cusson 1 \\ P.-G. Reinhard ${ }^{2}$ \\ H. St $\tilde{c k k e r}{ }^{3}$ \\ M. R. Strayer
}

'A. Greiner:

The rime-dependent Hartree-Fock (TOHF; thenry. using Skyrne forces, is widely used in microscopic descriptions of the mean-field dynanics of low-energy, heavy-ion reactions. 5 An approach wich allows extensions to emergies comparable to the nucleon rest mass ratses questions of covariance and retardation in the mean-field propagation. We present here a microscopic model of relativistic nuclear meanfield r.namics. The molel consists of nucleons obeying the time-dependent Dirac equation, a classical spin-zero attractive meson field (sigma), a spin-one repulsive mean field (mega), and a meson baryon interaction be* ween them. The resulting coupled field equations are solved simultaneously in mean-field approximation. Similar models have been studied for the static case by Walecka and others. ${ }^{6}$ The theory is treated in the Hartree approximation and this yields an effective Lagrangian. The masses and coupling constants for the musons are phenomennlogical and are adjusted to fit static nuclear matter properties. 5

An expression for the model Lagrangtan density is obtafned with model parameters as follows: baryon mass $\mathrm{n}_{\mathrm{h}} \mathrm{c}^{2}=938 \mathrm{MeV}, \mathrm{scal}$ ar weson mess $a_{5} c^{2}=500 \mathrm{meV}$, vector meson wass $a \mathrm{c} \mathrm{c}^{2}=780 \mathrm{HEV}$, scalar coupling constant $9_{5}=$ $18.030 \mathrm{Mel}$ fim, vector coupling constant $\boldsymbol{M}_{\mathbf{H}}=$ $33.141 \mathrm{MeV}$ fin. The meson masses are taken frow OREP adels, and the coupling constants are djusted to reproduce the binding energy and density of wclear atter.

The following equations of wotion energe fron the Lagrangian density:

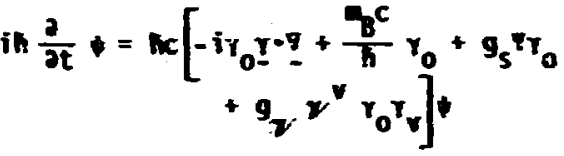

$$
\begin{aligned}
& \text { in } \frac{\partial}{\partial t} a=\left[-h^{2} \Delta+\left(m_{s} c^{2}\right)^{2}\right]+n g_{s}\langle\psi\rangle \\
& \text { in } \frac{\partial}{\partial t} ?=\text { I } \\
& \text { in } \frac{\partial}{\partial t} S_{v}=\left[-\hbar^{2} \Delta+\left(m_{v} c^{2}\right)^{2}\right] x_{\nu} \\
& + \text { fic g }\left\langle\phi^{-} \mathbf{r}_{u}\right\rangle \\
& \text { in } \frac{\partial}{\partial t} \gamma_{v}=S_{u}
\end{aligned}
$$

The gauge and continuity equations

$$
\partial_{v} v^{v}=0, \partial_{v}\left\langle\psi-v v_{v}\right\rangle=0
$$

are evaluated during the time evolution as a check of the numerical accuracy.

We use the classical equations of motion for the mesons in the mean-field theory. Thus the baryonic field can be treated exactly by single nucleon propagation and slater deterainants will remain so at all tines. We restrict, in the present work, the progation to the A cccupied bound states above the bryon vacuum. These approximations result in a theory free of mass and charge renormalization effects.

We represent the meson fieid and the baryon wavefunctions on - three-dimensional $(32 \times 32 \times 32)$ mesh in both cocrdinate and monentum space, with a mesh spacing of $0.5 \mathrm{fn}$ in soordinate space. The momentun space mesh is reached with the help of a fast Fourier transform. We enploy isospin degenerate nucleon wefunctions and treat the spin degrees of freedom in the usual fourcomponent spinor fomalisin.

We use the relativistic static Hartree wavefunctions for each of the 160 initial states. locatediat a separation oistance of IC fm from each other on the mesh, and Lorentz boosted to the adpropriate initial energy in the center of velocity frame. The tine evolution for the baryon wavefunclion is done using a fifti-order predictor-corrector schene in momentum space. and the meson equations are solved simultaneously 45 ing Green function techniques. The monentum space energy truncation was taken as? ciev. This limits the time step to less than 0.05 $\mathrm{fm} / \mathrm{c}$.

We have calculated heafion ${ }^{16} 0+160$ collisions dt. F./ALah $300 \mathrm{MeV}, 500 \mathrm{MeV}$, and $1200 \mathrm{MeV}$. The time evolustion is followed until the expanding sideways flow of matter hits the outer edge of 
the eesh. He show in Fig. 5.16 the time evolution of the baryon density. Mhose space integra? is the total conserved baryon mumer ( 32 in this case), for $E / A_{L} a b=600 \mathrm{tteV}$. The early time behavior of the collision is siailar to that of Skyrme IDif. A compression cone and side splashing develaps after about $t=6 \mathrm{fm} / \mathrm{c}$. The syoten then proceeds to spallate in all directions. This can be contrasted with Skrrme Totf where projectile-like fragments and target-like ones energe after the reaction. The resul:s of the present work are, in fact, reniniscent of fluid dynaical behavior. The behavior at the other two boubarding energies was found to be siailar.

1. Consultant from Duke University, Durhas, nc 27706.

2. Consultant from Iniversity of Erlangen, Erlangen, West Germany.

3. Michigan State University, Fast Lansing. MI 48824.

4. Consultant from iniversity of Frankfurt, Frankfurt, West Germany.

5. Pruceedings of the Horkshop on 'TOHF and

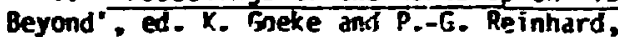
Lecture totes in Physics, Val. 17l, SpringerVerlag, Berlin, lggl.

6. 3. 5. Waieckd, nit. Thys. (N.Y.) 83, 49: $(1974)$

ORNL-OWG $84-16177$

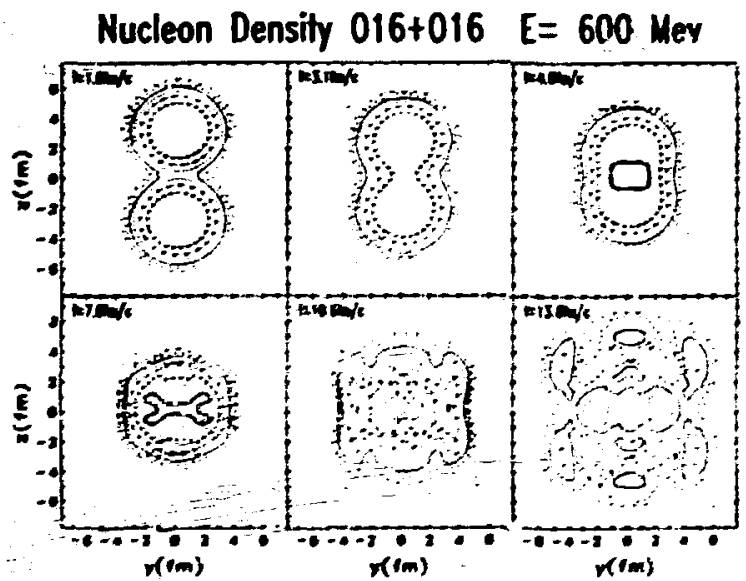

Fig.: 5.16. The conrdinate space haryon equidensity coneours fn the collifsion plane at various elimes nuiting the 600-MeV collision. The time in $\mathrm{fm} / \mathrm{c}$ is given fn the upper left corner of each frame. Fquitsensily contours are taken at values of $.245, .34, .08, .046, .026,015$, $.0 n e 5$. .055 nucleons/ $\mathrm{fm}^{3}$. The contour $\mathrm{l}$ ines corresponting to these eight cuta have the styles thick isolid, dashad, dot asshed, dot.ted and thin (solid, Hashom, Int-dashed, Hoted) so that each continur line ridn he identified at a glance.e. The absnlist.e marimum density reached was $0.29 n / 6.13$.

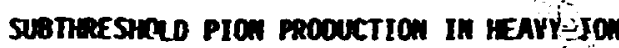
collisions

\author{
D. J. Ernst I A. R. Strayer \\ A. S. $\operatorname{lnar}^{2}$
}

In heavy-ion collisions low chergy pions have been detected ${ }^{3}$ at bombarding energies below the free nucleon-nucleon threshcld. The enission is thought to arise fron a cooperative process in which the relative motion of the centers of the imo jons is converted into the single degree of freedom of the produced pion. This makes subthreshold pion production a unique probe of the very early prethernalization stage of the collision.

We have developed a time-dependent, meanfield model of the emission process. In the model, mucleans in the target (projectile) are perturbed by the time-dependent mean field of the passing projectile (target). The timedependent pass oscillations wich are induced by the passing mucleus are coupled in lowest arder th pion production using a standard nonrelativis:ic $\mathrm{N+N+ \pi}$ coupling. Thus the relative motion is used to induce currents with bremstrahlung pians. Ising the time-dependent redction formal ism developed in Refs. 4 and 5 , the total inclusive pion emission cross section is found to be given by

$$
\begin{aligned}
& a=2 \pi \int_{0}^{\infty} b d b \Sigma(b) \int \frac{d^{3} k}{(2 x)^{3}} \frac{1}{\left(2 w_{x}\right)} \int \frac{d^{3} p}{(2 \pi)^{3}} \sum_{h} w_{h} \\
& \times\left(\frac{f o}{u}\right)^{2} \int_{i} \frac{d^{3} q d^{3} q}{(2 \pi)^{6} 2 w_{0}} \int \frac{d q_{0}}{2 \pi} \delta * q_{0} \\
& \times\left. a_{n}\left(\vec{q}+\overrightarrow{0}, q_{0}+y_{k}\right) \phi_{k}^{*}(\bar{l})\right|^{2},
\end{aligned}
$$

where $b$ is the impact picaneter of the heavy-ion collision. $E(b)$ is classical transmission coef ricient, $W_{h}$ is a spin-isospin degeneracy factor

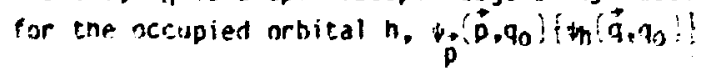
is the space and time Fourier transform of the final-state nucleon (initial state ntcleon), and $\phi_{p}(0)$ is a distortert pion wave function.

If we approximate the find nucleon wave function hy a plane ave and the pion wave function by a olane wave, the source current for the creation af the pion is proportional to the time-dependent wigner function. That is, the time-i'spendent. wave eunctions are fourier transformed in. the time varfable and evaluated at the energy requtred by energy conservation. Dne then constructs a Wigner function for these wave functions and the double differential pion emission cross section is proportional to this function.

He have modeled the external time-dependent perturhat ion by a harmonic-oscfllator, separable time-dependent field. This introduce: two parameters into the model .. a strennth function and t time scale. If we fit the strength to the tots! multip!icity for $C+C$ collisions at $85 \mathrm{MeV}$. 
we find epirically that the time scale $\tau^{-1}=$ Elab/A produces the energy dependence for the pion mitiplicity in $C+C$ collisions as shom in Fig. 5.17. Preliminary estimates for subthreshold kaon production indicate that these cross sections will be down many orders of magnitude as a result of the larger kaon mass and weaker coupling to the nucleon.

1. Consultant from Texas A \& H thiversity, College Station, $\mathrm{TX} 78712$.

2. Guest assignee from Uright Vuclear Structure Laboratory, Vale University, New Haven, CT 06511.

3. T. Johannson et al.., Phys. Rev. Lett. 48, 732 (1982); P. Braun-tunzinger et al.. Phys. Rev. Let.t. 52, 255 (1984).

4. A. S. Uar. H. R. Strayer, D. J. Ernst, and K. R. S. Devi, to be published in Physical Review C.

5. M. Baranger and I. Zahed, Phys. Rev. C 29, 1005 (1984).

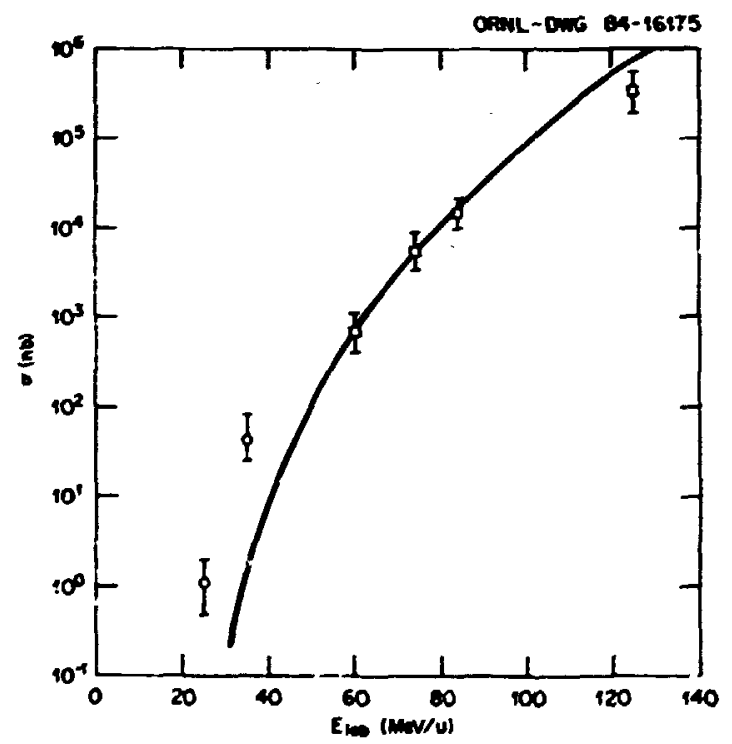

Fig. 5.17. The total ${ }^{12} \mathrm{C}+12 \mathrm{C}$ pion production cross section as a function of the hombarding energy.

\section{NUCLEAR STRUCTURE}

\section{THE UNISOR WUCLEAR STRUCTURE THEORY PROGRAM}

\section{Introduction}

G. A. Leander'

A theory program is suppor sed by UNISOR, the university isotope separator at ORNL. The aim is to conduct and stimlate theoretical research on muclear structure, especially in areas where experiments at the Holifield facility have or potentially could have an impact. Such areas of current interest are: low-energy structure in nuclei far from stability, the cold or 'yrast' sequence of states at very high spin, and the warimer structures wich are being studied by quasicontinuum spectroscopy. The focus lies on collectivity in nuclei, its basic principles, microscopic origin, and physical consequences. The wost significant results from the past year pertain to intrinsic reflection asymetry, wich was previously established to occur in certain nuclides. In particular, octupole deformation was shown to exert a major influence on highspin quasiparticles then it occurs, making nuclear rotation wore regular and collective in character. Furthermore, intrinsic electric dipole aoments have been shom to exhibit characteristic shell effects of their own, although they arise as a consequence of octupole deformation. These and other results are sumarized below under four topical headings with reference to the respective publications.

\section{Low-Energy Structure}
A. F. Barfield ${ }^{2}$
P. 1001 ler $^{7}$
F. Dönau ${ }^{3}$
J. Dudek"
B. E. Gnade 5
H. M. Howard 6
G. A. Leander 1
H. Mazarewicz 7,8
J. R. Nix ${ }^{9}$
Ph. Quentin'to
P. B. Semes 11
J. L. Hood II

Most microscopic theories for structure at Iow energies are based on single-particle orbits in a mean field, and an important activity is to follow up the results of spectroscopy in order to test and improve models for calculating such orbits. Data obtained during the last few years on the neutron-rich doubly agic nucleus $132 \mathrm{Sn}$ have been evaluated 17 in terms of current models (Hartree-Fock with Skyme III and Skyrme M forces, the Los Alamos-Lund folded lukawa, and the Warsaw Hoods-Saxon potentials) The successes and failures of each model in i32 Sn were significantly simflar to what is obtained in $208 \mathrm{~Pb}$. Assuming analogous correspendence hetween other doubly magic nuclei, the hitherto unobserved proton-rich not teus $1005 n$ can rather confidently bo-predicted to have a proton binding energy of 3-4 MeV.

An odd nucleon is an informative probe of collective as mell as single-particle properttes in the nucleus, and an on-going effort is therefore spent on the development and application of core-particle coupling models. In particular. the method of Conau and coworkers for coupling BCS quasiparticles to dynamical fields of the core has been combined with the interacting boson model (:BH) developed by Arima, Iachello, Scholten, and coworkers. This dynamiral BCS quasiparticle method has some obvious advantages over an alternative boson-fermion coupling model (IBFM) proposed by Iachello and Scholten: the BCS nethod can he applied with equal ease to any version of the IBM, methods exist to calculate the parameters of the coupling term microscoptcally, and there is no ambigutity in the form of 
the particle-transfer operator. The first step has been to compare the BCS and IBFM coupling schemes on a phenomenological basis.18 The difference lies in the way the Pauli principle is taken into account, and it is found that IBFH is more flexible if the strength of the exchange term is treated as a free parameter. There are specific values wich give energy spectra similar to those of BCS.

The dynamical BCS quariparticle method has been applied in the light $\mathrm{Hg}$ region, using the IBH2 cores with wixed boson numbers that were developed by the Tucson group. ONd- $\mathrm{Hg} \mathrm{i}_{13 / 2}$ spectra mere adequately reproduced. 19 Calculations in progress for light $\mathrm{Tl}$ and $\mathrm{Hg}$ isatopes will provide a more crucial test of specific features in these cores. 20 The latter calculations are also expected to suggest how to test experimentally a hypothesis that has been made in connection with IBM, namely, that protons couple mainly to the quadrupole field of the neutron bosons and neutrons to the proton bosons. As an aside in this context, a claim in the literature that there is evidence for acorrelations in experimental binding-energy systematics resulting from this mechanism was shown to be unfounded.21 Theoretical calculations for the light $\mathrm{Hg}$ cores are also being extended in connection with UNISOR measurements, using both models of prolate-oblate shape coexistence 22 and the IBM. 23,24

The particle-triaxial rotor model was applied for a variety of purposes.25,26 In particular, it was found that a very limited amount of spectroscopic data in certain isotopes might he enough to address the outstanding question of whether any well-deformed oblate nuclei exist beyond the sd shell. The prime candidate is the $N=Z$ nucleus $72 \mathrm{Kr}$, and it was seen that all the low-lying bands in its odd-A neighbors would have clear signatures of either oblate or prolate shape. An example is shown in Fig. 5.18, where cascarle to crossover ratios distinguish the two 3/2- bands. Another calculation, for IBSAu, strongly suggests that the very high structure ohserved in-beam by Larabee et al. contains an "extra" $h_{9 / 2}$ band. 26 similar to the

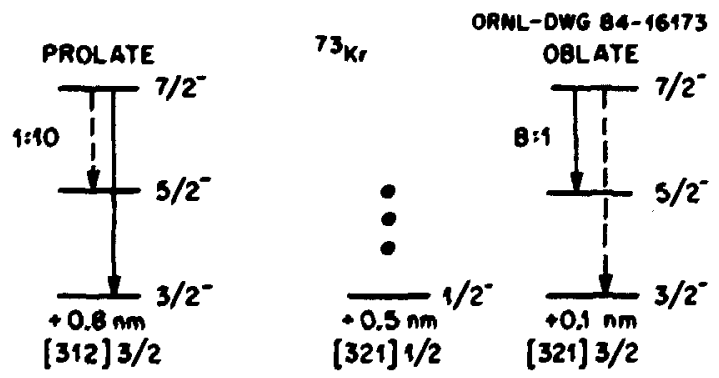

Fig. 5.18. l.arge oblate deformations are not knotwn to nccur except in light nuclef hut have long heen prefticted in the region of $72 \mathrm{Kr}$. Oh. late configurations would edsily he recognized in spect.rosenpy on oxt-mass nefghhors. For example, the calesulated niti-neutron $3 / 2^{-}$bands shnwn here have distinct. hranching ratios. one previously indicated by low-spin UNISOR data on 187 Au.

In the region of the very heavy elements $(Z)$ 100), the 1981 mass formula of mojller and Mix wes applied ${ }^{27}$ over a wider range of nuclei than in the original mass table, and lifetimes mere calculated for a-decay and spontaneous fissian. This work was motivated in part by the LEAP project a joint ORAL-LAML-LBL-LLil proposal to make a 254,255 Es target for massive transfer and heavy-ion fusion reactions. The present predictions differ somewhat from earlier work, minly due to the single-particle level scheme. One test of the level scheme wuld be a predicted kink in the a halr-life curve at $N=162$ due to a gap in the single-particle spectrum. It is similar to, but more pronounced than, the known kink at $N=152$. In the superheavy region the B-stability line was obtained at lower $N$ than previousiy, and the wost favorable case for lungevity is now ${ }^{290} 110$ wich could be reached from the LEAP target with a stable beam. The a halflife for 290110 is 167 days, and for doubly magic ${ }^{298} 114$ it is 1.2 minutes.

\section{Intrinsic Reflection Asymetry}
G. F. Bertsch 15
P. Olanders?
J. Durlek
G. A. Leander ${ }^{1}$
P. Möller?
Ph. Duent in 10
Y. Nazarewicz 7,8
I. Ragnarsson 7
E. Ruchowska12,13
M. R. Strayer

Octupole deformation which breaks the intrinsic reflection symmetry had previously been established in nuclei of the Ra-Th region, in part through an analysis of single-particle properties manifested by odd-A nuclei. 28 A somewhat different interpretation of the even-even nuclei in this region in terms of a clustering was tested, I $A$ using the case of $225 \mathrm{Ra}$ where the decoupling factors of the $K^{\pi}=1 / 2^{ \pm}$bands are sens $i$ tive indicators of octupole deformation. 29 However, it was found that a potential resembling a core plus an a particle does not give the constderable modification of the decoupling factors which are observed in experiment and which are ohtained with the smoother octupole-deformed equilfbrium shape derived hy the Strutinsky method. The Strutinsky calculations were al so examined critically in order to see to what extent the development of an octupole-deformed minimum could be influenced by different choices of single-particle and liquid-drop mode is.30 The most important single factor in the $R_{a}$ region turned out to be the spacing hetween the proton $17 / 2$ and $f_{13 / 2}$ shells, and that value seemed to depend on whether the parameters of the single-particle potential were fitted to dats in somewhat lighter and spherical or somewhat heavier and deformed nuclei.

For the neutron-rich nuclef around $14 \mathrm{~h} \mathrm{Ba}$, where octupole deformation has heen prevfously suggested hut not investigater unt $f$ now, all the models studied unanimously predict a soft, hut nevertheless octupole-deformed, equilibrfum shape. 30 Single-particle signatures of octupole deformatinn were calculated along the same lines ds in the Ra region, and the stiil rather scanty 
available data on odd-aass muclei were found to support the predicted asymetry. ${ }^{31}$

The consequences of octupole deformation at high spin were found to be significant. 32 Mormally, high-spin properties are dominated by the spin aligmment of a few quasiparticles from high-j shells. However, the octupole interaction mixes the high-j intruder shells with opposite-parity states in the valence shell, and thereby spreads the rotational strength over many valence orbitals. The in-band collective mowent of inertia is increased and "backbending" due to quasiparticle alignament no longer occurs, in agreement with experimental data for $222 \mathrm{Th}$

(Fig. 5.19). The spin alignment of rotational

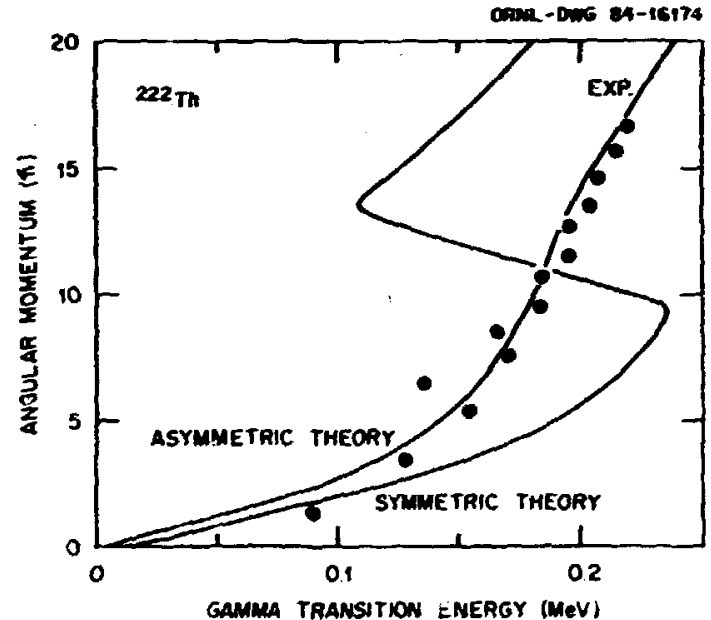

Fig. 5.19. The theoretical curves represent eformed shell -model-Bogol iuhov cranking calculations and the points experiment for the yrast line of $222 \mathrm{Th}$. The "symetric theory" has the customary constraint to reflection symetric intrinsic shapes and gives a backbend due to the alignment of $j_{15} /$ ? ieutrons. In the "asymmetric theory" the constraint is relaxed, ${ }^{222}$ Th acquires an octupole deformed equiliforium shape, the $j_{15 / 2}$ orbitals are fragmented, and the yrast line is smooth, as ooserved in experiment.

quasiparticles. which could be extracted from sidebands and bands in odd-A nuclef, 15 expecter to be rather uniferm and smaller than the aifgnment typical for high-j quasfparticles. The "sfgnature" quantum number commonly used at high spins is no longer relevant in the reflection asymetric case, and instead a more generally applfcable quantum number. "simplex", was proposed. Guastparticle bands are expected to exhibit stmplex spliftings and occastonal stmplex crosstings.

Octupole deformation is interesting not only per se but also because it leads to an "exotic state" of nuclear matter, where it is possible to ohserve phenomena wose existence requires broken reflection symetry. One such phenomenon is an intrinsic collective Fi moment. Wich arises when there is no symetry to make the proton and neutron centers of mass coincide exactly. Early estieates based on the liquiddrop wodel yielded El moments proportional to $B_{2} B_{3}$, hut with conflicting results for the sign. An attempt is being made to resolve this conflict using the extended Thomas-Fermi formalism. 32 The calculation ${ }^{33}$ shown in Fig. 5.20

ORNL-OWG S4-16582

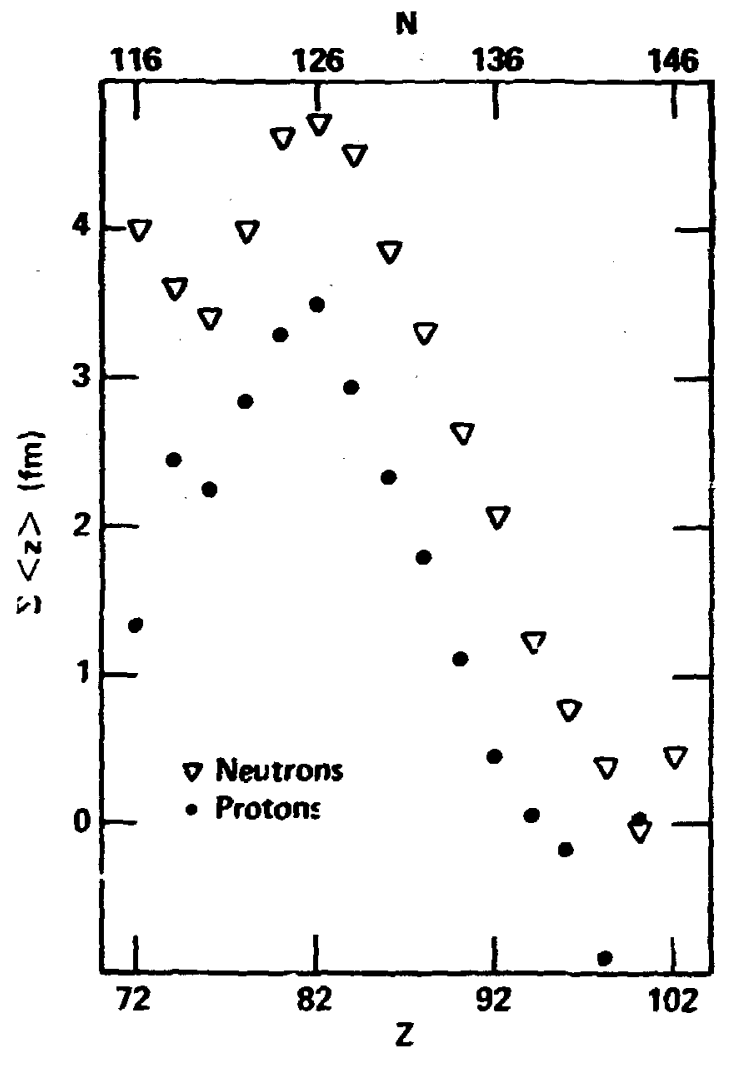

Fig. 5.20. A 'numerical experiment' which shows that ingle-particle effects on the El moment are neitier random nor small. Singleparticle states in a potential with fixed $B_{2}$ and $\delta_{3}$ are successively iflled, and the accumulated (z) exhibfts a coherent shell effect ahove the shell closures at $Z=82$ and $N=12 \hbar$.

reveals another large contribution to the El moment wich was not previousiy anticipater, namely, a single-particle shell effect at fixed $B_{2} B_{3}$. This shell effect must be strut insky renorma! izen to the liquit drop in a way that is consistent with the energy of the giant dipole resonance. ${ }^{34}$ The El shell effect could account for d systematic dependence on nucleon number that is emerging from recent data, and the ambiquity mentioned ahove in the sign uf the macroscopic contribution to the EI moment would then be endicically resolved. 33 
High Spins
P. Arve ${ }^{14}$
T. Bengtsson?
H. H. Guidry 16
Y. S. Chen 15
J. Dudek ${ }^{4}$
G. A. Leander:
W. Nazarewicz 7,8
P. Olanders 7
5. Frauendor ${ }^{3}$
I. Ragnarsson?

The deformed shell-morel-Bogol iubov-cranking theory was extended and explored for the case of reflection-asymetric mean fields, as described in the previous section (c.f. Fig. 5.19).

Recent ORM data on the yrast line of $158 \mathrm{Yb}$ were found to have a remarkable interpretation in terms of a configuration-dependent cranking model. 35 Both the data and the model exhibit collective band structure with near-constant level spacings in the high-spin regime $I=26-36$, leading to collective angular momentum being generated much more ef $f$ iciently than in welldeformed rotational nuclei like $168 \mathrm{Yb}$ (Fig. 5.21). Above $I=36$ there is an energy gap in

ORNL-DER, $84-1658.5$

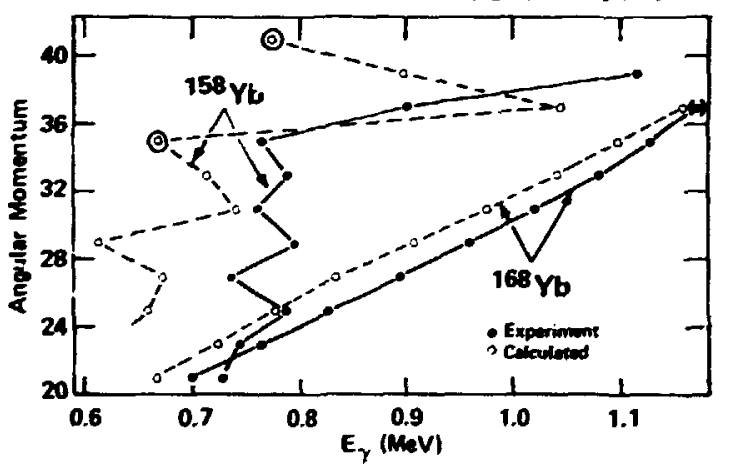

Fig. 5.21. Angular momen:'um versus E? gamaray energy for theoret:ia $i$ (dashed) and experimental (soijd) bands in 158 yb and hayb, respective:y. The generation of collective angular momentum up to $I=36$ is more energy efficient in transitional $159 \mathrm{Yb}$ than in well-deformed i68yh. This results from a gradual shape transition followed by hand termination in $158 \mathrm{yh}$.

the spectrum. In the model these features result from a gradual in-band transition from prolate shape at lower spias to a noncollect.fve oblate state at. $I=36$ which terminates the $\mathrm{col}$ lective hand. Rand terminations are commonplace in nurelei with only a few valence particles and also in the rotating harmonic oscillator model. hut. a band-terminating state had not. previously been thentified in expertment. for such a large number of valence particles and such large angular momentum as in lSaYh.

Severd high-sili theory projects, such as the stidty of signature splitting 35 and the develispment of mdels for ml radiation at high spin."7 are uafortionately not being pursued in cornection with ORNL at the present time, althnugh warranted hy the experisnential sieisation.

\section{Quasicontinuum Spectroscopy}

T. 0 .gtsson ${ }^{7}$ G. A. Leander

The study of muclear structure by means of the "quasicont inuum" of ganana rays wich emanate from unresolved levels at very high spin and nonzero intrinsic excitation energy is a developing field. In particular, energy-energy correlations seem to contain valuable information, but both the extraction of the correlations from experimental data and the theoretical interpretation of ridge-valley structures are subject to controversy. An interesting finding has been that the ridge separations obtained in experiments on a sequerce of nuclei around $A=125$ exhibit a systematic trend with nucleon number. which has a specific and semi-quant itative theoretical interpretation. 38,39 The data around :I8xe indicate a dynamical moment of inertia $(2)$ at high rotational frequencies that is well below the rigid-body estimate, wereas the data around $12 \mathrm{Ba}_{72} \mathrm{give}$ an effective $g(2)$

which increases up to or beyond the rigid-body value (Fig. 5.22). Previous cranking calculations suggested that the trend could be due to a well-deformed shape isomer wich comes low in energy specifically around $\mathrm{N}=72$. Configurationdegendent cranking calculations showed that the fl) values from the energy-energy correlations would indeed result if the decay pathways in the quasicontinuun resembled a representative sample of calculated near-yrast bands. More recently, the same kind of configuration-dependent cranking calculations were able to account for d cutof $f$ in the quasicontinuum $E$ ? spectrum of $158 \mathrm{Yb}$ above $\sim 800 \mathrm{keV}$

Development has continued on a Monte Carlo Code, GAMBLE, wich provides a statistical model framework for the evaluation of quasicont inuum correlations. New technical and physical features have been incorporated which will be applied during the coming year to study structuredependent aspects of the gama cascade pathway.

\section{INNISiR, Dak Ridge Associated}

Universities.

2. Iniversity of Arizona, Tueson, A2 85721 .

3. 7fK Rossendorf, Dresten, 6DR.

4. Centre de Recherches Nucléatres, Strashourg, France.

5. Texas Instrument:. Dallas, TX.

6. Lawrence Livermore National Laboratory. Livermore, CA 94550.

7. Lund University, Lund, Sweden.

8. Technfcal Iniversity, Warsaw, Poland.

9. Los Alamos National Labnratory, Los

Alamos, 87545. France.

11. Seorgita Institute of Technology. At lanta, GA 30332 .

12. KVI, Groningen. The Netherlands.

13. Iniverstey of Warsaw, Warsaw, Doland.

14. MORDITA, Copenhagen, Denmark.

15. institute of Atomic F.nerijy, Reijing. China.

$3 / 916$. 
17. 6. A: Leander et al., Phys. Rev. C 30 , 415 (1984).

18. G. A. Leander, P. B. Senwes, and

F. Dönau, in "Interacting Boson-Boson and Boson-

Fermion Systems," ed... by 0 . Scholten (Wurld

Scientific Publishers) in press.

19. P. B. Semes, G. A. Leander, and J. L.

Mood, ibid.

20. P. B. Semes et al., current work,

21. G. A. Leander, Phys. Rev. Lett. 52, 311

(1984).

22. J. D. Cole et al., Phys. Rev. C 30, in press.

23. A. F. Rarfield and B. R. Barrett, Phys.

Letc. $B$, in press.

24. A. F. Barfield, current work.

25. B. D. Kern et. al.. Phys. Rev. C 28, 2168 (1983).

26. A. J. Larabee et al., Bull. An. Phys.

Soc. 29, 1049 (1984).

27. G. A. Leander, P. Möller, J. R. Mix, and H. M. Howard, Proc. Int. Conf. on Atomic Masses and Fundamental Constants, Darustadt, 1984, in press.

28. 6. A. Leander and R. K. Sheline, Mucl. Phys. M413, 375 (1984).

29. R. K. Sheline et al., Phys. Lett. 1338, 13 (1983).

30. M. Mazarewicz et al., Mucl. Phys. A, in press.

31. G. A. Leander, H. Nazarewicz, P.

Olanders, I. Ragnarsson, and J. Dudek, to be published.

32. F. A. Leander, Ph. Duent in, and M. R. Strayer, current work.

33. F. A. Leander, in "Capture Gamma-Ray Spectroscopy and Related Topics," ed. hy 5. Raman (AIP Conference Series) in press.
34. 6. A. Leander and 6. F. Bertsch, current work.

35. I. Ragnarsson et al ., to be oubl ished.

36. Y. S. Chen, S. Fravendorf, and G. A. Leander, Phys. Rev. C 28, 2437 ( $1 \leq, 3$ ).

37. P. Arve, Y. S. Chen, and G. A. Leander, Physica Scripta T5, 157 (1983).

38. H. El-Saman et al., Proc. Int. Sym. on In-bean Muclear Spectroscopy, Debrecen, Hingary (Akadéaíai Ki adó,. Budapest) in press.

39. H. El-Samman et al., in press.

\section{ACCIDENTAL DEGEMERACIES Ni D OMMICA SUPERSTHETRIES 1}

\section{A. A. Balantekin'?}

Recently, several Hamiltonians appeared in the literature ${ }^{3}$ for wich energy levels exhibit a degeneracy which cannot be associated with the known syametry groups. We corsider the two Hamiltonians motivated by the nuc:ear shell model

$$
H=\frac{1}{2}\left(\vec{p}^{2}+\vec{r}^{2}\right)+\lambda(\vec{a} \cdot \vec{L}+3 / 2)
$$

and

$$
H=\frac{1}{2}\left(\vec{p}^{2}+\vec{r}^{2}\right)+\frac{1}{2 r^{2}}+\lambda \vec{O} \cdot L+\frac{3}{\partial r^{2}}
$$

For the first Hamiltonian wen we fix $j$, there is a degeneracy for the two cases $,-1, l=$ $j+1 / 2$, and $\lambda=-1, \ell=j-1 / 2$. For the second Hamiltonian, the degeneracy is between $\lambda=+1$, $j=l+1 / 2$, and $\lambda=-1, j=\ell-1 / 2$ with $\ell$ fixed. It is shown that the degeneracy of such

ORNL-DWG 84-16476
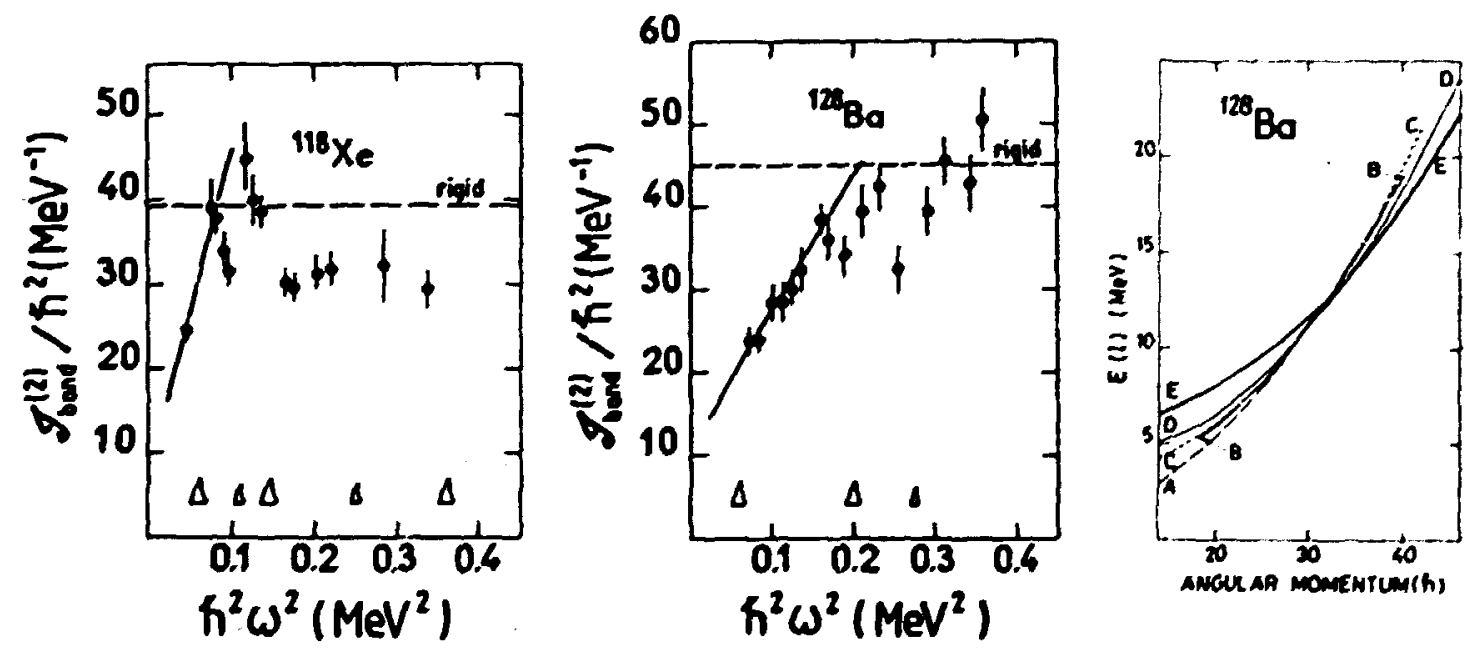

Fig. 5.22. These data for $11 \mathrm{BXe}$ and $128 \mathrm{Ba}$ provide some of the first evidencr. for connect $/ 89$ between nuclear structure calculattons at nigh spins and the dynamical moments of inertia, $f(?)$. derived irom quastgont inuum energy-energy correlations at high rotational frequencies. Thr continuad rise of $g(2)$ in $12 \mathrm{~B}_{\mathrm{Ba}}$ could reflect on increasing population in bands of type $E$ at high

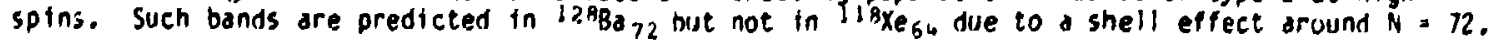




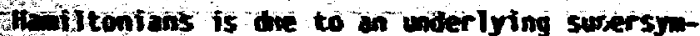

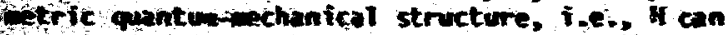
Q w witten os anticomutator of a fermianic tenseryed charge, 0 , with its Herwitian conjuyte, o

$$
H=\frac{1}{2}(0,0 t)
$$

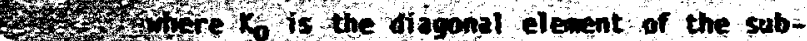
1 751 tebra $s p(2)$ wich acts on the bosonic sob-

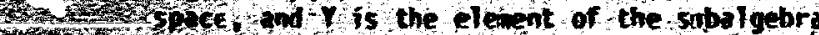
7. $30(2)$ wich acte on the fermionic subspace.

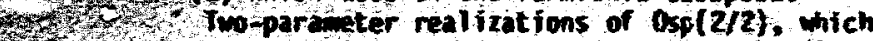
are potentfally useful in mulear and mlecular physfics, are currently under investigation.

1. Sumary of paper submitted to Annals of Phoreics.

2. Eugene P. Wigner Fellow.

3. H. Moshinsky and C. Quesne, Ann. Mhys. 148, 462 (1983); H. Ui and G. Takeda. Tohoky Unt versity preprint (1984).

\section{CHARACTER EAPAUSIOAS FOR DITART enoups}

\section{A. B. Balantek in}

Character expansion technfques are used to calculate certain incegrals, appearing in lattice gauge theorfes, to investigate the role of internal symmetrfes in certain high-energy reactfons, to fmipose the colorlessness condition in the calculation of a quart-gluon gas, to calculate the partition functions in the statistical theory of nuclear reactions, and to calculate the level density of a spherical mucleus as a function of angular momentum. A very general formula was presented to generate expanstons over U(N) characters.$^{2}$ He start from the power series expanston

$$
G(x ; t)=\sum_{n=0}^{\infty} A_{n}(x) t^{n}
$$

wher we normalize to " Taking $t_{f}, i=1, \ldots, 4$ to be the elgenvalues of the fundament sl representation matrix $U$ of $U(N)$, we get the follewing character expanston

$$
\begin{aligned}
& \left(\prod_{i \neq 1}^{n} \sigma\left(x ; t_{i}\right)\right)=\sum_{n_{2}+n_{2}{ }_{n-\infty}} \operatorname{det}\left\{n_{n_{j}+i-j}\right\}(x) \\
& x\left[n_{1} n_{2, \ldots, n_{n}} \mid(u)\right.
\end{aligned}
$$

where $\left[n_{1}, n_{2}, \ldots, \ldots, n_{n}\right]$ is the partition labeling the representations of $v(n), x_{\left[n_{1}, n_{2}, \ldots, n_{n}\right]^{(v)}}$

is the character of the representation

$\left[n_{1} n_{2}, \ldots, n_{n} \mid\right.$ and the 1 J-th prement of the mitix, wose interminant is calculated, is showin.

The possible cses of this forwala in lãttice gauge theorfes ond $i 50$ in the statistical nechanich il stems ore aurrently onder investigh tions:-

\section{RELATIVISTIC HEAYY-IOW PHYSICS}

\section{IMITIN ENERS DENSITY of GUAK-GUDN PLASH IN RELATIVISTIC KEAYY-IO' COLLISIONS'}

\section{Cheuk - Yin long}

We estimate the initial tapidity distribution and the initfal energy density in the central rapidity region of relativistic heavy-ion collistons by using a witiple-collistion model and the nuclear-thickness function of Glauber. The parameter of the rapidity distribution is determined from the experimental milfiplictity data of $p a$, id, aa, pA, $x^{+} A, k^{+} A$, Si $4 \mathrm{Ag}$, and Ca+C reactions. We find that the intefal energy density in the central rapidity region is high. For exanple. For the head-on collits on of $23 \mathrm{a}_{\mathrm{U}}$ on $23 \mathrm{E}$ at $30 \mathrm{GeV}$ per nucleon in the center of mass 5ystew, the maximum energy density is about 10 GeV/fin", wich may exceed the crittcal energy densfty for a phase transition from a confined hadron matter to an unconfined quark-gluon plasma The initial eneray density goes as A $/ 3 B^{3} / 3$ for the collitston of two nuclet wth mass numbers $A$ and $B$, and is rather insensftive to impact oarameters.

1. Abstract of paper: Phys. Rev. D 30,961 (1984). 


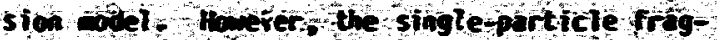
nentation dato for nonleading particles indicate That there is a reaction of the fraguentation cross section betase of subsequent collisions of the leading hopon along the collistom onsthe Wodificotion of the incoherent witiple tollitsion odel is sugpested. The adified nodelgives cross sections for the reactions $p+x$.

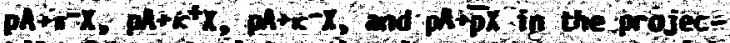
tile trosentation region in good ogreenent with experifuet. ucleon-auclews collisions reveal thit ancleon loses a Targe fraction of its energy in pussing

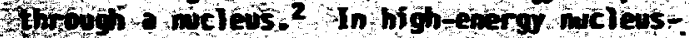

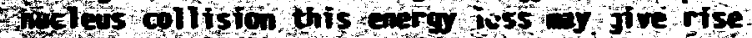

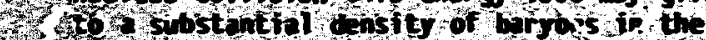

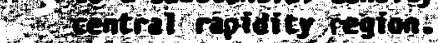

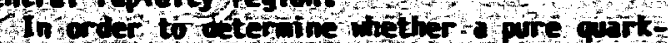

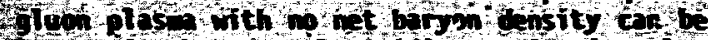
3 ond in the centrol caldity region on relati-

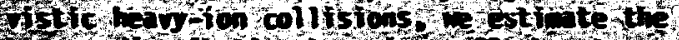

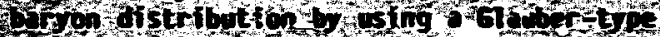

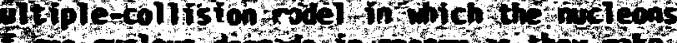

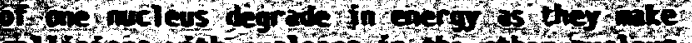

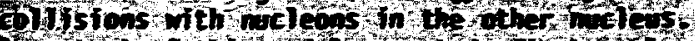

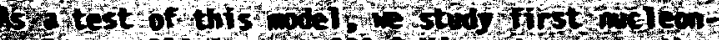

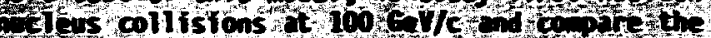

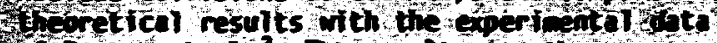
of Darton et als 3 me results are then ponera112d to study the bajon distribution in.

14 teis-nucleus collisions. It is found that in the head-on coletstion of two heavy nuctei $(A)$ 100), the baryon rapidity distributions have broad peaks and extend well into the central raptidity region. The energy density of the baryen in the central rapidity region is about 5-68 of the total energy density at a center-ofmass energy of 30 GeV per mucleon and decreases to about 2-37 at a center-of-mass energy of 100 GeV per nucleon. The stopping power for a baryon in muclear mitter is extracted.

1. Sumary of papers: Phys. Rev. Lett. 52, 1393 (1984) and Phys. Rev. 0 30. 972 (1984).

2. U. Busza and A. S. Coldhaber, Phys. Lett. 1398, 235 (1984).

- 3. D. S. Barton et al., Mys. Rev. 027. 2580 (1983).

\section{IUCLEON-MUCLEUS REACTIONS AT UTRA-RELATIVISTIC EAERGIES}

\section{Cheuk-Yin Wong}

In order to discern coherent processes from incoherent processes in nucleon-nucleus reactions at high energies, we study these reactions with an incoherent multiple collision modpl. In this model, the projectile mucleon ankes successive inelastic collistons with nucleons in the target nucleus, the probability of such collisions befing given by the thiekness function and the nuclcon-nucleon inelastic cross section. It is assumed that each baryon-baryon collistion produces particles and degrade momenta just as a baryon-baryon collision in free space, and that there are no secondary colltsions between the produced particies and the nucleons. We found that the inelastic proton data, the pseudnrepidity distribution dats diph/dn, and the total nucleon-nucleus absorption dats can be well explained by the incoherent mitiple colli.

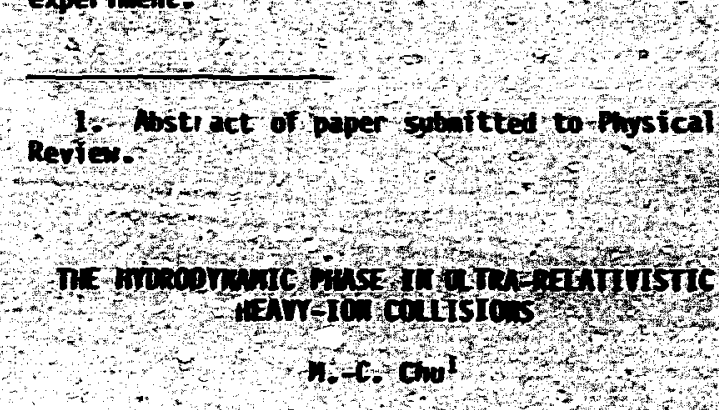

In recent vears, there has ben consturoble

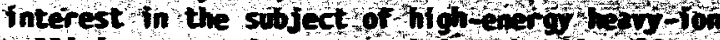
collisfons, a major reason being that tet offers the possibility of creating extended regions of extremely high-energy density. Ir fact, the energy density achievable ay bu high enough to carr atter through the decnnf inewent phase transitton. thus forning a quark-gluon plasma. 2-6 Such an exotic state of meter is belfeved to' simulate the conditions in the early universe and possibly in the interier of gravitationally collapsing astrophysical objects such as supernovae. The physics of quark-gluon plaswa therefore interests not only nuclear and partfele physfetsts but also astrophysicists as well.

If a quark-glion plasma is indeed formed in ultra-relativistic heavy-ion collisions, all the quanta excited in the plasma will rapidiy come into local thermal equilibrius, and a hydrodynamical description of the systen will be valid. It is of interest to study tie hydrodynamic evolution of a cylfindrically symietric quark-gluon plasma as formed in a central collision of two identical heavy ions.

At high enou th energies. two nuclef in a centra! collisicn will pass through each other. producing three regions in phase space: the target fragmentation region (TFR), the projectile fragmentation region (PFR), and the central rapidfty region (CRR). The TFR and PFR contain most of the net baryon number of the system and cont inue receding aray from each other at close to the speed of If ght after the collifston. In between TFR and PFR is the CRR with almost zero net baryon number but very high-energy density. Bjorken ${ }^{2}$ has given a rough sketch of the spacetime evolution of the CRR; fimediately after the collision, particles will be only makly interacting and undergo free streaming. Local thermol equifibrium will be established by a later proper time $\tau_{0}=1 \mathrm{fm} / \mathrm{c}_{\text {, }}$ and the subsequent hydrodynamic expansion of the plases will then be determined by the initial condteinns spectfied at $r$. $r_{0}$ and the laws of hydrodynamics. Further, assuming ...: existence of a central 
platede structure in the inclusive particle production versus rapidity, Bjorken brgues, that the fat obeys Lorentz-invariant boundary conditions. In particular, this means a iniform initial energy density, $\varepsilon_{0}$ and a scaling form of the Initial longitudinal veloc $=t y, v_{2}=z / t$.

The laws of relativistic bydrodynamics can be derived from

$$
2 P^{p}(x)=0
$$

with the stress-eitergy tensor

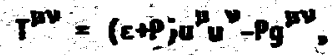

neglecting viscous and transport terns. Here $\varepsilon$ Is the local energy density. P the pressure, g $g^{\mathrm{wV}}$ the metric tensor, and the four-velocity of the noid.

2. Effect twe one-dineinstonal Dtion of the 1,irt-gloon plaswa with the bove inftial con-

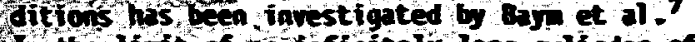
In the Ifitit of the taitely long cyllinder of plasw, these calculation show that the oredominantly longitudinal initial expanstion is Tater modified by the transwerse expansion. To study the more realistic case of a finit: cylinder, a two-dimensional calculation is therefore necessary. Specializing to cylindrical coordinates, we obtain the following set of equations:

$$
\begin{aligned}
& \frac{\partial}{\partial t}\left[(\varepsilon+P) r^{2}-P\right]+\frac{1}{r} \partial r\left[r(\varepsilon+P) r^{2} v_{r}\right] \\
& +\frac{\partial}{\partial z}\left[(c+P) r^{2} v_{z}\right]=0 \\
& \frac{\partial}{\partial t}\left[(c+P) r^{2} v_{z}\right]+\frac{1}{r} \frac{\partial}{\partial r}\left[r(c+P) r^{2} v_{r} v_{z}\right] \\
& +\frac{\partial}{\partial z}\left[(c+P) r^{2} v_{z}^{2}+P\right]=0 \\
& \left.\frac{\partial}{\partial t}\left[(\varepsilon+P) r^{2} v_{t}\right]+\frac{1}{r} \frac{\partial}{\partial r}\right) r\left[(c+P) r^{2} v_{t}^{2}+P\right] ! \\
& +\frac{\partial}{\partial z}\left[(c+P) r^{2} v_{t} v_{z}\right]=0 .
\end{aligned}
$$

Here, $v_{t}\left(v_{2}\right)$ is the now velocity porpendicular (paraliel) to the collision axis. We shall use the equation of state for a relativistic ideal gas, $P=c / 3$. wifh fs shom by lattice Hante Carlo calculations 8,9 to he a god approximation at temperacures above the critical temperature of the hadronization transition. The StefanBoltzmann law relates $c$ to temperature. $T$.

$$
\frac{c}{e_{0}}=\left[\begin{array}{l}
T \\
T_{0}
\end{array}\right]^{b} .
$$

where $T_{0}$ is the initial temperature.

Two mothods to solve Eas. (1) have been erfed. The first one ut flizes d finte element method in the r-z plane and propagates with a fourth-order predictor-corrector method in time.
In the second method, we rewrite Eq. (1) with the equation of state in a form resembling a Schroedfinger equation and then solve the equitions with the Peacean-kachford wethod. 16 . Both wethods are stable op to tive much later than wea the temperature drops to the critical temperature for madronization.

Prelfainary results from these calculations show that the quart-glon plases, if foried in an ultra-relativistic havy-ien collision, cools dom very rapidly. For the had-on collitsioe of 230y on 234, the energy density fin the plasia. will be lowered from $\varepsilon_{0}\left(-5 \mathrm{EeV} / \mathrm{fa}^{3}\right)$ Ref. 6) to 2 CeV/fin', wien hadronization is expected to start. after only buat $1 \mathrm{fo} / \mathrm{c}$. Wile the rate of coeling at $z=0$ is fond to caree with Bayn's calculations, that at the eads of the cylinoer is udified by a "pile-up" effect. resulting in slower cooling at large" 2 . eccuse of the rapid longitudinal expansion, the ef fect of the transverse expansion is w $y$ swal $\mathrm{fter}$ to hadroaization. The tehavier of the systen is also studied as a function of the highiy uicertain paraneter to. We found that the expansion of the plasma slows dom and becoses more uniform as the parameter $\tau_{0}$ is iacreased. Evolution of the system after the conset of hadroaization is an fuportant problew witch requires further study.

1. Guest assignee from California Institute of rechnology, Pasadena, Ca 91125, June-hugust, 1984.

2. J. D. Bjorken, Pijss. Rev. D 27, 140

(1983).

3. R. Anishetty. P. Koehler, and

L. NcLerran, Phys. Rev. D 22, 2793 (1980).

4. L. Miclerrin, in Quark Matcer Formition and Heavy-Ion rullisions, Prnc. Bhetereld Workshop, ed. M. Jacub and H. Satz (World Scientific, singapore, 1982), D. 63.

5. K. Ra; antle, in Quark Matter Formation and Heavy-Ion Collisions, Proc, hetefeld Workshop, ed, K. vacob and $H$. Satz (World Sciertific, Singapore, 1982), p. 39.

6. C. Y. Kong, Oak Ridge Mational Laboratory preprint, 1984.

7. G. Baym et al.., Mucl. Phys. M07, 541 (1983).

B. J. Engels, F. Korsch, I, Montvay, and H. Satz, Muri. Phys. Bzos [FS5], 545 (1982).

9. I. Montray and E. Pfetarinen, Phys. Lett. 1108, 148 (1982).

10. R. Varga, Matrix Iterative Malysts

(Prentice-Hali, Englewood CTfris, 1962), [. 273.

\section{THEORETICA:. ATOMIC PHYSICS}

\section{MLLTIPLE-VACAMCY PROOUCTION IN THE IDEPEDDENT-FERRI-PARTICLE MOOELI}

$$
\text { R. I. Backer A. L. Fard? }
$$

Neasuroments of $k x-r a y$ (or Auger) satellite intensities produced by fon impact have yfelded 


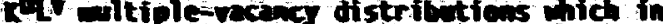
wost cases are narly bimajial. This toplfes that statistically, the hales bre nearly indeplient, wich is surpising in vier of the

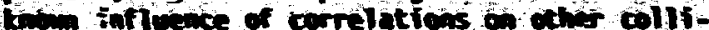
sten ricesses. We give a etailed deribation. of geveral exresstons for to-foinced altiple: recancy distribotigns in the fodepeidenteferint-

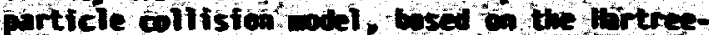

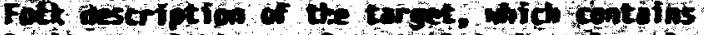

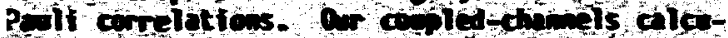

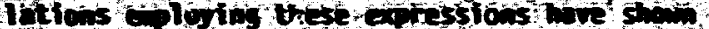

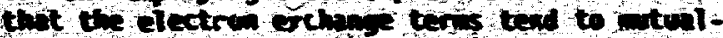

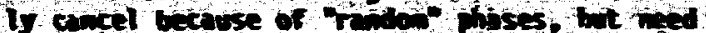

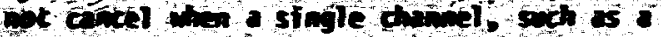
resoment electro coture. is-tominat.

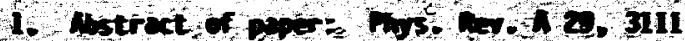
(193).

2. Texs $A$ \& $n$ bof wesity, colle, station. In Thas.

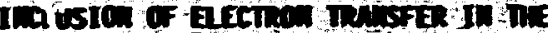

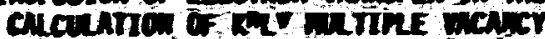 mosuciton or in finct ${ }^{2}$}

$$
\begin{aligned}
& \text { R. L. Becker A. L. Ford }{ }^{2} \\
& \text { J. F. Deading }
\end{aligned}
$$

Measureants of $k x$-ray or Auger satellite (and inpersatellite) intensities provide faforation on $X^{V}$ (and $R^{2} L V$ ) hole production in close collistons of ions with atoss. The Lshell vacarcy distributions usually are nearly bincmial. This distribution is spectified b; stingle parameter, $\overline{p_{1}}$, the mean $L-s h e l l$ vacancy probability. per electron. mich can depand on the speed $v$. the muclese charge $Z_{p}$ and the ionic charge $q$ of the profectile, and on the nuclear charge $Z_{T}$ of the target. Almost all calculations of $\bar{p}_{1}$. aside from our work, ${ }^{3-5}$ have been Iinited to first-order collistion approximaticns. horeover, all such calculations have included oniy impact tonfzation and, consequently, have neglected electron transfer to-the projectile. The first-order calculations have had enough success for proton and at, ha-particle inpact to remin in wogue for a decade, but they predict that $p_{2}$ is proportional to $z_{p}^{2}$, whereas $\bar{p}_{1}$ mist rewin 4 . Wich requires a inttary colliston approximation. We have coloined the "saturation" of $\bar{p}_{L}$ wth $Z_{p}$ in to untiory schimes, the pirst hagus approximation, and the mpre refined coupled-chamels approxtantion." 4 in beth scheres we used sfigle-centered expansion (SCE) in spin-orbiteis representing bound and unbound states of the target. Typically, we enploy cen radial functions for each of four ingular moments (s, p, d, and $f$ states).

The SCE provides on accurace basts for asymmetric systems $\left(Z_{0} \ll Z_{T}\right)$, but becomes inaceurote for nearly symmerfic systeins at intermodtate energies for which electron transfer to the profectile is very prcbeble. A full coupledchannel calculation with two-center expansion

(Ta) is stil watbittue for anselectro systes. We wave previously eneloped an inter

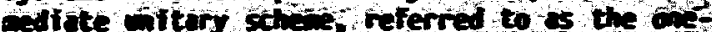

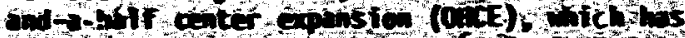

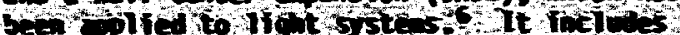

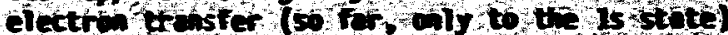

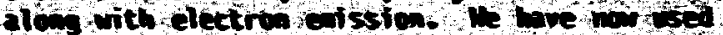

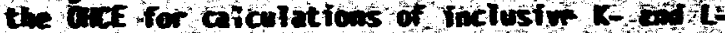

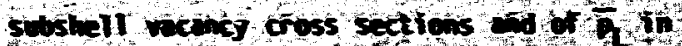

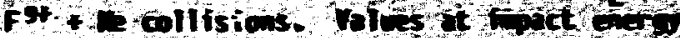

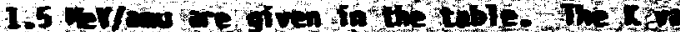

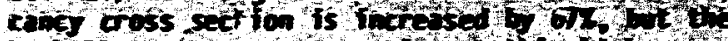

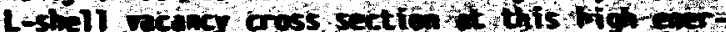

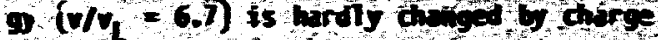
transto.

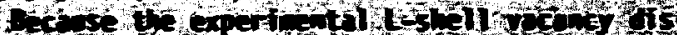

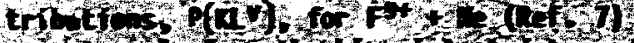

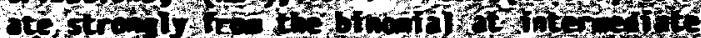

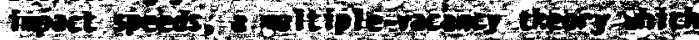

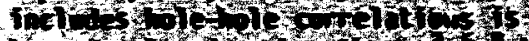
twe

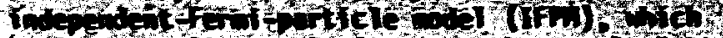

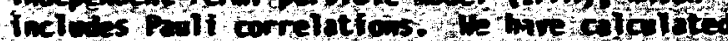
the effects of electro thastor o, to oft

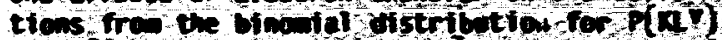

for Fot + Wa. It twined ot that the chersetrensfer from the k-shell to the now ine $k$ shell does not case inch deviation from binoail. The varfance is given in Table 5,1 . It reains to be seen what effect transfer to the

Table 5.1. Comparison of coupled channels cal. culations of vacancy production with and without inclustion of charge transfer (C.T.) to the arojectlle $x$-ihell: Fst + the at 1.5 weV/and. Inclustre racancy-oroduction cross sections. of in $10^{-20} \mathrm{~cm}^{2}$. A- $x-r \mathrm{y}$ satellite $(j=1)$ and hoper. satellite $(j-2)$ information: (1) Ean $L-s h e l l$ vacancy probability per electron, $\bar{p}_{1}(j) ;(2)$ ratin, $R^{(j)}$ of IFFi to binooial variance (both from oistributions with the IFPM value of $\bar{p}_{\mathrm{L}}(\mathrm{J})$.

Nithout r..T.

With C.T.

$\begin{array}{lll}q_{K} & 0.086 & 0.144 \\ \sigma_{25} & 2.337 & 2.391 \\ { }_{2 p 0} & 1.831 & 1.966 \\ { }_{2 p \pm 1} & 7.038 & 1.058 \\ q_{L} & 24.245 & 24.465 \\ \bar{p}_{L}^{(1)} & 0.759 & 0.754 \\ p_{L}^{(2)} & 0.781 & 0.799 \\ R^{(1)} & 0.976 & 0.939 \\ \dot{R}^{(2)} & 0.332 & 0.863\end{array}$


L-shell wight have. Hork has begun on that problew.

1. Sumary of poper presented at "x84," the International Conference on X-Ray and InnerShell Processes in Atoms, Molecules and Solids, leipzig, Ang. 20-24, 1984. See $x 94$ Nostracts, p. 41.

2. Texas A \& $n$ University. College Station, TX 77843.

3. R. L. Recker, A. L. Ford, and J. F. Reading, Mur.l. Instr. Neth. 214, 49 (1993).

4. R. L. Pecker, A. L. Ford, and J. F. Reading, Mucl. Instr. Neth. 13, 43 (1984).

5. R. L. Recker, A. L. Ford, and J. F. Reading, lucl. Instr, weth. B, 271 (1984).

6. J. F. reading, A. L. Ford, and R. L. - Pecker, d. Phys. R: Atom. Dol . Prys. 14, 1995 (1981): inid. 15, 3257 (1982); A. L. Ford, J. F. Reading, ind R. L. Becker, ibid. 15, 3257 (1982).

7. R. L. Kauffman, C. U. Ubods; K. R. Jamison, and P. Richard, Phys. Rev, A 11,872 (1975).

B. R. L. Recker, A, L. Ford, and J. F. Reatiry, Phys. Rev. A 29, 3111 (1984).

\section{THEORT OF MUTIPLE L-SHEL VACANT PROOUCTIO} In coImCIOESE WIH ELECTROM TRAMSFER I

\section{R. L. Becker}

Coincidence measurewents of the final ionic charges of both the projectile and the target provide a clean separation between reactions invol ving or not involving, charge transfer. Figure 5.23 shows such cross sections for $\mathrm{H}^{+}+$ Me. 2 They are dominated hy L-shell processes. Each cross section is labeled by two or three subscripts. The first two are the initial and final tonic charges of the projectile. A third subscript signifies the final number of vacancfes, $v$, in the target. The single-chargetransfer cross section with $v$ L-shell vacancfes can he calculated in the impact-parameter formalism. with the assumption that the transier is to the K-shell of the projectile $(\bar{x})$ by

$$
\sigma_{v} \equiv \sigma_{R_{,} L}^{K_{,} v^{8-v}}=2 \pi \int_{0}^{\infty} \text { dB B } p_{T_{,} L}^{K_{0} L^{8-v}}(B)
$$

where suserscripts label final occupancies and subscripts, final nonoccipancles. In the independent-Fermi-particle model (IFPY) the "number-exclusive hyperinclusive" (REHI) probsblitty in the integrand is given by an expression, irilar to that ${ }^{3}$ for $p K, L^{8-V}(B)$, approprlate for $K_{\text {a }}$ satellites. Figure 5,23 contains coupled-channels values at $E$. 75 and inn rev for olov, $v=1,2,3$ and their sui: $\sigma_{10}$. The prohahtlity for $v=0, p_{K, j}^{k}, l_{0}^{B}(B)$, is essentially zero in the IFPM. (There is a very sindil con. iribution from tronster from the k-shel!.)

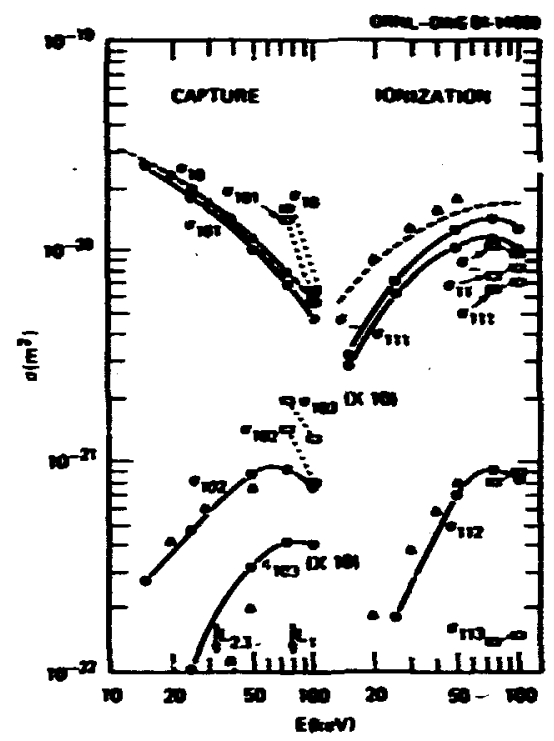

Fig. 5.23. Cross sections for the electroncapture and direct ionization channels in $\boldsymbol{H}^{+}+$ The collisions. The data and carves through them are taken from Fig. $2 a$ of Ref. 2. The cpen rectangles give OHCE-IFPm-coupled-channels values. The suiscripts are defined in the text.

Figure 5.24 gives the binomial and IFPM distri-

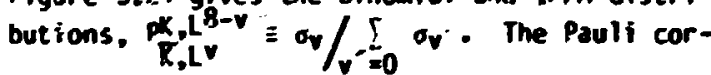
relations of the IFPM perait the supprission of

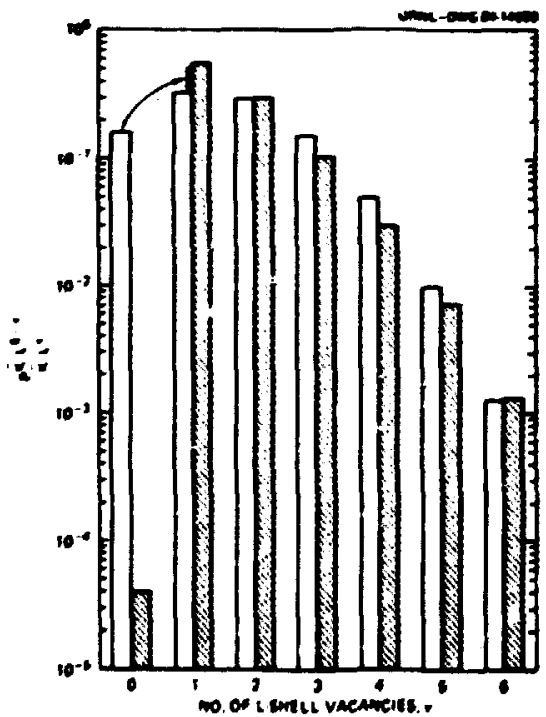

Fig. 5.24. L-shell vacancy distributicns (integrated over the impact parameter) for single charge transfer in $\mathrm{H}^{+}+$Ne collifions at Im keV. Shader, IFPM calculations, unshaded, binomial distribution with the IFPM value of $\bar{p}_{L}$. 
the $v=0$ components, in agreenent with experiment. These measurements ind IF/n calculations show for the first time a strikingly nonbinomial vacancy distribution explainable by Paulf corre1ations. Data and calculations ${ }^{2}$ for $\mathrm{He}^{+}+$the show the same features.

A wore complicated coincidence experinent could measure the L-shell vacancy distribution for collistions in which there is a charge transfer to the $\bar{R}$-shell and also a $K$ vacanicy. Rodoro et al." have, in fact, done this for the no-L-shell-vacancy case, $v=0$, by resriving the diagran line in neon under le 2 impac:. Uith If ${ }^{37}$ the diagran line ws warely see i. Only a little wore resolution would be needed to separate the satellites with varfous mubers of L-stell I vacancies. The theory of such a experiment would involve the KEI $R_{,}, K_{,} L^{8-v}(B)$. It is now being evaluate $J$.

1. A more extended discession is given in Section 4.2 of R. L. Becker. Wultiple Vacancy Pruduction by High Energy Heary Ions," invited paper at the Second Wortshop on High-Energy Ion-Atom Collision Processes, hug. 27-28, 1984, Debrecen, Hungary, to be pubifshed (Akadémiai Kfadó, Budapest).

2. R. D. OuBois, Phys, Rev. Lett, 52, 2248 (198i).

3. R. L. Becker, A. L. Ford, and J. F. Reading, Phys. Rev. A 29, 3111 (Ige4).

4. H. Rddbro, E. Horsdal-Pedersen, C. L. Cocke, and J. R. Macdonald, Phys. Rev. A 19. 1936 (I979).

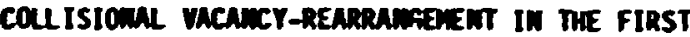

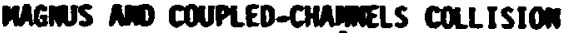 THEORIES ${ }^{3}$}

\section{R. L. Recker}

Measurements of L-subshell cross sections in gold and nefghboring heavy elements have shown variations with projectile charge, $Z_{p}$, and speed, $v$, which are not explained by first-order theortes with higher-order correct: ons for Coulamb deflection of the projectile and increased binding of the electrons and polarization of their wave functions during the collitston. Sarkadi and Mukoyama 2 took into account the collistional transfer of L-shell vacancies. from one subshell to another by a second interaction with the projectile, in a two-step model. They employ quantum-mechanical collision anplitudes to obtain probabilities for inclusive Lsubshell tonizations and for exitations (transfers) from one subshell to another. But they combine these probabilities in a completely classical way in set of kinetic equacions. Calculations with the model did repranuce the decrease $\sigma^{\circ} \sigma_{L_{3}} / \sigma_{L}$ wt in increasing $Z_{p}$ at low $v / v_{L_{3}}$, hut did not reproduce the ratio $\sigma_{L_{1}} / \sigma_{L_{2}}$, nor the alignment parameter $x_{20}$ of the $L_{3}$ subshell.
We show that ir the fully pantum-mechanical. unitary, first higius approximation, in sea classical collision theory one finds collisional-vacancy-rearrangement terms analogous to those of the two-step nodel. It also contains nonclassical terms, wich canot be expressed is products of probabilities. We give results .. merical calculations for argan, in the first .agnus and in the coupled-channels $a-$ proxination, wich fllustrate the deriations from first-order values of both ratios of subshell cross sections and 20 . At the relativeiy lom-inpact speeds of interest, charge transfer can be ifortant. We have begu calculatfons with our one-and-a-talf-center yersion of coupled-cnanmels theory to exhibit the effects of electron transfer.

1. Sumary of paper: Proceedings of the Second Horkshop on High-Energy Ion-itom Cr: Iision Processes, hug. 27-28, 1984, Debrecen, Hungary, to be published (Akadéaiai kiadó, Budapest).

2. L. Sarkadi and T. Mukoyana, J. Phys. B14, L255 (1981).

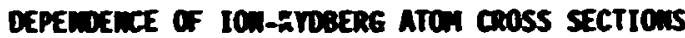
On ThE CRIEATATION of The RYOBERG STATE

$$
\text { R. L. Becker A. D. mackellar' }
$$

Classical trajectory monte Carlo (CTMC) calculations have proved very useful and surprisfingly accurate in estimating cross sect fons for collisions of charged particles (fons and electrons) with electrons in high Rydberg states. Aside from our wrk, the ralculations have been for classical ensembles corresponding to complete n-shells. However, preparation of Rydherg states by multiphoton absorption allows for the selection of the $l$ value. We have derived ${ }^{2}$ subensembles of the microcanonical hydrogenir. ensamble wich correspond to Rydberg states of given $(n, l)$ or given $(n, l, m)$. Results for the dependence of charge transfer. Fonization, and excitalion cross sections on the initial $i$ value have been published. ${ }^{3}$ The dependence of the charge transfer cross section on the initial m value is even are striking."

Figure 5.25 shows the CTMC charge transfer cross section for $\mathrm{H}^{+}+\mathrm{Na}^{*}(n=28)$ with $l=2$ and 27. Each $m$ value corresponds tn a classical range of values of a second fuler angle, $\theta$. For $m=0$ the ranje ends at $\theta=\pi / 2$, wilie for $m=l$ the range enjs at $q=0$. The figure shows also cross sections for spectified values of $(l, g)$ and of $(\ell,|m|)$. For $l=27$, specific $m$ values correspond to sich small intervals of that one value of $\theta$ in the triterval represents $n$ quite well. For $1=2,3=\pi / 2$ ceases to represent adequately $m=0$, and $\theta=0$ does not represent $m=2$ suffictently well. Hotice that for the nearly circylar orbtts $(t=27)$ the ratto $\sigma(m=0) / \sigma(m=27)$ exceeds an order of magnitude at high-impact speeds. 
1. University of Rentucky, Lexington, $\boldsymbol{K r}$ 40506.

2. R. L. Recker (unpubl ished).

3. R. L. Recker and A. D. Mackellar, J. Phys. B 17, 3923 (1984).

4. R. D. Mackel lar and R. L. Becker, 13th ICPEAC (Gerlin, 1983) Mbstracts, D. 660; R. L. Recker and A. D. Mackellar, Bu: 1. M. Phys. Soc. 29, 795 (1984).

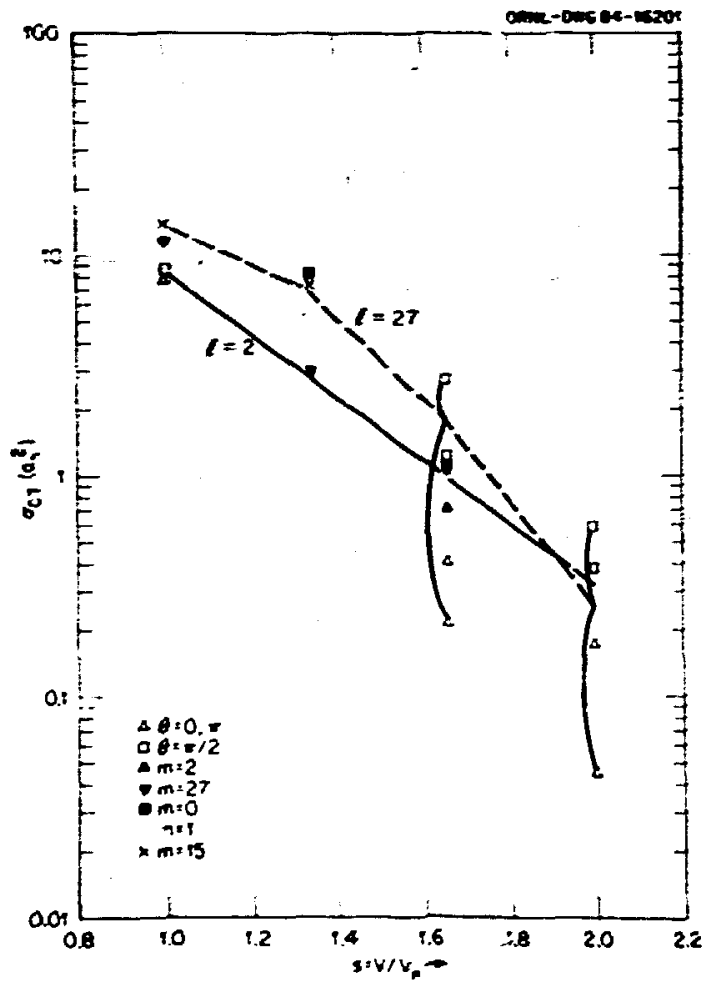

Fig. 5.25. C,TMC charge transfer cross section for $\mathrm{H}^{+}+\mathrm{Na}^{*}(n=28, l, m)$ in units of $\mathrm{an}_{n}^{2}=$ $n^{4} a_{0}{ }^{2}$ versus scaled impacl speed $s=v / v_{n}$ where $v_{n}=v_{0} / n$. The full (dashed) curve is for $\{=2(27)$ and a uniform weighting of $m$ values. Points: $\Delta, \theta=0, \pi ; \square, \theta=\pi / 2 ; \Lambda, m=2 ; 7, m=27 ; \square$, $m=0 ;+, \mid m i=1 ; x, m=15$.

\section{DUERICAL SOLUTYON OF THE TIME-DEPENDENT DIRAC EOUATION'}

\section{Bottcher M. R. Strayer}

A whe range of problems in atomic, nuclear, and particle physics can be reduced to the nuperical solution of systems of relativistic fermion wave equations, Dossibly coupled via fields. He have developed a direct numerical method of propagating solutions of the Dirac equation in tiine, with particular application to electron-positron pair production in heavy-ion collisions. Dur formulation contains a unified description of dmanical and spontaneous particie production, wich have hitherto been treatid as separate phenomina. We can also display pictures of the werefunction to assist the interpretation of various physical phenomena.

It is generally accepted that electronpositron pairs should be excited from the racuum in low-energy collisions of ions having a combined nuciear charge $\geq 173.2$ "Electrodynamic" positrons have indeed been observed; ${ }^{3}$ the most striking feature of these enission spectra is the presene of sharp Iines wich suggest the formation of long-lived nuclear complexes."

Calculatior of positron spectra requires that probabilities be calculated for the following single-particle processes: creation of a $\mathrm{k}$ shell vacancy, e.g.,

$$
\begin{aligned}
& 1)^{q+}+c^{n+}\left(1 s^{2}\right)+v^{q^{+}}+c^{(n-1)+}(I s) \\
& +e^{-\left(E^{-}\right)}
\end{aligned}
$$

and excitation of an electron from the Dirac sea into the sacancy

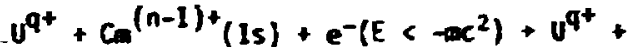

$$
\begin{aligned}
& \mathrm{Cm}^{\mathrm{n}+}\left(1 \mathrm{~s}^{2}\right)
\end{aligned}
$$

The ret outcome is the creation of an electronpositron pair with energies $\left(E^{-},-E\right)$, respective1y. Given the nuclear trajectory, both probabilities are deterained fran the solutions of the time-dependent Dirac equation for the notion of a single electron.

Since pair production occurs at very small internuclear separations (<100 fim), we are justified in using a monopole approximation to the two-center electrostatic potential. The wefunction then refiuces to a two-component pinor. whose components depend on a cingle radial coordinate $r$ (as well as time). These components are discretized hy expanding in finite elements. Most of our calculations are performed with 85 elements distributed nonuniformly over the interval $r=\left(0,12 a a_{0}\right)$, about one-third being inside the target nucleus. Propagation in time was achieved using a $(1,1)$ Padé approximant to the evolution operator over a small time interval.

To obtain results, we have had to iritroducs several novel numerical techniques: (1) then the collision is complete, transition probabilities are computed by projecting on eigenstates of the final-state Hamiltonian in the finite element hasis. (2) The accuracy of the solution at each time is controlled hy fterated matrix inversion. (3) Fermion coubling (the pathological appearance of high momentum components at low energiej) must be removed to calculate reli. able transition probabilities; we have achieved this by monifying the matrix representation of the kinetic energy operator.

As a first rest of the method, we calculated the probability $P_{K}$ of ejecting a K-shell electron from Po in a collision with $\mathrm{Sm}$ at a homharding penergy of $5.7 \mathrm{MeV} / \mathrm{amu}$. The variation of $P_{K}$ with impact parameter ${ }^{2}$ is rcasonahle. 
We next considered a Utin collision at 6.05 hel per particle with no muclear time delay $(T=0)$, and with a long tíce delay ( $T=50 \mathrm{a}^{2} \mathrm{t}_{0}$, $\left.t_{0}=2.42 \times 10^{-17} \mathrm{~s}\right)$. We tenote the transition probabilities from the initially occupied $15_{1 / 2}$ orbital to the positfre and negative contima by Pe-sPet: Figs. 5.26(a) and (b) show how Pe-, Pet ewives duriag the collision with and without a nuclear time delay. We note that one corsequence of the delay is to increase the flux of enitted pusitrons and decrease the flux of enitted electrons. The effect of the delay on the areft.ction is show in Figs. 5.26(c) and (d). The localized state in $5.26(c)$ decays into the outgoing waves seen to the right in fig. 5.26(d).

We have investigated the dependence of the energy spectra of the ejected electrons and positrons on a muciear tive delay T. Figures 5.27(a)-(d) show that regular oscillations in the positron spectra are characteristic of short time deTays, and that for $T>10 \alpha^{2} t_{0}$ these are superseded oy a sharp peak. The total positron probability grows approximately linearly, reaching value 0.23 wen the time telay $T=50$ $a^{2} t_{0}$, and thereafter does not change appreciably as $Y$ is-increased. The width of the peak decreases as $T$ increases and reaches a Iiniting value of $90 \mathrm{kel}$ due to the finite extent of the fiafte element lattice; the position of the peak for this case is constant at $2.23 \mathrm{mc}^{2}$ regardless of T. In Fig. 5.26(d) we also indicate the position of the peak in read-on U+Pu collisions (1.90 $\mathrm{mc}^{2}$ ) at about the same bombardir $q$ energy. and in U+Cin collisions at an inpact paraneter. $b=19 \mathrm{fm}\left(1.58 \mathrm{~m}^{2}\right)$.

1. Summary of paper submitted to Physical Review Letlers.

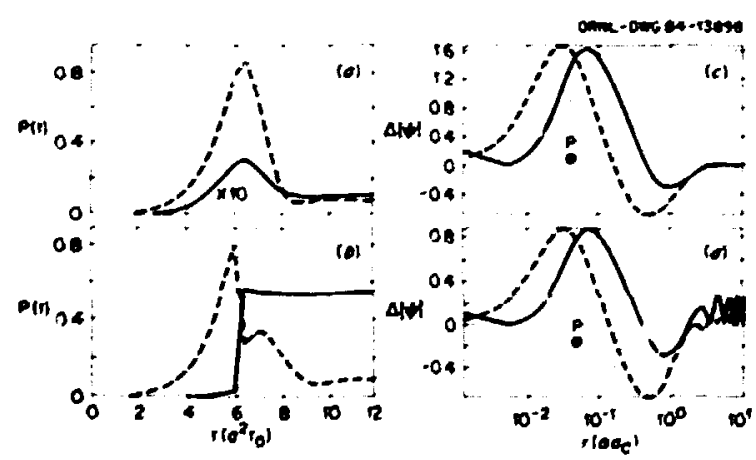

Fig. 5.25. ! $1+C_{m}$ hearton collitsion at a bomharding energy of $6.05 \mathrm{MeV}$ per particle. (a). (b): time evolution of the positron (full line) and electron (ootted Iine) in the finite element basis with and without a time delay of $T=5 n a^{2}$ $t_{0}$ occurring at $t=6 \alpha^{2} t_{0},(c)$ and (c) shows the change in the modulus of the $1 s_{1 / 2}$ wavefunction vs. the distance from the target nucleus hefore and after a time inlay of $i=50 a^{2}$ $t_{0}$; full and dotted lines refer to the small and large wavefunct ion components $G$ and $F$; denotes the position of the projectile nucleus.
2. Ouantion Electrodjmadics of Strong Fields," ad. W. Greimer, Pleme Press, how Tork, 1983.

3. J. Schmeppe, et al ., Myss. Rev. Letts. 51, 2651 (1983); H. Clemente, et al ., Phys. Let55. 137B. II (1984!.
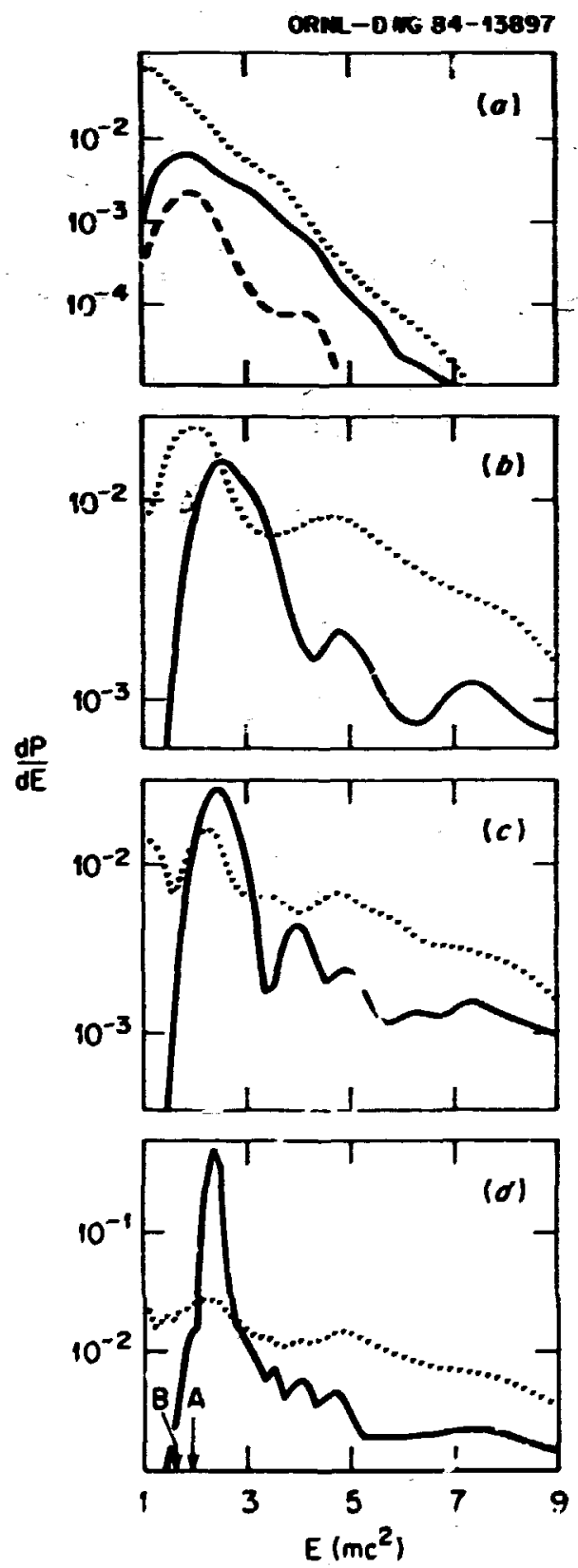

Fig. 3.7.7. $13+C m$ hadi-on collision at a hombarding energy of $6.05 \mathrm{MeV}$ per particle. Positron (full line) and electron (dotted line) spectra in the target frame in untes of (mc $\left.{ }^{2}\right)^{-1}$ per collision, for (a): no time delay; (b). (c). and (d), respect ively, $T=2.5,5,20 \mathrm{a}^{2}$ to. The positron spectrum for : $19 \mathrm{fm}$ is shown hy the dashed line in (a). 
4. U. Heinz, et al.. Mnn. Phys. (K.Y.) 151 , 227 (1983); J. Reinhardt et al .. Z. Phys. 1303, 103 (1981); M. R. Strayer et al.., Phys. Zev. C

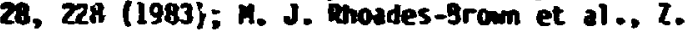
Plys - 1310, 297 (1983):

\section{PHEmaEmost of ACLER COLLISIOUS MITH Loms TINE DELATS}
M. A. Strayer
V. E. Oberacker' C. Bottcher

We have used the methods described in another section to interpret experiments on positron production in U+C. collisions.'

The energy spectrum of atomic positrons, integrated over wuclear scattering angles between $25^{\circ}$ and $65^{\circ}$ in the laboratory, consists of a broad structure ( $\mathrm{hm}$ ) between 0 and $1200 \mathrm{kel}$. on wich is superimposed a sall narrow feature (peak) at $316 \mathrm{keV}$ in the center of mass. The peak is associated with ingles close to $45^{\circ}$. We assume for the purposes of numerical estimation that the broad background is cure to the undelayed Rutherford trajectories with imact paraneters in the range $s=(4.5,19)$ fin. The pos $i$ tron yield involves the probability of creating a $K$-shell wle in the target, and that for filling the hole from the negative energy sea, both being strong functions of the impact parameter. Averaging over iapact parameters, we obtain a positron spectrum with a peak at $460 \mathrm{keV}$ in the center-of-mass frame, and reaching a maximum yield of $2.0 \times 10^{-7} / \mathrm{keV}$, in good agreement with the reasurements and adiabatic hasis calculations. 3

We shall identify the sharp peak with an inpact parameter $b=9 \mathrm{fm}$ appropriate to $45^{\circ}$ scattering of the heavy fons $m$ a Rutherford trajectory. This simple assumption predicts a peak at $390 \mathrm{keV}$ in the center of mass; a slightly modified trajectory would reproduce the experimental energy.

The ratio of the area under the peak to the drea under the remaining part of the spectrum, $(P / H)_{\text {expt }}=0.07 .4$ From our calculations, we know that the ratio $\lambda=$ (total positron probability with a time delay)/(the same quantity without a time delay) is insensitfve to the (mpact parameter, and $=1.5 T$. In derfye $(P / H)$ theory, we adopt a nodel in wich the nuclef have a probahility of coalescing in a range of relative orbital angular momenta (al) peak, thereby giving

$$
\left(\begin{array}{l}
P \\
H
\end{array}\right)_{\text {thenry }}=\frac{\lambda f \Delta\left(L^{2}\right)_{\text {peak }}}{\Delta\left(L^{2}\right)_{\text {hump }}} .
$$

Converting the angular momenta to scattering angles. we find $T f(\Delta L)_{\text {peak }} \times 55$ h. Since ah=l fm corresponds to $L=66 \%$, our estimate is in harmony with the estahlished models of the nuclear collision dynamics for this system.

He depart from earlier calculations in predicting sharp features in the efected electron spectra for some rangne of nuclear time delay. An example is given in Fig. 5,z,

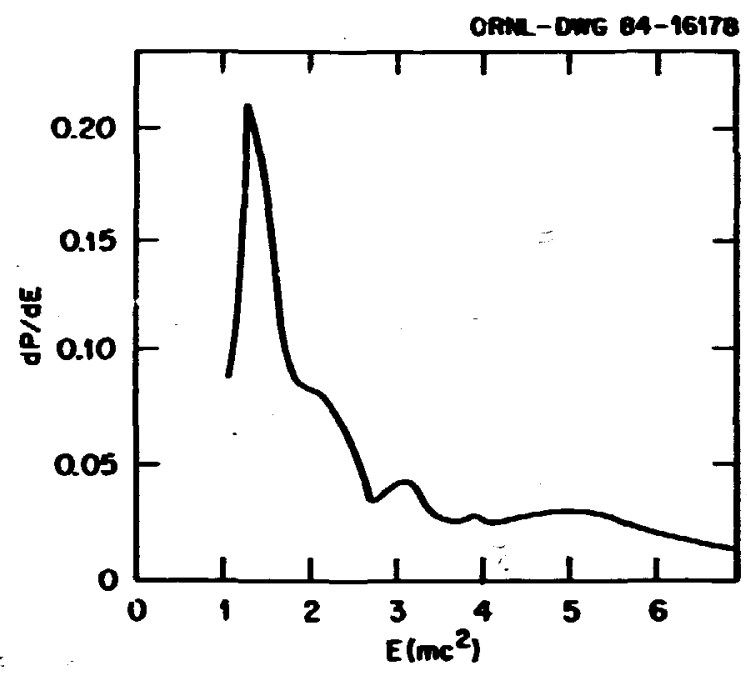

Fig. 5.28. Typical delta in electron spectrum as explafined in the text.

The niext stage il this investigation will be to synthesize thr predicted spectrum using realistic nuclear trajectories. We have already co. tructed suitable trajectories using model nu ir inte:actions with attractive pockets and inc!uding energy transfer to muclear excitations."

1. Consultant from Vanderbilt University, Nashville, TN 37235.

2. J. Schweppe et d?., Phys. Rev. Letts. 51, 2261 (1983).

3. J. Reinhardt et al., Z. Phys. A303, 103 (1981).

4. M. J. Rhoades-Brown et al., Z. Phys. A310, 287 (1983).

\section{TIME-DEPENDENT HARTREE-FOCK THEORY FOR HEAVY ION-ATOH COLLISIONS}

$$
\text { c. Bottcher }
$$

For several years, I have been able to sturty ion-atom collisions involving a single active elect:on, at intermediate energies (5-100) keV/amu), using numerical methods hased on a finfte-elenent representation of the wavefunction in positron space. I Other investlgators have published studies on two-electron systems. 2 The extension to "heavy" systems (which I understand as those having 10 or more electrons) raises new problems. In particular, the rapid $v$ ritation of the wavefunctions near the nuclef causes severe instabilities ard violations of unitarity.

The time-dependent Hartree-Fock (TOHF) method can he formulated as follows. The wavefunction of A electrons is approximated by a siater determinant of spin orbitals a with occupancy 
numbers we. Each orbital satisfies an independent particle Schrödinger equation

$$
h \phi_{\alpha}=1 \frac{\partial \phi_{a}}{\partial t}
$$

where (in atomic units)

$$
h=-\frac{1}{2} \nabla^{2}+v_{n}+v_{d}+v_{x}
$$

Ir. $5 q .(2), V_{n}$ is the muclear potential, $V_{d}$ the Iocal electrostatic interaction due to the other electrons, and $v_{x}$ the exchange interaction. We mate the following approximations: (1) $V_{d}$ is replaced by its average overall $a$, so that it is related to the electronic density by Poisson's equation.

$$
\nabla^{2} y_{d}=-2\left(\frac{A-1}{A}\right) D, 0=\Sigma \omega_{\alpha}\left|\phi_{a}\right|^{2}
$$

(2) $V$ is replaced by the Hartree-Slater local approximation. (3) Axial decoupling is assumed, $i . e$. , the wavefunction is taken to depend only on the cylindrical coordinates $(0, z)$ referred to the rotating internuclear axis.

The mumerical difficulties referred to above have been overcome by transforming to an orthogonal basis of finite elewents and by using an exact exponential propagator at each time step. Orthogonal finite elements mere also used to solve Poisson's equation. Excitation, capture, and ionization were distinguished by projecting on eigenstates of the initial and final

Hamiltonians constructed in the same basis used for the time propagation.

The process

$$
F^{9+}+\mathrm{He}+\mathrm{F}^{(9-m)+}+\mathrm{He}^{n+}+(n-m) e^{-}
$$

has been studied at two velocities, $v=1$ and 2 , and a range of impact parameters. Ionization and capture probabilities associated with the orbitals 250, 2pJ, and $2 p \pi$ are shown in Fig. 5.29. An interesting feature of these results is the "shakeoff" of $2 p$ o electrons around $b=1$ ap. This interpretation is borne out by the final state density contours show in fig. 5.30. At impact parameters $<0.5 \mathrm{a}$. shakeoff following quasi-resonant K-shell capture 1; important and will be addressed in future studies.

1. C, Bottcher, Phys, Rev, Letts, 48, 85 (1982); C. Bottcher, in "Electronic and Atomic Collisions." eds. J. Efchler et al. (Elsevier Science Publishers B.V., 1984) D. 1B7.

2. D. Etchenduer et al.., J. Phys. B 15, L17 (1982); K. C. Kulander et al .. Phys. Rev. A 25, 2968 (I982); K.R.S. Devi and J. D. Garefa, J. Phys. 16, 2837 (1983).

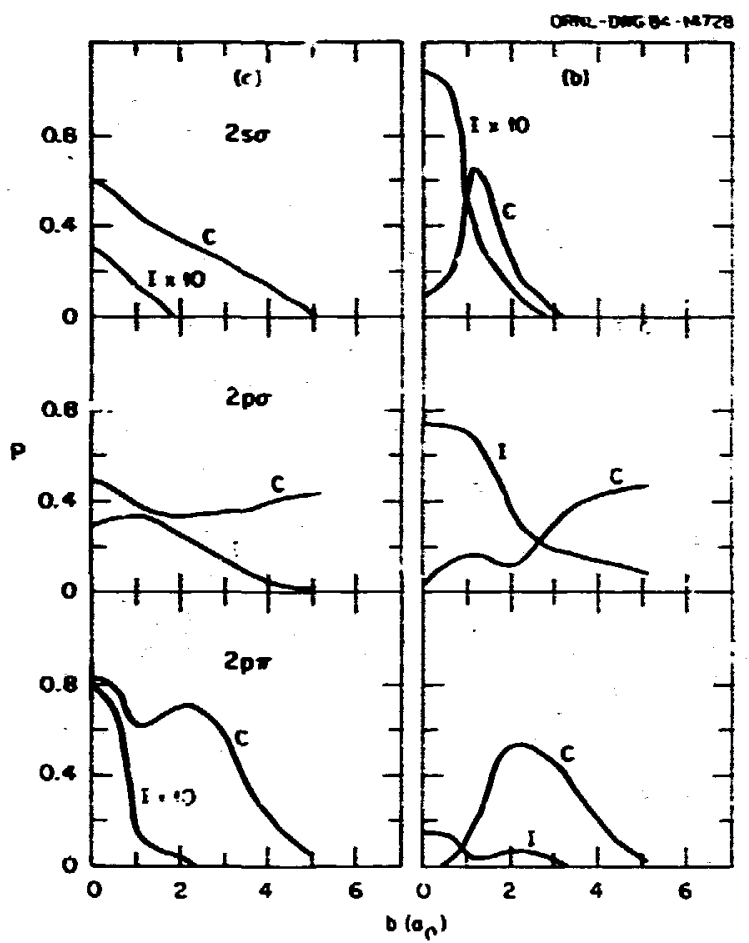

Fig. 5.29. The probabilities of capture (C) and fonization (I) for the 25\%, 200 , and $2 p \pi$ orbitals are plotted vs. fmpact parameter for two relative velocities: (a) $v=1,(b) v=2$.
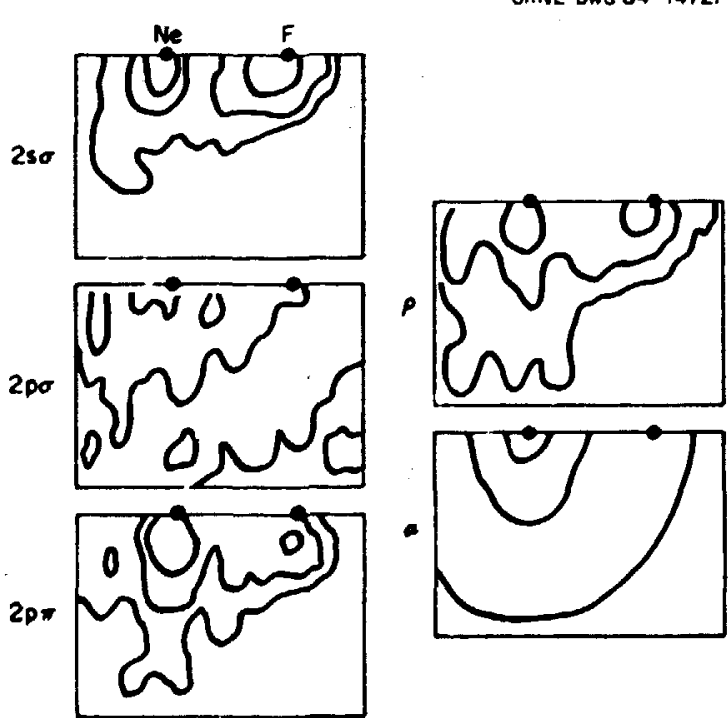

Fig. 5.30. The lefthand frames (see Fig. 5.29) show logarithmic density contours for the $2 s \sigma, 2 p \sigma$, and $2 p \pi$ orbitals. The righthand frames show contours of the total density (logarithmic) and the electronic self-consistent fli. Id (1 inear). 


\section{THESSHOD IOAIZRTIOM DHEORY}

\section{Bottcher}

In earlier work on low-energy ionization : csed mavepacket formulation and the timedependent Schrodinger equation. Further progress requires a athod of solving the stationary Schrodinger equation directly. This can be done by atching a set of solutions defined in a finite domain to asymptotic solutions on the lowndary.

All calctiations have so far been linited to the tro-dinension model of the e+H system, in which the electrons wre on a line passing through the proton at distances $r_{l}, r_{2}$ on opposite sides of the proton. Hyperspherical coordinates are defined by

$$
r_{1}=0 \cos \alpha, r_{2}=0 \sin \alpha
$$

We have show that eigenfunctions in a box $r_{1}$, $r_{2} \leq 93 a_{0}$ can be constructed with a finite element basis sufficiently large that the pripperties of breakup states near threshold are deseri'ed. In particular, it is possible to compute certain moments of the energy distribution between the two electrons with knowiedge of asymptotic solutinns, and the values agree with those predicted by the Wannier theory of threshold ionization to within $10 \%$.

It is well recognized that asymptotic solutions are best obtained by semiclassical methods. If I wite the wavefunction as $\psi=$ $\exp (i S), S$ satisfies the Hamilcon-Jacobi-Schrödinger equation

$$
(\vec{\nabla} s)^{2}=\left\{K^{2}+\frac{2 C(a)}{p}\right\}+i \nabla^{2} s
$$

where we have written the potential energy as $-C / p$. If I drop the term i $\nabla^{2} S$, I recover classical mechanics; the term discarded describes the diffraction of the deBrogite waves. Asymptotic solutions of (2) can always be described to a first approximation by a set of classical trafectories. I have found that all intuitively plausible solutions can be associated with sets of trajectories, each set having a common intersection (condensation point). Examples are given in Fig. 5.31: the set $W$ correspons to a so-called Hannier solution, and the set $T$ to a dipole (Temkin) solution.

In order to solve a scattering problem, I need a set of asymptotic functions $(p, a)$ complete in a for a fixed $\rho$. These can be obtained as follows for Wannier-type solutions. Suppose that at a very large distance

$$
S \sim K_{\rho}+\frac{C(a)}{K} \ln \left(\frac{p}{a}\right)+\lambda F(\alpha)
$$

where $F$ is almost arbitrary, e.g., $F(a)=1$ en $\sin 2 a$ leads to wavefunctions wich vanish rapidly as $a \rightarrow 0$. As $a, \lambda$ are varled, me obtains a double infinity of solutions sultable for matching at an fnner houndary. The conden. satton point is determfned hy a, a.g., the or -

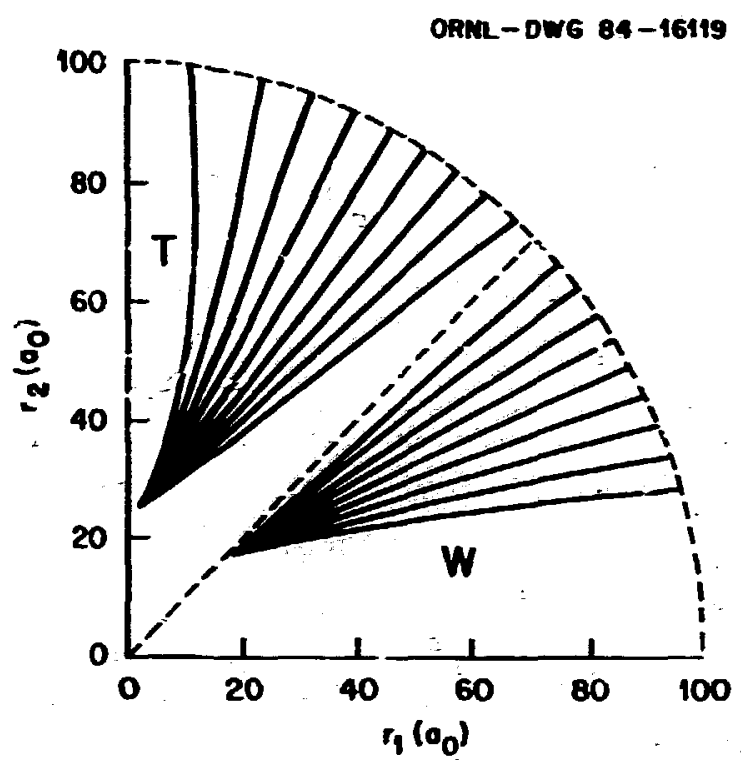

Fig. 5.31. Classical orbits corresponding to ionization in the e+pte system. The axes correspond to the distances of the two electrons from the proton.

bits in Fig. 5.3I correspond to $a=1, p_{C}=$ $25.02 / K^{2}$; $\lambda$ behaves like the index of a hyperspherical harminic. However, it is permissible to consider any linear combinations of the $\psi(a, \lambda)$; e.g., $\psi\left(a, \lambda_{0}\right)$ could be expanded in $\psi\left(a_{0}, \lambda\right)$ where $a_{0}, \lambda_{0}$ have fixed values.

From a numerical perspective, (2) is a highly (II-conditioned nonlinear PDE, but some progress towards solutions has been made.

A related subject is the use of a twodimensional model to obtzin qualitative insight into the four-body coulomb problem, f.e., two electrons in the field of two nuclei. This is discussed in another section of this annual report.

1. C. Bottcher, "Humerical Studies in Electron Impact Ionization," to appear in Advances in Atomic and Molecular Physics, 1985.

\section{FORMAL RELATIONSHTP BETMFEN MASER MHEORY AND QUANTUM FLUID RECHANICS}

$$
\text { C. Bottcher C. Feulliadel }
$$

For several years we have been investigating the hehavior of molecules coherently excited by radiation using the Bloch form of the densfty matrix equations. In particular we have studied the "ladder problem" in wich the molecule is modeled by an anharmonic oscillator. ${ }^{2}$ It seemed naturd to apply our formalism in an attempt to resolve a leng-standing controversy regarding the validity of classical solutions of this problem.? 
We started with the density antrix $p(\alpha, B)$ in the representation of vibrational states and transfored to a new representation $p(n, n)$. where $m, n$ are discrete eigenfunctions of the position operator $q$ with elgenvalues $q, 9 n$. Thus $p(\hat{0, \pi i})$ appraximates the density matrix in the position representation; diagonal elements correspond to particle density and off-diagonal elements can be related to the local current density, etc. Bloch equations in the position basis can be obtained from the usual Bloch equations by a linear transformation. The new equations can be related by a series of approxiintions to the classical Lfouvflle equations of wotion and hence to quantum fluid wechanics. Orr enalysis indicates that, unless the density of states is very large indeed, the inlierentiy quantal ef fect of stivilated enission is too larie to be discarded.

However, we have fourd that if the new equations are crimcated at the level of coherence between adjacent points $|\omega-n|<\& 1$, a remarkably good approximation to the exact (quantal) solution is obtained.

Figure 5.32 plots the faginary part of the dipole susceptibility in the model of Ref. 2
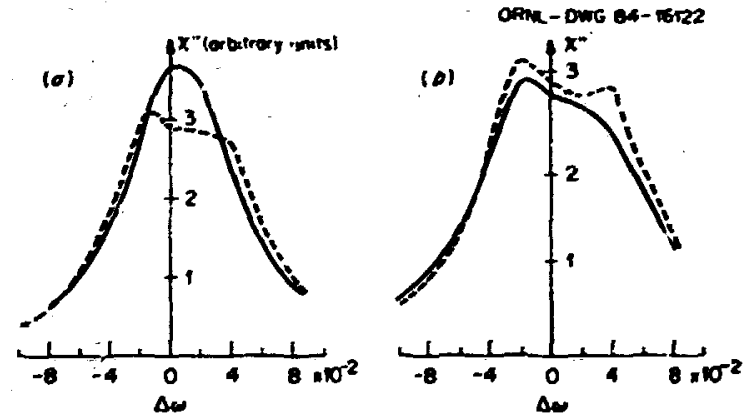

Fig. 5.32. Imaginary part of dipole susreptibility in the model of Ref. 2. (a) Origfral Bloch equations; full line, truncated to $a-B$ \& 1 ; Hasned 1 ine, exact, (b) Wew equatfons; full line, truncated to $m-n<1$; dasned line, exact.

with a reduced laser field, $1 \cdot 0.5$. In Fig. 5.32(a) show the drastic effect of truncating the original Bloch equations at the level $|a-\beta|<1$. Figure $5.32(b)$ shows that the effect on the new equations is much less severe. These equations are not equivalent to any prevfously recommended approximation, though their gelieral structure resembles quantum flufd mechanics. He are considering applications to the dissocfation of polyatonic molecules and to perturhed electron gases. The new approximation is suffi. cfently simple that it might be a competftive way of andyzing more complicater problems.

1. NORDA, N.S.T.L. Station, MS 39529.
2. C. Bottcher and C. Fevillade, Chem. Miss. L.stts. 95, 279 (1983).

3. J. R. Ickerhait et al., Optics Lett. 6, 377 (1981).

4. C. D. Cantrell et al., Optics rom. 60, 413 (1992).

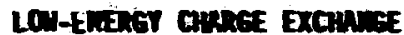

$$
\text { C. Bottcher ' T. G. Heill }
$$

We have continued our studies of charge capture from hydrogen atows by hishly stripped fons, using expanstions in one-electron mlecular elgenstates and a fuily gantal description of the nuclear notion. 2 When the adiabatic potential energy functions and coupling atrix elements have been calculated, we can solve the resulting coupled equations with sole facility. using fully vectorized pros rass developed for the Cray-1 and -15 .

In the past jear, we have moved on to study more highly fonized projectiles, notably the $8+$ isoelectronic sequence. Some results on $0^{\text {tt }}$ and Ar ${ }^{8+}$ are show in Fig. 5.33. We hope to study several members of this sequence with larger basis sets in order to make comparison with measurements in progress using the new ECRIS ion source at oRM. ${ }^{3}$

Since now routinely include -states in our basis sets, we have found a number of cases where $\sigma-\pi$ couplings affect the charge capture cross section by more than $30 \%$. Figure 5.34 shows results for $\mathrm{C}^{4+}$ and $0^{6+}$ projectiles. In

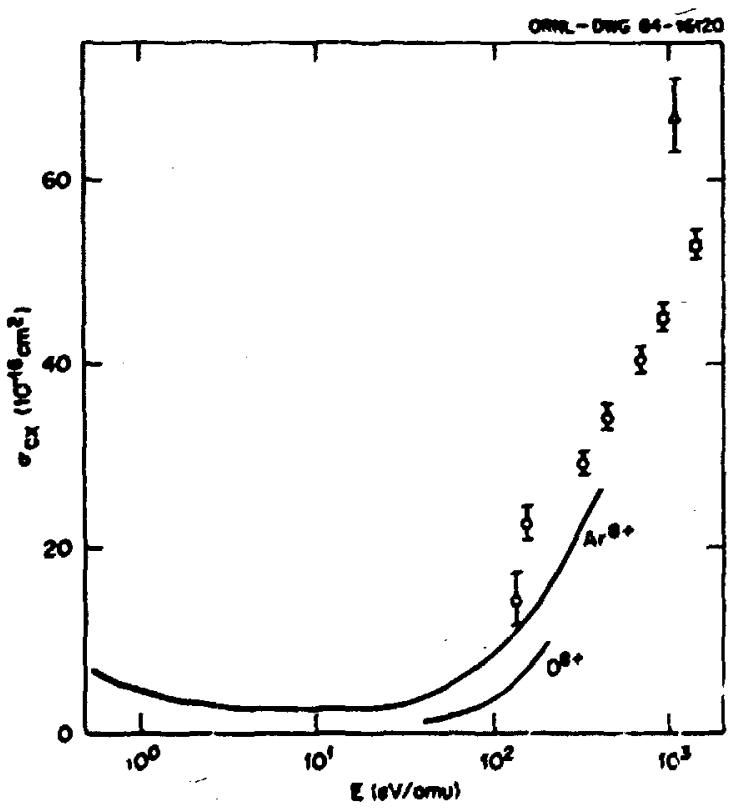

F1a. 5.33. Charge exchange cross sections for multicharged fons on hydrogen atoms. Fill Iines: calculated with a hats of 3 a-states: open circles: measurement of Ref. 3 on $\mathrm{n}^{\mathrm{Bt}}$ open trfangle: measurement of Ref. 4 on Art. 


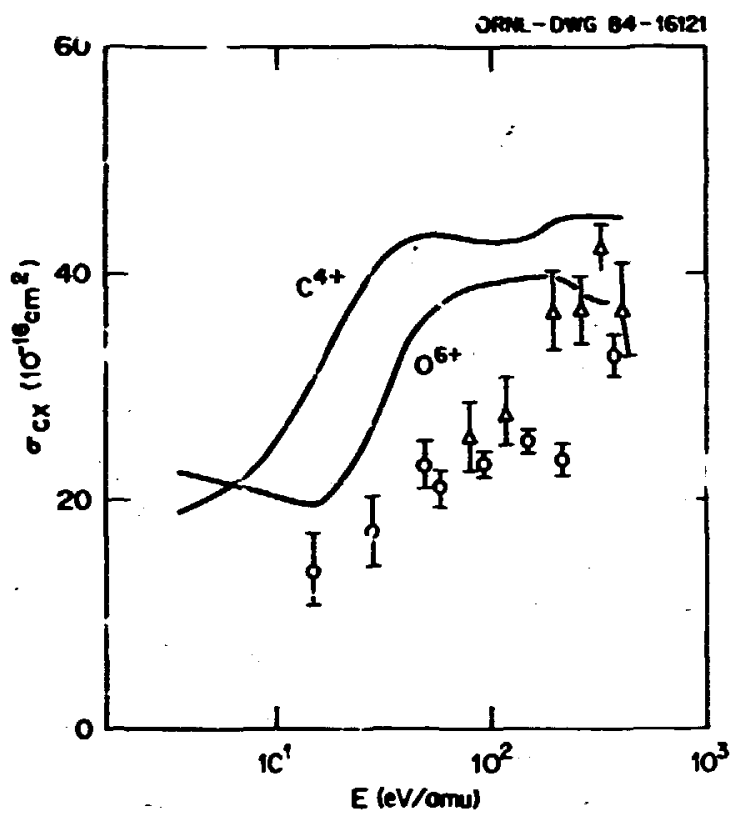

Fig. 5.34. Charge exchance cross sections for multicharged ions on hydrogen atoms. Fill lines: calculated with a basis of $30-$ and $3 \pi-$ states; open circles: measurements of Ref. 5 on $\mathrm{C}^{4+}$; open triangles: measurements of Ref. 6 on $0^{6+}$.

the case of $\mathrm{C}^{4+}$ the disagrepment with experiments appears to have worsened, a situation which merits further study.

He are also pursuing the more fundamencal objective of a very accurate calculation $m \mathrm{n}^{5+}$ + in in wich translation factors are included by matching to moving orbitals on some boundary. This matching requires an expansion in about 40 molecular eigenstates, for which we have now assembled the coupling matrix elements.

1. Consultant from University of Seorgia, Athens, $3 A$ A 30602 .

2. C. Boticher and T, G. Hefl, Chem. Phys. Letts. 86, 5.76 (1982).

3.. F. Meyer et al... private comunication.

4. D. H. Crandall et al., Phys. Rev. $A$ 22, $3 / 9$ (19BO).

5. P. A. Phaneuf, Phys, Kev, A 24, 1138 (1981).

6. R. A. Phaneuf et al., Phys. Rev. A 26, 1892 (1982).

\section{SUMMARY OF CODE DEVELOPMENT FOR ELECTRON-ION SCATTERING \\ C. Botteher D. C.e Griffint M. S. Pindzola'}

Bet,ween 1980 and 1983 we developed a program package to calculate distorter-wave excitation cross sections with internediate coupling wavefunctions based on a single configuration of H.trtree-fock orbitals. 3 The structure eigenvectors and radiative branching ratios ifor autoionizing states) are obtained from the Los Alamos atomic structure package developed by R. H. Cowan.

In the past year we have added codes to calculate ${ }^{2}$ rect ionization and dielectronic recombination. These codes are still based on Hartree-fock urbitals for bound states and distorted waves for continuw states, but use "conf iguration-averaged" approximutions in the structure calculations. They are mell suited to surveys of, e.g., isoelectronic senuences, and we have the capacity to stidy in more detafl individuil cases the e a breakdom of the approximations is suspecled.

At the same tire, a major effort has been devoted to extending the excitation code to handle configuration-interaction wavefunctions whi:h describe effects omitted by single configuration Hartree-fock theory, in particular, coupling between almost-degenerate configurations in the Hartree-Fock sea $\left(e .9 ., 3 s^{2} 3 p^{2}, 3 p^{4}, 3 p^{2} 3 d^{2}\right)$, and pair excitations out of the sed $\left(3 d^{l}, 3 d^{8} 4 f^{2}\right)$. The process of checking these rew codes is almost complete, and applfication to transition metal fons will be mase in the near future.

We hare had for some time the capacity to perform close-coupling calculations, but progress on the complex targets we are interested in has been hindered by the lack of an efficient angular momentum algebra packag: in other groups as well as ours. This defect has been remedied by adapting our distorted-wave algebra package, and pilot calculations are in progress.

Particular applications cf these codes are described in the follawing sections.

1. Consultant from Rollins College, Winter Park, FL 32789.

2. Consultant from Auburn University, Auburn, AL 36849.

3. C. Rottcher, 0. C. Griffin, and M. S. Pindzola, J. of Phys. B 16, L65 (1983).

\section{EXCITATION-AUTOIONITATION PROCESSES IN THE ELECTRON IMPACT IONIZATION OF SINGLY-CHARGED IONS}

$$
\text { D. C. Giriffinl M. S. Pindzola }
$$

\section{Bottcher}

We have examined the excitation. autoionization mechanism in eler.tron impact ionization of alkaline earth fons,

$$
\begin{aligned}
++n p^{6}(n+1) s & +n p^{5} n d(n+1) s+e \\
& +n p^{6}+e+e
\end{aligned}
$$

Our motfves were to examine the tata of Peert and Dolder ${ }^{3}$ which have never been fully interpreted, and to compare distorted-wave with 
close-coupling calculations, following a suggestion" that they wight seriously disagree for processes of the type (1). We also felt that the term dependence of the nd-orbital sho-ld have the same large effect as in the xenon isonuclear sequi nce wich we invest igated earlier.5 Indeed, it is the case that term dependence has a serious effect on the highest-lying state, that with a np'nd( ${ }^{1}$ ) parent, e.9., with a term-dependent $3 d$ orbital, the $3 p 53 d\left({ }^{1} p\right) 4 s^{2 p}$ state of $\mathrm{Ca}^{+}$lies at $33.6 \mathrm{eV}(37.6 \mathrm{eV})$ and has an excitation cross section at threshold of 25.4 mo $(106 \mathrm{~W})$. The mub rs in parentheses, just quoted, refer to the iveraged-config-ration 3d orbital.

We cárried out unitarized distorted-waye calculations in intermediate coupling for $\mathrm{Ca}^{+}$and $\mathrm{Ba}^{+}{ }^{6}$ The $\mathrm{Ca}^{+}$results can be compared with those of close-coupling calculations, and for fimpact energies twice threshold; the total excitation-autoionization cross sections agree to better than $20 \%$. No comparison is possible near threshold, since the close-coupling calculations ${ }^{4}$ retain only three out of nine possible levels of the multiplet and raglect term deper.dence; $m$ find that the discarded levels make comparable contributions to the cross section near threshold. However, both methods predict a large peak near threshold due to the $\left(L_{p}\right)^{2 p}$ state, of wich there is no experimental indication.

No other calculations have been performed on $\mathrm{Ba}^{+}$. Our results are shown in Fig. 5.35. As was the case in $\mathrm{Ca}^{+}$, the large calculated peak at $22 \mathrm{eV}$ due to (IP) $2 \mathrm{P}$ is not seen experimentally. He have, as yet, no explanation for this discrepancy, although we note that the peak is almost entirely dis to a single outgoing partial wave $(t=3)$.

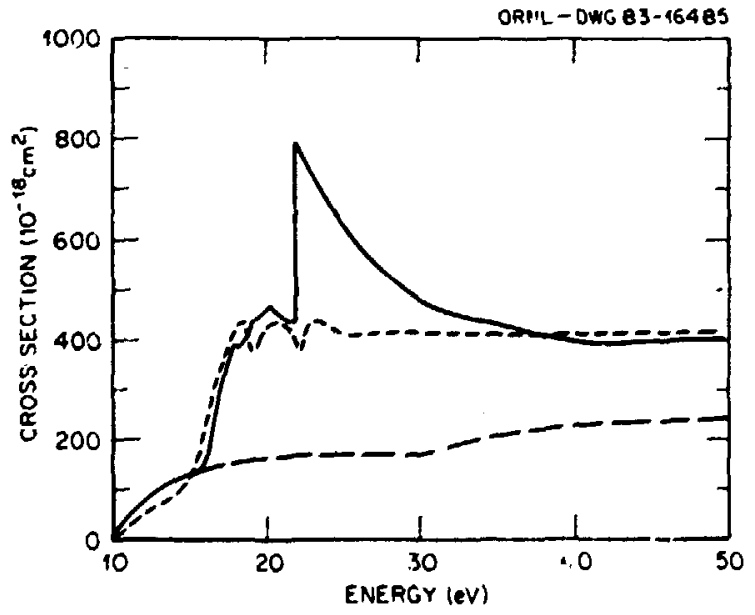

Fig. 5.35. Total iontzation cross section for Bat. Dashed line, direct tonization cross sections calculated from the Lotz equation (unscaled); dotted line, experimental results of Peart et a1. (1973); full line, unitarfzed, distorted-wave results (including transftions to all levels of $5 p^{5} 5 \mathrm{~d} 6 \mathrm{~s}$ ) added to the Lot $z$ value for direct iontzation.
We are now proceeding to close-coupling calculations on $\mathrm{Ca}^{+}$targets. The angular algebra part of our distorted-wave package has now been interfaced with the University College, London close-coupling package, (IMPNCT), opening up the possibility of large calculations with wany open shells. Exploratory studies suggest a high degree of mixing we to final-state interactions wich may remove the spurious peaks.

1. Consultani from Rollins College, Minter Park, FL 32789.

2. Consultant from Auburn thiversity, Auburn, N 36849.

3. B. Peart and K. T. Dolder, J. Phys. B: At - Nol. Phys. 8, 56 (1975).

4. P. 6. Burke et al., J. Phys. B: At. Mol. Phys. 16, L385 (1983).

5. D. C. Griffin et al., Phys. Rev. $A 29$. 1,29 (1984).

6. D. C. Eriffin et al.. J. Phys. B: At. Mol. Phys. 17, 3183 (1984).

\section{DIREC, AND IDTRECT IOAIzArion of TRANSITION METAL IONS}

\section{S. Dindzolal D. C. Griffin ${ }^{2}$ \\ C. Bottcher}

Au-ins, the past year we completed a survey of experimental and theoretical ciectron-impact ionization cross sections for transition metal ions in low stages of ionization. The a.rmic fons $\mathrm{Ti}^{+}, \mathrm{Ti}^{2+}, \mathrm{Ti}^{3+}, \mathrm{Fe}^{+}, \mathrm{Fe}^{2+}, \mathrm{Fe}^{3+}, \mathrm{Fe}^{4}$, $\mathrm{Ni}^{+}, \mathrm{Ni}^{2+}, \mathrm{Ni}^{3+}, \mathrm{Cu}^{+}, \mathrm{Cu}^{2+}$, and $\mathrm{Cu}^{3+}$ iere examined using electron-ion crossed beans measure.. ments and distorted-wave theory. In FIC. 5.36 we cumpare a single-configuration level to level distorted-wave calculation with experiment for $\mathrm{Ti}^{2+}, 3$ The theory predicts a rapid change in the cross section for $30 \mathrm{eV}$ to $35 \mathrm{eV}$ followed by a 10-eV plateau and then further jumps in the cross section around $45 \mathrm{eV}$. On a smaller scale, the experfmental measurements follow the same pattern. We believe that inclusion of configuration-interaction effects in the distorted-wave excitation calculations for $\mathrm{Ti}^{2+}$ will lower the cross section and thus improve the agreement between theory and experiment. In Fig. 5.37 we present the results of a singleconfiguralion level to level distorted-wave calculation for $\mathrm{Fe}^{4+}$ (in this case, no experiment has yet been performed). From our survey work, we expect that the total cross section results and the direct cross section results will bracket expriment. In order for sur predictions to be more precise, configuration-interaction effects in $\mathrm{Fe}^{4+}$ wfil need to be included in not only the indirect excitation process but also in the direct ionization calculation as well.

1. Consultant from Auburn University, Auburn, AL 35849 .

2. Consultant from Rollins College, Winter Park, FL 32789. tion. 


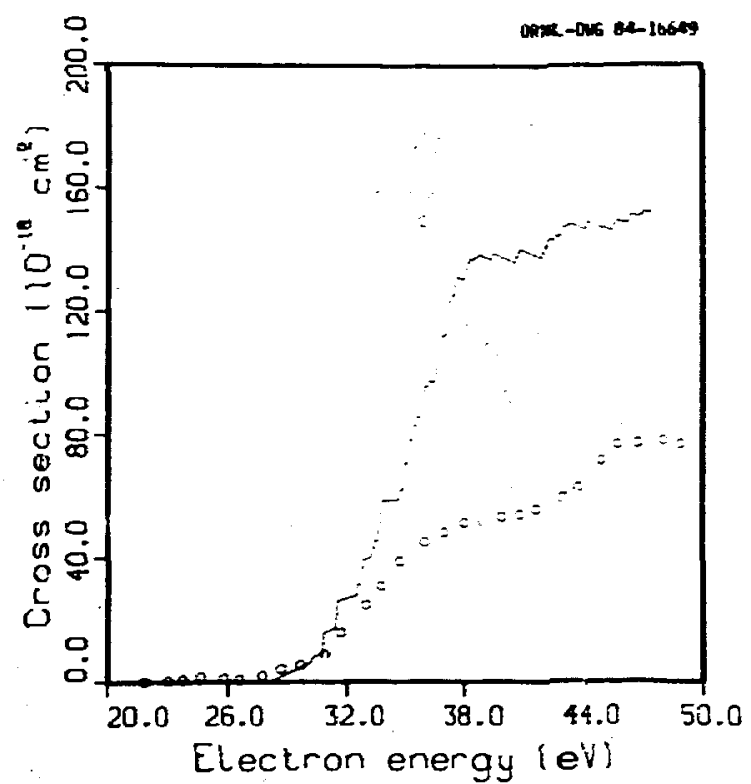

Fig. 5.36. Comparison of theory and experiment for electron imact ienization of $\mathrm{Ti}^{2+}$. Dotted line: direct ionization; full line: sum of direct and excitation-autoionization; open circles: measurements of Ref. 3 .

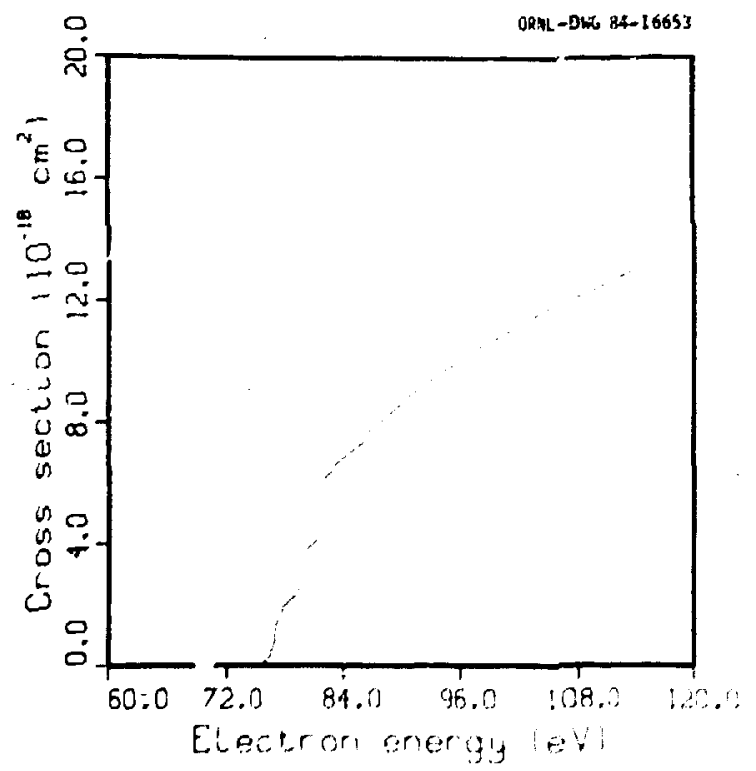

Fig. F... Predictions for electron impact ionfzation of $\mathrm{Fe}^{\mathrm{it}+}$. Dotted itne: direct iont. zation: full line: sum of direct and excitation-ionizition.

\section{DIERECTRONIC RECOBBIMTION IN ThE LITHIU ISOELECTROAIC SEQUENCE}

\author{
D. C. Giffint M. S. Pindzola ${ }^{2}$ \\ C. Bottcher
}

We have applied our recently de:sioped distorted-wave code for dielectronic recombination to calculate the cross sections assciated with the 25-2pitransition in the Li-like ions $\mathrm{B}^{2+}, \mathrm{C}^{3+}$, ard $\mathrm{O}^{5+}, 3^{3}$ We have compared calculated cross sections to selected configurations of the type $2 p n l$ in pure LS coupling, intermediate coupling, and a configuration-averaged approximation. The wost precise (intermediate coupling) results tend to be $50 \%$ higher than LS coupling, but agree fairly will vith configuration-averaged results.

The explanation can be seen if we write the process, e.g. in $8^{2+}$, as

$$
\begin{aligned}
e\left(k^{-} 2^{-}\right)+B^{2+}\left(i s^{2} 2 s\right) & +B^{+}\left(1 s^{2} 2 p n z, L\right) \\
& +B^{+}\left(1 s^{2} 2 s n z\right)+h v
\end{aligned}
$$

In pure L5-coupling the total angular mentum $L=L \pm 1=L$ wile in intermediate coupling; the spin-orbit interaction wixes $L=l=l^{\prime} \pm 1$. The former states typically have autoionizing rates $10^{3}$ - $10^{5}$ times the radiative rate, so that if the latter are mixed to the extent of 1 part in $10^{3}$, they will contribute equally to the cross section.

The interpretation of experiments on dielectronic recombination is presently in a state of flux. However, we note that measuremenis seem to be much larger than early theoretical predic. tions suggested, and that the effect of spinorbit interactions and external fip:os is usually to enhance the calculated cross section.

The sensitivity of comparisons with experiment to assumptions about experimental conditions is fllustrated in Fig. 5.38. Figure 5.38(a) shows the predicted cross section for $8^{2+}$ as a function of experfmental energy resolution, wille Fig. 5.38(b) shows the variation with the field ionization cutoff.

1. Consultant from Rollins College, Winter Park, FL 327?9.

2. Consultant from Auburn University, Auburn, AL 36849 .

3. D. C. Griffín et al., Phys. Rev. A (1985) in press.

4. P. F. Dittner et al ., Phys. Rev. Letts. 51. 31 (1983).

THE EFFES, O ELECTRIC FIELOS ON DIELECTRONIC RECUABIMATION IN IONS OF THE LITHIUA AND SODIUM ISOELECTRONIC SEQUENCES

$$
\begin{gathered}
\text { D. C. Griffinl M. S. Pindzola } 2 \\
\text { C. Bottcher }
\end{gathered}
$$

Dielectronic recombination (DR) is particuIarly sensitfye to the presence of external 


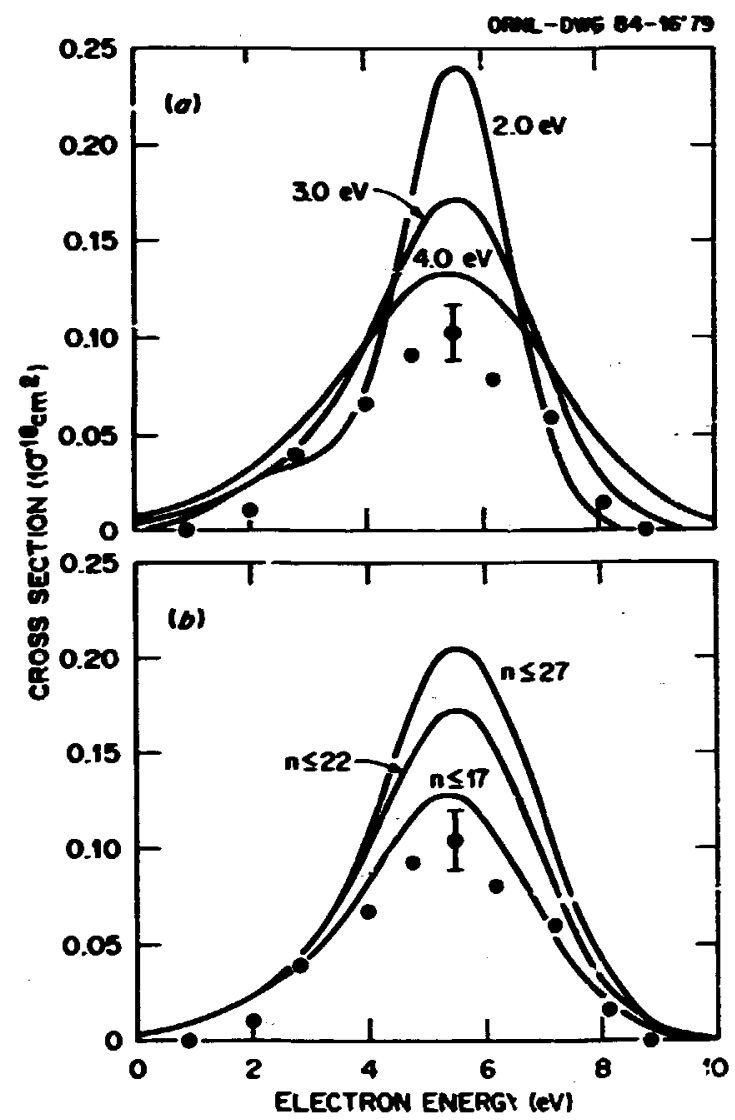

Fig. 5.38. Predicted cross section for dielectronic recombination of $8^{2+}$, (a) Variation of predicted cross section with electronic energy spread; (b) variation with analyzer field cutoff in principal quantum number, $n$; fflled circles are measurements from Ref. 4 .

electric fields. Such fields can cause a redistribution of angular momentum among the doublynxcited, resonant, Rydberg states infch, in turn, fncreases the number of states for which the rate of resonant-recombination is apprectable. He have just completed a systematic study of the ffeld enhancement of dielectrontc recombination in the Li and $\mathrm{Ma}$ isoelectronic sequen:es. In particular, we have applied the linear Stark approximation to examine these field effects for the dielectronic recombination transi tions assocfated with the $2 s+2 p$ excitation in the Li-like tons $\mathrm{B}^{2+}, \mathrm{C}^{3+}, \mathrm{O}^{5+}$ and $\mathrm{Fe}^{23+}$, and the $35 \rightarrow 3 p$ excitation in the $\mathrm{Ma}-1$ ifke fons $\mathrm{Mg}^{+}$, $\mathrm{S}^{5+} \mathrm{Cl}^{6+}$ and $\mathrm{Fe}^{\text {ist. }}$.

Although this technique does not allow one to determfne ffeld mixing as a function of electric field strength, it does provide physical insight into the nature of field effects in dielectronic recombination, and allows one to study fmportant trands in the maxtmum ffeld enhancement of the DR cross section as a function of fontzation stage. We find that the magnitude of the field enhancement decreases as we move up an iscelectronic sequence, and is of the order of two or three in highly ionized systens. Furthermore, we show that DR transitions through doublyexcited states near threshold can produce large narrow peaks in the cross section at low energies, wich are especially proainent in high stages of ionization, and are not affected by electric fields.

An especially striking exaple of the effect just referred to is provided by the $0 R$ cross sectial for $\mathrm{Fe}^{23+}$, shown in Fig. 5.39 as a function of electrm energy. In this plot, the narrow resonances associated with recombination

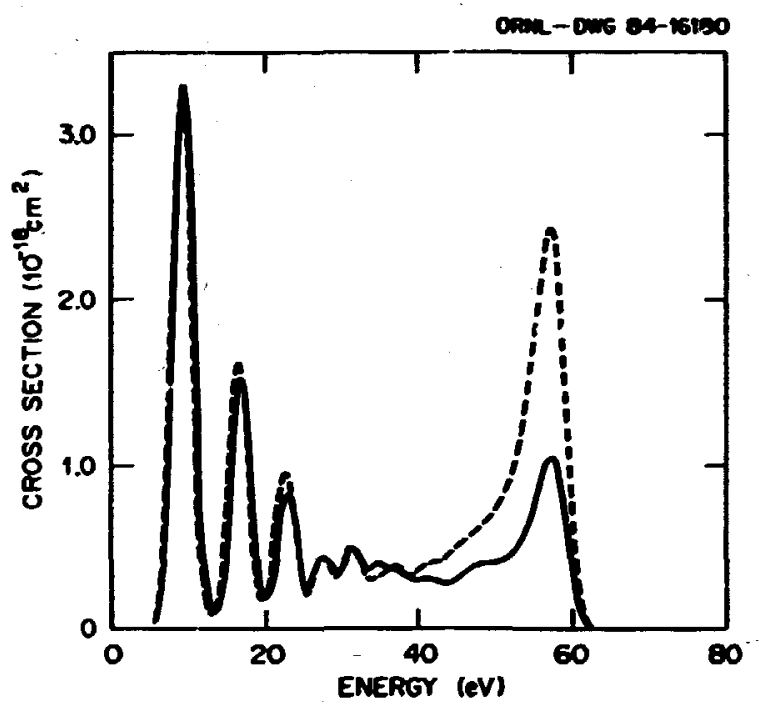

Fig. 5.39. Predicted dielectronic rerombination cross section of $\mathrm{Fe}^{23+}$ convoluted into a 3-eV Gaussian electron energy distribution. The full and dashed lines pertain to the cross section with and without an external electric field.

transitions through the doubly-excited configuratfons 2 pne are convoluted with $3.0-e V$ Gaussian to simulate an experimental electronenergy spread. The field enhancenent in the high-energy peak is approxfmately equal to 2.3 . The promine it low-energy peak is due to transftfons through the resonani states for wich the principal quantum of the Rydberg electron is $n=12$.

1. Consultant from Rollins College, Winter Park, FL 32789.

2. C,onsultant from Auburn Universfty, Auhurn, AL 36849 . 


\title{
6. NUCLEAR SCIENCE APPLICATIONS
}

\begin{abstract}
Nuclear sri ince has coman frontiers with other basic sciences. The current prcgram concentrates on three areas. (1) In cooperation with the Engineering Physics and Wothematics, Malytical Chemistry, and Compting and Telecomanications Divisions, a prograa is currently underway te obtain verification of the neutronic and irradiation performance of higher actinides in fast spectrun reactsrs. This progran utilizes the Dounreay Prototype Fast Reactor and will be completed by 1988. (2) In association with the Aralytical Chemistry Division, the technique of studying the heavy-ion-induced $x$-ray satellite spectra has been advanced to the point that it can be applied to netal alloys and other materials of interest to the Metals and Ceramics Division. This program utilizes the Ex Tandem and the HIRF. Significant advances in this area are expected with the construction by early 1985 of an ultra-high-l esolution Van Hams spectrometer. (3) Morking with personnel from the Aerospace Corporation, a progran to study single-event upsets in integrated circuits has been started. With encouragement from MASA, plans are being drawn up to build a bean line at HHIRF dedicated to this program.
\end{abstract}

\section{ACTIVITIES :H SUPPORT OF THE US/UR JOIUT EXPERIIEENT IN THE DOUNRFAY PROTOTYPE FAST REACTOR}
S. Raman
H. L. Adairl
J. L. Botts 2
D. A. Costanzoz
B. L. Broadhead ${ }^{3}$
J. K. Dickens*
J. F. Emery ${ }^{2}$
R. L. Walker ${ }^{2}$

The Inited States and the United Kingdom are engaged in a join research program in which samples of the higher actinides are irradiated in the Dounreay Prototype Fast Reactor in Scotland. The purpose of the program is (1) to study the materials behavior of selected hijher actinide "fuels" and (2) ton determine the integral cross sections of wide variety of the higher isotopes. Samples of the actinides are incorponrated in fuel pins inserted in the core. For the fuel study, the actinifes selected are ${ }^{241} \mathrm{Am}$ and ${ }^{244} \mathrm{Cm}$ in the form of $\mathrm{Am}_{2} \mathrm{O}_{3}, \mathrm{Cm}_{2} \mathrm{O}_{3}$, and $\mathrm{Am}_{6} \mathrm{Cm}(\mathrm{RE}) \mathrm{O}_{2}$, where (RE) represents a mixture of lanthanides. For the cross-section determinations, the samples are milligram quantities of actinide oxides of $24 \mathrm{Acm}$, $245 \mathrm{Cm}, 244 \mathrm{~cm}, 243 \mathrm{Cm}$, 243 Am, $231 \mathrm{Am}, 244 \mathrm{PU}_{\mathrm{U}}, 242 \mathrm{Pu}$, $261 \mathrm{Pu}, 269 \mathrm{Pu}, 239 \mathrm{Pu}^{2}, 23 \mathrm{HPu}, 237 \mathrm{~Np}, 238 \mathrm{~J}, 236 \mathrm{JJ}$ $235 \mathrm{~J}, 234 \mathrm{ls}, 2331 \mathrm{~s}, 232 \mathrm{Th}, 230 \mathrm{Th}$, and $231 \mathrm{~Pa}$ encap. sulated in vanadium. The development and appitication of the technology for preparing the actinide samples have been described in a report hy quinby, et 1.5 The characterization of the starting materials used in the samples for the crosssection determinations (denoted as physies specimens) and in the dosimeters has been described in a report by Walker, et al. ${ }^{6}$ Preanalysis calculations were carried out ${ }^{7}$ concerning actinide huildup and burnout in order to atd the experimentalists in the planning and preparation of their respective medsurements.

The first fucl pin expertinent was removed from the reactor in late 1983 after an trradiation of 63 effective full-power ddys and shipped to DRNL where subsequent analysis began in May 1984. The experimental results of gamma-ray characterization of Pission prodiscts Prom severd of the dosimeters have recently been cumpleted. These results include fission product activities for $238 \mathrm{U}, 235 \mathrm{~J}$, and ${ }^{2}{ }^{3} \mathrm{P}_{\mathrm{u}}$ at each of the th. ee dosimeter locations. Calculations mere performed in a manner similar to the pre-analysis calculations reported in Ref. 7 with the following exceptions: (1) the actual operating history of the reactor was used; (2) all actinide and fission product cross $\mathrm{sec}-$ tions were collapsed to one group with the sper. trum near the axial centerline of the physics specimen section; and (3) the flux levels employed in the calculations were from the actual reactor run.

Shown i. Table 6.1 are preliminary results for six different fission products produced by the fissioning of $232 \mathrm{U}, 235 \mathrm{~J}$, and $23{ }^{3} \mathrm{Pu}_{\mathrm{u}}$ at each of the three different dosimeter positions in fuel pin 1. The results are given as the ratio of the experimental value $(E)$ to the calculated value $(C)$. In all cases the agreement is very good with $E / C$ values between 0.81 and 1.14. Also encouraging is that within each incation a given dosimeter's $E / C$ values are vary nearly independent of the particular fission prodict. This indicates that sme of the remaining dis:repancies are systematic in nature. Thus, for example, if the flux used in the calculations was decreased $10 \%$ in the top (Positions 1 and 2) and middle (Positions 17 and 18) dosinetry incations, the resulting disagreements would then be in\% or less. The variation in $E / C$ values as function of the fission products Por a given dosimeter is larger for the bottommost (Posteinns 33 and 34) locations than those of the top and middie locations. This is dip to a known defteinncy in the spertrum used to obtatn the l-group cross sec:tans, and will be corrected in future andiyses.

The IJS/UK joint program is ongning wt:h more detalled calculations and additional measurements underway. The preltiminary results obtained thus far are very encouraging. The gamut of activities in support of this program involves not only several JRNL divisions but also close interaction and cooperation with Hanford Finginerring Development Lahordtory (HF.IL) and the Bunnedy Muclear Power Devalopment Fistabli shient. 
1. Operations Division, ORIL.

2. Analytical Chemisiry Division, ORuL.

3. Computing and TElecowunications

Division, DRm.

4. Engineering Pi:ysics and Mathematics Division, ORhe.

5. T, C. Quinby et al.. -Preparation of Actinide Speciurens for the US/JK Joint Experiment in the Dourrayy Prototype Fast Reitor." Oall-5858 (1982).

5. 2. L. Halker et al., Cnaracterization of Act inide Physics Spectmens for the US/UK Joint Experiment in the Dounreay Prototype Fast Reactor." 0AM-5986 (1983).

7. B. L. Broadhead, H. B. Fove, and S. Raman, -Preanalysis Calculations in Support of the US/Ur. Joint Exferiment in the Dounreay Prototype Fast Reactor," 0201-6058 (1984).

Table 6.1. US/K dosimeter results for Fuel Pin 1. Listed oelow are the ratios of experimertal value to the calculated value.

\begin{tabular}{|c|c|c|c|c|c|c|c|c|c|}
\hline \multirow{2}{*}{$\begin{array}{l}\text { Fission } \\
\text { Product }\end{array}$} & \multicolumn{3}{|c|}{$238 y$} & \multicolumn{3}{|c|}{$235 \mathrm{~V}$} & \multicolumn{3}{|c|}{$239 p:$} \\
\hline & Pos. I & Pos. 17 & Pos. 33 & Pos. 1 & Pos. 117 & Pos. 33 & 05.12 & Pos. 118 & Pos. 34 \\
\hline $\begin{array}{l}95 \mathrm{wb} \\
95 \mathrm{Zr} \\
103 \mathrm{Ru} \\
106 \mathrm{Ru} \\
137 \mathrm{Cs} \\
144 \mathrm{Ce}\end{array}$ & $\begin{array}{l}0.89 \\
0.90 \\
0.93 \\
0.89 \\
0.93 \\
0.93\end{array}$ & $\begin{array}{l}0.81 \\
0.81 \\
0.85 \\
0.81 \\
0.80 \\
0.88\end{array}$ & $\begin{array}{l}0.95 \\
0.94 \\
1.83 \\
0.96 \\
1.00 \\
1.14\end{array}$ & $\begin{array}{l}0.95 \\
0.95 \\
0.90 \\
0.84 \\
0.94 \\
0.95\end{array}$ & $\begin{array}{l}0.96 \\
0.96 \\
0.87 \\
0.84 \\
0.94 \\
0.98\end{array}$ & $\begin{array}{l}1.08 \\
1.08 \\
1.00 \\
0.93 \\
1.07 \\
1.02\end{array}$ & $\begin{array}{l}0.84 \\
0.83 \\
0.85 \\
0.85 \\
0.87 \\
0.83\end{array}$ & $\begin{array}{l}0.86 \\
0.85 \\
0.87 \\
0.86 \\
0.89 \\
0.85\end{array}$ & $\begin{array}{l}0.96 \\
0.97 \\
0.39 \\
0.75 \\
1.00 \\
0.97\end{array}$ \\
\hline
\end{tabular}

\section{ULTRAHIGH-RESOLUTION STUDIES OF HEAVY-IOM- IROUCED X-RAY SATELLITE EHISSIOM}

$$
\begin{array}{ll}
\text { C. R. Vane } & \text { G. Morford } \\
\text { E. Källne! } & \text { S. Riman } \\
\text { J. Källne! } & \text { M. S. Sinith }
\end{array}
$$

When an energetic heavy ion collides with a target atom, several L-shell and M-shell electrons are ejacted along with a K-shnll electron. The initial vacancy distribution of, say, the $L$ shell is altered by vacancy refilling prior to $k_{\alpha} x$-ray emission. What is seen experimentally at moderately sigh resolution is a series of KLn satellite peaks where $n$ cenotes the number of L-shell vacancies. Figure 6.1 shows sulfur $\mathrm{K}_{\mathrm{\alpha}} \mathrm{L}^{n}$ for $\mathrm{Ha}_{2} \mathrm{SO}_{4}$ and MaSCN excited by $36 \mathrm{MeV} \mathrm{Clq+}$ ions from the $0 \mathrm{kNL}$ EN tandem. Experimental resolution here is $\sim 7 \mathrm{eV}$ FWHM. These peaks actually represent over a hundred individual transitions. He have studied this fine structure from six sulfur compounds when bombarded by 34-MeV ${ }^{35} \mathrm{Cl}$ ions. The data, composed of six normalized then pieced-zogether, narrow band spectra (20-to-40 el each), are displayed in Fig. 5.2. These spectra were taken with a highthroughput, high-resolution Bragg crystal specttrometer ${ }^{4}$ in the Van Hamos geometry. They show that the individual satellite peaks from $S$ compounds not only differ in their gross shapes but also contain pronounced $\sim \mathrm{l-eV}$ fine structures that exhibit intensity variations. For example, the area of the prominent sharp peak at $\sim 2340 \mathrm{eV}$ varies nearly lineariy with target sulfur effective charge. It changes by $+63 \%$ in going from $\mathrm{NaSCN}$ to $\mathrm{Ha}_{2} \mathrm{SO}_{4}$, nearly 2.5 times the sensitivity found $f_{0}$. the ent 1 re $K_{\mathrm{a}} L^{2}$ area measured earlier at lower resolution. : The observed profiles agree qualitatively with Hartree-Fock predictions ${ }^{6}$ for the lower degrees of L-shell ionization (KL ${ }^{0}, \mathrm{KL}^{b}$, and $\mathrm{KL}^{2}$ ), but less well for the higher degrees. In order to systematically investigate these remarkable fine structures, we have designed and begun construction of a new Van Hamos spectrometer' of much higher collection efficiency employ.
ORIL-DIG 84-15665

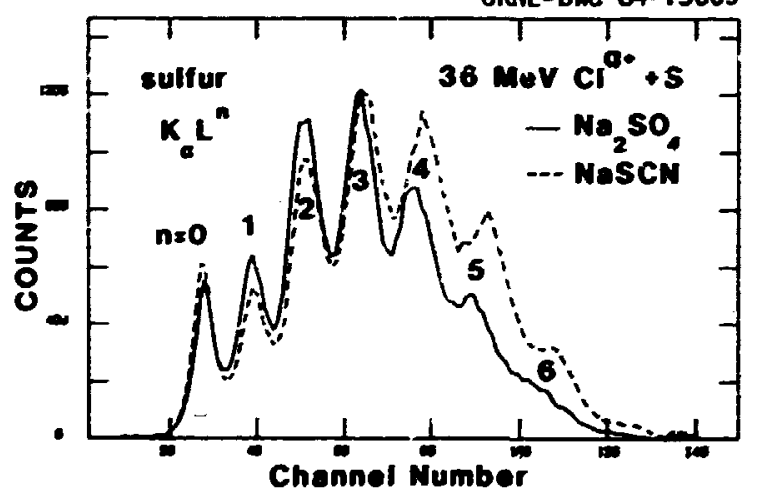

Fig. 6.1. Moderacely high resolution $(\sim 7 \mathrm{eV}$ FWHM at $2.3 \mathrm{keV}$ ) sulfur $K_{a} x$-ray spectra generated by $36 \mathrm{MeV} \mathrm{Clq}+$ ions on $\mathrm{KSCN}_{3}$ and $\mathrm{Ma}_{2} \mathrm{SO}_{4}$.

ing a large curved crystal $(10 \mathrm{~cm} \times 10 \mathrm{~cm} ; 25.4 \mathrm{~cm}$ radius of curvature) and a position-sensitive detector. The main features of the spectrometer are shown schematically in Fin. 6.3. The design resolution was $1 \mathrm{eV}$ :t $2.5 \mathrm{keV}$ with a dynamic range of 200-300 eV. The positions of the crystal and the detector are remotely variable on linear tracks allowing Bragg angle adjustment from $30^{\circ}$ to $70^{\circ}$ for a particular crystal. With this instrument, we intend to study both the chemically sensitive and temporal variations in $x$-ray spectra from a wide variety of targets bonbarded with heavy ions from the Holifield Heavy Ion Research Facility.

1. Now at JET Joint European Undertaking, 1.b: ngdon, Engl and.

2. Undergraduate student from the Edinboro State College, Fininboro, PA, under the nRAU Summer itudent Program.

3. How a Jraduate student at Yale University, New Haven, C,T O6511. 
4. E. Kallne and J. Käline, Dhysica Scripta T3, 185 (1983).

5. r.. R. Vane et al., Mucl. Instmim. Methods Phys. Res. B3, 88 (1984); iee Fig. 4 of this paper.

6. R. L. Hatson, H.F. Calculations of he-like 5, private comanication.

7. C. R. Vane, H. S. Snith and S. Raman, ORML report, in preparation.

OFNL-DEG 84-14557

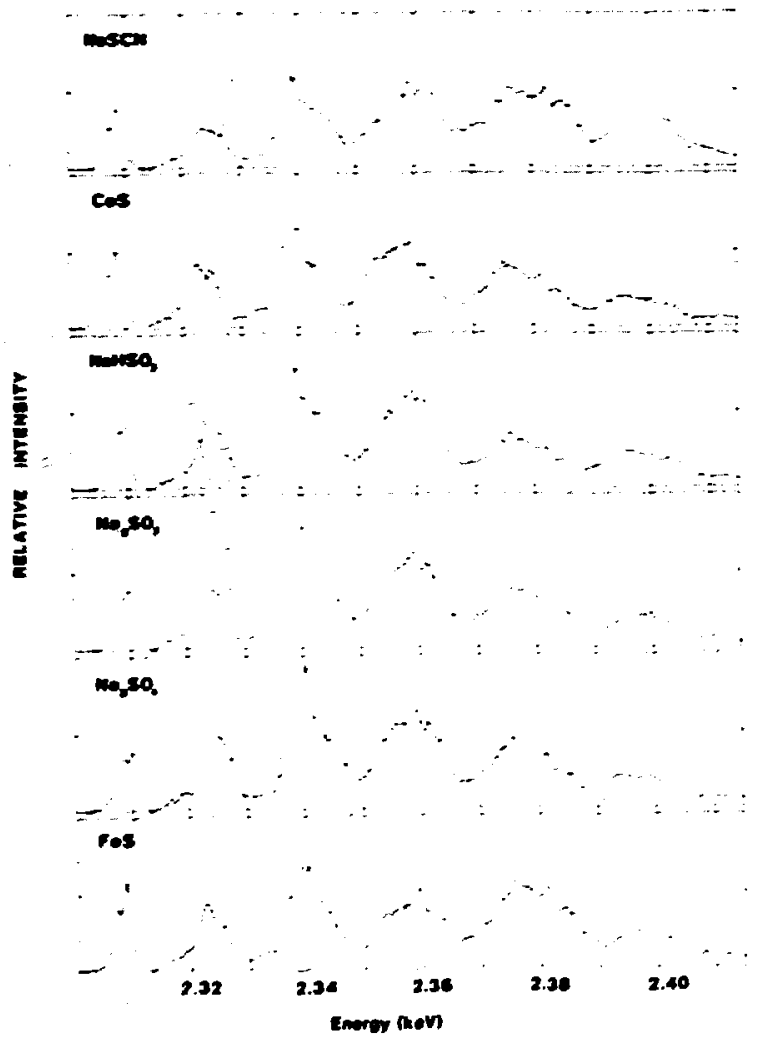

Fig. 6.2. Very high resolution $(\sim 1$ eV FWH at $2.3 \mathrm{keV}$ ) sulfur $k_{a} x$-ray spectra generated by $34-$ MeV CIq ions on six compound:. Spectra were pieced together from 20- tu 40-eV wije segments taken with narrow-bandwidth Van Hamos geometry spectrometer.

\section{EFFEC .F THE CHEMICAL ENVIROHNENT ON} THE L A'D M HEAVY-ION-INDUCED X-RAY SATE! LITE EMISSION SPECTRA
T. M. Rosset 1
J. M. Dale!
D. D. Hulett!
P. L. Peomiller
H. F. Krause
S. Raman
C. R, Vane
J. P. Young'

I le effect of the chemical environment on heavy-ton-induced $k_{\text {a }} x$.ray satellite yielils has been studied exiensively for a variety of second and third row elements. 2 issing high-resolition measurements, it ha; been shown thet the sensi. tivity of the satellite lines to the chemical milifer is due to the modification of the vacancy

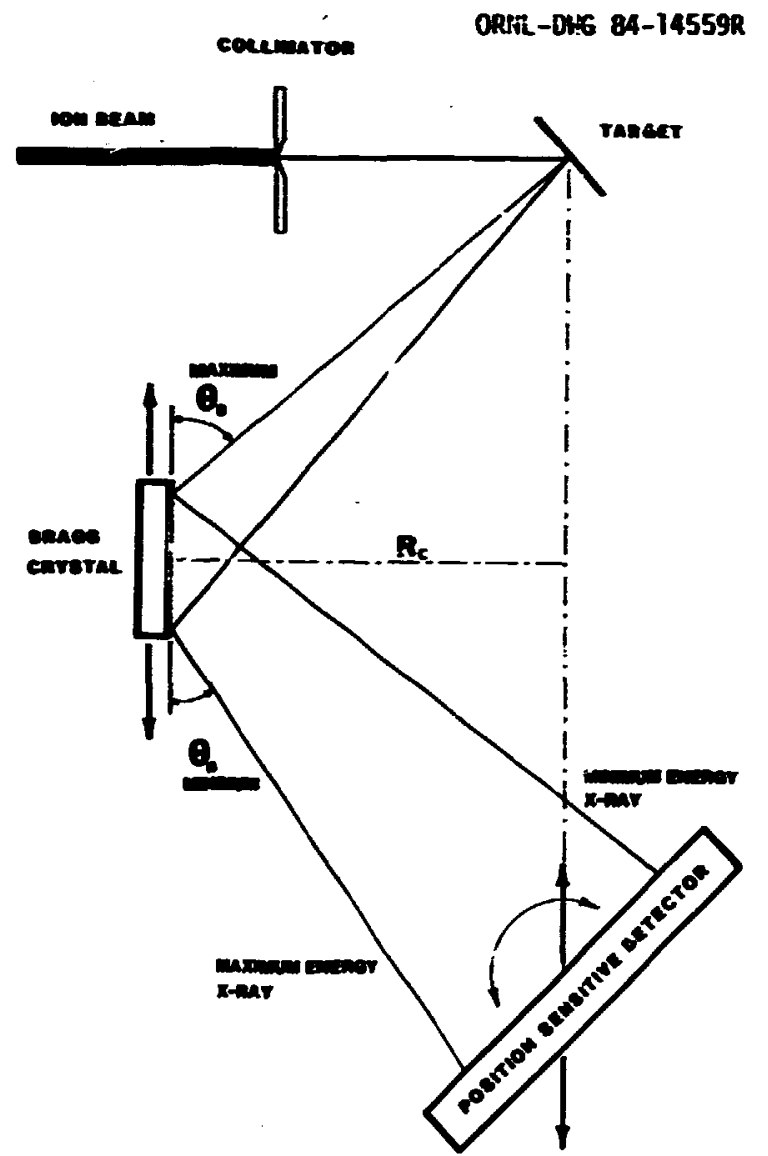

Fig. 6.3. Schematic of new Van Hamos spectrometer. Arrows indicate positioning of remotely adjusted components.

distribution created at the time of collision by inter- and intra-atomic relaxation processes during the lifetime of the $K$ vacancy. Because the relaxation processes depend primarily on the avallability of weakly bound electrons, a changs. in the valence electron distribution due to a change in the chemical enyironment alters the vacancy transfer rates and thus alters the observed satellite yield. 3 Recently, simflar investigations have heen extended co the $L x$-ray satellites of cransition metal elements. 4,5 Variations in the measured satellite intensity distrihution were observed and correlated to chemical differences among a series of molybdenum alloys and compounds. 5 These results suggest that hedvy-ion-induced $x$-ray satellite emission (HIXSE) is capable of analyzing the chemical nature of intermediate $Z$ elements as effectively as low $Z$ elements, As part of the effort to confirm the sensitivity of HIXSE to intermediate 2 elements and to expand this method to new reyions of the periodic table, we have examined the effect of the chemical environment on the $2 r$ and $10 L$ and the $A u$ and $\mathrm{Pb} H \mathrm{X}$-ray satellite lines at the Dax Ridge EN-Tandem Van de fraaff accelerator.

The $Z r L$-ray satellite spectra of $7 r \hat{s}_{2}$ and $Z r$ produced by $36-\mathrm{MeV} \mathrm{Cl}$ ton hombardment are showin in 
Fig. 6.4. Esed on previous investigations 5 and Dirac-Feck calculations of transftion energies, 6 the first band corresponds principally te the L2\% satellite lines and the second band to the $L_{B} h_{m}$ transition. The siniler peaks following the LIII absorption edge at $2.22 \mathrm{keV}$ are minl: due to the L L exhibit vathations in their satellite intensity profile due to differences in the chenical enviroment of the $\mathrm{Zr}$ im.

In order to aplify these veriations and extract chesical information, difference spectra were generated using the $\mathrm{Zr}$ metal spectrum as the subtrahend. Tro examles of these difference spectra are show in Fig. 6.5. It can be seen that the deviation fro zero (no chenical effect) is larger for $\mathrm{ZO}_{2}$ than $\mathrm{ZrAl} 2$. Mis is in accord with calculated values of the bulk valenceelectron densities. The valence-electron density, Dy, defined as the nuber of valence electrons/ $h$ : approximately describes the concentration of electrons avaflable for vacancy refilling. 3.7 It is thus a measure of the effect of the chemical environment of the enitting target ion. The energy region which corresponds approximately to the $\mathrm{L}_{\mathrm{\alpha}} \mathrm{m}$ satellite lines $(2.10-2.17 \mathrm{keV})$ was found to be particularly sensitive to changes in $\mathrm{D} v$. This is also in good accord with previous observations for to compounds, 5 and with data for $t_{b}$.

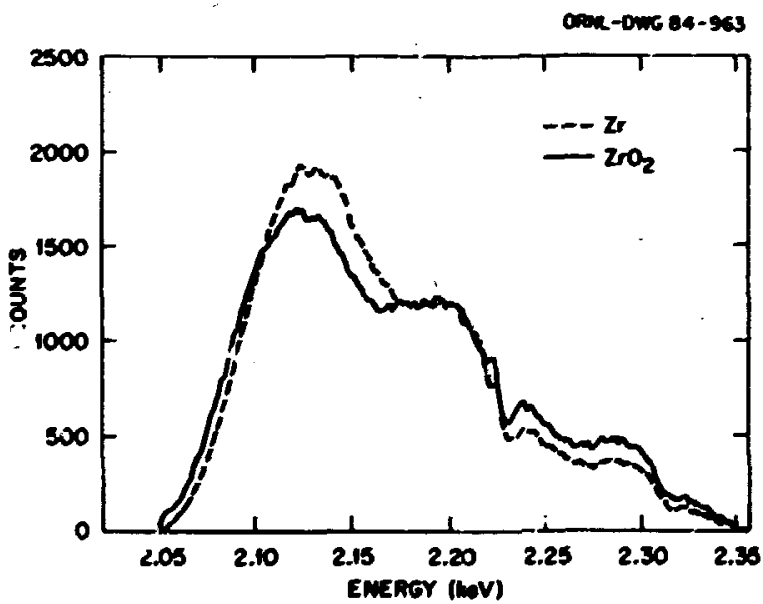

Fig. 6.4. The corrected and normalized $\operatorname{Zr} L x-$ ray satelitite spectra produc $3 d$ by the bombardment of $\mathrm{Zr}$ metal (dashed Iine) and $\mathrm{ZrO}_{2}$ solid line) with 36-HeV Cl ions.

In addition to examining compounds, a sertes of Zr-Tt alloys were also investigated. Although the difference spectra are dissimilar to the difference spectrs of the compounds, is was ohserved i or Mo-Ti alloys, 5 the alloy spectro exhibi: a systematic decrease in the absolute value of the difference as the valence electron density of the minuend approaches the subtrahend. These results provide additional evidence that HIXSF, can dis. tinguish between sifferent types of chemical sond..

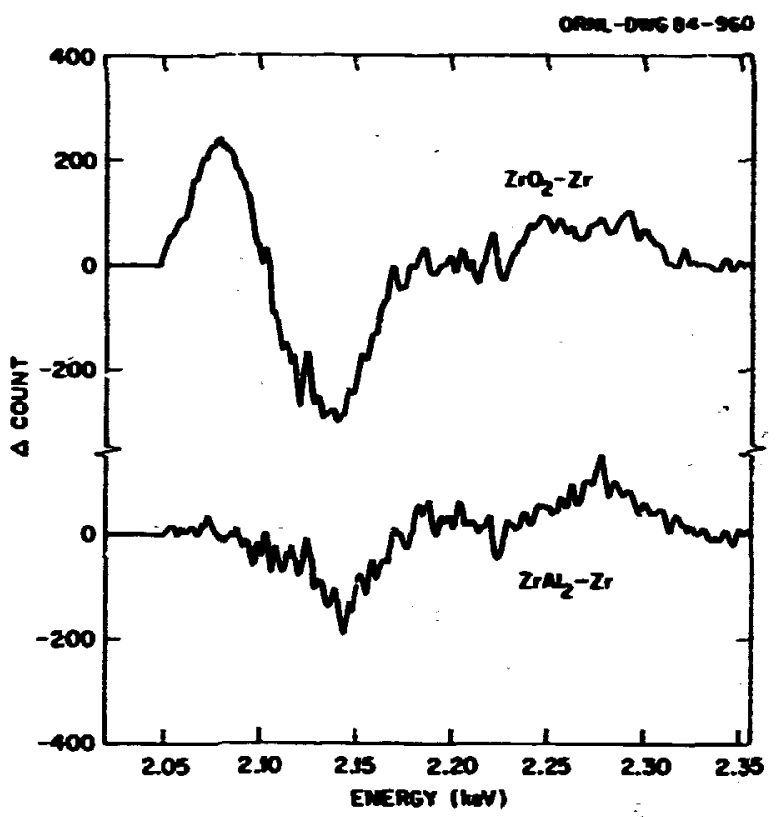

Fig. 6.5. The $Z \boldsymbol{r} x$-ray satellite difference spectra of $\mathrm{ZrO}$ and $\mathrm{ZrAl}_{2}$. The $\mathrm{Zr}$ metal spectru is the subtrahend. The projectile was $36-\mathrm{MeV} \mathrm{Cl}$ ions.

ing. Together with the $\mathrm{Zr}$ and 10 compound data, it confirms the sensitivity of the $L$ x-ray satellites to the chemical environment of intermediate $I$ elements.

The Po and Au $X$-ray satellite spectra were also examined with 36-meV ol ions. The spectra, Iike those of $\mathrm{Zr}, \mathrm{Bb}$, and $\mathrm{H}_{0}$, exhibit variations in the satellite intensity profile due to differences in the chemical er.vironnent of the target ion. Like the $L$ spectra, the first two bands in the $M$ spectra are composed of overlapping hypersatellites of the $M_{\alpha}$ ans $M_{\beta}$ transitions. Attempts to extract chemical information from these spectra are being pursued.

1. Analytical Chemistry Division.

2. S. Raman and C. R. Vane, Mucl. Instrum. Methods Phys. Res. B3, 71 (1984).

3. R. L. Watson et al. Phys. Rev. M15, 91\%, (1977).

4. M. IXa et al. Inner-Shell and X-ray Physics of Atoms and Solids, eds., D. J. Fabian, H. Kletnpoppen and L. M. Watson (Plenum, New York, 1981) D. 205.

5. T. M. Rosseel et al.. Nucl. Instrum. Methods Phys. Res. B3, 94 (1984); J. Phys. F: Met. Phys. 14, L37 (1984).

6. C. Hestor. Modification of a Relativis. tic Hartree-Fock Program from J. P. Desclaux, Comp. Phys. Comm. 9, (1975).

7. A valence electron is dcfined here as any electron beyond the rare gas structure $d$ and $q$ electrons from a filled subshell. 


\section{SIDLATIOA OF COSHIC-RAY UPSET OF AICROELECTROMIC OEVICES}
W. A. Molasinskil
J. Adolphsen 2
G. Brucker ${ }^{3}$
M. Knolls
R. Pogal
S. Raman

$$
\text { C. R. Vane }
$$

Several types of microelectronic chips have been bowbarded with beans of $\mathrm{Aw}, \mathrm{Ag}$, and $\mathrm{C}$ ions as part of a continuing investigation concerned with the effects of heavy cosmic rays on wodern computers in space. With the present level of miniaturization, sensitivity of circuits in these computers has reached the point where individual energetic heavy ions such as those in the Fe or C-11-0 peaks in the cosinic ray flux can produce upsets in the computer operation. These upset.s. termed Single Event lpsets (SEU), can produce effects whose level of seriousness ranges from innocuous to disastrous.
The runs were carried out in collaboration with the MSA Goddard Space Flight Center, the Air Force Space Technology Center, Sandia butional Laboratories, RCA, and the Aerospace Corporation. Various logic circuits representing several forws of silicon technology and different levels of complexity were frradiated to provide data needed in the design of devices resistant to SEU. Besides providing thr needed data, the runs successfully demonstrated the feasibility of running diffuse, spatially uniform beans, with intensities appropriate for this type of experiment.

1. The Merospace Corporation, Los Angeles, CA. 2. MuSA Goddard Space Flight Center, Greenbelt, 1.

3. RCa, Princeton, Mu.

4. Sandia Mational Laboratory, Albuquerque, 


\section{PLASMA DIAGNOSTICS FOR FUSION PROGRAM}

\section{MUTICHAMEL POLARITETRY USITS FARSDAY YOTATIOH}

\author{
C. H. Mạ \\ D. P. Hutchinson
}

P. A. Staats

Silultaneous masurements of electron density and Faraday rotation on all five channels of the FIR interfercmeter/polarimeter system on ISX-B tokanak have been achieved. The achievement is due ainly to two major improvements to the detectors and the faraday modulator of the previous system: (1) The He-cooled Putley detectors are replaced by high responsivity Schottky

\%. diodes and (2) a novel technique is used to improve the performance of the polarization modulator. The use of the Schottky diodes in the present system has not only increased the detection sensitivity but has alsu el iminated the need for a wire-grid analyzer for polarization determination. The previous Faraday modulator utilized an air core coll. A ferrite disk was mounted in the center of the cofl. The modulation frequency was limited to 3-5 kHz due to the high inductance and large stray capacitance of the 600-turn coil. In the present system, the miti-turn coil has been replaced by a single-turn copper coil which acts as the secondary of a radio frequency (rf) transformer. A modulation frequency of $92 \mathrm{kHz}$ has been achieved by using a serfes resonant cfrcuit for the primary winding. The increase of the modulation frequency has not only greatly improved the time resolution of the polarimeter but has also reduced the of coupling between the modulator and the detection circuit.

A schematic diagram of the modiffed interferometer/polarimeter system is shown in Fig. 7.1. Briefly, the system consists of a pair of cw 447 fodomethane lasers, optically pumped by separate $\mathrm{CO}_{2}$ lasers. The FIR cavities are tuned such that the two FIR lasers oscillate at frequencies differing by $\Delta f$ of the order of $1 \mathrm{MHz}$. The linearly polarized beam of the source laser is passed through a ferrite polarization modulator. a mechanical polarization rotator into the dielectric waveguide, and is then divided into five beams wich are projected through the plasma. Emerging from the plasma chamber, each beam enters again into a waveguide and is directed onto a signal detector. Part of the beam from the reference laser is $\mathrm{mixed}$ first in a reference detector with a portion of the source laser, which is split off before passage through the modulator, and the remainder is guided to the signal detect or to $m i x$ with the plasma-probing beam. Schottky dtodes are

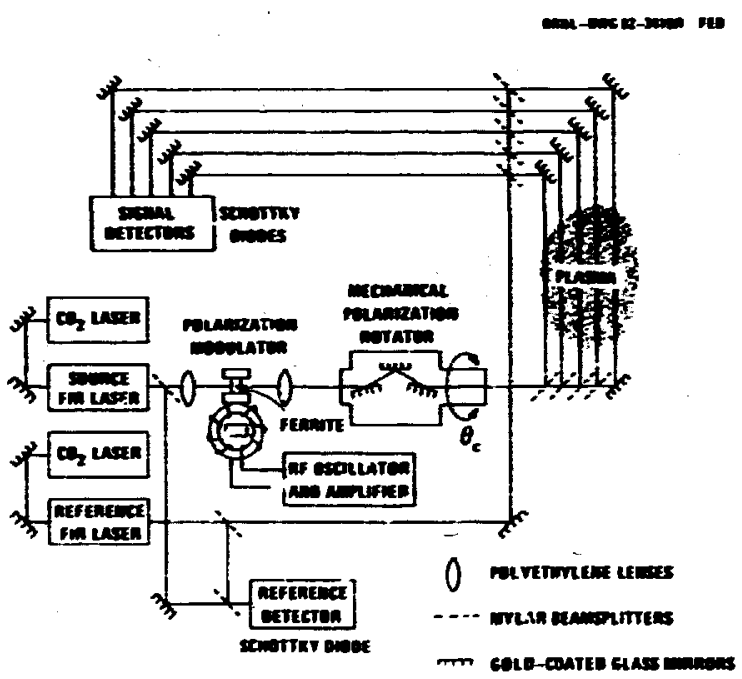

Fig. 7.1. Schenatic of the multichord FIR interferometer/polarimeter system for simultaneous measurements of line-dveraged electron density and Faraday rotation in ISX-B tok anak plasma.

utilized for all detectors. The output of the reference detector is a sinusoid at frequency $\Delta f$ and is used as reference signal for phase detection. The output of each signal detector is filtered, ampliffied, and fed into a digital phase detection circuit to extract the phase shift due to flasma density. An envelope detection circuit is utilized to demodulate the phase-modulated signal, and provides a sinusoidal signal at the modulation frequency wicese amplitude is proportional to $J_{1}\left(\theta_{m}\right) \cdot \operatorname{Sin}(\theta)$. where $\theta_{m}$ is the amplitude of the modulation angle, $\theta$ is the sum of the rotation angles due to mechanical polarization rotator, $\theta_{c \text { p }}$ and Faraday rotation in plasma, $\theta_{p}$. J J $\left(\theta_{m}\right)$ is the Bessel function of the first kind with order one. This signa! is synchronously detected by a lock-in ampiffier. yielding an output voltage, $V_{\text {out }}=V_{0} \cdot \sin (\theta)$. The calibration constant, $v_{0}$, can be obtained by setting the mechanfical polarization rotator at a few degrees $\left(64^{\circ}\right)$ and measuring the value of $V_{\text {out }}$ without plasma in the chamber. The voltage $V_{0}$ as determined by this technique calfbrates the polarimeter in a manner that does not require the absolute 
knowledge of laser power, detector responsivity, rodulation angle, or any system losses.

The sy'stem has been routinely exployed to study the ohmic- as well as neutral-beam-heated plasea discharges in ISX-B tokanak for over cre year. The standard deviati. I of the output of the interferometer of a constant phase shift is less than $5 \times 10^{-2}$ fringe. Since one fringe corresponds to a line-averaged density of $9.2 \times 10^{12} \mathrm{~cm}^{-5}$, density variations as small as $4.6 \times 10^{1 \mathrm{i}} \mathrm{cm}^{-3} \mathrm{can}$ be measured. The polarimeter shows a sensitivity of the order of one williradian and a time resolution of one msec. Figure 7.2 shows the time-resolved traces of (a) Faraday rotation and (b) line-averaged electron density of a typical tokdak plasma discharge. A neutral-hydrogen bean of approxiately I was injected into the plasma $70 \mathrm{msec}$ after the initiation of the plasma discharge. The position of each channel, relative to the center of the tokanak vacuum chamber is also indicated in the inset. The negative Faraday rotation on the central channel (channel 2) is due to the outward shift of the plasma centroid. The fast response of the system is demonstrated in Fig. 7.3. During this plasma discharge, a solid hydrogen pellet was injected into the
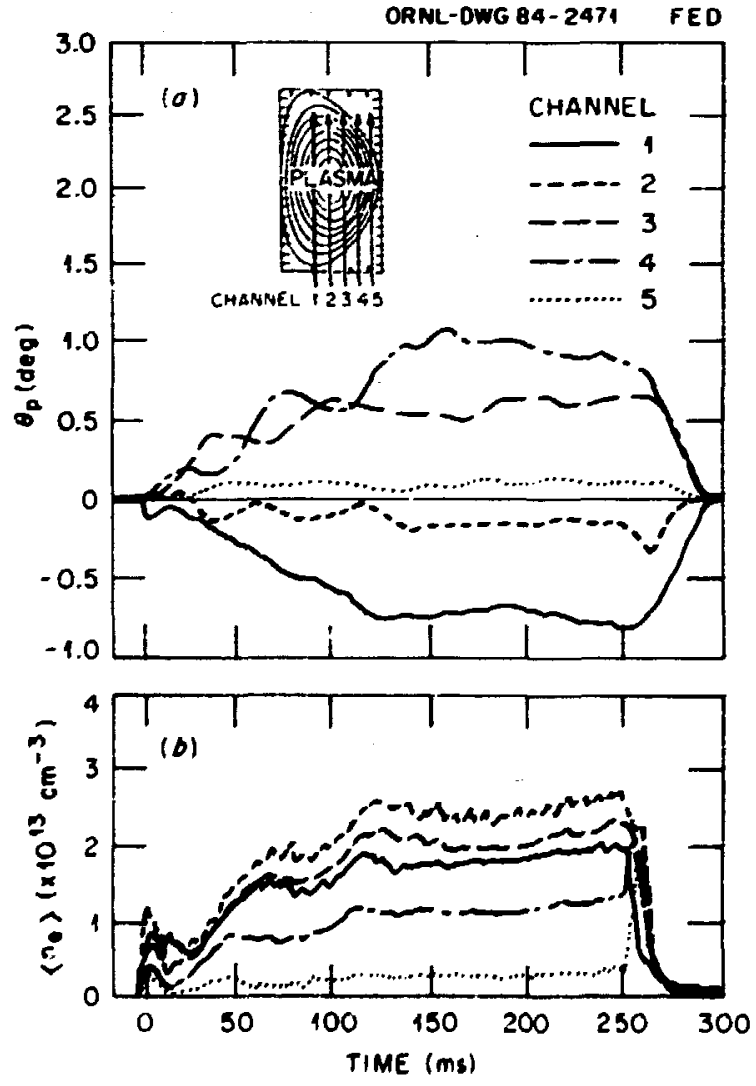

Fig. 7.2. Time variation of (a) Faraday rotation and (b) line-averaged electron densfty measured by the multichord FIR interferomter/ polarimeter system on ISX-B tokamak.

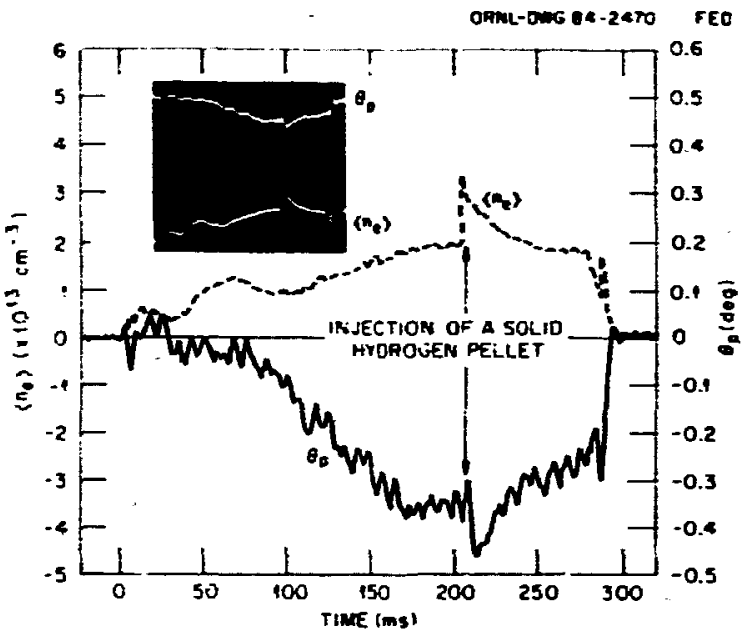

Fig. 7.3. Typical display of tíme-variation of line electron density and Faraday rotation signal on central channel of the FIR interferometer/polarimeter systen. The abrupt changes of the line density and Faraday rotation are caused by the injection of a solid hydrogen pellet at the time of $204 \mathrm{msec}$ after the beginning of the discharge.

plasma at the time of approximately 204 msec. The pellet caused an aorupt density increase of $21.4 \times 10^{13} \mathrm{~cm}^{-3}$, and a change of Faraday rotation of $=0.15^{\circ}$ on the central channel. The changes of density and Faraday rotation occur during a period of approximately $400 \mathrm{usec}$. The t ime delay ( $\cong 3 \mathrm{msec}$ ) between two curves is due to the RC constant of the lock-in amplifier ( 1 $\mathrm{msec}$ ). It is believed that this is the first simultaneous measurement of electron density and Faraday rotation in pellet-injected discharges. Data analysis codes are under development to reconstruct the asymmetric spatial profiles of electron density and plasma current from the 1 ine-averaged chordal measurements.

We have continued to support the implementa$t$ ion of the FIR dfagnostics on the TEXT tokamak at the University of Texas, Austin. T. Price has simultaneously measured both density and Faraday rotation on all six channels of che TEXT system. Although the system is not completely calibrated, the Faraday signals are of the proper polarity on all six channels and of roughty the proper magnitude.

$C$. $H$. Ma has been engaged in the develoment of the FIR polarimeter for the TFTR tokamak at Princeton Plasma Physics Laboratory. Experiments have been conducted to determi ne the performance characteristics of the polarimeter. A signal-to-noise ratio of $20 \mathrm{db}$ has been achieved for a simulated Faraday rotation angle of $5^{\circ}$ with four milliwats of laser power. An analy$s$ is has also been carried out in order to identify some possible problems in the measurements. and to establish the calibration procedure for the system. 


\section{DIAENOSTICS DEVELOTEMT CENTER}
C. i. Barnett
E. W. Thomas 1
R. O. $\operatorname{Legg}^{1}$

A collaborative effort has been initiated with $E$. H. Thomas (Georgia Institute of Technology) and P. Bakshi (Boston College) to determine the feasibility of xeasuring plasma edge turbulence and electric fields in magnetically-confined plasmas. Fast-response diode arrays with $H_{a}$ filters have been constructed to observe time correlations of $H_{a}$ enission from adjacent locations in the plasma edge using techniques developed by Zmeben et al. 3 The first series of measurewents will establish the physical extent of coherent eaission regions, both poloidally and toroidally. The second series will include the replacerent of the $\mathrm{H}_{\alpha}$ filter with a wonochromator. By imagitig a poloidal strip of the plasma on a vertical entrance slit and placing the diode array in the vertical detector plane we can deterni ne the electric field from the Stark-broadened profiles or shifts of the $H_{a}$ line. Since the conrentional analysis of the Start effect in plasmas is in terms of the static Holtsmark field, it is necessary to include the line shape and shifts due to quasistatic and high-frequency fields. Modeling of the line shape for different magnitudes and frequencies for these fields has been completed. In addition, the analysis indicates that by determining the polarization of the $\mathrm{H}_{8}$ line the direction of the driving electric field can be determined. Two 8-diode arrays have been fabricated and tested with their associated anplifiers, circuitry, and signal recording. Computer programs are being written for cross-correlation analysis. Initial tests will be performed using a rapidly pulsed proton beam in $\mathrm{H}_{2}$ gas, after wich the apparatus will be moved to the TEXT tokamak at the University of Texas at Austin.

To measure the ion temperature in a high temperature D-D plasma, we have proposed to make use of the oxygen total neutron cross section which has a deep resondnce at about $2.35 \mathrm{MeV},{ }^{4}$ At low plasma temperatures the 2.45-MeV D-D neutron spectra is narrow. As the plasma temperature increases, kinetic broadening increases the width of the neutron distribution with the low-energy tafl spilling over into the oxygen resonance. By measuring the atcenuation of the neutrons through a liquid oxygen cell, th, plasma fon temperature can be obtained if the ions have a Maxwellifan velocity distribution. Prel iminary calculations 5 had shown the experiment to be feasible for a 2-keV plasma fon temperature using an available liquid oxygen cell. More irecise calculations by Alsmiller et al.6 for a global neutron source from a 6-keV $0-0$ plasma indicated that the overall efficiency of the neutron detector at the rear of the absorption cell would be $4 \times 10^{-11}$ counts per source neutron. This can be compared to a source strength on the ISX-B tokamak of $2 \times 10^{10}$ neutrons/sec or approximately 0.16 counts/ discharge at the rear detector. Since the calculations are belfeved to be accurate to within
20\%, the technique does not appear to be feasible.

High speed (4000 fps) franing camera studies have been completed on the TEXT tokanak during stable and turbulent operation. Photographs were taken at three viewing ports: perpendicular to the plasim at the liniter port, longitudinal to the plasma viewing the inside of the toroidal liniter, and perpendicular to the plasen $180^{\circ}$ fro limiter. Semiquant itat ive data from the films were obtained by visual observation and frame-by-frame scanning with an optical densitoneter. The observations are sumarized as follows:

- Striations or particle density channels are always present at the beginning or termination of the discharge and ouring turbulent operation.

- During stable periods of operation, no fluctuations were observed.

- Spallation of acro-size particles or molten pieces of metal were observed during the formation of the discharge, at disruption or with unstable operation, and at the end of discharge.

- Macro-particles mere observed at $180^{\circ}$ fram the limiter, apparently coming from the wall.

- Particles coming from the wall or liniter usually flowed in the direction of the plasia current with a luminous plume extending in front of and behind the particles.

A low-energy Cs neutral particle analyzer? has beren loaned to the University of Wisconsin for use in the measurement of tokamak plasma ion temperature. In recalibrating the conversion and detection efficiency of the analyzer we found that the sensitivities of two of the channel mitfipliers were a factor of $2-5$ less than the other two. The electrostatic analyzer is being rebuilt and the multipliers are being replaced. These changes in calibration demonstrate the need for periodic: recalibration of analyzers after several months of operation.

1. Consultant, Georgia Institute of Technology, At lanta, Seorgía.

2. Consultint, Boston College, Boston, Massachusetts.

3. S. J. Zewben, J. McChesney, and R. H. Gould, Muclear Fusión 23, 825 (1983).

4. P. H. Stelson and C. F. Barnet.t, "Plasma Ion Temperature Measured by Neutron iransmission Through Liquid Oxygen," Bul1. Amer. Phys. Soc. 23, 882 (1978).

5. C. H. Johnson et al. Measurement of the 2.35-MeV Window in $0+n$," Mational Bureau Special Publication 594, Nuclear Cross Section for Tecinology, 1980.

6. R. G. Alsmiller, Jr., et al., "Background

Calculdtions for the Measurement of the Ion

Temperature in a Deuterfum Plasma,"

ORNL/TM-9230, July 1984.

7. 0. M. Thomas, "Low Energy Neutral

Particle Spectrometer," ORNL-6004, p. 266, Dec. 1983. 


\section{FEASIBILITY OF MPH PRRTICLE OTAGNOST:CS BY $\mathrm{CO}_{2}$ LASER THONSOW SEATIERINS}
D. P. Hutchinson
J. Sheffield 1
R. L. Vander Sluis
D. J. Sigmar

Introduction

The behavior of alpha particles in an ignited fusion plasma is of considerable importance in fusion researci since it is the eneray transferred to the O-T plasma from the energetic alphas that will be used to sustain the plasma temperature. Several methods have been proposed to weasure the density and/or velocity distribution of the alphas. 2-5 Among the suggested techniques are the utilization of charge exchange reactions of the alphas with high energy neutral diagnostic beams, $2^{2-4}$ the scattering of farinfrared radiation. 4 and the detection of the alphas that escape from the plasia. 4,5 : We have evaluated a method that uses the scattering of a high power $\mathrm{CO}_{2}$ laser by the Debye sphere of electrons associated with each alpha particle to determine both the density and velocity distribution of these energetic fusion products.

\section{Sciattered Power Spectrum}

The incident radiation at the wenumber $\boldsymbol{K}_{\mathbf{i}}$ and frequency wi is scattered and Doppler shifted by electrons in the plasma to the scattered wavenumber $F_{s}$ and frequency $w_{s}$. Tre radiation of a particular $\bar{K}_{5}$ and $\omega_{5}$ reaching the detector come from a periodic distribution of electrons in the plasma with vavenumbers $k=$ $\bar{k}_{s}-\bar{k}_{f}$ and frequency $\omega=w_{s}-\omega_{j}$. The fons are massive and scatter relatively little of the incident radiation. However, when $|\bar{k}|>\lambda_{D}$, the Debye length, radiation is scattered from the electrons witch are a Debye shield on each ion. For the conditions used in this diagnostic, the shift in frequency corresponds to the Doppler shift caused by the fon-thermal speed $v_{i} \approx \omega / k_{i}$, and the electrrn-thermal speed $v_{e}=\omega / k$. The scattered power in the frequency range $\omega+\omega$ $+d w$ and solid angle do from a length $L$ of the incident beam of electromagnetic radiation is given by Sheffield,?

$$
P_{S}(\vec{R}, \omega) d \phi d \omega=P_{i} r_{0}^{2} n_{e}^{L d Q} \frac{d \omega}{2 \pi} Q(\bar{R}, \omega)
$$

where $Q(k, \omega)=\frac{2 \pi}{k}\left|1-\frac{G_{e}}{E}\right|^{2} f_{e}(\omega / k)$

$$
+\frac{2 \pi}{k}\left|\frac{G e}{e}\right|^{2} \sum_{z} z f_{f}(\omega / k)
$$

and where it is assumed that the scattered frequency shift w $\ll$ w. The electron and ton velocity distribution functions are $f_{e}(w / k)$ and $f_{f}(w / k)$, respectively; $r_{0}{ }^{2}$ is the Thomson cress section, $7.95 \times 10^{-26} \mathrm{~cm}^{2} ; n_{e}$ is the electron densfty; $P_{i}$ is the incident laser power: and $G_{e}$ is the electron susceptibility.
We assuse that the electron and bulk ion velocity distributions of the plasing are Maxmellitan and the bulk plasm is ade up of i sotopes of hydrogen $(z=1)$. The high energy spectrum of the alphas is assumed to be isotropically distributed in velocity space and $f_{\alpha}\left(v_{a}\right)=$ constant for $v<v_{\infty}$, the velocity of a 3.5 PeV a-particle. Wove $v_{e}$ the alpha distribution function is zero. Also, ignore $k$ inetic broadening of the alpha velocity distribution due to the finite energy of the interacting $0-T$ ions.

In order to determine the range of scattering angles and frequencies over wich wore detailed calculations should be performed, we first place simplifying constraints upon the scattered spectru: (1) consider only phase ve!ocities near $\omega / k=v_{\alpha}$ and (2) pick a region where the alpha particle scattering and electron scattering are equal. Assuming a 10.6-y wavelength laser, this corresponds to a scattering angle $\theta=0.70^{\circ}$. The signal received from the electron scattering for $v_{e}>v_{\alpha}$ will be used to ... calibrate the alpha-scattered signal, so that for known $n_{e}, T_{e}$ we may deternine $n_{e}$.

Based on this simplified calculation, the scattering geometry depicted in Fig. 7.4 ms chosen. The scattered signal is assumed to emerge from the plasma device through an annular window centered on the inp t laser bean. The viewing window has an angular span from $0.5^{\circ}$ to

OAML-Dwa esc-13es

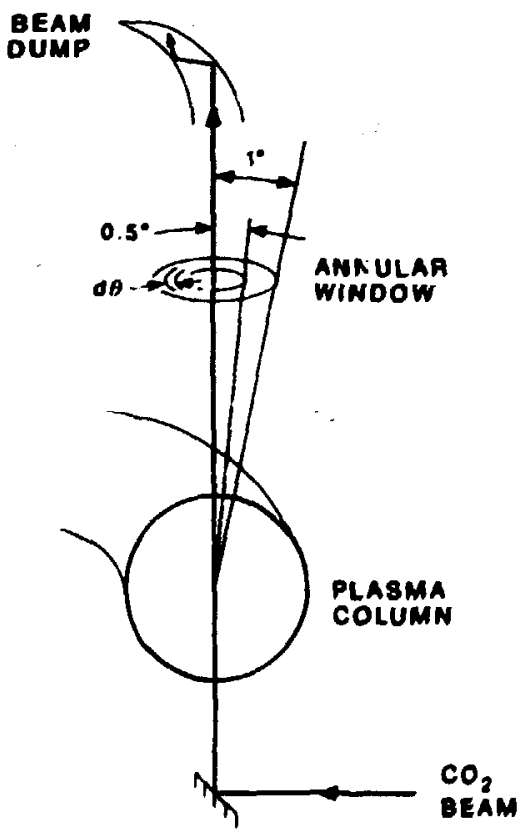

Fig. 7.4. The scattering geometry shown in this figure was chosen to accomnodate the small scattering angle necessary to measure the alpha particle velocity distribution. 
1.0\%. A plot of the total scattered-spectru expected from a TFTR-like plasma as a function of scattered frequency shift based on the geometry depicted in Fig. 7.4 is shown in Fig. 7.5. This calculation assumes a flat alpha particle velocity distribution and indicates that alphsparticle produced scattering is dominant ever a range of scattered frequencies fro 6-22 612 . Figure 7.6 depi-cs a calculation of the composite spectrum as a function of frequency in watts of scattered power per unit frequency for scattering frequencies of from 0.1 to $25 \mathrm{Gtz}$ about the line center of the incident laser for three simple velocity distributions. The distributions wodeled are (1) $f_{f}(v) \equiv \nu / v$; (2) $f_{\mathrm{e}}(v) \equiv$ constant; and $(3)^{\mathrm{f}} \mathrm{f}_{\mathrm{e}}(v) \equiv v$. The aplitude of the distributions was normalized to the vaive $n d v_{e}$ at $v_{e^{*}}$. The first distribution proportional to $1 / r$ mould represent a buildup of particles toward zero velocity assuafing 1 ittle or $n$ diffusion in real space: the second assumes a model based on calculations of IFIR1 ike ${ }^{6}$ plaswas that predict a fiat alpha velocity distribution; and the third distribution proportional to $\checkmark$ could result if alpha particles were lost during the slawing down process. These distributions are not assumed to accurately codel a true plasma case but mere chosen to investigate the sensitivity of the proposed diagnostic technique to changes in the alphaparticie velocity distribution. Figure $\mathbf{7 . 6}$ shows the scattered power plotted for scattering frequencies greater than $6 \mathrm{GHz}$, the approximate f requency where the scattering from the injected beam components goes to zero. The composite scattered power from the electrons is constant

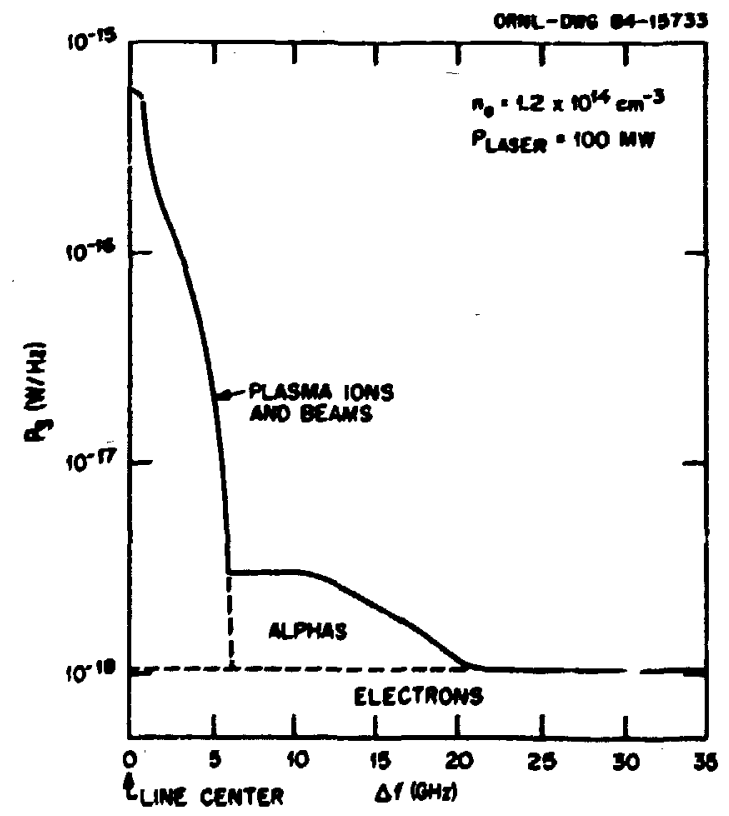

fig. 7.5. A piot of the total scatcered spectrum calculated from Eq. (6) is shown as a function of scattered Prequency shift.

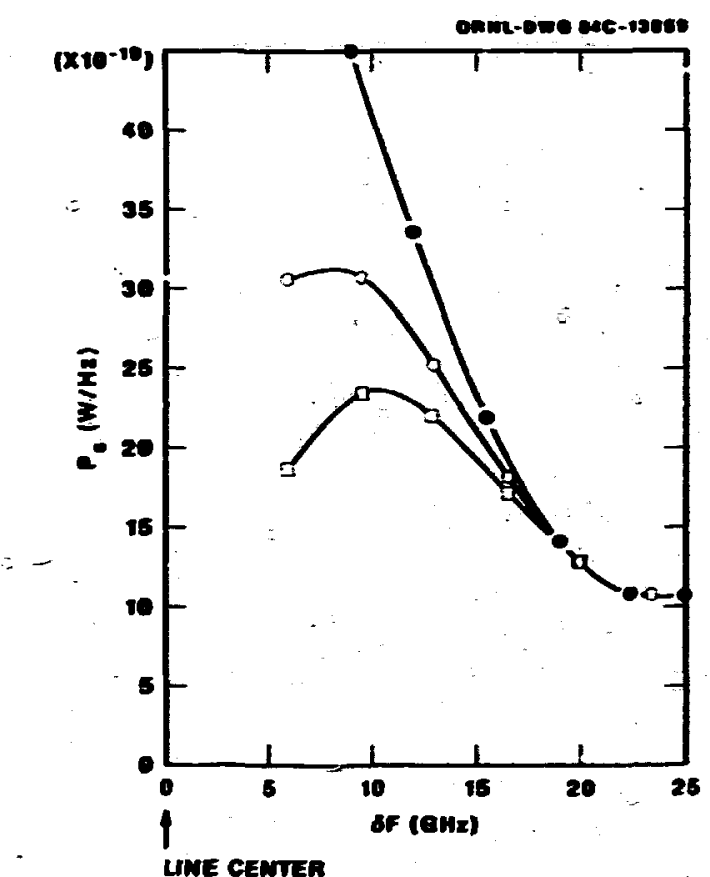

Fig. 7.6. The scattered power versus the shifted frequency of is plotted for three simple aparticle distributions. The a-particle distribution proportional to $1 / v$ is represented by the open circles, the $f(v)=$ constant distribution by the cpen squares, and the distribution proportional to $r$ is represented by the open triangles. The electron density assumed for these calculations is $1.2 \times 10^{14} \mathrm{~cm}^{-3}$.

over this range and has a value of approximately $1.05 \times 10^{-17} \mathrm{~W} / \mathrm{Hz}$. The 1/f portion of the scattered spectruin from 10. to $2 C \mathrm{GHz}$ is due to a veraging over the window. The scattering frow the alpha particles is $2-3$ times the scattering from the electrons over this frequency range.

\section{Detection System}

The scattered power from the alpha particles covers the frequency range from 6 to approximately $20 \mathrm{GHz}$ on either side of the 10.6 itne of the $\mathrm{CO}_{2}$ laser, whose center frequency is approximately $28,306 \mathrm{GHz}$. The frequency resolution required is too high to use a grating for dispersion as in a conventional Thomson scattering measurement and too small to use a FabryPerot interferometer. Aiso, the noise level of a liquid-nitrogen-coolcd HgCdTe detector operating in the viceo mode is too high to allow measurement of this power level. If ive assume a requitied frequency resolution of $6 \mathrm{GHz}$, the video noise-equivalent-power (NEP) $r$ of this detector is on the order of $5 \times 10^{-8}$ wets. 8 Referring to Fig. 7.7, a bar graph is presented where the scattered power integrated over a 6-GHz bandwidth is plotted as a function of the irequency of a number of channels centered on the Prequenctes $7,11,17$, and $53 \mathrm{GH}$. "rom the 


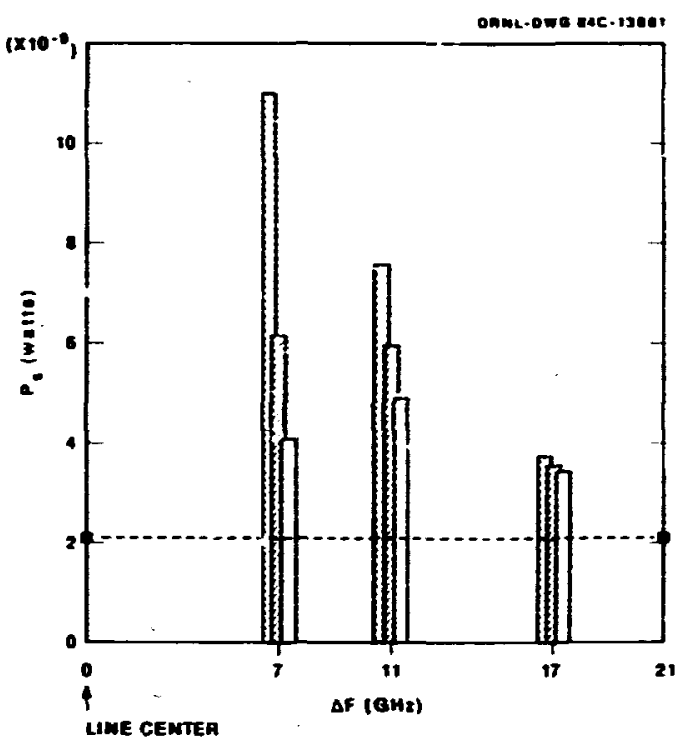

Fig. 7.7. The scattered power integrated over a 2 Iiz $^{\prime}$ bandwidth is plotted as a function of the shifted irequency of a number of channels centered on the frequencies $7,11,17 \mathrm{GHz}$ from the center of the incident laser iine. The channel at $53 \mathrm{GHz}$, shich observes only the electrons, is shown as a dotted line. The $1 / v$ distribution is iepresented by the bars slanted cown to the right, the $f(v)=$ constant distribution is depicted by the bars slanted up to the right, and the open bars represent the $f(v)=v$ distribution.

center of the laser 1 ine. (The reason for this channel selection will be stated later.) The power level observed by the detectors varies from approximately $2 \times 10^{-9}$ wats to $1 \times 10^{-8}$ watts resu'ting in a signal-to-noise ratio (S/N) of 0.02 to 0.1 . This $S / N$ is clearly unacceptable. By using these detectors as mixers in a heterodyne mode, the NEP reduces to $1 \times 10^{-19}$ $\mathrm{W} / \mathrm{Hz}$ i7 resulting in a detector noise power of $2 \times 10^{-10}$ watt.s over a $6-6 \mathrm{~Hz}$ bandwidth. This detector $n_{2}:-t$ level translates into an input $(\mathrm{S} / \mathrm{N})_{\mathrm{j}}$ of 3 to 16 for a $100 \mathrm{MW} \mathrm{CO}_{2}$ laser. The post-dezection signal-to-noise ratio $(>i, i)$ pd for a heterodyne receiver is primarily deterinined by the post-detectior, avers ing time and the input detector bandwidth. Assuming that the sigrial is averaged over a laser pulse width of $1 \mathrm{\mu s}$ from a detector with a bandwidth of $6 \mathrm{GHz}$, the postdetection signal-to-noise ratio (S/N)pd will be improved by a factor $\sqrt{B \tau+I}$, where $B=$ predeteation bandwidth $=6 \mathrm{GHz}$ and $\tau=$ laser pulse width $=1 \mu \mathrm{s}$.

$$
(S / N)_{p d}=\frac{(S / N)_{i}}{(S / N)_{1}+J} \sqrt{B \tau+T}=77.5 \text {. }
$$

so that a system S/N of approximately 75 will be achieved.
In order to convert the system to heterodyne detection, a series of $\mathrm{CO}_{2}$ laser local oscillators anst be :ound whose frequencies differ from the incident laser frequency by only a few gigahertz. A $\mathrm{CO}_{2}$ rotational line spacing of $53 \mathrm{GHz}$ occurs in the vicinity of the highest gain transition, the 10P20 line. Fortunately, a number of $c-w$ lasers dre availabl ? within the required frequency range. We have constructed lasers operating on the first sequence bands of $\mathrm{CO}_{2}$ and $\mathrm{N}_{2} \mathrm{O}$ mich produce power levels of several watts, far more than the 1-2 aid local oscillator requirement of the higdTe detectors. ${ }^{9}$ It is expected that other useful local oscillator frequencies will becone available when isotopic $\mathrm{CO}_{2}$ lasers are considered.

\section{Testing and Calibration}

A very interesting and attractive feature of the proposed alpha particle diagnostic is that a simpler scattering experiment way be conducted on a non-ignited plasma device to determine the feasibility of the measurement. Referring to Fig. 7.6, since the scattered power oue to the alpha particles is roughly equal to the smallangle Thomson scattering measurement of the electron scattering in the absence of alpha particles, the sensitivity of the diagnostic may be determined. In fact, since the electron density and temperature will be known from other diagnostics, an absolute calibration is possible. Also, oecause a detector observing frequencies shifted $53 \mathrm{GHz}$ from line center will only see scattering from the electrons, even in a burning plasma with alphas present, the experiment will be self-calibrating for every measurement.

1. Fusion Energy Division.

2. D. E. Post et al., "Techniques for Measuring the Alpha-Particle Distribution in Magnetically Confined Plarmas," J. Fusion Energy, 1, 129-142 (1981:.

3. U. Schumacher and E. Springmann, "Some Considerations Concerning Fusion Alpha Particle Diagnostics," IPP 1/215, Max-Planck-Institut Für Plasmaphysik Report, April 1908.

4. K. N. Sato and M. Sasao, "Alpha Particle Di agnostics for the R-Project," Institute of PI asma Physics, Kagoya University Annual Report, March 1982.

5. G. Gerdin and B. W. Wehring, Technical Progress Report on the TFTR Alpha Extraction and Weasurement Project, DOE/ER/53197-1, U.5. DOE, september 1983.

6. Wayne Holberg, ORNL, prívate communication.

7. J. Sheffield, Plasma Scatterting of Electromagnetic Radiation. ACademic Press, NY, 1975.

8. Santa Barbara Research Catalog, p. 6 (1983).

9. B. J. Peyton et al." "High-Sensitivity Receiver for Infrared Laser Communications," IEEE J. Quantum Electronics, QE-8(2),

Feb. 1972. 


\author{
H. O. Cohn \\ 6. T. Condol \\ J. E. Braul \\ T. Handler \\ H. H. Bugg
}

\section{CHNH PHOTOPRODUCTION AT 20 GeY}

The high energy physics progran for this period has centered on a hybrid bubble chamer experiment at SLAC to study charm photoproduction with 20 GeV photons obtained from a backscattered laser beam. The project is a collaboration with groups from SLAC, LBL, MIT. Brown University, Tufts University, University of Tennessee, and other institutions in Great Britain, Japan, and Israel. Over $10 \%$ of the photographs obtained mere scanned and measured on the spiral reader at ORNL. Charm candidates were selected by careful examinatior of the vertex of interactions in liquid hydrogen on high resolution photographs taken with specfal cameras where the lens was diffraction Ifmited with a resolution of about 45 microns. Any event where one or more tracks did not extrapolate to the vertex were considered charm candidates. Geometric reconstruction and momentum determination was made from pictures taken with a three view conventional optics arrangement.

Several papers resulted from this experiment covering the charm photoproduction cross section, ${ }^{2}$ charm lifetimes, ${ }^{3}$ and inclusive photoproduction of neutral strange particles." The inclusive charm cross section at a shoton eners: of $20 \mathrm{GeV}$ was found to be $60 \pm 8-21 \mathrm{nb}$. Here, the first error is due to the sensitivity of the experiment, while the second expresses $t$ systematic errors, principaliy due to $t_{\text {. }}$ lifetimes and 2-prong branching ratfo uncertainties. Evidence was found for a non-D $\bar{D}$ component to charm photoproduction, consistent with $135 \pm$ 20) $\mathrm{s} \mathrm{AC}_{\mathrm{C}}^{+}$production and some $\mathrm{D}^{* \pm}$ prodrction.

\section{CHARH LIFETIME}

The charm-lifetime results are based on 42 neutral, 45 charged, and 13 topologically anbiguous decays of charmed mesons and were reported by the collaboration to be:

$$
\begin{aligned}
& \tau_{0^{0}}=(6.4+1.1 \pm 0.5) \times 10^{-13} \mathrm{sec} \\
& \tau_{0^{ \pm}}=\left(8.2_{-1.9}^{+1.3} \pm 0.6\right) \times 10^{-13} \mathrm{sec}
\end{aligned}
$$

and their ratio $\tau_{D^{ \pm}} / \tau_{D^{\circ}}=1.3+0.5$. The charged Iffetime agrees well with the previously reported world average value, hovever, our neutral lifetime value is considerably longer thar. that of $\left(\begin{array}{c}+0.8 \\ -0.6\end{array}\right) \times 10^{-13}$ given by the data particle group." One event with proper flight time of $55 \times 10^{-13}$ sec has been observed in this experiment. This possibility of it not poing a true charm event has been examined carefully with the conciusion that it is highly likely the event is genuine. The probability of observing such an event in our experiment is about 27 , based on our measured iffetime. On the other hand, the probability of observing this or a longer decay in this experiment is about $4 \times 10^{4}$ if the Iifetime is taken to be the world average of $4.4 \times 10^{-13} \mathrm{sec}$.

\section{SEARCH FOR CROSS SECTION ENHANCEMENT} NEAR THRESHOLD

It has been suggested ${ }^{6}$ that an enhancement of several microbarns in cross section for rp * charm baryon + charm meson exists a few hundred MeV above threshold. "The collaboration has searched for such an enhancement ${ }^{7}$ by exposing the SLAC one-meter hydrogen bubble chamber to a photon beam with energy peaked at $10.5 \mathrm{GeV}$, which is $700 \mathrm{HeV}$ above the $\mathrm{yp}+\mathrm{D}^{-} \Sigma_{\mathrm{c}}{ }^{++}$threshold. Except for reducing the beam energy, the experiment was performed in identical manner to the run at $20 \mathrm{GeV}$. A total of 98,000 pictures were taken. No charm decays were detected from which an upper limit to the charm cross section of $94 \mathrm{nb}(90 \%$ confidence level) at $\mathrm{E}=10 \mathrm{GeV}$ was ontained.

\section{PhOTOPRODUCTION OF THE CHARGEO $\lambda_{3}(1980)$ kesON}

Little work has been done on the photoproduction of the spin 2 mesons. The only wellestablisned observations have been of the charged $A_{2}$ through the reaction, $Y_{p} \rightarrow A_{2} n_{n}$. In a routine search of the data from a massive bubble chamber experiment, wose primary goal was to study charmed particle production, we have observed an enhancement in the bosonic spectrum of the reaction $\mathrm{Yp}^{+\pi^{+} \pi^{+} \pi-n}$, which is difficult to interpret other than photoproduction of the $A_{3}$.

For the current study, only the $\sim 80,000$ three-prong events have been examined. All events, for which a three-constraint fit was possible, were eliminated from further consideration $\left(y p+p t^{+} \pi^{-}, p^{+} K^{-}, p p^{-}\right)$. Further, if on 
event contained an identified proton, either by ionization measurements or because it was a heavily ionizing particle wich stopped in the chamber volume, it was removed from our sasple. Finally, any event with an identified gama in the downstream system was el iminated. Our data are derived fion the sample of these events which fit the hypothes is $y p+\pi^{+} x^{+} \pi-n$ with a photon energy in the momentum range 15-22 feV/c. The full three-pion spectru contains evidence only for $A_{2}$ production. However, when the restriction is made that $\left|t_{\gamma, 3 x}\right|$ be less than $0.1 \mathrm{GeV}^{2}$, the three-pion spectru Jisplays clear evidence for not only the $\boldsymbol{h}^{+}$but also for a higher mass enhancesent.

This spectrum can be fitted, in an acceptable fashion, to a polynomial background together with simple Breit wigner resonances for the $A_{2}$ and a higher mass meson $\left(A_{3}\right)$. Although the fitting progran prefers a marrow $A$ resonance $\left(r_{A_{3}}=57 \mathrm{MeV}\right)$. The fit quality is virtually undiminished for an $A$ whose width is constrafined to be $200 \mathrm{Mel}$. The dipion mass spectrum for $A^{+}$events shows that both $p^{\circ}$ and $f^{\circ}$ are present as the decay products of the $A_{3}$. Since the only knom particle in the mass region with $I^{G}=1^{-}$is the $A_{3}$ and since the $A_{3}{ }^{+}$decays both to $p^{\circ} \pi^{+}$and $f^{*} \pi^{+}$, the simplest assumption is that we are observing the photoproduction of the $A_{3}$. Because its production is enhanced at small ' $t$ '. it is sugges ted that the $A$ is produced by pion exchange. Such a conclusion would further imply that the $A_{3}{ }^{+}$has a finite decay width to $\pi^{+} \gamma$. Our initial estimate is that this width is $\sim 1.5 \mathrm{MeV}$.

\section{CAPTURE OF SLON AMTIPROTONS}

Another area of study concerned the behavior of slow antiproton capture by nuclei. The data were derived from an old bubble chamber exposure to slow antiprotons which stopped in plates of carbon, titanium, lead, and tantalum placed in the bubble chamber. Three papers variously reported the low energy antiproton-nucleus reaction cross sections, $A^{\circ}$ production from low energy antiproton anninilations in complex nuclei, 9 and multinucleon captures of slow $\bar{p}$ in complex nuclet and search for the $H$ dibaryon. 10 The later paper reports on a search for doubly strange final states which should become feasible in slow $\bar{p}$ captures in nuclet if the incident $\bar{p}$ interacts with clusters of nucleons.
Because of the destruction of a unit of baryon number of the capturing cluster, the reactions possess comparable $Q$ values to the ordinary strangeness producing annihilation reactien. Should double strangeness production occur in $\bar{p}$ annihilations, a doubly strange six-quark dibaryon (H) predicted by the MIT bag model ${ }^{11}$ ay occur. A saple of $80,000 \bar{p}$ annihilations in muclei with $\bar{p}$ monenta of less than $400 \mathrm{MeV} / \mathrm{c}$ were searched for the production of two $A^{\circ}$, two $K^{+}$or $a A^{*}$ and $K^{-}$in a single interaction. No examples of such processes were found leading to upper limits for frequencies of production of final states containing $2 k^{*}, 2 k^{+}, k-k^{*}$ of $5 \times$ $10^{-4}$ per $\bar{p}$ annihilation. If th $: H$ particle should exist as an object with nass less than $z_{n}$. it would be stable against decay by the strong interaction, in which case the dominant weak decay mode would 'e the $\delta$.p with a lifetime somerhat longer than the $A^{\circ}$. Such decay modes would leave a unique signature in a hydrogen bubble chamber of "Vee" containing two baryonic prongs, and the negative prong would decay to a $x$ - or yield a $A^{*}$ from the reaction $\bar{\Sigma} p+A^{*} n$. No such events were found leading to a $H$ proasction upper liait of $9 \times 10^{-5}$ assuming its lifetime is comparable to that of the $\Lambda^{\circ}$.

1. Adjunct staff members from the University of Tennessee, Knoxville, TK 37996.

2. K. Abe et al., Phys. Rev. 300, 1 (1984).

3. $K$. Abe et al., Proceedings of XXII International Cinference on High Erergy Physics, Leipzig (J0 34), to be published.

4. K. Abe et al., Phys. Rev. 290, 1877 (1984).

5. Review of Particle Properties, Rev. Mod. Phys. 56, 16. 2, Part II, (April 1984).

6. H. Rubinstein and L. Stodolsky, Phys. Lett. 768, 479 (1978). (1984).

7. K. Abe et al., Phys. Rev. 300, 694 (1984).

8. H. 0. Cohn et al., Phys. Rev. C29, 232

9. G. T. Condo, T. Handler, and H. D. Cohn, Phys. Rev. C29, 1531 (1984).

10. G. T. Condo et al., Phys. Lett, 1448, 27 (1984). (1977).
11. R. L. Jaffe, Phys. Rev. Lett. 38, 195 


\section{COMPILATIONS AND EVALUATIONS}

\section{CONTROLLED-FUSION ATOAIC DATA CENTE?}
L. F. Barnett
H. B. Gilbodyl
D. C. Gregory
P. M. Griffin
C. C. Havener
A. M. Howald
H. T. Hunter
R. K. Janev ${ }^{2}$

H. I. Kirkpatrick E. W. Thomas"

C. R. Rhon ${ }^{3}$

E. H. McDaniel

R. H. McKnight 5

F. H. Meyer

T. J. Morgan 6

R. A. Phaneuf

R. S. Pindzola'

The data center continues to maintain a current annotated bibliography of atomic and molecular collision processes of interest in fusion research. During the 24-year history of the data center we have consistently been 1-2 years behind in entering current references into our data base. This year, references are entered directly into our computer file every three months and within a few months we anticipate a monthly input. A universal data storage and retrieval system (InquIRE) has been implemented by $M$. Uright of the Comput ing and Telecomunications Division. Bibliographical information can now be retrieved on-line using ten different search elements. At the present time three outside users are experimenting with retrieval using telecomunications lines tu determine the feasibility of extending the data base to a larger group of outside users. Over the years, as computer technology has advanced, we have user several different storage and retrieval programs, with the result that we are at present only able to conduct on-l ine searches of references entered since 1978. Ef forts are being made to convert the various earlier bibliographic formats to one compatible with the INOUIRE format.

Cooperative efforts have continued with the At omic Data Center for Fusion at the Institute of Plasma Physics (IPP, Magova, Japan), Atomic and Nuclear Data Center : :-ie Japan Atomic Energy Research Institute (JAERI, Tokaf-mura, Japan), and the International Atonic Energy Agency Atomic Data Center (IAEA, Vienna, Austria). During this reporting period, an agreement was reached that the ORNL bibliography would serve as the mafor input to these centers. ORNL will provide computer tapes every three months. Our staff cont inues to meet with and advise the IAEA data center on their activities.

To increase the accuracy and efficiency of extracting numerfcal data from the 1fterature, we have purchased a digitizer tablet and a small stand-alone computer with telecommurications capabilities. The required software has been witten and implemented to perform the following steps: (1) digitize numerical data and store on floppy disc; (2: concatenate individual data sets for a given process into one set for comparison and evaluation; (3) redigitize the recommended cross section curve; (4) calculate Maxwellian reaction rate coefficients from cross section data; (5) provide a seven-parameter fit to rate coefficients as a function of teperature; and (6) prepare computer-generated curves of recomended data and data tables for publication. Interfacing the center's computer with the central PDP-10 permits steps 4-6 to be completed.

During the present reporting period the decision has been made to update and expand Vols. 1 and 2 of the present compilation, "Atomic Data for Controlled Fusion" (ORML-5206 and -5207) to a new series with five or more volumes. Previously, the complations contained only graphs and data tables of recomended mumeriral data. Future volumes will add tables and graphs of reaction rate coefficients for interactions of beam-Maxwellian or Maxwell lian-Maxwellian distributions. Progress has been made on the first volume, "Heavy Particle Collisions," which will be done in-house. An interagency agreement with Jean Gallagher of the data center at the Joint Institute of Laboratory Astrophysics has been initiated to complie cross section data for "Electron Collisions." Reaction rate coeffic fent tables and graphs will be generated by the ORil data center staff. Volume 3 of the series "Collision of Atomic Particles with Surfaces" has been completed and is now in the final stages of review before publication. Particle interactions with surfaces is st 111 an inexact scfence and much of the data is qualitative. The fourth volume "Spectroscopic Data for Iron" has been complled by $W$. Wiese and $h t s$ colleagues at the National Bureau of Standards. He have recefved the compilation, except for one chapter on atomic energy levels, wich wafts computer reformatting for compatibility prifor to publication. Work has been initiated on volume 5 , "Colltstons of Carbon and Oxygen Ions with Electrons, $\mathrm{H}, \mathrm{H}_{2}$, and He," authored by $\mathrm{R}$. A. Phaneuf of the ORNL Data Center, R. K. Janev, Institute of Physics-Belgrade, and $M . S$. Pindzola, Auburn Untversity. Data have been comptled from the 1 tterature, digitized, evaluated, and cross sections have been recommended for electron capture by $\mathrm{Cq}^{+}$and $\mathrm{Oq}^{+}$tons from atomic hydrogen and helium. The completion date of these ifive volumes is antictpated to be 
Decemer 1985. Additional volumes are being planned for collisions of other impurity ions present in high temperature fusion plasmas.

1. Consultant, Oueen's University, Belfast, Morthern Ireland.

2. Consultant, Institute of Physics, Belgrade, Yugoslavia.

3. Sumer student participant, 1984. Present address: University of Virginia, Charlottesville, Virginia.

4. Consultant, Ceorgia Institute of Technology, At lanta, Ceorgia.

5. Consulcant, Mational Bureau of Standards, Gaithersburg, Maryl and.

6. Consuita it, Mesieyan University, Middletown. Co necticut.

7. Consul ant, Auburn University. Auburn, Al abana.

\section{WUCLEAR DATA PROJECT}
S. J. Ball
M. J. Martin
Y. A. Ellis-Akovali
M. R. Meginnis

H. R. Schmorak

The Nuclear Data Project (KDP) is one of five data evaluation centers comprising the U.S. Nuclear Data Metwork (USNDN). The Project is responsible for the evaluation of nuclear structure information in the mass region $A>195$. The NDP mintains a complete computer-indexed 1 ibrary of reports and published articles in experimental nuclear structure physics as mell as copies of the Evaluated Muclear Structure Data and Huclear Structure Reference files (ENSDF, MSR).
The Project houses the position of Editorin-Chief of the Muclear Data Sheets. All ass chains from the 14 centers in the International Nuclear Data Metwork are edited here, and the Editor-in-Chief has the ultimate responsibility for the quality of the mass chains entered into ERSOF and, thus, for what is published in the Wuclear Data Sheets.

\section{Activities}

Data Evaluation. During this report period, WDP staff embers prepared revised evaluations for 9 mass chains. References to the publications based on these evaluations are given in the publications section of this report. EMSDF now includes:

1948 Adopted Level Properties data-sets $(A=1-263)$

2396 decay scheme data sets

4257 reaction data sets

Mass Chain Editing and Reriew. NDP staff members edited and/or reviewed 12 mass chains. Information Services. Mop staff members responded to about 25 requests by researchers outside the evaluation center for specific information. Responses took the form of searches of the ENDSF and NSR files and personal consultation. A list of reports and praprints received by the NDP is prepared and distributed monthly to division staff members.

Research. NDP staff members have participated in research with other groups in the division. References to these activities are given in the publications section of this report. 


\section{ACCELERATOR-COLLIDER STUDY}

\author{
6. D. Alton \\ H. H. Ackins 1 \\ C. Baktash \\ J. R. Beene \\ J. A. Biggerstaff \\ E. D. Hudson \\ C. H. Jones \\ R. C. Juras 2 \\ 1. Y. Lee \\ J. A. Martin \\ J. H. MeConnell
}

\section{InTROOUCTIO}

This section describes a study done for an accelerator facility to be ised to accelerate counter-rotating beans of heavy nuclei to kinetic energies of $10 \mathrm{GeV}$ per nucleon and to bring these beams into head-on collistion. The extrese conditions occurring in the resulting extended system of hot hadronic matter should result in creation in the laboratory of a qualitatively new form of matter, a quark-gluon plasma. This form of matter is thought to consist of quarks and gluons wich are free to propagate over large space-time distances, in sharp contrast to the situation encountered in everyday experience in wich quarks and gluons are confined to volumes the size of protons and neutrons. The production of very hot plasma could re-create in the laboratory conditions wich have not existed in the universe since a short time, $10 \mathrm{\mu S}$, after the Big Bang, at wich t ime quarks and gluons were first confined in hadrons as the universe cooled. Production of very dense, as opposed to very hot, plasma could allow simulation in the laboratory of conditions thought to prevail today in the cores of neutron stars.

The proposed facility is designed to be able to scan through the expected phase transition separating the normal quark-confining, hadronic phase of nuclear atter and the quarkdeconfining, plasma phase of nuclear matter. The nature of the phase transition, such as its order, latent heat, and location in temperaturedensity space, can be studiad using this scanning capability of the facility. The conditions necessary to produce the phase transition. such as requited collision eneray as a function of the mass of the colliding partners, can be determined. All of this information constitutes essential experimental input to the theoretical description of the transition from hadronic to quark matter. This study is made all the more compelling by noting that such a transition represents the artifictal reversal, in the laboratory, of one of the symetry-breaking transitions postulated to be responsfble for the observed nierarchy of forces in physics.
J. B. McGrory
H. T. Milner
S. H. Mosko
F. E. Obenshain
D. K. Olsen
F. Plasil
R. L. Robinson
R. R. Strayer
C. N. Thongs $^{3}$
C. Y. Hong
G. R. Young

Such symetry-breaking transitions are one of the win cornerstones of modern gauge field theories of matter.

The facflity is also mell suited for the study of the haryon-rich mifestation of the quark-gluon plasma. This is due to good luminosity and energy variability of the accelerators in the region of $5 \mathrm{GeV} / \mathrm{u}$. At about this collision energy, two heavy nuclei should overlap with maximum pileup of baryon density. creating a "cold" plasea wich may be similar in structure to the matter in the cores of neutron stars.

The facility consists of a series of three accelerators optinized for the acceleration of heavy ions. The existing tandem electrostatic acclerator will be used as injector, providing beams of heavy ions ranging from $12 \mathrm{C}$ to $197 \mathrm{Au}$ at corresponding kinetic energies ranging from $10 \mathrm{MeV} / \mathrm{u}$ to $2 \mathrm{MeV} / \mathrm{u}$. This will be followed by a small, room temperature, booster synchrotron with a bending product of $16 \mathrm{Tm}$. The size of this synchrotron is given by the requirement that all the above heavy ions be completely fonized by passage through a stripper foil after their exit from this accelerator. The th:rd accelerator in sequence will be a pair of superconducting accelerator-collider rings. These will intersect at six points and have a bending product of $90.5 \mathrm{Tm}$, corresponding to $10 \mathrm{ceV} / \mathrm{u}$ for $197 \mathrm{Au}^{79+}$ ions. Expertmental halls will be located at (initially) four of the six intersection woints and will have facilities for various type detectors ranging from single-arm spectrometers to large $4 \pi$ detector facilities. A plan view of the facility as it would be located on the ORNL site is shown in Fig. 10.1.

The peak initial luminosity of the facility will range from $\mathrm{L}_{0}=3.5 \times 10^{28} \mathrm{Cin}^{-2} \mathrm{sec}^{-1}$ for $12 \mathrm{C}+12 \mathrm{C}$ collitistons to $\mathrm{L}=3.3 \times 10^{26}$ $\mathrm{Cm}^{-2} \sec ^{-1}$ for $197 \mathrm{Au}+199$ Au collístons. Assuming that central collistons of heavy fons (the most likely ones to produce the transition to quark-gluon plasma) occur for impact parameters $b$ of $b<0.5: \mathrm{m}$, these values correspond to 275 central collistons per second for $12 \mathrm{C}+12 \mathrm{C}$ and to 2.6 central collitstons per second for $197_{A u}+197 \mathrm{Au}$. These rates are high enough to 
ORM-016 64-:7175

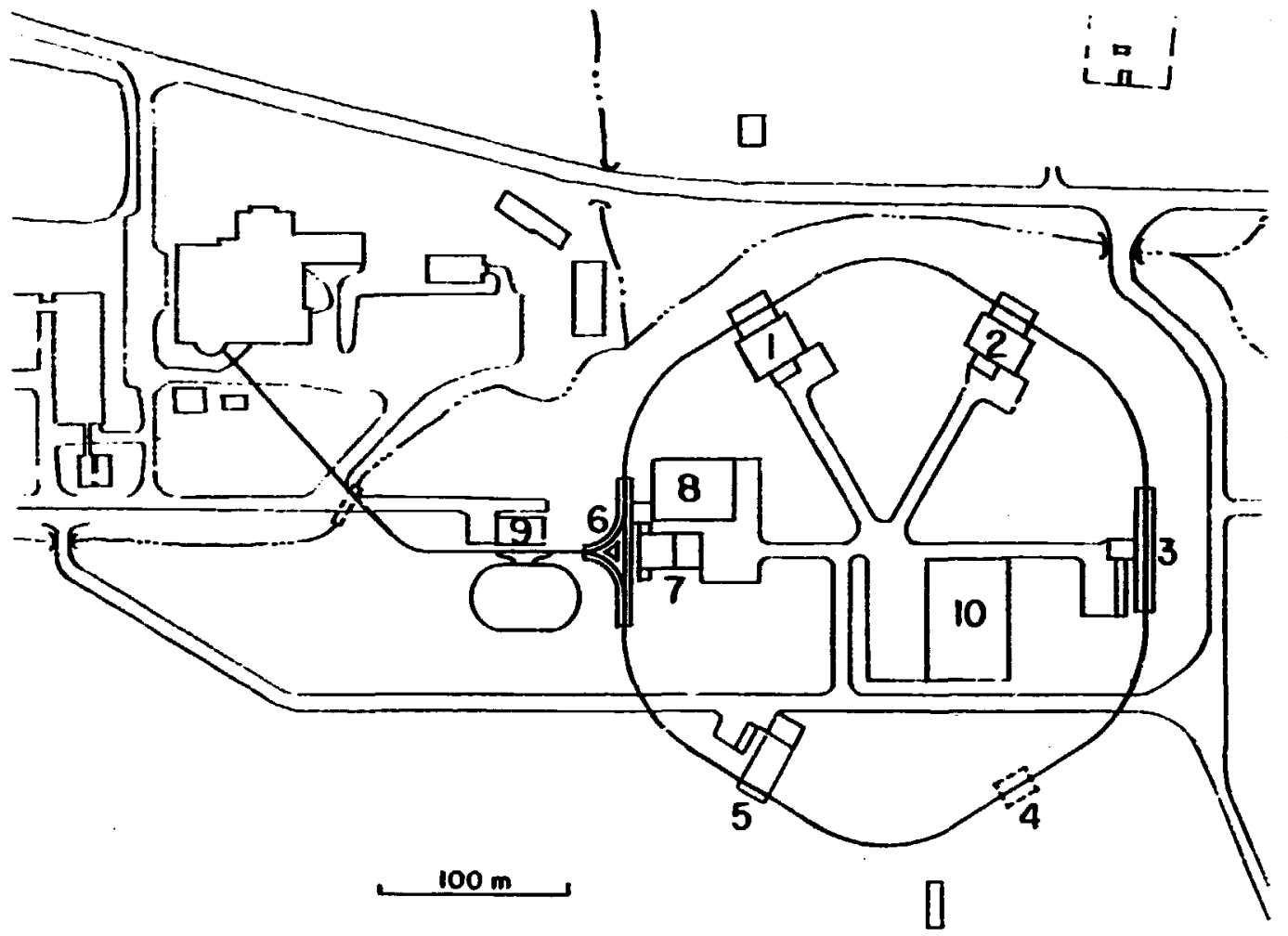

Fig. 10.1. ORML Heavy-Ion Collider Buflding Layout

I Large experiment area 1 Large experiment area $\$ 2$ Narrow-angle experiment hall Pad for future use dide-angle experiment hall Beam injection hall

\author{
7 Cryogenics and HVAC equipment buildings \\ 8 Control and office building \\ 9 Booster ring support building \\ 10 Magnet factor: and warehouse \\ II Electrical yard (not shown)
}

allow thorough investigation of several exclusive reaction channels and to allow broad variation of beam parameters over reasonable running times for experiments.

\section{SCIENTIFIC MOTIVATION}

\section{A. Theoretical Considerations}

The primary scientific objective of the proposed facility is the formation and study of the quark-gluon plasma, wich is predicted to be formed in collisions of relativistic heavy nuclet. In addition to the intrinsic nterest of studies of the behavior of matter under extreme conditions of density and temperature, there are two specific motivations: (1) the production of the quark-gluon plasma addresses the problem of quark confinement, wich is one of the most perplexing phenomena of modern physics: and (2) the production of the plasma may re-create in the laboratury conditions that are similar to those believed to have extsted throughout the unfverse in the early stages (up to 10 us) after the Btg Bang.

Confinement in DCD ts believed to result from a process known ds infrared slavery: when a quark tries to leave a nucleon, the potential energy of the color force field increases without bound. The quark is either pulled back into the nucleus or the color field is neutralized by the production of a quark-antiquark pair. In either event, tne individual quark does not get free. When the yuark is well within the confines of the micleon, it moves relatively freely. For quarks in this regime, the color force field becames weak. It has thus proved useful to model the nucleons as hags of quarks. The physical vacuum exterior to the bag exerts a confining pressure on the small region of space inside the bag. The quarks move freely within the has, where they exist in a regton of "perturbative QCI) vacium," but are unable to overcome the confining force of the exterfor vacuum and escape from the bay. In more precise terms, phenomenological models of the strong interaction postulate "color conf inement," so that the fundamental entittes of (XV), quarks and gluons, are permanently confined in bound states. Only those states for which the net color yuantum number is zero (colorless states, or color singlets) can extst separately; hence, colored particles like quarks and gluons cannot appear as free particles. In wat cousld prove to be one 
of the marked successes of 400 , recent calculations using the lattice-gauge approximation seem to show that color confinement is an inherent property of YCD, resulting fram its basic structure. If this is correct, CCD actually predicts quart confinement, and the necessity of introducing it ad hoc disappears. These calculations show another remarkable property. At high towperatures (and presumbly high densities), a transition occurs to a new phase in mich color is no longer conf ined in individual hadronj. Instead, quarks and gluons are free to move nearly independent ly over the wole vo sue for which the conditions for the phase transition have been achieved. The resulting assembly of "asyiptotically free" quarks and gluons is referred to as the quark-gluon plasma. These calculations are still in their infancy, however, and quark confinement remins the least understood aspect of $\mathrm{CCD}$ and therefore the most in meed of experimental investigation.

At low baryon density and teaperature, watter is composed of quarks confined in hadrons, but as the energy density is raised (by increasing the temperature or the baryon density or both), matter becomes deconfined into a quark-gluon plasia. The exact location and the nature of the deconfinesent transition continue to be a subject of intense investigation.4,5 In an SU(3) Yang-Mills system of gluons, Monte Carlo lattice calculations predict a critical temperature of $T_{c}=160-180 \mathrm{MeV}$ and a phase transition of the first order ${ }^{6,7}$ Results for a system with quarks and gluons have been obtained by retaining only the leading term in the fermion coupling. ${ }^{B}$ In this cas:, the phase transition is less sharp and becomes second order in nature. The enargy density of the quark-gluon plasma is close to the Stefan-Boltzman value for an ideal gas of massless gluons and quarks. i .e., about $2.0 \mathrm{GeV} / \mathrm{fm}^{3}$ at a temperature of about $190 \mathrm{MeV}$.

In general, the study of the quark-gluon plasma will help us understand the properties of matter under conditions of extreme temperature and density. Specifically, it may provide us with a good way to measure the momentum scale parameter $\Lambda$ of YCD. Furthermore, by simulating primordial conditions, it may help our understanding of the evolution of the early universe, as well as the structure of neutron stars and the dynamics of supernova explosions. It has been suggested that, at an early epoch, matter in the universe was in the form of a quark-gluon plasma with very small net baryon density. Our familiar hadron-dominated world of the present epoch is the result of a phase transition from the quark-gluon plasm to hadronic matter. Df particular importance to the investigation of the early universe are the order of the phase transition and the temperature and density at which it occurs.9 A first-order transition is accompai ed hy large fluctuations at the transition putint and may produce planet-size biack holes, wich could have important implications for the question of the "missing mass" of the universe.

in contrase to the quark-gluon plasma associated with the evolution of the early universe, the plasmas in the interior of neutron stars and in sapernova explosions have a large net baryon density but a relatively low temperature.
The occurrence of quark-gluon plasad, homever, depends on the critical density at the phase transition, and the possibility of a stable neutron star with a quark-gluon interior exists only if the critical density is less than about nine times the normal density of nuclear matter. In any case, it is clear that in view of their relevance to cosmology and to astrophysics, studies of quark-gluon plasmas with both low and high baryon densities would be of great interest.

The nature of the quark-gluon plasma is expected to vary as a function of the beam energy and of the region of rapidity at wich it is produced. There result two basic experimental approaches to obtain deconfinement. The first concentrates on the pileup of barjon density such that the hadrons begin to overlap, leading to the formation of a relatively cool quart-gluen plasea. This situation corresfonds to densities given by $-5-10 p_{n a}$ and by texperatures of less than $100 \mathrm{MeV}$. The optinum energies finvolved in this case wust not be excessive in order to avotd the relativistic kineantical problems of longitudinal growth and of transparency. The former causes the hadrons produced in the collision to be formed over too large a region of space to attain high density. The latter reflects the increasing probability of one nucleus to pass through another with increasing relative energy. Various estimates 10,11 put this energy at around $b \mathrm{GeV}$ per nucleon in the c.m. system.

Alternatively, instead of compressing the matter, high hadron densitfes may be reached by raising the teriperature of the system. This situation may be reached experimentally at higher energies. In this case, there are two separate regions: the mid-rapidity, central region, corresponding to a high hadron (pion) density but to a relatively low net baryon density, and the fragnentation regions at the extremes of the rapidity range, corresponding to high baryon densities. The critical boubarding energy needed for the formation of a quark-gluon plasma has been estimated by many authors. $11-13$ Une estimate ${ }^{\lfloor 3}$ gives the energy density produced in a head-on collision of two equal nuclei, each with a mass number $A$ as

$$
c=0.06 \wedge 0.70\left(0.48 \mathrm{In} E_{c_{0} m_{0}}+0.37\right) \mathrm{GeV} / \mathrm{fm}^{3} \text {. }
$$

where $E_{C}$. is the center-of-mass energy per nucieon in units of seV. Inis estimate is based on multiplicity data obtained from a large mumber of nucleus-nucleus reactions. The region of collision energy and of mass number A leading to an energy density exceeding the critical energy density of about $2 \mathrm{GeV} / \mathrm{fm}^{3}$ is indicated by the shaded region in Fig. 10.2. Thus, in a collision of two nuclei, each with $E_{c . m .}$. $10 \mathrm{GeV}$ per nucleon and $A>100$, the energy density produced will be high eñough, for the hadronic-matter to quark-gluon plasma phase transition to take place in the high temperature, low-baryon density mode.

The possible formation of a baryon-rich quark-gluon plasma at the extremes of the rapidity range, fie.., in the fragmentation region, is also of interest. 14 it was suggested that at $E_{1 a b} \geq 25-50$ GaV per nucleon, the baryons in the fragmentation region may be 


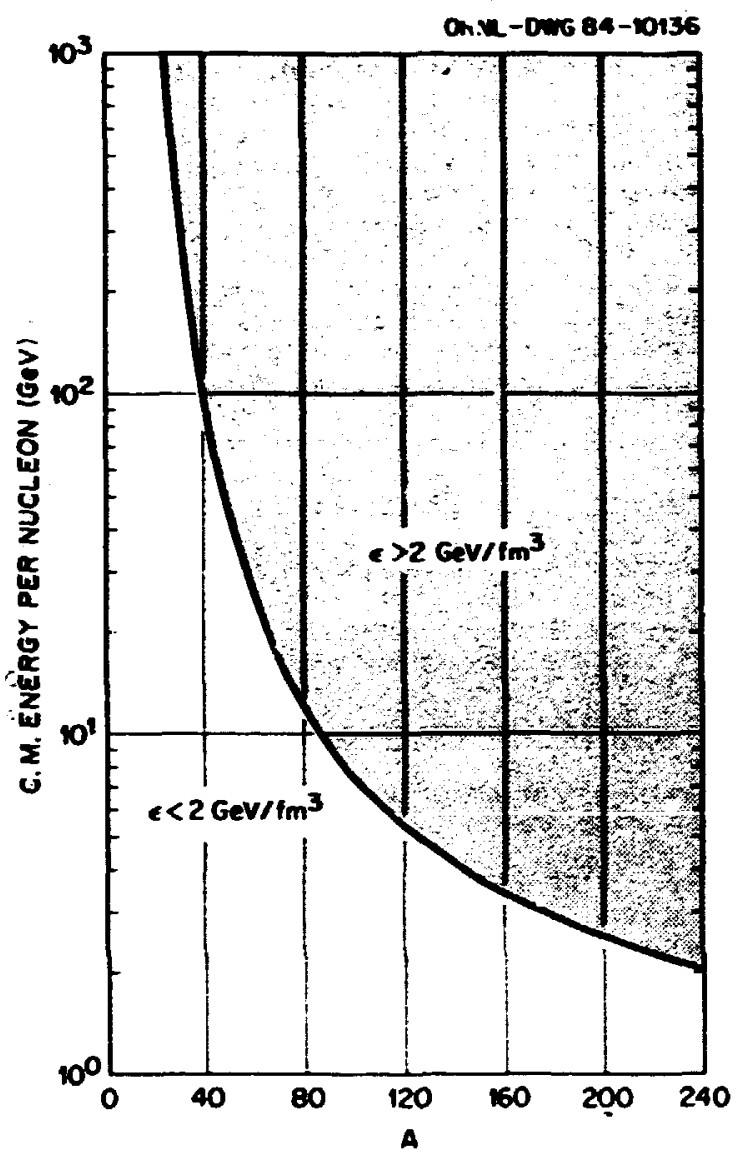

Fig. 10.2. In a head-on collision of two equal nuclei, the region of energy and mass zombinations which lead to an energy density greater than $2 \mathrm{GeV}, \mathrm{fim}^{3}$ is shown shaded.

compressed and excited. The excitation energy in the resulting nuclear fragments may reach 3.5 $\mathrm{GeV} / \mathrm{fm}^{3}$ and may thus exceed the critical energy required for a phase transition.

\section{B. Experimental Considerations}

The experimental investigation of the quarkgluon plasma that is expected to be created in collisions between relativistic nuclei is ifkely to be subject to several complications. In general, any specific signal fron the quark-gluon plasma will have to be detected in the presence of a large Dackground originating from the later stages of the decay of the system. Since the equilibrium, nonequilibrium, and critical hehavfor of the system dramatically affect the composition of the final-state particles and the character of their spectra, and since all of the processes involved are not well-known, the unambiguous isolation of the signals drising from the quark-gluon plasma from the signals arising from the hadronization and freezing-out processes is one of the major challenges that experimentalists have to face.
1. Observation of the Onset of the Quark-Gluon Plasma

Initiaily, the focus should be on those observables that carry the most unamiguous and direct signals from the quart-gluon plasm. Ideally, one would hope to observe such fingerprints of the plasma as fractionally charged fragments or long-lived exotic configurations of yarks and/or gluons. However, in the absence of any firm theoretical prediction for the existence of such exotic products, we shouls ais at the study of those penetrating probes that (a) have higher production rates in the quark-gluon plasma compared to the rates in hadronic matter and (b) survive through the hadronization and freezing-out processes with minimal interaction.

\section{a. Direct Radiation}

Due to the near absence of final-state interactions, the electromagnetic probes (leptons and photons; should provide a relatively clean signal from the early stages of the nucleusnucleus collision. In a hot plasma, the $q 9$ annihilation process yives rise to a significant number of dileptons $(q \bar{q}+\bar{\mu})$, even beyond the kinematic liait for collisions. 15 Unfortunately, several sources of background are anticipated. At large invariant dilepton masses (II $\geq 2 \mathrm{GeV}$ ), the Drell-Yan process gives rise to a significant continuum background. Similarly, at lower asses, both the continuum lepton-pair creation (e.g... $\pi^{+} \pi^{-} \rightarrow$ il at $M=0.8 \mathrm{GeV}$, Dalitz-pairs from $\pi^{0}, \omega$, and $\zeta$, and GetheHeitler pair production at $M<0.6 \mathrm{GeV}$ ), and the uiscrete peaks due to the decãy of vector mesons $(\rho, w$, at $H=0.7-1,0 \mathrm{GeV})$ form sources of background. Shuryak 16 has suggested that the intermediate mass region of $<H<2 \mathrm{GeV}$ is an ideal region to study dilepton production.

Similarly, direct photon production arising from $q-q$ and $q-g$ scattering processes provides a valuable source of information about the interior of the not plasma. Here again, other sources of gamma rays (e.g.. two-photon decay of $\pi^{0}$ and $\eta^{0}$ ) may interfere with a positive identification. However, one may take advantage of the distinguishing features of these sources of background in order to discriminate against them. For example, the $\pi^{0}$ decay photons are kinematically correlated with each other and have a Hagedorn distribution with an average kinetic energy of $160 \mathrm{MeV}$. In contrast, direct gammas measure the temperature or heat capacity of the quark-gluon plasma or of had-onic matter and are not limited in energy.

\section{b. Ereeze-Out Products}

Observation of strange and charmed hadrons (and their antiparticles), wich are produced on $1 ;$ rarely in ordinary hadrontic interactions, constitutes an alternative and complementary approach to the above identification techntque. Ordinary nuclear matter contains only the up (u) and down (d) quarks. Therefore, because of the high chemical potential of these ligint quarks, 
ss̄ pir production proceeds much more easily in a quart-giuon plasm. According to Rafelski, 17 this results in the following apprexiate enhancement ratios:

$\frac{\langle s\rangle \text { plase }}{\langle s\rangle \text { gas }}=50-100$, and $\frac{\langle\bar{s}\rangle}{\overline{\text { Qu }} \text { or } \overline{d\rangle}}=5$. Upon cooling dom, the quark constituents of the plasm will freeze out into various hadrons, which are expected to reflect the composition of the hot plasm. Therefore, a large (relative to the gas phase) production cross section for strange, autistrange, and altiply strange hadrons would be a likely indication of the formtion of the quark-gluon plasma. It should be noted that the shorter the plasma lifetime, or the smoother the phase transformation is, the wore unique this sigal becopes, coepared to other indicators. Rafelskil7 has estimated that the decay of antihyperons formed in a plasea should provide several Vee's. When normalized to, for exaple, the cross section measured in pp interactions, high rates of decay of this type could constitute a signature of the onset of the formation of the puark-gluon plasma.

\section{Discontinuities Associated with Phase Transitions}

Should the transition from madronic metter to the quark-glunn plasm be a first-order transition, wany ceasurable properties are expected to exhibit a discontinuity across the phase transition. One obvious manifestation of a first-order phase transition is the constant temperature with increasing input energy (bean energy). This is due to the need to supply the latent heat for the formation of the quark matter. It has been suggested that the transverse momentum is a measure of the pressure of the central plasm and that the ultiplicity, at a given rapidity, is a measure of energy deposition. On this basis, one may expect the transverse momentum $\left(P_{1}\right)$ as a function of mitiplicity (at a given rapidity) to show saturation effects similar to those seen in other firstorder phase transitions.

In the quark-glucn plasma, the basic scattering processes are $-f$ ferent from those of nucleon-nucleon scatteriı. Therefore, a change of the pattern of mass and energy flow of emitted particles may signal the formation of a new form of atter. Furthermore, the midrapidity $(y=0)$ region is expected to be especially rich in hadronized particles originating from the plasms. Thus, the mitiplicity distribution, dn/dy, in the mid-rapidity region, night be directly related to the probability of plasma formation.

\section{Properties and Dynamics of the Plasma}

Complications associated with the characterization of the quark-gluon plasma are expected to resuit from the effects of dynamics and from the rapidly changing space-time evolution. For example, starting with a formation $t$ twe of $\tau_{0}=1 \mathrm{fm} / c$, the energy density is expected to subsequently drop by a factor of nearly 2 in $1 \mathrm{fm} / \mathrm{c}$. It may take a $\mathrm{few} \mathrm{fm} / \mathrm{c}$ for the plasma to equiliforate (thermal phase).
However, in the interio period, the plasm expands with a Hubble constant estinated to be 107 larger than the cosmological tubble constant, and its evolution will be governed by. nonequilibrium processes. To study these coaplicated phases and their interfaces (initial compression and fornation of a plasma, nonequilibrium expansion, thermalization, hadronization, and freezing-out processes), one needs to measure and correlate many hydrodynamical observables. The bulk properties of these systems my be characterized oy tenperature, volume, relaxation times, and energy and mss densities. However, may of these parameters with wich we hope to characterize the plasim and its space-tive evolution are, at best, only loosely connected to observables. A great deal cf careful systeatic mork will have to be undertaken before acceptable diagnostic wethods can be established. In the following, some possible experiments to deterinine a few basic properties of the quark-gluon plasmare discussed.

\section{a. Spatial- Characteristics}

An important property is the size and the shape of the region over wich the phase change has occurred. This quantity is prifarily sensitive to the mechanisin of energy deposition and thermalization in the reaction process; however. knowledge of the spatial extent of the plasma region will be important if we are to correctly interpret other peasurements.

Intensity interfercmetry id, is is a mellestablished technique for studying the spacetime structure of an enission source. Pairs of identical particles are detected, and a correlation $C\left(\vec{p}_{1}, \vec{p}_{2}\right)$ is deternined. This function depends not onily on the space-time extent of the source, but also on its degree of coherence. thus providing both geometrical and dynamical information on particle production to the reaction. The sensitivity of such mezsurements increases rapidly as the witiplicity of a particular species of particle increases. Since very large pion multiplicities are expected under conditions required for quark-gluon production, pion interiarometry would appear to be a potentially important tool. Unfortunately. pion; and other strongly interacting particles will carry little information about the hottest regions where the plasma can exist, because of absorption and rescattering effects in surrounding cooler matter. Therefore, two- and higher order interferometry of pions (speckle interferometry) will yield information only on the size and the shape of the system at the freezeout time. Oirect information, however, will be carried by electromagnetically interacting particles, f.e., hy photons and leptons wich are coptously produced in the plasma, as discissed in the previous section. Such experiments could yfeld reasonably direct information on plasma size, as well as provide tool for investigat. ing long-range order and correlation lengths in the plasma.

It is possible that $K^{+}$correlations may aiso be an tmportant tool. The $K^{+}$satisfy the requirement of a weak interaction with normal hadronte matter and so can carry information out of the hot, dense region. It is possible that 
these correlations could provide information on the space-tiane structure of the plasan region near the freeze-out, i.e.., on the reconf inement process.

\section{b. Ienperature}

It will be important to study the evolution of the temperature of the plasma as a function of bombarding energy. The best tool is probably provided by the measurement of the shapes of spectra of particles enitted at various stages of the reaction (e.c., ghotons and dileptons for the plasm and pions for the freeze-out stage). Once the critical energy density for the phase transition is reached, additiosal energy rould go to "welting" madrons into plasm, i.e., providing the latent heat of deconf inement rather than increasing the temperature. This effect is one of the potential signatures of the phase transition discussed in an earlier section. It would be of interest to continue an investigation of this temperature to higher $c_{\text {. }}$. . energies until it again begins to rise. The length of this temperature plateau would be a measure of the latent heat of deconf inement.

Because of the complicated dynamics of the plasaa, the interpretation of this type of data is al so not expected to be straight forward. Since the observed particle spectra are spacetime integrated, they receive important contributions from both the early stages (i.e.. thermally unequilibrated) and later stages (hadronization and freeze-out) of the evolution. Proper deconvolution of these spectra requires not only a better theoretical understanding of the nonequilibrium process, but also a systematic experimental effort, in order to isolate the thermal component.

\section{c. Lifetime}

The yuark-gluon plasma is expected to exist for a very short time. Its lifetime will depend on the dynamics of expansion and on energy transfer. A possible method to determine the plasma iffetime would consist of the masurement of the antal jield of photons, leptons, and strange particles. The number of photons and leptons produced is related to the plasma size, temperature, and lifetime. If the size and temperature are deterained independently, it may be possible to dedice the lifetime of the plasma. A similar thod could be applied to the abundance of strangeness. It has been shown's that the time constant for the strange quarks to reach an equilibrium concentration is comparable to the lifet ime of the quark-gluon plasma. Therefore, the lifetine can be derived from the ratio of the number of strange quarks to the number of baryons among the products.

\section{ACCELERATOR FACILITY}

The accelerator facility proposed here provides a means of accelerating hadvy nuclei, with masses up to at least 200 atomic mass units (amu). to kinet ic eneryies of at least in GeV per anu (sev/"i) and hringing two counterrotsting heams of such nuclei into collision.
The resulting reaction energy avaflable in the center-of-mass system corresponds to that produced by a beam of $255.6 \mathrm{GeV} / \mathrm{u}$ colliding with a stationary target. It is belfeved such centerof - ass energies and masses of collision partners are mell in excess of the minim values needed to produce a quark-gluon plasim.

The nuclei in each bean will thus have total energies up to at least 11.7 times their rest ass equivalent energy. The facilfty can easily vary the final kinetic energy of each bean dom to a lower value of only haif of a GeV/u. The facility can also accelerate a different type of mucleus in each of the two collider rings. A large nuber of nuclef, with masses spanning the entire periodic table, can be produced by the ion source and accelerated. It should thus be possible to vary the collision conditions sufficiently to produce systems wich both do and do not exhibit the expected transition from hadronic atter to the quark-gluon plasma state.

Several collision points, or intersection regions, will be provided about the collider circuaference so that up to four or possibly five experiments can proceed simultaneously. Although each group of experimenters aust, of necessity, observe collisions between the same muclei at the same energy, provisions will be ade to tune the properties of each intersection region, such as luminosity and crossing angle, to the needs of specific groups and to integrate the experimental apparatus of each group into the structure of the collider.

The three accelerators required are arranged in a cascade with those preceding the collider rings serving to provide bunched beaws of fully ionized heavy ions at sufficiently high energy for injection into the collider rings. In order, the facility of 11 include an ion source to produce negative heavy fons, a tandem electrostatic accelerator to provide initial acceleration and first electron stripping of the heavy tons, a small booster synchrotron to bunch the bean and produce ions mich my subsequently be completely fonized, and a pair of intersection accelerator co'lider rings to boost the ions to their final relativistic energies and bring them into collision. The collider will include additional large halls at the intersection regions to house experimental apparatus. Transfer lines connecting the various accelerators and a cantral control area complete the factlity.

The generiz advantages of a tandem accelerator as an injector in a miti-component system for the acceleration of heavy lons have been widely recognized. Specifically, they include:

(1) High beam quality (low emittance and energy dispersion, high energy and intensity).

(2) flexibility (easily changed beam energy, species, intensity, and time structure).

(3) Long source lifet ine and generally stable operation.

(4) Simplicity (bean extraction, time modulation, and contral).

Operation of the HHIRF tandem accelerator (see Fig. 10.3) as an injector for the booster synchrotron requires acceleration of high intensity, low frequency beam pulses. Typical parameters used for the collider deston calculations are: peak injected current, 200 uA; pulse 


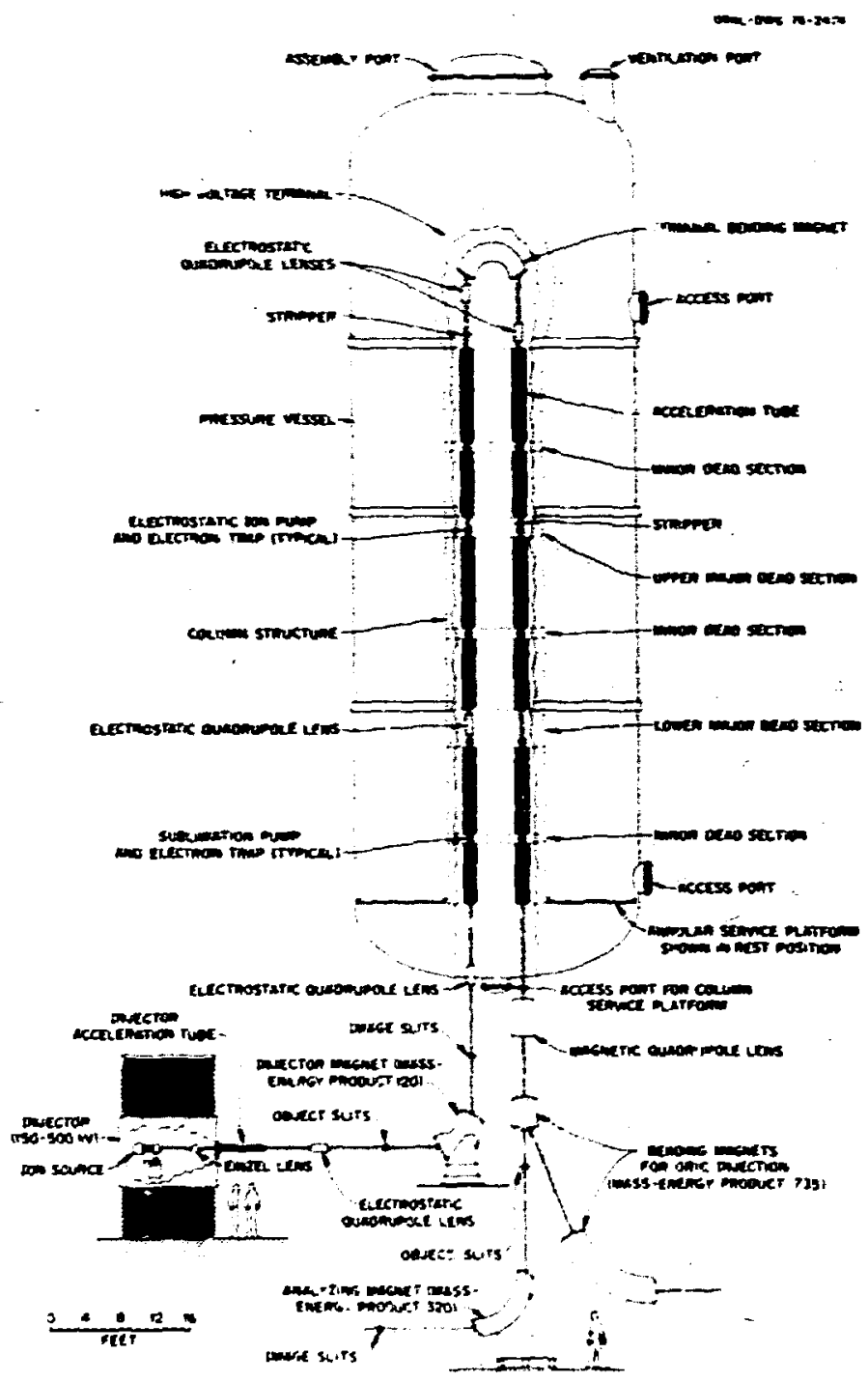

Fig. 10.3. HIRF tandew electrostatic accelerator.

duration, 100 usec: repetition rate, $1 \mathrm{~Hz}$. Operation in this mode is ade possible by the recent developiment of hi gh-current, negat ive heavy-tion sources 20,21 and by the relatively large terainal capacitance of large tandea accelerators which allows acceleration of intense bean pulses without significant terminal voltage decrease.

The HIRF tander accelerator is well suited to operation in this mode for several reasons:

(1) Use of quadrupole lens in che low-energy acceleration tube increases the acceptance of the accelerator by a factor of about two to a value of $16 \pi \mathrm{mm}$ mrad iteyl/2 at a terminal potential of 22 MW.22 This may be important since high-current, pulsed soirces may have higher emitcance values than lowcurrent sources. [Hote, however, that the estimated eniteance for o 7Ux beam fraction for the Middieton Mark VII axtal geometry surface fontzation source is $1.6 \mathrm{~m} \mathrm{~m} \cdot \mathrm{mrad}$ Mevitiz (Ref., 21).]

(2) Use of a $180^{\circ}$ terminal mognet allows complete terminal charge-state separation.
Thus, unused post-terminal charge states do not contribute to terminal voltage decrease.

(3) The large size of the terminal leads to a large terminal capacitance, $300 \mathrm{pf}$, wich also reduces terminal voltage decrease. As an example, we consider acceleration of 197 Aul7t with an injected bean pulse of 200 uA for duration of 100 usec. Assuming a $15 \%$ charge state fraction, the total charge perturbation will be

$$
\begin{gathered}
(200+0.15 \times 17 \times 200) \times 10^{-6} \frac{\text { Coulond }}{\text { sec }} \\
\times 10^{-4} \mathrm{sec}=7 \times 10^{-8} \text { coulonb. }
\end{gathered}
$$

With cerminal caparitance of 300 pf. Ints leads to a terminal woltage decrease of

$$
\Delta V=\frac{7 \times 10^{-8} \text { coulono }}{300 \times 10^{-12} \mathrm{~F}}=240 \mathrm{~V} \text {. }
$$

- value mich will resule in a beam margy change, $\triangle E / E$, of the order of $10^{-5}$ at both the 
terminal and after acceleration through the entire accelerstor. This is a negl igible additional energy spread for injection into the booster synchrotron.

An iaportant capability of tandem electrcstatic accelerators as injectors for heavy-ion colliders is their ability to accelerate any species of negative ion for wich a source may be developed. Table 10.1 lists several ions for wich high dc current source operation has been dewonstrated.20 "Iso listed is the cube root of the wss muber of the given nucleus. Since this is proportional to the nuclear radius, and since the probability of attaining conditions for producing a quark-gluon plasm also depends on nuclear radius, it is critical to have available a large range of nuclei of differing radi $i$ which can be accelerated by the tandem. It is seen fro the table that this condition has been met in the ion source development al ready performed.

The booster synchrotron will be a siall conventiona? machine, of circumference $144 \mathrm{~m}$, employing warm iron magets and a pair of ferrite-tuned of cavities to accelerate all nuclei up to mass of 200 and to kinetic energies of $500 \mathrm{HeV} / \mathrm{u}$. At such kinetic energies, all electrons can be removed from greater than $80 x$ of the nuclei in a beam of gold ions (mass $2 y /$; by passage through a thin stripping foil. Such a beas can then be accelerated and stored by the collider rings. For lighter ions the stripping efficiensy improves at $500 \mathrm{MeV} / \mathrm{u}$ compared to that for gold, reaching neariy $100 \%$ for ions of 100 and and lighter.

Table 10.1. Ions available from negative ion source

\begin{tabular}{|c|c|c|}
\hline Ion & 2 & $\mathrm{~A}^{1 / 3}$ \\
\hline H & 1 & 1 \\
\hline$c$ & 6 & 2.29 \\
\hline 0 & 8 & 2.52 \\
\hline$S$ & 16 & 3.17 \\
\hline $\mathrm{Cl}$ & 17 & 3.27 \\
\hline $\mathrm{Ni}$ & 28 & 3.87 \\
\hline $\mathrm{Cu}$ & 29 & 3.98 \\
\hline Se & 34 & 4.34 \\
\hline $8 r$ & 35 & 4.33 \\
\hline Ag & 47 & 4.78 \\
\hline I & 53 & 5.03 \\
\hline$r_{b}$ & נון & 5.54 \\
\hline$P_{t}$ & 73 & 5.79 \\
\hline$A_{u}$ & 79 & 5.82 \\
\hline
\end{tabular}

As the largest ion kinetic energies are required for the heaviest ions to rewove all electrons by passage through a stripping foil. the necessary size of the booster is determined by the charge state of 197 hu ions (taken as the representat ive ass -200 bean in this report) after they pass through a thin carbon foil at the exit of the tandem accelerator. For a tandew terminal of $22 \mathrm{M},{ }^{193} \mathrm{Au}$ ions will strip to charge state $17^{+}$at the stripping foil inside the tandem terminal and exit the tandew at 396 MeV, or $2.01 \mathrm{MeV} / \mathrm{u}$. Gold ions at this energy strip to a most probable charge state of $44^{7}$, meaning a manetic rigidity $\mathrm{B}_{p}=16 \mathrm{~T} \cdot \mathrm{m}$ for the booster synchrotron will correspond to an exit energy of $488 \mathrm{MeV} / \mathrm{u}$ for $197 \mathrm{Au}^{46+}+$ ions.

The tandem beans will be transferred, after stripping at the exit, to the booster via an achronatic transfer line constructed of FODO cells with bending distributed symietrically about points $180^{\circ}$ apart in betatren phase. The required gnet strencihs are quite low due to the seall (CI Ta) rigitities of the tandem beams. A combination of gnetic septum, electrostat ic deflector, and collapsing orbit buap will be used to inject the bean into the booster ring.

A simple F000 cell lattice has been chusen for the booster, consisting of 18 cells, of which 12 incorporate two 2.6-m-long dipole magnets and of wich the reasining 6 have no dipoles. The resulting free straight sections are used for injection, extraction, and accelpration of the beam. The lattice is arranged with six-fold periodicity as show in Fig. 10.4.
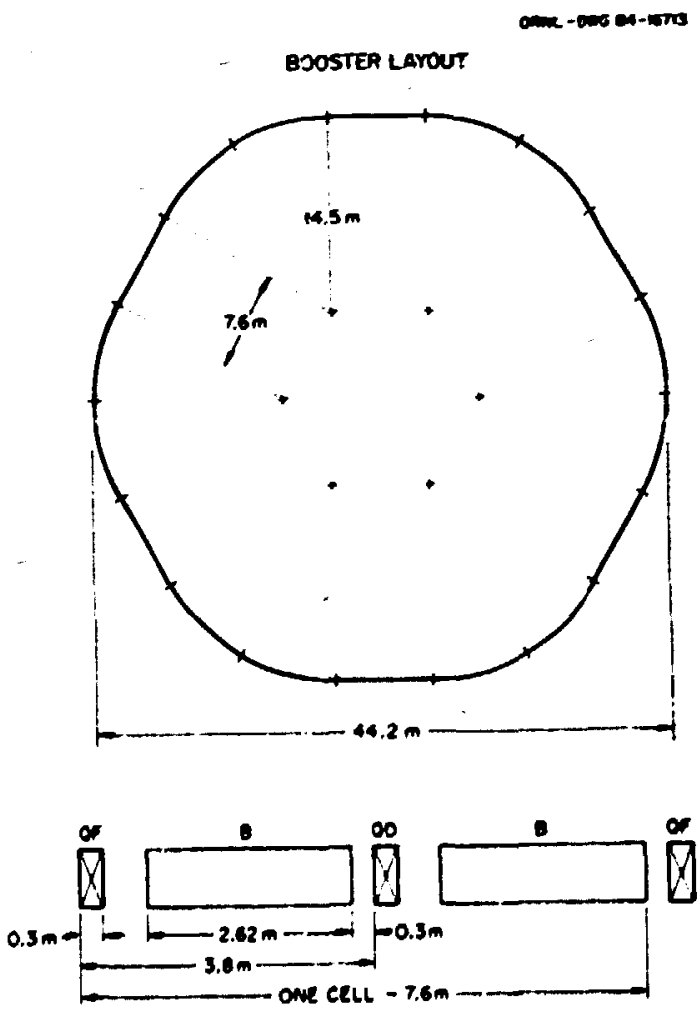

Fiy. 10.4. Booster layout and full cell. 
$A$ layout of one of the full cel's containing dipoles is also shom in Fig. 1).4. The betatron phase advance of each cell is chosen to be $37^{\circ}$, yielding betatron tunes $v_{X} \sim v_{y} \sim 4.3$. Parameters of the booster are given in Table 10.2 .

The betatron acceptance in each plane is estimated to be greater than $40 \pi$ marad wile the eaittance of beans produced by the tanden is measured to be less than 2a m-arad. By stacking in horizontal betatron space using a collapsing orbit buep, it should be possible to inject in excess of ten successive turns; to be conservative, in the following we have assumed ten such turns ay be injected. The injected nuiber of iciss per booster cycle resulting from this assumption is then given in Table 10.3. An instantaneous source current of $200 \mu \mathrm{A}, a$ tanden

Table 10.2. Booster parameters

\begin{tabular}{ll} 
Circumference & $136.8 \mathrm{~m}$ \\
Wumber of cells & 18 \\
Cell lenrth & $7.6 \mathrm{~m}$ \\
Dipole length & $2.62 \mathrm{~m}$ \\
Dipole field & $1.6 \mathrm{~T}$ \\
Quadrupole length & $0.3 \mathrm{~m}$ \\
Quaurupole poletip field & $0.9 \mathrm{~T}$ \\
Phase advance/cell & $87^{\circ}$ \\
Tune, H and V & 4.3 \\
Mmax $/ \beta_{\text {min }}$ & $12.5 \mathrm{~m} / 2.5 \mathrm{~m}$ \\
Tmax $/ \eta_{\text {min }}$ & $2.7 \mathrm{~m} / 0.5 \mathrm{~m}$ \\
Max. Bo & $16 \mathrm{Tm}$ \\
Bend radius & $10 \mathrm{~m}$ \\
\hline
\end{tabular}

terminal potential of $22 \mathrm{w}$, and selection of the optime charge state after carbon foil stripping in the tanden terminal and at the tandew exit have been assumed. An overali transaission efficiency of $75 \%$ is used for the tandea, in accord with operationi i experience. The space-charge limit at injection into the booster for a time depression $\Delta v=0.1$ and a bunching factor $B_{f}=0.5$ is also given; it is seen that in no case is this limit exceeded.

The booster synchrotron will eaploy conventional, room :emperature, iron-dor: ated magnets. These are needed because of the rapid booster cycle $(0.5 \mathrm{~Hz})$ and are acceptable tre to the modest power requirement of the booster.

(Because the booster is needed only for a few minutes to refill the collider every feu hours, it can be "turned off" ietween refills for further power savings.) A description of the standard dipole and quadrupole magnets is given in Table 10.4, and cross sections of each are shom in Fig. 10.5. To eliminate sagitta, the dipoles wili be constructed of $1 / 16^{\prime}$ laminations stacked along an arc of the proper radius. Other agnets included in sall correction packages will, include trim dipoles and quadrupoles, skew quadrupoles, and sextupoles. Orbit bump manets, injection and ejection mgnetic septua magnets, and the ejection kicker complete the set of mgnets required for the booster.

The vacuum requi rements of the booster are ade especially severe by the need to avoid charge-changing reactions of the partly stripped heavy ions with residual gas atoms and molecules in the vacuum chamber. Since these criss sections are of the order of megabarns at injection, pressurec of $10^{-11}$ tor at $20^{\circ} \mathrm{C}$ are required in the vacuum pipe of the booster. These will be obtained by having an all stainless steel vacuum systen capable of withstanding $200^{\circ} \mathrm{C}$ bakeout. All possible joints will be field melded; those wich must be demounted will be made with metal seal ultrahigh vacuum flanges. Metal-seal sector valves will be used to isolate each quadrant of the machine. Properly trapped roughing and turbomolecular pump will be used for evacuating to $10^{-6}$ torr, followed by a combination of high-speed sputter ion pumps and titanium sublimator pumps for attaining the ultrahigh final vacuum of $10^{-1}$ torr. The vacuum chamber will be thermally insulated from the magnet ?aminations to prevent the

Table 10.3. Booster currents

\begin{tabular}{|c|c|c|c|c|c|}
\hline Ion & $\begin{array}{l}\text { Kinetic energy } \\
\text { at tanderm exit } \\
\text { (HeV/u) }\end{array}$ & $\begin{array}{l}\text { Charge stat: } \\
\text { at tandem exit }\end{array}$ & $\begin{array}{l}\text { Current at } \\
\text { tandem exit } \\
\text { (particle } \mu A \text { ) }\end{array}$ & $\begin{array}{l}\text { Number infected } \\
10 \text { eurns } \\
\left(x 10^{9}\right)\end{array}$ & $\begin{array}{c}\text { Space } \\
\text { charge limit } \\
\left(x 10^{9}\right)\end{array}$ \\
\hline${ }^{12} \mathrm{C}$ & 11.0 & $6^{+}$ & 75 & 14.75 & 42.9 \\
\hline 325 & 7.56 & $15^{+}$ & 22.5 & -5.32 & 12.7 \\
\hline${ }^{32} \mathrm{Cu}$ & 4.89 & $24^{*}$ & 9.0 & 2.64 & 6.37 \\
\hline $127 I$ & 2.71 & $35^{+}$ & 5.4 & 2.11 & 3.38 \\
\hline $197 \mathrm{Av}$ & 2.01 & $44^{+}$ & 4.05 & 1.90 & 2.27 \\
\hline
\end{tabular}


Table 10.4. Booster magnets

\begin{tabular}{ll}
\hline & \\
Dipole & \\
Length & $2.0 \mathrm{~m}$ \\
Aperture & $12 \mathrm{~cm}(\mathrm{~h}) \times 5 \mathrm{~cm}(\mathrm{v})$ \\
Good field aperture & $7 \mathrm{~cm}(\mathrm{~h}) \times 5 \mathrm{~cm}(\mathrm{v})$ \\
Max field & $1.57 \mathrm{~T}$ \\
Coil turns/pole & 9 \\
Max current & $3472 \mathrm{\lambda}$ \\
Inductance & $3.2 \mathrm{mH}$ \\
Resistance & $5.1 \mathrm{mQ}$ \\
Peak power & $96.5 \mathrm{kH}$ \\
Height & $2 \mathrm{Tons}$ \\
Munber & 32 \\
& \\
& \\
Quadrupole & \\
Lengtli & \\
Pole contour & $0.3 \mathrm{~m}$ \\
Max gradient & $\times y=10.5 \mathrm{~cm}^{2}$ \\
Pole tip field & $19.4 \mathrm{~T} / \mathrm{m}$ \\
Coil turns/pole & $0.63 \mathrm{~T}$ \\
Max current & 6 \\
Inductance & $3839 \mathrm{~A}$ \\
Resistance & $0.48 \mathrm{mH}$ \\
Peak power & $1.51 \mathrm{mQ}$ \\
Meight & $29.7 \mathrm{kH}$ \\
Number & $0.36 \mathrm{Tons}$ \\
& 48 \\
\hline & \\
\hline &
\end{tabular}

latter's temperature from rising ahove $65^{\circ} \mathrm{C}$ to prevent damage to coil insulation. 'd magnet lamination insulation.

After the beam has been accelerated by the booster and given its final bunching in $\mathrm{rf}$ phase, the ejector kicker is triggered to bring the beam across a magnetic septum and into ar: extraction channel. The beam is yuickly brought to a focus in both planes and passed through a stripping foil (except for very light beams with $A<20$ amu, wich are al ready fully stripped upon injection into the booster). Such a foil must be $\sim 100 \mathrm{mg} / \mathrm{cm}^{2}$ of $\mathrm{Cu}(\sim 0.1 \mathrm{~mm}$ thick) for heavy beams such as $197 \mathrm{Au}$ and correspondingly thinner for less massive beams. The beam is then led through an achromatic transfer line to a splitter and septum magnet wich deflects it into one of the two $90^{\circ}$ bends leading to injection inte the two rings of the collider.

The accelerator-collifider consists of two interleuved, roughly hexagonal shape rings of magnets. The general site layout is shown in Fig. 10.1. The rings are each 864 meters in circumference ( 6 times the booster cicumference) and cross at six equally spaced intersection regions. Each ring has a bending power of $90.535 \mathrm{im}$, suffictent to constrain $197 \mathrm{Au} 79+$ of $k$ inetic energy $10 \mathrm{GeV} / \mathrm{u}$ to ti,e reference orbit. Each ring has ferrite-tuned rf cavities to accelerate the heans from the injection energy of $48 \mathrm{~S} \mathrm{MeV} / \mathrm{u}$ to any desired final energy up to 11) GeV/u, which corresponds to a maximum revolution frequency change of $32 \%$. The ring circumferences are divided roughly equally into six bending arcs, consisting of simple fuoo cells, and six lang straight sections containing
BOOSTER DIPOLE CAOSS-SECTION

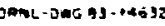

B.man = 26.310

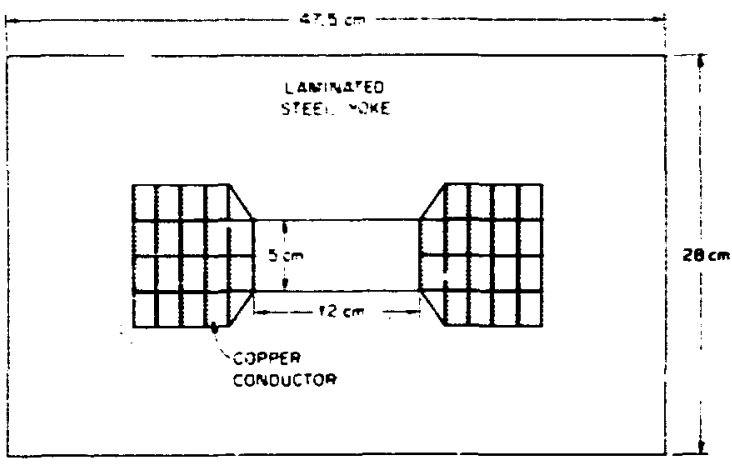

BOOSTER OUI JRUPOLE CROSS-SECTION

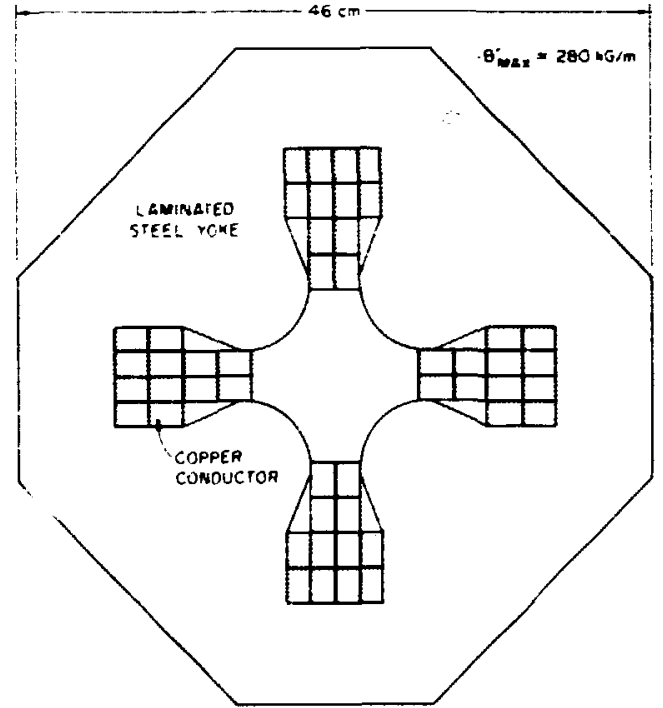

Fig. 10.5. Booster dipole and yuadrupole cross sections.

necessary dispersion suppressors, matching quadrupoles, low beta insertions, and beam merging and reseparation inagnets. The optical elements for the long straight sections must be designed to permit varying the crossing angle of the beams and thus the length of the luminous region and to permit varying the luminosit/ from one intersection region to another to accomodate different experimental requifements. A spectal butlding for experimental apparatus will surround four of the intersection regions, with two factlities for large detector arrays, one for wide-angle spectrometers and one for narrowangle spectrometers. A fifth intersection region will be provided with an open pad beneath the collider ririgs for future development, and the sixth will have a somewhat enlarged tunnel to concentrats machine functions such as injection, ejection, rf cavities, and spectal equipment. A list of parameters is in Table 10.5.

The arcs are made up of six FO00 cells each, $12 \mathrm{~m}$ in length. A phase advance near $90^{\circ}$ per cell has been chosen, simplifying certain aspects of injection and extraction component 
Table 10.5. wilider parameters

\begin{tabular}{ll}
$\begin{array}{l}\text { Circumference } \\
(6 \times \text { booster) }\end{array}$ & $964 \mathrm{~m}$ \\
Mumber, length of arcs & $6,72 \mathrm{~m}$ \\
Mumber of cells/arc & 6 \\
Length of cell & $12 \mathrm{~m}$ \\
Phase advance/cell & $92^{\circ}$ \\
Dipole length & $4 \mathrm{~m}$ \\
Dipole field & $2 \mathrm{~T}$ \\
Quadrupole iength & $0.8 \mathrm{~m}$ \\
Quadrupole poletip field & $1.2 \mathrm{~T}$ \\
Bax in arcs & $20 \mathrm{~m}$ \\
Mumber, length of IR's & $6,72 \mathrm{~m}$ \\
Free space at crossing & $15 \mathrm{~m}$ \\
Bax at crossing & $63 \mathrm{~m}$ \\
nat crossing & $0 \mathrm{~m}$ \\
Maximum rigidity & $90.53 \mathrm{Tm}$ \\
Bending radius & $45.3 \mathrm{~m}$ \\
& \\
\hline
\end{tabular}

placement and yielding a maximum betatron amplitude of $20 \mathrm{~m}$. The sixth cell in each arc contains the dispersion suporessing elements for the following intersection region. A matched cell with no bending follows this, followed by the matching and low beta sections leading to the beam-merging magnets and the crossing point. A layout of one sextant of the ring lattice is shown in Fig. 10.6, which al so shows the layout of one full regular cell.

Because of the large normalized emittance ( $\sim 9 n \mathrm{~mm} m \mathrm{mad}$ ) for light-ion beans just after injection into the booster and because of the need to have large emittances for heavy-ion beams in order to limit the emittance growth rates due to intrabeam scattering, the collider rings are designed to accomnodate beams of $10 \mathrm{n}$ mm・mrad normalized enittance. Allowing $\pm 5 \mathrm{~mm}$ for closed orbit distortion leads to a minimum aperture requirement of $4 \mathrm{~cm}$ good field, or a coft 10 of $6 \mathrm{~cm}$ assuming good field extends to $2 / 3$ of the coil I0. The magnets planned for the collider are of the type knuwi genertically as "super ferric" magnets, f.e.., iron-dominated whdow frame magnets with supercondurting coils. The dipoles are plannes to be 4 meters long and to have a very conservative ffeld of $2 T$. The magnets will be constructed with a curvature of $45 \mathrm{~m}$ to remove the $4.4 \mathrm{~cm}$ sagitta that would result otherwise in straight dipole magnets. The small aperture required and high-current density of present superconductor will result in a compact yoke design. It is therefore planned to assemble two dipoles in a common yoke. Quadrupoles will be kept separate from one another to allow independent positioning of the quadrupoles in the two rings. Development work will also concentrate on mounting dipoles, quadrupoles, correction packages, and beam pickup elements for one-half cell into a common cryostat. This will simplify transfer of heltum between ring elements and minimize the number of cold to warm joints needed for power and control leads.
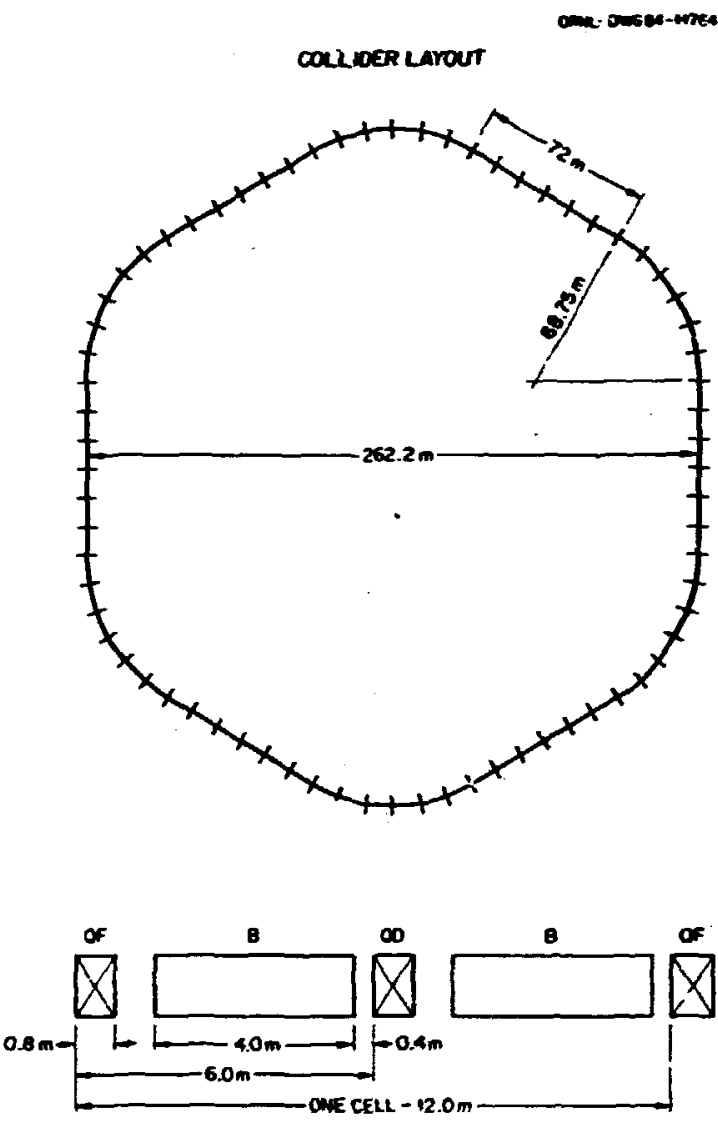

Fig. 10.6. Collider layout and full cell.

In order to provide for short bunches for collider operation, the of system in the collider will run at a hamonic of that in the booster. Given the ratio of circumferences between booster and collifoer $(1: 6)$, matching of frequencies at transfer $(1.572 \mathrm{MHz})$ results in a harmonic $E$ system in the collider. Since this corresponds to a 636-ns separatior, between bunches and since injection kickers can be made to operate much faster, 100 ns for a 500-6 field, a higher harmonic choice is favored. Present plans call for an $h=144$ system operating between 37.73 at injection $(\beta=0.7549)$ and $49.78 \mathrm{wHz}$ at top energy $(\beta=0.9964)$. With a 106-ns injection kicker, every fourth $r f$ bucket would be filled in the system, resulting in 36 bunches distributed about the circumference of each ring. Thus 72 booster cycles, or 144 seconds, would be required to reriil both collider rings. Ferrite-tuned single-gap cavities will be provided in each ring for accelerating and maintaining the length of each beam Dunch. An acceleration cycle of one minute to full energy is planned. followed by storage for several hours in colltder mode.

The colltder magnets will be of the cold iron. cold bore type. This conserves space instide the cotl for beam ptpe, compared to warm bore designs due to the lack of extra insulation. The vacuum attained in such magnets is $10^{-11}$ torr at $4^{\circ} \mathrm{K}$ (based on FNAL experience). 
This is adequate to provide for bean survival times of more than one day for the process of electron capture by the beam ions from residual gas ionized by the beam. However, the bean gas background at experiments will be rather high at such pressures, so transitions to a warm bore, baked stainless steel vacuum system are planned for the intersection regions of the machine. The components of this part of the vacuum system will be similar to those used in the booster ring.

Projected performance of the facility for five representative heavy ions is given in Table 10.6. Listed therein are maximum energy, number of stored ions, and maximum initial luminosity for head-on collisions in the intersection rejions. Preliminary estimates of luminosity iifet ime due to intrabean scattering indicate that for gold beams the luminosity will decay to 25\% of this value after 2 hours; for light beams the projested decay times for luminosity are in excess of one day. The principal loss mechanism for light beams (carbon, sulfur, copper) will be nuclear cross sections which are of the order of 1-5 barns. For very heavy beams such as gold, the cross sections for electromagnetic Coulomb disintegration and $K$-shell pair production are the dominant loss modes. They increase with collision energy and total more than 50 barns at $10 \mathrm{GeV} / \mathrm{u} \times 10 \mathrm{GeV} / \mathrm{u}$.

\section{EXPERIMENTAL FACILITIES AMD SUPPORT BUILOINGS}

The present design of the collider ring accomodates six straight sections, one of wich will be used for bean injection and $r f$ systems. The remaining straight sections will provide for up to five intersection regions (IR). Four of the IR will be equipped with shielded experimental halls, wile the fifth one will be left as a large open pad. This open area is reserved for second generation detectors whose space requirements cannot be foreseen at present. The physical dimensions of the five experimental areas are given in Table 10.7, while Figs. 10.7 and 10.8 show plan and elevation views of the large hall; Fig. 10.1 shows their location on the ring.

A brief survey of existing large collider detectors shows their dimensions are about $11 \mathrm{~m}$ long by $8 \mathrm{~m}$ high by $8 \mathrm{~m}$ wide. Such detectors are easily accomodated between the last insertion quadrupoles, as there will be 15-m free space along the beams center $2 d$ at the intersection point. Furtreinol ?, to allow for forwardangle instrumentation, the accelerator tunnel will be enlarged for a considerable distance about the intersection point so that counters can be placed at smail angles to the colliding beams. To achieve a high luminosity, it is necessary to use near the detectors special low beta insertion quadrupole magnets other than those used in the standard lattice. If the magnetic field in the detectors is not collinear with the beams, special compensating dipoles will be ri:eded to avoid beam-optics disturbances. The beam tube in the center of the IR will be of thin-wall beryllium to reduce interaction with the scattered particles, notably conversion of electrons and photons. A vacuum of $10^{-11}$ torr at $20^{\circ} \mathrm{C}$ will be mintained in the IR.

The experimental halls will be equipped with standard laboratory facilities such as clean and general power. lighting. climate control, communications, cooling water, gas handling equipment, fire al arms and sprinkler systems, radiation monitors, and drainage. If roon temperature magnets are used for the detectors, nne or two megawatts of power may be needed. But if superconduct ing magnets are used, little power will be needed to energize the magnets. However, liqiid helium refrigeration equipment will then be needed to cool the magnets to $4^{\circ} \mathrm{K}$. Up to several hundred kilowatts of power will be provided to power the large number of detector modules and the associated electronics that comprise a typical detector system to be housed in the intersection region halls.

Provisions will be made for a large staging area adjacent to each of the two large experimental halls, to allow for the assembly, testing, modification, and repair of the detector systems. The staging areas will have the same elevation as the experimental halls. Loading docks and service roads provide for the access and transport of equipment to and from these areas. Each area will also have laboratory space to handle work on smaller detectors, office space for experimentalists, and a large counting room adjacent for readout electronics and computers. Signals corresponding to machine

Table 10.6. Collider performance

\begin{tabular}{|c|c|c|c|c|}
\hline Ion & $\pi \mathrm{enm}_{\mathrm{Nr}}^{\mathrm{E}}$ & Ions/Bunch & $\begin{array}{l}T / A_{\max } \\
(\mathrm{GeV} / \mathrm{u})\end{array}$ & $\begin{array}{c}L_{0} \quad \text { (head-on) } \\
\beta^{\star}=3 m \\
\left(\mathrm{~cm}^{-2} \sec ^{-1}\right)\end{array}$ \\
\hline${ }^{12} \mathrm{C}$ & 10 & $1.110^{10}$ & 12.7 & $3.610^{28}$ \\
\hline $32 s$ & 10 & $4.010^{9}$ & 12.7 & $4.610^{27}$ \\
\hline${ }^{63} \mathrm{Cu}$ & 10 & $2.010^{9}$ & 11.6 & $1.1 \quad 10^{27}$ \\
\hline $127 !$ & 10 & $1.610^{9}$ & 10.4 & $5.010^{26}$ \\
\hline $137 \mathrm{Au}$ & 10 & $1.2 \quad 10^{9}$ & 10.10 & $3.310^{26}$ \\
\hline
\end{tabular}


Table 10.7. Locations, dimensions ( $)$, and crane capacities (tons) of the experimental areas

\begin{tabular}{|c|c|c|c|c|c|c|}
\hline Area & Location & Length & Midth & $\begin{array}{l}\text { beam } \\
\text { height }\end{array}$ & $\begin{array}{l}\text { Ceiling } \\
\text { height }\end{array}$ & $\begin{array}{l}\text { Crane } \\
\text { capacity }\end{array}$ \\
\hline Large $i 1$ and 2 & 1811 o'clock & 14 & 12 & 5 & 10 & - \\
\hline Fomard bldg. (2) & & 3 & 5 & 3 & 6 & - \\
\hline Staging area & & 20 & 20 & 5 & 15 & 40 \\
\hline Narrow-angle & 3 o'clock & 10 & 12 & 2 & 7 & 10 \\
\hline Forward stubs (2) & $\cdot$ & 25 & 12 & 2 & 7 & 10 \\
\hline $\begin{array}{l}\text { Open pad } \\
\text { (future use) }\end{array}$ & 5 o'clock & 20 & 15 & 3 & - & - \\
\hline Nide-angle & 7 o' clock & 15 & 30 & 4 & 10 & 20 \\
\hline Injection hall & $90^{\prime}$ clock & 72 & 6 & 1.2 & 3 & - \\
\hline
\end{tabular}

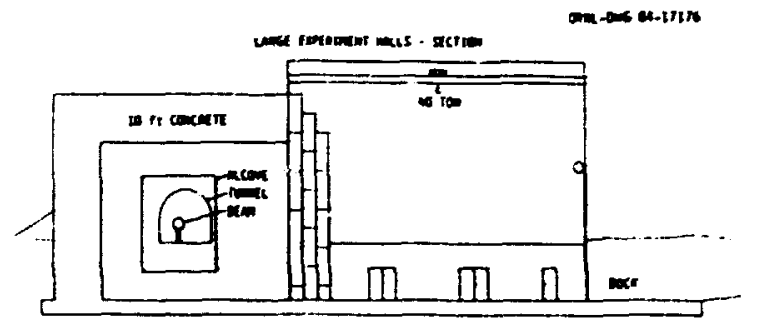

Fig. 10.7. Larye factlity hall - section view.

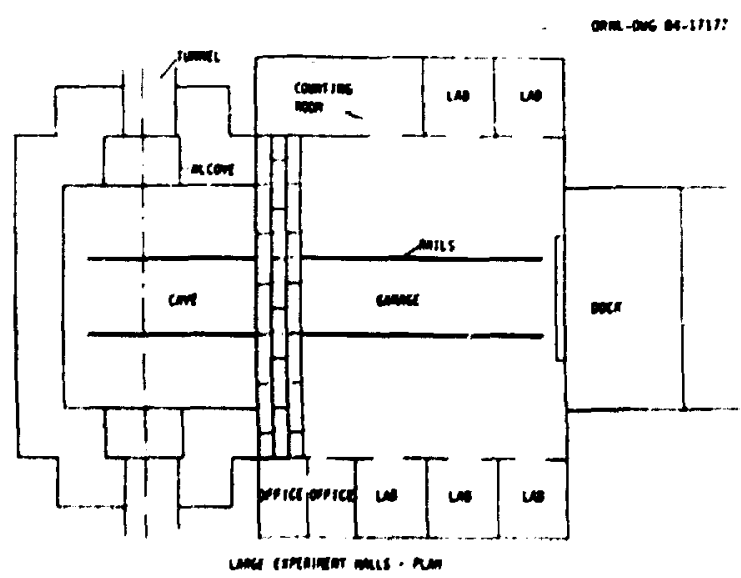

Fig. 10.8. Large facflity hall - plan view. parameters such as bean crossing time, beam position and profile, luminosity, vacuum, radiation level, and status (filling, rampling, colliding, and dumping) will be provided for each counting area.

lt is envisioned that the $4 \pi$ detectors will be assembled on raflroad-type carriages in the staging area, and rolled into position on rails. This will minimize the shielding and crane-coverage requirements. The shtelding between the hall and staging area will be easily movable. In this scheme, the detector removal from and installation on the beam itne will take a short time and have minimal impact on the accelerator operation. The total weight of a typical 4x detector may be a few hundred tons, and some parts may weigh as much as 30 tons. Therefore, a crane of appropriate stze will be installed in the staging area. The average floor loading for a completely assembled detector will be approximately 10 tons $/ \mathrm{m}^{2}$.

1. Computing and Telecomunications Division.

2. Instrumentation and Controls Division.

3. Oak Ridge Assoctated Unt versittes, Oak Ridge, Tennessee.

4. For a revtew, see J. Kogut, Hucl. Phys. A418, 381C (1984).

5. G. Baym, Nucl. Phys. A418, 433c (1984).

6. J. Engels et al., Mucl. Phys, B205, 545 (1982); $i$. Montvay and $E$. Piezarinen, Phys. Lett. 1108,148 (1982). 
7. J. Kogut et al.. Phys. Rev. Lett. 48, 1140 (1982).

8. J. Engels, F. Karsh, and H. Satz, Phys. Lett. 1138, 398 (1982).

9. D. Schrama and $x$. Olive, hucl. Phys. 1418, 289c (1994).

10. M. Busza and A. S. Coldhaber, Phys. Lett. 1393, 235 (1984).

11. H. Gyulassy, Proceedings of the Bielefeld Morkshop, World '́cientific, 1982, p. 81.

12. J. D. Bjorken, Phys. Rev, D 27, 140 (1983).

13. C. Y. Wong. Phys, Rev, O (in press).

14. R. Anishetty, P. Koehler, and L.

Mclerran. Phys. Rev. 0 22, 2793 (1980).

15. K. Kajantie and $H$. I. Miettinen, Z. Phys.

C14, 357 (1982); J. Badier et al., Proc.

P:elefeld Workshop, World Scientific (1982). p. 463.
16. E. Y. Shuryak and 0. Y. Zhirov, Phys. Lett. 898, 253 (1980).

17. J. Rafel ski, Rucl. Phys. A418, 215c (1984)

18. M. Gyulassy, S. X. Kauffimann, and L. W. Wilson. Phys. Rev. C 20, 2267 (1979) and references therein; $\boldsymbol{H}$. Boggid and I. Otterlund, Proc. Bielefeld Workshop, Horld Scient ific (1982), p. 485 and references therein.

19. M. A. Zjac et al... Proc. 5th High Energy Heavy Ion Sumer Study, LBL-12652.

20. G. D. Alton and G. C. Blazey, Mucl. Instrun. Methods 166, 105 (1979).

21. R. Middleton, Mucl. Instrum. Methods Phys. Res. 214, 139 (1983).

72. J. D. Larson and C. M. Jones, Mucl. Instrum. Wethods 149, 489 (1977). 


\title{
11. PUBLICATIONS
}

\author{
List Prepared by Shirley J. Ball
}

The following list of publications includes primarily those articles by Physics Division staff mers and associates mich appeared in print from October 1983 through Septenber 1984. Articles pending publication as of Septemer 30, 1984, are listed inmediately following this section.

\section{BOOR, JOURIM, NO PROCEEOIMSS MRICLES}

Abe, K.. T. C. Bacon, J. Ballaa, L. Berny, A. V. Bevan, H. H. Binghan, J. E. Brau, K. Braune, O. Brick, M. M. Bugg, J. Butler, M. Camerón, J. F. Carmll, C. V. Cautis, J. S. Chima, H. O. Cohn, D. C. Colley, 6. T. Condo, S. Dado, R. Diamond, P. J. Dornan, R. Erickson, T. Fieguth, R. C. Field, L. Fortney, B. Franek, M. Fujiwara, R. Gearhart, T. Glanzman, J. J. Goldberg, G. P. Gopal, A. T. Coshaw, E. S. Hafen, V. Hagopian, G. Hali, E. R. Hancock. T. Handler, H. J. Hargis, E. L. Hart, P. Haridas, K. Hasegawa, I. Hayashino, D. Q. Huang, R. I. Hulsizer, S. Isaacson, M. Jobes, G. E. Kalimus, D. P. Kelsey, J. Kent, T. Kitagaki, J. Lannutti, A. Levy. P. W. Lucas, M. Macvermott, H. A. Mann, T. Maruyana, R. Merenyi, R. nilburn, C. Milstene, K. C. Hof feit, J. J. Murray, A. Mapier, S. Hoguchi, F. Uchiai, S. O'Neale, A.P.T. Palounek, I. A. Pless, M. Rabin, P. Rankin. M. J. Robetson, A. H. Rogers, E. Ronat, H. Rudnicka, T. Sato, J. Schneps, S. J. Sewell, J. Shank, A. M. Shapiro. C. K. Sinclair, R. Sugahara, A. Suzuki, K. Takanashi, K. Tamai, S. Tanaka, S. Tether, H. B. Wald, H. O. Walker, M. Midgoff, C. G. Wilkins, S. Molbers, C. A. Moods, Y. Wu, A. Yamaguchi, R. K. Yamanoto, S. Yamashita, G. Yekutieli, Y. Yoshimura, G. P. Yost, and H. Yuta

"Charm Photoproduction at $20 \mathrm{GeV}, "$ Phys. Rev. 0 30, 1-21 (1984)

Abe, K., T. C. Bacon, J. Ballam, A. V. Bevan, H. H. Bingham, J. E. Brau, K. Braune, D. Brick, H. M. Bugg, J. Butler, W. Cameron, J. T. Carroll, C. V. Cautis, J. S. Chima, H. U. Conn, D. C. Colley, G. T. Condo, S. Dado, R. Diamond, R. Erickson, T. Fieguth, R. C. Field, B. Franek, N. Fujiwara, K. Furuno, R. Gearhart, D. Gershoni, J. J. Goldberg, G. P. Gopal, A. T. Goshaw, E. S. Hafen, G. Hall, E. R. Hancoci, T. Handler, H. J. Hargis, P. Haridas, E. L. Hart, K. Hasegawa, T. Hayashino, I. Hideta, R. I. Hulsizi:r, M. Jobes, G. E. Kalmus, D. P. Kelsey, J. Kent, T. Kitagaki, A. Levy, P. H. Lucas, W. A. Mann, R. Merenyi, R. Milburn, C. Milstene, K. C. Moffeit, J. J. Murray, A. Napier, S. Moguchi, F. Ochiai, S. O'Neale, Y. Ontani, A.P.T. Palounek, I. A. Pless, P. Rankin, A. H. Rogers, E. Ronat, H. Rudnicka, H. Sagowa, T. Sato, J. Schneps, J. Shank, A. M. Shapiro, R. Sugahara, A. Suzuki, K. Takahashi, K. Tamai, S. Tanaka, S. Tether, H. O. Malker, M. Hidgoff, C. G. Wilkins, S. Wolbers, C. A. Woods, A. Yamaguchi, R. K. Yamamoto, S. Yamashita, Y. Yoshimura, G. P. Yost, and H. Yuta

"Inclusive Photoproduction of Neuiral Strange Particles at $20 \mathrm{GeV}, "$ Phys. Rev. 0 29, 1877-87 (1984)

Abe, K., T. C. Bacon, J. Ballam, A. '. Bevan, H. H. Bingham, J. E. Brau, K. Braune, D. Brick, H. M. Bugg, J. M. Butler, H. Cameron, H. O. Cohn, D. C. Colley, S. Dado, R. Diamond, P. Dingus, R. Erickson, R. C. Field, B. Franek, N. Fujiwara, R. Gearhart, T. Glanzman, J. J. Goldberg, A. T. Goshaw, G. Hall. E. R. Hancock, T. Handler, H. J. Margis, E. L. Hart, K. Hasegawa, R. I. Hulsizer, M. Jobes, G. E. Ka lmus, U. P. Kelsey, T. Kitagaki, W. Kowald, A. Levy, P. W. Lucas, W. A. Mann, E. McCrory, R. Merenyi, R. Milburn, C. Mi Istene, K. C. Moffeit, J. J. Murray, A. Mapier, S. Noguchi, F. Ochiai, S. O'Meale. A.P.T. Palounek, I. A. Pless, P. Rankin. H. J. Robertson, H. Sagawa, T. Sato, J. Schneps, F. J. Seweil, J. Shank, A. M. Shapiro, R. Sugahara, A. Suzuki, K. Takahashi, K. Tamai, S. Tanaka, S. Tether, W. D. Walker, M. Widgotf, C. G. Milkins, S. Holbers, C. A. Moods, A. Yamaguchi, R. K. Yamanoto, S. Yamashita, Y. Yoshimura, G. P. Yost, and H. Yuta

"Study of the $p^{\prime}(1,00)$ Mass Region Using yp $+\pi^{+} \pi^{*} p$ at $20 \mathrm{GeV}, "$ Phys. Rev. Lett. 53, $751-754$ (1984)

Abe, X., T. C. Bacon, H, H. Bingham, J. E. Brau, X. Braune, D. Brick, H. M. Bugg, J. M. Butler, H. Cameron, H. O. Cohn, D. C. Colley, S. Dado, P. Dingus, R. Erickson, R. C. Field, B. Franek, R. Gearhart, T. Glanzman, I. M. Godfrey, G. Hall, E. R. Hancock, H, J. Hargis, E. L. Hart, M. J. Harwin, K. Hasegawa, R. I. Hulsizer, M. Jobes, T. Lafka, G. E, Kalmus, D, P. Kelsey, T. Kitagaki, A, Levy, R. Merenyi, J. J. Murray, V. O'Vell, I. A. Pless, P. Rankin, E. Ronat, H. Sagawa, S. J. Sewell, J. Shank, J. Shimony, K. Tamai, S. Tanaka, U. A. Waide, M. Widgnff, S. Holbers, C. A. Woods, A. Yamaguchi, R. K. Yamamoto, G. P. Yost, and H. Yuta

"Search for a Threshold Enhancement in the yp $\rightarrow$ Charmed Baryon + Charmed Meson Cross Section," Phys. Rev. O 30, 694-96 (1984) 
Alton, G. D.

"Ionization Phenomena and Sources of Ions," pp. 44-178 in Applied Atoaic Collision Physics, Yol. 4: Condensed Hatter, Acadewic Press, Orlando, Florida, 1983

Alton, G. D. (Invited Paper)

"Lonization Phenomena and Sources of Megative Ions," p. 85 in Vol. I, Proceedings, International Ion Beam Engineering Congress, Kyoto, Japan, September 12-16, 1983

Andersen, L. H., H. Frost, P. Hvelplund, H. Znudsen, and S. Datz "Correlated Two-Electron Effects in Highly Charged Ion-Atom Collisions: Transfer Ionization and Transfer Excitation in 20-HeV Aul5t+ He Collisions," Phys. Rev. Lett. 52, $518-21$ (1984)

Arve, P., Y. S. Chen, and G. A. Leander "Hicroscopic Calculations of Nuclear Mobbling Rotation," Proceedings, Hordic Symposium on Muclear Physics, Fuglso, Denmark, August 16-20, 1982, Phys. Scr. T5, 157-ól (1983)

Atkins, W. H. "Oata Acquisition Software for the Holifield Heavy Ion Research Facility," Proceedings, Conference on Real-Time Computer Applications in Particle and Huclear Physics, Berkeley, California, May 16-19, 1983, IEEE Tráns. Muci. Sci. US-30, 3797-801 (1983)

Auble, R. L. "Muclear Data Sileets for $A=198, "$ Mucl. Data Sheets 40, 301-83 (1984)

Auble, R. L., Edicor "Holifield Heavy Ion Research Facility Kewsletter," Issue Ho. 25 (1984)

Auble, R. L., J. B. Ball, F. E. Bertrand, C. B. Fulmer, D. C. Hensley, I. Y. Lee, R. L. Robinson, P. H. Stel son, C. Y. Hong, D. L. Hendrie, H. D. Holmgren, and J. D. Silk -Light-Ion Emission from Reactions Induced by 0.8-2.4 GeV 160 . Projectiles," Phys. Rev. C 28, 1552-64 (1983)

Austin, S. M., G. A. Baym, F. Bsehm, D. A. Bromley, H. H. Chen, D. Cline, K. A. Erb, H. A. Grunder, C. M. Hoffman, A. K. Kerman, M. H. Macfarlane, P. G. Roos, J. P. Schiffer, A. 2. Schwarzschild, I. Sick, and E. W. Vogt "Report of the Panel on Electron Accelerator Facilities," DOE/NSF Nuclear Science Advisory Committee Report, DOE/ER-0164 (April 1983)

Austin, S. M., G. A. Baym, F. Boehm, D. A. Bromley, H. H. Chen, D. Cline, K. A. Erb, H. A. Grunder, C. M. Hoffman, A. K. Kerman, M. H. Macfarlane, P. G. Roos, J. P. Schiffer, A. Z. Schwarzschi!d, I. Sick, and E. W. Vogt "A Long Range Plan for Nuclear Science," DUE/NSF Muclear Science Advisory Committee Report (December 1983)

Awes, T. C., R. L. Ferguson, R. Novotny, F. E. Obenshain, F. Plasil, S. Pontoppidan, V. Rauch, G. R. Young, and $H$. Sann "Energy Division in Damped Reactions," Phys. Rev. Lett. 52, $251-54$ (1984)

Awes, T. C., R. L. Ferguson, R. Wovotny, F. E. Obenshain, F. Plasil, V. Rauch, H. Sann, and G. R. Young (Invited Paper) "Projectile Fission in 58 $\mathrm{Ni}$-Induced Reactions at 15.3 MeV/u," Pp. 101-04 in Proceedings, Workshop on Nuclear Dynamics III, Copper Mountain, Colorado, March 5-9, 1984, Indiana University Muclear Chemistry Report, INC-40007-24 (April 1984)

Ball. J. B.. and R. L. Auble (Invited Paper) "Emission of High-Energy Light Particles from Intermediate-Energy Heavy-Ion Reactions," pp. $359-73$ in Proceedings, Second Indo-U.S. Symposium on Nuclear Physics at Cyclotron and Intermediate Energy, Bombay, India, May 24-28, 1982, Bhabha Atomic Research Centre, Bombay, India, 1983

Barnett, C. F. "Preface," in Applted Atomic Collision Physics, Vol. 2: Controlled Fusion, Academic Press, New York, 1984

Barnett, C, F. "Introduction," pp. 1-25 in Applied Atomic Collision Physics, Vol, 2: Controlled Fusion, Acadenitic Press, New York, 1984

Barnett, C. F. "Particle Plasma Uiagnostics," pp. 249-30s in Applied Atomic Collision Physics, Vol, 2: antrolled Fusion. Academic Press, New York, 1984 
Barnett, C. F., and D. C. Gregory

-adi's Controlled Fusion Atomic Bata Center." 156-59 in Proceedings, Wortshop on Electronic and Ionic Collision Cross Sections Weeded in the Holing of Radiation Interaction with Watter. Argonne, Illinois, December 6-8, 1983, Argonne Mational Loboratory Report, Ml-84-28 (hay Ig94)

Eecker, R. L., A. L. Ford, and J. F. Reading Thitiple-Vacancy Production in the Independent-Fermi-Particle Nodel," Phys. Rev. A 29, 3111-21 (1944)

Cecker, R. L., A. L. Ford, and J. F. lleading

"Rate of Saturation of Target L-Shell Vacancy Probability. M. with Projectile Charge as Given by Coupled-Channels Calculations," Proceedings, Third International Conference on Particle-Induced $x$ - $R$ ay Exission, Heidelberg, Lest Cermany, buly 18-22, 1993, Mucl . Instru. Wethods Phys. Res.. Sect. B, 231 [B3], 43-46 (1994)

Cecker, R. L., A. L. Ford, and J. F. Reading (Invited Paper)

"The Roles of Pauli Correlations, Channel Couplings, and Shake-Off in Ion-Induced Rl and $K^{2}$ LV Multiple-Yacancy Production," Proceedings. Mhird Hortshop on Ianer Suell Ionization by Light Ions, Linz, Austria, lugust 4-5, I983, Mucl. Instrum. Methods Fuys. Res., Sect. 8, 232 [84], 2il1-78 (198)

Beene, J. R., R. L. Nuble, F. E. Sertrand, M. L. Halbert, D. C. Mensley, U. J. Horen, R. L. Robinson, R. O. Sayer, and T. P. Sjoreen (Invited Paper) "Meutron and Gim Decas of Giant Resemances in 201Po Excited by 381 teV 170 lons." pp. 161-78 in Muclear Phrsics with Heavy Ions (Proceedings, International Conference on huclear Physics with Heavy Ions, Stony brook, hew York, Lpril 14-16, 1983), Harnood Acadewic, the Vork, 1994

Bemis, C. E.. Jr., J. R. Beene, J.L.C. Ford, Jr., D. Shapira, and B. Shivakumar (Invited Paper) -Develope it of Optically Puimed Polarized Jet Targets for Use in Heavy-Ion Reaction Studies," Pp. 141-! in Proceedings, Workshop on Polarized Targets in Storage Rings, Arganne, Illinois, May 17-18, 1984, Argonne Mational Leooratory Report, All-85-50 (August 1984)

Berman, B. L., S. Oatz, R. H. Feari.k, R. L. Swent, R. H. Pantell, H. Park, J. O. Kephart, and R. K. Klein

- Planar Channeling Radiation from Relativistic Positrons and Electrons in Lif," Proceedings, 10th International Conference on Atomic Collisions in Solids, Bad Iburg, F.R.G., July 18-22, 1983, MuCl. Instrum. Methods Phys. Res.., Sect. B, 230 [B2], 90-94 (1984)

Bertrand, F. E.

"Giant Resonances - Why Protons?." pp. 181-232 in Proceedings, Conference on Studying Muclei with Medium Energy Protons, Ednonton, Canada, July 11-13, 1983, TRIum Meson Facility Report TRI-83-3 (1983)

Bertrand, F. E., J. R. Beene, and T. P. Sjoreen

"Heavy-Ion Excitation and Photon Decay of Giant Resonances," Proceedings, International Symposium on Highiy Excited States and Huclear Structure, Orsay, France, September 5-8, 1983, J. Phys. (Paris) Colloq. 45, Cf-99-114 (1984)

Blecher, M., K. Gotow, R. L. Burman, M. V. Hynes, M. J. Leitch, M. S. Chant, L. Rees, P. G. Roos, F. E. Bertrand, E. E. Gross, F. E. Denshain, T. P. Sjoreen, G. S. Blarpied, B. K. Preedom, and B. G. Ritchie "Isospin Effects in $*$ Elastic Scattering from ${ }^{12} \mathrm{C},{ }^{13} \mathrm{C}$, and ${ }^{14} \mathrm{C}$ at 65 and 80 MeV," Phys. Rev. $C 28$, 2033-41 (1983)

Bottcher, C. (Invited Paper)

"Humerical Solution of the Few-Bory Schrodingur Equation," DD. 187-200 in Proceedings, XII:

International Conference on the inysics of Electronic and Atomic Collisions, Berlin, West Germany, July 27-August 2, 1983, Elsevier. Ansterdam, 1984

Bottcher, $C$.

"Mumerical Calculations on Electron-Impact Ionization," pp. 241-66 in Advances in Atomic and Molecular Physics. Vol. 20, Academic Press, Mew York (1984)

Braga, R. A., B. E, Gnade, R. H. Fink, and H. K. Carter "Half-Life of the $h_{9 / 2}$ Shell-Model Intruder-State Isomer 197mAu," Hucl. Phys. A410, $441-44$ (1983)

Braun-Munzinger, P., P. Paul, L. Ricken, J. Stachel, P, H, Zhang, G, R, Young, F, E, Ubenshain, and E. Grosse

"Pion Production in Heavy-Ion Collitstons at $\varepsilon_{1 \text { ab }} / A=3 b$ MeV," Phys. Rev. Lett. 52, $255-58$ (1984) 
Breinig, M., 6. J. Vixon, P. Engar, S. B. Elston, and I. A. Stllin

"First bservation of the Thomas Peak in Contimum Capture: Capture of 4 Atons froa Ch, Molecules by Fast Mr+ Frojectiles," Phys. Rev. Lett. 51, 1251-54 (1983)

Brick, B., H. Rudnicka, A. M. Shapiro, M. Midgoff, R. E. Ansorge, M. M. Keale, D. R. Mard, B. M. Mysan, R. A. Burnstein, H. A. Rubin, E. D. Alyea, Jr., L. Bachan, C.-Y. Chien, P. Lucas, A. Pevsner, J. T. Hober, T. Frank, E. S. Hafen, P. Haridas, D. Huang, R. I. Hulsizer, V. Kistiakousky, P. Lutz. S. H. U. I. A. Pless, T. H. Stoughton, V. Suchorebrow, S. Tether, P. C. Trepagnier, M. von Randow, 1. We, R. R. Yamanoto, F. Grard, J. Hanton, V. Henri, P. Herquet, J. M. Lesceux, R. Windmolders, M. Debock, F. Crijns, H. Xittel, M. Retzger; C. Pols, M. Schouten, R. Van de Halle, H. D. Cohn, F. Carminati, R. Dolfini, S. Matti, R. Diharco, P. F. Jacques, R. Kaleltar, R. J. Plano, P. Stamer, I. L. Watt5, E. B. Brucker. E. L. Roller, S. Taylor, S. Dado, J. Eolderg, G. Alexander, O. Benary. J. Grumbus, R. Heifetz, A. Lev, J. E. Brau, H. H. Dugg, 6. T. Condo, T. Handler, E. L. Hart, A. H. Rogers, Y. Eisenberg, U. Larshon, A. Shapira, T. Ludlan, R. Steiner, and H. Taft

"Search for Long-Lived +2 Hadrons," Phys. Rev. 0 30, 1134-36 (1964)

Brick, D., H. Rudnicka, A. R. Shapiro, R. Midgoff, R. E, Ansorge, M. W. Meale, D. R. Mard, B. A. Whyman, R. A. Burnstein, H. A. Dubin, E. D. Aljea, Jr., L. Bachan, C.-T. Chien, P. Lucas, A. Persner, J. I. Bower, T. Frank, E. S. Hafen, P. Haridas, D. Huang, R. I. Hulsizer, V. Kistiakousky, P. Luti, S. H. U. I. A. Pless, T. B. Stoughton, V. Suchorebrow, S. Tether, P. C. Trepagnier, Y. M, R. R. Tannoto, F. Grard, J. Hanton, V. Henri, P. Herquet, J. H. Lesceux, P. Pilette, R. Mindwiders, F.

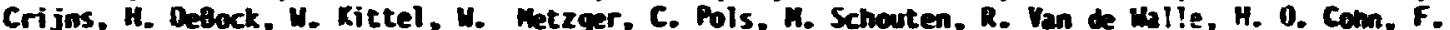

Carminati, R. Dolfini, S. Ratti, R. Dikarco, P. F. dacques, M. Ralelkar, R. J. Plano, P. Staner, T. L. Wak:5, E. B. Brucker, E. L. Xoller, S. Taylor, L. Eerny, S. Oado, J. Ziloberg, S. Toaff, G. Alexander, O. Benary, J. Grunhaus, R. Heifetz, A. Ley, J. E. Erau, M. A. Bugg, G. T. Condo, T. Handler, E. L. Hart, A. H. Rogers, V. Eisenberg, U. Ka,shon, A. Shapira, T. Ludian, R. Steiner, and H. Iaft -Planar Events in Hadron-Proton Collisions at 147 EeV/C and Their Jet-Like Structures," Z. Phys. C24, $19-29(1984)$

Burydörfer, J., M. Breiniy, S. B. Elston, and I. A. Sel iin Calculation of Electron-Loss-to-Continum Cusps: An Algebraic Approach," Phys. Rev. A 28, $3217-93$ (1983)

Carlton, R. F., J. A. Harvey, and C. H. Johnson "s- and p-Wave Meutrons on 30Si and 345: Spherical Optical Model Analysis," Phys. Rev, C 29, $1988-92$ (1984)

Carter, H. $\mathrm{K}$.

"Laser Spectroscopy on $\beta$-Unstable Muclei and Future Directions for Laster Spk-roscopy," pp. 906-11 in Vol. II, Proceedings, Third LAiPf II Workshop, Los Alanos, New Mexico, July 13-20, 1983, Los Alanos National Laboratory Report Uh-9933-E (1983)

Carter, H. K., P. Juncar, J. A. Bounds, C. R Bingham, D. J. Pegg, and W. Fairbank, Jr. "Sensitive Collinear Laser Spectroscopy on Fast Atom and Ion Beams." Proc. Soc. Photo-0ptical Eng. 426, 60-64 (1983)

Castel, B., and A.li.M. van Hees "Oistribution of EI Spin-flip Strength," Phys. Rev. C 28, 2571-73 (1983)

Chen, Y. S., S. Frauendorf, and G. A. Leander "Shape of Rotating (tuasi-Particle Urbits and Signature Splitting in La, Ce, and Pr Nuclei," Phys. Rev. C 28, 2437-46 (1983)

Church, U. A., K. W. Jones, B. M. Johnson, M. Meron, and I. A. Sell in -Production and Scorage of Highl, Charyed lons at Room Temperature," J. Phys. B17, L401-06 (1984)

Church, U. A., R. A. Kenefick, H. S. Burns, C. S. U, R. Holmes, S. Huldt, S. Berry, M, Breiniy, S. Clston, J.-P. Rozet, I. A. Sellin, U. Taylor, and B. Thomas "Charye Transfer to Multicharged Recoli Ions in a Penning Trap," Phys, Rev. Lett. 51, 1636-39 (1983)

Cohn, H. W.. J. E. Brau, W. M. Busuy, G. T. Condo, T. Handler, and E. L. Hart "Low-Eneryy p-Nucleus Reaction Cross Sections." Pnys. Kev. C 29, 332 (1984)

Condo, G. T., W. B. Bugg, T. Handler, and H. U. Cohn "Multinucleon Captures of Slow Antiprotons in Complex thuclei and s Search for the H Dibaryon," Phys. Lett. 1448, 27-29 (1984)

Condo, G. T., T. Handler, and H. J. Cohn " $A^{j}$ Prodiction Prom Low-Energy Antiproton Annintlations in Complex Nuclet," Pnys. Rev. C, 29, 1531-33 (1984) 
Datz, 5 .

"Atonic Collisions in the High-Energy Regies," p. 369-94 in Atonic Pursics, Yol. 8, Plemu, hew Tort, 19R?

Oat2, S... Editor

Applied Atonic Collision Puysics, Ml. 4: Condensed Matter, Academic Press, Orlando, Florida, 1983

Datz, S. (Invited Paper)

"Dielectronic Decobimation: An Introduction," pp. 795-800 in Proceedings, XIII International

Conference on the Physics of Electronic and Atomic Collisions, Berlin, West Germiny, July 27-August

2. 1983, Horth-foll land, hosterdan, 1994

Datz, S., R. M. Fearick, H. Part, R. H. Pantell, R. L. Sinent, d. O. Kephart, and B. L. Berman -Electror and Pssitron Channeling Radiation from Type-Ia and Type-II: Diamonds," Proceedings, Iuth International Conference on Atomic Collisions in Solids, Bad Iourg, F.R.6., July 18-22, 1983, Mucl. Instrum. Hethods Phys. Mes.. Sect. 6, 230 [12], 74-79 (ight)

Qavies, K.T.R., H. Menud, h. Strayer, K.R.S. Devi, and T. Raffray "Geometry and Oynamics of a Zero-Temperature Ferwi-6as Madel for Preequilibriug Emission of hucleons

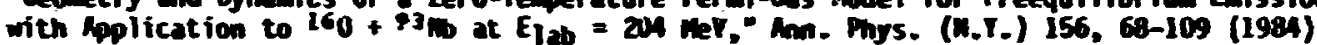

Davies, R.T.R., A. J. Siert, and J. R. Mix (Invited Paper) "Dramical fusion Threstolds in Macroscopic and Microscopic theories," pp. 57-76 in Muclear Physics with Heavy lons (Proceedings, International Conference on Muclear Physics with Heavy Tons, Stony Brock, Thew Vort, April 14-16, 1983), Kanmood Acadenic, Mew Tork, 1944

Dittner, P. F., S. Datz, P. D. Miller, C. O. Maak, P. H. Stelson, C. Bottcher, M. Meskovic, and C, M. Fow (Inv :ed Paper) -Cross Sections for Dielectronic Recomination of $\mathrm{B}^{2+}$ and $\mathrm{C}^{3+}$ via $1 \mathrm{~s}^{2} 2 \mathrm{~s}+1 \mathrm{~s}^{2} 2 \mathrm{p}$ Excitation." pp. 819-26 in Proceedings, XIII International Conference on the Physics of Electronic and Atomic Collisions, Berlin, Mest Eerman, hly 27-hugust 2, 1983, Dorth-Hol land, Ansterdam, 1984

Donangelo, R., M. M. Guidry, R. E. Weese, and M. J. Rhoades-Brown "Angular Localization and Approximations to the Deformed Huclear Potential in Heavy-Ion Reactions," Phys. Rev. C 29, 1925-27 (1984)

Ellis-Akovali, Y. A. "Huclear Data Sheets for A = 230," Mucl. Data Sheets 40,385-423 (I984)

Ellis-Akovali, Y. A. "Huclear Data Sheets for $A=234$, " Mucl. Data Sheets 40, 523-603 (1983)

Gaarde, C., J. S. Larsen, H. Sagawa, N. Untsuka, J. Rapaport, T. H. Taddeucri, C. D. Coodaan, C. C. Foster, C. A. Soulding, D. Horen, T. Masterson, and E. Sugarbaker "Spin Dipole Strength in $12 \mathrm{~N}$, " Mucl. Phys. 1422, 189-204 (1984)

Gavron, A., P. Eskola, A. J. Sierk, J. Boissevain, H. C. Britt, K. Eskola, M. M. Fowler, H. Onm, J. B. Wi Ineliny, S. Mald, and K. L. Ferguson

"Hew Evaluation of Fission -Fragment Angular Distributions in Heavy-Ion Reac: : ans," Phys, Rev. Lett. 52, $589-92(1984)$

Gomez del Campo, J., J. A. Biggerstaff, R. A. Dayras, O. Shapira, A. H. Snell, P. H. Stelson, and R. G. Stokstad

"Comparison of Fusion Cross Sections for the ${ }^{10} B+160$ and $12 C+14 N$ Systems," Phys. Rev. C 29, 1722-34 (1984)

Gomez del Campo, J., R. H. Fearick, J. A. Biggerstaff, C. D. Moak, P. D. Miller, N. Meskovic, D. Shapira, and J.P.F. Sellschop (Invited Paper)

"Crystal Blocking Measurements in Heavy-Ion Reactions," Proceedings, Sixth Symposium on Nuclear Physics, Oaxtepec, Hexico, January 4-7, 1983, Notas de Fisica 6, 115-26 (1983)

Griffin, D. C., C. Bottcher, M. S. Pindzola, S. M. Younger, D. C. Gregory, and D. H. Crandall "Electron-Impact Ionization in the Xenon Isonuclear Sequence," Phys. Rev. A 29, 1729-41 (1984)

Griffin, D. C., M. S. Pindzola, and C. Botteher

"Calculations of the Contributions of Excitation-Autoionization to the Electron-Impact lontzation of $\mathrm{Ca}^{+}$and $\mathrm{Bd}^{+}$in the Uistorted-Have Approximation." J. Phys. B17, 3183-92 (1984)

Groeneveld, K. U., H. Meckbach, I, A, Sellin, and J. Burydorfar "Collisional Electron Transfer tnto the Continuum of lonic Projectiles," Comments At. Mol. Phys. 4, $187-200(1984)$ 
Gross, E. E., T. P. Cleary, J.L.C. Ferd, Jr., D. C. Hensley, X. S. Toth, F. T. Bater, A. Scott, C. R. Binghain, and J. A. Vrba

Huclear moments of the first Excited State of $22 \mathrm{Me}$ from $22 \mathrm{Me}$ (132 MeV) + $200 \mathrm{~Pb}$ Scattering." Phys. Rev. C 29, 459-63 (1904)

Gross, E. E., and H. P. Fenell

"Resulution of a Coulomb Reorientation Abiguity in 100,- Mucl. Phys. M411, 329-36 (1983)

Hamilton, J. H., A. Y. Rayyz, C. F. Maguire, R. B. Piercey, R. Bengtsson, P. Moller, J. R. Mix, J.-1. Znang, R. L. Robinson, and S. Fravendorf

"Effects of Reinforcing Shell Gaps on the Conpetition Between Spherical and Highly Deformed Shapes," J. Phys. 610, 287-L91 (1934)

Helmer, R. G., and C. W. Reich

"Levels in 204 Po from the Decay of 204 At, " Phys. Rev. C 27, 224b-60 (1983)

Meinsley, 0. C.

"Parallel Processor for Fast Event Analysis," Proceedings, Coaference on Real-Time Computer Applications in Particle and Muclear Physics, Berkeley, California, Gy 16-19, 1983, IEEE Trans. Mucl. Sci. IS-30, 3913-16 (1983)

Horen, D. J., F. E. Bertrand, E. E. Gross, T. P. Sjoreen, D. R. Mclaniels, J. R. Tinsley, J. Lisantti, L. W. Suenson, J. B. Micclell land, T. A. Carey, S. J. Seestrom-thorris, and $\boldsymbol{K}_{\text {. dones }}$ "Excitation of E'z Transitions in "C Ca by 334-Hel Protons," Phys. Rev. C 30, 709-11 (1984)

Horen, O. J., R. L. Macklin, J. A. Harvey, and H. H. Hill "Weasurements of the Keutron Iransaission and Capture Cross Sections in 204Pb," Pnys. Rev. C 29. 2126-34 (1944)

Hudson, E. O., K. S. Lord, H. L. Mallory, and T. A. Antaye

"Dual Arc Penning Ion Source Gas Flow Experiments," pp. I30-32 in Proceedings, Tenth International Conference on Cyclotrons and Their Applications. East Lansing, Michigan, Aprif 30 -flay 3 , I984, IEEE Conf. Record 84CHI996-3, Institute of Electrical and Electronics Engineers, Mew York, 1984

Huyse, M.

"Ionization in a Hot Cavity," Hucl. Instrum. Methods Phys. Res. 215, 1-6 (1983)

Hynes, M. V., J.L.C. Ford, Jr., T. P. Sjoreen, J. L. Blankenihip, and F. E. Bertrand "Design a id Performance of a Vertical Urift Chamber for Hedvy-Ion Applications," Hucl. Instrum. Methods inys. Hes., Sect. A, 224, 89-96 (1984)

Itikawa, Y., S. Mara, T. Kato, S. Makazaki, M. S. Pindzola, and D. H. Crandall "Recomended Data on Excitation of Carbon and Oxygen Ions by Electron Collisions," Report IPPJ-AM-27. Institute of Plasima Physics, Magoya University, Magoya, Japan (June 1983), 135 pp.

Johnson, C. H., M. M. Larson, C. Mahaux, and R. R. Winters

"Reply to 'Calculation of the Lorentz-Heighted Average S Matrix from High-Resolution, Low-Energy Neutron Scattering Data"," Pnys. Rev. C 29, 1563-65 (1984)

Johnson, N. R., C. Baktash, and I. Y. Lee (Invited Paper) "Bismuth Germanate's Role in the Nex Revolution in Gamma-Ray Spectroscopy," Proceedings, 1983 Muclear Science Symposium, San Francisco, California, October 19-21, 1983, IEEE Trans. Mucl Sci. M5-31. 243 (1984)

Jones, C. M. (Invited Paper)

"Large Electrostatic Accelerators, DP. 496-503 in Proceedings, Tenth International Conference on Cyclotrons and Their Applications, East Lansing. Michigan. Aprit 30-hay 3, 1984, IEEE Conf. Record 84CFIS96-3, Institute of Electrical and Electronics Engineers, New York, 1984

Jones, C. M., G. D. Alcon, J, B. Ball, J. A. Henjamin, J. A. Biggerstaff, K. A. Erb, E. D. Hudson, R. C. Juras, P. K. Kloeppel, K. S. Lord, C. A. Ludemann, J. E. Mann, J. A. Mart in, S. H. Mosko, E. G. Richardson, R. U. Sayer, and N. F. Liegler

"Status Report on the Holifield Heavy Ion Research Facility," Proceedings, Sixth Tandem Conference, Chester, England, April 18-22, 1983, Nucl. Instrum. Methods Phys. Res. 220, 1-9 (1984)

Kahane, S., S. Kaman, G. G. Slaur-"er, C. Coceva, and M. Stefanon "Electric Uipole Transitions irom Neutron Capture in 167 Er Kesonances," Pnys. Kev, C 30, 807-19 (1984) 


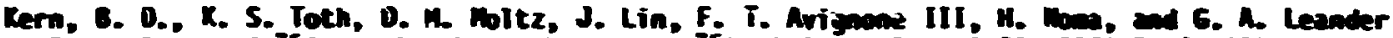

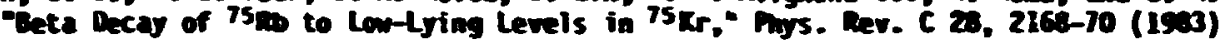

Robos, A. M., B. A. Bron, R. Lindsay, and 6. R. Sotchler Toidim Dodel Amalysis of Elastic and Inelastic a-farticle Scattering Using a Densfty-Dependent Force," Mucl. Mys. M25, 205-32 (1594)

Robos, A. M., and 6. A. Satchler

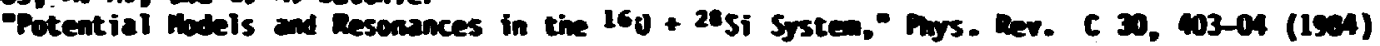

Larabee, A. J., L. H. Courtines, S. Fravendor f, L. L. Miedinger, J. C. Mudingten, H. P. Fenell, M. R. Johnson, I. T. Lee, and F. K. Fitionen

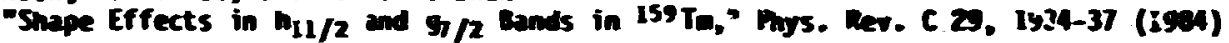

Leander. 6. A. "Coment on 'Muclef: A Superfluid Condensate of a Particles? a Study Mithin the Interactias-iosoa Model"." Muss. Rer. Lett. 52, 311 (1594)

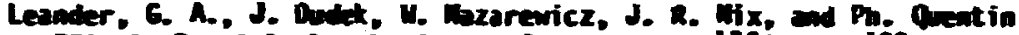

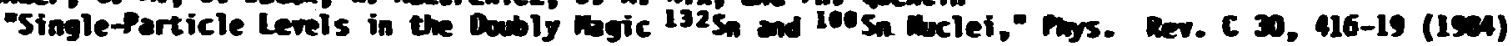

Leander, C. A., and R. X. Sullime "Intrinsic Reflection Assmmetry in Ou-A nuclef," mecl: Muss. M13, 375-416 (194)

Lee, I. Y., C. Bektash, and J. X. Saladin

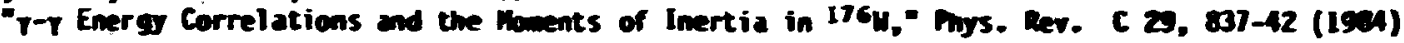

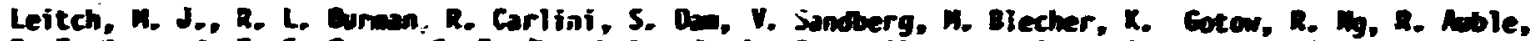
F. E. Bertand, E. E. Gross, F. E. Obenshain, J. Wu, G. S. Bianied, B. F. Preedom, B. G. Ritchie, W. Bertozi. M. V. Hymes, M. A. Rovash, and R. P. Redurine "Pien-fucleus Elastic Scattering at au MeV," Mhys. Rev. C 29, 561-6B (19e4)

Lonnroth, T., and C. Baktash -In-Bean Study of $213_{R n,} 215_{R a}$, and $217_{R a}$ : Strongly Emanced E3 Transitioas in Odd-A $\|=127$ Isotones," Phys. Scr. 28, 459-66 (1983)

Ludemann, C. A. "A Aicroprocessor Multi-task Monitor," Proceedings, Conference on Real-Tíne Computer hpplications in huclear and Particle Physics, Berkeley, California, May 16-19, 1983, IEEE Trans. Mucl. Sci. IS-30, 3858-63 (1983)

Lynch, W. B., C. B. Chitrood, W. B. Tsang, D. J. Fields, D. R. Klesch, C. R. Gelske, G. R, Toung, T. C. Ames, R. L. Ferguson, F. E. Coenshain, F. Plasil, R, L. Robinson, and A. D. Panagiotou "Formation and Decay of a Localized Region of Migh Excitation in Heavy-Im-Induced Reactions," Phys. Rev. Lett. 51, 1850-53 (1983)

Lynch, W. 6., C. B. Chitwood, M. B. Isang, D. J. Fields, D. R. Klesch, C. K, Geloke, G, R, Young, T. C. hues, R. L. Ferguson, F. E. Doenshain, F. Plasil, R, L. Robinson, and A. D. Panagiotou -Response to Comment on 'Formation and Decay of a Localized Region of High Excteation in Heavy-Ion-Induced Reactions"," Phys. Rev. Lete. 52, 2302 (1984)

Ma, W. C., A. V. Ramayya, J. H. Haditon, S. J. Robinson, M. E. Barclay, Z. Zneo, J. D. Cole, E. F. Zganjar, and E. H. Soejewski "Ground-State Shape and Crossing of Kear spherical and Deformed Bands in 102 $\mathrm{kg}, "$ Phys. Lett. 1393, 276-78 (1984)

Mackellar, A. D., and B. Castel "s- and p-Wave Meutrons on 30si and 34s: Coupled Channels Optical hodel," Phys. Rev. C 29, 1993-95 (1984)

Hackellar, A. D., G. R. Satchler, and $C_{,}-Y$, Wong "An Exploratory Study of Antiproton-Stucleus Scattering," Z. Phys. A316, 35-42 (1984)

Maguire, C. F., G. L. Bomar, K. E. Barclay, R. B. Piercey, A. V. Ramayya, J.L.F, Ford, Jr., D. Shapira, E. R. Flynn, J. D. Moses, J. C. Peng, and $W_{\text {. Stein }}$ "Systematic Spectroscopic-Factor Discrepancy in Hedvy-lon Proton-Pickup Reactions on "OCa," Phys. Rev. Lett. 52, 743-46 (1984) 
hartin, J. A. (Invited Paper)

-Tive Intermatioal Cyclotron Confereace's Tweaty-five Tears of Progress," p. 1-2 in Proceedings. Terth Intermatioal Conference on Crclotrons and Their Aglications, East Lansing, Michigan, hpril

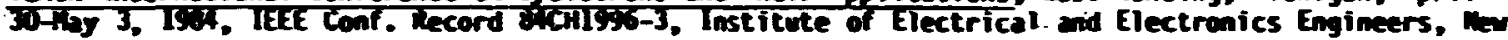
Tort, 1904

- Mcloy, K. M., and G. R. Satchler "hiclear thainbows and Heary-Ion Scattering," Mucl. Mys. M17, 157-73 (19a4)

Mehta, R., . L. Ouggan, F. D. Welaniel, M. C. Andrews, G. Lapicki, P. U. Miller, L. A. Rayburn, and A. R. Zander -Oirect Ionization and Electron Capture in n-Shell x-loy Probetion by Flworime Ions," Phys. Rev. A 23, 2722-26 (1923)

Mignerey, A. C., C. Merouane, S. Bradley, D. Eenton, H. Erewer, J. V. Silk, K. Kriatouski, V. E. Viola. L... T. C. Mes, F. E. Coenshain, and S. Peateppidan

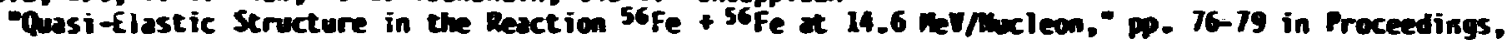

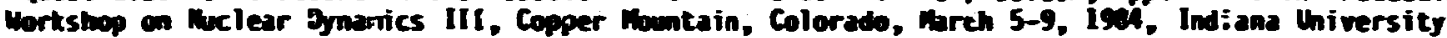
Wuclear Gevistry Report, In -10007-24 (April 1994)

Mazarewicz, H., P. Ulanders, I. Ragarsson, J. Oudek, and 6. A. Leander

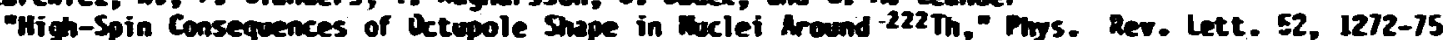
(1964)

O'Kelley, G. O., R. L. Rable, L. D. Hulett, H. J. Kim, M. T. Mi Iner, S. Ramo, O. Shahal, C. R. Vane, J. P. Toung, and G. Lapicki - Inlications of Heavy-Ion-Induced Satellite X-iay Emission II: Production of $X$ and $L \mathrm{X}$-Rays by 0.9-2.6 Weitu Argon Ions in Thick Targets of Vanadiua, Copper, Wiobium, Tantalum, and Platimum," iroceedings. Third International Conference on Particle-Induced $X$-2ay Emission, Heidelberg, West Gerasang, Wily W6-22, I983, Mucl. Instrum. Methods. Mys. Res.., Sect. B, 231 [83], 78-87 (1984)

Park, H., R. H. Pantel I, R. L. Siment, J. D. Kephart, B. L. Berman, S. Datz, and R. M. Fearick "Comparison of Channeling Radiation from Diamonds with and without Platelets," J. Appl. Phys. 55, $358-64(1984)$

Praneuf, R. A.

"Atomic Processes Involving Impurities in the Plasma Edge," Proceedings, Workshop on Impurity Control Physics, atlanta, Eeorgia, Way 30-31, 1994, pp. 101-25 in Report ETR-IiNToR/ICP/3 (1984)

Pindzola, M. S., D. C. Griffin, C. Bottcher, D. H. Crandall, R. A. Phaneuf, and D. C. Gregary "Electron-Impact Double Ionization of Rare-tas Ions," Phys. Rev. A 29, 1749-56 (1984)

Plasil, F. "Comment on 'Fission-fragment Angular Distributions'," Phys. Rev. Lett. 52, 1929 (1984)

Plasil, F., T. C. hes, B. Cheynis, D. Drain, R. L. Terguson, F. E, Dobenshain, ג. J. Sierk, S. G. Steadman, and G. R. Young "Angular-thementum-Dependent Fission Barriers in the Rare-Earth Region," Phys. Rev. C 39, $1145-48$ (1984)

Plasil, F., T. C. hes, B. Cheynis, D. Drain, R. L. Ferguson, F. E, Obenshain, A. J. Sierk, S. G. Steadran, G. R. Young, A. Gavron, J. Boissevain, H. C. Britt, K. Eskola, P. Eskola, M. M. Fowler, Z. Fraenkel, H. Unm, J. van der Plicht, and S. Hald (Invited Paper) "Recent Results in Havy-lan-Induced Fission." pp. 1-4 in Proceedings, Workshop on Muclear Dynamics III. Copper Mountain, Colorado, March 5-), 1984, Indiana University Muclear Chemistry Report. Ime-40007-24 (April I984)

Pontoppidan, S., P. R. Christensen, U. Hansen, F. Videbaek, H. C. Britt, B. H. Erkkila, Y. Patin, R. H. Stokes, M. P. Webb, R. L. Ferguson, F. Plasil, and G. R. Young "Quasielastic Transfer Reactions Induced by $56 \mathrm{Fe}$ on $58 \mathrm{Ni}, 64 \mathrm{NI}$, and $122 \mathrm{Sn}$," Phys. Rev. C 28, 2299-311 (1983)

Poskanzer, A., H-b. Ritter, B. Ludewiyt, K. Foley, S. Borenstetn, E. Platner, H. Love, D. Keane, and F. Plasil

"Event Parameters - Fixed Target," po. 38-44 in Proceedings, Horkshop on Detectors Por Relativistic Nuclear Collisions, Berkeiey, Califorinis, March 26-30, 1984, Lawrence Berkeley Laboratory Repori. LBL-Ia225 (August 1984)

Raman, 5. "Ion-Induced X-Ray Emission," pp. 407-28 in Applied Atomic Collision Phystics, Vol. 4: Condensed Matter, Academic Press, Orlando, Flortda, 1983 
Manan,.S., Editor

- Actinide newsletter," Issue bo. 7 (March 1384)

Rama, S., E. Kallne, J. Kallne, T. Makajima, C. W. Mestor, Jr., P. H. Stelson, C. R. Yane, and T. A. Watkievicz

-Implications of Heary-Ion-Induced Satellite $X$-Ray Emission V: Fine Structure of the Sulfur $\mathbf{R} \cdot \mathbf{X}-\mathrm{kay}$ Satellite Peaks," Proceedings, Mird International Conference on Particle-Ind sed X-Ray Eaission, Heidelberg, Mest Cermany, July 18-22, 1933, Mucl. Instru. Methods Phys. Res... Sect. 3, 231 [B3], 100-03 (1934)

Raman, S., M. Ratynsti, E. I. Jurney, M. E. Bunker, and J. H. Stamer ${ }^{-36} S(n, y)$ 37s Reaction, with Thermil Meutrons and Decay of $37 \mathrm{~S}$ to Levels in $37 \mathrm{Cl}$, Phys. Rev. C 30, 26-30 (1984)

Rama, S., and C. R. Vane (Invited Paper)

"Iplications of Heavy-Ion-Induced Satellite R-Ray Emission I: Introduction," Proceedings, Third International Conference on Particle-Induced X-Ray Epission, Heidelberg, West Germany, Wuly 18-22, 1983, Mucl. Instrum. Methods Phys. Res., Sect. 8, 231 [B3], 71-77 (1984)

Rapaport, J., T. Taddeccci, T. P. Welch, C. Gaarde, J. Larsen, D. J. Horen, E. Sugarbaker, P. Koncz,

C. C. Foster, C. D. Goodman, C. A. Goulding, and T. Masterson "Excitation of Giant Spin-Isospin Multipole Vibrations in 54,56Fe and 58,60 Mi," Mucl. Phys. M410. 371-98 (19:3)

Reading, J. F., A. L. Ford, 3. S. Swith, J. Alexander, and R. L. Becker (Invited Paper) -Progress in Mumerical Calculations of Ion-Atom Collisions." Proceedings, Third Horkshop on Inner Shell Ionization by Light Ions, Linz, Austria, August 4-5, 1983, Mucl. Instrum. Methods Phys. Res., Sect. B, $232[84], 266-70$ (1984)

Reading, J. F., A. L. Ford, J. S. Smith, and R, L, Becker (Invited Paper!

"Learning from Mumerical Calculations of Ion-Atom Collisions," Pp. 201-11 in Proceedings, XIII International Conference on the Physics of Electronic and Atomic Collisions, Berlin, Mest Germany, July 27-August 2, 1983, Horth-Hol land, Anstercan, 1084

Riedinger, L. L.

"Aligned Bands and Nuclear Shapes in the $N=90$ Region," Proceedings, Nordic Symposium on Nuclear Physics, Fuglso, Denark, Augrist 16-20, 1982, Phys. Scr. T5, 36-44 (1983)

Rosseel, T. M., J. M. Dale, H. H. Ounn, L. D. Hulett, S. Kahane, H. F. Krause, S. Raman, G. G. Slaughter, C. R. Vane, and J. P. Young - Implications of Heavy-Ion-Induced Satellfte X-Ray Emission IV: Chemical Effects in L X-Ray Sacellites of Molybdenum Compounds and Allisys." Proceedings, Third Inter.lational Conference on Particle-Induced $x$-Ray Emission. Heidelbeig, West Germany, July 18-22, 1983, Nucl. Instrum. Methods Pi.ys. Res., Sect. B, 231 [B3], 94-99 (1964)

Rosseel, T. M., J. P. Young, J. M. Dale, A. DasGupta, L. D. hulett, H, F. Krause, C. T. Liu, S. Raman, and $C ., 2$, Vane

"Application of Heavy-Ion-Induced X-Ray Satellite Emission to Alloys,"J. Phys. F14, L3i-L41 (1984)

Satchler, G. R: (Invited Paper)

"Hucleus-Hucleus Potentials," Proceedings, International Conference on Heavy-Ion and Hiclear Physics, Catania, Italy, March 21-26, 1983, Mucl. Phys. 1409, 3c-20c (1983)

Satchler, G. R.

"The Disturted-Waves Theory of Uirect Muclear Reactions with Spin-0rbit Effects," [Nuc1. Phys, 55, 1-33 (1964)], Current Conten:s (Citation Classics Section) 23, No. 36, 18 (1983)

Satchler, G. R., and W. T. Pinkston "Properties of the Transition Amplitude for Two-Mucleon Transfer Reactions," Mucl. Phys. 1411, 144-50 (1983)

Satchler, G. R.., and W. T. Pinkston

"Comment on $J$ lependence in $(\alpha, d)$ Transfer Reactions," Phys. Lett. 1348, 7-10 (1984)

Sayer, R, 0 .

"RIP - General Purpuse Interactive Display at NHIKF," Proceedings, Conference on Real-Time Computer Applications in Particle and Nuclear Physics, Berkeley, Californid, May 16-19, 1983, IEEE Trans. Hucl. Sci. NS-30, 3833-37 (1983) 
Schmorak, M. R.

"Huclear Cata Sheets for $A=231,235$, and 239," Nucl. Data Sheets 40, 1-147 (1984)

Schuck, C., N. Bendjaballah, R. M. Diamond, Y. Ellis-Akovali, K. H. Lindenberger, J. O. Mewton, F. S. Stephens, J. U. Garrett, and 3. Herskind

"Evidence for Reduced Neutron Pairing Correlations in $165 \mathrm{Yb}, "$ Phys. Lett. 1428, 253-57 (1984)

Seiwert, M., M. Greiner, V. Uberacker, and M. J. Rhoades-Brown

"Test of the Proximity Theorem for Deformed Nuclei," Phys. Rev. C 29, 447-85 (1984)

Shapira, 0., and K. A. Erb (Invited Paper)

"Holecular Degrees of Freedom: Resonances and Urbiting," pp. 305-28 in Nuclear Physics with Heavy

Ions (Proceedings, International Conference on Nuclear Physics with Heavy Ions, Stony Brook, New

York, April 14-15, 1983), Harwood Academic, New York, 1984

Shapira, D., K. A. Erb, J.L.C, Ford, Jr., J. Gomez del Campo, B. Shivakumar, R. Hovotny, D. Schull, S. T. Thornton, K. L. Parks, and R. Cecil (Invited Paper)

"Orbiting in Collis: ons Between Liyht Heavy Ions $(A T+A p<50)$." Proceedings, Sixth Symposium on Nuclear Physics, Oaxtepec, Mexico, January 4-7, 198, Notas de Fisica 6, 325-54 (1983)

Shel ine, R. K., D. Decaran, K. Nybo, T. F. Thorsteinsen, G. Louhoiden, E. R. Flynn, J. A. Cizewski,

D. K. Burke, G. Sletten, P. Hill, H. Kaffrell, H. Kurcewicz, G. Myman, and G. A. Leander "Evidence for Near-Stable Uctupole Deformation in ${ }^{225}$ Ra," Phys. Lett. 133B, 13-16 (1983)

Sjoreen, I. P., F. E. Bertrand, R. L. Auble, E. E. Gross, D. J. Horen, D. Shapira, and D. B. Wright "Inelastic Excitation of Giant Resonances by 400-MeV 160," Phys. Rev. C 29, 1370-76 (1984)

Sjoreen, T. P., J.L.C. Ford, Jr., J. L. Blankenship, R. L. Auble, F. E. Bertrand, E. E, Gross, D. C. Hensley, D. Schull, and M. V. Hynes

"The Vertical Urift Chamber as a High Resolution Focal Plaile Detector for Heavy Ions," Nucl. Instrum. Methods Phys. Ris., Sect. A, 224, 42I-31 (1984)

Strayer, M. R., R. Y. Cusson, A. S. Umar, P.-G. Reinhard, D. A. Bronley, and W. Greiner "Time-Dependent Hartree-Fock Picture of Muclear Molecular Resonances," Phys. Lett. 1358, 261-65 (1984)

Swent, R. L., R. H. Pantell, H. Park, J. O. Kephart, R. K. Klein, S. Datz, R. H. Fearick, and B. L. Berman

"Planar and Axial Channeling Radiation from Relativistic Electrons in LiF," Phys. Rev. B 29, 52-60 (1984)

Tabor, S. L., G. Neuschaefer, J. A. Carr, F. Petrovich, C. C. Chang, A. Guterman, M. T. Collins, D. L. Friesel, C. Glover, S. Y. Van der Werf, and S. Raman

"The ( $\left.{ }^{3} \mathrm{He}, t\right)$ Reaction at $197 \mathrm{MeV}$ on ${ }^{12} \mathrm{C}, 24 \mathrm{Mg},{ }^{28} \mathrm{Si}$, and ${ }^{40} \mathrm{Ca}, " \mathrm{Nucl}$. Phys. A422, 12-44, 1984)

Taddeucci, T. N., T. A. Carey, C. Gaarde, J. Larsen, C. O. Goodman, D. J. Horen, T, Masterson, J. Rapaport, T. P. Welch, and E. Sugarbaker

"Méasurement of the Transverse Spin-Transfer Coefficient $O_{N N}\left(0^{\circ}\right)$ for $\left(p_{p o l}, n_{D o l}\right)$ Reactions at 160 MeV," Phys. Rev. Lett. 52, 1960-63 (1984)

Taddeucci, T. N., J. Rapaport, C. C. Foster, C. D. Goodman, C. Gaarde, J. Larsen, C. A. Goulding, D. J. Horen, T. Masterson, and E. Sügarbaker

"Spin Excitations in $40 \mathrm{Ca}(p, n), "$ Phys. Rev. C 28, 2511-14 (1983)

Thornton, S. T., R. L. Parks, D. Shapira, D. Schull, J.L.C. Ford, Jr., B. Shivakumar, and J. Gomez del Campo

"Observation of a Critical Angular Momentum for Deep Inelastic Processes with Light Heavy Ions," pp. 97-100 in Procepdings, Workshop on Nuclear Dynamics 1II, Copper Mountain. Colorado, March 5-9, 1984, Indiana University Nuclear Chemistry Keport, INC-40007-24 (April 1984)

Toth, K. S., D. M. Moltz, E. C. Schloemer, M. D, Cable, F. T. Avt gnone, III, and Y, A. Ellis-Akovali "Beta-Delayed iroton Activities: 147 Jy and ${ }^{149} \mathrm{Er}, "$ Pnys. Rev. C 30, 712-14 (1984)

Umar, A. S., M. R. Strayer, and D. J. Ernst "A Time-Dependent External-Field Model for Particle Emisston in Heavy-Ion Reactions," Phys. Lett. 1408, 290-94 (1984)

van der Plicht, J., H. C. Britt, M. M. Fowler, L. Fraenkel, A. Lavron, J. B. Wtlhelmy, F. Plasil, T. C. Awes, and G. K. Young

"Fission of Polontum, Usmium, and Erbium Composite Systems," Phys, Rev, C 28, 2022-32 (1983) 
Vane, C. R., L. D. Hulett, Jr., S. Kahne, F. D. McDaniel, H. T. Milner, G. D. O'Kelley, S. Raman, T. M. Rosseel, G. G. Slaughter, S. L. Varghese, and J. P. Young

"Implications of Heavy-Ion-Induced Satellite $X$-Ray Enission III: Chemical Effects in High-Resolution Sulfur $K_{\alpha} X$-Ray Spectra," Proceedings, Third International Conference on Particle-Induced $X$-Ray

Emission, Heidelbery, West Germany, July 18-22, 1983, Mucl. Instrum. Methods Phys. Res., Sect. B, 231 [B3], 88-93 (1984)

Willett, S. J., S. K. Korotky, R. L. Phill ips, D. A. Bromley, and K. A. Erb

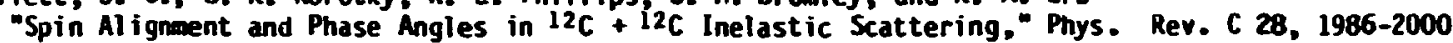
(1983)

Nillis, R. D., S. L. Allman, C. H. Chen, G. O. Alton, and G. S. Hurst "Pumping of Inert Gases by Electron-Lipact Ionization Sources and Associated Memory Effects," J. Vac. SGi. Technol. A2, 57 (1984)

Wong, C. Y. "Baryon Distribution in Relativistic Heavy-Ion Col'isions," Phys. Rev. Lett. 5E,, $1393-96$ (1984)

Nong, C. Y.

"Initial Energy Density of Quark-Gluon Plasma in Rela:ivistic Heavy-Ion Collisions," pp. 207-10 in Proceedings, Morkshop on Muclear Dynamics III, Copper Mountain, Colorado, March 5-9; 1984, Indiana University Muclear Chemistry Report, IKC-40007-24 (April 1984)

Mong. C. Y. (Invited Paper)

Baryon Distribution in Relativistic Heavy-Ion Collisions," pp. 203-05 in Proceedings, Horkshop on Muclear Dynamics III, Copper Mountain, Colorado, March 5-9, 1984, Indiana University Huclear Chemistry Report, InC-40007-24 (April 1984)

Nong, C. $Y$. "Initial Energy Density of Quark-Gluon Plasma in Relativistic Heavy-Ion Collisions," Phys. Rev. D 30, $961-71$ (1984)

Nong, C. Y.

"Baryon Distribution in Relativistic Heavy-lon Collisions," Phys. Rev. D 30, $972-84$ (1984)

Hong, C. Y., A. K. Kerman, G. R. Satchler, and A. D. Mackell ar "Ambiguity in Antiproton-Nucleus Potentials from Antiprotonic-Atom Data," Phys. Rev. C 29, 574-80 (1984)

Young, G. R., J. A. Martii, and J. B. Ball

"A 10-GeV on 10-GeV Heavy-Ion Collider," Proceedings. Third International Conference on Ultra-Relatívistic Nucleus-Nucleus Collisions, Upton, New York, September 26-30, 1983, Nucl. Phys. A418, 361c-69c (1984)

\section{PROGRESS REPORT}

Ball, J. B., Division Director Physics Division Progress Report for Period Ending September 30, 1983, ORNL-6004 (December 1983)

\section{TOPICAL REPORTS}

Barnett, C, F., D. H. Crandall, H. B. Gllbody, D. C. Gregcry, M. I. Kirkpatrick, E. W. McDantel, R. H. McKnight, F. W. Meyer, T. J. Morgan, R. A. Phaneuf, M. S. Pindzola, and E. W. Thomas 1982 Bibliography of Atomic and Molecular Processes, ORNL-6052 (May 1994)

Bottcher, C., D. C. Griffin, M. S. Pindzola, and R. A. Phaneuf Indirect Processes in Electron-Ion Scattering, ORNL/TM-8868 (Uctober 1983)

Hal Dert, M. L.

New Vistas of Heavy-Ion Reactions with the Spin Spectrometer, ORNL/TM-8986 (January 1984)

$01 \operatorname{sen}$, U. K., J. A. Martin, and U. J. Horen

A Preliminary Study of Possible UHELA Keplacement Options, URNL/TM-8669 (June 1984) 


\section{Mrticles pending publcation as OF SEPTEmber 30, 1984}

Abe, K., R. Armenteros, T. C. Bacon, J. Ballan, H. H. Bingham, J. E. Brau, K. Braune, D. Brick, M. M. Bugg, J. H. Butler, W. Caneron, H. U. Cohn, D. C. Colley, G. T. Condo, P. Dingus, R. Erickson, R. C. Fiel , B. Franek, R. Gearhart, T. Glanzman, I. M. Codfrey, J. J. Goldberg, G. Hail, E. K. Hancock, H. J. Hargis, E. L. Hart, M. J. Harwin, K. Hasegawa, M. Jobes, T. Kafka, G. E. Kalmus, D. P. Kelsey, T. Kitagaki, M. A. Mann, R. Merenyi, R. Milburn, K. C. Moffeit, J. J. Murray, A. Mapier, V. R. O'Dell, P. Rankin, H. Sagawa, J. Schneps, S. J. Sewel I, J. Shank, A. M. Shapiro, J. Shimony, K. Tamai, S. Tanaka,

D. A. Haide, M. Midgoff, S. Wolbers, C. A. Moods, A. Yamaguchi, 6. P. Yost, and H. Yuta "Charm Photoproduction at 20 beV Including Preliminary Lifetime Results with Improved Optical Resolution," Proceedings, xxII Internation?l Conference on High-Energy Physics, Leipzig, East Gerwany, July 19-25, 1984

Abe, K., R. Armenteros, T. C. Bacon, J. Ballan, H. H. Binghan, J. E. Brau, X. Braune, D. Brick, M. M. Bugg, J. M. Hutler, N. Cameron, H. O. Cohn, D. C. Colley, G. T. Condo, P. Dingus, R. Erickson, R. C. Field, B. Franek, R. Learhart, T. Glanzman, I. M. Godfrey, J. J. Goldberg, G. Hail, E. R. Harcock, H. J. Hargis, E. L. Hart, M. J. Harwin, K. Hasegawa, M. Jobes, T. Kafka, G. E. Kalmus, D. P. Kelsey, T. Kitagaki, M. A. Mann, R. Merenyi, R. Milburn, K. C. Moffeit, J. J. Murray, A. Mapier, V. R. O'Dell, P. Rankin, H. Sagawa, J. Schneps, S. J. Sewell, J. Shank, A. M. Shapiro, J. Shimony, K. Tamai, S. Tanaka, D. A. Waide, M. Midgoff, S. Wolbers, C. A. Moods, A. Yanaguchi, G. P. Yost, and H. Yuta - Comparison of Lambda and Antil andada Inclusive Photoproduction at 20 bev with Cuark-Diquark Fusion Hodel," Proceedings, XxII International Conference on High-Energy Physics, Leipzig, East Gemany, July 19-25, 1984

Alton, G. D., R. H. Compton, and D. J. Pegg "Electron Decachment Spectroscopy of $20+120 \mathrm{keV} \mathrm{H}$ and He- Projectiles Interacting with Thin He and Ar Targets," Physical Review A

Awes, T. C., R. L. Ferguson, F. E. Obenshain, F. Plasil, G. R. Young, P. Braun-thunzinger, R. Freifelder,

P. Paul, L. Ricken, J. Stachel, P. de Young, and P.-H. Zhang

"A Large Acceptance Spectrometer for $\pi^{0}$ Mesons," Proceedings, Conference on Instrumentation for Heavy-Ion Huclear Research, Oak Ridge, Tennessee, Uctober 22-24, 1984

Balantekin, A. B.

"Aceidental Degeneracies and Supersymetric Quantum Mechanics," Annals of Physizs (New York)

Becker, R. L.

"Vacancy-Rearrangement Theory in the First Magnus Approximation," Proceedings, Second Workshop on High Energy Ion-Atom Collisions, Debrecen, Hungary, August 27-28, 1984

Becker, R. L., A. L. Ford, and J. F. Reading (Invited Talk) "Multiple Vacancy Production by High-Energy Heavy Ions," Proceedings, Second Workshop on High Energy Heavy-Ion Collisions, Debrecen, Hungary, August 27-28, 1984

Becker, R. L., and A. D. MacKellar "Theoretical Initial-(n,l) Dependence of Ion-Rydberg Atom Collision Cruss Sections," Journal of Physics B

Bemis, C. E., Jr., J. K. Beene, J.L.C. Ford, Jr., O. Shapira, and B. Shivakumar (Invited Paper) "Uevelopment of Polarized Targets for Subbarrier Fusion Studies," Proceedings, International Conference on Fusion Reactions Below the Coulomb Barrier, Cambridge, Massachusetts, June 13-15, 1984

Berman, B. L., and S. Datz "Channeling-Radiation Expertments," Topics in Current Physics, "Coherent Radiation Sources"

Berry, S. O., G. A. Glass, 1. A. Sellin, K.-U. Groeneveld, D. Hofmann, L. H. Andersen, M. Breinig, S. B. Elston, P. Enyar, M. M. Schauer, N. Stolterfoht, H. Schmidt-Bocking, G. Nolte, and G. Schiwietz "Shape of the Electron Capture to the Continuum Cusps for $\mathrm{H}, \mathrm{H}_{2}$, and He Tarjets in the Velocity Range 6.3 to 18.0 a.1..," Physical Review A

Bertrand, F. E., and J. R. Beene

"Giant Resonance Gamma Decay in 208 Po," Physics News in 1984

Bertrand, F. E., J. R. Beene, and M. L. Halbert

"Photon Decay of Giant Resonances," Proceedings, International Symposium on Nuclear Spectrnscopy and Nuclear Interactions, Osaka, Japan, March 21-24, 1984

Bertsch, G. F. (Invited Paper) "Nonrelativistic Theory of Heavy-Ion Collisions," Proceedings, School on Heavy-lon Physics, Erice, Sicily, July 17-23, 1984 
Bottcher, C.

"Transition State Theory for Monstationary Atomic and Kolecular Systems," Proceedings of the Royal Society of London A

Britt, H. C., M. M. Fowler, Z. Fraenkel, A. Gavron, J. Van der Plicht, J. B. Nilheliay, F. Plasil, T.

Awes, and G. R. Young (Invited Paper)

"Heavy-Ion-Induced Fission Reactions," Proceedings, International School-Seninar on Heavy-Ion

Physics, Alushta, Crimea, U.S.S.K., April 14-21, 1983

Carlton, R. F., J. A. Harvey, and C. H. Johnson

-Optical Model Scattering Functions for Low Enérgy Meutrons on $86 \mathrm{Kr}$." Proceedings, Meutrcn-Mucleus

Collisions: A Probe of Huclear Structure, Glouster, Ohio, September 5-8, 1984

Casson, H. H., D. P. Hutchinson, C. H. Ma, P. A. Staats, and J. B. Milgen

"Far-Infrared Laser Diagnostic on EBT and Extreme Far-Forward Laser Scattering on ISX," Proceedings, 5th Topical Conference on High Teqperature Plasma Diagnnstics, Tahoe City, California, Septemer $16-20,1984$

Chitwood, C. B., J. Aichelin, D. H. Boal, G. Bertsch, D. J. Fields, C. K. Gelbke, H. G. Lynch, H. B. Tsang, J. C. Shill cock, T. C. Ares, R. L. Ferguson, F. E. Obenshain, F. Plasil, R. L. Robinson, and G. R. Young - Final State Interactions Between Noncompound Light Particles for 160 Induced Reactions on 197 Au at $E / A=25$ MeV." Physical Review Letters

Cale, J. D., J. H. Hami lton, A. V. Ramayya, H. Lourens, B. van Mooijen, H. Kawakami, L. A. Mink, E. H. Spejewski, H. K. Carter, R. L. Mekodaj, G. A. Leander, L. L. Riedinger, C. R. Bingham, E. F. Zganjar, J. L. Mood, R. H. Fink, K. S. Toth, B. D. Kern, and K.S.R. Sastry "The Decay of $188 \mathrm{Tl}$ and Doserved Shape Coexistence in the Bands of $188 \mathrm{Hg}$, Physical Review C

Cusson, R. Y., P.-G. Reinhard, M. R. Strayer, J. A. Maruhn, and M. Greiner "Density as a Constraint and tite Separation of Internal Excitation Energy in TDHF," Zeitschrift fur Physik A

Datz, S., and C. D. Moak "Heavy-Ion Channeling," Heavy-Ion Science

Davies, K.T.R., K.R.S. Devi, S. E. Koonin, and M. R. Strayer "TOHF Calculations of Heavy-Ion Collisions," Heavy-Ion Science

Demas, M. G., C. H. Ma, D. P. Hutchinson, and P. A. Staats "Determination of Spatial Distribution of Plasma Electrons by Multi-beam FIR Interferometry," IEEE Journal of Plasma Science

Dennis, L. C., and S. Raman

"Location of a Doorway State Using the Channel $n+297 \mathrm{~Pb}, "$ Proceedings, Fifth International Symposium on Gama-Ray Spectroscopy and Related Topics, Knoxville, Tennessee, September 10-14, 1984

El-Saman, H., V. Barci, T. Bengtsson, A. Gizon, J. Gizon, L. Hildingsson, O. Jerrestam, H. Klamra, R.

Kossakowski, Th. Lindblad, Y. Cono, T. Bengtsson, and G. A. Leander "Dynamic Moments of Inertia in Xe, Cs, and Ba Muclei," Proceedings, International Symposium on In-Beam Nuclear Spectroscopy, Debrecen, Hungary, May 30-June 1, 1984

El-Samman, H., V. Barci, A. Gizon, J. Gizon, L. Hildingsson, D. Jerrestam, H. Klamra, R. Kossakowski, Th. Lindblad, T. Bengtsson, and G. A. Leander "On the Difference of the Dynamic Moment of Inertia alband (2) for Xe and Ba Nuclei," Nuclear Physics A

Elston, S. B., S. D. Berry, M. Breinig, R. DeSerio, C. E. Gonzalez Lepera, I. A. Sellin, K, -0.

Groeneveld, D. Hoffman, P. Kuschir, I. B. Nemirovski, and L. I. Liljeby "Doubly Differential Emission nistributions from Electron Loss to the Continuue from Fast Heavy Projectiles in Gas Targets," Pricecedings, Symposium on the Physics of Electron Ejection in Ion-Atom and Ion-Sol id Collitsions, Aarhus, Denmark, June 29-30, 1984

Erb, K. A. (Invited Paper) "Uuast-Molecular Single-Nucleon Effects in Meavy-Ion Collisions." Proceedings, Sth Adriatic International Conference on Nuclear Phystcs: Fundamental Problems in Heavy-Ion Collisions, Hvar. Yugoslavia, September 24-29, 1984

Erb, K. A., and U. A. Bromley "Heavy-Ion Resonances," Heavy-Ion Sctence 
Farid, H. E., and G. R. Satchler "One Effect of IIsing Relativistic Rinematics in the Anzlysis of Heavy-ion Elastic Scattering." Physics Letters B

Farid, M. E., and B. R. Satchler "Some Optical-Hodel Analyses of the Elastic Scattering of $40 \mathrm{Ar}$ at $1760 \mathrm{MeV}$." Muclear Physics A

Fetell, M. P., M. R. Johnson, F. X. HeGowan, J. S. Hattula, I. Y. Lee, C. Baktash, Y. Schutz, J. C. Mells, L. L. Riedinger, M. M. Lididry, and S. C. Pancholi "The Collectivity of $160,161 Y_{b}$ at Hign Spin." Physics Letters $B$

Ford, J.L.C. (Invited Paper) "Heavy Ions, Taryets, and Research at HiRf," Proceedings, 1983 Workshop of the Intemational Muclear Target Development Society, Argonne, Illinois, September 7-9, 1983

Fulmer, C. B., 6. R. Satchler, K. h. Erb, D. C. Hensley, R. L. Muble, J. B. Ball, F. E. Bertrand, and E. E. Gross "Elastic and Inelastic Scattering of 158 MeV 9Be Ions," Huclear Physics A

Gavron, A., J. Boissevain, H. C. Britt, K. Eskola, P. Eskola, M. M. Fowler, h. Mh, J. B. Wi lhelmy, I. C. Awes, R. L. Ferguson, F. E. Obenshain, F. Plasil, G. R. Toung, and S. Hald "Fission Cross Sections up to $20 \mathrm{MeV} / \mathrm{amu}$." Physical Review C

Glass, 6. A., P. Engar, S. B. Berry, A. Breinig, R. DeSerio, S. B. Elston, and I. A. Sellin "Electron Capture to the Continuum from Atomic Hydrogen," Proceedings, Eighth Conference on the Application of Accelerators in Research and I, idustry, Denton, Texas, Hovember 12-14, 1984

Goodnan, C. D,. R. C. Byrd, I. J. Van Heerden, T. A. Carey, D. J. Horen, J. S. Larsen, C. Gaarde, J. Rapaport, T. P. Nelch, E. Sugarbaker, and T. N. Taddeucci

"Heasurements of camow-Tel ler Strength Distributions in Mass 13 and 15," Physical Review Letters

Gregory, D. C. (Invited Paper)

"Indirect Processes in Electron-Impact Lonization of Multiply Charyed Jons," Proceedings, Eighth Conference on the Application of Accelerators in Research and Industry, Denton, Texas, Moveaber $12-14,1984$

Griffin, D. C., M. S. Pindzola, and C. Bettcher

"Distorted Have Calculations of Dielectronic Recombination Cross Sections in the Li Isoelectronic Sequence," Pnysical Review A

Guidry, M. W., R. E. Neese, C. R. Bingham, L. L. Riedinger, J. A. Vrba, I. Y. Lee, N. R. Johnson, G. R. Satchler, P. A. Butler, R. Donangelo, J. U. Rasmussen, D. L. Hillis, and H. H. Kluge

"Heavy-I on Inelastic Scattering from Deformed Ruclei," Nuclear Physics A

Horen, D. J.

"Isotopes," McGraw-H. 11 Encyclopedia of Science and Technology, Sixth Edition

Hutchinson, D. P., C. H. Ma, P. A. Staats, and K. L. Vander Sluis

"Far-Infrared Interferometry/Polarimetry on the ISX-B Tokamak," Proceedings, Third International

Conference on Infrared Physics, Zurich, Switzerland, July 23-27, 1984

Hutchinson, U. P., K. L. Vander Sluis, and J. Sheffield

"Feasibility of Alpha Particle Measurement by $\mathrm{CO}_{2}$ Laser Thomson Scattering," Proceedings, Ninth

International Conference on Infrared and Millimeter Waves, Takarazuka, Osaka, Japan, October 22-26, 1984

Hutchinson, D. P., K. L. Vander Sluis, J. Sheffield, and D. J. Sigmar

"Feasibility of Alpha Particle Measurement by $\mathrm{CO}_{2}$ Laser Thnmson Scattering," Proceedings, 5th Topical

Conference on High Temperature Plasma Diagnostics, Tahoe City, California. September 16-20, 1984

Johnson, C. H. (Invited Paper)

"Optical Models from Low-Energy s-, p-, and d-Have Cross Sections," Proceedings, Neutron-Nucleus

Collisions: A Probe of Nuclear Structure, Glouster, Ohio, September 5-8, 1984

Juncar, P., H. K. Carter, R. L. Mlekodaj, J. A. Bounds, C. R. Bingham, D. J. Pegg, and J. D. Cole "Hew Mechod to Measure Doppler Shift: First Results and Proposal, "Physical Review Letters 
Kin, H. J., T. C. hes, J. R. Beene, C. E. Benis, Jr., R. L. Ferguson, I. Y. Lee, F. K. McGowan, F. E. Obenshain, F. Plasil, V. Rauch, R. L. Robinson, and S. Steadan

"Calibration and Initial Experiments with the ORM -HIT Recoil Mass Spectroneter," Proceedings, International Conference on Fusion Reactions Below the Coulomb Barrier, Cambridge, Massachusetts, June 13-15, 1984

Klein, R. K., J. O. Kephart, R. H. Pantell, H. Part, B. L. Berman, R. L. Swert, S. Datz, and R. M. Fearick

"Electron Channeling Radiation from Diamond," Physical Review B

Robos, A. M., and 6. R. Satchler

"R Global Uptical Potential Analysis of $160+20$ Si Elastic Scattering," Muclear Physics A

Leander, 6. A.

-Sheil Effects on the El moments of Ra-Th Muclei." Proceedings, Fifth International Symposium on

Capture Gama-Ray Spectrosciopy and Related Topics, Knoxville, Tennessee, September 10-14, 1984

Leander, 6. A., P. Miller, J. R. Mix, and H. M. Howard

"Calculated Mosses and Half-iives for Muclei in the Region $100<Z<110$," Proceedings, Seventh International Conference on Atowic Masses and Fundamental Constants, Darmstadt, West Germany, Septemer 3-7, 1984

Leander, G. A., P. B. Semes, and F. Donau (Invited Paper)

"Unpaired Mucleons as Probes of Core Collective Fields," Proceedings, International Morkshop on Interacting Boson-Boson and Boson-Fermion Systems, Guli Lake, Michigan, May 28-30, 1984

Lisantti, J., J. R. Tinsley, D. M. Drake, I. Bergquist, L. M. Swenson, D. K. McDaniels, F. E. Bertrand, E. E. Gross, D. J. Horen, T. P. Sjoreen

Analyzing Power for the Inelastic Continuum with 2w0-tieV Protons," Physics Letters B

Ludewann, C. A., and B. J. Casstevens

"Operator Interface to the URIC Control Systew," Proceedings, European Physical Society Conferznce on Computing in Accalerator Design and Technolgy. Berlin, West Germany, September 20-23, 1983

Ma, C. H., D. P. Hutchinson, P. A. Staats, and K. L. Yander Sluis

"FIR Interferometer/Polarimeter System on ISA-B Tokamak," Prcceedings, 5th Topical Conference on High Temerature Plasma Diagnostics, Tahoe City, California, September 16-20, 1984

Marston, J. B., and 5. E. Koonin

"Mean-field Calculations of Fluctuations in Muclear Collisions," Physical Review Letters

Martin, M. J.

"Huclear Decay Data for Selected Radionuclides," Mational Council on Radiation Protection and Measurements Report, Appendix

MeDaniels, D. K., J. R. Tinsley, J. Lisantti, D. M. Drake, I. Bergqvist, L. W. Swenson, F. E. Bertrand,

E. E. Gross, D. J. Horen, T. Sjoreen, R. Lil jestrand, and H. Wilson "Cross Section and Analyzing Power Measurements for the Giant Resonance Region in $208 \mathrm{~Pb}$ with $200-H e V$ Protons," Physical Review C

Meyer, F. H.

"The CQNL ECK Multicharged IOn Source," Froceedings, International Conference on the Physics of Highly Ionized Atoms, Oxford, England, July 2-5, 1984

Moltz, D. M., J. P. Sullivan, R. E. Tribble, C. A. Gagliardi, K. S. Toth, and F. T. Avignone "Meutron-Defictent Mass Surface Between the $1 f_{7 / 2}$ and $19 g / 2$ Shells: The Masses of $77 \mathrm{Kr}$ and $79 \mathrm{Kr}$," Proceedings, Seventh International Conference on Atomic msses and Fundanental Constants, Darwstadt, West termany, September 3-7, 1984

Moltz, D. M., K. S, Toth, F. T. Avignone, III, H. Moma, B. O. Kern, R. E. Tribble, and J. P. Sullivan "Beta Decay of $76 \mathrm{KD}$ and the Level Structure of $76 \mathrm{Kr}$," Muclear Physics A

Mazarewicz, M., P. Olanders, I. Ragnarsson, J. Uudek, and G. A. Leander "Octupole Shapes in Huclei and Some Rotational Consequences Thereof," Proceedings, XIX Winter School on Physics Selected Topics in Kuclear Structure, Zakopane, Poland, April 13-15, 1984

Mazarewicz, H., P. Ulanders, I, Ragnarsson, J. Dudek, G. A. Leander, P. Moiler, and E. Ruchowskd "Analysis of Octupole Instability in Medium Mass and Heivy Nuclef," Nuclear Physics A 
Neskovic, $n$.

"The Effect of Transverse Correlations in Lon Channeling in Very Thin Crystals," Physical Reviev Letters

Park, H., J. U. Kephart, R. K. Klein, K. H. Pantell, B. L. Berman, S. Datz, and R. L. Suent "Electron Channeling Radiation from Diamonds with and without Platelets," Journal of Applied Physics

Phaneuf, R. A.

"Electron Capture by Multiply Charged Lons fron Hydrogen Atons at Low Energies," Proceedings, VIII International Seminar on Ion-Aton Collisions, Utrecht, The Netherlands, August 4-5, 1983

Phaneuf, R. A., and D. H. Crandall

"Collisions of Low-Eneryy Multiply Charged Ions," Proceedings, Atomic Physics Contractors" Workshop, Gaithersburg, Maryland, April 26-27, 1984

Plasil. F. (Invited Paper) "Recent Advances in Fusion-Fission Reactions," Proceedings, Tsukuba International Symposium on Heavy-Ion Fusion Reactions. Ibaraki, Japan, September 3-5, 1984

Ragnarsson, I., T. Bengtsson, W. Mazarewicz, J. Dudek, G. A. Leander, and C. Baktash "Evidence for Band Termination at Very High Spin in $158 \mathrm{Yb}$." Physical Review C

Raman, S., R. F. Carlton, J. C. Wells, E. T. Jurney, and J. E. Lynn "Thermal Keutron Capture Gama Rays from Sulfur isotopes: Experiment and Theory," Physical Review C

Raman, S., and B. Fogelberg (Invited Paper: "Dverlapping $\beta$ Decay and Resonance Neutron Spectroscopy." Proceedings, International Symosiun on Nuclear Spectroscopy and Muclear Interactions, Usaka, Japan, March 21-24, 1984

Ramayya, A. V., H. C. Ha, J. H. Hamilton, S. J. Robinson, K. Zhao, J. D. Cole, E. F. Zganjar, and E. H. Spejewski

"Competing Nuclear Structures in $182-188 \mathrm{Hg}$," Proceedings, International Symposium on In-Beam Huclear Spectroscopy, Uebrecen, Hungary, May 14-18, 1984

Rapaport, J., R. Alarcon, B. A. Brown, C. D. Goodman, D. Horen, T. Masterson, E. Sugarbaker, and T. H. Taddeucci

"The $5 I V(p, n)^{5 l} \mathrm{Cr}$ Reaction at $E_{p}=160 \mathrm{MeV}, "$ Nuclear Physics A

Rapaport, J., C. Gaarde, J. Larsen, C. Goulding, C. D. Goodman, C. Foster, D. J. Horen, T. Masterson, E. Sugarbaker, and T. H. Taddeucci The $19 \dot{F}(p, n) 19 \mathrm{Me}$ and $39 \mathrm{~K}(p, n)^{39} \mathrm{Ca}$ Reactions at Intermediate Energies and Quenching of the Gamow-Teller Strength," Nuclear Physics A

Remaud, B.

"A Linearized Kinetic Equation for the Transport of Fast Mucleons Through Muclei," Physics Letters B

Sellin, I. A.

"Forward Electron Production in Heavy Ion-Atom and Ion-Solid Collisions," Proceedings, International Conference on X-Ray and Inner Shell Processes in Atoms, Molecules, and Solids. Leipzig, East Germany. August 20-24, 1984

Sellin, I. A. "Convoy Electron Production by Heavy Ions in Solids," Proceedings, Eighth Conference on the Application of Accelerators in Research and Industry, Denton, Texas, November 12-14, 1984

Sellin, I, A., S. U. Berry, M. Breinig, C. Bottcher, R. Latz, M. Burkhard, H. Folger, H.-J. Frischkorn, K. -0 . Groeneveld, V. Hofmann, and P. Koschar

"Anomalous Kean Free Paths for Scattering of Convoy Electrons Generated by Fast, Highly Ionized Ions in Thin Solid Targets," Proceedings, Symposium on the Physics of Electron Ejection in Ion-Atom and Ion-Solid Collisions, Aarhus, Uenmark, June 29-30, 1984

Sellin, I. A., S. B. Elston, and S. D. Berry

"Recent Advances in Forward Electron Production Studies in Ion-Atom and Ion-Solid Collisions," Proceedinys, Second Workshop on High-Energy Ion-Atom Collision Processes, Debrecen. Hungary, August $27-2 B, 1984$

Semmes, P. B., G. A. Leander, and J. L. Hood (Invited Paper)

"Particle-Core Coupling Calculations for the Positive Parity States in the Odd-Mass Hy Isotopes as a Test of IBA Core Descriptions," Procecdings, International Workshop on Interacting Boson-Boson and Bosnrifermion Systems, Gull Lake, Michtgan, May 28-30, $19: 4$ 
Smapira, L., Conference Chairman

-Prograa and Abstracts for Conference on Instrumentation for Heavy-Ion Muclear Research," Oak Ridge, Tennessee, October 22-24, 1984

Shapira, D., J.L.C. Ford, Jr., R. Hovotny, B. Shi w.kunar, R. L. Parks, and S. I. Thernton

"The HutRf Supersonic Gas-Jet Target Facility." .irilear Instruments and Methods in Physical Research, Section A

Shapira, D., J. Comez del Canpo, J.L.C. Ford, Jr., B. Shivakumar, P. H. Stel son, B. A. Harmon, R. A.

Parks, and S. T. Thornton

"Huclear Fiysics Experiments with the Dal-Hidaf Supersonic Gas-Jet Target," Proceedings, Eighth Conference on the Application of Accelerators in Research and Industry, Denton, Texas, Noverier 12-14, 1984

Shapira, D., D. Schull, J.L.C. Ford, Jr., B. Shivakumar, R, L. Parks, R. A. Cecil, and S. Thornton -Observation of Angular Mowentuin Saturation in Deep-Inelastic Processes Involving Light Heavy Ions," Physical Review Letters

Stone, R. E., C. R. Binghan, L. L. Riedinger, R. M. Lide, H. K. Carter, R. L. Mekodaj, and E. H. Spejewski

The Decay of Mass-Separated $199 \mathrm{MP}$ and 1999Po," Mnysical Review C

Strayer. M. R. (Invited Paper)

"Chaos near the Coulomb Barrier? - Muclear Molecules," Proceedings, International Conference on

Fusion Reactions Belou the Coulonb Barrier, Cabridge, Massachusetts, June 13-15, 1984

Strayer, M. R. (Invited Paper)

- Huclear Molecules in Toff. - Proceedings, 5th Adriatic International Conference on Muclear Physics:

Fundamental Problems in Heavy-Ion Collisions, Hvar, Yugoslavia, September 24-29, 1984

Thorn, C. E., J. W. Ulness, E. K. Marburton, and S. Raman

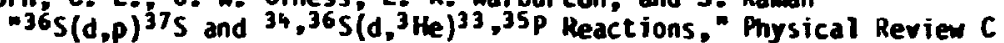

Toth, K. S., Y. A. Ellis-Akovali, C. R. Binghan, D. M. Moltz, H. K. Carter, R. L. Mlekodaj, E. H. Spejewski, and D. C. Sousa

"Decay Properties of $186 \mathrm{~Pb}$ and the Lead Alpha-Decay Rate Anomaly," Proceedings, Seventh International Conference on Atomic Masses and Fundamental Constants, Darmstadt, Mest Gerany, September 3-7, 1984

Toth, K. S., Y. A. Ellis-Akovali, C. R, dingham, D. M. Moltz, D. C. Sousa, h. K. Carter, R. L. Mlekodaj, and E. H. Spejewski

"Evidence from a Decay That $Z=82$ Is Not Magic Midway Between $N=82$ and $N=126$," Physical Review Letters

Toth, K. S., O. M. Moltz, E. C. Schloemer, M. O. Cable, F. T. Avignone, III, and Y. A. Ellis-Akovali "Beta-Delayed Proton Activities: $147 \mathrm{Dy}$ and $149 \mathrm{Er}, "$ Proceerings, Seventh International Conference on Atomic Masses and Fundamental Constants, Darmstadt, West Ger...3ny, September 3-7, 1984

Isang, M. B., H. G. Lynch, C. B. Chitwood, D. J. Fields, D. R. Klescr, C. K. Gelbke, G. R. Young, T. C. Awes, R. L. Ferguson, F. E. Obenshain, F. Plasil, and R. L. Robinson Azimuthal Correlations Between Light Particles Enitted in 160 -Induced Reactions on $12 \mathrm{C}$ and $197 \mathrm{Au}$ at $400 \mathrm{MeV}, "$ Physics Letters B

Uchai, H., C. H. Nestor, Jr., S. Raman, and C. R. Vane "Energy Shifts of $L X$ Rays from $70<Z<90$ Elements due to Multiple $M$ Vacancies," Atomic Data and Nuclear Data Tables

Umar, A. S.

"A Time-Uependent Mean-Field Theory for Prompt Mucleon Emission in Heavy-Ion Keactions," Proceedings, International Conference on Fusion Reactions Below the Coulomb Barrier, Cambridge, Massachusetts. June $13 \cdot 15,1984$

Umar, A. S., M. K. Strayer, D. J. Ernst, and K,K.S. Devi Mean-field Tneory of Prompt, High-Energy Hucleon Emission," Physical Review $C$

Hells, J. C., N. K. Johnson, J. Hattula, M. P. Fewell, D. R. Haenni, I, Y. Lee, F. K. MeGowan, J. W, Johnson, and $L$. L. Rtedinger

"Evidence for Collective Behavior in ${ }^{128} \mathrm{Ce}$ Prom Lifetime Measurements," Physical Review $\mathrm{C}$

Welton, T. A.

"Fluctuation as Explanation in Quantum Electrodynamics," Annals of Physics (New York) 
Winters, R. R., C. H. Johnson, and A. U. Mackellar

- Uptical Model for Low-Energy Meutrons on $60 \mathrm{Ni}$." Physical Review C

Nong, C. $Y$.

"Wucleon-Mucleus Reactions at UItra-Relativistic Energies," Physical Review D

Hong, C. Y.

Rotating Torodial Muclei in Heavy-Ion Reactions," Physical Review C

Young, G. F. (Lnvited Paper)

- Rear-Threshold Production of Neutral Pi Mesons in ihecry-Ion Reactions, Proceedings, 7th Oaxtepec Symposiua on Muclear Physics, Oaxtepec, Mexico, January 4-6. 1984

Ziegler, K. F., E. G. Richardson, J. E. Mann, P. K. Kloeppel, R. C. Juras, C. H. Janes, J. A.

Biggerstaff, J. A. Benjamin, and G. D. Alton

"Status of the Oak Ridge 25 URC Accelerator," Proceedings. Symposium of Northeastern Accelerator

Personnel, Rochester, New Vorix, October 3-5, 1983

Ziegler, K. F., E. G. Richardson, J. E. ihann, R. C. Juras, C. M. Jones, D. L. Haynes, J. A. Benjanin, and 6. D. Alton

"Status Report on the ORH 23 UKC Accelerator." Proceedings, 1984 Syposiuw of Northeastern Accelerator Personnel with Postaccelerator Workshop, Stony Brook, Wew York, Uctober 15-18, 1994

Zumbro, J. D., E. B. Shera, Y. Tanaka, C. E. Bewis, Jr., R. A. Maumann, M. V. Hoehn, H. Reuter, and R. M. Steffen "E2 and E4 Deformations in $233,234,235,238 \mathrm{U}$," Physical Review Letters 


\title{
12. PAPERS PRESENTED AT SCIENTIFIC AND TECHNICAL MEETINGS \\ October 1983 Through September 1984
}

\author{
List Prepared by Shirley J. Bail
}

1983

\author{
Syposiua of Mortheastem Accelerator Personnel, Rochester, Mew York, October 3-5, 1983
}

K. F. Ziegler, E. G. Richardson, J. E. Mann, P. K. Kloeppel, R. C. Juras, C. H. Jones, J. A. Biggerstaff. J. A. Benjamin, and 6. D. Alton

"Status of the Oak Ridge $25 \mathrm{mc}$ Accelerator"

\section{Merican Physical Society Meeting. Wotre Dame, Indiana, October 13-15, 1983}

C. Baktash, Y. Shutz, I. Y. Lee, F. McGowan, M. R. Johnson, M. P. Fevell, H. L. Halbert, D. C. Hensley,

L. Courtney, A. Larabee, L. L. Kiedinger, and D. G. Sarant ites

"Study of the Spin-Induced Shape Changes in $158 \mathrm{Yb}$, " Bull. Am. Phys. Soc. 28, 991 (1983)

M. E. Bunker, J. H. Starner, and S. Raman

Decay of 37S," Bull. An. Phys. Soc. 28, 968 (1983)

C. B. Chitwood, H. G. Lynch, M. B. Tsang, D. Fields, D. Klesch, A. D. Panagiotou, C. K. Gelbke, T. C.

Awes, R. Ferguson, F. Obenshain, F. Plasil, R. Robinson, and G. R. Young

"Two-Proton Correlations at Smail Rehative Momentum for 160-Induced Reactions on 197 Au at

$E / A=25 \mathrm{MeV}, "$ Bull. Am. Phys. Soc. 28, 973 (1983)

L. H. Courtney, L. L. Riedinger, A. J. Larabee, C. R. Bingham, C. Baktash, M. Halbert, D. Hensley,

N. R. Johnson. I. Y. Lee, Y. Schutz, J. Hattula, J. Haddington, B. Herskind, D. Haenni, D. Sarantites,

A. Dilmanian, M. Rajagopalan, S. Hjorth, and A. Johnson

"Discrete Gamma-Ray Studie's Using Spin Spectrometer." Bull. Am. Phys. Soc. 28, 982 (1983)

Y. A. Ellis-Akovali, K. S. Toth, D. M. Moltz, and D. C. Sousa

"Single-Particle States near $\hat{N}=82$; Search for the $N=81$ Isotope, ${ }^{149} \mathrm{Er}, "$ Bull. Am. Phys. Soc. 28 , 981 (1983)

J. Gomez del Campo (Invited Talk)

"Measurements of Huclear Deexcitatior. Times Using the Crystal Blocking Technique," Bull. Am. Phys. SOC. 28, 987 (1983)

M. L. Halbert (Invited Talk)

"Hew Vistas of Heavy-Ion Reactions with the Spin Spectrometer," Bull. Am. Phys. Soc. 26, 964 (1983)

H. Ikezoe, D. G. Kovar, G. Rosner, G. Stephens, E. Ungricht, B. Wilkins, T. Awes, G. R. Young, C.

Maguire, Z. Kui, H. C. Ma; S. Robinson, D. Watson, and G. Word

"Coincidence Measurements Between Evaporation Kesidues and Light Particles Produced in $160+40 \mathrm{Ca}$ at $E_{1 a b}(160)=160 \mathrm{MeV}, "$ Bul1. Am. Phys. Soc. 28, 974 (1983)

C. H. Johnson. N. H. Hill, J. A. Harvey, and D. J. Horen "Afterpulses of Several usec for an RCA-8854 Multtplier,"Bull. Am. Phys. Soc. 28, 992 (1983)

D. Klesch, C. B. Chitwood, D. Fields, C. K. Gelbke, H. G. Lynch, M. B. Tsang, A. D. Panagtotou, T. C. Awes, R. Ferguson, F. Ubenshain, F. Plasil, R. Robinson, and G, R. Young "Single and Two-Particle Measurements for Light Particle Emisston of 160-Induced Reactions at 400 MeV," Bull. Am. Phys. Soc. 28, 973 (1983)

A. J. Larabee, L. H. Courtney, S. Fravendorf, L. L. Riedinger, J. C. Haddington, M. P. Fewell, N. R. Johnson, I. Y. Lee, and F. K. McGowan

"Shape Effects in $h_{1 / / 2}$ and $9 / 2$ Bands in $159 \mathrm{Tm}, "$ Bull. Am. Phys. Soc. 28, 982 (1983) 
U. C. Ha, A. V. Ramayya, R. Zhao, J. H. Hailton, H. E. Barclay, S. Robinson, J. D. Cole, E. F. Zganjar,

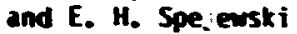

"High Spin States in 182,184Hg," Bull. Na. Phys. Soc. 28, 989 (1983)

A. D. Mackellar and C. H. Johnson

"Coupled Channel OP Analysis with Parity Dependence for $n+{ }^{0} \mathrm{Ca}, "$ Bull. Am. Phys. SoC. 28, 982 (1933)

0. H. Holtz, K. S. Toth, J. P. Sullivan, R. E. Tribble, C. A. Gagliardi, and F. T. Avignone III "hass of 75kr," Bull. Am. Phys. Soc. 28, 970 (1983)

S. Raan (Invited Talk)

-Uverlapping B Decay and Resonance Neutron Spectroscopy," Bull. Na. Phys. Soc. 28, 972 (1983)

A. V. Ranayya, M. C. Ma, J. H. Hanilton, H. E. Barclay, K. Zhao, S. Robinson, J. D. Cole, E. F. Zganjar, and $E$. H. Spejewski

-Identification of $182 \mathrm{Hg}$ with Near-Spherical and Deforwed States," Bull. Am. Phys. Soc. 28, 989 (1983!

Zhao Kui, C. F. Maguire, J.L.C. Ford, Jr., D. Shapira, F. E. Bertrand, E. Gross, R. O. Sayer, and T. P. Sjoreen

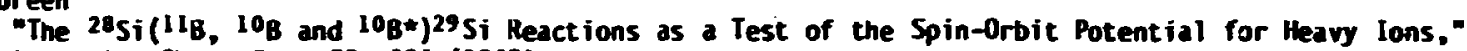

Bull. Nm. Phys. Soc. 28, 994 (1983)

1983 Muclear Science Symposium, San Francisco, Californta, October 19-21, 1983

D. C. Hens ley

"Data Acquisition with the Event Handler

H. R. Johnson, C. Baktash, and I. Y. Lee (Invited Talk)

"Bismuth Germanate's Role in the Mew Revolution in Gawa-Ray Spectroscopy"

1983 Annual Neeting of the Southeastern Section of the American Physical Society, Columbia, South Carolina, Noveaber 3-5, 1983

B. D. Kern, K. S. Toth, O. M. Moltz, J. Lin, F. T. Avignone, III, and G. Leander "Beta Decay of $75 \mathrm{~kb}, "$ Bull. Am. Phys. Soc. 28, 130 (1984)

R. A. Phaneuf (Invited Talk)

"Electron Capture by Multiply Charged Ions from Hydrogen Atoms at Low Energies," Bull. Am. Phys. Soc. 29, 123 (1984)

I. A. Sellin (Invited Talk)

"Some 01d, New, Borrowed, and Blue Topics in Accelerator-Related Atomic Physics," Bull. Am. Phys. Soc. 29, $123(1984)$

American Physical Society Meeting. San Francisco, Calffornia, November 20-23, 1983

B. L. Berman, S. Datz, J. U. Kephart, R. K. Klein, R. H. Pantell, H. Park, R. L. Swent, M. U. Alguard, and $M$. V. Hynes

"Channeling Radiation from LiH," Bull. Am. Phys. Soc. 28, 1322 (1983)

H. Park, R. H. Pantell, R. L. Swent, J. O. Kephart, R. K. Klein, B. L. Berman, S. Datz, and R. H. Feartck

"Comparison of Channeling Radiation from Diamonds with and without Platelets," Bull. Am. Phys. Soc. 28, $1323(1983)$

Horkshop on Electronic and Iontc Collisions Cross Sections Meeded in the Modeling of Radiation Interaction with Matter. Argonne, Illinots, December 6-8, 1983

D. C. Gregory

"The Cont.rolled fusion Atomic Data Center" 
1904

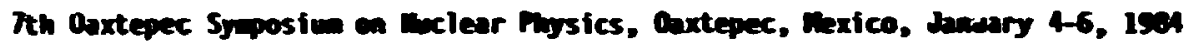

6. R. Young (Invited Talk)

- Mear-Threshold Production of heutral Pi nesons in Heary-Ion Reactions"

International bortshop en Myperfine Interactions, Rapor, India, damary 16-25, 1594

C. E. Rewis, Jr. (Invited Talk)

-Laser Optical Puping in Iuclear Physics: Fission Isomers, Oriented Targets, and Hyperfine Puping in Single-Electron Atos:

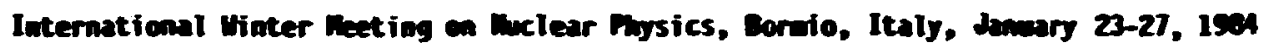

T. C. hues, R. L. huble, F. E. Certrand, R. L. Haibert, O. C. Hensley, I. Y. Lee, V. O. Rouch, R. L. Robinson, D. 6. Sarantites, and 6. R. Toung

-Fast Particles in Colncidence with $y$ Rajs from the $12 \mathrm{C}+60 \mathrm{ni}$ and $12 \mathrm{C}+120 \mathrm{Sa}$ Reactions"

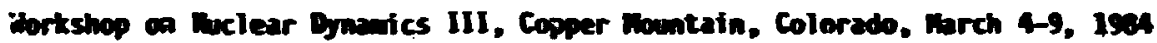

T. C. Mres, R. L. Ferguson, R. Movotny, F. E. Uenshain, F. Plasil, Y. Rauch, H. Sann, and 6. R. Youny (Invited Talk)

-Projectile Fission in Salli-Induced Reactions at $15.3 \mathrm{meV} / \mathrm{u}^{*}$

A. C. Mignerey, C. Merouane, S. Bradley, D. denton, H. Breuer, J. D. Silk, K. Twiatowski. V. E.

Viola, Jr.. T. C. Ares, F. E. Wenshain, and S. Pontoppidan

"Quasi Elastic Structure in the Reaction $50 \mathrm{Fe}+56 \mathrm{Fe}$ at $14.6 \mathrm{NeV} / \mathrm{fucl}$ eon"

F. Plasil, T. C. Mes, B. Cheynis, D. Brain, R. L. Ferguson, F. E. Obenshain, A. J. Sierk, S. 6.

Steadan, G. R. Young, A. Gavron, J. Boissevain, H. C. Britt, K. Eskola, P. Eskola, M. M. Fowler, Z.

Fraenkel, H. Him, J. van der Plicht, ans S. Wald (Invited Talk)

"Recent Results in Heavy-Ion-Induced Fission"

S. T. Thornton, R. L. Parks, D. Shapira, D. Schull, J.L.C. Ford, Jr., and B. Shivakumar "Osservation of a Critical Angular Momentum for Deep Inelastic. Processes with Light Heavy Ions"

C. Y. Wong (Invited Talk)

-Initial Energy Density of Quark-Gluon Plasma in Relativistic Heavy-Ion Collisions"

C. Y. Wong (Invited Talk)

-Baryon Distribution in Relativistic Heavy-Ion Collisions"

International Symposium on Muclear Spectroscopy and Muclear Interactions, Osaka, Japan, March 21-24, 1984

F. E. Bertrand, J. R. Beene, and M. L. Halbert "Photon Decay of Giant Resonances"

S. Raman (Invited Talk)

"Overlapping Decay and Resonance Keutron Spectroscopy"

Workshop on Detectors for Relativistic Auclear Collisions, Berkeley, California, March 26-30, 1984

A. Poskanzer, H-G. Ritter, B. Ludewigt, K. Foley, S. Borenstein, E. Platner, d. Love, D. Keane, and F. PIasti

"Event Parameters - Fixed Target"

American Chemical Society, Division of Muclear Chemistry and Technology, St. Louis, Hissouri, Aprt1 8-13, 1984

R. L. Ferguson (Invited Talk) "Heavy-Ion-Induced Fission of Medium-Energy Compound Systenis with Projectile Energies up to 25 MeV per Nucleon" 
xix minter school en Mysics Selected Topics in Meclear Structure, 2akopane, Poland, April 13-15, 1984

W. Mazarenicz, P. Olanders, I. Ragnarsson, J. Oudek, and 6. A. Leander "Octupole Shapes in maclei and Some Rotational Consequences Thereof"

Merican Pussical Society Reeting, Mashington, D. C., April 23-26, 1984

J. R. Beene (Invited Faper)

"Crystal Ball Studies of Giant Resonance Decay," Bull. An. Phys. Soc. 29, 620 (1994)

P. Brauntunzinger, P. Paul, L. Ricken, J. Stachel, P. H. Zhang, 6. R. Yow: ,, F. E. Wenshain, and E. Grosse

-Collectivity in Subthreshold Pion Production," goll. M. Phjs. Soc. 29, 672 (1984)

Y. A. Ell is-Akcr-Ii, K. S. Toth, O. M. Holtz, F. T. Avignone, E. C. Schloever, and M. D. Cable -Search for the it Isomeric Transition in $149 \mathrm{Er}$. Buil. Am. Phys. Soc. 29, 718 (?994)

C. B. Fulner, D. C. Hensley, R. L. Ruble, J. B. Ball, K. A. Erb, E. E. Gross, 6. R. Satchler, D. Shapira, and Y-d. Ohan Elast ic Scattering of 140-hey 'Be on 12C and 160," Bull. Am. Phys. Soc. 29, 624 (1984)

J. Comez del Capo, R. Ribis, K. Reskovic, D. Shapira, J. A. Biggerstaff, C. D. Moak, P. D. Miller, J.P.F. Sell lschop, and R. W. Fearick -Crystal alocking Measurements for Elastic Transfer Reactions and a-Particle Erission for the $160+$ mat je Sjystem," Bul1. An. Phys. Soc. 29, 698 (1984)

D. J. Horen, F. E. Bertrand, E. E. Gross, T. Sjoreen, J. B. NcClelland, T. A. Carey, S. J.

Seestron-tiorris, $K_{\text {. Thomas, }}$. K. McDaniels, j. R. Tinsley, d. Lisantti, and L. Simenson "Excitation of E2 Transitions in ${ }^{40} \mathrm{C} 2$ by 334-teV Protons," Bull. Am. Phys. $50 \mathrm{C}$. 29,628 (1984)

C. H. Johnson, H. W. Hill, J. A. Harvey, and D. J. Horen -Afterpulsas from Residual Gases in RCA-69854 and RCA-4522 mitipliers, Bull. Am. Phys. Soc. 29, 564 (1984)

G. A. Leander (Invited ialk) "Learning to Read the Muclear Structure Story Told by Yrast y Rays," Eull. An. Phys. Soc. 29, 726 (1984)

Y. G. Lynch, C. B. Chitmood, O. J. Fields, C. K. Gelbke, D, R. Klesch, M. H. Isang, G. R. Young, T. C. Ames, R. L. Ferguson, F. E. Ubenshain, F. Plasil, and R. L. Robinson "Large-Angle Correlations Between Honequilibriun Light Particles Enitted in 160 -Induced Reactions at $E / A=25$ HeV," Bull. An. Phys. Soc. 29, 672 (1994)

A. D. Hackellar, C. H. Johnson, and R, R, Minters -Deformed Optical Model Potential for s-Have Meutrons on $60 \mathrm{Ni}$ in the Kilovolt Energy Regton," Bull. Aa. Phys. Soc. 29, 636 (1984)

G. A. Petitt, A. Gavron, J. R. Rezne, B. Chenis, R. L. Ferguson, F. E. Obenshain, F. Plisil, G. R. Young, $M$. Jazskelainen, D. G. Sarantites, and C. F. Maguire "Systematics of Tonequilibrium teutron Enission in Inelastic Reactions ${ }^{22} \mathrm{C}+150 \mathrm{Gd}$ and $20 \mathrm{Me}+150 \mathrm{Md}$," Bul1. An. Phys. Soc. 29, 673 (1984)

R. V. Ribas, N. R. Johnson, I. Y. Lee, D. R. Haenni, L. L. Riedinger, R, M. Diamond, F, S. Stephens, S. Shih, and $H$. Kluge "Coulamb Excitation of 160Dy with Lead Ions," Bull. An. Phys. Soc. 29, 688 (1964)

D. Shapira, B. Shivakumar, J. Gomez del Campo, J.L.C. Ford, Jr., P. H. Stelson, B. A. Harmon, and S. T. Thornton

"On ene Equilibration of the Mass Degree of Freedom in Orbiting Processes," Bull. Am. Phys. Soc. 29, $626(1984)$

B. Shivakumar, D. Shapira, J. Gomez del Campo, J.L.C. Ford, Jr., P. H. Stelson, B. A. Harmon, and S. T. Thornton

"Backward Angle Yields of Products from ${ }^{28}$ St + 14N Collitsions," Bull. Am. Phys. SoC. 29, 626 (1984)

K. S. Toth, D. M. Moltz, F. T. Avi ynone, E. C. Schloemer, M. D. Cable, and Y. A. Ellis-Akovalt "Identification of Iinger via Its B-Delayed Proton Activity," Bull. An. Phys. SoC. 29, 716 (1984) 
D. Wang, R. Alarcon, P. Weich, J. Rapaport, D. Horen, T. M. Taddeucci, C. O. Coodan, E. Sugarbaker, and C. Sarde

-The 57,50fe(p,n)57,58co Reactions at $120 \mathrm{MeV}, "$ Bull. Am. Phys. Soc. 29, 717 (1984)

R. R. Minters, C. H. Johnson, and A. D. Mackellar

- A Spherical Optical Potential for s-Wave Reutrons on 60Ni in the Kilovolt Energy Region," Bull. M. Phys. Soc. 29, 636 (1984)

J. D. Zumbro, R. A. Mumann, E. B. Shera, M. V. Hoehn, W. Reuter, C. E. Bemis, Jr., Y. Tanaka, and R.

M. Steffen

"Precision huonic-Atom Determinations of the Charge Paraeters for $233,234,235,238 U, " 3 u 11$. Am. Phys. Soc. 29, 720 (1984)

Atomic Myssics Contractors' Workshop, Eathersburg, Maryland, April 26-27, is84

R. A. Phaneuf and D. H. Crandall

"Collisions of Low-Energy Multiply Charged Ions"

Tenth International Conference on Cyclotrons and Their Applications, East Lansing, Hichigan, April $30-\ln 3$ 3, 1984

E. D. Hudson, R. S. Lord, M. L. Mallory, and T.- A. Antaya

"Dual Arc Penning Ion Source Gas Flow Experiments," Bul1. Am. Phys. Soc. 29, 837 (1984)

C. M. Jones (Invized Talk)

"Review of the Current Status of Large Electrostatic Accelerators," Bull. Am. Phys. Soc. 29, 849 (1984)

J. A. Martin (Invited Talk)

"Conference Introduction: The International Cyclotron Conference's Twenty-five Years of Progress," Bul1. H. Phys. Soc. 29, 833 (1984)

International Symposium on In-Bean thuclear Spectroscopy, Debrecen, Hungary, Hay 14-18, 1984

H. El-Saman, V. Barci, T. Bengtsson, A. Gizon, J. Gizon, L. Hildingsson, D. Jerrestan, W. KIanra, R. Kossakouski, G. A. Leander, and Th. Lindblad

"Collective thoment of Inertia of $110,122 \times \mathrm{e}$ and $128,130 \mathrm{Ba}$ "

A. V. Ranayya, H. C. Ma, J. H. Hanilton, S. J. Robinson, K. Zhao, J. D. Cole, E. F. Zganjar, and E. h. Spejewski

"Competing Muclear Structures in $182-18 \mathrm{Hg}^{\circ}$

Workshop on Polarized Targets in Storage Rings, Argonne, Illinois, May 17-18, 1944

C. E. Beats, Jr., J. R. Beene, J.L.C. Ford, Jr., D. Shapira, and B. Shivakumar (Invited talk) "Development of Optically Pumped Polarized Jet Targets for Use in Heavy-Ion Reaction Studies"

Thirteenth International Colloquium on Group Theoretical Methods in Physics, College Part, Maryland, May 21-25, 1984

H. H. Khalfl and M. M. Shal aby

"An Alternative Residue Expansion for the Scattering Amplitude"

Conference on the Intersections Between Particle, and Wuclear Physics, Steanboat Sp-ings, Colorado, May 23-30, 1984

G. R. Young (Invited Talk)

"Accelerator-Colliders for Relativistic Heavy lons or in Search of 'uminosity" 
International Workshop on Interacting Boson-Boson and Boson-Fermion Systews, Gull Lake, Michigan, May 28-30, 1984

G. A. Leander, P. B. Semes, and F. Donau (Invited Talk)

"Unpaired 'ucleons as Probes of Core Collective Fields"

P. B. Semmes, G. A. Leander, and J. L. Mood (Invited Talk) -Particle-Core Coupling Calculations for the Positive Parity States in the Odd-Hass Hg Isotopes as a Test of IBA Core Descriptions"

Horkshop on Impurity Control Physics, Atlanta, Ceorgia, May 30-31, 1984

R. A. Phaneuf

"Atomic Processes Involving Impurities in the Plasma Edge"

American Physical Socfety (Division of Electron and Atomic Physics), Storrs, Ccnnecticut, May 30-June 1. 1984

R. L. Becker anc A. D. Mackellar

"Calculations of Collisions of Ions with Rydberg Atoms in High |m| States," Bull. Am. Phys. Soc. 29, 795 (1984)

C. Bottcher

"A Classical Theory of Recombination in Perturbed Environments," Bull. Am. Phys. Soc. 29, 792 (1984)

C. Bottcher

"Time-Dependent Self-Consistent Field Study of a Heavy-Ion Collision: F9+ Me,"Bull. Am. Phys. Soc. 29, 813 (1994)

C. Bottcher and T. G. Heil

"Charge Transfer in Few-Electron Systems Using One-Electron Eigenstate Expansions," Bull. Am. Phys. Soc. 29, 810 (1984)

S. Datz (Invited Talk)

"Beams Experiments on Dielectronic Recombination," Bull. Am. Phys. Soc. 29, 784 (1984)

S. B. Elston, M. Breinij, I. Sellin, S. Berry, D. Hofmann, P. Koschar, and I. B. Memi rovsky "Two-Dimensional Energy/Angular Distributions of ELC Electrons for $41-\mathrm{MeV}$ and 82-MeV $05+$ Projectiles on He and Me," Bull. Am. Phys. Soc. 29, 777 (1984)

P. Focke, I. B. Nemirorsky, E. Gonzalez Lepera, W. Meckbach, I. A. Sellin, and K. O. Groeneveld "Bgam Fofl Convoy Electron Distribution as a Function of Energy and Angle of Emission," Bull. Am. Phy 5. Soc. 29, 817 (1984)

A. M. Howald, R. A. Phaneuf, D. H. Crandall, and D. C. Gregory "single, Double, and Triple Ionization of $\mathrm{Xe}^{6+}$ by Electron Impact," Bull. Am. Phys. Soc. 29, 804 (1984)

F. H. Meyer "The ORNL ECR Multicharged Ion Suurce," Bull. Am. Phys. Soc. 29, 810 (1984)

R. A. Phaneuf, C. C. Havener, and H. F. Krause "Ion-Atom Merged Beams," Bul1. Am. Phys. Soc. 29, 812 (1984)

M. S. Pindzola, O. C. Griffin, and C. Bottcher "DraCula - A Dielectronic Recombination Cross Section Code," Bull. Am. Phys. Soc. 29, 805 (1984)

J. F. Reading (with A. L. Ford and R. L. Becker) (Invited Talk) "Charge Transfer and the Development of Techniques for Numerical Calculations of Ion-Atom Collisfors," Bull. Am. Phys. Soc. 29, 821 (1984)

Conference of the Belgian Physical Society, Louvain-la-Neuve, Belgium, June 7-8, 1984

P. UeFrance, U. Belic, F. Broutllard, S. Chantrenne, O. Gregory, J. Jureta, and S. Rachaff "Experimental Study of the Ionization of Multicharged Ions" 
International Conference on Fusion Reactions Below the Coulob Barrier, Caporidge, Massachusetts, June 13-15, 1984

C. E. Bemis, Jr., J. R. Beene, J.L.C. Ford, Jr., D. Shapi ra, and B. Shivakunar (Invited Talk) "Development of Polarized Targets for Sutuarrier Fusion Studies"

H. J. Kin, T. C. Awes, J. R. Beene, C. E. Bemis, Jr., R. L. Ferguson, I. Y. Lee, F. K. McGowan, F. E. Obenshain, F. Plasil, V. Rauch, R. L. Robinson, and S. Steadaan

"Calibration and Initial Experiments with the ORRL HIT Recoil Mass Spectrometer"

H. R. Strayer (Invited Talk)

"Chaos near the Coulow Barrier? - Nuclear Molecules"

A. S. Uhar

"A Tir.e-Dependent Mean-Field Theory for Prompt Mucleon Eaission in Heary-Ion Reactions"

Symositum on the Physics of Electron Ejection in Ion-Atom and Ion-Solid Collisions, Mrhus, Denmark, June 29-30, 1984

S. B. Elston, S. U. Berry, M. Breinig, R. DeSerio, C. E. Conzalez Lepera, I. A. Sellin, K.-0.

Groen veld, D. Hoffman, P. Koschar, I. B. Newi rovski, and L. I. Li jeby

"Oc ibly Differential Emission Distributions from Electron Loss to the Continuum from Fast Heavy Projectiles in Gas Targets"

I. A. Sellin, S. D. Berry, M. Breiníg, C. Bottcher, R. Latz, M. Burkhard, H. Folger, H. -J. Frischkorn,

K. -0. Groeneveld, D. Hofmann, and P. Koschar "Anomalous Mean Free $P_{i}$ : hs for Scattering sf Convoy Electrons Generated by Fast. Highly Ionized Ions in Thin Solid Targets"

International Conference on the Physics of Highly Ionized Atoms, Oxford, England, July 2-5, 1984

F. W. Meyer

"The ORNL ECR Multicharyed Ion Source"

School on Heavy-Ion Physics, Erice, Sicily, July 17-23, 1984

G. F. Bertsch (Invited Talk)

"Nonrelativistic Theory of Heavy-Ion Collisions"

XXII International Conference on High-Energy Physics, Leipzig, East Germany, July 19-25, 1984

K. Abe, R. Armenteros, T. C. Bacon, J. Ballam, H. H. Bingham, J. E. Brau, K. Braune, D. Brick, H. M. Bugg, J. M. Butler, W. Cameron, H. G. Cohn, D. C. Colley, G. T. Condo, P. Dingus, R. Erickson, R. C. Field, B. Tranel, R. Gearhart, T. Glanzman, I. M. Godfrey, J. J. Goldberg, G. Hail, E. R. Hancock, H. J. Hargis, E. L. Hart, M. J. Harwin, K. Hasegawa, M. Jobes, T. Kafka, G. E. Kalmus, D. P. Kelsey, T. Kitagaki, W. A. Mann, R. Merenyi, R. Milburn, K. C. Moffeft, J. J. Murray, A. Napier, V. R. O'Dell, P. Rankin, H. Sagawa, J. Schneps, S. J. Sewell, J. Shank, A. M. Shapiro, J. Shimony, K. Tamat, S. Tanaka,

D. A. Waide, M. Widgoff, S. Hcibers, C. A. Woods, A. Yamaguchi, G. P. Yost, and H. Yuta "Charm Photoprociuc:ion at $20 \mathrm{GeV}$ Including Preliminaiy Lifetime Results with Improved Optical Resolution"

K. Abe, R. Armenteros, T. C. Bacon, J. Ballam, H. H. Bingham, J. E. Brau, K. Braune, D. Brick, H. M. Bugg, J. M. Butler, H. Cameron, H. U. Cohn, D. C. Colley, G. T. Condo, P. Dingus, R. Ertckson, R. C. Fteld, B. Franek, R. Gearhart, T. Gianzman, I. M. Godfrey, J. J. Goldberg, G. Hall, E. R. Hancock, H. J. Hargis, E. L. Hart, M. J. Harwin, K. Hasegawa, M. Jobes, T. Kafka, G. E. Kalimus, D. P. Kel sey, T. Kitagakt, W. A. Mann, R. Merenyt, R. Milburn, K. C. Moffett, J. J. Murray, A. Napler, V. R. O'Dell, P. Rankin, H. Sagawa, J. Schreps, S. J. Sewell, J. Shank, A. M. Shapiro, J. Shimony, K. Tamat, S. Tanaka,

D. A. Haide, M. Widgoff, S. Woibers, C. A. Hoods, A. Yamagucht, G. P. Yost, and H. Yuta "Comparison of Lambda and Antilambda Inclusive Photoproduction at $20 \mathrm{GeV}$ with Quark-Diquark Fusion Model"

9th International Conference on Atomic Physics, Seattle, Washington, July 23-27, 1984

C. Bottcher and M, R. Strayer

"Hurierical Solutions of the Dirac Equation Describing Collisions Between Very Heavy Ions" 
C. Bottcher, R. Latz, M. Burghardt, H.-J. Frischkorn, K.-U. Groeneveld, D. Hofiann, P. Koschar, S. D. Berry, M. Breinig, and I. A. Sellin "Anowalous Mean Free Paths of Convoy Electrons in Foils: A Couloab Focussing Effect?"

P. Engar, M. Breiniy, R. DeSario, I. A. Sellin, C. E. Gonzalez-Lepera, S. D. Berry, M. Bloewer, and T. Undermood "Field-Ionization of Foil-Excited Rydbery States of Fast Oxygen Ions"

P. M. Griffin and C. C. Havener "EUV Radiation from keV Electron Impact on Tantalum"

Third International Conferenct on Infrared Physics, Zurich, Switzerland, July 23-27, 1984

D. P. Hutchinson, C. H. Ma, P. A. Staats, and K. L. Vander Sluis "Far-Infrared Interferonetry/Polarimetry on the ISX-B Tokamak"

Tenth International Conference on Particles and Huclef, Heidelberg, West Germany, July 30-hugust 3, 1984

C. S. Mishra, G. S. Blanpied, B. M. Preedom, B. G. Ritchie, R. S. Moore, M. Blecher, K. Gotpx, R. L. burman, M. V. Hynes, E. Piasetzky, N. S. Chant, P. G. Roos, F. E. Bertrand, T. P. Sjoreen, F. E. Obenshain, and E. E. Gress

"Elastic $\pi^{ \pm}$Scattering from ${ }^{14} \mathrm{C}$ at $50 \mathrm{MeV}$

B. Fick, H. Bleciner, K. Gotow, D. Mright, G. Blanpied, J. A. Escalante, C. S, Mishra, B. M. Preedom,

R. L. Burman, M. Hynes, C. Ciangaru, N. Chant, P. Roos, B. G. Ritchie, F. E. Bertrand, E. E. Gross,

F. E. Ubenshain, and W. Burger " ${ }^{ \pm}$Elastic Scattering Sensitivity to the Keutron Radius"

International Conference on X-Ray and Inner Shell Processes in Atoms, Molecules, and Solids, Leipzig, East Germany, August 20-24, 1984

R. L. Becker, A. L. Ford, and J. F. Reading "Inclusion of Electron Transfer in the Calculation of KnLV Multiple Vacancy Production by Ion Impact"

I. A. Sellin

"Forward Electron Production in Ion-Atom and Ion-Solid Collisions"

American Chemical Society, Division of Muclear Chemisty and Technology, Philadelphia, Pennsylvania, August 26-31, 1984

G. A. Leander (Invited Talk)

"Spectroscnpy of Rotating Reflection-Asymmetric Muclef"

Second Morkshop on High Energy Ion-Atom Collisfons. Debrecen. Hungary, August 27-28, 1984

R. L. Becker "Vacancy-Rearrangement Theory in the First Magnus Approximation"

R. L. Becker, A. L. Ford, and J. F. Reading (Invited Talk) "Multiple Vacancy Production by High-Energy Heavy Ions"

I. A. Sellin "Hortzons of Forward Electron Production in Ion-Aton and Ion-Soltd Collisions"

IMS-RIKEN International Symposfum on Heavy-Ion Physics, Mt. Fuj1, Japan, Rugust 27-31, 1984

J. R. Beene, F. E. Bertrand, M. L. Halbert, D, C. Hensley, R. L. Auble, D. J. Horen, R. L. Robinson, T. P. Sjoreen, and R. U. Sayer (Invited Talk) "Crystal Ball Studies of Giant Resonance Vecay"

Tsukuba International Sympostum on Heavy-Ion Fuston Reactions, Ibarakt, Japan, September 3-5, 1984

F. Plasil (Invited Talk)

"Recent Advances in Fusion-fission Reactions" 
H. Yanada, D. 6. Sarantites, R. L. Robinson, M. L. Halbert, D. C. Hensley, V. Rauch, R. L. Muble, F. E. Bertrand, and J. H. Hanflton

"Partial Fusion Reaction in Crystal Ball Spin Spectio eier"

Seventh International Conference on Atomic Masses and Fundamental Constants, Darastadt, West Cermany, September 3-7, 1984

6. A. Leander, P. Mller, J. R. Mix, and M. M. Howard

"Calculated Masses and Half-Lives for Muclei in the Region $100<Z<110^{*}$

D. H. Moltz, K. S. Toth, F. T. Avignone, J. P. Sullivan, R. E. Tribble, and C. A. Gagliardi "Keutron-Deficient Mass Surface Between the $1 f_{7 / 2}$ and $199 / 2$ Shells"

K. S. Toth, Y. A. Ellis-Akovali, C. R. Binghan, D. M. Moltz, H. K, Carter, R. L. Mlekodaj, E. H. Spejews $i$, and $D$. C. Sousa

"Decay Properties of $186 \mathrm{~Pb}$ and the Lead Alpha-Decay Rate Ancilaly*

K. S. Toth, D. H. Moltz, E. C. Schloewer, H. D. Cable, F. T. Avignone, III, and Y. A. Ellis-Akovali "Beta-Delayed Proton Activities: 1970y and $149 \mathrm{Er}$

Meutron-Mucleus Collisions: A Probe of lluclear Structure, Glouster, Ohio, September 5-8, 1984

R. F. Carlton, J. A. Harvey, and C. H. Johnson "Optical Model Scattering Functions for Low Energy Meutrons on 86 Kr"

C. H. Johnson (Invited Talk)

"Optical Models from Low-Energy s-, p-, and d-Wave Cross Sections"

Fifth International Sympsium on Gama-Ray Spectroscopy and Related Topics, Knoxville, Tennessee, September 10-14, 1984

J. R. Beene, F. E. Bertrand, M. L. Halbert, D. C. Hensley, R. L. Auble, D. J. Horen, R. L. Robinson,

T. P. Sjoreen, and R. O. Sayer (Invited Talk)

"Crystal Ball Studies of Giant Resonance Meutron and Gamma Decay"

L. C. Dennis and S. Raman

"Location of a Doorway State Using the Channel $n+207 \mathrm{~Pb}$ "

B. Fogelberg, J. Harvey, M. Mizumoto, and S. Raman

"Observation of Extremely Low s-wave Strength in the Reaction $136 \times e+n$ "

Z. Gacsi, J. Sa, J. L. Meill, E. T. Jurney, and S. Raman

"Decay Scheme of Ii6sn from $\left(n, n^{\prime} y\right)$ and $(n, y)$ Results"

G. A. Leander "Nuclear Structure of Neutron-Rich Fission Products"

G. A, Leander

"Shell Effects on the El Moments of Ra-Th Nuclei"

N. K. Sherman, H. F. Davidson, S. Raman, H. Delbianco, G. Kajrys "Comparison of Photoabsorption by 160 and $180^{\circ}$

5th Topical Conference on High Temperature Plasma Diagnostics, Tahoe City, California, September 16-20, 1984

C. A, Bennett, D. P. Hutchinson, K. L. Vander Sluis, and P. A. Staats

"Oevelopment of Sequence and Regular Band Lasers for Use as Local Oscilliators in Thomson Scattering Alpha Particle Diagnostics"

H. H. Casson, D. P. Hutchinson, C. H. Ma, P. A. Staats, and J. B. Wilgen "Far-Infrared Laser Diagnostic on EUT ano Extreme Far-Forward Laser Scattering on ISX"

D. P. Hutchinson, C. H. Ma, K. L. Vander Sluis, and P. A. Staats

"Feasibility of Alpha Particle Measurement by $\mathrm{CU}_{2}$ Laser Thomson Scattering" 
C. H. Ma, D. P. Hutchinson, P. A. Staats, and K. L. Vander Sluis "FIR Interferometer/Polarimeter System on ISX-B Tokamak"

1984 Federation of Malytical Chemistry and Spectroscopy Societies, Philadelphia, Pennsylvania, September 16-21, 1984

C. R. Vane

"Environental Effects in High-Resolution X-Ray Satellite Spectra"

Sth Mriatic International Conference on Muclear Physics: Fundanental Problens in Heavy-Ion Collisions, Hvar, Yugoslavia, September 24-29, 1984

K. A. Erb (Invited Talk) "Puasi Holecular Single-ltucleon Effects in Heavy-Ion Collisions"

H. R. Strayer (Invited Ialk) "Huclear Molecules in MHF" 


\section{GENERAL INFORMATION}

MWOUMCEREWTS

A. Baha Balantekin was appointed Eugene P. Uigner Fellow ef fective September 10, 1984. George F. Bertsch ws appointed UT/Onk Distinguished Scient ist ef fect ive hugust 6, 1984.

PERSOMEL COUMEES

New Staff Nembers

A. Scientific Staff

M. Breinig, University of Tennessee, Knoxville, Tennessee (part-time)

8. L. Burks, University of North Carolina, Chapel Hill, Worth Curolina

D. T. Dowling, Tennessee Technological University, Cookeville, Iennessee

R. A. Erb (transferred from Central Management Offices)

C. W. Glover, Indiana University, Blocanington, Indiana

D. L. Haynes (transferred from Engineering Division)

H. T. Hunter, University of Washington, Seattle, Washington

8. Administrative and Technical Staff

H. R. Dinehart, Accelerator Operations

J. R. Ellis, Secretary (transferred from Central Managenent offices)

J. R. Heath, Secretary

C. A. Irizarry, Accelerator Operations

R. L. McPherson, Accelerator Operations

\section{Staff Transfers and Terminations}

A. Scientific Staff

J. A. Benjamin (present address: Brookhaven Mational Laboratory, Upton, New York)

J. L. C. Ford, Jr. (deceased)

C. B. Fulmer (deceosed)

R. S. Lord (ret frement)

J. E. Mann (retirement)

T. P. Sjoreen (transferred to Solid State Division)

C. L. Viar (retfrement)

B. Adainistrative and Technical Staff

V. K. Hill, Secretary (retirement)

J. C. Sharp, Laboratory Technician (transferred to Laboratory Protection Division)

P. T. Singley, Accelerator Operations (transferred to Energy Oivision) 
TEMPORAT ASSIGinETTS

Short-Tern Assignments

Scientific Staff

M. B. Neskovic, Boris Kidric Institute of Muclear Science, Belgrade, Yugoslavia

Guest Assignments

A. Graduate Students

S. D. Berry, University of Tennessee, Knoxville, Tennessee

H. J. Bloemer, University of Tennessee, Knoxville, Yennessee

H. P. Carpenter, University of Tennessee, Xnoxville, Tennessee

M-C. Chu, California Institute of Technology, Pasadena, California

L. H. Courtney, University of Tennessee, Xnoxville. Tennessee

M. E-A. H. Farid, Ass iut University, Assiut, Egypt

G. A. G!ass, Univerity of Tennessee, Knoxville, Tennessee

R. M. Kincaid, University of Tennessee, Knoxville, Tennessee

A. J. Larabee. Uaiversity of Tennessee, Knoxville, Tennessee (on leave from Rashaster University)

S. E. Lasley, University of Tennessee, Knoxville, Tennessee

X. T. Lis, University of Tennessee, Knoxville, Tennessee

R. S. Moore, Indiana University, Bloomington, Indiana

K. J. Nyherg, University of Tennessee, Knoxville, Tennessee (on leave from Research Institute for Atomic Physics, Stockholm, Sweden)

J. Shimony, University of Tennessee, Knoxville, Tennessee

B. Shivakumar, Yale University, Mew Haven, Connecticut

R. T. Short, University of Terinessee, Knoxville, Tennessee

K. M. Teh, Vanderbilt University, Mashville, Tennessee

J. S. Thompson, University of Tennessee, Knoxville, Tennessee

H. Uchai, Emory Uni versity, At lanta, Georgia

S. A. Unar, Yale University, Mew Haven, Connect Icut

T. A. Underwood, University of Tennessee, Knoxville, Tennessee

B. Co-op Students

D. E. Hoglund, Virginid Polytechnic Institute and State University, Blacksburg, Virginia

M. A. Halker, Morehouse College, Atlanta, Georgia

C. ORAU Graduate Research Participant

J. A. Bounds, University of Tennessee, Knoxville, Tennessee

D. ORAU Postgraduate Research Training Program

C. C. Havener, North Carolina State University, Raleigh, North Carolina

A. H. Howald, University of Wisconsin, Madison, Wisconsin

E. ORAU Faculty Research Participant

C. A. Bennett, Ir., University of North Carolina, Asheville, Morth Carolina 
F. Caw Student Research Participants

J. W. Cooke, Eckerd College, St. Petersburg, Florida

L. T. Daly, Mount Holyoke College, South thadley. Hassachusetts

F. A. Harrison, Dartmouth College, Hanover, Men Haspshire

L. B. Lurio, Columbia University, Mew York, Mew York

6. L. Morford, Edinboro thi versity of Pennsylvania, Edinboro, Pennsylvania

D. H. Ol ive, Carson-lieman Coll lege, Jefferson City, Tennessee

D. Thouson, Lanrence University, Appleton, Misconsin

6. Creat Lakes Col lege Association Science Progran

D. R. Shepard, Lmence Iniversity, Appleton, Misconsin

H. Southern Colleges and Universities Union Science Progran

T. L. Hart, Centenary College of Laisiana. Shreveport, Louisiana

$$
\text { Sumer Assigneats }
$$

A. Undergraduate Student

E. H. Lee, Massachusetts Instit.ute if Technology, Cabridge, Mussachusetts

B. Graduate Students

E. A. Goode, University of Tennessee, Xnoxville, Tennessee

C. R. Mhan, University of Virginia, Charlottesville, Virginia

J. B. Marston, Princeton University, Princeton, Mew Jersey 
PHTSICS DIVISION SEMIMARS: OCTOBER I983-SEPTERBER 1984

Those sewinars arranged by the Physics Division and announced in the ORRL Technical Calendar are listed below. During the period of this report, I. Y. Lee served as Seminar Chairman.

Date

Speaker

Title

1983

oct. 6

Oct. 20 Karl L. Kratz, Univ. of Mainz, Mainz, Federai Republic of Geraany

Oct. 27 Cheuk -Y in Hor.g

Physics Division Staff

Mov. 3 Julrich Heinz, Vanderbilt University, Mashville

Mov. 1n G. F. Bertsch, Michigan State University, East Lansing

Nov. 16 Erhard Salzborn, Justus Liebig University, Giessen, F.R.G.

Dec. 1 Gunther Rosner, Argonne National Laboratory

Dec. 8 Rolf Scharenberg, Purdue University, Laf ayette, Indiana

Dec. 15 F. T. Avignone, Iniversity of South Carolina, Columbia

1984

Feb. 2 J. Wilcyznski, Michigan State University. East Lansing

Feb. 3 Cheuk -Yin Wong, ORNL Staff Memier

Feb. 23 S. Pontoppidan, Niels Pohr Institute, Copenhagen, nenmark

March 2 Mark J. Rhoades-Brown, State University of New York at Stony Brook, New York

March 13 Helmut 0. Oeschler, Institut für Kernphysik, Darmstadt, F.R.G.

March 15 T. Ddssing, Lawrence Berkeley Laboratory

March 16 U. Winkler, University of Heidelberg, F.R.G.

March 20 Peter Twin, Daresbury Lahoratary Waritington, U.K.

March 22 Rainer Novotny, University of Giessen, F.R.G.

April 10 Surrender Satni Argonne National Laboratory
What do we Know About Shell Model States in the Tin Region

Beta Strength Function Phenomena of Short-Lived Neutron-Rich Isotopes

Energy Density of Quark-Gluon Plasma in

Relativistic Heavy-Ion Collisions

Quark-Gluon Transport Theory

From IDHF to Mewtonian Mechanics in Heavy Ion Collisions

Experiments with Multiply Charged Ions: Transfer Ionization and Electron Impact Ionization

Complete and Incomplete fusion

Muclear Fragmentation as a Critical Phenomena

Double Beta Decay: Status and Future Projections

Role of the Entrance-Channel Angular Momentum in the Mechanism of Heavy -Ion Reactions at Low Energy

Stopping Power of Nuclear Matter

Angular Momentum Transfer in Deeply Inelastic Heavy -I on Coll isions

A Self-Consistent Description of Low Energy Heavy-Ion Reactions

Linear Momentum Transfer Studies at Energies Around $100 \mathrm{MeV} / \mathrm{u}$.

Dynamics of Angular Momentum in Damped Nuclear Reactions

Three-Nuclei Fragmentation in Light-Ion Reactions at $11 \mathrm{MeV} / \mathrm{n}$

Probe the r-Ray Continuum with TERESA

Recent Results from the Darmstadt-Hefdelberg Crystal Ball

Resonance. Phenomena in Neavy -Ion Reactions 
Date

Speaker

April 5 K. U. Schroeder University of Rochester

April 9 Frederick E. Milis Ferni lational Accelerator Laboratory

April 12 Indrek Martinson University of Lund, Sweden

Aprii 19 Fred Petrorich Florida State University Tallahassee

May 17 Teng Lek thoo Argonne Mational Laboratory

May 18 Armon McPherson Morth Carolina State University Raleigh

May 24

Mike M. Euidiry University of Tennessee Oak Ridge Mational Laboratory

May 31 Fleming Videbaek Niels Bohr Institute, Copenhagen

June 1 Michel Vergnes Institute of Muclear Physics Orsay, France

June 7 Cheuk-Yin Wong

Physics Division Staff

June Il Roland A. Dayras

Center for Huclear Study (CEN)

Saclay, France

June 14

Peter Morsch

Institute for Muclear Physics

Nuclear Research Installation (KFA)

Jülich, F.R.G.

June 19 I. Hertel

Free University of Berlin

Berlin, F.R.G.

June $26 \quad k$. Goeke

Nuclear Research Installation (KFA)

Julich, F.R.G.

June 28 D. Guerreau

GANIL, Caen, France

July 12 Claire Schuck

Orsay, France

July 17 Robert L. Varner

University of North Carolina

July 19 K. D. Hildenbrand

GSI, Darmstadt, F.R.G.

July 26 Hans Ströher

Universiey of Giessen, F,R.G.
Iitle

Mass and Energy Iransport in Heavy Ion Collisions

Electron Beam Cooling

Optical Studies of Ion-Atom Interactions

Orerview of the Scattering of Elementary Probes from Muclei

Entrance Channel Dependence in Compound Wucleus Decay

A New Radiometric Standard for the EUY

Direct Reactions and High-Spin Physics

Strong Coupling Effects in Heavy-Ion QuasiElastic Reactions

Change of Structure in the Germanium Region \& the Interacting Boson Model

Incoherent Multiple Collision Description of Nucleon-Nucleus Reactions at Ultra-Relativistic Energy

Projectile Fragmentation at GaNIL Energies

New Giant Resonance Results from Jutich

Collision of Laser Excited Atons

Quantized ATDHF for Low-Energy Heavy-Ion Reactions

Present Status of GANIL and Recent Experimental Results

Rotational Inertia and Pairing in good Rotors of the Rare Earth Region

A Global Parameterization of the Nucleon-Nucleus Optical Model

Aspects of Deep-Inelastic Reactions Between Mo and Mo at Incident Energies from 12 to $18 \mathrm{MeV} / \mathrm{u}$

Electrofission of Pre-actinfo Nuclei 
Date

Speaker

Aug. 2

Peter noiler

Lund Inst $i^{\prime}$ ute of Technology

Lund, Swe sen

Aug. 9 Volker E. Oberacker

Vandertilt University

Sept. 4 H. Kl apisch

Racah Institute of Physics Hebrew University of Jerusalen Is rael

Sept. 6

D. H. Madkarni

Bhabha Atomic Pesearch Center

Trombay, Bombay. India

Sept. 13 Jim Feagin

California State University Fullerton

Sept. 18 H. V. KI apdor Max Plank Institute Heidelberg, F.R.G.

Sept. 20 Patrick J. Daly Purdue University

Sept. 25 Osamu Miyamura Osaka University Toyonaka, Usaka, Japan
Title

Mass Formula Comining the best Features of the Droplet Model and the Finite Range Model A Nicroscopic Approach to the Calculations of
Heavy Ion Potentials

An Efficient Progran for Relativistic Distorted Waves for Highly Ionized Heavy Atons

Results of Recent Fission Experíments from Trombay

Mannier Threshold Theory

Neutrino Spectra in the Reactor Core \& Reactor Decay Heat

Spectroscopy of $\mathrm{N}-\mathrm{O2}$ Wuclei Near the Proton Drip Line

Cosmic Ray Events Above TeV/amu: Is There Any Phase Transition? 
SCIENTIFIC REETINGS SPCWSORED BY THE PHYSICS DIVISION ANO HELD DURINS THE REPORTING PERIOO

\section{Fifth International Symposium on Capture Gana-Ray}

Spectroscopy and Related Topics

Knoxville, Tenn., Septenber 10-14, 1984

S. Raman, organizer

Information Meeting, December 1983

The most recent Physics Division Information Meeting was held on December 12-14, 1983.

The members of the Advisory Cormittee were:

S. E. Xoonin, California Institute of Technology

E. Merzbacher, University of North Carolina

R. E. Pollock, Indiana University

O. K. Scott, Michigan State University 


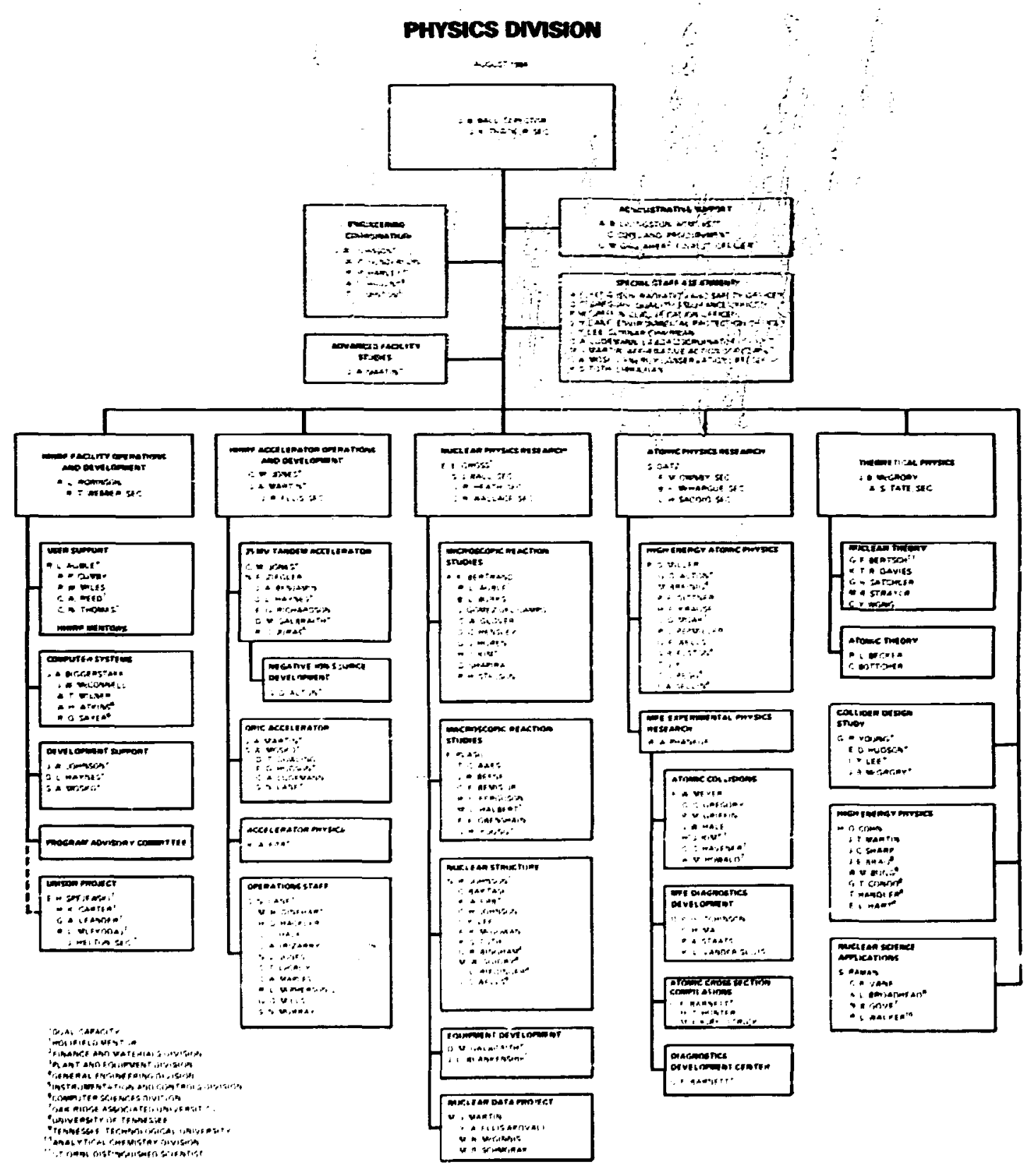


ORNL -6120

Distribution Category

UC-34

I RTERMAL DISTRIBUTION

1. G. D. Alton

2. T. D. Anderson

3. B. R. Appleton

4. R. L. Auble

5. S. I. Auerbach

6. T. C. Ames

7. C. Baktash

B. A. B. Balantekin

9-129. J. B. Ball

130. S. J. Ball

132. C. F. Barnett

132. R. L. Becker

133. J. R. Beene

134. C. E. Benis, Jr.

135. J. A. Benjamin

136. F. E. Bertrand

137. G. F. Bertsch

138. J. A. Biggerstaff

139. C. R. Bingham

140. Biology Library

141. C. Bottcher

142. J. E. Brau

143. W. M. Bugg

144. B. L. Burks

145-146. Centrai Research Library

147. H. D. Cohn

148. G. T. Condo

149. R. P. Cumby

150. S. Datz

151. K. T. R. Davies

152. P. F. Dittner

153. D. T. Dowling

154. B. G. Eads

155. J. R. Ellis

156. Y. A. Ellis-ikovali

157. S. B. Elston

158. .K. A. Erb

159. R. L. Ferguson

160. C. W. Glover

161. J. Somez del Campo

162. D. C., Gregory

163. P. M. Griffin

164. E, E, Gross

165. M. H. Guidry

166. R. L. Hahn

167. M. L. Halbert

168. T. Handler

169. B. A. Harmon

170. E. L. Hart

171. J. A. Harvey

172. L. J. Hawkins-Saddif

173. D. L. Haynes

174. J. R. Heath

175. D. C. Mensley

176. R. D. Hippler

177. D. J. Horen

178. E. D. Hudson

179. D. P. Hutchinson

180. L. H. Jenkins

181. C. H, Johnson

182. J. H. Johnson

183. N. R. Johnson

184. C. M. Jones
125. P. R. Kasten

186. S. V. Kaye

187. H. J. Kim

188. H. F. Krause

189. E. H. Krieg, Jr.

190-191. Laboratory Records Department

192. Laboratory Records, ORNL R.C.

193. G. A. Leander

I94. I. Y. Lee

195. A. B. Livingston

196. R. S. Lord

197. C. A. Ludenann

198. C. H. Ma

199. J. E. Mann

200. J. A. Martin

201. M. J. Martin

292. J. H. McConnell

203. F. K. HcGowan

204. J. B. McGrory

205. B. F. Mcharque

205. G. S. Mctleilly

207. F. H. Meyer

208. R. W. Miles

209. P. D. Miller

210. H. T. Hilner

211. R. L. Mlekodaj

212. S. H. Mosko

213. F. R. Mynatt

214. F. E. Obenshain

215. D. K. Olsen

216. ORNL - Y-12 Technical Library Document Reference Section

217. F. M. Ounby

218. D. C. Parzyck

219. R. H. Peelle

2.20. D. J. Pegg

221. P. L. Pepmiller

222. F. G. Perey

223. R. A. Phaneuf

224. J. J. Pinajian

225. F. Plasil

226. H. Postma

227. M. L. Poutsma

228. S. Raman

229. E. G.' Richardson, Jr.

230. L. L. Riedinger

231. R. L. Robinson

232. T. M. Rosscel

233. M. J. Seltmarsh

234. G. R. Satchler

235. R, D. Sayer

236. M. R. Schmorak

237. H. D. Schults

238. I. A. Sellin

239. D. Shapira

240. J. Sheffield

241. B. Shivakumar

242. T. P. Sjoreen

243. A. H. Sneli

244-246. E. H. Spefewsk f

247. P. A. Staats

248. P. H. Stelson

249. M. R. Strayer 
250. A. S. Tate

251. J. K. Thacker

252. K. S. Toth

C53. D. B. Trauger

254. K. L. Vander Sivis

255. C. R. Vane

256. C. R. Hallace

297. R. T. Mebber

258. G. F. Hells

259. J. C. Wells, Jr.

260. K. K. Wilkinson

261. C-Y. Wong

262. G. R. Young

263. N. F. Ziegl':r

264. A. Zucker

\section{EXTERNAL MISTRIBUTION}

265. B. J. Allen, Physics Division, Australian Atomic Energy Commission, Sutherland, N.S.W., Australia

266. C. H. Annett, U.S. Army - CAORA (Physics), 5445 Charlotte, Kansas Ciciy, Missouri 64110

267. Charles A. Barnes, Division of Physics and Astronony, California Institute of Technology, Pasadena, California 91125

268. G. A. Bastin, Centre de Spectrometrie Mucleaire et de Spectrometrie de Masse, B. P. 104, 91405 Orsay, France

269. H. Behrens, Zentralstelle fur Atomkernenergie-Dokumentation, Kernforschungszentrum Karlsruhe 7514, Eggenstein-Leopoldshafen-2, Federal Republic of Germany

270. Ingmar Bergstrom, Nobel Institute of Physics, Stockholm 50, Sweden

271. Biblioteca do Depto. de Fisica Huclear, Instituto de Fisica da USP, Caixa Postal 20516. 0100 - Suo Paulo, SP, Brasil

272. Bibliotheque - Madame Belle, Universite de Grenoble, Institut des Sciences Mucleaires, 53, rue des Martyrs, B. P. 21, 38 Grenoble, France

273. J. P. Blaser, Swiss instit.e for Nuclear Research (SIN), CH-5234 Viliigen, Switzerland

274. S. D. Bloom, University of california, Lawrence Livermore National Laboratory, P. 0. Box 808, Livermore, California 94550

275. H. G. Blosser, Cyclotron Laboratory, Michigan State University, East Lansing, Michigan 48824

275. H. Bohn, Physik-Departinent E12, Technische Universitat, 8046 Garching bei Munchen, Federal Republic of Germany

277. A. Bohr, Copenhagen Iniversity, Niels Bohr Institute, Blegdamsvej 17, Copenhagen, Denmark

278. J. S. Briggs, Theoretical Physics Division, Atomic L.ergy Research Establ:shment, Harwell, Didcot, Oxford:hire OX 11 ORA, England

279. J. A. Brink, Library Division, The Merensky Institute of Physics, University of Stellenbosch, Stellenbosch, Republic of South Africa

280. 7. A. Bromley, Nuclear Structure Laboratory, Yale University, New Haven, Connecticut 06520

281. T. A. Cahill, Director, Crocker Nuclear Lahoratory, University of California, Davis, California 95616

282. Jose L. S. Carvalhs, Instituto de Radioprotecao e Dosimetria, C.N.E.N., Ay, das Americas km 1l,5 Barra de Tijuca - R. J., 22700 - Rio de Janeiro, R. J. Brazil

283. David Clark, Lawrence Berkeley Laboratory, University of California, Berkeley, California 94720

284. D. H. Crandall, Branch Chief for Experimental Research, Applied Plasma Physics Division, Office of Fusion Energy, ER 542, G-226, GTN, U.S. Department of Energy, Wasnington, D.C. 20545

285. F. L. Culler, office of the President, Electric Power Restarch Instituce, P. 0. Box 10412, 3412 Hillview Avenue, Palo Alto, California 94303

286. T. J. Curtin, Director, Office of Kesearch and "irants Administration, Texas Women's University, Box 22939. TWU Station, Denton, Texas 76204

287. R. Y. Cusson, Physics Department, Duke University, Durham, North Carolina 27706

288. Solange de Barros, Head, Department of Nuclear Physics, Universidade Federal do Rio de Janeiru, Instituto de Fisica - Department Fisica Nuclear, Centro de Technología - Bloco A, Il ha co Fundan - Rio de Janeíro, Brasil

289. Adriano de Lima, Physics Laboratory, University of Coimbra, Coimbra, Portugà

290. R. M. Diamond, Chemistry Division, Lawrence Berkeley Laboratory, Berkeley, California 94720

291. Diacio Dietzsch, Depto. de Fisica Experimental, Instituzo de Fisica, Iniversidade de Sao Paulo, Cx. Postal 20516. Sao Paulo, S.P., Brazil

292. I. Facchini, Physics Department. University of Milan, Via Salidini 50, Milan, Italy

293. H. Faraggi-Mathieu, Centre d'Etudes Aucleaires de Saclay, B. P. No. 2, Gif-Sur-Yvette (S. and 0$.$) , France$

294. M. P. Fewell, Australian National University, Canberra, 2600 Australia

295. G, N. Flerov, Laboratory for Nuclear Reartions, Dubna Joint Institute for Nuclear Research, Dubna, Hoscow Oblast, IJ,S.S.R. 
296. J. D. Fox, Department of Physics, Florida State University, Tallahassee, Florida 32306

297-306. QCA-ORS, Attention: R. R. Winters, Denison University, Granville, Ohio 43023

307. H. E. Gove, Nuclear Structure Laboratory, University of Rochester, BIdg. 510A, Rochester, New York 14627

308. Walter Greiner, Institut fur Kernphysik der Johann Holfgang Goethe Universitat, Frankfurt an Main. Federai Republic of Germany

309. H. Grunder, Lawrence Berkeley Laboratory, Berkeley, California 94120

310. M. Grypeos, Iniversity of Thessaloniki, Department of Theoretical thysics, Thessaloniki, Greece

311. J. H. Hamilton, Department of Physics, Vanderbilt University, Hashville, Tennessee 37203

312. K. Harada, Division of Physics, Japan Atomic Energy Research Institute, Tokai Research

Institute, Postal Area Mumber 319-11. Tokai-mura, Maka-gun, Ibaraki-ken, Japan

313. J. S. Hattula, Department of Physics, University of Jyvaskyla, Seminaarinkatu 15, $\$ F-40100$ Jyvaskyla 10, Finland

314. R. L. Heath, Idaho Kational Engineering Laboratory, EGsG Idaho, Inc., P. 0. Box 1625, Idaho Falls, Idaho a3401

315. H. R. McK. Hyder, Nuclear Physics Laboratory, Oxford University, Keble Road, Oxford OXI 3RH, England

316. Institute for Energy Analysis, P. D. Box 117, Oak Ridge. Tennessee 37830

317. Institute of Physics, High Energy and Nuclear Physics Library, C. Postal 20.516,0100 - SaO Paulo, S.P., Brasil

318. Beth Jinkerson, ORAU/UPD

319. R. Kamermans, Fysisch Laboratorium, Rijksuniversiteit ltrecht, P. 0, B0x 80.000, 3508 TA UTRECHT, The Hetherlands

320. H. Kamitsubo, Head, Cyclotron Laboratory, Institute of Physical and Chemical Research, Wako-shi, Saitama, 351 Japan

321. ก. G. Kanke, Ruhr-Universitat Bochum, Dynamitron Tanden Laboratory, Universitattskr, 150 Gibaunde NT, Postfach 1021 48, 4360 Bochum 1, Federal Republic of Garmany

322. H. Kawakami, Institute for Nuclear Study, University of Tokyo, Midori-cho, Tokyo, Japan

323. P. K. Kloeppel, Associate Professor, Department of Physics, Monmoutr. College, Monmouth, Illinois 61462

324. M. Langevin, Institut de Physique Nucleaire, B. P. Mo. 1, 91406 Orsay Cedex, France

325. J. A. Lenhard, DOE-ORC, Federal Office Building, Dak Ridge. Tennessec 37830

326. Michel Letournel, Centre de Recherches Nucleaires, Service des Acce!erateurs, B. P. 20 CRO, 67037 St rasbourg Cedex, France

327. Librarian, Atomic Energy Centre, P. 0. Box No. 164, Ramna, Dacca, Bancladesh

328. Librarian, Chen Kin-hai, Institute of Modern Physics, Academia Sinica. $P .0$. Box 31, Lanzhou, People's kepublic of China

329. Librarian. Cyclotron Laboratory, Michigan State University, East Lan',ing, Michigan 48824

330. Librarian, Cyclotron Laboratory, RlKEN (The Inscitute of Physical and Chemical Research), Wako-shi, Sa itama 351, Japan

331. Librarian, Department of Physics, Georgia State University, Atlanta, Georgia 30303

332. Librarian, GANIL, B. P. No. 5027, 14004 Caen Cedex, France

333. Librarian, GSI, Postfach 110541,6100 Darmstadt, Federal Republic of Germany

334. Liorary of the Institute of Atomic Energy, Beijing, People's Republic of China

335. Librarian, MERT Division Library, Cak Rídge Associated Universities, P. 0. Box 117, Dak Ridge, Tennessee 37831-0117

336. Librarian. Physics Nepartment, 374 Bausch and Lomb Building, University of Rochester. Rochester, New York 14627

337. Librarian, Institut des Sciences Nucleaires, B. D. No. 257 - Centre de Tri, 38044 Grenoble Cedex, France

338. A. D. Mackellar, Department of Physics and Astronomy, University of Kentucky, Lexington, Kentucky 40506-0055

339. G. Madurga, Departameto de Física Atomica y Muclear, Facultad de Ciencias, Universidad de Sevilla, Sevilla, Spain

340. Mario Hariscotti, Comision Nacional de Energia Atomica, Departmento de física, Avenida del Líbertador 8250,1429 Ruenos Aires, Argentina

341. Niels Marquardt, Institut fur Experimentalphysik III der Ruhr-Universitat Bochum, Postfach 102148 Gehaude $18 / 3$, 4631) Bochum, Feceral Republic of Germany

342. J. V. Martinez, Division of Chemical Sciences, Hail Stop J309 CTN, IJ.S. Department of Energy, Washington, n.C. 20545

343. W. R. Mcilurray, Deputy Chief Scientist, Kerninstituut Van Die Suldelike Universiteite, Southern Universities Nuclear Institiste Republic of Souch Africa

344. G. K. Mehta, Professor of Physics, Van de Graff Laboratory, Indian Institute of Technolony Kanpur, Kanpur 26,8 015, India

345. A. Michaudon, Chef du Service de Physique Nucleaire, Commissariat a 1 Energie Atonifue, Centre d'Etude: de Bruyeres le Chatel, P. 0. Box 61, Montrouge 921?0, France

346. R. lifddleton, Department of Physics, Iniversity of Pennsylvania, Philadelphia, Pennsylvania 19104

347. E. Mignecc, INFN Lahoratorío Nazionale del Sur, Corso Italia 57, 95127 Catania, Italy 
348. J. C. O. Milton, Physics Division, Atomic Energy of Canada Ltd., Chalk River, Canada KOJ 1J9

349. 6. C. Morrison. Department of Physics, University of Birmingham, Biraingham B15 2TT, England

350. S. C. Mukherjee, Librarian, Saha Institute of Muclear Physics, 92, Acharya Prafulla Chandra Road, Calcutta - 9, India

351. K. Hagatani, Institute for Nuclear Study, University of Tokyo, Ianashi, Tokyo, 188 Japan

352. Y. Hakamura, JPL. Systens Analysis Section, California Institute of Technology, 4800 Dak Grove Orive, Pasadena, California 91103

353. Ranier M. Hovotny, University Giessen, II. Physical Institute, Heinrich-Buff-Ring 16, 6300 Giessen, Federal Republic of Germany

354. Masumi Oshima, Japan Atomic Energy Research Institute, Tokai Establishment, Iokai-mura, Maka-gun. Ibaraki-ken 319-11, Japan

355. Peter Paul, Department of Physics, S.U.H.Y. at Stony Brook, Stony Brook, New York 11794

356. Max Peisach, Southern Universities Huclear Institute, P. 0. Box 17, Faure, 1131, Republic of South Africa

357. Elliott S. Pierce, Assistant Director, Molecular Sciences, Department of Energy, Washington, D.C. 20545

359. A. - Pilt, Tandem Accelerator Laboratory, Mchaster University, Hamilton, Ontario, Canada LB5 4K1

359. R. E. Pollock, Department if Physics, Indiana Univorsity, 8loomington, Indiana 47405

360. B. Povh, lwax Planck Institıt fur Kernphysik, 69 Heidelberg, Saupfercheckweg, Postfach 124B, Federal Republic of Germany

361. James Purcell, Department of Physics, Georgia State thiversity, At lanta, Georgia 30303

362. Jacobo Rapaport. Department of Physics, Onio University, Athens, Onio 45701-0640

363. John Rasmussen, Lawrence Berkeley Laboratory, Building 70A, Berkeley, California 94720

364. Patrick Richard, Physics Department, Kansas State University, Manhattan, Kansas 66506

365. E. T. Ritter, Division of Nuclear Physics, ER 23, Mail Station J-309, U.S. Department of Energy, Washington, D.C. $205 \div 5$

366. L. Rosen, Los Al amos Mational Laboratory, P. 0. Box 1663, Los Alamos, Mew Mexico 87544

367. Ranier Santo, Sektion Physik der Universitat Munchen, 8046 Garching, Beschleuigerlaboratorium (Forschungsgelande), Federal Republic of Germany

368. D. G. Sarantites, Department of Chemistry, Washingt on University, St. Louis, Missouri 63130

369. У. Sarantseva, Head, Publishing Department, Joint Institute for Huciear Research, Head Post Office, P. 0. Box 79, Moscow, U.S.S.R.

370. J. P. Schiffer, Physics Division, Argonne Hational Laboratory, 9700 South Cass Avenue, Argonne, Illinois 60439

371. A. Schwarzschild, Brookhaven llational Laboratory, Upton, York 11973

372. Hermann Schweickert, Cyclotron Laboratory, Kernforschungszentrum Karlsruhe, Institut fur Applied Physik, P. 0. Box 3640, 0-7500 Karlsruhe 1. Federal Republic of Germany

373. S. Seki, Tandem Accelerator Center, Iniversity of Isukuba, Ibaraki 305, Japan

374. J. C. C. Sharp, Information Of ficer, Daresbury Laberatory, Science Research Council. Daresbury, Harrington WA4 4AD, England

375. R, H. Siemssen, Kernfysisch Versneller Instituit dier Rijksıniversiteit Zernikelaan 25, 9747 AA Froningen, The Ketherlands

375. C. Signorini, INFN Laboratorio Nazionale di Legnaro, Via Romea 4, 35020 Legnaro-Padova, Italy

377. S. J. Skorka, iandem Accelerator Laboratory, University of Munich, Munich, Federal Republic of liermany

378. Hsu Loke Soo, Department of Physics, Nanyang Iniversity, Singapore 22, Republic of Singapore

379. C. Speth, Institut fur Kernphysix, KFA, Julich, Postfach 1913, D-5170 Julich, Federal Republic of Germany

390. T. Springer, Institut Max von Laue - Paul Langevin, $156 \times$ Centre de Tri, 38402 Gorenoble Cedex, France

381. R. G. Stokstad, Lawrence Berkeley Lahoratory, BIdg. 893, Berkeley, California 94720

382. T. T. Sugihara, College of Science, Oregon State University, Corvallis, Oregan 97331

383. Kazusuke Sugiyana, Department of Wuclear Engineering. Faculty of Engineering. Tohoku Intiversity, Sendai, Japan

384. Shiqyed Tanakd, Japan Atomic Enerny Research Institute, Tokai-mura, Ibaraki-ken, Japan

385. J. Teillac, Lahoratoire de Physique Phicleaire d'Orsay, Facultes des Sciences, B. P. No. 1 , Orsay (S. and 0.). France.

386. S. T. Thornton, Dhysics nepartment, "niversity of Virginia, Charlottesville, Virginia 22901

387. T. A. Tombrello, nivision of Physics and Astronomy, California Institute of Technology, 106-38. Pasadena. California 911?.5

398. P. J. Iwin, Dareshury Nuclear Physics Laboratory, Dareshury, Nr. Warrington, Lancashire, England

389. 3. P. Unik, Arqonne Whtional Lahoratory, Bullding 20n, Argonne, Illinois 60439

390. Robert Vandenbosch, Jepartment of Chemistry, Iniversity of Washington, sedttle, Washington 98195

391. A. van der Woude, Kernfysisch Versneller Institut der Rijksuniversiteit, Inniversiteitscomplex Paddepoel, Troningen, The ketherlands

392. H. Verheul, Natuurkundig Lahoratorium der Vrije Iniversiteit. de Boeleladn 1081, Amsterdam, The Hetheriands 
393. VICKSI, Sekretariat, Hahn-Heitner Institut fur Kernforschung Berlin GmbH, Postfach 390128 , D-1000 Berlin 39, Federal Republic of Germany

394. W. von Oertzen, Hahn-Heitner Inst itut fur Kernforschung, Berlin GmbH, Federal Republic of Germany

395. George Vourvopoulos, Department of Physics, Hestern Kentucky Univer sity, Bowl ing Green, Kentucky 42101

396. A. H. Wapstra, Institute Voor Kernphysisch Onderzoek, Ooster Ringdijk 18, Aasterdam, The Netherlands

397. Harlan L. Matson, Deputy Staff Director, Subcomittee on Energy Development and Applications, Committee on Science and Technology, U.S. House of Representatives, B374 Rayburn House Office Building, Washington, D.C. 20515

398. H. E. Wegner, Department of Physics, 901A, Brookhaven Mational Laboratory, Upton, New York 11973

399. D. C. Meisser, Department of Muclear Physics, Institute of Advanced Studies, The Australian Mat ional thiversity, P. 0. Box 4, Canberra ACT 2600, Australia

400. W. G. Heitkamp, Muclear Physics Laboratory, G-10, University of Hashington, Seatt le, Washington 98195

401. Joseph Heneser, Department of Physics, B1dg. 510, Brookhaven Mational Laboratory, Upton, New York 11973

402. E. P. Migner, Department of Physics, Princeton University, Princeton, New Jersey 08540

403. B. H. Wildenthal, Department of Physics, Drexel University. Philadelphia, Pennsylvania 19104

404. H. B. Villard, Program Director for Intermediate E ergy Physics, Hational Science Foundation, $1800 \mathrm{G}$ Street $\mathrm{WH}$, Washington, D.C. 20050

405. H. Yamada, School of Physics, The University of itelbourne, Parkville, Victoria, Australia 3052

406. Takashi Yamazaki, Research Center for Nuclear Physics, Osaka University (Suita Campus), Ibaraki, Osaka, 567 Japan

407. D. Youngblood, Cyclotron Institute, Texas A81 thiversity, College Station, Texas 77940

408. Alexander Xenoul is, Van de Gradff Laboratory, Wuclear Research Center, Demokridos, Aghia Paraskev: Attikis, Athens, Greece

409. E. F. Zganjar, Department of Physics and Astronmy, Loụisiana State University, Baton Rouge, Louisiana 70803

410. K. Ziegler, Hahn-Meitner Institut fur Kernforschung Berlin CmbH, Postfach 3901 28, D-1000 Berlin 39, Federal Republic of Germany

411-506. Given distribution as shown in TID-4500 under Physics catego:, (25 copies - NTIS) 